\title{
Kinetic Resolution via Rh-catalyzed C-C Activation of Cyclobutanones at Room Temperature
}

\author{
Lin Deng ${ }^{\dagger}$, Yue Fu ${ }^{\ddagger}$, Siu Yin Lee ${ }^{\dagger}$, Chengpeng Wang ${ }^{\dagger}$, Peng Liu** and Guangbin Dong*,† \\ ${ }^{\dagger}$ Department of Chemistry, University of Chicago, Chicago, Illinois 60637, United States \\ ${ }^{\ddagger}$ Department of Chemistry, University of Pittsburgh, Pittsburgh, Pennsylvania 15260, United States \\ *Email: gbdong@uchicago.edu \\ *Email: pengliu@pitt.edu
}

\section{Table of contents}

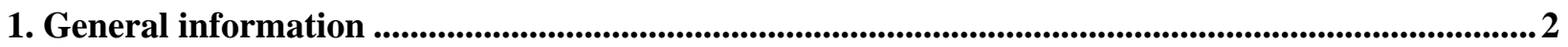

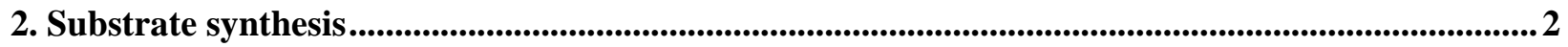

3. Rh-catalyzed kinetic resolutions between cyclobutanones and alkynes.............................................. 11

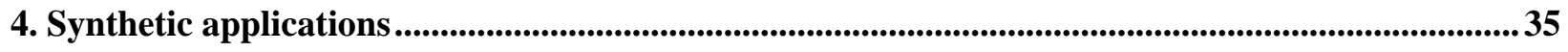

5. Computational Studies .......................................................................................................................................... 40

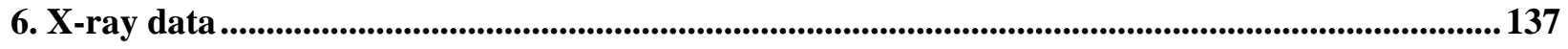

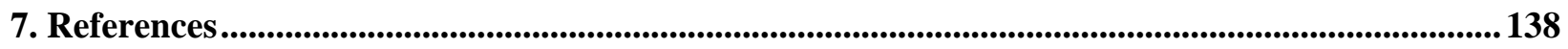

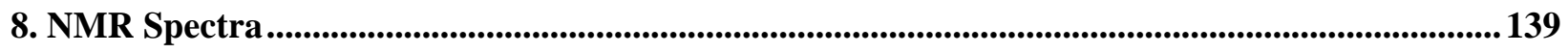




\section{General information}

Unless noted otherwise, all solvents were dried by filtration through a Pure-Solv MD-5 Solvent Purification System (Innovative Technology). The solvents for the $\mathrm{C}-\mathrm{C}$ Activation reactions were distilled freshly over sodium or calcium hydride and carefully freeze-pump-thawed. All the $\mathrm{C}-\mathrm{C}$ Activation reactions were carried out under nitrogen atmosphere with a stir bar in a sealed vial. Reaction temperatures were reported as the temperatures of the bather surrounding the flasks or vials. Sensitive ligands and rhodium catalysts and solvents were transferred under nitrogen into a nitrogen-filled glovebox with standard techniques. Analytical thin-layer chromatography (TLC) was carried out using $0.2 \mathrm{~mm}$ commercial silica gel plates (silica gel 60, F254, EMD chemical). Vials (17 x $60 \mathrm{~mm}$ (7.5mL) with PTFE lined cap attached) were purchased from Qorpak and flame-dried or put in an oven overnight. Highresolution mass spectra (HRSM) were obtained on a Agilent 6224 Tof-MS and are reported as m/z (relative intensity). Accurate masses are reported for the molecular ion $[\mathrm{M}+\mathrm{Na}]^{+},[\mathrm{M}+\mathrm{H}]^{+}$. Infrared spectra were recorded on a Nicolet 380 FTIR using neat thin film technique. Nuclear magnetic resonance spectra $\left({ }^{1} \mathrm{H}\right.$ NMR and ${ }^{13} \mathrm{C}$ NMR) were recorded with a Bruker Avance 400 instrument $\left(400 \mathrm{MHz},{ }^{1} \mathrm{H}\right.$ at $400 \mathrm{MHz},{ }^{13} \mathrm{C}$ at $101 \mathrm{MHz}$ ) and Bruker Avance 500 instrument (500 MHz, ${ }^{19} \mathrm{~F}$ at $470 \mathrm{MHz}$ ). Unless otherwise noted, all spectra were acquired in $\mathrm{CDCl}_{3}$. Chemical shifts are reported in parts per million (ppm, $\delta$ ), downfield from tetramethylsilane (TMS, $\delta=0.00 \mathrm{ppm}$ ) and are referenced to residual solvent $\left(\mathrm{CDCl}_{3}, \delta=7.26 \mathrm{ppm}\left({ }^{1} \mathrm{H}\right)\right.$ and $77.00 \mathrm{ppm}\left({ }^{13} \mathrm{C}\right) ; \mathrm{CD}_{2} \mathrm{Cl}_{2}, \delta=5.32 \mathrm{ppm}\left({ }^{1} \mathrm{H}\right)$ and $\left.53.84 \mathrm{ppm}\left({ }^{13} \mathrm{C}\right)\right)$. Coupling constants were reported in Hertz $(\mathrm{Hz})$. Data for ${ }^{1} \mathrm{H}$ NMR spectra were reported as follows: chemical shift ( $\mathrm{ppm}$, referenced to protium; $\mathrm{s}=$ singlet, $\mathrm{d}=$ doublet, $\mathrm{t}=$ triplet, $\mathrm{q}=$ quartet, hept=heptuplet, $\mathrm{dd}=$ doublet of doublets, $\mathrm{td}=$ triplet of doublets, $\mathrm{ddd}=$ doublet of doublet of doublets, $\mathrm{m}=$ multiplet, coupling constant $(\mathrm{Hz})$, and integration). Analytical HPLC was carried out on an Angilent 1260 infinity HPLC with DAD, Chiralpak IA-IF, served as columns, and mixtures of $n$-hexane and $i$-PrOH were used for elution.

\section{Substrate synthesis}

Substrates 1a, 1b, 1c, 1e, 1f and $\mathbf{1 h}$ were synthesized through Mitsunobu reaction followed by deprotection of the ketal. S1, ${ }^{1} \mathbf{1 a}-\mathbf{I},{ }^{2} \mathbf{1 b}-\mathbf{I},{ }^{3} \mathbf{1 c}-\mathbf{I},{ }^{3} \mathbf{1 d - I},{ }^{2} \mathbf{1 f - I},{ }^{4} \mathbf{1 h - \mathbf { I } ^ { 5 }}$ and $\mathbf{1 e ^ { 6 }}$ are literature known compounds, and their characterization data matches the reported data. 


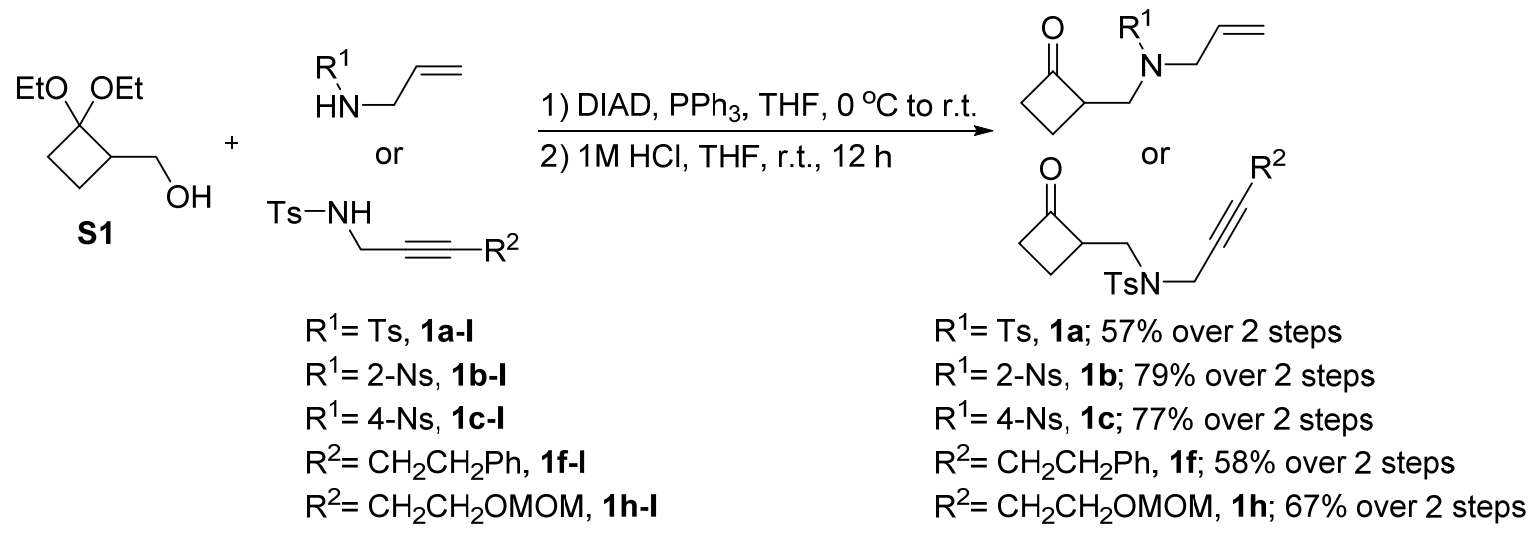

The procedure for the Mitsunobu reaction and deprotection sequence is as follows (using substrate 1a as an example): A solution of S1 (574.9 mg, $3.3 \mathrm{mmol}, 1.1$ equiv.) and DIAD (727.9 mg, $3.6 \mathrm{mmol}, 1.2$ equiv.) in THF $(5 \mathrm{~mL})$ was added to a solution of 1a-I (633.9 mg, $3 \mathrm{mmol}, 1.0$ equiv.) and triphenylphosphine (943.6 mg, $3.6 \mathrm{mmol}, 1.2$ equiv.) in THF $(5 \mathrm{~mL})$ at $0{ }^{\circ} \mathrm{C}$. The mixture was warmed to room temperature and stirred overnight. The mixture was concentrated, and the residue was dissolved in THF $(6 \mathrm{~mL}) .1 \mathrm{M} \mathrm{HCl}(2 \mathrm{~mL})$ was added, and the resulting mixture was stirred at room temperature overnight. The mixture was diluted with ethyl acetate and washed by water, $\mathrm{NaHCO}_{3}$ and brine. The organic layer was dried and concentrated, and the residue was purified by column chromatography to give 1a as a colorless oil in 57\% yield (506 mg) over 2 steps.

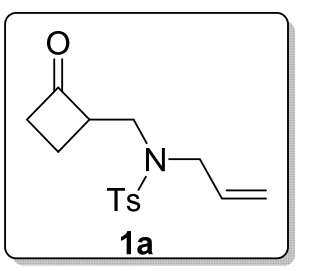

Compound 1a was isolated as a colorless oil in 57\% yield over 2 steps. $\mathrm{R}_{f}=0.4($ EtOAc/Hexane $=1 / 3) .{ }^{1} \mathbf{H}$ NMR (400 MHz, $\mathbf{C D C l}_{3}$ ): $\delta 7.75$ - $7.62(\mathrm{~m}, 2 \mathrm{H}), 7.34-7.28(\mathrm{~m}, 2 \mathrm{H}), 5.57$ (ddt, $J=17.2,10.1,6.4 \mathrm{~Hz}$, $1 \mathrm{H}), 5.28-5.05(\mathrm{~m}, 2 \mathrm{H}), 3.91-3.73(\mathrm{~m}, 2 \mathrm{H}), 3.67-3.52(\mathrm{~m}, 1 \mathrm{H}), 3.43(\mathrm{dd}, J=14.5,8.6 \mathrm{~Hz}, 1 \mathrm{H}), 3.25$ (dd, $J=14.5,5.8 \mathrm{~Hz}, 1 \mathrm{H}$ ), 3.06 (dddd, $J=17.8,10.6,8.2,2.5 \mathrm{~Hz}, 1 \mathrm{H}), 2.93$ (dddd, $J=17.7,9.8,5.1,2.6$ $\mathrm{Hz}, 1 \mathrm{H}), 2.43$ (s, 3H), 2.21 (dtd, $J=11.5,10.3,5.1 \mathrm{~Hz}, 1 \mathrm{H}), 2.01-1.83$ (m, 1H). ${ }^{13} \mathbf{C}$ NMR (101 MHz, $\mathbf{C D C l}_{3}$ ): 208.76, 143.49, 136.56, 132.77, 129.77, 127.16, 119.32, 59.52, 51.22, 45.92, 44.75, 21.52, 15.72. IR: v 2925, 1778, 1598, 1447, 1343, 1160, 1091, $934 \mathrm{~cm}^{-1}$; HRMS calcd. For [M+H] $]^{+}: 294.1158$ Found: 294.1163.

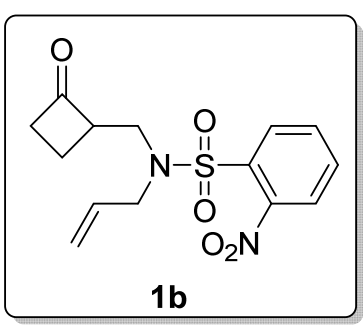


Compound $\mathbf{1 b}$ was isolated as a colorless oil in $79 \%$ yield over 2 steps. $\mathrm{R}_{f}=0.3($ EtOAc/Hexane $=1 / 2) .{ }^{1} \mathbf{H}$ NMR (400 MHz, $\left.\mathbf{C D C l}_{3}\right): \delta 8.08-8.01(\mathrm{~m}, 1 \mathrm{H}), 7.76-7.62(\mathrm{~m}, 3 \mathrm{H}), 5.64$ (ddt, $J=17.2,10.1,6.3 \mathrm{~Hz}$, $1 \mathrm{H}), 5.39-5.01(\mathrm{~m}, 2 \mathrm{H}), 4.08-3.91(\mathrm{~m}, 2 \mathrm{H}), 3.71-3.42(\mathrm{~m}, 3 \mathrm{H}), 3.07$ (dddd, $J=17.7,10.4,8.2,2.2$ $\mathrm{Hz}, 1 \mathrm{H}), 2.94$ (dddd, $J=17.8,9.8,5.1,2.4 \mathrm{~Hz}, 1 \mathrm{H}), 2.22$ (dddd, $J=11.5,10.5,9.7,5.1 \mathrm{~Hz}, 1 \mathrm{H}), 1.97$ 1.77 (m, 1H). ${ }^{13} \mathbf{C}$ NMR (101 MHz, $\left.\mathbf{C D C l}_{3}\right): \delta$ 208.22, 147.91, 133.63, 133.41, 132.17, 131.76, 131.03, 124.26, 119.77, 59.25, 50.40, 45.59, 44.82, 15.68. IR: v 2926, 1778, 1545, 1373, 1163, 1084, $775 \mathrm{~cm}^{-1}$; HRMS calcd. For $[\mathrm{M}+\mathrm{Na}]^{+}: 347.0672$ Found: 347.0684.

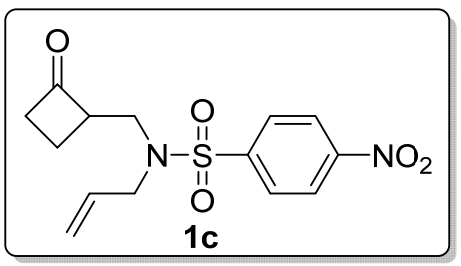

Compound 1c was isolated as a white solid in $77 \%$ yield over 2 steps. Melting Point: $85-87{ }^{\circ} \mathrm{C}$. $\mathrm{R}_{f}=0.3$ (EtOAc/Hexane=1/2). ${ }^{1} \mathbf{H}$ NMR (400 $\left.\mathbf{M H z}, \mathbf{C D C l}_{3}\right): \delta 8.40-8.30(\mathrm{~m}, 2 \mathrm{H}), 8.07-7.92(\mathrm{~m}, 2 \mathrm{H}), 5.55$ (ddt, $J=16.6,10.1,6.3 \mathrm{~Hz}, 1 \mathrm{H}), 5.32-5.07(\mathrm{~m}, 2 \mathrm{H}), 4.00-3.84(\mathrm{~m}, 2 \mathrm{H}), 3.67-3.54(\mathrm{~m}, 1 \mathrm{H}), 3.49$ (dd, $J=14.5,7.9 \mathrm{~Hz}, 1 \mathrm{H}), 3.34$ (dd, $J=14.5,6.2 \mathrm{~Hz}, 1 \mathrm{H}), 3.10$ (dddd, $J=17.9,10.6,8.3,2.4 \mathrm{~Hz}, 1 \mathrm{H}$ ), 2.96 (dddd, $J=17.8,9.7,5.0,2.6 \mathrm{~Hz}, 1 \mathrm{H}), 2.34-2.18(\mathrm{~m}, 1 \mathrm{H}), 2.05-1.74(\mathrm{~m}, 1 \mathrm{H}) .{ }^{13} \mathbf{C}$ NMR (101 MHz, $\left.\mathbf{C D C l}_{3}\right): \delta 207.94,150.03,145.67,131.73,128.34,124.45,120.15,59.21,51.04,45.89,44.88,15.66$. IR: v 2925, 1778, 1530, 1351, 1163, 1088, $772 \mathrm{~cm}^{-1}$; HRMS calcd. For [M+Na] $]^{+}: 347.0672$ Found: 347.0677.

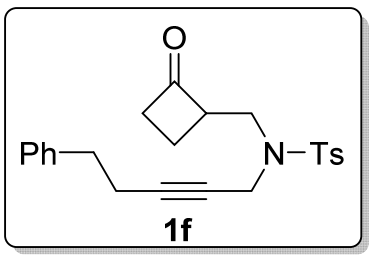

Compound 1f was isolated as a light yellow solid in 58\% yield over 2 steps. Melting Point: $98-100{ }^{\circ} \mathrm{C}$. $\mathrm{R}_{f}$ $=0.4\left(\right.$ EtOAc/Hexane=1/3). ${ }^{1} \mathbf{H}$ NMR (400 $\left.\mathbf{~ M H z , ~} \mathbf{C D C l}_{3}\right): \delta 7.72-7.65(\mathrm{~m}, 2 \mathrm{H}), 7.30-7.23(\mathrm{~m}, 4 \mathrm{H})$, $7.23-7.17(\mathrm{~m}, 1 \mathrm{H}), 7.11-7.02(\mathrm{~m}, 2 \mathrm{H}), 4.15(\mathrm{dtd}, J=18.4,2.2,0.8 \mathrm{~Hz}, 1 \mathrm{H}), 4.04(\mathrm{dt}, J=18.4,2.2 \mathrm{~Hz}$, 1H), $3.61-3.48$ (m, 1H), $3.44-3.24$ (m, 2H), 3.07 (dddd, $J=17.8,10.6,8.2,2.5 \mathrm{~Hz}, 1 \mathrm{H}), 2.94$ (dddd, $J$ $=17.7,9.8,5.1,2.7 \mathrm{~Hz}, 1 \mathrm{H}), 2.54(\mathrm{t}, J=7.4 \mathrm{~Hz}, 2 \mathrm{H}), 2.40(\mathrm{~s}, 3 \mathrm{H}), 2.28-2.11(\mathrm{~m}, 3 \mathrm{H}), 1.90$ (ddt, $J=$ 11.4, 9.7, 7.9 Hz, 1H). ${ }^{13} \mathbf{C}$ NMR (101 MHz, $\left.\mathbf{C D C l}_{3}\right): \delta 208.48,143.44,140.22,135.73,129.36,128.37$, 128.22, 127.83, 126.36, 85.66, 73.11, 59.02, 45.01, 37.79, 34.49, 21.52, 20.47, 15.47. IR: v 2925, 1778, 1598, 1454, 1348, 1162, 1090, $750 \mathrm{~cm}^{-1}$; HRMS calcd. For [M+Na]+: 418.1447 Found: 418.1451.

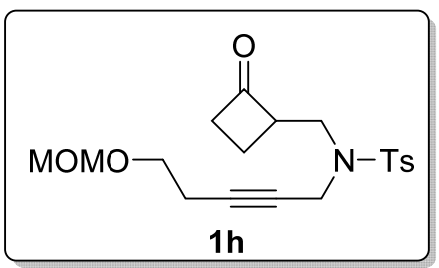


Compound $\mathbf{1 h}$ was isolated as a colorless oil in $67 \%$ yield over 2 steps. $\mathrm{R}_{f}=0.4($ EtOAc/Hexane $=1 / 3) .{ }^{1} \mathbf{H}$ NMR (400 MHz, $\mathbf{C D C l}_{3}$ ): $\delta 7.80-7.66(\mathrm{~m}, 2 \mathrm{H}), 7.32-7.27$ (m, 2H), 4.57 (s, 2H), 4.19 (dtd, J = 18.4, 2.2, $0.9 \mathrm{~Hz}, 1 \mathrm{H}), 4.09-4.00(\mathrm{~m}, 1 \mathrm{H}), 3.68-3.54(\mathrm{~m}, 1 \mathrm{H}), 3.44$ (ddd, $J=14.0,8.7,0.9 \mathrm{~Hz}, 1 \mathrm{H}), 3.39-$ $3.31(\mathrm{~m}, 6 \mathrm{H}), 3.10$ (dddd, $J=17.8,10.6,8.3,2.5 \mathrm{~Hz}, 1 \mathrm{H}), 2.98$ (dddd, $J=17.7,9.8,5.2,2.6 \mathrm{~Hz}, 1 \mathrm{H}), 2.43$ (s, 3H), $2.32-2.17(\mathrm{~m}, 3 \mathrm{H}), 1.99$ (ddt, $J=11.5,9.7,7.9 \mathrm{~Hz}, 1 \mathrm{H}) \cdot{ }^{13} \mathbf{C}$ NMR (101 MHz, $\left.\mathbf{C D C l}_{3}\right): \delta$ 208.56, 143.54, 135.69, 129.40, 127.83, 96.33, 83.08, 73.54, 65.58, 59.00, 55.27, 45.11, 45.00, 37.79, 21.49, 19.93, 15.47. IR: v 2925, 1778, 1598, 1443, 1348, 1162, 1028, $918 \mathrm{~cm}^{-1}$; HRMS calcd. For $[\mathrm{M}+\mathrm{H}]^{+}: 380.1526$ Found: 380.1539 .

Substrate 1d was synthesized from $\mathbf{1 b}-\mathbf{I I}$ (prepared from $\mathbf{S 1}{ }^{1}$ and $\mathbf{1 b}-\mathbf{I}^{3}$ ) through deprotection of 2-Ns followed by Ms protection.

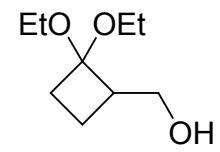

S1

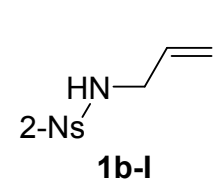

1b-I

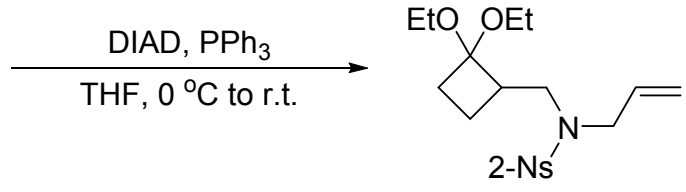

$1 \mathrm{~b}-\mathrm{II}, 66 \%$

Synthesis of 1b-II: A solution of S1 (191.4 mg, $1.1 \mathrm{mmol}, 1.1$ equiv.) and DIAD (242.4 mg, $1.2 \mathrm{mmol}$, 1.2 equiv.) in THF ( $2 \mathrm{~mL})$ was added to a solution of 1b-I $(242.2 \mathrm{mg}, 1 \mathrm{mmol}, 1.0$ equiv.) and triphenylphosphine (314.5 mg, $1.2 \mathrm{mmol}, 1.2$ equiv.) in THF $(2 \mathrm{~mL})$ at $0{ }^{\circ} \mathrm{C}$. The mixture was warmed to room temperature and stirred overnight. The mixture was concentrated and the residue was purified by column chromatography to give $\mathbf{1 b}-\mathbf{I I}$ as a colorless oil in $66 \%$ yield $(263 \mathrm{mg}) . \mathrm{R}_{f}=0.4$ (EtOAc/Hexane=1/2). ${ }^{1} \mathbf{H}$ NMR (400 $\left.\mathbf{~ M H z , ~} \mathbf{C D C l}_{3}\right): \delta 8.08-8.00(\mathrm{~m}, 1 \mathrm{H}), 7.74-7.56(\mathrm{~m}, 3 \mathrm{H}), 5.66$ (dddd, $J=17.1,10.1,6.9,5.7 \mathrm{~Hz}, 1 \mathrm{H}), 5.39-5.03(\mathrm{~m}, 2 \mathrm{H}), 4.04$ (ddq, $J=16.0,5.5,1.2 \mathrm{~Hz}, 1 \mathrm{H}), 3.92$ (ddt, $J=16.0,6.9,1.3 \mathrm{~Hz}, 1 \mathrm{H}$ ), 3.54 (ddd, $J=14.8,9.2,0.9 \mathrm{~Hz}, 1 \mathrm{H}$ ), $3.45-3.23$ (m, 5H), 2.65 (ddddd, $J$ $=10.2,9.0,7.8,5.1,1.2 \mathrm{~Hz}, 1 \mathrm{H}), 2.14$ (dddd, $J=12.1,9.9,4.3,1.0 \mathrm{~Hz}, 1 \mathrm{H}), 1.93$ (dddd, $J=12.2,9.5,8.1$, $1.3 \mathrm{~Hz}, 1 \mathrm{H}), 1.80(\mathrm{dtd}, J=11.2,9.2,4.3 \mathrm{~Hz}, 1 \mathrm{H}), 1.35(\mathrm{ddt}, J=11.2,10.0,8.0 \mathrm{~Hz}, 1 \mathrm{H}), 1.17(\mathrm{t}, J=7.1$ $\mathrm{Hz}, 3 \mathrm{H}), 1.15$ (t, $J=7.1 \mathrm{~Hz}, 3 \mathrm{H}) .{ }^{13} \mathbf{C}$ NMR (101 MHz, $\mathbf{C D C l}_{3}$ ): $\delta 148.03,133.88,133.32,132.86$, $131.48,130.88,124.09,119.00,102.09,56.62,56.32,50.35,47.02,43.51,29.35,17.28,15.31$, 15.22. IR: v 2976, 1546, 1353, 1162, 1056, $771 \mathrm{~cm}^{-1}$; HRMS calcd. For $[\mathrm{M}+\mathrm{Na}]^{+}: 421.1404$ Found: 421.1409.
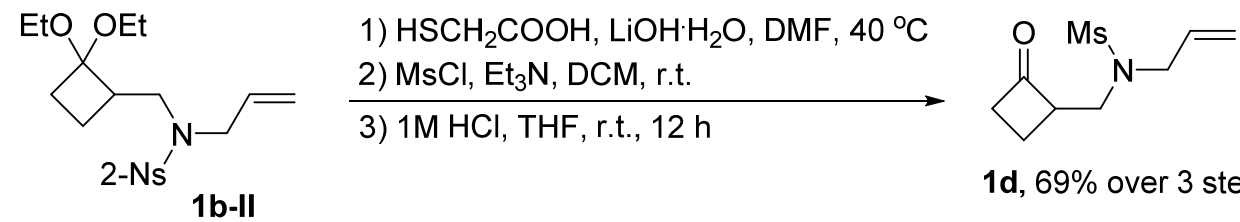

1d, $69 \%$ over 3 steps

Synthesis of 1d: 1b-II (199.3 mg, 0.5 mmol, 1.0 equiv.) was dissolved in DMF (8 mL). $\mathrm{LiOH}_{2} \mathrm{O}(230.8$ $\mathrm{mg}, 5.5 \mathrm{mmol}, 11$ equiv.) and $\mathrm{HSCH}_{2} \mathrm{COOH}$ (276.4 mg, $3 \mathrm{mmol}, 6$ equiv.) were added to the stirring solution, and the mixture was stirred at $40{ }^{\circ} \mathrm{C}$ overnight. The mixture was diluted with ethyl acetate and washed with $\mathrm{NaHCO}_{3}$. The organic phase was dried and concentrated, and the residue was dissolved in $\operatorname{DCM}(5 \mathrm{~mL})$. $\mathrm{Et}_{3} \mathrm{~N}$ (101.2 mg, $1 \mathrm{mmol}, 2.0$ equiv.) and $\mathrm{MsCl}$ (63.0 mg, $0.55 \mathrm{mmol}, 1.1$ equiv.) were 
added to the stirring solution, and the mixture was stirred at room temperature overnight. The mixture was washed with $1 \mathrm{M} \mathrm{HCl}$ twice and $\mathrm{NaHCO}_{3}$. The organic layer was dried and concentrated, and the residue was dissolved in THF $(1.5 \mathrm{~mL}) .1 \mathrm{M} \mathrm{HCl}(0.5 \mathrm{~mL})$ was added to the solution, and the mixture was stirred at room temperature overnight. The reaction mixture was diluted with ethyl acetate and washed with water, $\mathrm{NaHCO}_{3}$ and brine. The organic layer was dried and concentrated, and the residue was purified by column chromatography to give $\mathbf{1 d}$ as a colorless oil in $69 \%$ yield $\left(75 \mathrm{mg}\right.$ ) over 3 steps. $\mathrm{R}_{f}=0.4$ (EtOAc/Hexane=1/3). ${ }^{\mathbf{1}} \mathbf{H}$ NMR (400 MHz, $\mathbf{C D C l}_{3}$ ): $\delta 5.82$ (ddt, $\left.J=17.2,10.1,6.4 \mathrm{~Hz}, 1 \mathrm{H}\right), 5.35-5.26$ $(\mathrm{m}, 2 \mathrm{H}), 3.89(\mathrm{dt}, J=6.5,1.4 \mathrm{~Hz}, 2 \mathrm{H}), 3.68-3.55(\mathrm{~m}, 1 \mathrm{H}), 3.47(\mathrm{dd}, J=14.7,7.5 \mathrm{~Hz}, 1 \mathrm{H}), 3.40(\mathrm{dd}, J=$ 14.6, $6.9 \mathrm{~Hz}, 1 \mathrm{H}$ ), 3.09 (dddd, $J=18.0,10.6,8.1,2.5 \mathrm{~Hz}, 1 \mathrm{H}), 3.02-2.91$ (m, 1H), 2.89 (s, 3H), 2.24 $(\mathrm{dtd}, J=11.5,10.3,5.2 \mathrm{~Hz}, 1 \mathrm{H}), 1.90$ (ddt, $J=11.5,9.8,7.9 \mathrm{~Hz}, 1 \mathrm{H}) \cdot{ }^{13} \mathbf{C}$ NMR (101 MHz, $\left.\mathbf{C D C l}_{3}\right): \delta$ 208.75, 132.38, 119.73, 59.24, 50.47, 45.57, 44.81, 39.25, 15.40. IR: v 2925, 1774, 1325, 1147, 1084, $787 \mathrm{~cm}^{-1}$; HRMS calcd. For [M+Na] $]^{+} 240.0665$ Found: 240.0665.

Substrate $\mathbf{1 g}$ was synthesized from $\mathbf{S} \mathbf{1}^{1}$ and $\mathbf{1 h}-\mathbf{I}^{5}$ using stronger deprotection conditions.

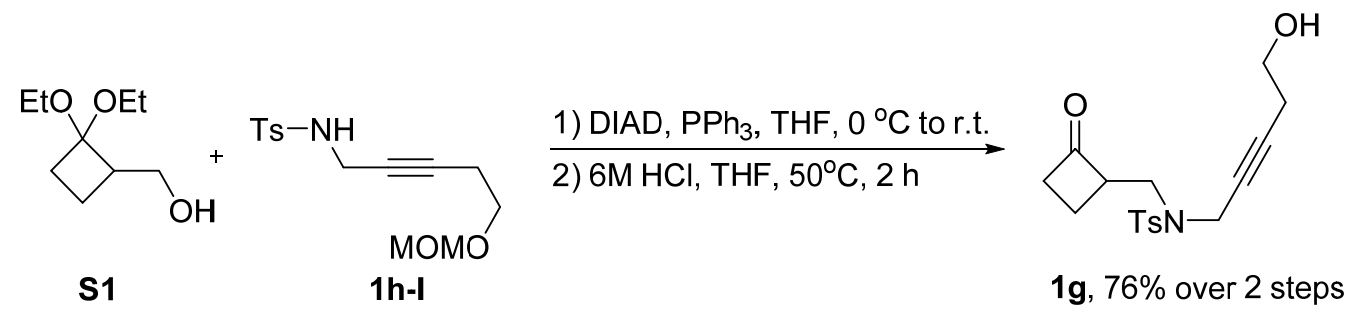

Synthesis of 1g: A solution of S1 (514.5 mg, $2.95 \mathrm{mmol}, 1.2$ equiv.) and DIAD (552.5 mg, $2.73 \mathrm{mmol}$, 1.1 equiv.) in THF $(5 \mathrm{~mL})$ was added to a solution of $\mathbf{1 h}-\mathbf{I}(735.0 \mathrm{mg}, 2.47 \mathrm{mmol}, 1.0$ equiv.) and triphenylphosphine $\left(715.7 \mathrm{mg}, 2.73 \mathrm{mmol}, 1.1\right.$ equiv.) in THF $(5 \mathrm{~mL})$ at $0{ }^{\circ} \mathrm{C}$. The mixture was warmed to room temperature and stirred overnight. The mixture was concentrated, and the residue was dissolved in THF $(6 \mathrm{~mL}) .6 \mathrm{M} \mathrm{HCl}(2 \mathrm{~mL})$ was added, and the resulting mixture was stirred at $50{ }^{\circ} \mathrm{C}$ for $2 \mathrm{~h}$. The reaction mixture was diluted with ethyl acetate and washed by water, $\mathrm{NaHCO}_{3}$ and brine. The organic layer was dried and concentrated, and the residue was purified by column chromatography to give $\mathbf{1 g}$ as a colorless oil in $76 \%$ yield $(627 \mathrm{mg})$ over 2 steps. $\mathrm{R}_{f}=0.4($ EtOAc/Hexane $=2 / 1) .{ }^{1} \mathbf{H}$ NMR (400 MHz, $\left.\mathbf{C D C l}_{3}\right): \delta 7.76-7.67(\mathrm{~m}, 2 \mathrm{H}), 7.36-7.28(\mathrm{~m}, 2 \mathrm{H}), 4.18(\mathrm{dtd}, J=18.4,2.2,0.9 \mathrm{~Hz}, 1 \mathrm{H}), 4.13-3.99(\mathrm{~m}$, $1 \mathrm{H}), 3.70-3.55(\mathrm{~m}, 1 \mathrm{H}), 3.53-3.43(\mathrm{~m}, 3 \mathrm{H}), 3.37$ (dd, $J=14.0,5.9 \mathrm{~Hz}, 1 \mathrm{H}), 3.11$ (dddd, $J=17.9,10.6$, 8.2, $2.5 \mathrm{~Hz}, 1 \mathrm{H}$ ), 2.98 (dddd, $J=17.8,9.8,5.2,2.6 \mathrm{~Hz}, 1 \mathrm{H}), 2.43$ (s, 3H), $2.32-2.15$ (m, 3H), 1.99 (ddt, $J$ $=11.5,9.8,8.0 \mathrm{~Hz}, 1 \mathrm{H}), 1.65$ (br, 1H). ${ }^{13} \mathbf{C}$ NMR (101 $\left.\mathbf{M H z}, \mathbf{C D C l}_{3}\right): \delta 208.89,143.77,135.67,129.46$, $127.75,83.24,74.44,60.68,58.99,45.10,45.00,37.76,22.89,21.51,15.36$. IR: v 3531, 2925, 1777, $1598,1346,1161,1090,750 \mathrm{~cm}^{-1}$; HRMS calcd. For $[\mathrm{M}+\mathrm{H}]^{+}: 336.1264$ Found: 336.1271.

Substrate 1i was synthesized from $\mathbf{1 g}$ through a three-step procedure. 


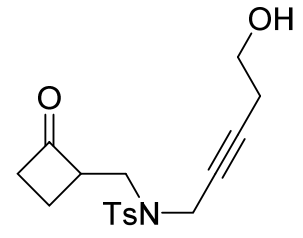

19

1) $(\mathrm{EtO})_{3} \mathrm{CH}, \mathrm{p}-\mathrm{TsOH}, \mathrm{EtOH}$, r.t.

2) $\mathrm{BnBr}, \mathrm{NaH}, \mathrm{DMF}$, r.t.

3) $1 \mathrm{M} \mathrm{HCl}$, THF, r.t., $12 \mathrm{~h}$

Synthesis of 1i: 1 g (185.0 mg, $0.55 \mathrm{mmol}, 1.0$ equiv.) was dissolved in EtOH (5 mL). (EtO) ${ }_{3} \mathrm{CH}(274 \mu \mathrm{L}$, $1.65 \mathrm{mmol}, 3.0$ equiv.) and $\mathrm{TsOH}(18.9 \mathrm{mg}, 0.11 \mathrm{mmol}, 0.2$ equiv.) were added to the stirring solution, and the mixture was stirred at room temperature overnight. The mixture was quenched by saturated $\mathrm{NaHCO}_{3}$ solution $(20 \mathrm{~mL})$ and extracted with ethyl acetate. The combined organic layers were washed with brine, dried and concentrated. The residue was dissolved in DMF (5 mL), and NaH (44 mg, 1.1 mmol, 2.0 equiv.) was added to the stirring solution. After $10 \mathrm{~min}, \mathrm{BnBr}(67.1 \mu \mathrm{L}, 0.565 \mathrm{mmol}, 1.02$ equiv.) was added and the mixture was stirred at room temperature overnight. The mixture was quenched by water and extracted with ethyl acetate. The combined organic layers were washed with brine, dried and concentrated, and the residue was dissolved in THF $(6 \mathrm{~mL}) .1 \mathrm{M} \mathrm{HCl}(2 \mathrm{~mL})$ was added, and the resulting mixture was stirred at room temperature overnight. The mixture was diluted with ethyl acetate and washed by water, $\mathrm{NaHCO}_{3}$ and brine. The organic layer was dried and concentrated, and the residue was purified by column chromatography to give $1 \mathbf{i}$ as a colorless oil in $66 \%$ yield (154 $\mathrm{mg}$ ) over 3 steps. $\mathrm{R}_{f}=$ 0.4 (EtOAc/Hexane=1/3). ${ }^{1} \mathbf{H}$ NMR (400 MHz, $\left.\mathbf{C D C l}_{3}\right): \delta 7.78-7.66(\mathrm{~m}, 2 \mathrm{H}), 7.40-7.33(\mathrm{~m}, 2 \mathrm{H}), 7.33$ - $7.28(\mathrm{~m}, 3 \mathrm{H}), 7.25-7.22(\mathrm{~m}, 2 \mathrm{H}), 4.46(\mathrm{~s}, 2 \mathrm{H}), 4.18(\mathrm{dtd}, J=18.3,2.3,0.8 \mathrm{~Hz}, 1 \mathrm{H}), 4.11$ - $3.98(\mathrm{~m}$, 1H), $3.72-3.52$ (m, 1H), 3.42 (ddd, $J=14.1,8.6,0.8 \mathrm{~Hz}, 1 \mathrm{H}), 3.38-3.27$ (m, 3H), 3.07 (dddd, $J=17.8$, 10.6, 8.2, $2.5 \mathrm{~Hz}, 1 \mathrm{H}$ ), 2.95 (dddd, $J=17.8,9.7,5.2,2.6 \mathrm{~Hz}, 1 \mathrm{H}), 2.39$ (s, 3H), $2.29-2.14$ (m, 3H), 2.05 - $1.86(\mathrm{~m}, 1 \mathrm{H}) .{ }^{13} \mathbf{C}$ NMR (101 $\left.\mathbf{M H z}, \mathbf{C D C l}_{3}\right): \delta 208.58,143.45,137.86,135.68,129.37,128.45,127.82$, $127.79,127.64,83.16,73.51,72.94,67.99,59.02,45.15,44.99,37.84,21.51,19.86,15.48$. IR: v 2923, 1777, 1597, 1453, 1348, 1161, 1092, $746 \mathrm{~cm}^{-1}$; HRMS calcd. For [M+H] ${ }^{+}: 426.1734$ Found: 426.1744.

Substrate $\mathbf{1} \mathbf{j}$ was synthesized from $\mathbf{1 g}$ through a three-step procedure.

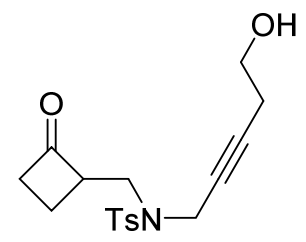

$1 \mathrm{~g}$
1) $(\mathrm{EtO})_{3} \mathrm{CH}, \mathrm{p}-\mathrm{TsOH}, \mathrm{EtOH}$, r.t.

2) $\mathrm{CH}_{3} \mathrm{COCl}$, pyridine, DCM, r.t.

3) $1 \mathrm{M} \mathrm{HCl}, \mathrm{THF}$, r.t., $12 \mathrm{~h}$

$1 \mathrm{j}, 62 \%$ over 3 steps

Synthesis of 1j: 1g (143.0 mg, $0.43 \mathrm{mmol}, 1.0$ equiv.) was dissolved in $\mathrm{EtOH}(5 \mathrm{~mL})$. (EtO) ${ }_{3} \mathrm{CH}(213 \mu \mathrm{L}$, $1.28 \mathrm{mmol}, 3.0$ equiv.) and $\mathrm{TsOH}(14.8 \mathrm{mg}, 0.086 \mathrm{mmol}, 0.2$ equiv.) were added to the stirring solution, and the mixture was stirred at room temperature overnight. The mixture was quenched by saturated $\mathrm{NaHCO}_{3}$ solution $(20 \mathrm{~mL})$ and extracted with ethyl acetate. The combined organic layers were washed with brine, dried and concentrated. The residue was dissolved in DCM $(5 \mathrm{~mL})$, and pyridine $(104 \mu \mathrm{L}$, 1.29 mmol, 3.0 equiv.) was added to the stirring solution. After $10 \mathrm{~min}$, acetyl chloride $(61 \mu \mathrm{L}, 0.86$ mmol, 2.0 equiv.) was added, and the mixture was stirred at room temperature overnight. The mixture 
was quenched with water and extracted with ethyl acetate. The combined organic layers were washed with brine, dried and concentrated, and the residue was dissolved in THF (6 mL). $1 \mathrm{M} \mathrm{HCl}(2 \mathrm{~mL})$ was added, and the resulting mixture was stirred at room temperature overnight. The mixture was diluted with ethyl acetate and washed by water, $\mathrm{NaHCO}_{3}$ and brine. The organic layer was dried and concentrated, and the residue was purified by column chromatography to give $\mathbf{1} \mathbf{j}$ as a colorless oil in $62 \%$ yield (100 $\mathrm{mg}$ ) over 3 steps. $\mathrm{R}_{f}=0.3$ (EtOAc/Hexane=1/3). ${ }^{\mathbf{1}} \mathbf{H}$ NMR (400 $\left.\mathbf{M H z}, \mathbf{C D C l}_{3}\right): \delta 7.86-7.59$ (m, 2H), $7.35-$ $7.28(\mathrm{~m}, 2 \mathrm{H}), 4.20(\mathrm{dtd}, J=18.4,2.2,0.9 \mathrm{~Hz}, 1 \mathrm{H}), 4.06(\mathrm{dtd}, J=18.5,2.2,0.6 \mathrm{~Hz}, 1 \mathrm{H}), 3.93-3.79(\mathrm{~m}$, $2 \mathrm{H}), 3.72-3.55(\mathrm{~m}, 1 \mathrm{H}), 3.44(\mathrm{ddd}, J=14.0,8.5,0.9 \mathrm{~Hz}, 1 \mathrm{H}), 3.34(\mathrm{ddd}, J=14.0,6.0,0.6 \mathrm{~Hz}, 1 \mathrm{H}), 3.11$ (dddd, $J=17.9,10.6,8.2,2.5 \mathrm{~Hz}, 1 \mathrm{H}), 2.98$ (dddd, $J=17.7,9.8,5.2,2.6 \mathrm{~Hz}, 1 \mathrm{H}$ ), 2.43 (s, 3H), $2.34-$ 2.17 (m, 3H), 2.03 (s, 3H), $2.02-1.92(\mathrm{~m}, 1 \mathrm{H}) .{ }^{13} \mathbf{C}$ NMR (101 MHz, $\mathbf{C D C l}_{3}$ ): $\delta 208.52,170.59,143.71$, $135.65,129.43,127.78,81.91,74.12,61.83,58.99,45.08,45.01,37.70,21.49,20.79,18.88,15.43$. IR: $\mathrm{v}$ 2925, 1778, 1739, 1597, 1348, 1240, 1162, $816 \mathrm{~cm}^{-1}$; HRMS calcd. For [M+Na] : 400.1189 Found: 400.1186.

Substrate $\mathbf{1 k}$ was synthesized from $\mathbf{1 g}$ through TBS protection.

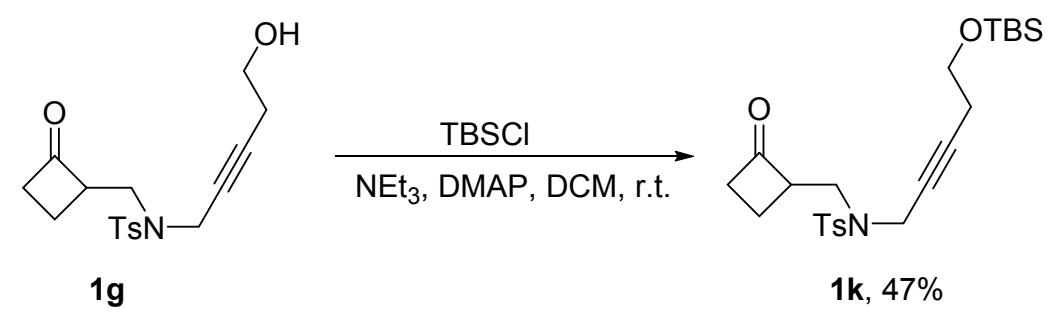

Synthesis of 1k: A flame-dried Schlenk flask was charged with 1 g (167.7 mg, 0.5 mmol, 1.0 equiv.), DMAP (6.1 mg, $0.05 \mathrm{mmol}, 0.1$ equiv.) and DCM $(5 \mathrm{~mL})$, and the mixture was cooled to $0{ }^{\circ} \mathrm{C} . \mathrm{NEt}_{3}(210$ $\mu \mathrm{L}, 1.5 \mathrm{mmol}, 3$ equiv) was added to the stirring solution. TBSCl (113.0 $\mathrm{mg}, 0.75 \mathrm{mmol}, 1.5$ equiv.) was dissolved in DCM ( $3 \mathrm{~mL})$, and the solution and added to the reaction mixture. The reaction was warmed to room temperature and stirred overnight. The mixture was quenched by saturated $\mathrm{NH}_{4} \mathrm{Cl}$ solution (20 $\mathrm{mL}$ ) and extracted with ethyl acetate. The combined organic layers were washed with brine, dried and concentrated, and the residue was purified by column chromatography to give $\mathbf{1 k}$ as a colorless oil in $47 \%$ yield (106 mg). $\mathbf{R}_{f}=0.4$ (EtOAc/Hexane=1/4). ${ }^{1} \mathbf{H}$ NMR (400 $\left.\mathbf{M H z}, \mathbf{C D C l}_{3}\right): \delta 7.82-7.63(\mathrm{~m}, 2 \mathrm{H}), 7.32$ $-7.27(\mathrm{~m}, 2 \mathrm{H}), 4.32-4.14(\mathrm{~m}, 1 \mathrm{H}), 4.05(\mathrm{dt}, J=18.3,2.2 \mathrm{~Hz}, 1 \mathrm{H}), 3.69-3.56(\mathrm{~m}, 1 \mathrm{H}), 3.51-3.40(\mathrm{~m}$, $3 \mathrm{H}$ ), 3.34 (dd, $J=14.1,6.0 \mathrm{~Hz}, 1 \mathrm{H}$ ), 3.10 (dddd, $J=17.8,10.6,8.2,2.5 \mathrm{~Hz}, 1 \mathrm{H}$ ), 2.98 (dddd, $J=17.8,9.7$, 5.2, $2.6 \mathrm{~Hz}, 1 \mathrm{H}), 2.42(\mathrm{~s}, 3 \mathrm{H}), 2.24(\mathrm{dtd}, J=11.5,10.3,5.2 \mathrm{~Hz}, 1 \mathrm{H}), 2.11(\mathrm{tt}, J=7.3,2.2 \mathrm{~Hz}, 2 \mathrm{H}), 1.98$ (ddt, $J=11.5,9.8,8.0 \mathrm{~Hz}, 1 \mathrm{H}), 0.87$ (s, 9H), 0.04 (s, 6H). ${ }^{\mathbf{1 3}} \mathbf{C}$ NMR (101 MHz, $\mathbf{C D C l}_{3}$ ): $\delta$ 208.50, $143.46,135.72,129.35,127.82,83.31,73.49,61.45,59.05,45.09,44.99,37.81,25.81,22.84,21.52$, 18.23, 15.48, -5.33. IR: v 2929, 1780, 1598, 1350, 1163, 1095, $838 \mathrm{~cm}^{-1}$; HRMS calcd. For [M+Na] $]^{+}$ 472.1948 Found: 472.1956.

Substrate 11 was synthesized from $\mathbf{S 2}^{6}, \mathbf{S 3}^{7}$ and $\mathbf{S 6}$ (commercially available) through a multi-step sequence. 


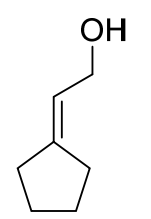

S2

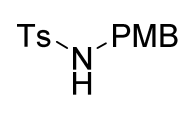

S3

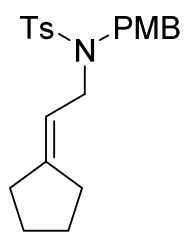

S4, $72 \%$

Synthesis of S4: A solution of S2 (2.59 g, $23.1 \mathrm{mmol}, 1.2$ equiv.) and DIAD (4.28 g, $21.2 \mathrm{mmol}, 1.1$ equiv.) in THF $(30 \mathrm{~mL})$ was added to a solution of $\mathbf{S 3}(5.59 \mathrm{~g}, 19.2 \mathrm{mmol}, 1.0$ equiv.) and triphenylphosphine $\left(5.55 \mathrm{~g}, 21.2 \mathrm{mmol}, 1.1\right.$ equiv.) in THF $(30 \mathrm{~mL})$ at $0{ }^{\circ} \mathrm{C}$. The mixture was warmed to room temperature and stirred overnight. The mixture was concentrated and the residue was purified by column chromatography to give $\mathbf{S} 4$ as a colorless oil in $72 \%$ yield $(5.33 \mathrm{~g}) . \mathrm{R}_{f}=0.3($ EtOAc/Hexane $=1 / 5)$. ${ }^{1}$ H NMR (400 MHz, CDCl $)$ ): $\delta 7.77$ - $7.66(\mathrm{~m}, 2 \mathrm{H}), 7.35$ - $7.28(\mathrm{~m}, 2 \mathrm{H}), 7.22-7.12(\mathrm{~m}, 2 \mathrm{H}), 6.89-$ $6.75(\mathrm{~m}, 2 \mathrm{H}), 4.92(\mathrm{tp}, J=6.9,2.3 \mathrm{~Hz}, 1 \mathrm{H}), 4.25(\mathrm{~s}, 2 \mathrm{H}), 3.79(\mathrm{~s}, 3 \mathrm{H}), 3.70(\mathrm{dt}, J=6.9,1.4 \mathrm{~Hz}, 2 \mathrm{H}), 2.44$ (s, 3H), $2.16-2.03(\mathrm{~m}, 2 \mathrm{H}), 1.97-1.81(\mathrm{~m}, 2 \mathrm{H}), 1.65-1.44(\mathrm{~m}, 4 \mathrm{H}) .{ }^{13} \mathbf{C}$ NMR (101 MHz, $\left.\mathbf{C D C l}_{3}\right): \delta$ $159.05,148.28,142.95,137.67,129.61,129.53$, 128.39, 127.22, 113.87, 113.74, 55.27, 49.86, 45.87, 33.64, 28.46, 26.17, 25.92, 21.51. IR: v 2954, 1612, 1513, 1340, 1157, $905 \mathrm{~cm}^{-1}$; HRMS calcd. For $[\mathrm{M}+\mathrm{H}]^{+}: 386.1784$ Found: 386.1788.

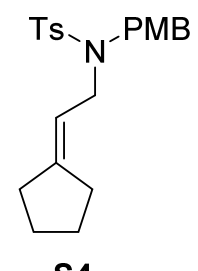

S4
1) $\left.\left.\mathrm{Cl}_{3} \mathrm{CCOCl}, \mathrm{Zn}-\mathrm{Cu}, \mathrm{DME}, \mathrm{Et}_{2} \mathrm{O},\right)\right)$ )

2) $\mathrm{Zn}, \mathrm{AcOH}$, r.t.

3) TFA, DCM, r.t.

4) $(\mathrm{EtO})_{3} \mathrm{CH}, \mathrm{TsOH}, \mathrm{EtOH}$, r.t.

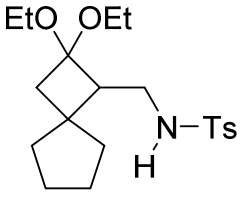

S5, $35 \%$ over 4 steps

Synthesis of S5: A solution of trichloroacetyl chloride (5.03 g, $27.7 \mathrm{mmol}, 2.0$ equiv.) in ether (15 mL) was added to a stirring suspension of S4 (5.33 g, $13.8 \mathrm{mmol}, 1.0$ equiv.), DME (2.5 g, $27.7 \mathrm{mmol}, 2.0$ equiv.) and $\mathrm{Zn}-\mathrm{Cu}(3.60 \mathrm{~g}, 55.2 \mathrm{mmol}, 4$ equiv.) in ether $(15 \mathrm{~mL})$. The reaction mixture was stirred under sonication for $2 \mathrm{~h}$ and then diluted with ether. The mixture was filtered through a short plug of silica gel, and the filtrate was concentrated. The residue was dissolved in AcOH $(20 \mathrm{~mL})$, and the solution was added to a stirring suspension of zinc dust ( $9.0 \mathrm{~g}, 138 \mathrm{mmol}, 10$ equiv.) in $\mathrm{AcOH}(10 \mathrm{~mL})$. The mixture was stirred at room temperature overnight. The mixture was filtered, and the filtrate was concentrated. The residue was dissolved in ethyl acetate and washed with $\mathrm{NaHCO}_{3}$ and brine. The organic layer was dried and concentrated, and the residue was dissolved in DCM $(12 \mathrm{~mL})$. TFA (4 mL) was added slowly to the solution, and the mixture was stirred at room temperature for $1 \mathrm{~h}$. The mixture was then concentrated, and the residue was dissolved in $\mathrm{EtOH}(10 \mathrm{~mL})$ followed by the addition of TsOH $(241 \mathrm{mg}, 1.4 \mathrm{mmol}$, 0.2 equiv.) and $(\mathrm{EtO})_{3} \mathrm{CH}(3.11 \mathrm{~g}, 21 \mathrm{mmol}, 3$ equiv.). The reaction was stirred at room temperature overnight. Then, the reaction mixture was concentrated, and the residue was purified by column chromatography to give S5 as a colorless oil in 35\% yield $(1.83 \mathrm{~g})$ over 4 steps. $\mathrm{R}_{f}=0.4$ (EtOAc/Hexane=1/3). ${ }^{1} \mathbf{H}$ NMR (400 $\left.\mathbf{M H z} \mathbf{C D C l}_{3}\right): \delta 7.89-7.57(\mathrm{~m}, 2 \mathrm{H}), 7.32(\mathrm{~d}, J=8.0 \mathrm{~Hz}, 2 \mathrm{H}), 5.07$ (dd, $J=10.0,2.9 \mathrm{~Hz}, 1 \mathrm{H}), 3.46-3.23(\mathrm{~m}, 4 \mathrm{H}), 3.20-3.06$ (m, 1H), 2.89 (ddd, $J=12.2,11.0,2.9 \mathrm{~Hz}$, $1 \mathrm{H}), 2.44(\mathrm{~s}, 3 \mathrm{H}), 2.34$ (ddd, $J=11.0,5.0,2.8 \mathrm{~Hz}, 1 \mathrm{H}), 2.00-1.83(\mathrm{~m}, 2 \mathrm{H}), 1.78-1.66(\mathrm{~m}, 1 \mathrm{H}), 1.58-$ 
$1.38(\mathrm{~m}, 6 \mathrm{H}), 1.36-1.23(\mathrm{~m}, 1 \mathrm{H}), 1.16(\mathrm{t}, J=7.1 \mathrm{~Hz}, 3 \mathrm{H}), 1.10(\mathrm{t}, J=7.1 \mathrm{~Hz}, 3 \mathrm{H}) .{ }^{13} \mathbf{C}$ NMR (101 MHz, $\left.\mathbf{C D C l}_{3}\right): \delta 143.18,136.99,129.65,126.97,100.73,56.76,56.29,49.24,43.50,41.99,40.21,39.56,33.41$, 23.27, 22.79, 21.53, 15.27, 15.25. IR: v 3288, 2972, 1408, 1330, 1162, $1052 \mathrm{~cm}^{-1}$; HRMS calcd. For $[\mathrm{M}+\mathrm{Na}]^{+}: 404.1866$ Found: 404.1874.

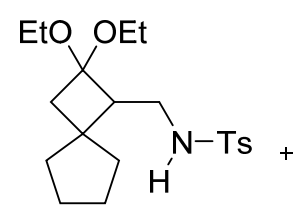

S5

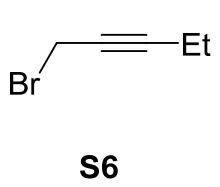

1) $\mathrm{NaH}, \mathrm{DMF}, \mathrm{r.t}$

2) $1 \mathrm{M} \mathrm{HCl}, \mathrm{THF}$, r.t., $12 \mathrm{~h}$

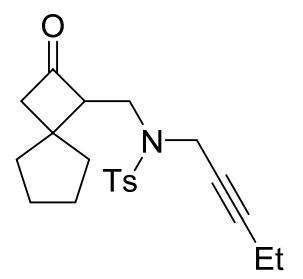

1I, $67 \%$ over 2 steps

Synthesis of 1l: S5 (381.5 mg, 1.0 mmol, 1.0 equiv.) was dissolved in DMF (5 mL), and NaH (80 mg, 2.0 mmol, 2.0 equiv.) was added to the stirring solution. After $10 \mathrm{~min}, \mathbf{S 6}(310 \mu \mathrm{L}, 3.0 \mathrm{mmol}, 3.0$ equiv.) was added and the mixture was stirred at room temperature overnight. The mixture was quenched with water and extracted with ethyl acetate. The combined organic layers were washed with brine, dried and concentrated. The residue was dissolved in THF $(6 \mathrm{~mL})$, and $1 \mathrm{M} \mathrm{HCl}(2 \mathrm{~mL})$ was added to the solution. The resulting mixture was stirred at room temperature overnight. The mixture was diluted with ethyl acetate and washed by water, $\mathrm{NaHCO}_{3}$ and brine. The organic layer was dried and concentrated, and the residue was purified by column chromatography to give $\mathbf{1 l}$ as a colorless oil in $67 \%$ yield $(250 \mathrm{mg}$ ) over 2 steps. Melting Point: $58-59{ }^{\circ} \mathrm{C} . \mathrm{R}_{f}=0.4$ (EtOAc/Hexane=1/5). ${ }^{1} \mathbf{H}$ NMR (400 $\left.\mathbf{M H z}, \mathbf{C D C l}_{3}\right): \delta 7.83-$ $7.64(\mathrm{~m}, 2 \mathrm{H}), 7.32-7.27(\mathrm{~m}, 2 \mathrm{H}), 4.29$ (dtd, $J=18.5,2.3,0.8 \mathrm{~Hz}, 1 \mathrm{H}), 4.01$ (dt, $J=18.4,2.2 \mathrm{~Hz}, 1 \mathrm{H})$, $3.65-3.42(\mathrm{~m}, 2 \mathrm{H}), 3.34-3.11(\mathrm{~m}, 1 \mathrm{H}), 3.03-2.83(\mathrm{~m}, 1 \mathrm{H}), 2.69(\mathrm{dd}, J=16.8,1.7 \mathrm{~Hz}, 1 \mathrm{H}), 2.42(\mathrm{~s}$, $3 \mathrm{H}), 2.05-1.62(\mathrm{~m}, 9 \mathrm{H}), 1.51$ (dddd, $J=12.5,7.6,3.4,1.3 \mathrm{~Hz}, 1 \mathrm{H}), 0.86(\mathrm{t}, J=7.5 \mathrm{~Hz}, 3 \mathrm{H}) .{ }^{13} \mathbf{C}$ NMR (101 MHz, $\mathbf{C D C l}_{3}$ ): $\delta$ 207.03, 143.44, 135.33, 129.34, 127.93, 88.04, 71.36, 62.29, 57.86, 42.28, 40.66, 39.72, 37.27, 32.21, 23.92, 23.84, 21.49, 13.40, 12.08. IR: v 2940, 1775, 1598, 1451, 1349, 1162, 906 $\mathrm{cm}^{-1}$; HRMS calcd. For $[\mathrm{M}+\mathrm{Na}]^{+}: 396.1604$ Found: 396.1603.

Substrate 1m was synthesized from S7 (commercially available), S8 (commercially available) and S1 ${ }^{1}$ through a multi-step sequence.

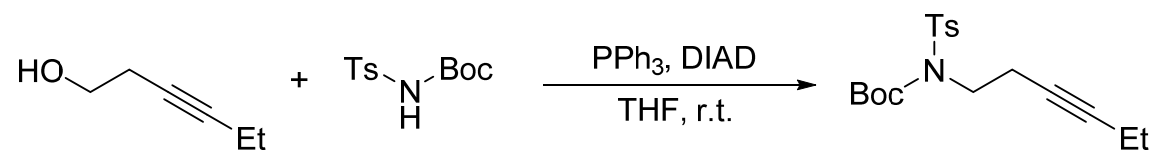

S7

S8

S9, $87 \%$

Synthesis of S9: A solution of S7 (1.08 g, $11 \mathrm{mmol}, 1.1$ equiv.) and DIAD (2.42 g, $12 \mathrm{mmol}, 1.2$ equiv.) in THF (25 mL) was added to a solution of $\mathbf{S 8}(2.71 \mathrm{~g}, 10 \mathrm{mmol}, 1.0$ equiv.) and triphenylphosphine (3.14 g, $12 \mathrm{mmol}, 1.2$ equiv.) in THF $(25 \mathrm{~mL})$ at $0{ }^{\circ} \mathrm{C}$. The mixture was warmed to room temperature and stirred overnight. The mixture was concentrated and the residue was purified by column chromatography to give S9 as a white solid in 87\% yield (3.04 g). $\mathrm{R}_{f}=0.6$ (EtOAc/Hexane $\left.=1 / 3\right) .{ }^{1} \mathbf{H}$ NMR (400 MHz, $\left.\mathbf{C D C l}_{3}\right): \delta 7.91-7.71(\mathrm{~m}, 2 \mathrm{H}), 7.36-7.28(\mathrm{~m}, 2 \mathrm{H}), 4.16-3.81(\mathrm{~m}, 2 \mathrm{H}), 2.82-2.53(\mathrm{~m}, 2 \mathrm{H}), 2.43(\mathrm{~s}$, 
3H), 2.14 (qt, $J=7.5,2.4 \mathrm{~Hz}, 2 \mathrm{H}), 1.34$ (s, 9H), 1.09 (t, $J=7.5 \mathrm{~Hz}, 3 \mathrm{H}) .{ }^{13} \mathbf{C}$ NMR (101 MHz, CDCl $\left.\mathbf{3}\right): \delta$ 150.76, 144.09, 137.35, 129.20, 127.85, 84.28, 83.87, 75.35, 45.72, 27.83, 21.59, 20.22, 14.05, 12.39. IR: v 2979, 1728, 1357, 1291, 1157, $970 \mathrm{~cm}^{-1}$; HRMS calcd. For $[\mathrm{M}+\mathrm{Na}]^{+}: 374.1397$ Found: 374.1398.

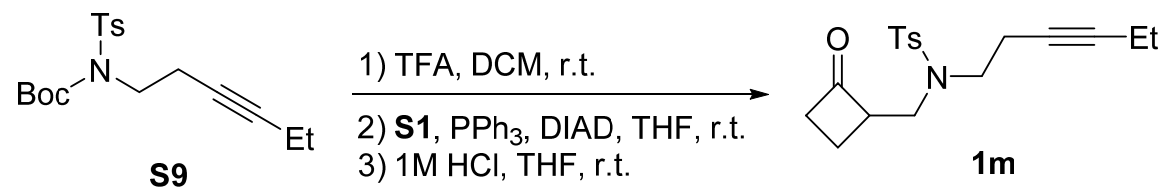

Synthesis of 1m: S9 (702 mg, 2 mmol, 1.0 equiv.) was dissolved in DCM (5 mL). Trifluoroacetic acid ( $1.14 \mathrm{~g}, 10 \mathrm{mmol}, 5.0$ equiv.) was added dropwise, and the solution was stirred at room temperature for 6

h. The mixture was quenched with $\mathrm{NaHCO}_{3}$ and extracted with DCM. The combined organic layers were dried and concentrated, and the residue was dissolved in THF $(5 \mathrm{~mL})$ followed by the addition of $\mathrm{PPh}_{3}$ (629 mg, $2.4 \mathrm{mmol}, 1.2$ equiv.). A solution of $\mathbf{S 1}$ (383 mg, $2.2 \mathrm{mmol}, 1.1$ equiv.) and DIAD (485 mg, 2.4 mmol, 1.2 equiv.) in THF ( $5 \mathrm{~mL})$ was added to the mixture at $0{ }^{\circ} \mathrm{C}$. The mixture was warmed to room temperature and stirred overnight. The mixture was concentrated, and the residue was dissolved in THF $(4 \mathrm{~mL}) .1 \mathrm{M} \mathrm{HCl}(1.5 \mathrm{~mL})$ was added, and the resulting mixture was stirred at room temperature overnight. The mixture was diluted with ethyl acetate and washed by water, $\mathrm{NaHCO}_{3}$ and brine. The organic layer was dried and concentrated, and the residue was purified by column chromatography to give $\mathbf{1 m}$ as a colorless oil in $45 \%$ yield (300 mg) over 3 steps. $\mathrm{R}_{f}=0.4($ EtOAc/Hexane $=1 / 3) .{ }^{\mathbf{1}} \mathbf{H}$ NMR (400 MHz, $\mathbf{C D C l}_{3}$ ): $\delta 7.78-7.63(\mathrm{~m}, 2 \mathrm{H}), 7.33-7.27(\mathrm{~m}, 2 \mathrm{H}), 3.61$ (dddt, $\left.J=15.7,10.1,5.3,2.7 \mathrm{~Hz}, 1 \mathrm{H}\right)$, $3.49(\mathrm{dd}, J=14.5,8.7 \mathrm{~Hz}, 1 \mathrm{H}), 3.38-3.17(\mathrm{~m}, 3 \mathrm{H}), 3.07$ (dddd, $J=17.8,10.6,8.3,2.4 \mathrm{~Hz}, 1 \mathrm{H}), 2.94$ (dddd, $J=17.7,9.7,5.0,2.6 \mathrm{~Hz}, 1 \mathrm{H}), 2.42$ (s, 3H), 2.37 (dddt, $J=8.9,6.7,4.5,2.4 \mathrm{~Hz}, 2 \mathrm{H}), 2.24$ (dtd, $J$ $=11.5,10.3,5.1 \mathrm{~Hz}, 1 \mathrm{H}), 2.10(\mathrm{qt}, J=7.5,2.4 \mathrm{~Hz}, 2 \mathrm{H}), 1.93(\mathrm{ddt}, J=11.5,9.7,8.0 \mathrm{~Hz}, 1 \mathrm{H}), 1.07(\mathrm{t}, J=$ $7.5 \mathrm{~Hz}, 3 \mathrm{H}) .{ }^{13} \mathbf{C}$ NMR (101 MHz, $\left.\mathbf{C D C l}_{3}\right): \delta 208.44,143.50,136.36,129.76,127.12,83.82,75.66$, 59.58, 48.06, 47.36, 44.79, 21.48, 19.46, 15.88, 14.00, 12.30. IR: v 2975, 1778, 1456, 1340, 1159, 968 $\mathrm{cm}^{-1}$; HRMS calcd. For $[\mathrm{M}+\mathrm{H}]^{+}: 334.1471$ Found: 334.1478.

\section{Rh-catalyzed kinetic resolutions between cyclobutanones and alkynes}

\section{General procedure:}

In a nitrogen-filled glove box, a 4-mL vial was charged with the cyclobutanone substrate $(0.1 \mathrm{mmol})$, $\left[\mathrm{Rh}\left(\mathrm{CH}_{2}=\mathrm{CH}_{2}\right)_{2} \mathrm{Cl}\right]_{2}(1.9 \mathrm{mg}, 0.005 \mathrm{mmol}, 5 \mathrm{~mol} \%)$, (R)-DTBM-segphos (11.8 $\left.\mathrm{mg}, 0.01 \mathrm{mmol}, 10 \mathrm{~mol} \%\right)$ and $\mathrm{AgSbF}_{6}(3.4 \mathrm{mg}, 0.01 \mathrm{mmol}, 10 \mathrm{~mol} \%$ ), followed by $1000 \mu \mathrm{L} \mathrm{1,4-dioxane.} \mathrm{After} \mathrm{a} \mathrm{homogeneous}$ solution was formed, the vial was capped and the reaction mixture was maintained at room temperature (1a to $1 \mathbf{k}$ ) or $40{ }^{\circ} \mathrm{C}$ (1) for $12-13 \mathrm{~h}$. After the reaction was completed, the mixture was concentrated and the residue was purified by silica gel chromatography.

Both $2 a$ to 2 and recycled 1 a to 11 were collected and measured the yield. The H-NMR spectra of recycled $1 a$ to 11 are identical to the original spectra. The HPLC data and optical rotation data were collected and shown in the following session. 


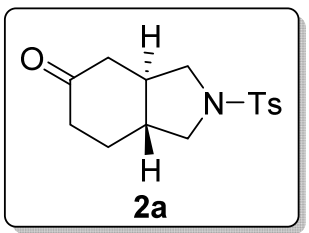

2a (13.5 mg) was isolated as a white solid in $46 \%$ yield. Melting Point: $186-188{ }^{\circ} \mathrm{C} . \mathrm{R}_{f}=0.2$ (EtOAc/Hexane=1/3). ${ }^{1} \mathbf{H}$ NMR (400 $\left.\mathbf{~ M H z , ~} \mathbf{C D C l}_{3}\right): \delta 7.81-7.68(\mathrm{~m}, 2 \mathrm{H}), 7.39-7.30(\mathrm{~m}, 2 \mathrm{H}), 3.68(\mathrm{dd}$, $J=9.6,7.1 \mathrm{~Hz}, 1 \mathrm{H}), 3.59(\mathrm{dd}, J=9.7,7.0 \mathrm{~Hz}, 1 \mathrm{H}), 2.96(\mathrm{dd}, J=10.7,9.7 \mathrm{~Hz}, 1 \mathrm{H}), 2.86(\mathrm{dd}, J=10.7,9.6$ $\mathrm{Hz}, 1 \mathrm{H}), 2.53$ (ddd, $J=14.3,4.0,1.9 \mathrm{~Hz}, 1 \mathrm{H}), 2.48-2.40$ (m, 4H), 2.26 (dddd, $J=15.4,13.1,6.7,0.9 \mathrm{~Hz}$, $1 \mathrm{H}), 2.19-2.07$ (m, 2H), $1.96-1.84(\mathrm{~m}, 1 \mathrm{H}), 1.81-1.68(\mathrm{~m}, 1 \mathrm{H}), 1.44$ (tdd, $J=14.7,12.7,5.7 \mathrm{~Hz}, 1 \mathrm{H})$. ${ }^{13}$ C NMR (101 MHz, $\left.\mathbf{C D C l}_{3}\right): \delta 208.27,143.56,134.35,129.80,127.28,52.61,51.90,44.17,43.88$, 42.75, 39.81, 26.47, 21.55. IR: v 2937, 1705, 1598, 1343, 1160, 1027, $816 \mathrm{~cm}^{-1}$; HRMS calcd. For $[\mathrm{M}+\mathrm{Na}]^{+}:$316.0978. Found: 316.0977.

Chiral HPLC for 2a (Chiralpak ID, hexane:isopropanol $=70: 30,1 \mathrm{~mL} / \mathrm{min}, 230 \mathrm{~nm}$ ), $\mathrm{t}_{\text {minor }}=64.8 \mathrm{~min}$, $\mathrm{t}_{\text {major }}=39.2 \mathrm{~min} .[\alpha]_{\mathrm{D}}^{22.6}=72.6\left(\mathrm{c}=1.79, \mathrm{CH}_{2} \mathrm{Cl}_{2}\right)$ at $98 \%$ ee.

\section{Racemic Sample 2a}

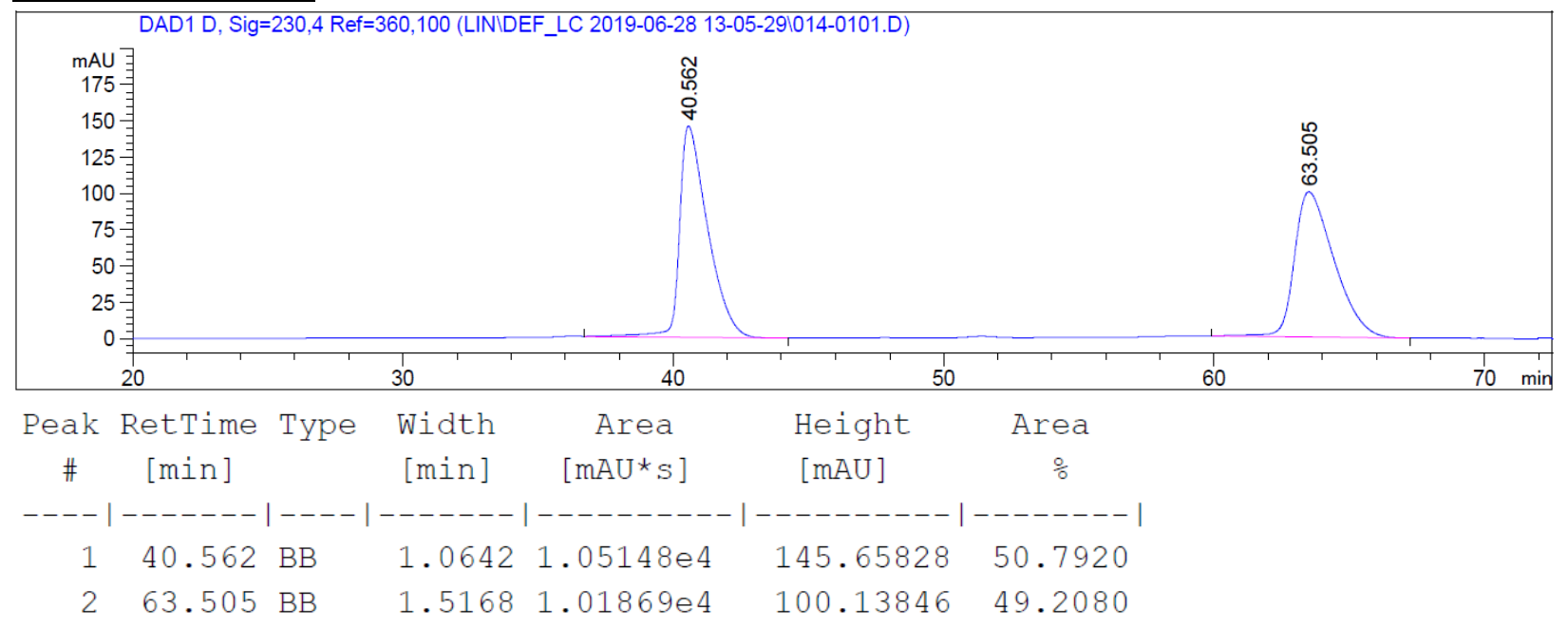

\section{Enantiomeric Sample 2a}

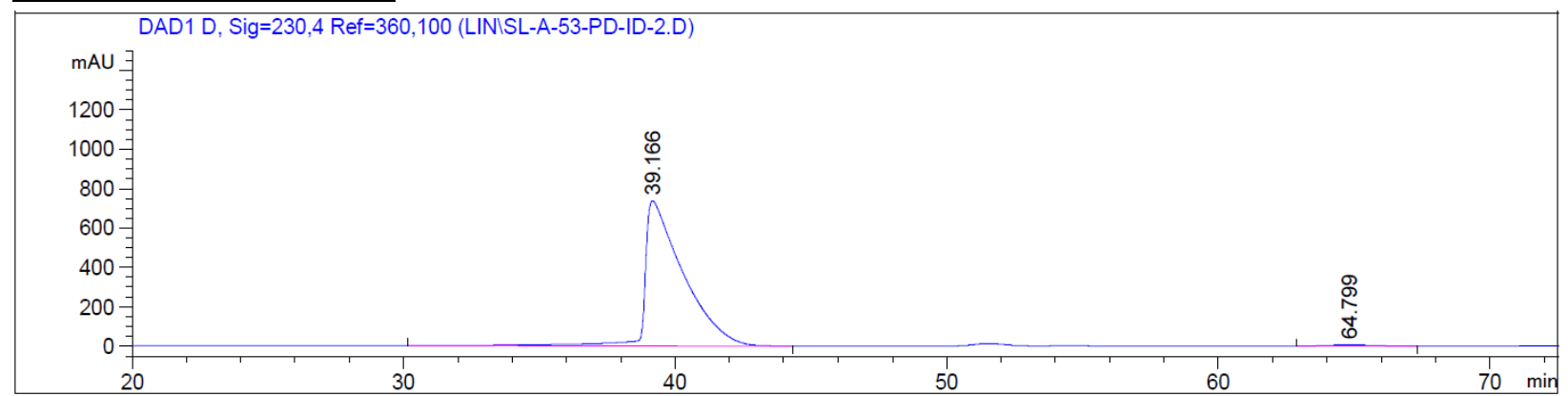




\begin{tabular}{|c|c|c|c|c|c|c|}
\hline $\begin{array}{c}\text { Peak } \\
\#\end{array}$ & $\begin{array}{c}\text { RetTime } \\
\text { [min] }\end{array}$ & Type & $\begin{array}{l}\text { Width } \\
\text { [min] }\end{array}$ & $\begin{array}{c}\text { Area } \\
{\left[m A U^{*} \mathrm{~s}\right]}\end{array}$ & $\begin{array}{l}\text { Height } \\
{[\mathrm{mAU}]}\end{array}$ & $\begin{array}{c}\text { Area } \\
\frac{\circ}{\circ}\end{array}$ \\
\hline & & & ---1 & $-----------\mid$ & ----1 & --- \\
\hline 1 & 39.166 & $\mathrm{BB}$ & 1.3731 & 7.20310 e4 & 733.85266 & 99.1963 \\
\hline 2 & 64.799 & $\mathrm{BB}$ & 1.1507 & 583.57434 & 6.20788 & 0.8037 \\
\hline
\end{tabular}

1a $(13.8 \mathrm{mg})$ was recycled as a colorless oil in $47 \%$ yield.

Chiral HPLC for 1a (Chiralpak IA, hexane:isopropanol $=90: 10,1 \mathrm{~mL} / \mathrm{min}, 260 \mathrm{~nm}$ ), $\mathrm{t}_{\text {minor }}=15.0 \mathrm{~min}$, $\mathrm{t}_{\text {major }}=14.2 \mathrm{~min} .[\alpha]_{\mathrm{D}}^{22.6}=-30.4\left(\mathrm{c}=1.38, \mathrm{CH}_{2} \mathrm{Cl}_{2}\right)$ at $98 \%$ ee.

\section{Racemic Sample 1a}

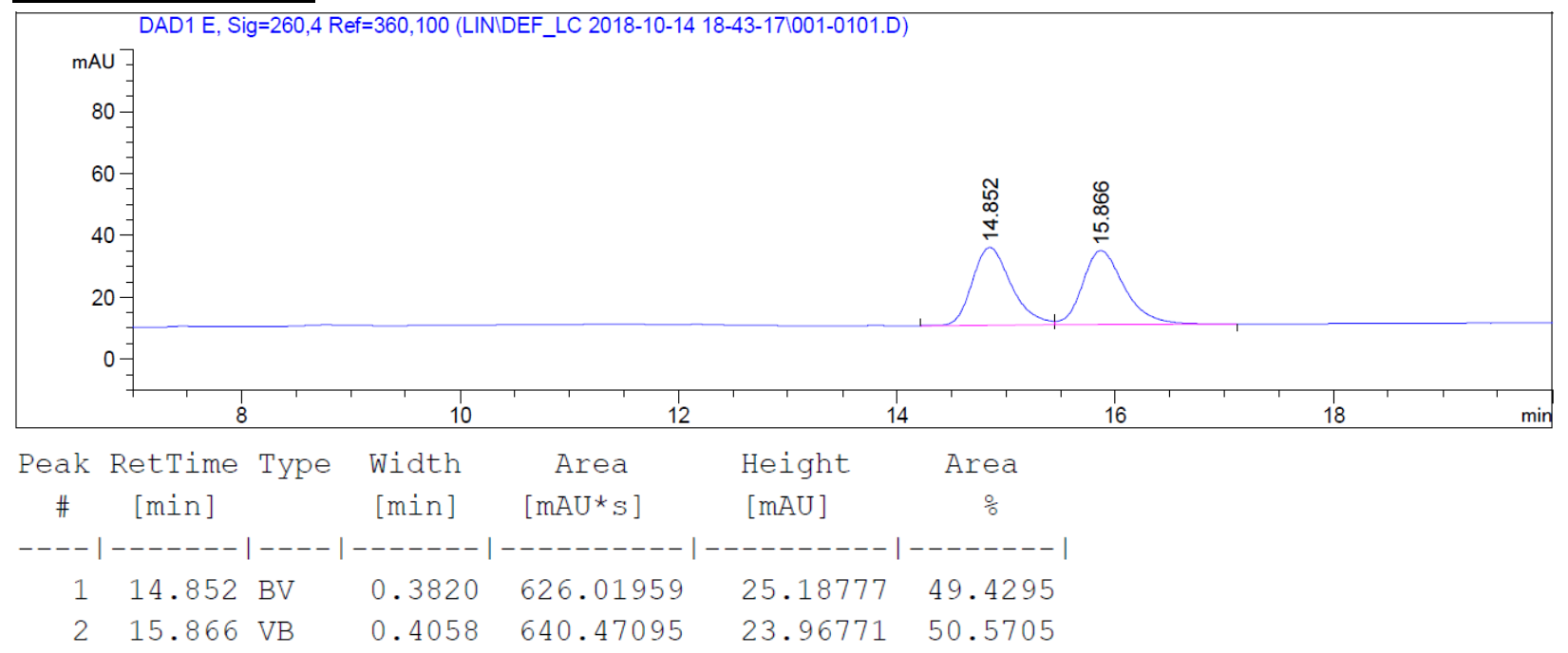

\section{Enantiomeric Sample 1a}

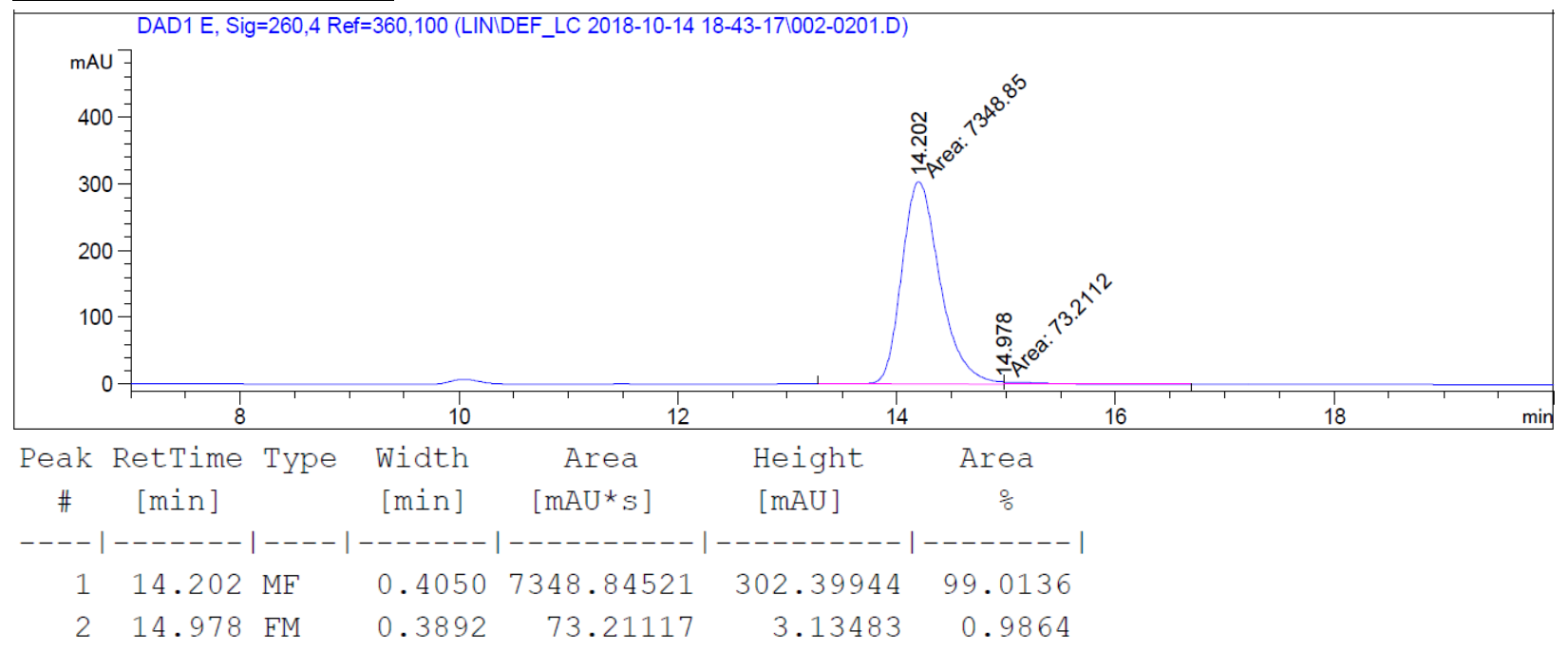




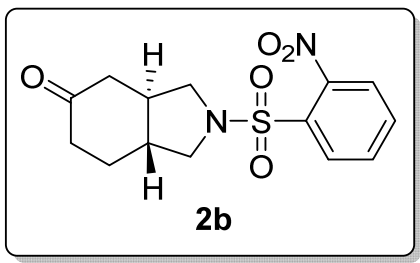

2b (14.3 mg) was isolated as a light yellow solid in $44 \%$ yield. Melting Point: $187-189{ }^{\circ} \mathrm{C} . \mathrm{R}_{f}=0.3$ (EtOAc/Hexane=1/1). ${ }^{1} \mathbf{H}$ NMR (400 MHz, $\left.\mathbf{C D C l}_{3}\right): \delta 8.14-7.99(\mathrm{~m}, 1 \mathrm{H}), 7.76-7.67(\mathrm{~m}, 2 \mathrm{H}), 7.66-$ $7.61(\mathrm{~m}, 1 \mathrm{H}), 3.81(\mathrm{dd}, J=9.3,6.8 \mathrm{~Hz}, 1 \mathrm{H}), 3.73(\mathrm{dd}, J=9.3,6.7 \mathrm{~Hz}, 1 \mathrm{H}), 3.17-3.01(\mathrm{~m}, 2 \mathrm{H}), 2.61$ (ddd, $J=14.2,3.8,1.9 \mathrm{~Hz}, 1 \mathrm{H}), 2.51$ (ddt, $J=15.5,4.9,1.9 \mathrm{~Hz}, 1 \mathrm{H}), 2.34$ (dddd, $J=15.5,13.1,6.6,0.9 \mathrm{~Hz}$, 1H), $2.27-1.97(\mathrm{~m}, 4 \mathrm{H}), 1.63-1.49(\mathrm{~m}, 1 \mathrm{H}) .{ }^{13} \mathbf{C}$ NMR (101 MHz, $\left.\mathbf{C D C l}_{3}\right): \delta 208.06,133.57,132.46$, 131.62, 130.84, 124.01, 52.56, 51.96, 44.40, 43.81, 42.97, 39.81, 26.39. IR: v 2925, 1714, 1544, 1373, $1349,1164,779 \mathrm{~cm}^{-1}$; HRMS calcd. For $[\mathrm{M}+\mathrm{Na}]^{+}: 347.0672$. Found: 347.0675.

Chiral HPLC for $\mathbf{2 b}$ (Chiralpak ID, hexane:isopropanol $=75: 25,1 \mathrm{~mL} / \mathrm{min}, 210 \mathrm{~nm}$ ), $\mathrm{t}_{\text {minor }}=112.1 \mathrm{~min}$, $\mathrm{t}_{\text {major }}=122.3 \min .[\alpha]_{\mathrm{D}}^{22.6}=-278.2\left(\mathrm{c}=0.87, \mathrm{CH}_{2} \mathrm{Cl}_{2}\right)$ at $95 \%$ ee.

\section{Racemic Sample 2b}

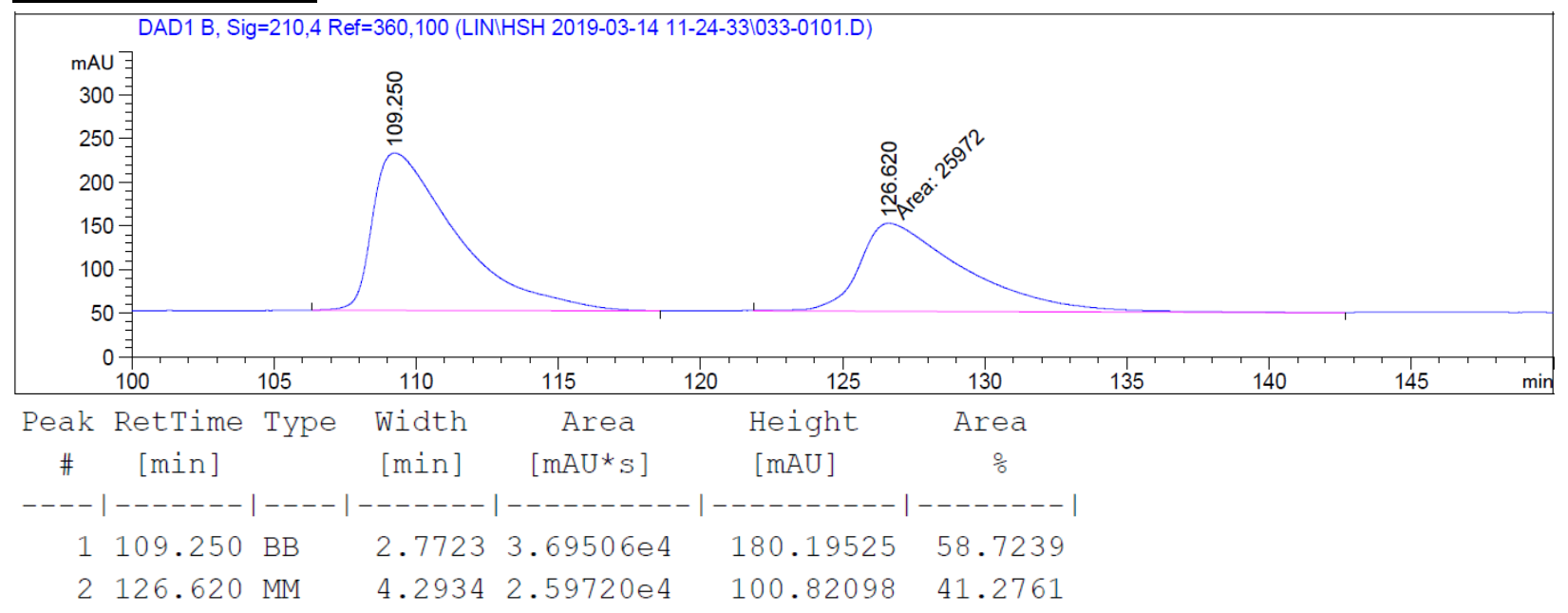

Enantiomeric Sample 2b

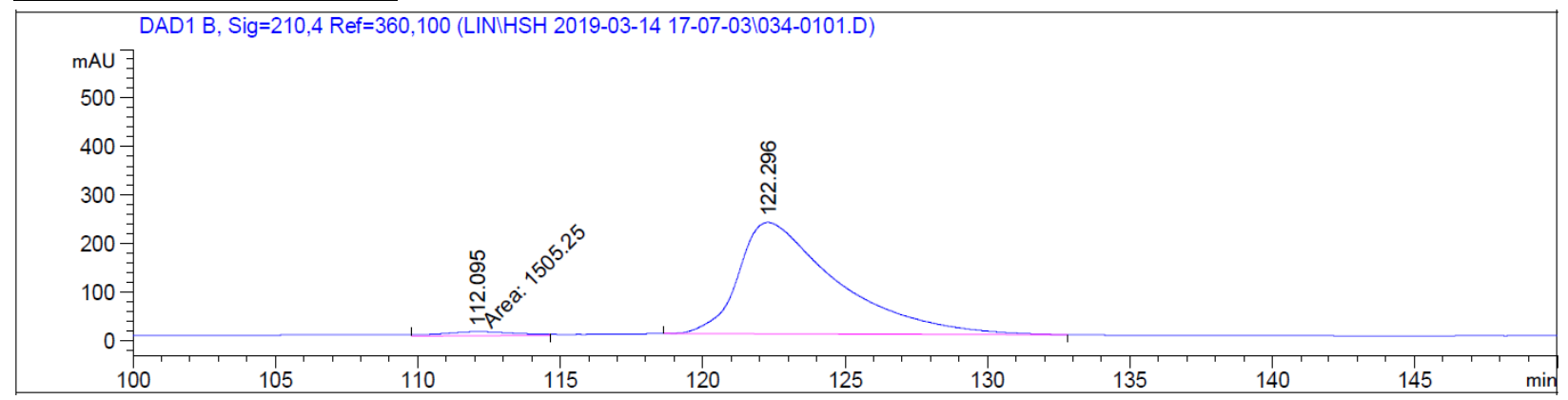




\begin{tabular}{|c|c|c|c|c|c|c|}
\hline $\begin{array}{c}\text { Peak } \\
\#\end{array}$ & $\begin{array}{c}\text { RetTime } \\
\text { [min] }\end{array}$ & Type & $\begin{array}{l}\text { Width } \\
\text { [min] }\end{array}$ & $\begin{array}{c}\text { Area } \\
{\left[\mathrm{mAU}^{*} \mathrm{~s}\right]}\end{array}$ & $\begin{array}{l}\text { Height } \\
{[\mathrm{mAU}]}\end{array}$ & $\begin{array}{c}\text { Area } \\
\quad \%\end{array}$ \\
\hline \multicolumn{7}{|c|}{$---|-------|----|-------|----------|----------|--------\mid$} \\
\hline 1 & 112.095 & & 2.9839 & 1505.25464 & 8.40771 & 2.6500 \\
\hline 2 & 122.296 & $\mathrm{BB}$ & 3.2182 & $5.52972 e 4$ & 229.56522 & 97.3500 \\
\hline
\end{tabular}

1b (15.2 mg) was recycled as a colorless oil in $47 \%$ yield.

Chiral HPLC for $\mathbf{1 b}$ (Chiralpak IC, hexane:isopropanol $=80: 20,1 \mathrm{~mL} / \mathrm{min}, 210 \mathrm{~nm}$ ), $\mathrm{t}_{\operatorname{minor}}=75.3 \mathrm{~min}$, $\mathrm{t}_{\text {major }}=50.8 \mathrm{~min} .[\alpha]_{\mathrm{D}}^{22.6}=-11.4\left(\mathrm{c}=0.35, \mathrm{CH}_{2} \mathrm{Cl}_{2}\right)$ at $90 \%$ ee.

\section{Racemic Sample 1b}

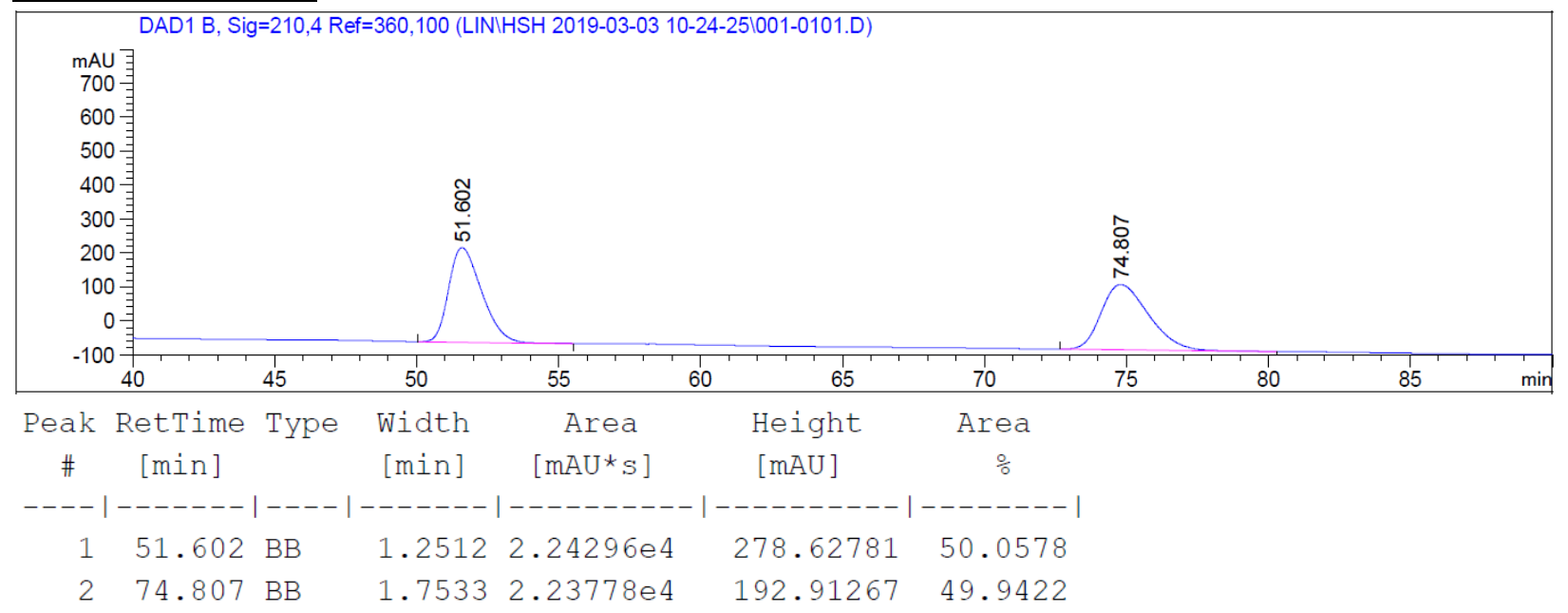

\section{Enantiomeric Sample 1b}

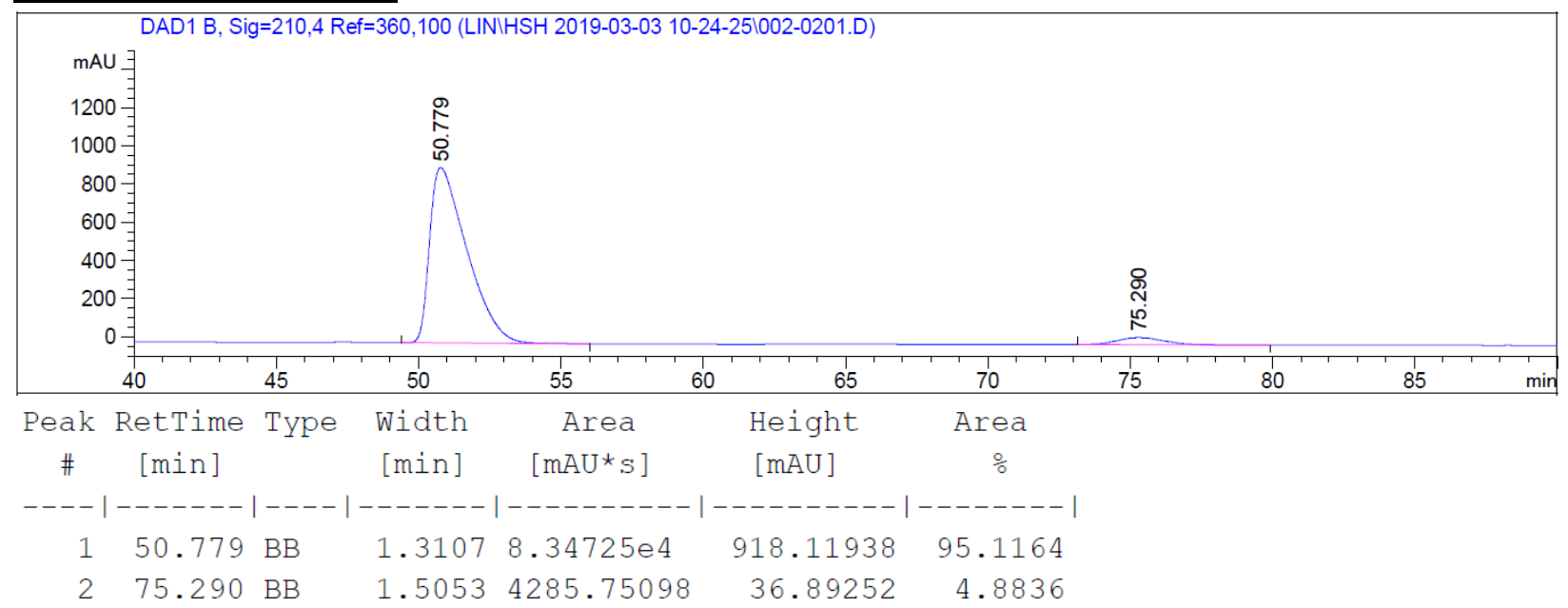




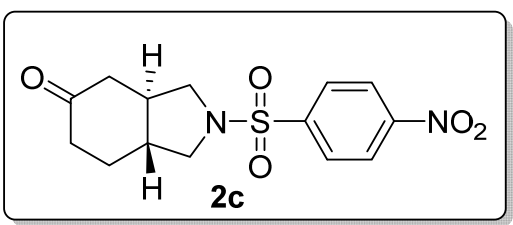

2c (16.2 mg) was isolated as a white solid in 50\% yield. Melting Point: $210{ }^{\circ} \mathrm{C}$ (decomposed). $\mathrm{R}_{f}=0.3$ (EtOAc/Hexane=1/1). ${ }^{1} \mathbf{H}$ NMR (400 MHz, DMSO-d $\left.{ }_{6}\right): \delta 8.46-8.40(\mathrm{~m}, 2 \mathrm{H}), 8.19-8.04$ (m, 2H), 3.62 $(\mathrm{dd}, J=9.5,7.0 \mathrm{~Hz}, 1 \mathrm{H}), 3.55(\mathrm{dd}, J=9.4,6.8 \mathrm{~Hz}, 1 \mathrm{H}), 2.90(\mathrm{dd}, J=10.6,9.5 \mathrm{~Hz}, 1 \mathrm{H}), 2.83(\mathrm{dd}, J=10.7$, $9.5 \mathrm{~Hz}, 1 \mathrm{H}), 2.36-2.22(\mathrm{~m}, 3 \mathrm{H}), 2.18$ (ddt, $J=15.3,4.9,1.8 \mathrm{~Hz}, 1 \mathrm{H}), 2.04-1.70(\mathrm{~m}, 3 \mathrm{H}), 1.53-1.32$ (m, 1H). ${ }^{13}$ C NMR (101 MHz, DMSO-d ${ }_{6}$ ): $\delta 209.05,150.35,142.75,129.14,125.21,52.90,52.27,43.59$, 43.42, 41.94, 39.71, 25.94. IR: v 2923, 1704, 1528, 1461, 1347, 1161, $855 \mathrm{~cm}^{-1}$; HRMS calcd. For $[\mathrm{M}+\mathrm{H}]^{+}:$325.0853. Found: 325.0852 .

Chiral HPLC of 2c (Chiralpak ID, hexane:isopropanol $=75: 25,1 \mathrm{~mL} / \mathrm{min}, 230 \mathrm{~nm}), \mathrm{t}_{\text {minor }}=78.4 \mathrm{~min}$, $\mathrm{t}_{\text {major }}=50.4 \mathrm{~min} .[\alpha]_{\mathrm{D}}^{22.6}=-33.3\left(\mathrm{c}=0.75, \mathrm{CH}_{2} \mathrm{Cl}_{2}\right)$ at $94 \%$ ee.

\section{Racemic Sample 2c}

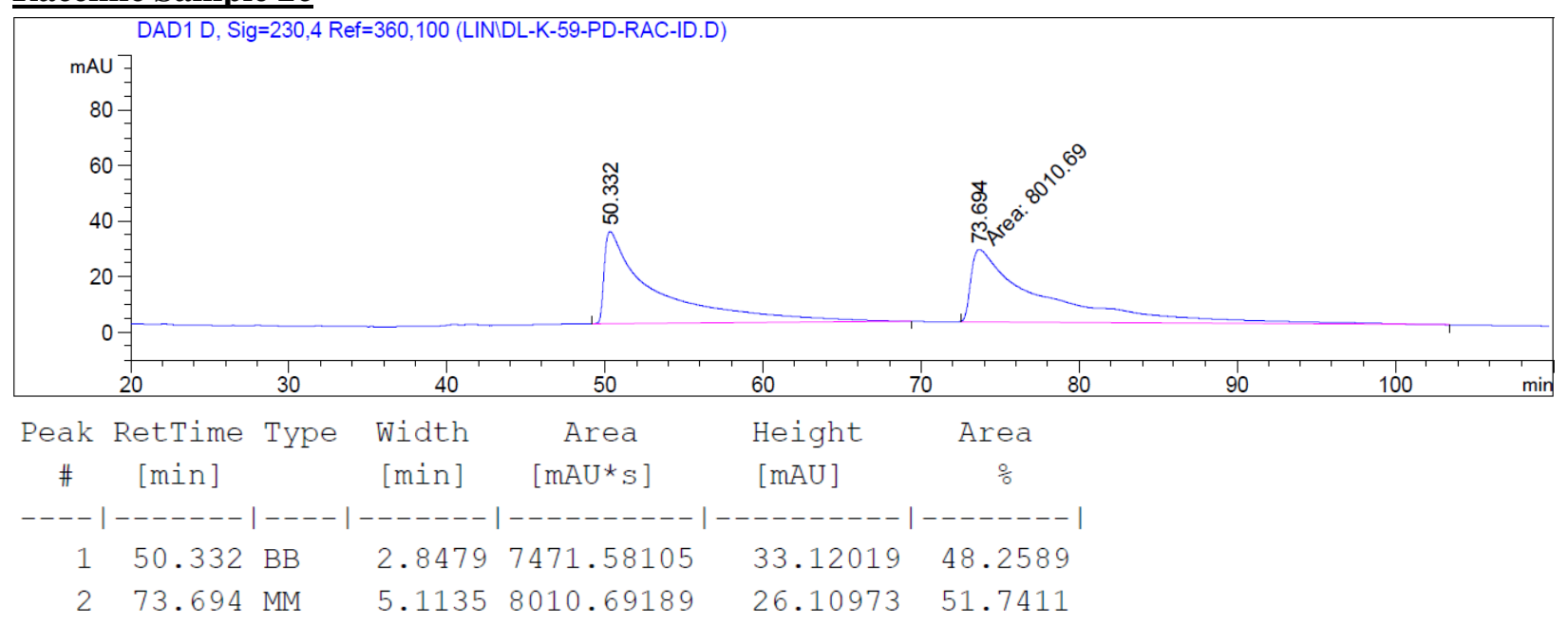

\section{Enantiomeric Sample 2c}

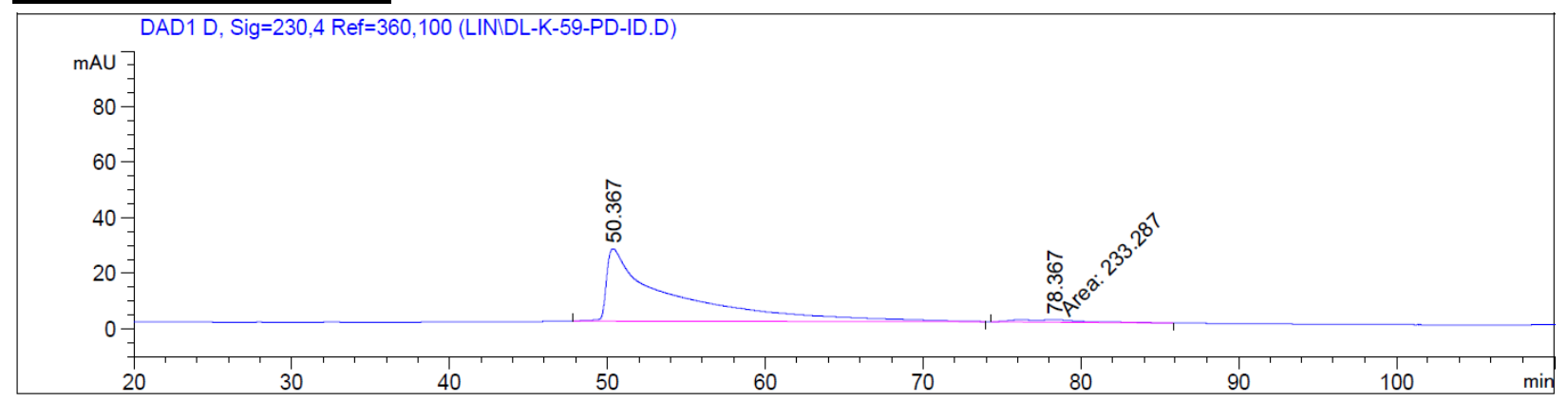




\begin{tabular}{|c|c|c|c|c|c|c|}
\hline $\begin{array}{c}\text { Peak } \\
\#\end{array}$ & $\begin{array}{c}\text { RetTime } \\
\text { [min] }\end{array}$ & Type & $\begin{array}{l}\text { Width } \\
\text { [min] }\end{array}$ & $\begin{array}{c}\text { Area } \\
{\left[\mathrm{mAU}^{\star} \mathrm{s}\right]}\end{array}$ & $\begin{array}{c}\text { Height } \\
{[\mathrm{mAU}]}\end{array}$ & $\begin{array}{c}\text { Area } \\
\frac{\circ}{\circ}\end{array}$ \\
\hline & -- & & -- & ---------- & $\mid----------$ & $--------\mid$ \\
\hline 1 & 50.367 & $\mathrm{BB}$ & 3.4522 & 7271.30029 & 26.08440 & 96.8914 \\
\hline 2 & 78.367 & MM & 4.6921 & 233.28732 & $8.28652 e-1$ & 3.1086 \\
\hline
\end{tabular}

1c (13.6 mg) was recycled as a white solid in $42 \%$ yield.

Chiral HPLC for 1c (Chiralpak IA, hexane:isopropanol $=90: 10,1 \mathrm{~mL} / \mathrm{min}, 210 \mathrm{~nm}$ ), $\mathrm{t}_{\text {minor }}=35.7 \mathrm{~min}$, $\mathrm{t}_{\text {major }}=29.8 \mathrm{~min} .[\alpha]_{\mathrm{D}}^{22.6}=-82.4\left(\mathrm{c}=0.74, \mathrm{CH}_{2} \mathrm{Cl}_{2}\right)$ at $96 \%$ ee.

\section{Racemic Sample 1c}

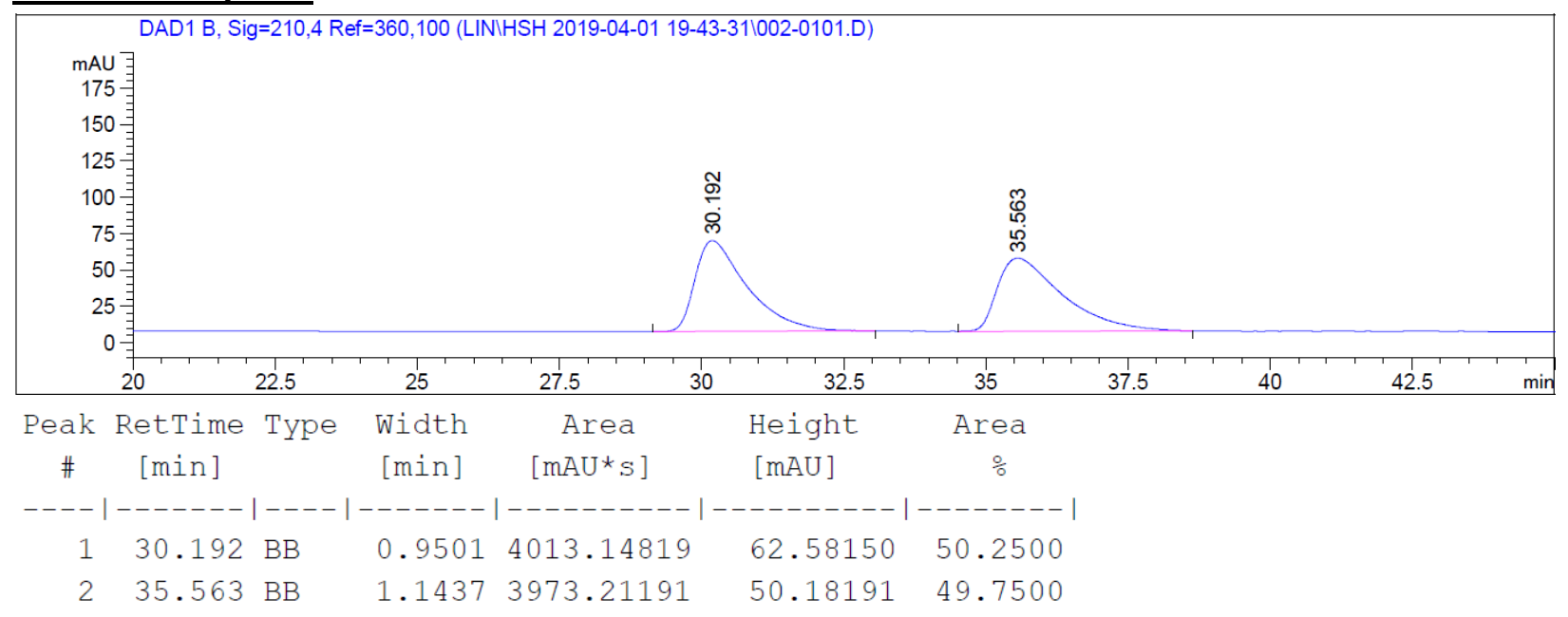

\section{Enantiomeric Sample 1c}

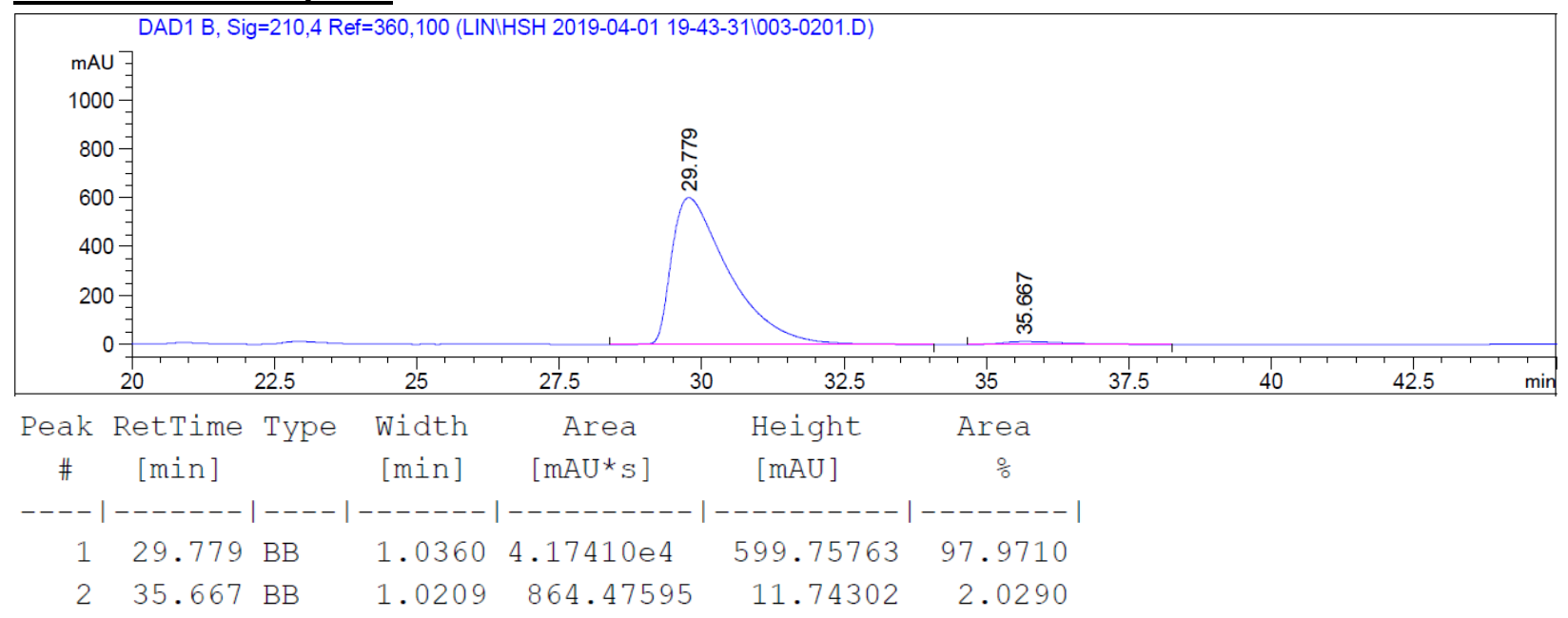




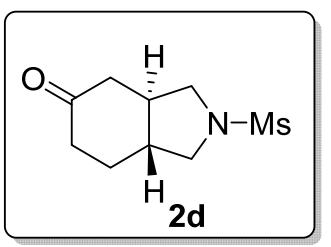

2d (9.5 mg) was isolated as a white solid in $44 \%$ yield. Melting Point: $170-172{ }^{\circ} \mathrm{C} . \mathrm{R}_{f}=0.3$ (EtOAc/Hexane=1/1). ${ }^{\mathbf{H}} \mathbf{H}$ NMR (400 MHz, $\left.\mathbf{C D C l}_{3}\right): \delta 3.71(\mathrm{dd}, J=9.5,6.8 \mathrm{~Hz}, 1 \mathrm{H}), 3.62(\mathrm{dd}, J=9.6$, $6.8 \mathrm{~Hz}, 1 \mathrm{H}), 3.06(\mathrm{dd}, J=10.4,9.5 \mathrm{~Hz}, 1 \mathrm{H}), 2.97$ (dd, $J=10.5,9.4 \mathrm{~Hz}, 1 \mathrm{H}), 2.86(\mathrm{~s}, 3 \mathrm{H}), 2.64$ (ddd, $J=$ $14.2,3.8,1.9 \mathrm{~Hz}, 1 \mathrm{H}), 2.53$ (ddt, $J=15.5,4.9,2.0 \mathrm{~Hz}, 1 \mathrm{H}), 2.36$ (dddd, $J=15.5,13.2,6.6,0.9 \mathrm{~Hz}, 1 \mathrm{H}$ ), $2.30-2.18$ (m, 2H), 2.18 - 1.97 (m, 2H), $1.64-1.50$ (m, 1H). ${ }^{13} \mathbf{C}$ NMR (101 MHz, CDCl $): \delta 208.09$, 52.43, 51.73, 44.60, 43.92, 43.14, 39.85, 35.75, 26.50. IR: v 2956, 1704, 1328, 1190, 1143, $792 \mathrm{~cm}^{-1}$; HRMS calcd. For $[\mathrm{M}+\mathrm{H}]^{+}: 218.0845$. Found: $218.0847 .[\alpha]_{\mathrm{D}}^{22.7}=71.9\left(\mathrm{c}=1.14, \mathrm{CH}_{2} \mathrm{Cl}_{2}\right)$ at $99 \%$ ee.

Note: Due to the low UV absorption of $\mathbf{2 d}$, the chiral HPLC data were obtained by converting $\mathbf{2 d}$ (both racemic and enantiomeric samples) to its indole derivative 2d' as shown below:

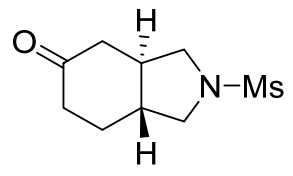

2d

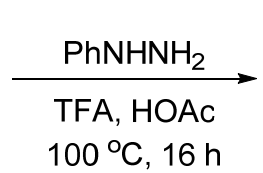

$100^{\circ} \mathrm{C}, 16 \mathrm{~h}$

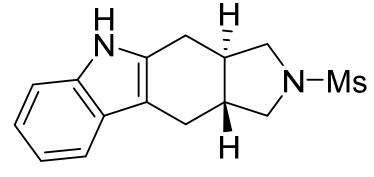

2d'

Chiral HPLC of 2d' (Chiralpak IB, hexane:isopropanol:THF = 75:15:10, $1 \mathrm{~mL} / \mathrm{min}, 230 \mathrm{~nm}), \mathrm{t}_{\text {minor }}=89.6$ $\min , \mathrm{t}_{\text {major }}=70.3 \mathrm{~min}$.

\section{Racemic Sample 2d'}

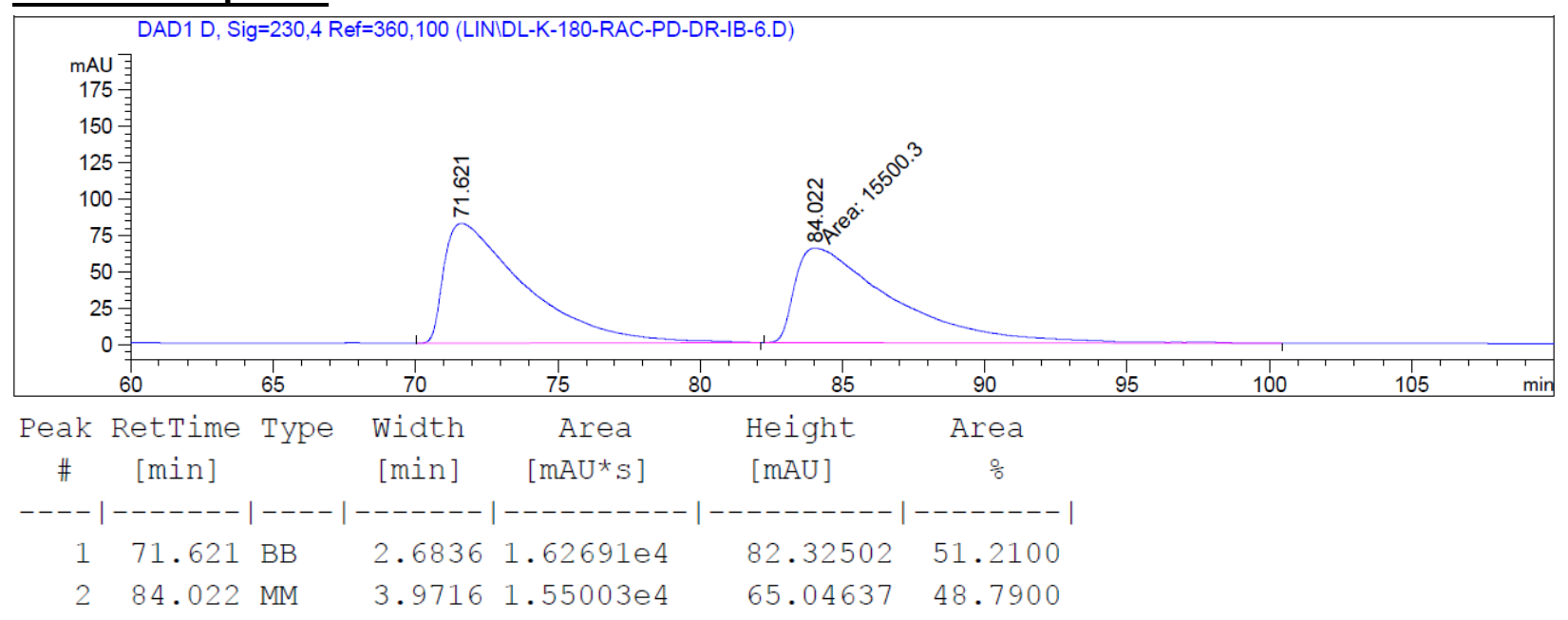

\section{Enantiomeric Sample 2d'}




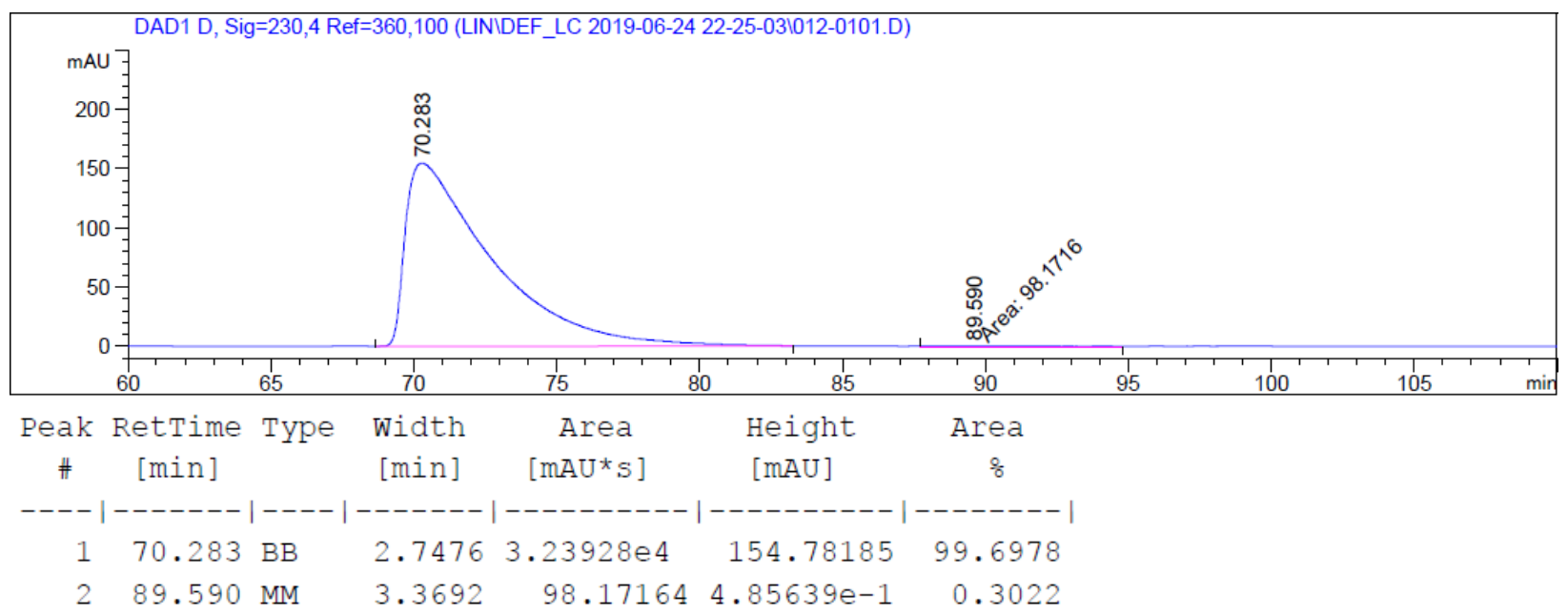

1d (10.0 mg) was recycled as a colorless oil in $46 \%$ yield.

Chiral HPLC for 1d (Chiralpak IF, hexane:isopropanol $=80: 20,1 \mathrm{~mL} / \mathrm{min}, 210 \mathrm{~nm}$ ), $\mathrm{t}_{\operatorname{minor}}=19.7 \mathrm{~min}$, $\mathrm{t}_{\text {major }}=20.7 \mathrm{~min} .[\alpha]_{\mathrm{D}}^{22.7}=254.5\left(\mathrm{c}=1.21, \mathrm{CH}_{2} \mathrm{Cl}_{2}\right)$ at $82 \%$ ee.

\section{Racemic Sample 1d}

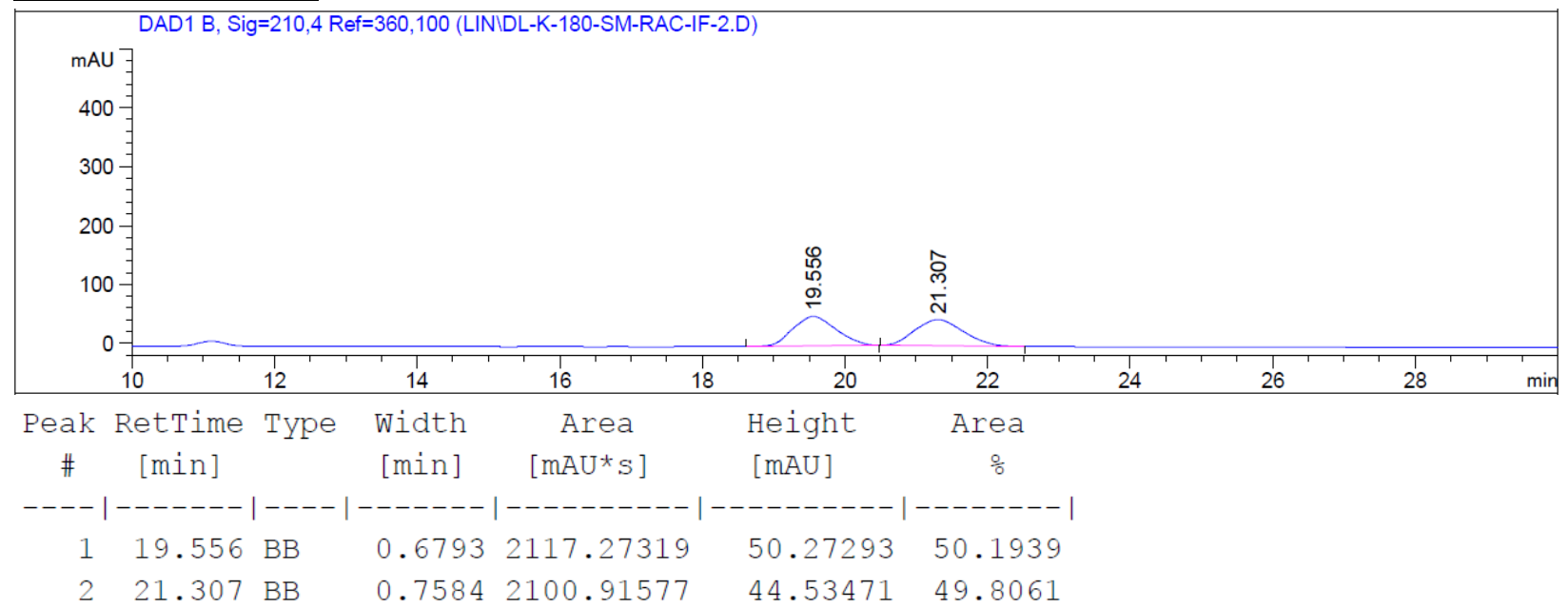

\section{Enantiomeric Sample 1d}

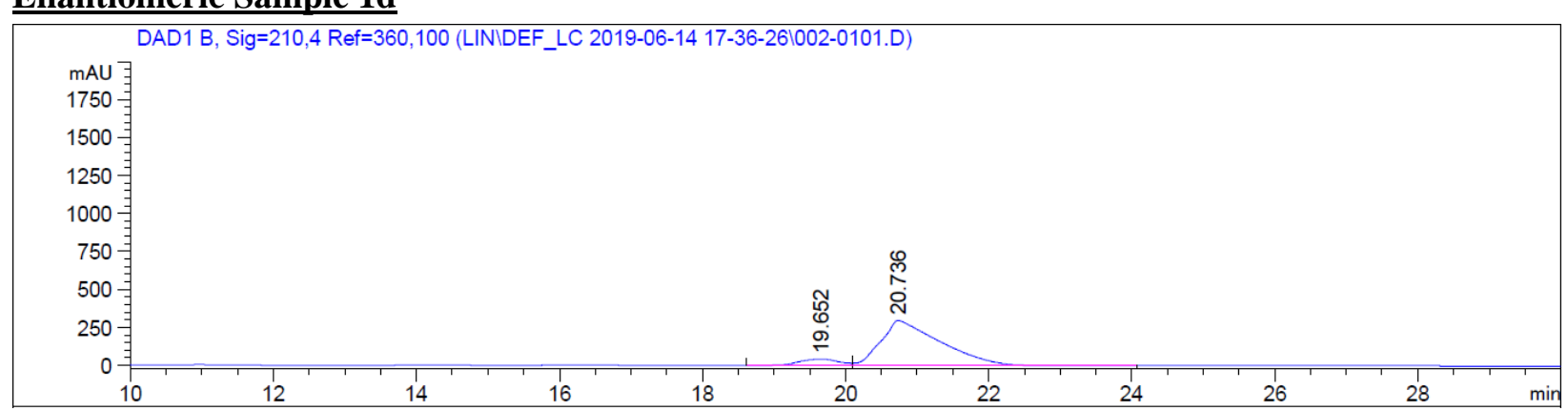



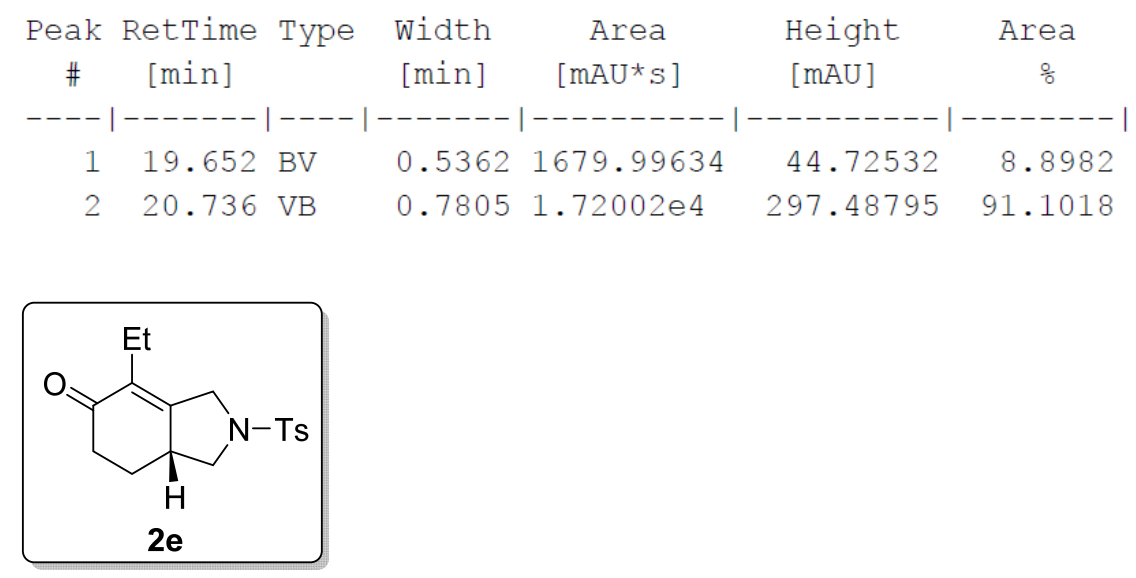

2e (15.2 $\mathrm{mg})$ was isolated as a colorless oil in $48 \%$ yield. The characterization data of $\mathbf{2 e}$ is identical as the reported data. ${ }^{5}$ The HPLC data of $\mathbf{1 e}$ and $\mathbf{2 e}$ are shown below.

Chiral HPLC of 2e (Chiralpak IB, hexane:isopropanol $=92: 8,1 \mathrm{~mL} / \mathrm{min}, 254 \mathrm{~nm}$ ), $\mathrm{t}_{\text {minor }}=32.5 \mathrm{~min}$, $\mathrm{t}_{\text {major }}=27.1 \mathrm{~min} .[\alpha]_{\mathrm{D}}^{22.6}=-205.0\left(\mathrm{c}=1.00, \mathrm{CH}_{2} \mathrm{Cl}_{2}\right)$ at $98 \%$ ee.

\section{Racemic Sample 2e}

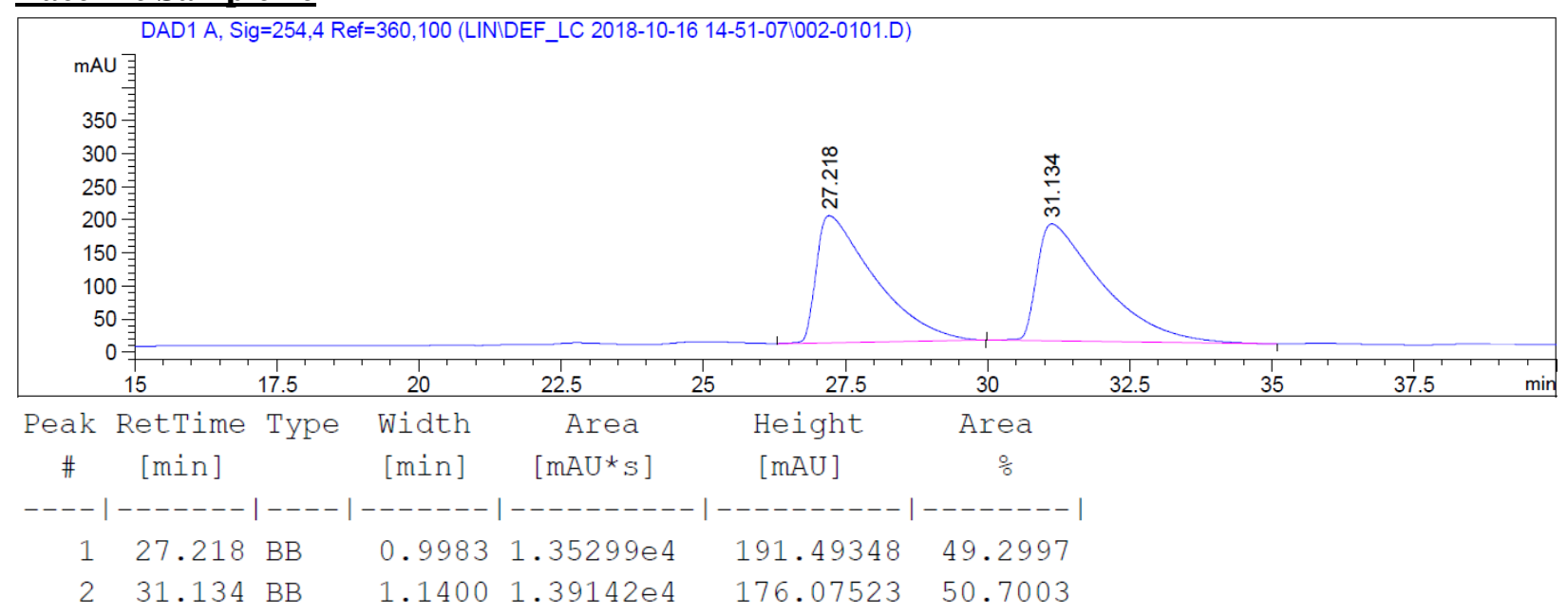

\section{Enantiomeric Sample 2e}

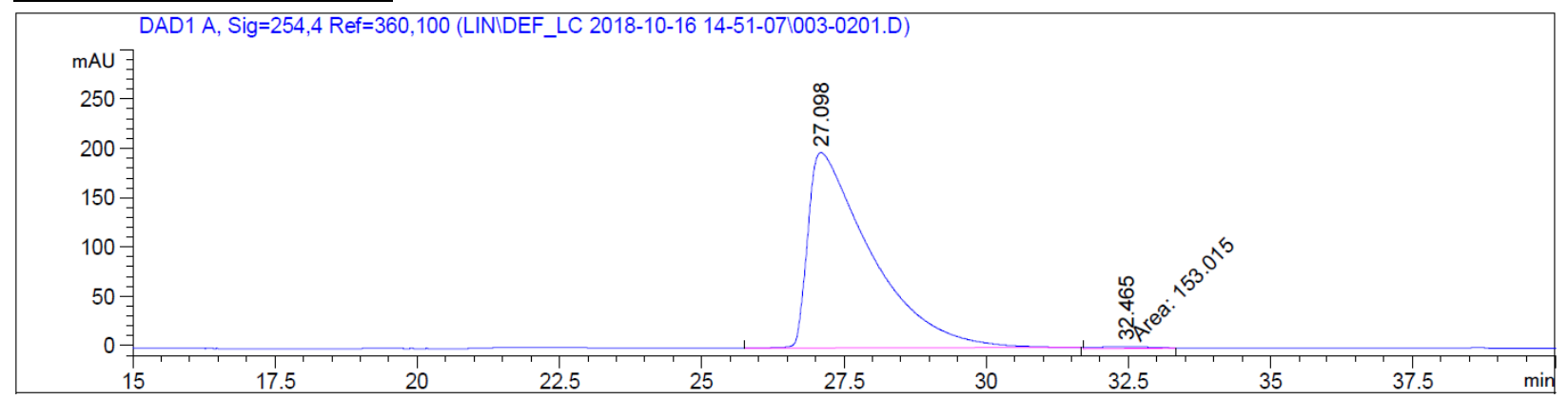




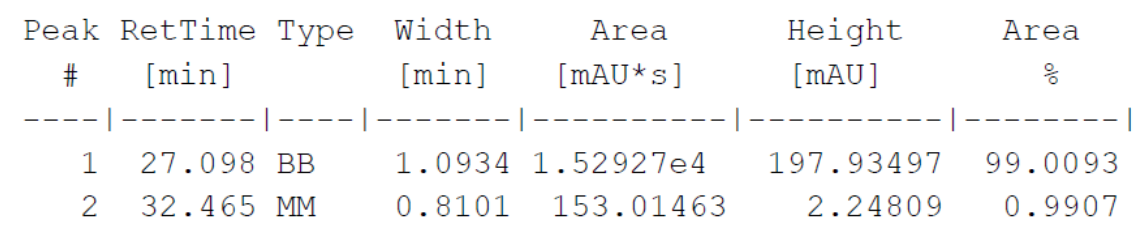

1e (12.6 mg) was recycled as a colorless oil in $40 \%$ yield.

Chiral HPLC for 1e (Chiralpak IA, hexane:isopropanol $=95: 5,1 \mathrm{~mL} / \mathrm{min}, 254 \mathrm{~nm}$ ), $\mathrm{t}_{\text {minor }}=22.9 \mathrm{~min}$, $\mathrm{t}_{\text {major }}=20.7 \mathrm{~min} .[\alpha]_{\mathrm{D}}^{22.6}=-12.7\left(\mathrm{c}=0.79, \mathrm{CH}_{2} \mathrm{Cl}_{2}\right)$ at $98 \%$ ee.

\section{Racemic Sample 1e}

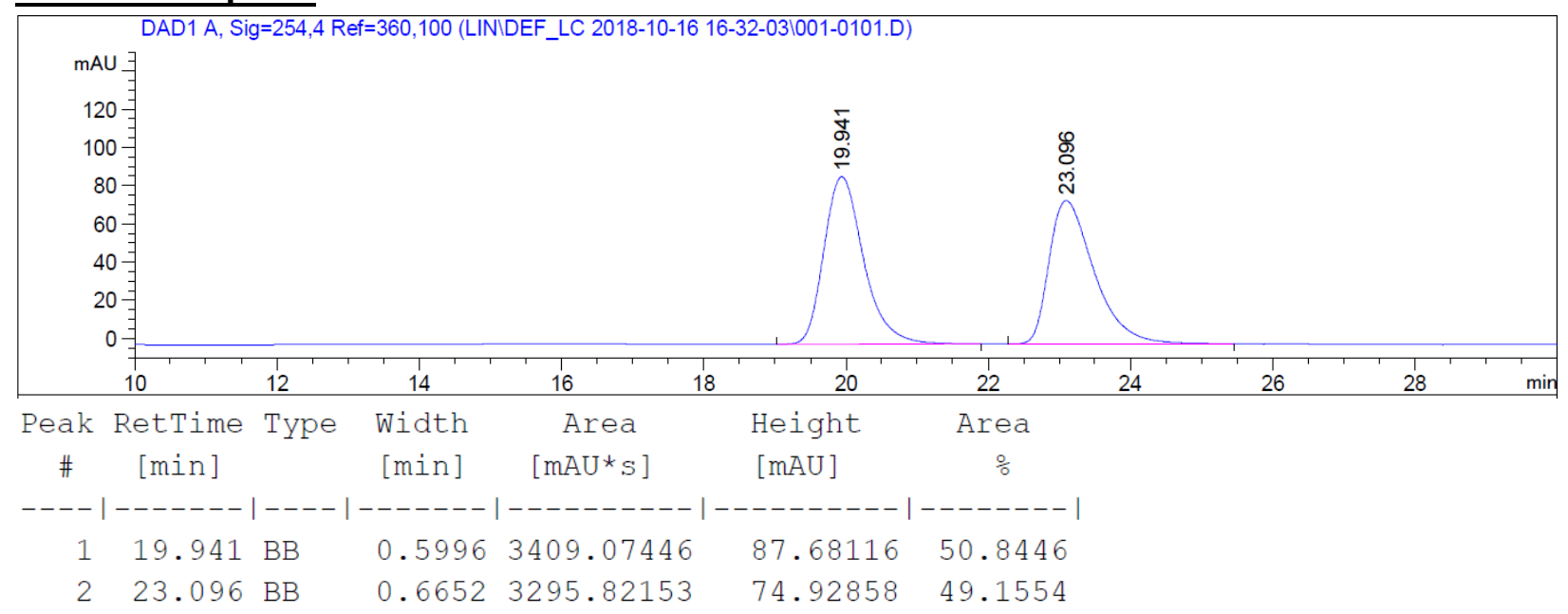

\section{Enantiomeric Sample 1e}

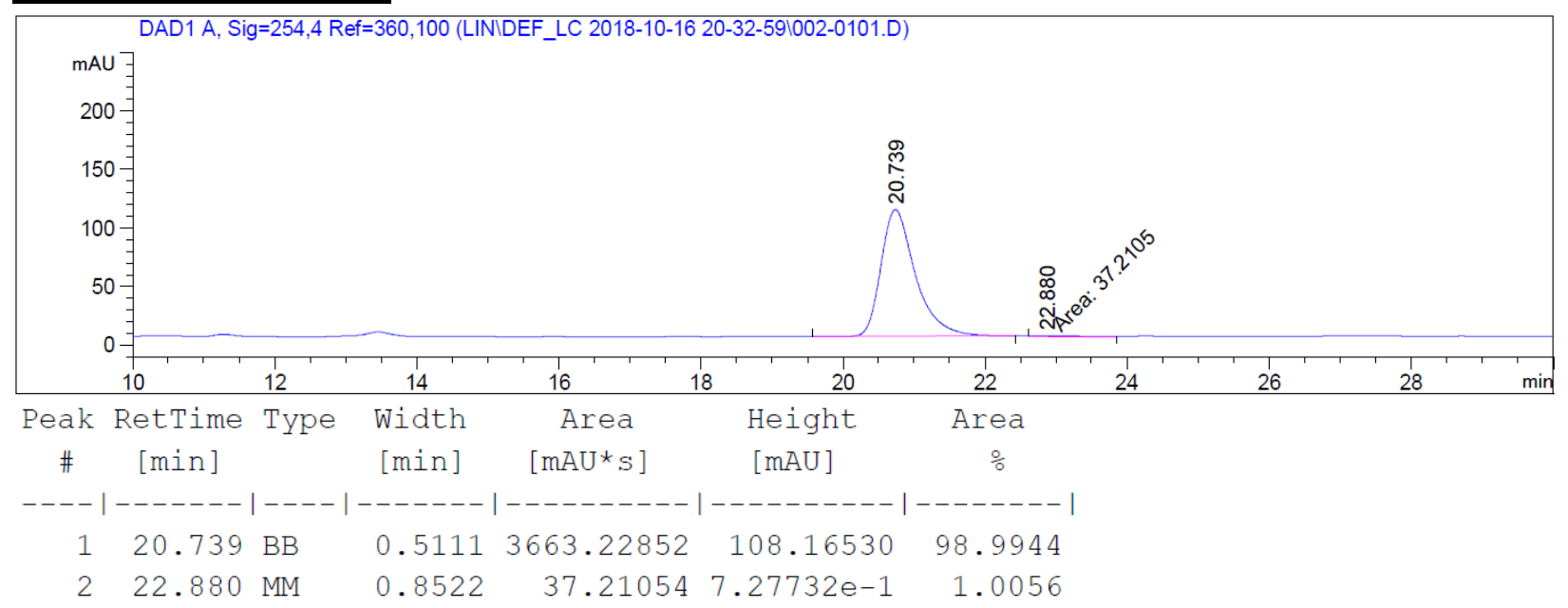




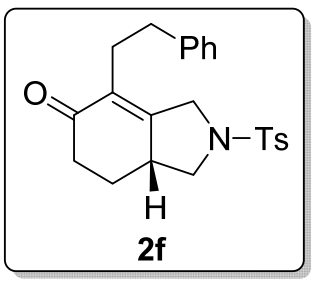

2f $(16.6 \mathrm{mg})$ was isolated as a colorless oil in $42 \%$ yield. $\mathrm{R}_{f}=0.3$ (EtOAc/Hexane=1/4). ${ }^{\mathbf{1}} \mathbf{H}$ NMR (400 MHz, $\left.\mathbf{C D C l}_{3}\right): \delta 7.79-7.60(\mathrm{~m}, 2 \mathrm{H}), 7.41-7.32(\mathrm{~m}, 2 \mathrm{H}), 7.24-7.17(\mathrm{~m}, 2 \mathrm{H}), 7.15-7.01(\mathrm{~m}, 3 \mathrm{H}), 3.90$ - 3.72 (m, 2H), 3.45 (dd, $J=17.0,2.4 \mathrm{~Hz}, 1 \mathrm{H}), 2.92-2.77(\mathrm{~m}, 1 \mathrm{H}), 2.72-2.54(\mathrm{~m}, 2 \mathrm{H}), 2.55-2.39$ (m, 6H), $2.36-2.24$ (m, 2H), 2.10 (dtd, $J=12.3,4.8,2.4 \mathrm{~Hz}, 1 \mathrm{H}), 1.58-1.45$ (m, 1H). ${ }^{13} \mathbf{C}$ NMR (101 MHz, $\left.\mathbf{C D C l}_{3}\right): \delta 197.00,158.41,143.95,141.24,132.81,131.89,129.87,128.59,128.22,127.67,126.09,53.19$, 50.13, 40.63, 36.77, 34.16, 28.88, 26.41, 21.60. IR: v 2925, 1666, 1453, 1346, 1164, 1094, $816 \mathrm{~cm}^{-1}$; HRMS calcd. For $[\mathrm{M}+\mathrm{H}]^{+}: 396.1628$. Found: 396.1627.

Chiral HPLC of $2 \mathbf{f}$ (Chiralpak IB, hexane:isopropanol $=92: 8,1 \mathrm{~mL} / \mathrm{min}, 210 \mathrm{~nm}$ ), $\mathrm{t}_{\text {minor }}=40.4 \mathrm{~min}, \mathrm{t}_{\text {major }}=$ $32.4 \mathrm{~min} .[\alpha]_{\mathrm{D}}^{22.6}=-115.7\left(\mathrm{c}=1.59, \mathrm{CH}_{2} \mathrm{Cl}_{2}\right)$ at $96 \%$ ee.

\section{Racemic Sample 2f}

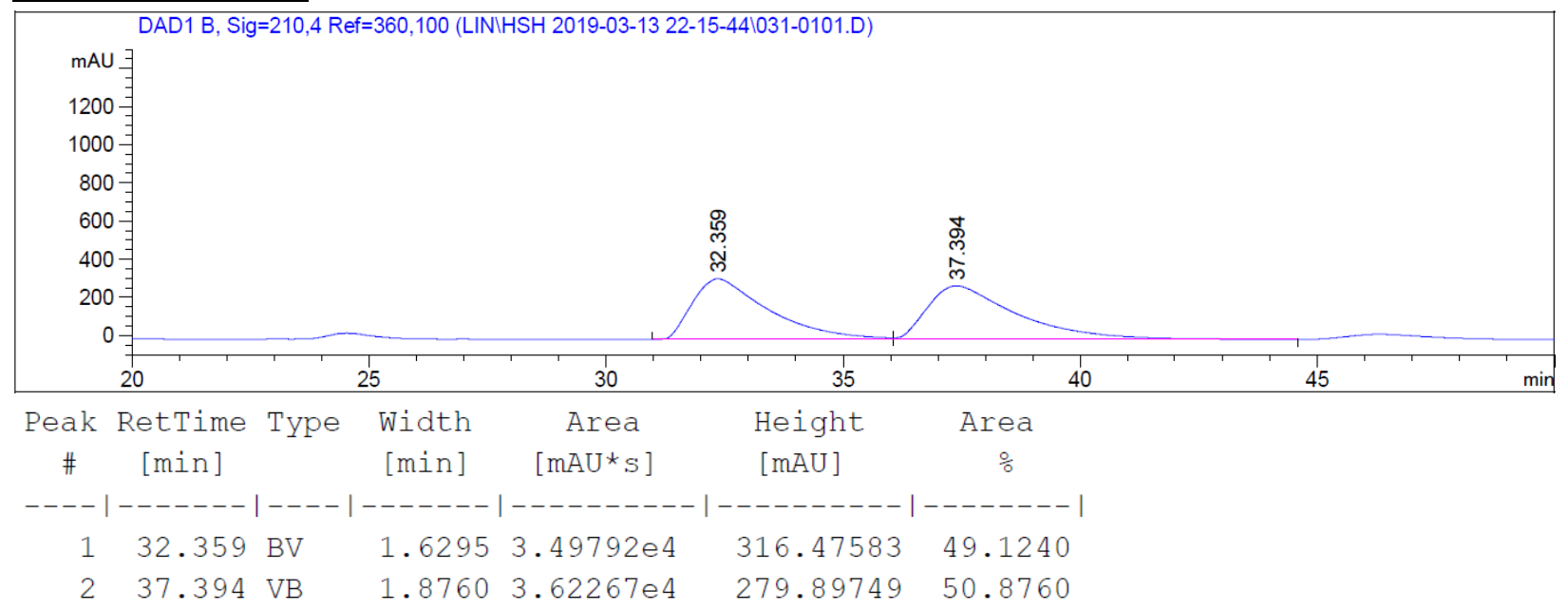

\section{Enantiomeric Sample 2f}

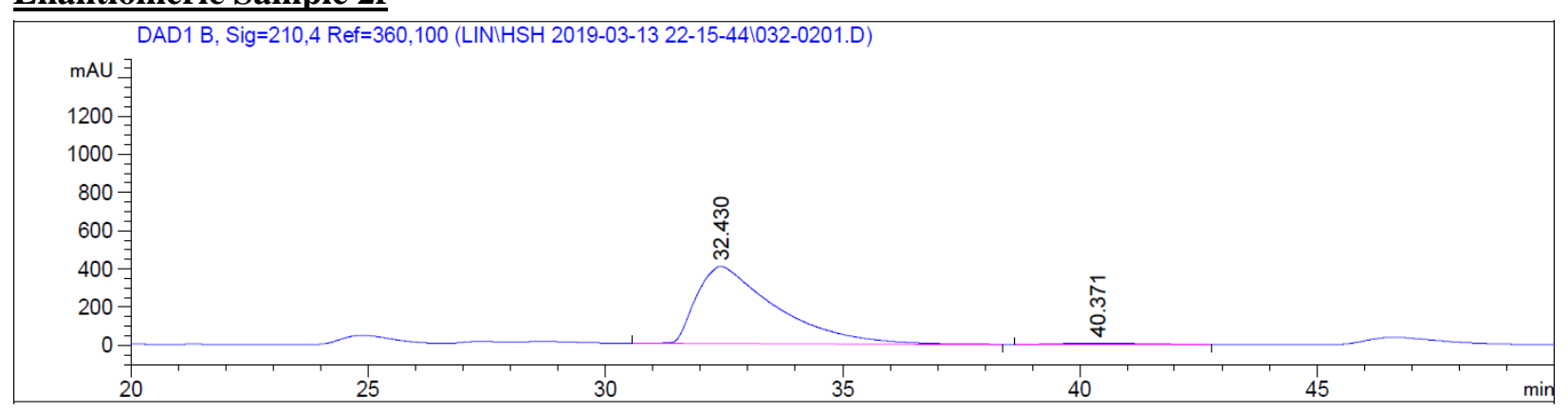




\begin{tabular}{|c|c|c|c|c|c|c|}
\hline $\begin{array}{c}\text { Peak } \\
\#\end{array}$ & $\begin{array}{c}\text { RetTime } \\
\text { [min] }\end{array}$ & Type & $\begin{array}{l}\text { Width } \\
\text { [min] }\end{array}$ & $\begin{array}{c}\text { Area } \\
{\left[m A U^{\star} s\right]}\end{array}$ & $\begin{array}{l}\text { Height } \\
{[\mathrm{mAU}]}\end{array}$ & $\begin{array}{c}\text { Area } \\
\%\end{array}$ \\
\hline & 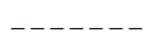 & & & ---------- & ---------- & $--------\mid$ \\
\hline 1 & 32.430 & $\mathrm{BB}$ & 1.6659 & $4.66369 e 4$ & 404.27081 & 98.1967 \\
\hline 2 & 40.371 & $\mathrm{BB}$ & 1.3748 & 856.43756 & 7.63441 & 1.8033 \\
\hline
\end{tabular}

1f $(17.7 \mathrm{mg})$ was recycled as a colorless oil in $45 \%$ yield.

Chiral HPLC for 1f (Chiralpak IA, hexane:isopropanol $=95: 5,1 \mathrm{~mL} / \mathrm{min}, 210 \mathrm{~nm}$ ), $\mathrm{t}_{\text {minor }}=31.5 \mathrm{~min}$, $\mathrm{t}_{\text {major }}=28.9 \mathrm{~min} .[\alpha]_{\mathrm{D}}^{22.6}=-109.6\left(\mathrm{c}=1.35, \mathrm{CH}_{2} \mathrm{Cl}_{2}\right)$ at $90 \%$ ee.

\section{Racemic Sample 1f}

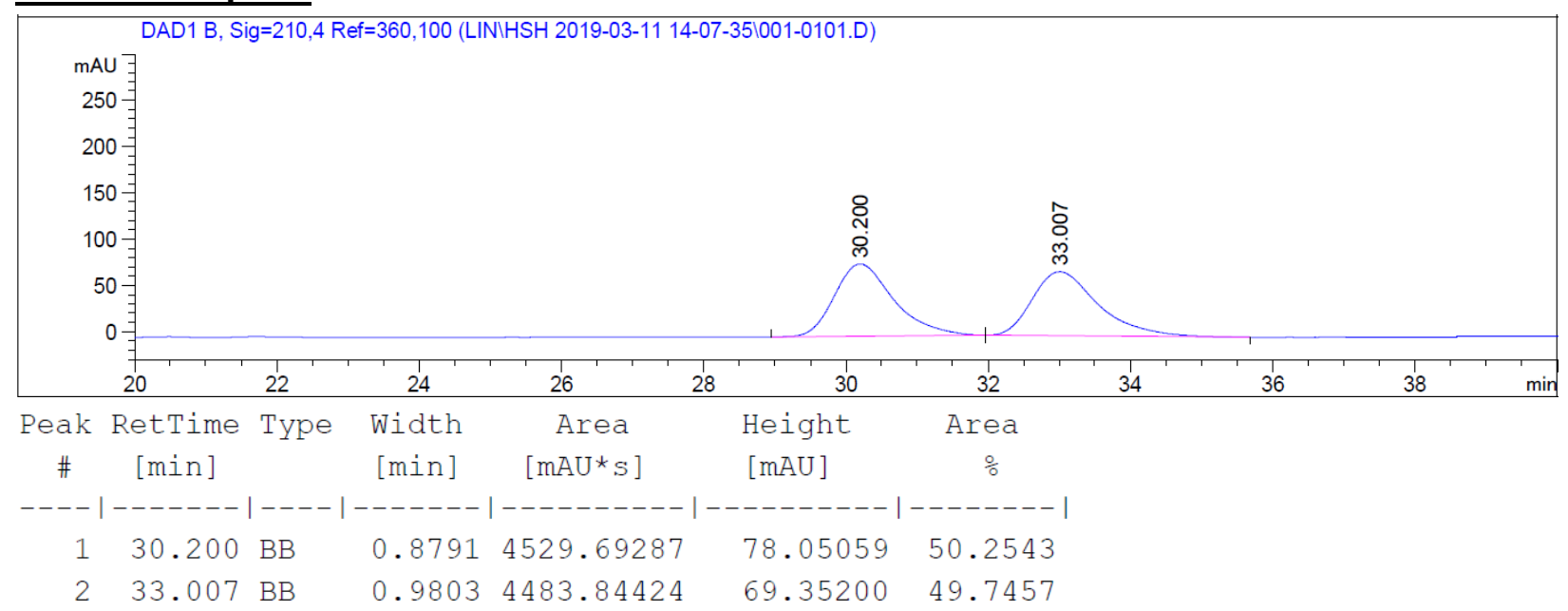

\section{Enantiomeric Sample 1f}

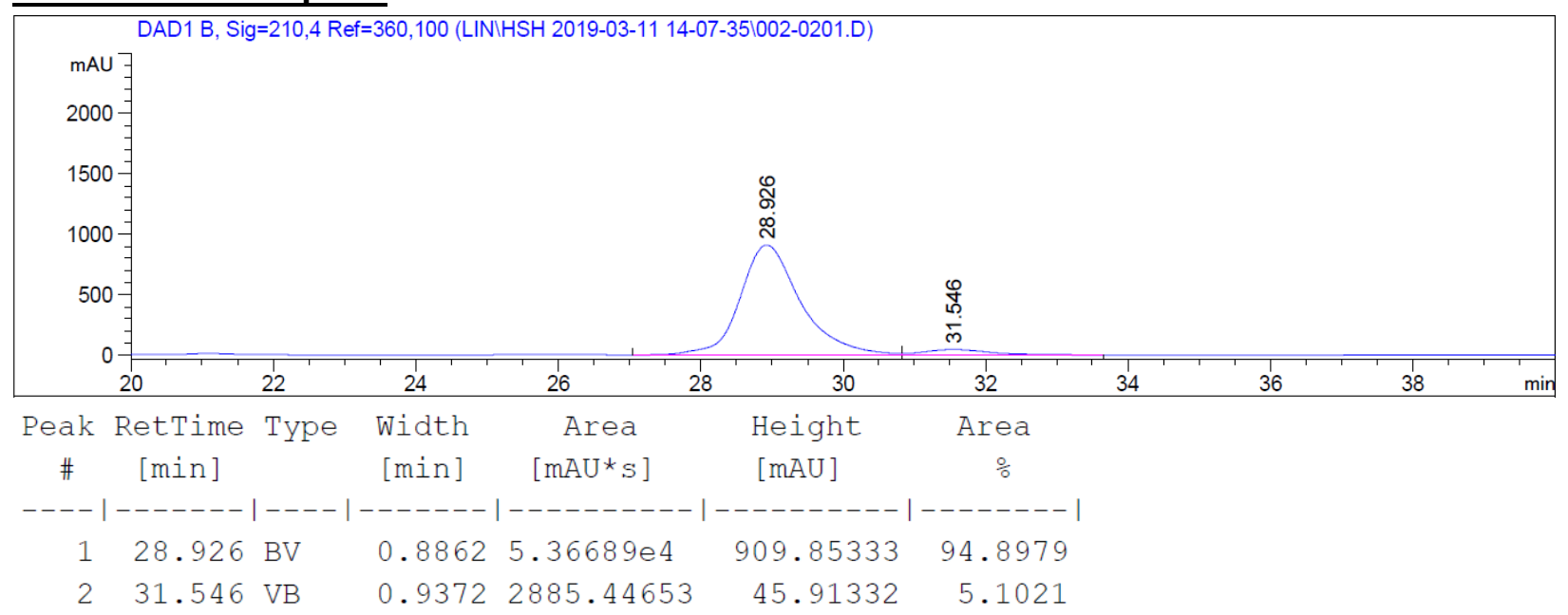




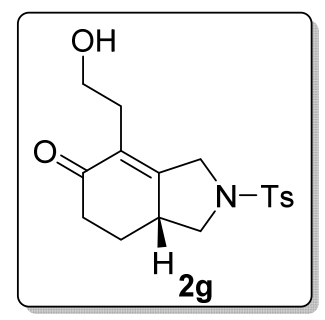

$2 \mathrm{~g}$ (15.4 mg) was isolated as a white solid in $46 \%$ yield. Melting Point: $110-113{ }^{\circ} \mathrm{C} . \mathrm{R}_{f}=0.2$ (EtOAc/Hexane=1/1). ${ }^{1} \mathbf{H}$ NMR (400 MHz, $\left.\mathbf{C D C l}_{3}\right): \delta 7.82-7.64(\mathrm{~m}, 2 \mathrm{H}), 7.46-7.34(\mathrm{~m}, 2 \mathrm{H}), 4.33$ (d, $J=17.2 \mathrm{~Hz}, 1 \mathrm{H}), 4.02-3.84(\mathrm{~m}, 2 \mathrm{H}), 3.62(\mathrm{q}, J=5.3 \mathrm{~Hz}, 2 \mathrm{H}), 3.22-2.87$ (m, 1H), 2.61 (dd, $J=10.9$, $9.3 \mathrm{~Hz}, 1 \mathrm{H}), 2.53$ (ddd, $J=17.5,4.5,2.5 \mathrm{~Hz}, 1 \mathrm{H}), 2.49-2.41(\mathrm{~m}, 4 \mathrm{H}), 2.41-2.25(\mathrm{~m}, 2 \mathrm{H}), 2.21-2.11$ (m, 1H), 1.98 (br, 1H), 1.60 (dddd, $J=14.5,12.7,11.6,4.4 \mathrm{~Hz}, 1 \mathrm{H}) .{ }^{13} \mathbf{C}$ NMR (101 MHz, $\left.\mathbf{C D C l}_{3}\right): \delta$ 198.34, 160.23, 144.16, 132.44, 130.34, 129.92, 127.75, 61.44, 53.36, 50.67, 40.79, 36.58, 29.95, 26.39, 21.59. IR: v 3449, 2927, 1664, 1597, 1344, 1163, 1043, $816 \mathrm{~cm}^{-1}$; HRMS calcd. For $[\mathrm{M}+\mathrm{Na}]^{+}: 358.1083$. Found: 358.1084 .

Chiral HPLC of $\mathbf{2 g}$ (Chiralpak ID, hexane:isopropanol $=80: 20,1 \mathrm{~mL} / \mathrm{min}, 254 \mathrm{~nm}$ ), $\mathrm{t}_{\text {minor }}=93.3 \mathrm{~min}$, $\mathrm{t}_{\text {major }}=80.2 \mathrm{~min} .[\alpha]_{\mathrm{D}}^{22.6}=-440.1\left(\mathrm{c}=1.67, \mathrm{CH}_{2} \mathrm{Cl}_{2}\right)$ at $98 \%$ ee.

\section{Racemic Sample 2g}

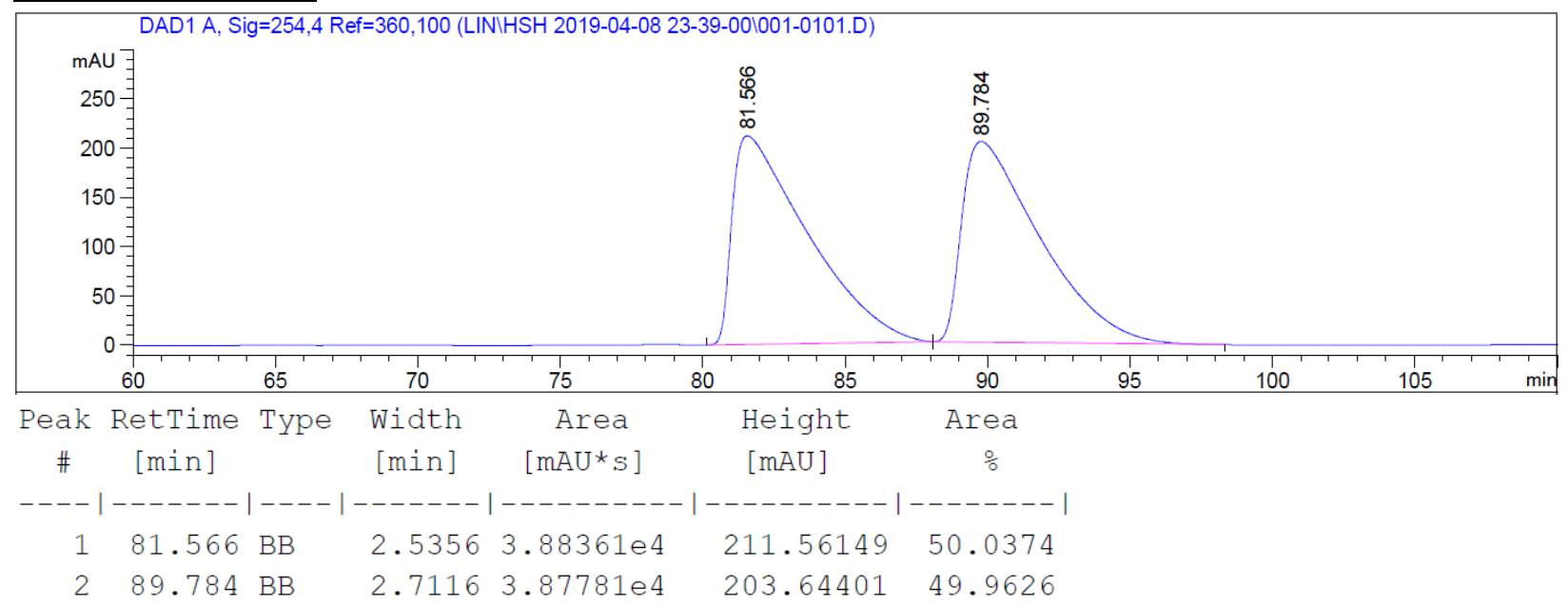

\section{Enantiomeric Sample 2g}

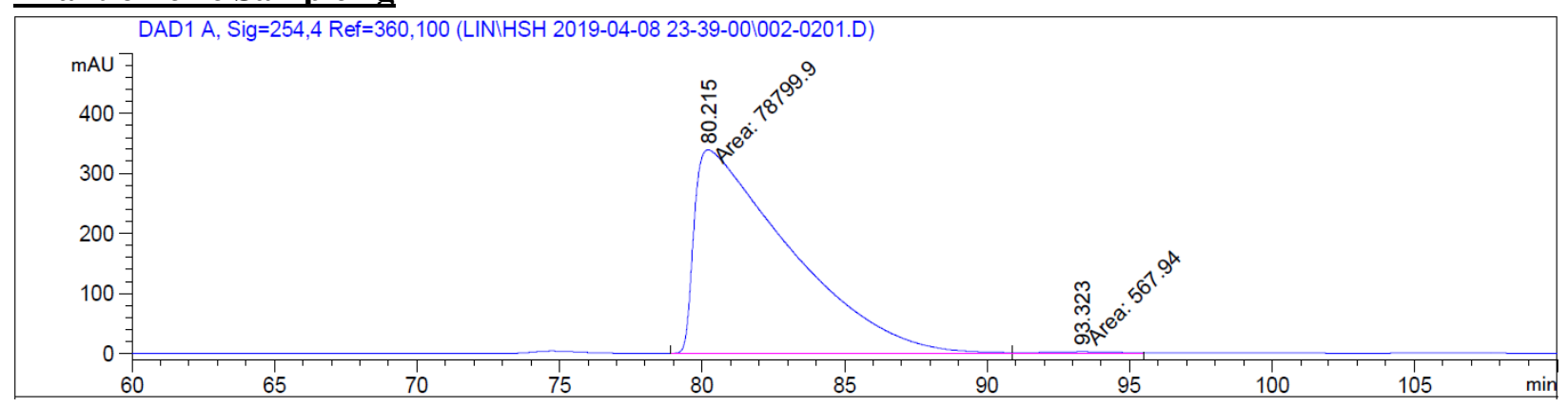




\begin{tabular}{|c|c|c|c|c|c|c|}
\hline $\begin{array}{c}\text { Peak } \\
\#\end{array}$ & $\begin{array}{c}\text { RetTime } \\
\text { [min] }\end{array}$ & Type & $\begin{array}{l}\text { Width } \\
\text { [min] }\end{array}$ & $\begin{array}{c}\text { Area } \\
{\left[\mathrm{mAU}^{*} \mathrm{~s}\right]}\end{array}$ & $\begin{array}{l}\text { Height } \\
\text { [mAU] }\end{array}$ & $\begin{array}{c}\text { Area } \\
\%\end{array}$ \\
\hline & & & - & $\mid-----------$ & ------ & $-------\mid$ \\
\hline 1 & 80.215 & MM & 3.8759 & $7.87999 e 4$ & 338.84863 & 99.2844 \\
\hline 2 & 93.323 & MM & 3.3607 & 567.94049 & 2.81659 & 0.7156 \\
\hline
\end{tabular}

$1 \mathbf{g}(16.0 \mathrm{mg})$ was recycled as a colorless oil in $48 \%$ yield.

Chiral HPLC for $1 \mathrm{~g}$ (Chiralpak IA, hexane:isopropanol $=90: 10,1 \mathrm{~mL} / \mathrm{min}, 230 \mathrm{~nm}$ ), $\mathrm{t}_{\text {minor }}=32.3 \mathrm{~min}$, $\mathrm{t}_{\text {major }}=28.4 \mathrm{~min} .[\alpha]_{\mathrm{D}}^{22.6}=-38.1\left(\mathrm{c}=1.89, \mathrm{CH}_{2} \mathrm{Cl}_{2}\right)$ at $88 \%$ ee.

\section{Racemic Sample 1g}

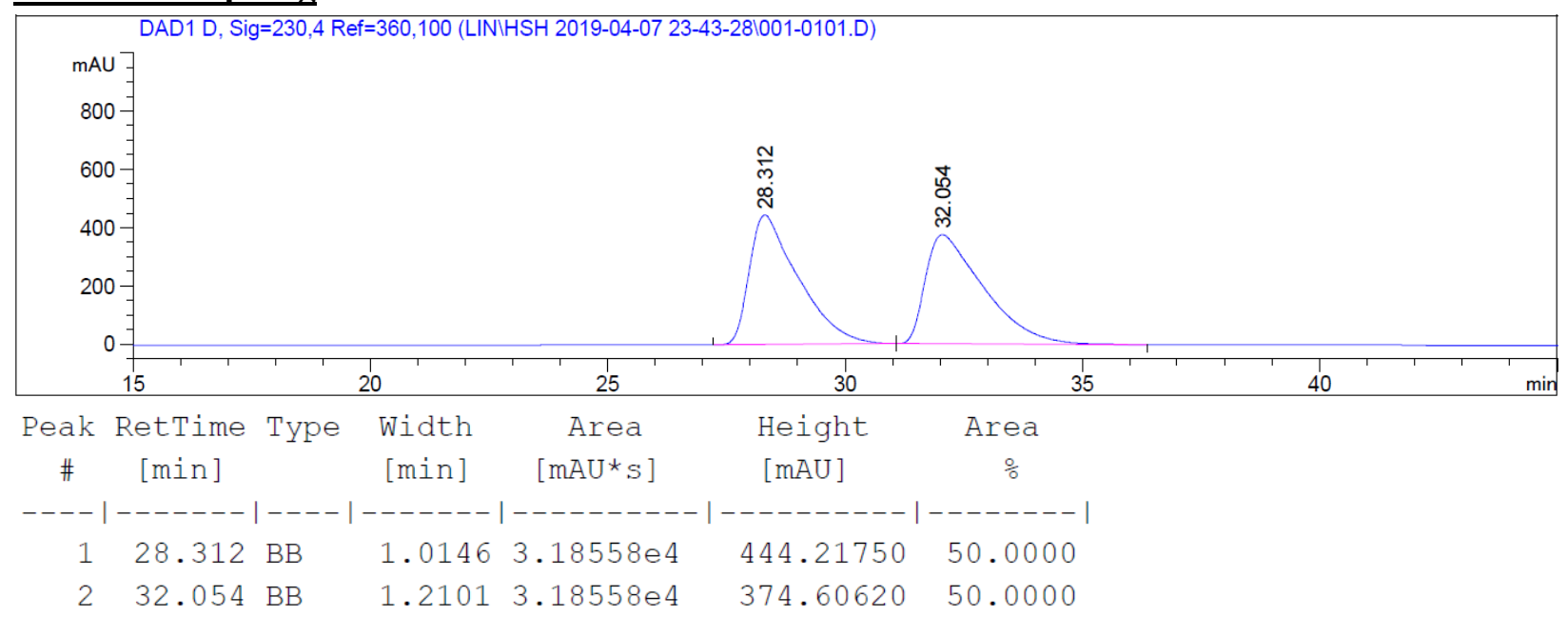

\section{Enantiomeric Sample 1g}

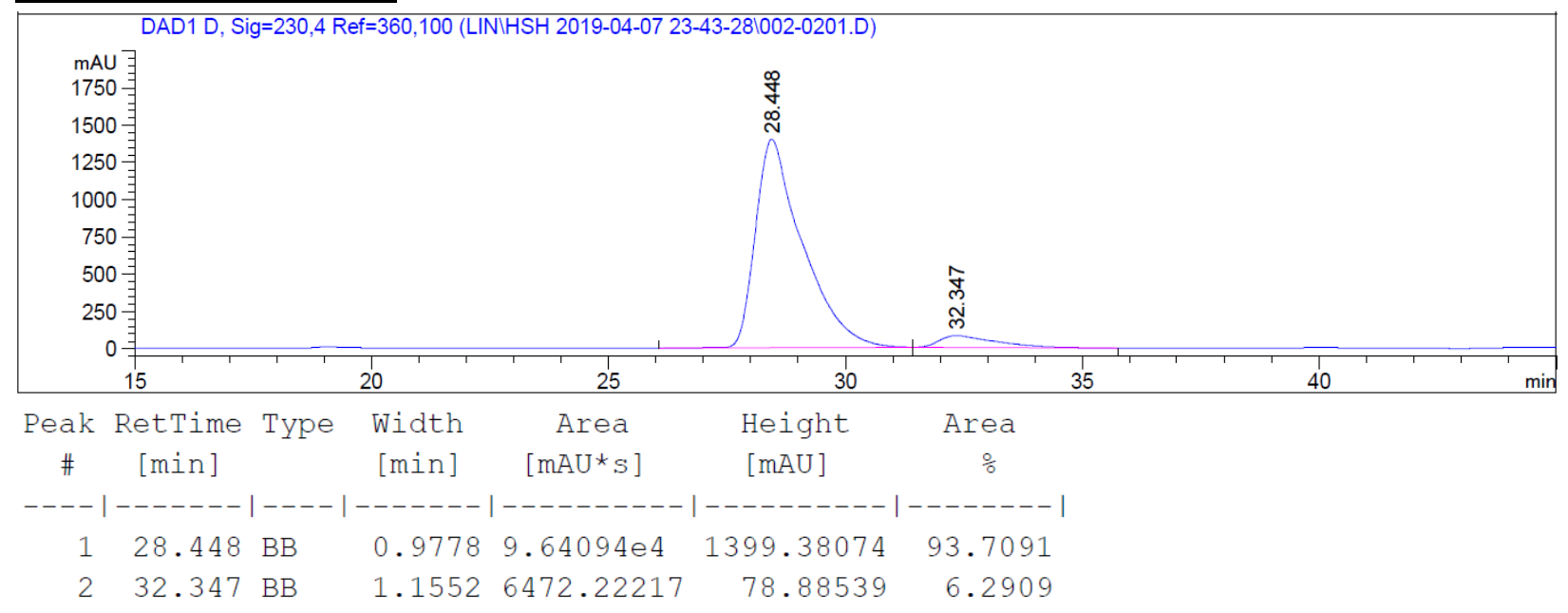




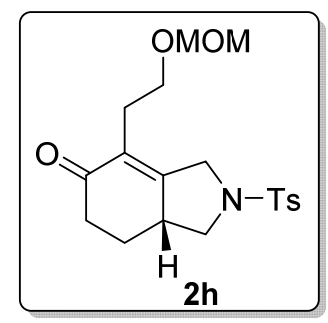

2h (18.2 mg) was isolated as a colorless oil in $48 \%$ yield. $\mathrm{R}_{f}=0.3$ (EtOAc/Hexane=1/2). ${ }^{\mathbf{1}} \mathbf{H}$ NMR (400 MHz, $\left.\mathbf{C D C l}_{3}\right): \delta 7.76-7.69(\mathrm{~m}, 2 \mathrm{H}), 7.42-7.33(\mathrm{~m}, 2 \mathrm{H}), 4.48-4.42(\mathrm{~m}, 2 \mathrm{H}), 4.40-4.31(\mathrm{~m}, 1 \mathrm{H}), 4.02$ - $3.83(\mathrm{~m}, 2 \mathrm{H}), 3.57-3.43(\mathrm{~m}, 2 \mathrm{H}), 3.22(\mathrm{~s}, 3 \mathrm{H}), 3.09-2.90(\mathrm{~m}, 1 \mathrm{H}), 2.57(\mathrm{dd}, J=10.9,9.3 \mathrm{~Hz}, 1 \mathrm{H})$, $2.54-2.40(\mathrm{~m}, 5 \mathrm{H}), 2.38-2.25(\mathrm{~m}, 2 \mathrm{H}), 2.15(\mathrm{dtd}, J=12.3,4.8,2.4 \mathrm{~Hz}, 1 \mathrm{H}), 1.55$ (dddd, $J=14.6,12.7$, 11.6, 4.4 Hz, 1H). ${ }^{13} \mathbf{C}$ NMR (101 MHz, $\left.\mathbf{C D C l}_{3}\right): \delta$ 196.88, 159.93, 144.02, 132.44, 129.92, 129.84, 127.81, 96.14, 66.05, 55.01, 53.51, 50.82, 40.74, 36.60, 27.01, 26.46, 21.57. IR: v 2923, 1666, 1468, $1346,1164,1041,816 \mathrm{~cm}^{-1}$; HRMS calcd. For $[\mathrm{M}+\mathrm{H}]^{+}: 380.1526$. Found: 380.1516.

Chiral HPLC of $\mathbf{2 h}$ (Chiralpak IF, hexane:isopropanol $=85: 15,1 \mathrm{~mL} / \mathrm{min}, 210 \mathrm{~nm}$ ), $\mathrm{t}_{\text {minor }}=53.4 \mathrm{~min}$, $\mathrm{t}_{\text {major }}=71.4 \min .[\alpha]_{\mathrm{D}}^{22.6}=-461.1\left(\mathrm{c}=0.95, \mathrm{CH}_{2} \mathrm{Cl}_{2}\right) 98 \%$ ee.

\section{$\underline{\text { Racemic Sample 2h }}$}

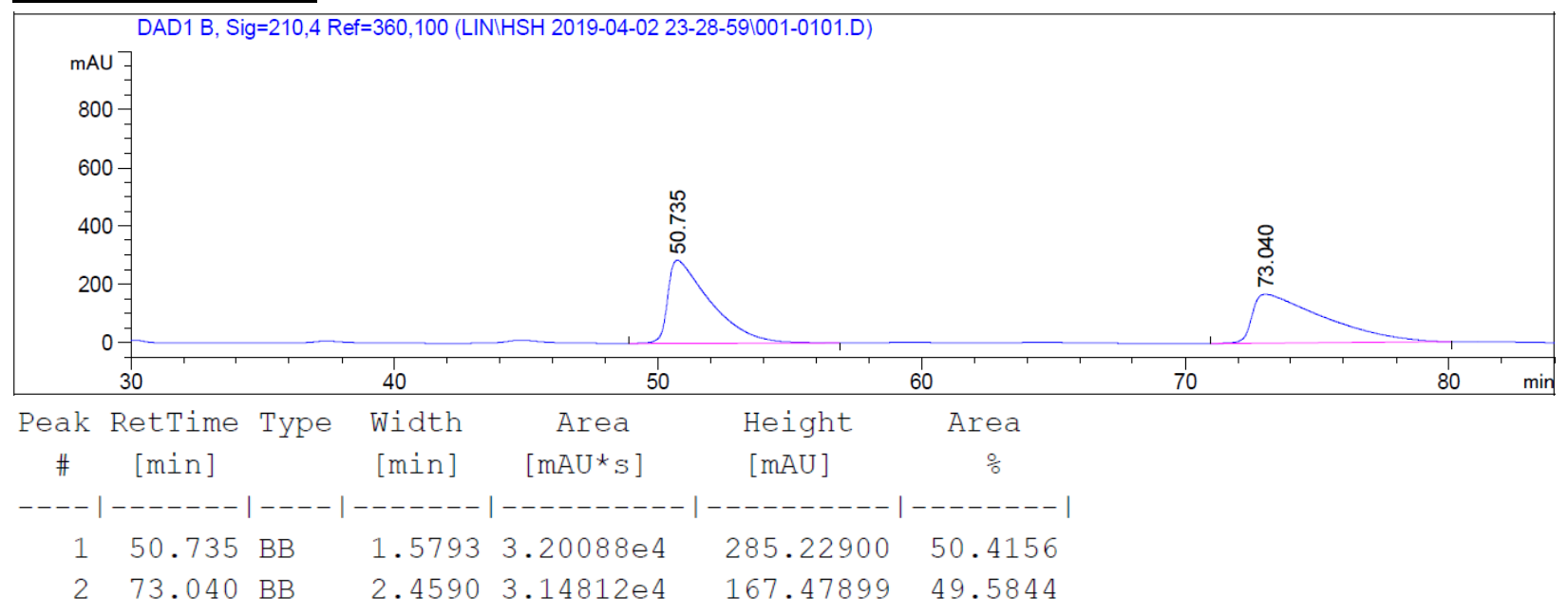

\section{Enantiomeric Sample 2h}

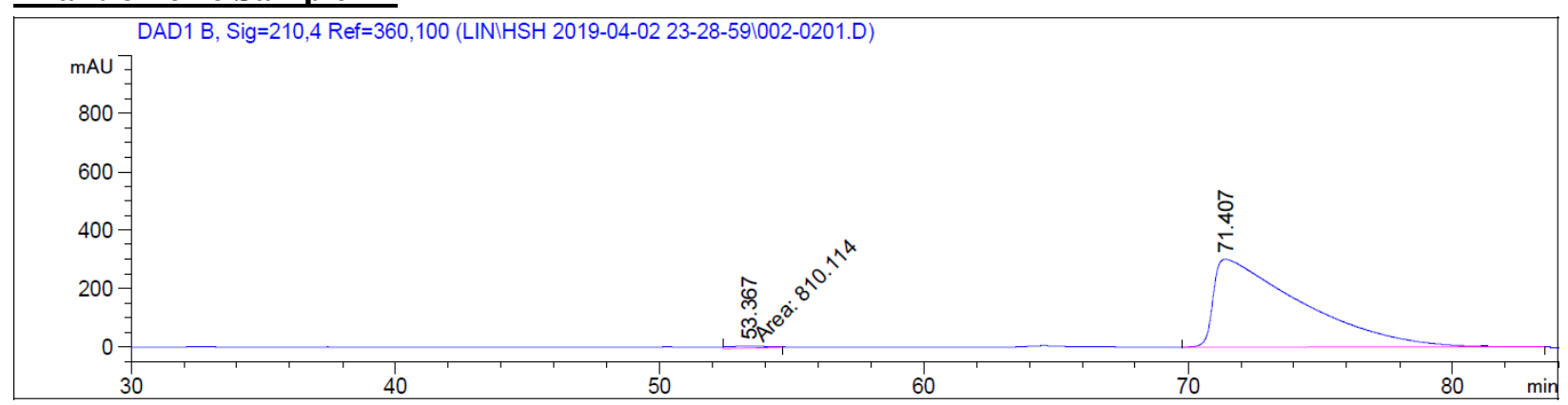




\begin{tabular}{|c|c|c|c|c|c|c|}
\hline $\begin{array}{c}\text { Peak } \\
\#\end{array}$ & $\begin{array}{c}\text { RetTime } \\
\text { [min] }\end{array}$ & Type & $\begin{array}{l}\text { Width } \\
\text { [min] }\end{array}$ & $\begin{array}{c}\text { Area } \\
{\left[\mathrm{mAU}{ }^{\star} \mathrm{S}\right]}\end{array}$ & $\begin{array}{l}\text { Height } \\
{[\mathrm{mAU}]}\end{array}$ & $\begin{array}{c}\text { Area } \\
\%\end{array}$ \\
\hline & ------- & -- & ------- & ------------ & ---------- & $--------\mid$ \\
\hline 1 & 53.367 & MM & 1.1920 & 810.11365 & 8.17228 & 1.1493 \\
\hline 2 & 71.407 & BB & 2.9724 & $6.96760 \mathrm{e} 4$ & 301.02255 & 98.8507 \\
\hline
\end{tabular}

1h (19.0 mg) was recycled as a colorless oil in 50\% yield.

Chiral HPLC for $\mathbf{1 h}$ (Chiralpak IA, hexane:isopropanol = 92:8, $1 \mathrm{~mL} / \mathrm{min}, 214 \mathrm{~nm}$ ), $\mathrm{t}_{\text {minor }}=30.1 \mathrm{~min}$, $\mathrm{t}_{\text {major }}=22.8 \mathrm{~min} .[\alpha]_{\mathrm{D}}^{22.6}=-22.7\left(\mathrm{c}=2.42, \mathrm{CH}_{2} \mathrm{Cl}_{2}\right)$ at $98 \%$ ee.

\section{Racemic Sample 1h}

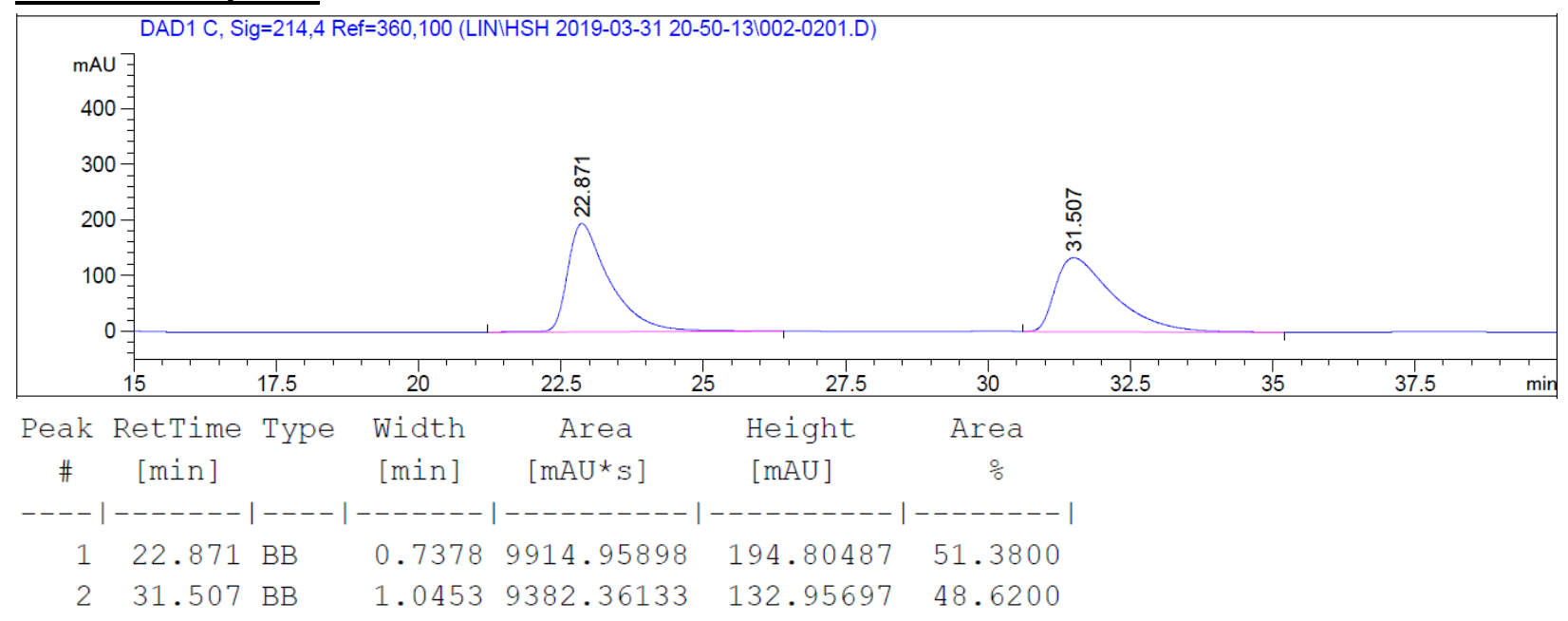

\section{Enantiomeric Sample 1h}

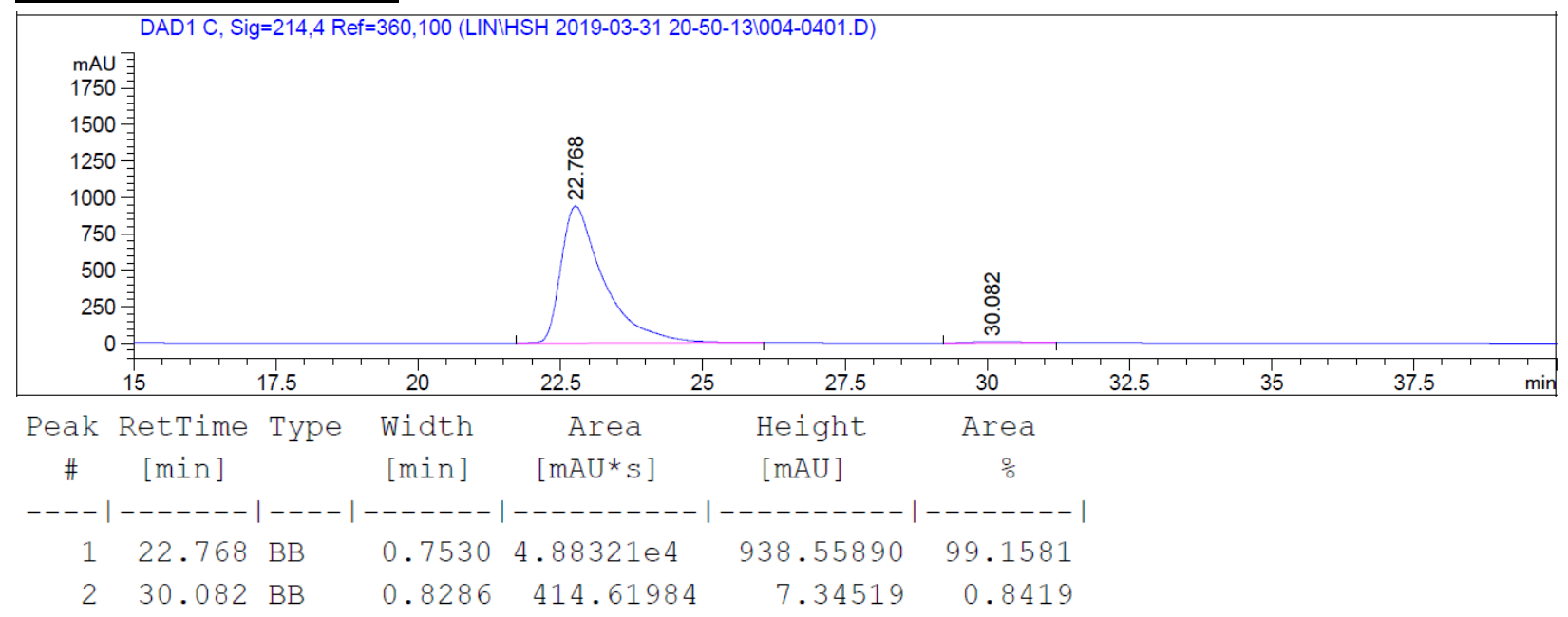




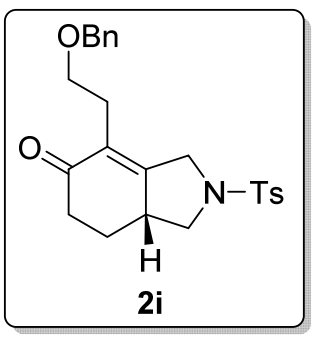

$2 \mathbf{i}(16.1 \mathrm{mg})$ was isolated as a colorless oil in $38 \%$ yield. $\mathrm{R}_{f}=0.4($ EtOAc/Hexane $=1 / 3) .{ }^{\mathbf{1}} \mathbf{H}$ NMR (400 MHz, $\left.\mathbf{C D C l}_{3}\right): \delta 7.75-7.64(\mathrm{~m}, 2 \mathrm{H}), 7.37-7.24(\mathrm{~m}, 5 \mathrm{H}), 7.22-7.16(\mathrm{~m}, 2 \mathrm{H}), 4.47-4.24(\mathrm{~m}, 3 \mathrm{H}), 3.97$ $(\mathrm{dd}, J=17.3,2.4 \mathrm{~Hz}, 1 \mathrm{H}), 3.89(\mathrm{dd}, J=9.3,8.0 \mathrm{~Hz}, 1 \mathrm{H}), 3.48-3.39(\mathrm{~m}, 2 \mathrm{H}), 3.00-2.86(\mathrm{~m}, 1 \mathrm{H}), 2.59$ $(\mathrm{dd}, J=10.9,9.3 \mathrm{~Hz}, 1 \mathrm{H}), 2.52-2.42(\mathrm{~m}, 2 \mathrm{H}), 2.41-2.21(\mathrm{~m}, 5 \mathrm{H}), 2.11(\mathrm{dtd}, J=12.3,4.9,2.4 \mathrm{~Hz}, 1 \mathrm{H})$, 1.52 (dddd, $J=14.5,12.7,11.6,4.4 \mathrm{~Hz}, 1 \mathrm{H}) .{ }^{13} \mathbf{C}$ NMR (101 MHz, $\left.\mathbf{C D C l}_{3}\right)$ : $\delta$ 196.96, 159.92, 143.97, $138.31,132.56,130.00,129.82,128.35,127.74,127.53,127.39,72.74,68.54,53.45,50.93,40.71,36.58$, 27.14, 26.42, 21.53. IR: v 2925, 1667, 1598, 1453, 1347, 1164, 1095, $816 \mathrm{~cm}^{-1}$; HRMS calcd. For $[\mathrm{M}+\mathrm{H}]^{+}:$426.1734. Found: 426.1734 .

Chiral HPLC of 2i (Chiralpak IB, hexane:isopropanol $=92: 8,1 \mathrm{~mL} / \mathrm{min}, 254 \mathrm{~nm}$ ), $\mathrm{t}_{\text {minor }}=53.6$ min, $\mathrm{t}_{\text {major }}=$ 41.9 min. $[\alpha]_{\mathrm{D}}^{22.6}=-299.0\left(\mathrm{c}=1.94, \mathrm{CH}_{2} \mathrm{Cl}_{2}\right)$ at $98 \%$ ee.

\section{Racemic Sample 2i}

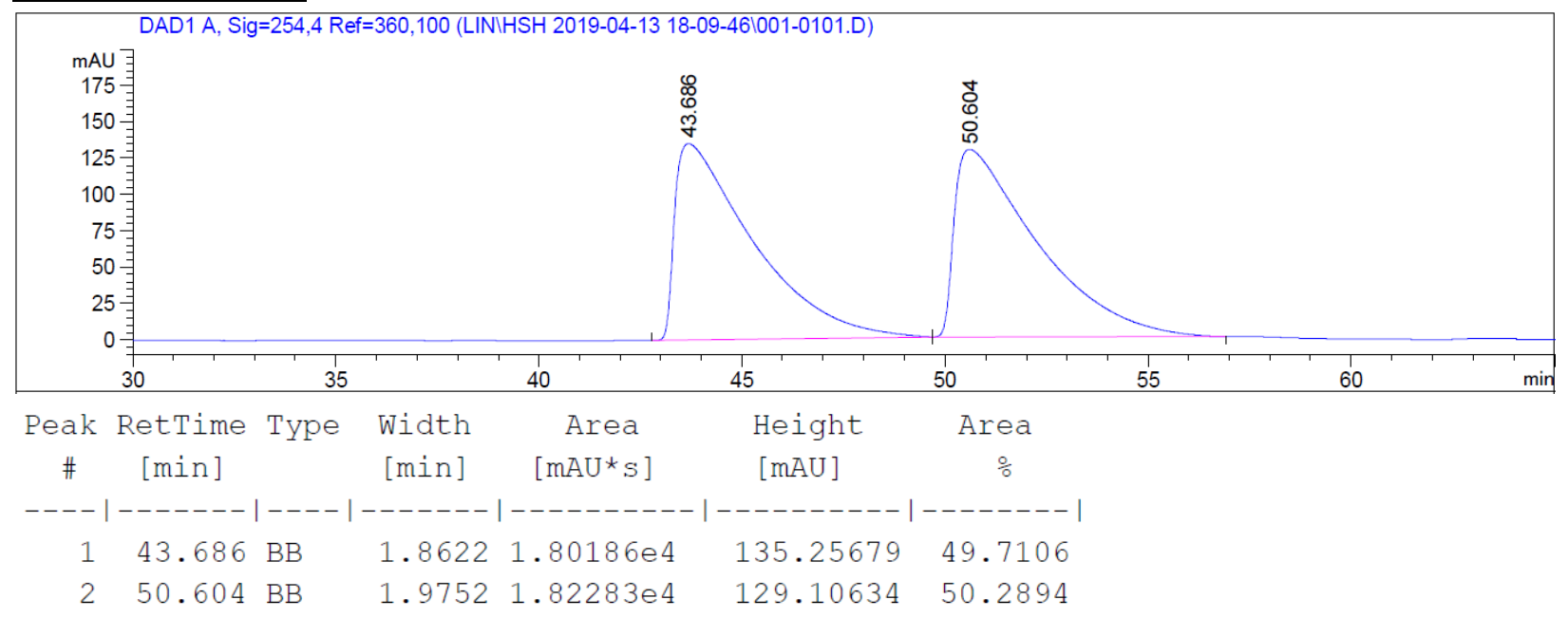

\section{Enantiomeric Sample 2i}

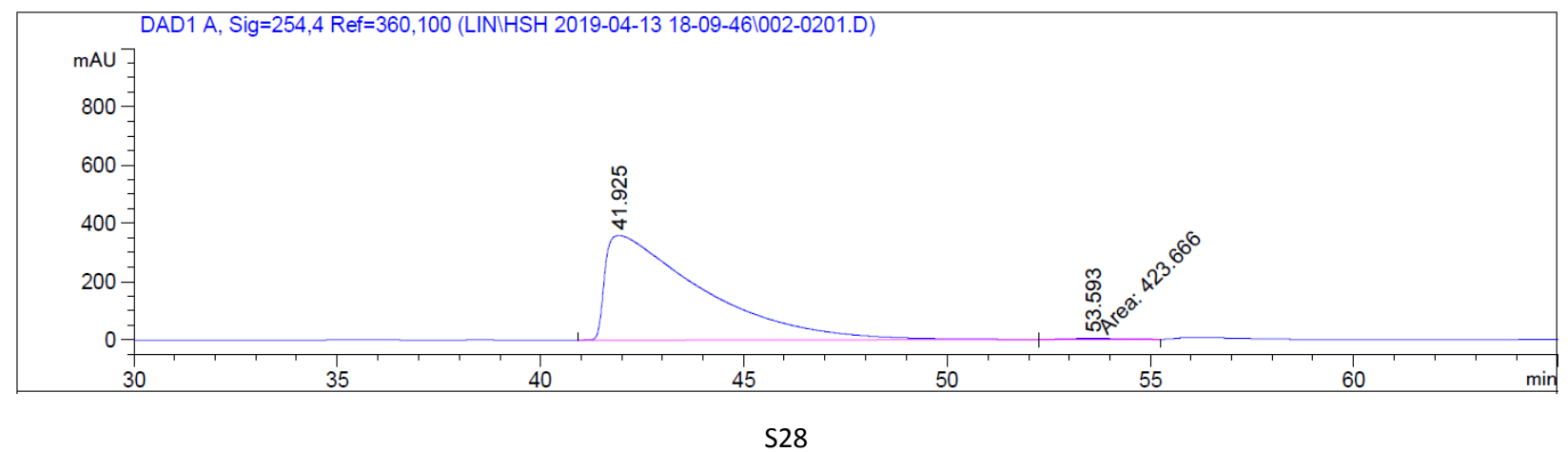




\begin{tabular}{|c|c|c|c|c|c|c|}
\hline $\begin{array}{c}\text { Peak } \\
\quad \#\end{array}$ & $\begin{array}{c}\text { RetTime } \\
\text { [min] }\end{array}$ & Type & $\begin{array}{l}\text { Width } \\
\text { [min] }\end{array}$ & $\begin{array}{c}\text { Area } \\
{\left[m A U^{*} \mathrm{~s}\right]}\end{array}$ & $\begin{array}{l}\text { Height } \\
{[\mathrm{mAU}]}\end{array}$ & $\begin{array}{c}\text { Area } \\
\quad \%\end{array}$ \\
\hline-- & ------- & & ---- & ----------- & -----------1 & $--------\mid$ \\
\hline 1 & 41.925 & $\mathrm{BB}$ & 2.2364 & $5.90058 e 4$ & 358.84818 & 99.2871 \\
\hline 2 & 53.593 & MM & 1.7877 & 423.66602 & 3.94976 & 0.7129 \\
\hline
\end{tabular}

$1 \mathbf{i}(19.0 \mathrm{mg})$ was recycled as a colorless oil in $45 \%$ yield.

Chiral HPLC for 1i (Chiralpak IB, hexane:isopropanol $=92: 8,1 \mathrm{~mL} / \mathrm{min}, 210 \mathrm{~nm}$ ), $\mathrm{t}_{\text {minor }}=24.5 \mathrm{~min}$, $\mathrm{t}_{\text {major }}=21.4 \mathrm{~min} .[\alpha]_{\mathrm{D}}^{22.6}=-77.2\left(\mathrm{c}=2.06, \mathrm{CH}_{2} \mathrm{Cl}_{2}\right)$ at $98 \%$ ee.

\section{Racemic Sample 1i}

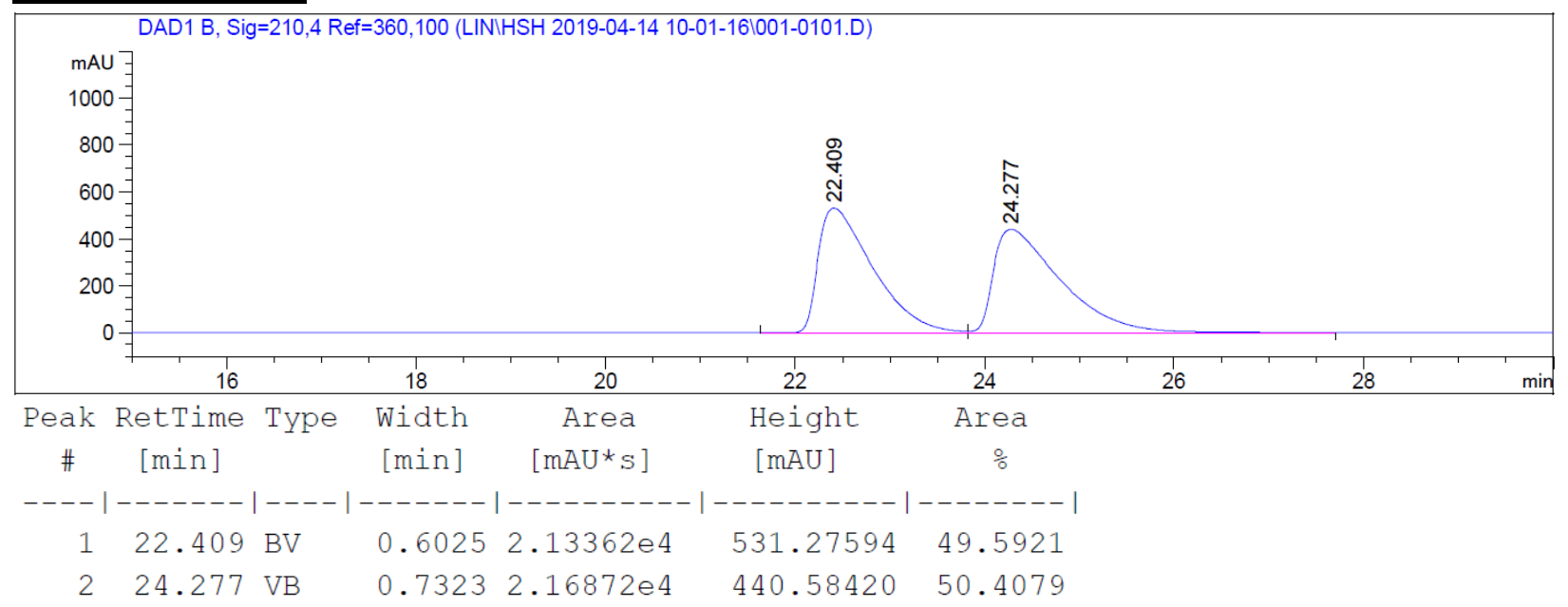

\section{Enantiomeric Sample 1i}

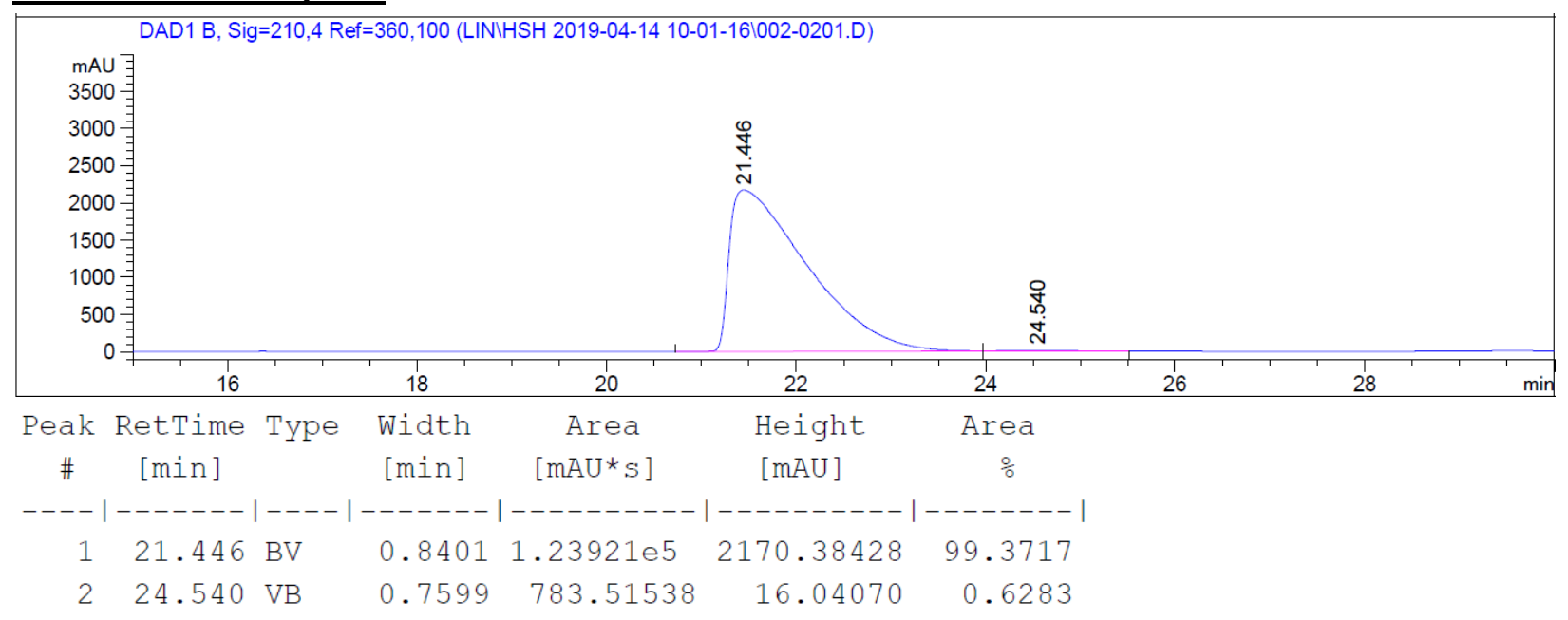




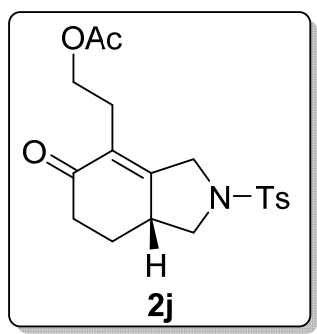

$2 \mathbf{j}(17.5 \mathrm{mg})$ was isolated as a colorless oil in $46 \%$ yield. $\mathrm{R}_{f}=0.3($ EtOAc/Hexane $=1 / 2) .{ }^{\mathbf{1}} \mathbf{H}$ NMR (400 MHz, $\mathbf{C D C l}_{3}$ ): $\delta 7.81-7.69(\mathrm{~m}, 2 \mathrm{H}), 7.46-7.33(\mathrm{~m}, 2 \mathrm{H}), 4.30$ (d, $\left.J=17.2 \mathrm{~Hz}, 1 \mathrm{H}\right), 4.09$ (ddd, $J=10.8$, 6.7, $6.0 \mathrm{~Hz}, 1 \mathrm{H}), 4.00$ (ddd, $J=10.9,6.9,6.0 \mathrm{~Hz}, 1 \mathrm{H}), 3.92(\mathrm{dd}, J=9.3,7.9 \mathrm{~Hz}, 1 \mathrm{H}), 3.86$ (dd, $J=17.1$, $2.4 \mathrm{~Hz}, 1 \mathrm{H}), 3.07-2.94(\mathrm{~m}, 1 \mathrm{H}), 2.60(\mathrm{dd}, J=10.9,9.3 \mathrm{~Hz}, 1 \mathrm{H}), 2.57-2.42(\mathrm{~m}, 5 \mathrm{H}), 2.42-2.27(\mathrm{~m}$, 2H), 2.15 (dtd, $J=12.3,4.9,2.4 \mathrm{~Hz}, 1 \mathrm{H}), 1.90(\mathrm{~s}, 3 \mathrm{H}), 1.65-1.51(\mathrm{~m}, 1 \mathrm{H}) .{ }^{13} \mathbf{C}$ NMR (101 MHz, $\left.\mathbf{C D C l}_{3}\right): \delta 196.63,170.71,159.80,144.17,132.51,129.94,129.16,127.77,62.55,53.39,50.49,40.81$, 36.47, 26.33, 26.20, 21.56, 20.78. IR: v 2953, 1738, 1668, 1598, 1347, 1241, 1164, 1043, $817 \mathrm{~cm}^{-1}$; HRMS calcd. For $[\mathrm{M}+\mathrm{H}]^{+}: 378.1370$. Found: 378.1368 .

Chiral HPLC of $\mathbf{2} \mathbf{j}$ (Chiralpak IB, hexane:isopropanol $=80: 20,0.7 \mathrm{~mL} / \mathrm{min}, 254 \mathrm{~nm}$ ), $\mathrm{t}_{\text {minor }}=43.9 \mathrm{~min}$, $\mathrm{t}_{\text {major }}=36.8 \mathrm{~min} .[\alpha]_{\mathrm{D}}^{22.6}=-271.3\left(\mathrm{c}=1.74, \mathrm{CH}_{2} \mathrm{Cl}_{2}\right)$ at $96 \%$ ee.

\section{Racemic Sample 2i}

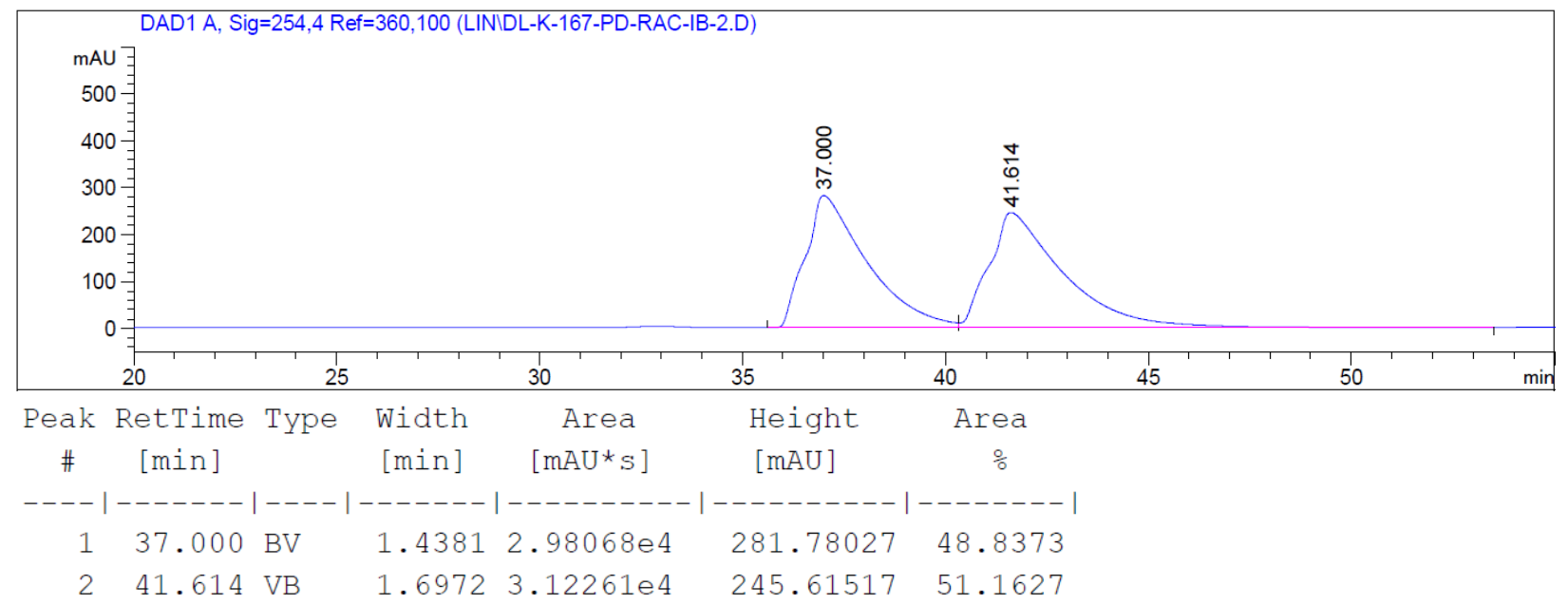

\section{Enantiomeric Sample 2i}

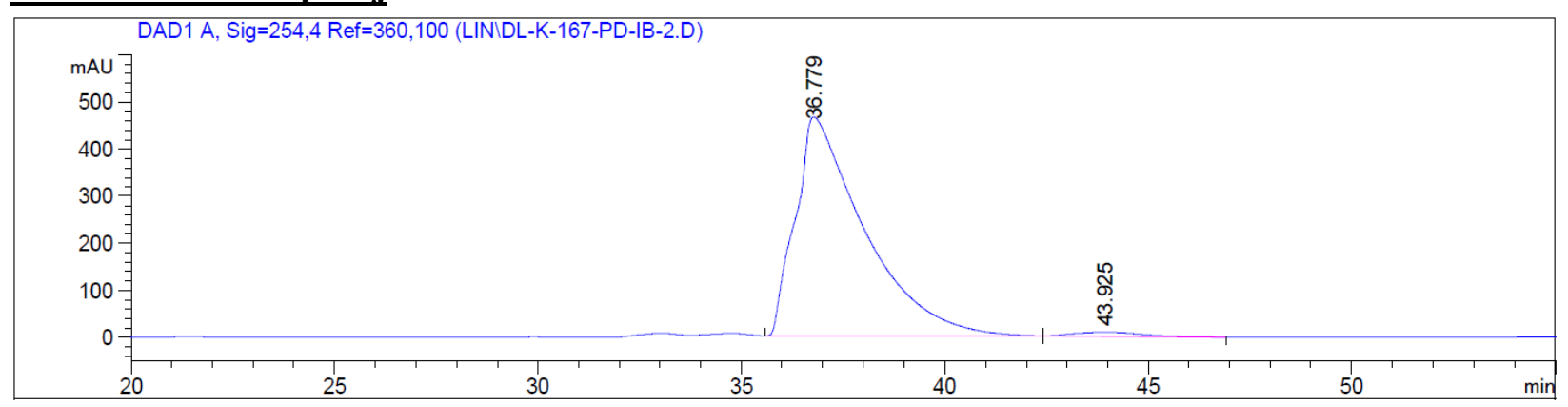




\begin{tabular}{|c|c|c|c|c|c|c|}
\hline $\begin{array}{c}\text { Peak } \\
\#\end{array}$ & $\begin{array}{c}\text { RetTime } \\
\text { [min] }\end{array}$ & Type & $\begin{array}{l}\text { Width } \\
\text { [min] }\end{array}$ & $\begin{array}{c}\text { Area } \\
{\left[\mathrm{mAU}^{*} \mathrm{~s}\right]}\end{array}$ & $\begin{array}{l}\text { Height } \\
{[\mathrm{mAU}]}\end{array}$ & $\begin{array}{c}\text { Area } \\
\frac{\circ}{\circ}\end{array}$ \\
\hline-- & ----- & & ----- & | ---------- & ---------- & $--------\mid$ \\
\hline 1 & 36.779 & BB & 1.6022 & $5.47271 e 4$ & 464.62576 & 98.2376 \\
\hline 2 & 43.925 & $\mathrm{BB}$ & 1.4614 & 981.81592 & 9.18286 & 1.7624 \\
\hline
\end{tabular}

$\mathbf{1 j}$ (19.0 mg) was recycled as a colorless oil in 50\% yield.

Chiral HPLC for $\mathbf{1 j}$ (Chiralpak IA, hexane:isopropanol $=85: 15,1 \mathrm{~mL} / \mathrm{min}, 214 \mathrm{~nm}$ ), $\mathrm{t}_{\text {minor }}=24.4 \mathrm{~min}$, $\mathrm{t}_{\text {major }}=15.9 \mathrm{~min} .[\alpha]_{\mathrm{D}}^{22.6}=-76.6\left(\mathrm{c}=1.97, \mathrm{CH}_{2} \mathrm{Cl}_{2}\right)$ at $98 \%$ ee.

\section{Racemic Sample 1j}

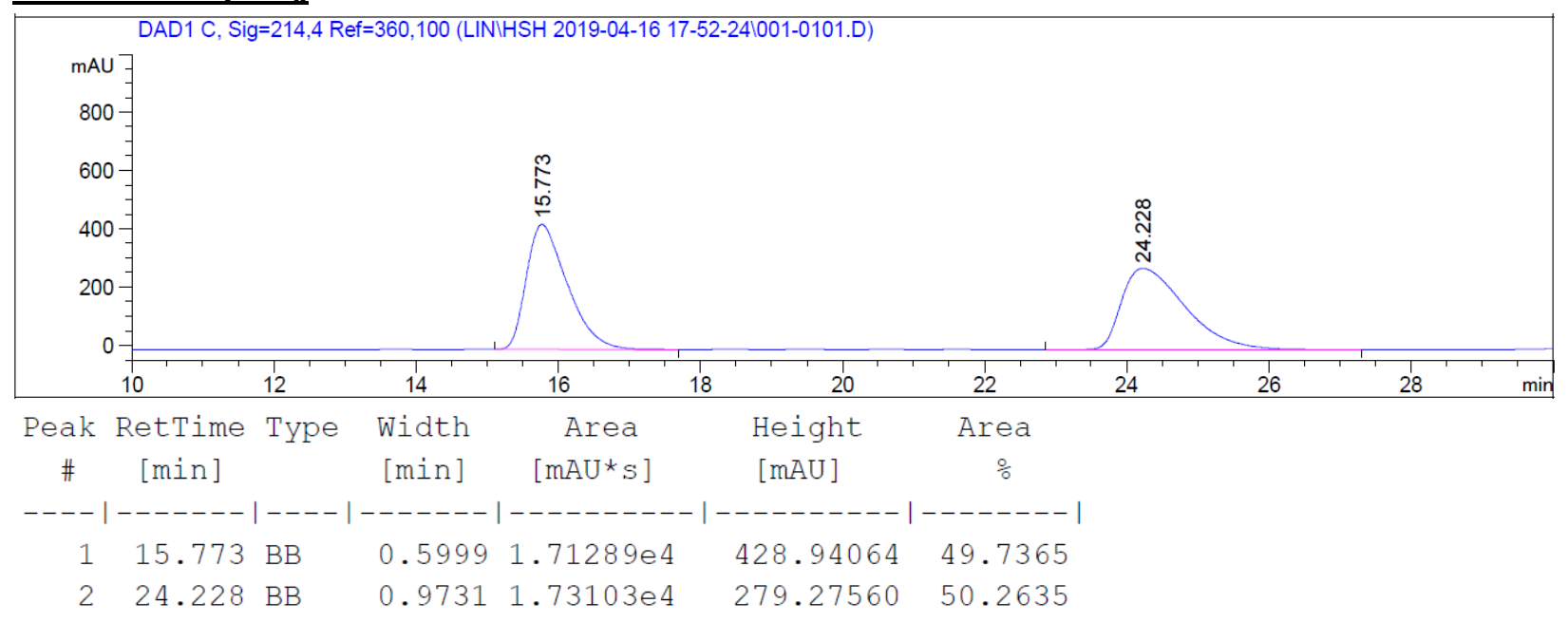

\section{Enantiomeric Sample 1j}

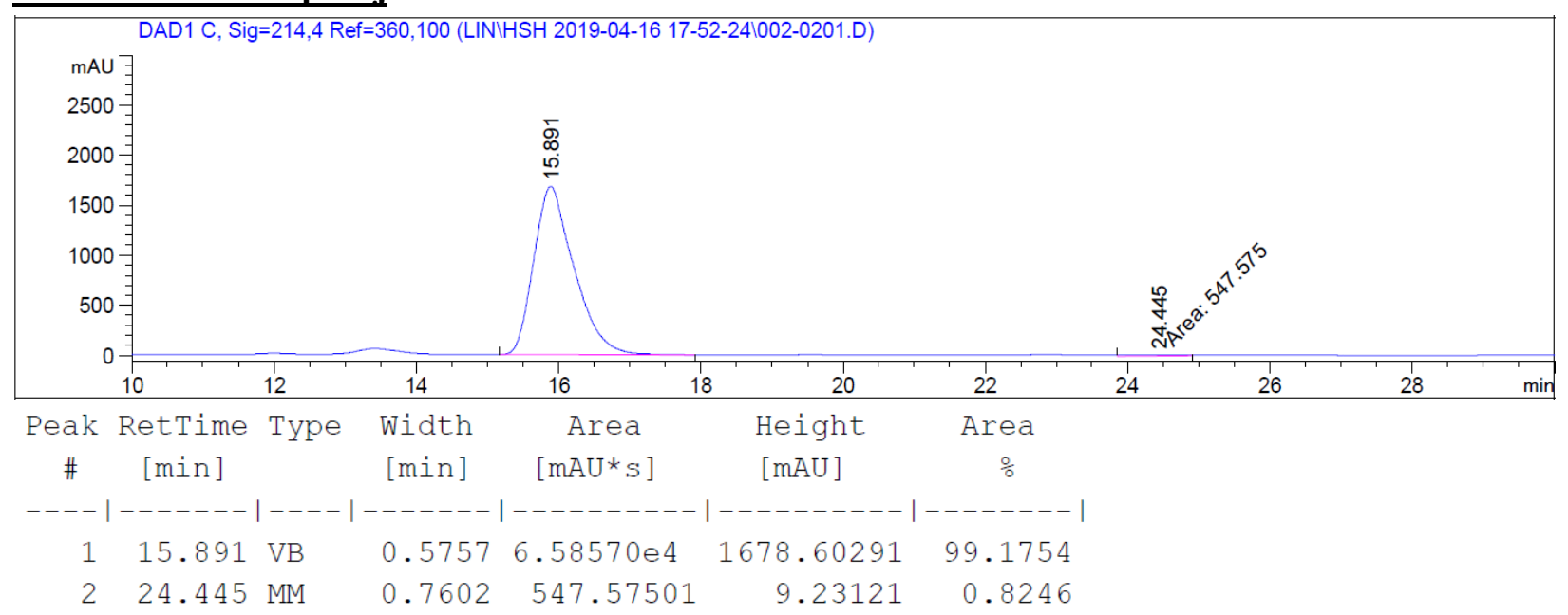




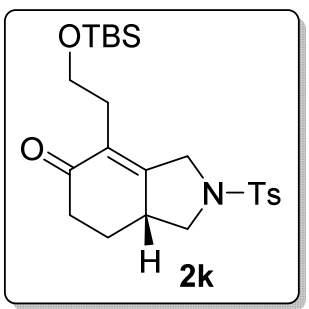

2k (19.0 mg) was isolated as a colorless oil in $43 \%$ yield. $\mathrm{R}_{f}=0.4$ (EtOAc/Hexane=1/3). ${ }^{\mathbf{1}} \mathbf{H}$ NMR (400 MHz, $\left.\mathbf{C D C l}_{3}\right): \delta 7.89-7.64(\mathrm{~m}, 2 \mathrm{H}), 7.49-7.31(\mathrm{~m}, 2 \mathrm{H}), 4.33(\mathrm{dt}, J=17.0,1.2 \mathrm{~Hz}, 1 \mathrm{H}), 4.01-3.81(\mathrm{~m}$, $2 \mathrm{H}), 3.71-3.53(\mathrm{~m}, 2 \mathrm{H}), 3.09-2.88(\mathrm{~m}, 1 \mathrm{H}), 2.61$ (dd, $J=10.9,9.3 \mathrm{~Hz}, 1 \mathrm{H}), 2.50$ (ddd, $J=17.2,4.3$, $2.4 \mathrm{~Hz}, 1 \mathrm{H}), 2.44(\mathrm{~s}, 3 \mathrm{H}), 2.42-2.21(\mathrm{~m}, 3 \mathrm{H}), 2.14(\mathrm{dtd}, J=12.4,4.8,2.4 \mathrm{~Hz}, 1 \mathrm{H}), 1.57$ (dddd, $J=14.5$, 12.6, 11.6, $4.4 \mathrm{~Hz}, 1 \mathrm{H}), 0.79$ (s, 9H), -0.06 (s, 3H), -0.08 (s, 3H). ${ }^{13} \mathbf{C}$ NMR (101 MHz, $\left.\mathbf{C D C l}_{3}\right): \delta 197.02$, 160.02, 143.93, 132.79, 130.00, 129.89, 127.73, 61.41, 53.32, 50.85, 40.87, 36.69, 30.10, 26.41, 25.86, 21.55, 18.24, -5.40, -5.51. IR: v 2928, 1669, 1472, 1350, 1256, 1165, 1096, $837 \mathrm{~cm}^{-1}$; HRMS calcd. For $[\mathrm{M}+\mathrm{Na}]^{+}:$472.1948. Found: 472.1959.

Chiral HPLC of 2k (Chiralpak IA, hexane:isopropanol $=90: 10,1 \mathrm{~mL} / \mathrm{min}, 254 \mathrm{~nm}$ ), $\mathrm{t}_{\text {minor }}=14.6 \mathrm{~min}$, $\mathrm{t}_{\text {major }}=11.9 \mathrm{~min} .[\alpha]_{\mathrm{D}}^{22.6}=-125.5\left(\mathrm{c}=1.45, \mathrm{CH}_{2} \mathrm{Cl}_{2}\right)$ at $94 \%$ ee.

\section{Racemic Sample 2k}

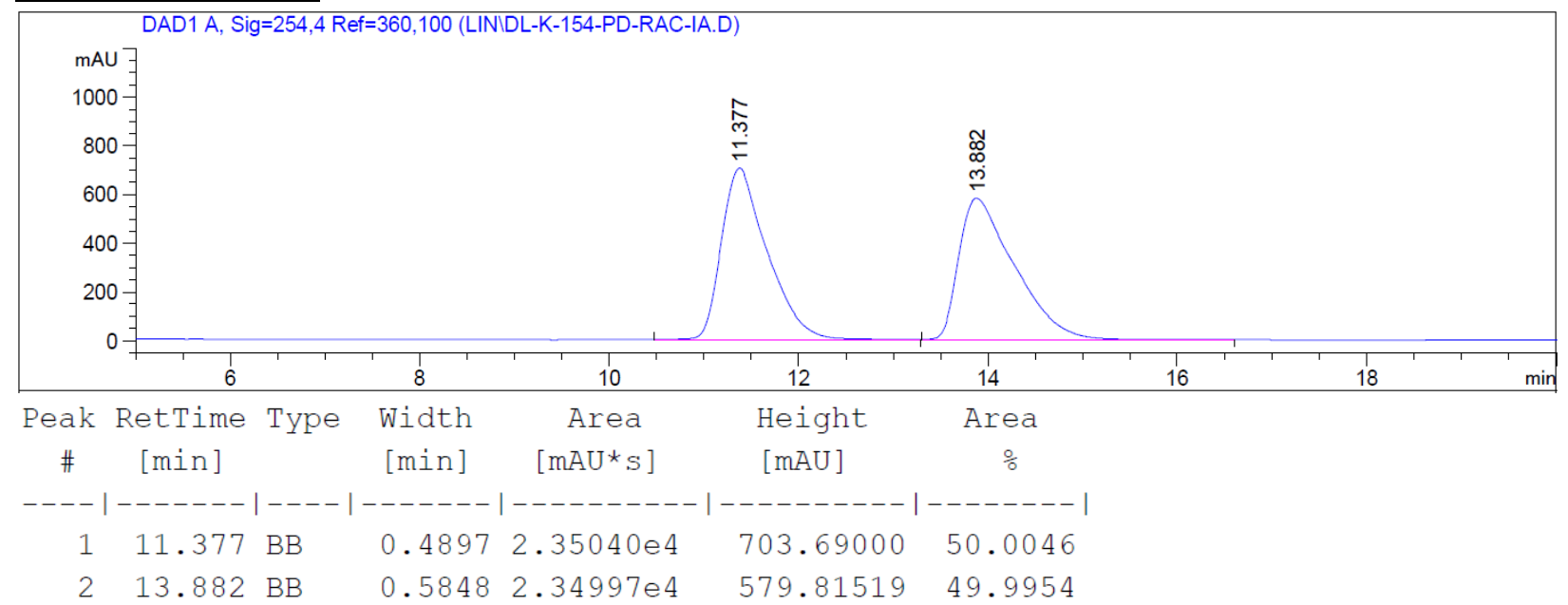

\section{Enantiomeric Sample 2k}

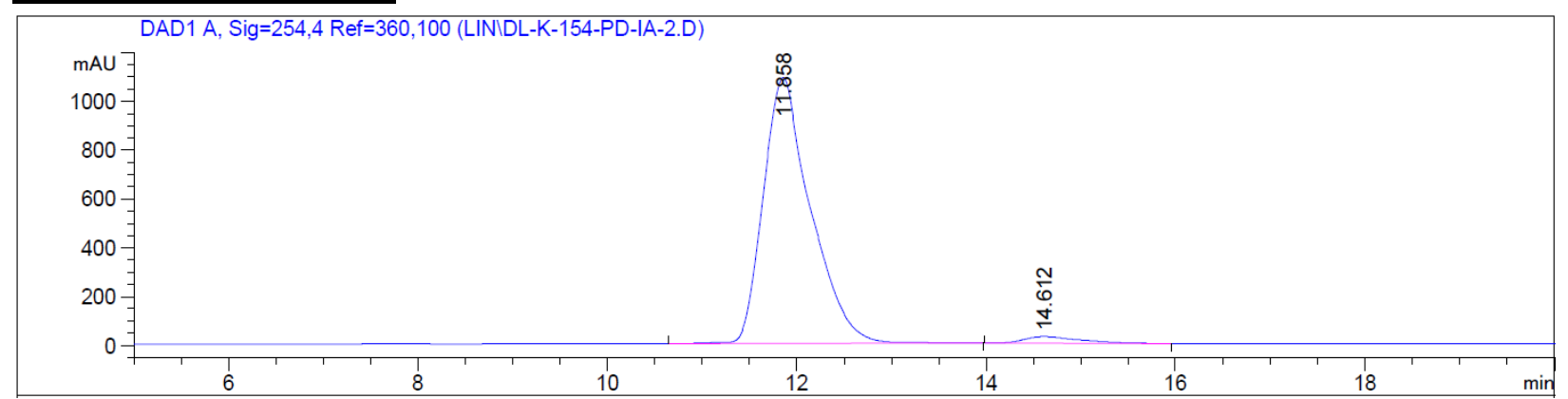




\begin{tabular}{|c|c|c|c|c|c|c|}
\hline $\begin{array}{c}\text { Peak } \\
\#\end{array}$ & $\begin{array}{c}\text { RetTime } \\
\text { [min] }\end{array}$ & Type & $\begin{array}{l}\text { Width } \\
\text { [min] }\end{array}$ & $\begin{array}{c}\text { Area } \\
{\left[\mathrm{mAU}{ }^{\star} \mathrm{s}\right]}\end{array}$ & $\begin{array}{l}\text { Height } \\
{[\mathrm{mAU}]}\end{array}$ & $\begin{array}{c}\text { Area } \\
\frac{\circ}{\circ}\end{array}$ \\
\hline-- & ------ & -1 & ---- & $-----------\mid$ & $-----------\mid$ & $--------\mid$ \\
\hline 1 & 11.858 & BB & 0.5090 & $3.78350 \mathrm{e} 4$ & 1089.98828 & 97.2285 \\
\hline 2 & 14.612 & $\mathrm{BB}$ & 0.5789 & 1078.47021 & 26.94623 & 2.7715 \\
\hline
\end{tabular}

1k (17.8 mg) was recycled as a colorless oil in $40 \%$ yield.

Chiral HPLC for 1k (Chiralpak IF, hexane:isopropanol $=90: 10,1 \mathrm{~mL} / \mathrm{min}, 210 \mathrm{~nm}$ ), $\mathrm{t}_{\text {minor }}=18.1 \mathrm{~min}$, $\mathrm{t}_{\text {major }}=15.6 \mathrm{~min} .[\alpha]_{\mathrm{D}}^{22.6}=-63.4\left(\mathrm{c}=1.72, \mathrm{CH}_{2} \mathrm{Cl}_{2}\right)$ at $99 \%$ ee.

\section{Racemic Sample 1k}

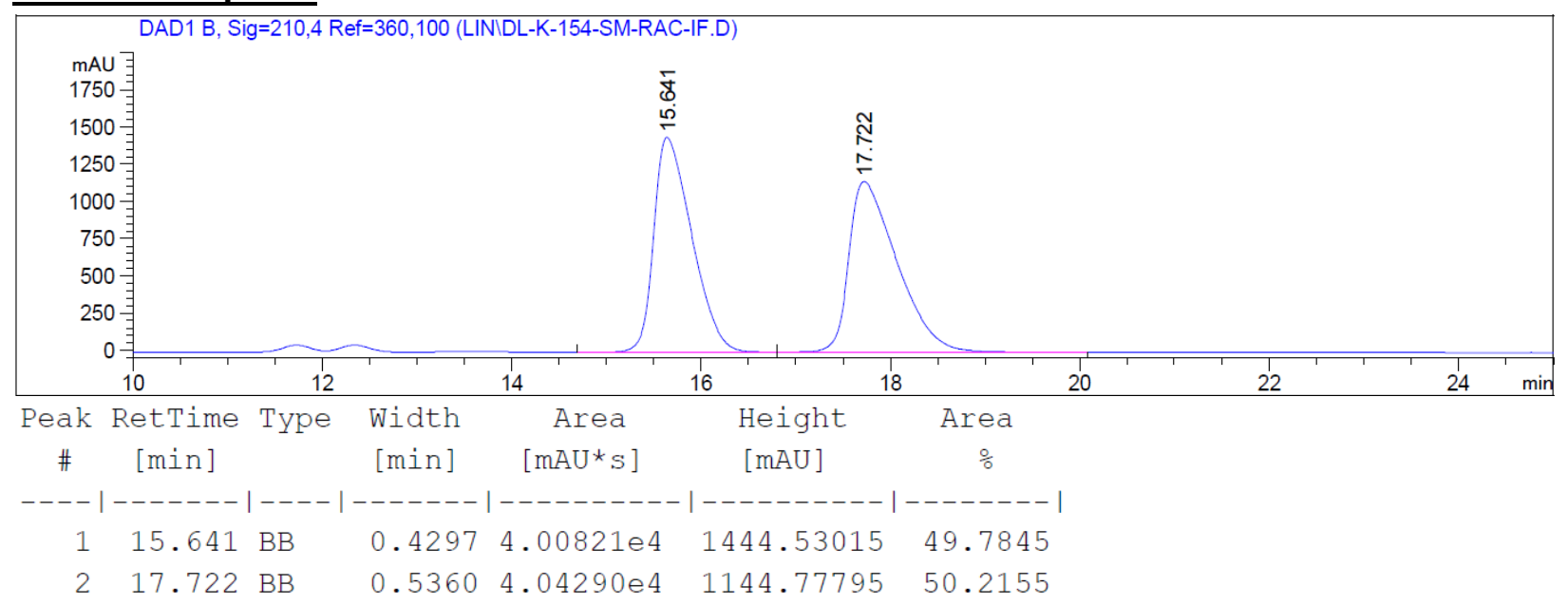

\section{Enantiomeric Sample 1k}

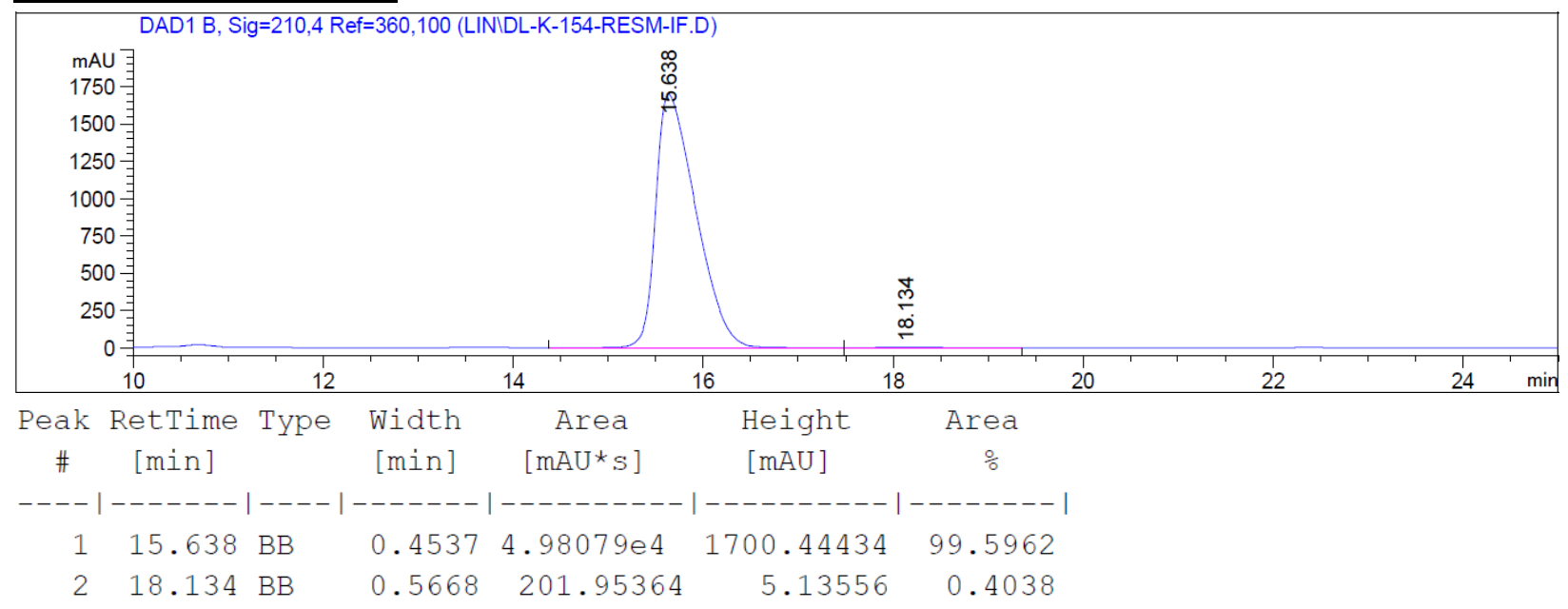




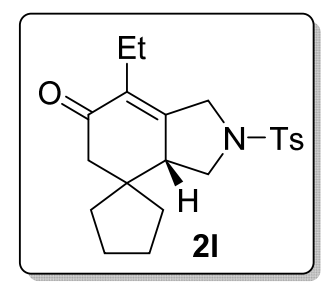

2l (11.9 mg) was isolated as a colorless oil in $32 \%$ yield. $\mathrm{R}_{f}=0.4\left(\right.$ EtOAc/Hexane=1/3). ${ }^{\mathbf{1}} \mathbf{H}$ NMR (400 MHz, $\left.\mathbf{C D C l}_{3}\right): \delta 7.80-7.66(\mathrm{~m}, 2 \mathrm{H}), 7.44-7.34(\mathrm{~m}, 2 \mathrm{H}), 4.22(\mathrm{~d}, J=16.9 \mathrm{~Hz}, 1 \mathrm{H}), 3.93-3.68(\mathrm{~m}, 2 \mathrm{H})$, $3.26-3.07(\mathrm{~m}, 1 \mathrm{H}), 2.85(\mathrm{dd}, J=10.6,9.3 \mathrm{~Hz}, 1 \mathrm{H}), 2.49-2.41(\mathrm{~m}, 4 \mathrm{H}), 2.26(\mathrm{~d}, J=16.0 \mathrm{~Hz}, 1 \mathrm{H}), 2.15$ $(\mathrm{dq}, J=14.9,7.6 \mathrm{~Hz}, 1 \mathrm{H}), 2.09-1.98(\mathrm{~m}, 1 \mathrm{H}), 1.71-1.54(\mathrm{~m}, 3 \mathrm{H}), 1.52-1.39(\mathrm{~m}, 3 \mathrm{H}), 1.32-1.23(\mathrm{~m}$, 1H), $1.18(\mathrm{ddd}, J=13.7,8.1,6.4 \mathrm{~Hz}, 1 \mathrm{H}), 0.89$ (t, $J=7.5 \mathrm{~Hz}, 3 \mathrm{H}) .{ }^{13} \mathbf{C}$ NMR (101 MHz, $\left.\mathbf{C D C l}_{3}\right): \delta$ 197.57, 155.24, 144.13, 134.29, 132.65, 129.90, 127.69, 51.69, 50.44, 49.72, 48.88, 46.80, 38.94, 30.17, 25.56, 24.68, 21.61, 19.51, 12.75. IR: v 2954, 1668, 1598, 1452, 1348, 1165, 1093, $816 \mathrm{~cm}^{-1}$; HRMS calcd. For $[\mathrm{M}+\mathrm{Na}]^{+}:$396.1604. Found: 396.1613.

Chiral HPLC of $2 \mathbf{l}$ (Chiralpak ID, hexane:isopropanol $=90: 10,1 \mathrm{~mL} / \mathrm{min}, 254 \mathrm{~nm}$ ), $\mathrm{t}_{\text {minor }}=45.7 \mathrm{~min}$, $\mathrm{t}_{\text {major }}=37.4 \mathrm{~min} .[\alpha]_{\mathrm{D}}^{22.6}=294.4\left(\mathrm{c}=0.72, \mathrm{CH}_{2} \mathrm{Cl}_{2}\right)$ at $99.5 \%$ ee.

\section{Racemic Sample 2l}

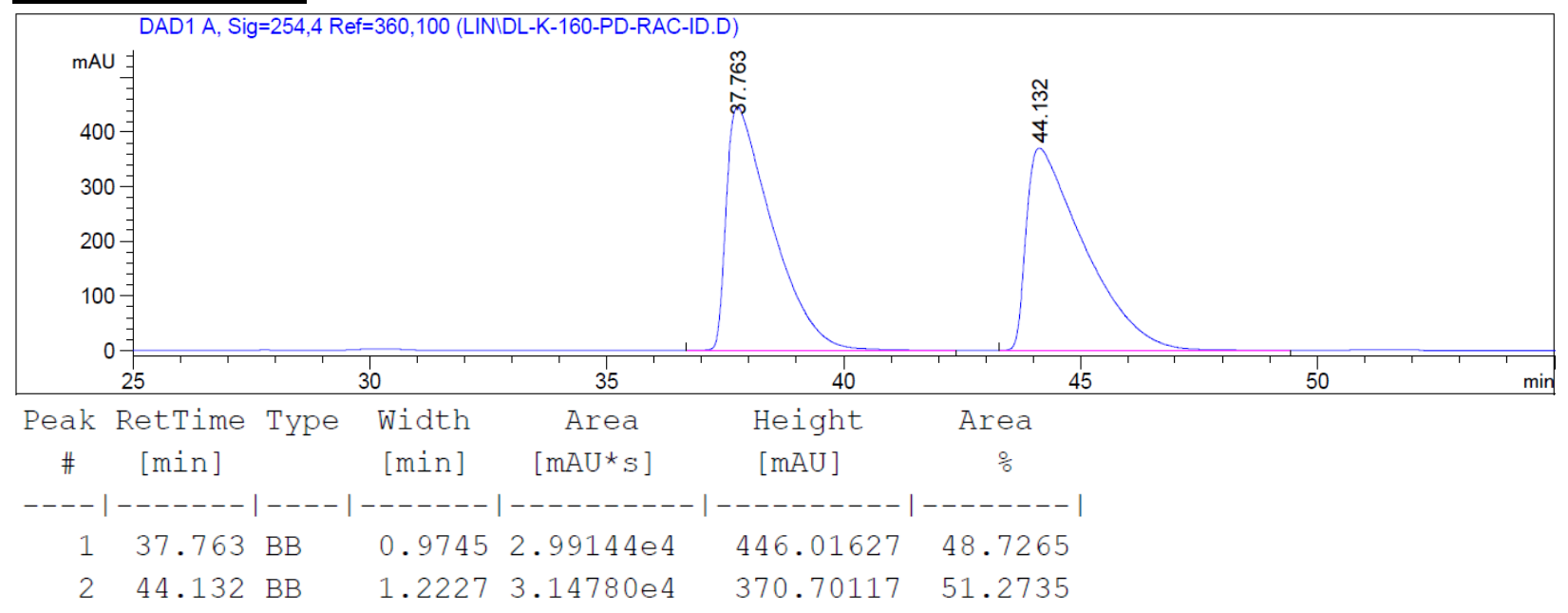

\section{Enantiomeric Sample 2l}

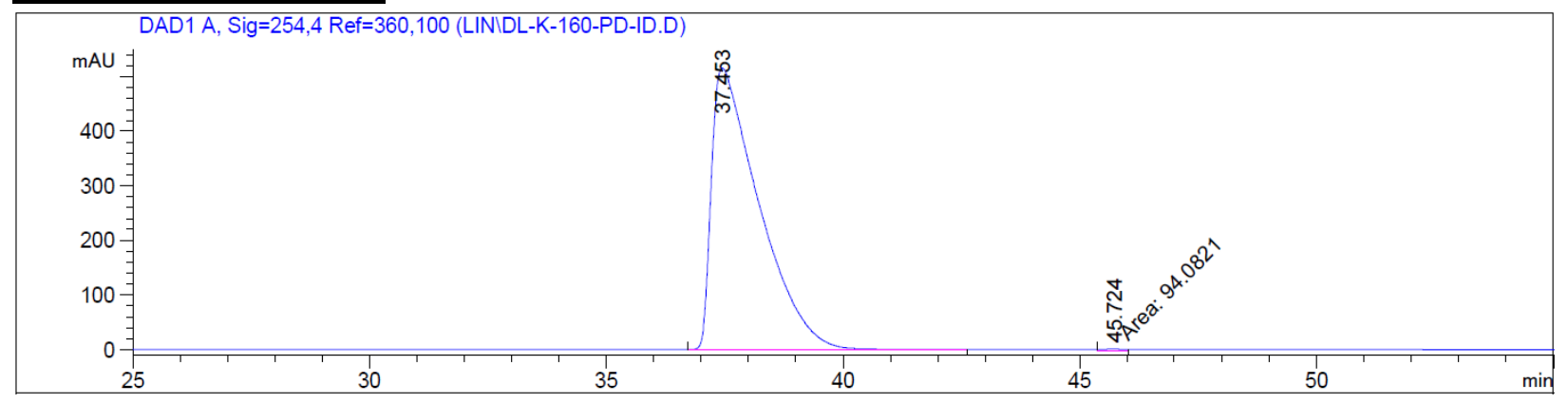




\begin{tabular}{|c|c|c|c|c|c|c|}
\hline $\begin{array}{c}\text { Peak } \\
\#\end{array}$ & $\begin{array}{c}\text { RetTime } \\
\text { [min] }\end{array}$ & Type & $\begin{array}{l}\text { Width } \\
\text { [min] }\end{array}$ & $\begin{array}{c}\text { Area } \\
{\left[\mathrm{mAU}{ }^{*} \mathrm{~s}\right]}\end{array}$ & $\begin{array}{l}\text { Height } \\
{[\mathrm{mAU}]}\end{array}$ & $\begin{array}{c}\text { Area } \\
\%\end{array}$ \\
\hline & ---- & 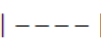 & ------- & |--------- & $----------\mid$ & -------- \\
\hline 1 & 37.453 & $\mathrm{BB}$ & 1.0162 & $3.59899 \mathrm{e} 4$ & 515.68945 & 99.7393 \\
\hline 2 & 45.724 & MM & 0.6383 & 94.08212 & 2.45648 & 0.2607 \\
\hline
\end{tabular}

11 (16.0 mg) was recycled as a colorless oil in $43 \%$ yield.

Chiral HPLC for 11 (Chiralpak IF, hexane:isopropanol $=92: 8,1 \mathrm{~mL} / \mathrm{min}, 210 \mathrm{~nm}$ ), $\mathrm{t}_{\text {minor }}=25.3 \mathrm{~min}$, $\mathrm{t}_{\text {major }}=26.9 \mathrm{~min} .[\alpha]_{\mathrm{D}}^{22.6}=-443.6\left(\mathrm{c}=0.78, \mathrm{CH}_{2} \mathrm{Cl}_{2}\right)$ at $65 \%$ ee.

\section{Racemic Sample 11}

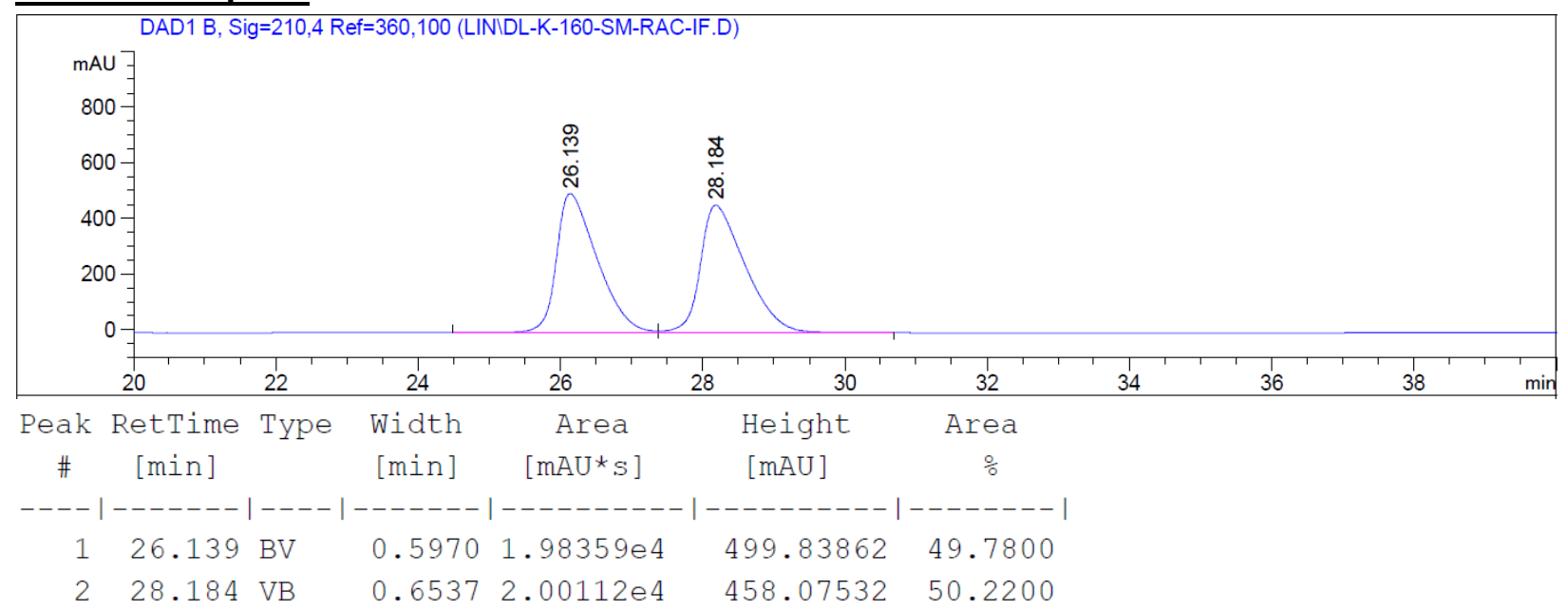

\section{Enantiomeric Sample 1l}

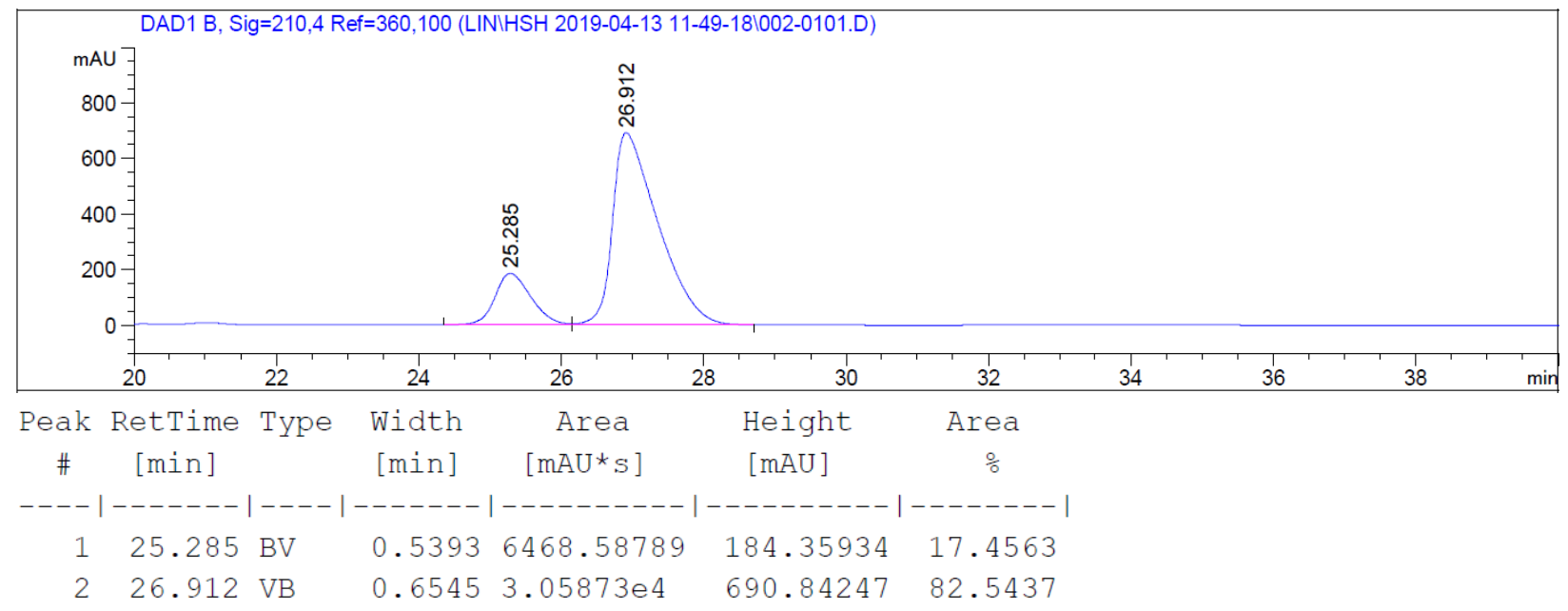

\section{Synthetic applications}




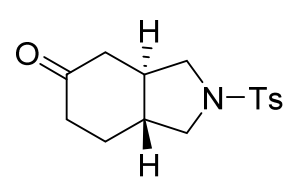

2a

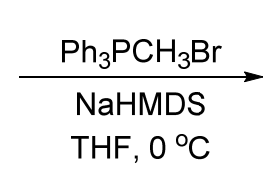

THF, $0^{\circ} \mathrm{C}$

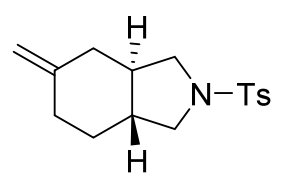

$3,68 \%$

A flame-dried Schlenk tube was charged with $\mathrm{Ph}_{3} \mathrm{PCH}_{3} \mathrm{Br}(268 \mathrm{mg}, 0.75 \mathrm{mmol}, 15$ equiv.) and THF (3 $\mathrm{mL}$ ), and the mixture was cooled to $0{ }^{\circ} \mathrm{C}$. NaHMDS ( $2 \mathrm{M}$ in THF, $0.375 \mathrm{~mL}, 0.75 \mathrm{mmol}, 15$ equiv.) was added dropwise, and the mixture was stirred at $0{ }^{\circ} \mathrm{C}$ for $30 \mathrm{~min}$. 2a (14.7 $\mathrm{mg}, 0.05 \mathrm{mmol}, 1$ equiv.) was dissolved in THF $(0.2 \mathrm{~mL})$, and the solution was added to the mixture at $0{ }^{\circ} \mathrm{C}$. After the full conversion of 2a, the mixture was quenched with $\mathrm{NH}_{4} \mathrm{Cl}$ and extracted with ether. The combined organic layers were dried and concentrated. The residue was purified by column chromatography to give 3 as a white solid in $68 \%$ yield $\left(9.9 \mathrm{mg}\right.$ ). Melting Point: $112-114{ }^{\circ} \mathrm{C} . \mathrm{R}_{f}=0.5$ (EtOAc/Hexane $\left.=1 / 5\right) .{ }^{1} \mathbf{H}$ NMR (400 MHz, $\mathbf{C D C l}_{3}$ ): $\delta 7.82-7.64(\mathrm{~m}, 2 \mathrm{H}), 7.37-7.28(\mathrm{~m}, 2 \mathrm{H}), 4.79-4.63(\mathrm{~m}, 2 \mathrm{H}), 3.60-3.47(\mathrm{~m}, 2 \mathrm{H}), 2.85(\mathrm{dd}, J$ $=10.9,9.4 \mathrm{~Hz}, 1 \mathrm{H}), 2.77(\mathrm{dd}, J=10.9,9.4 \mathrm{~Hz}, 1 \mathrm{H}), 2.47-2.37(\mathrm{~m}, 4 \mathrm{H}), 2.33$ (ddt, $J=13.8,3.9,1.9 \mathrm{~Hz}$, 1H), $2.04-1.92(\mathrm{~m}, 1 \mathrm{H}), 1.92-1.84(\mathrm{~m}, 1 \mathrm{H}), 1.82-1.72(\mathrm{~m}, 1 \mathrm{H}), 1.55-1.30(\mathrm{~m}, 2 \mathrm{H}), 1.06(\mathrm{dtd}, J=$ 12.9, 11.8, 4.2 Hz, 1H). ${ }^{\mathbf{1 3}} \mathbf{C}$ NMR (101 $\left.\mathbf{~ M H z , ~} \mathbf{C D C l}_{3}\right)$ : $\delta$ 146.19, 143.19, 134.68, 129.65, 127.28, 110.04, 52.65, 52.50, 45.30, 44.06, 37.10, 33.71, 28.62, 21.53. IR: v 2936, 1342, 1160, 1093, 1039, $659 \mathrm{~cm}^{-1}$; HRMS calcd. For $[\mathrm{M}+\mathrm{Na}]^{+}: 314.1185$. Found: $314.1194 .[\alpha]_{\mathrm{D}}^{22.7}=228.0\left(\mathrm{c}=0.93, \mathrm{CH}_{2} \mathrm{Cl}_{2}\right)$ at $98 \%$ ee.

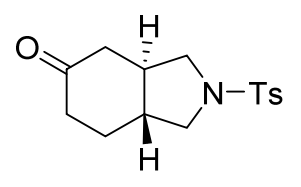

2a
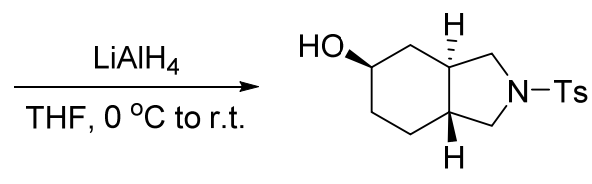

4, $78 \%$

A flame-dried Schlenk tube was charged with $\mathrm{LiAlH}_{4}(9.5 \mathrm{mg}, 0.25 \mathrm{mmol}, 5$ equiv. $)$ and THF (0.25 mL). 2a (14.7 mg, $0.05 \mathrm{mmol}, 1$ equiv.) was dissolved in THF $(0.25 \mathrm{~mL})$, and the solution was added to the tube dropwise. The mixture was stirred at room temperature for $1 \mathrm{~h}$. The mixture was quenched with water, filtered and concentrated, and the residue was purified by column chromatography to give $\mathbf{4}$ as a white solid in $78 \%$ yield (11.5 mg). d.r. $>20: 1$ based on the NMR spectrum of the crude mixture. Melting Point: $154-155{ }^{\circ} \mathrm{C} . \mathrm{R}_{f}=0.3$ (EtOAc/Hexane=1/1). ${ }^{1} \mathbf{H}$ NMR (400 MHz, $\mathbf{C D C l}_{3}$ ): $\delta 7.79-7.66$ (m, 2H), $7.35-7.28(\mathrm{~m}, 2 \mathrm{H}), 3.62(\mathrm{tt}, J=10.3,4.5 \mathrm{~Hz}, 1 \mathrm{H}), 3.56-3.46(\mathrm{~m}, 2 \mathrm{H}), 2.83(\mathrm{dd}, J=10.7,9.4 \mathrm{~Hz}, 1 \mathrm{H})$, 2.77 (dd, $J=10.7,9.4 \mathrm{~Hz}, 1 \mathrm{H}), 2.43$ (s, 3H), 2.09 (dddd, $J=11.9,4.7,2.9,1.8 \mathrm{~Hz}, 1 \mathrm{H}), 2.00$ (ddtd, $J=$ $12.7,4.7,3.2,1.8 \mathrm{~Hz}, 1 \mathrm{H}), 1.83(\mathrm{dq}, J=12.8,3.3 \mathrm{~Hz}, 1 \mathrm{H}), 1.53(\mathrm{br}, 1 \mathrm{H}), 1.47-1.18(\mathrm{~m}, 3 \mathrm{H}), 1.16-0.96$ (m, 2H). ${ }^{13} \mathbf{C}$ NMR (101 MHz, $\left.\mathbf{C D C l}_{3}\right): \delta$ 143.24, 134.66, 129.67, 127.25, 70.14, 52.25, 52.22, 43.77, 42.94, 37.16, 34.68, 25.67, 21.52. IR: v 3397, 2931, 1337, 1162, 1093, $664 \mathrm{~cm}^{-1}$; HRMS calcd. For $[\mathrm{M}+\mathrm{H}]^{+}: 296.1315$. Found: 296.1327. $[\alpha]_{\mathrm{D}}^{22.7}=383.5\left(\mathrm{c}=1.09, \mathrm{CH}_{2} \mathrm{Cl}_{2}\right)$ at $98 \%$ ee. 


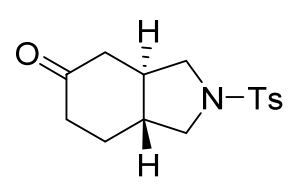

2a

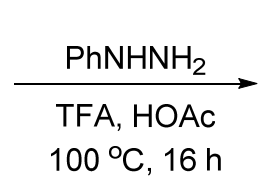

$100{ }^{\circ} \mathrm{C}, 16 \mathrm{~h}$

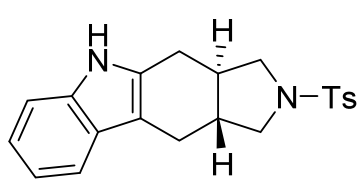

$5,75 \%$

A 4-mL vial was charged with 2 a $(14.7 \mathrm{mg}, 0.05 \mathrm{mmol}, 1$ equiv. $)$, AcOH (0.6 mL), TFA $(0.2 \mathrm{~mL})$ and phenylhydrazine ( $14.1 \mathrm{mg}, 0.13 \mathrm{mmol}, 2.6$ equiv.), and the mixture was stirred at $100{ }^{\circ} \mathrm{C}$ for $16 \mathrm{~h}$. After cooling to room temperature, the mixture was poured into water with ice. The mixture was extracted with DCM, and the combined organic layers were dried and concentrated. The residue was purified by column chromatography to give 5 as a white solid in $75 \%$ yield $(12.0 \mathrm{mg})$. Compound 3c was observed as the only regioisomer. Melting Point: 232-235 ${ }^{\circ} \mathrm{C} . \mathrm{R}_{f}=0.4$ (EtOAc/Hexane=1/3). ${ }^{1} \mathbf{H}$ NMR (400 MHz, $\left.\mathbf{C D C l}_{3}\right): \delta 7.83-7.74(\mathrm{~m}, 2 \mathrm{H}), 7.72(\mathrm{br}, 1 \mathrm{H}), 7.44-7.36(\mathrm{~m}, 1 \mathrm{H}), 7.36-7.30(\mathrm{~m}, 2 \mathrm{H}), 7.29-7.23(\mathrm{~m}$, $1 \mathrm{H}), 7.12(\mathrm{ddd}, J=8.1,7.0,1.4 \mathrm{~Hz}, 1 \mathrm{H}), 7.10-7.03(\mathrm{~m}, 1 \mathrm{H}), 3.91-3.66(\mathrm{~m}, 2 \mathrm{H}), 3.15-3.00(\mathrm{~m}, 2 \mathrm{H})$, $3.01-2.86(\mathrm{~m}, 2 \mathrm{H}), 2.60-2.46(\mathrm{~m}, 1 \mathrm{H}), 2.45-2.32(\mathrm{~m}, 4 \mathrm{H}), 2.19-1.94(\mathrm{~m}, 2 \mathrm{H}) .{ }^{13} \mathbf{C}$ NMR (101 MHz, $\left.\mathbf{C D C l}_{3}\right): \delta 143.40,136.18,134.45,132.45,129.72,127.35,126.99,121.57,119.48,117.70,110.55$, 109.60, 52.79, 52.78, 41.71, 41.36, 26.67, 24.10, 21.53. IR: v 3397, 2880, 1750, 1465, 1334, 1158, 743 $\mathrm{cm}^{-1}$; HRMS calcd. For $[\mathrm{M}+\mathrm{H}]^{+}: 367.1475$. Found: $367.1482 .[\alpha]_{\mathrm{D}}^{22.7}=-2.46\left(\mathrm{c}=1.22, \mathrm{CH}_{2} \mathrm{Cl}_{2}\right)$ at $98 \%$ ee.

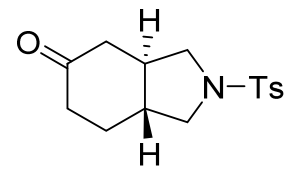

2a

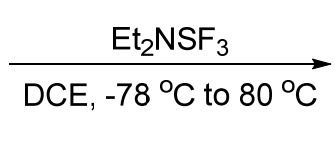

A flame-dried Schlenk tube was charged with 2a (14.7 mg, $0.05 \mathrm{mmol}, 1$ equiv.) and distilled DCE (0.5 $\mathrm{mL}$ ), and the mixture was cooled to $-78{ }^{\circ} \mathrm{C} . \mathrm{Et}_{2} \mathrm{NSF}_{3}(32.2 \mathrm{mg}, 0.2 \mathrm{mmol}, 4$ equiv.) was added dropwise, and the mixture was warmed to room temperature. The mixture was further heated at $80{ }^{\circ} \mathrm{C}$ for $2 \mathrm{~h}$. After cooling to room temperature, the mixture was concentrated, and the residue was purified by column chromatography to give 6 as a white solid in $58 \%$ yield $(9.1 \mathrm{mg})$. Melting Point: $133-135{ }^{\circ} \mathrm{C} . \mathrm{R}_{f}=0.7$ (EtOAc/Hexane=1/2). ${ }^{1} \mathbf{H}$ NMR (400 MHz, $\left.\mathbf{C D C l}_{3}\right): \delta 7.80-7.65$ (m, 2H), $7.43-7.30$ (m, 2H), $3.66-$ $3.50(\mathrm{~m}, 2 \mathrm{H}), 2.89$ (ddd, $J=10.9,9.4,1.2 \mathrm{~Hz}, 1 \mathrm{H}), 2.81(\mathrm{dd}, J=10.9,9.4 \mathrm{~Hz}, 1 \mathrm{H}), 2.45$ (s, 3H), $2.31-$ $2.06(\mathrm{~m}, 2 \mathrm{H}), 1.87$ (ddq, $J=13.2,5.6,2.8 \mathrm{~Hz}, 1 \mathrm{H}), 1.78-1.59(\mathrm{~m}, 2 \mathrm{H}), 1.55-1.40(\mathrm{~m}, 2 \mathrm{H}), 1.38-1.26$ (m, 1H). ${ }^{13}$ C NMR (101 MHz, $\left.\mathbf{C D C l}_{3}\right): \delta 143.49,134.45,129.79,127.25,123.10(\mathrm{dd}, J=244.7,240.8$ $\mathrm{Hz}), 51.74(\mathrm{~d}, J=4.0 \mathrm{~Hz}), 51.72(\mathrm{~d}, J=3.9 \mathrm{~Hz}), 43.03(\mathrm{~d}, J=1.7 \mathrm{~Hz}), 41.06(\mathrm{~d}, J=10.5 \mathrm{~Hz}), 36.46(\mathrm{t}, J$ $=24.5 \mathrm{~Hz}), 33.35(\mathrm{dd}, J=26.5,23.3 \mathrm{~Hz}), 23.73(\mathrm{~d}, J=10.8 \mathrm{~Hz}), 21.54 .{ }^{19} \mathbf{F}$ NMR (470 MHz, CDCl $): \delta$ $91.40(\mathrm{~d}, J=242.3 \mathrm{~Hz}),-97.49(\mathrm{dtt}, J=242.5,34.3,11.2 \mathrm{~Hz})$. IR: v 2935, 1598, 1342, 1163, 1037, 664 $\mathrm{cm}^{-1}$; HRMS calcd. For $[\mathrm{M}+\mathrm{H}]^{+}: 316.1177$. Found: $316.1187 .[\alpha]_{\mathrm{D}}^{22.7}=260.0\left(\mathrm{c}=0.50, \mathrm{CH}_{2} \mathrm{Cl}_{2}\right)$ at $98 \%$ ee. 


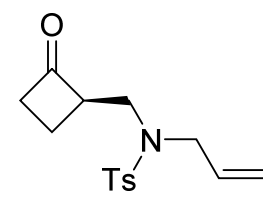

$1 \mathrm{a}$

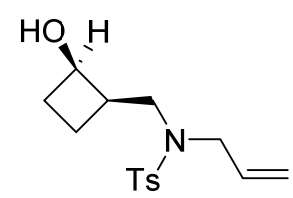

$7,80 \%$

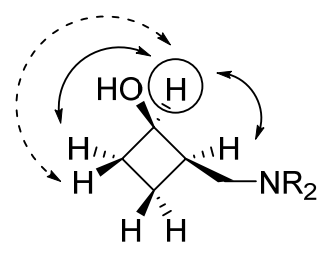

NOE

A flame-dried Schlenk flask was charged with 1a (10.0 mg, $0.034 \mathrm{mmol}, 1$ equiv.) and THF (2 mL), and the mixture was cooled to $-78{ }^{\circ} \mathrm{C}$. L-selectride ( $1 \mathrm{M}$ in THF, $0.068 \mathrm{~mL}, 0.068 \mathrm{mmol}, 2$ equiv.) was added dropwise, and the mixture was stirred at $-78{ }^{\circ} \mathrm{C}$ for $1 \mathrm{~h}$. The mixture was quenched with water and warmed to $0{ }^{\circ} \mathrm{C} . \mathrm{H}_{2} \mathrm{O}_{2}$ (35\% solution, $\left.0.2 \mathrm{~mL}\right)$ was then added, and the mixture was further stirred for 30 min. The mixture was diluted with ethyl acetate and washed with water, $\mathrm{Na}_{2} \mathrm{SO}_{3}$ and brine. The mixture was dried and concentrated, and the residue was purified by column chromatography to give 7 as a colorless oil in 80\% yield (8.0 mg). d.r. $>20: 1$ based on the NMR spectrum of the crude mixture. The cis configuration was determined by COSY and NOE analysis. $\mathrm{R}_{f}=0.3($ EtOAc/Hexane $=1 / 2) .{ }^{1} \mathbf{H}$ NMR (400 MHz, $\left.\mathbf{C D C l}_{3}\right): \delta 7.80-7.66(\mathrm{~m}, 2 \mathrm{H}), 7.35-7.29(\mathrm{~m}, 2 \mathrm{H}), 5.62(\mathrm{ddt}, J=17.2,10.1,6.4 \mathrm{~Hz}, 1 \mathrm{H}), 5.26-$ $5.06(\mathrm{~m}, 2 \mathrm{H}), 4.44(\mathrm{tt}, J=6.4,3.2 \mathrm{~Hz}, 1 \mathrm{H}), 3.89$ (ddt, $J=15.8,6.2,1.5 \mathrm{~Hz}, 1 \mathrm{H}), 3.74$ (ddq, $J=15.7,6.4$, $1.2 \mathrm{~Hz}, 1 \mathrm{H}), 3.61$ (ddd, $J=14.5,9.6,0.7 \mathrm{~Hz}, 1 \mathrm{H}), 2.97(\mathrm{~d}, J=3.4 \mathrm{~Hz}, 1 \mathrm{H}), 2.87(\mathrm{dd}, J=14.4,5.4 \mathrm{~Hz}$, $1 \mathrm{H}), 2.72-2.60(\mathrm{~m}, 1 \mathrm{H}), 2.43(\mathrm{~s}, 3 \mathrm{H}), 2.28-2.16(\mathrm{~m}, 1 \mathrm{H}), 1.91-1.82(\mathrm{~m}, 1 \mathrm{H}), 1.80-1.71(\mathrm{~m}, 2 \mathrm{H}) .{ }^{13} \mathrm{C}$ NMR (101 MHz, $\left.\mathbf{C D C l}_{3}\right): \delta$ 143.43, 136.37, 133.15, 129.75, 127.14, 118.77, 68.54, 51.46, 46.67, 39.00, 28.41, 21.51, 20.14. IR: v 3516, 2939, 1334, 1157, 1090, $767 \mathrm{~cm}^{-1}$; HRMS calcd. For [M+Na] ${ }^{+}$: 318.1134. Found: 318.1140 .

Chiral HPLC for 7 (Chiralpak IF, hexane:isopropanol $=90: 10,1 \mathrm{~mL} / \mathrm{min}, 210 \mathrm{~nm}$ ), $\mathrm{t}_{\text {minor }}=28.0 \mathrm{~min}$, $\mathrm{t}_{\text {major }}=23.4 \mathrm{~min} .[\alpha]_{\mathrm{D}}^{20.6}=181.2\left(\mathrm{c}=0.80, \mathrm{CH}_{2} \mathrm{Cl}_{2}\right)$ at $99 \%$ ee.

\section{Racemic Sample 7}

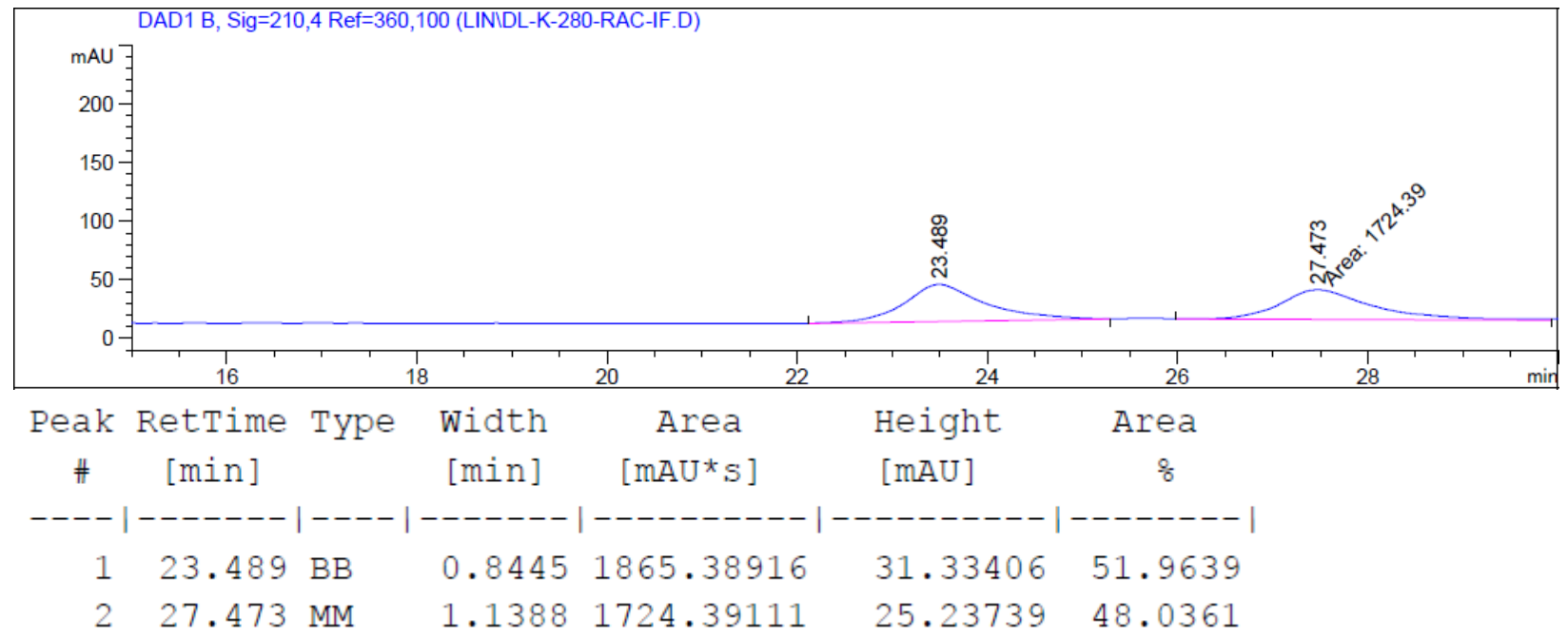

\section{Enantiomeric Sample 7}




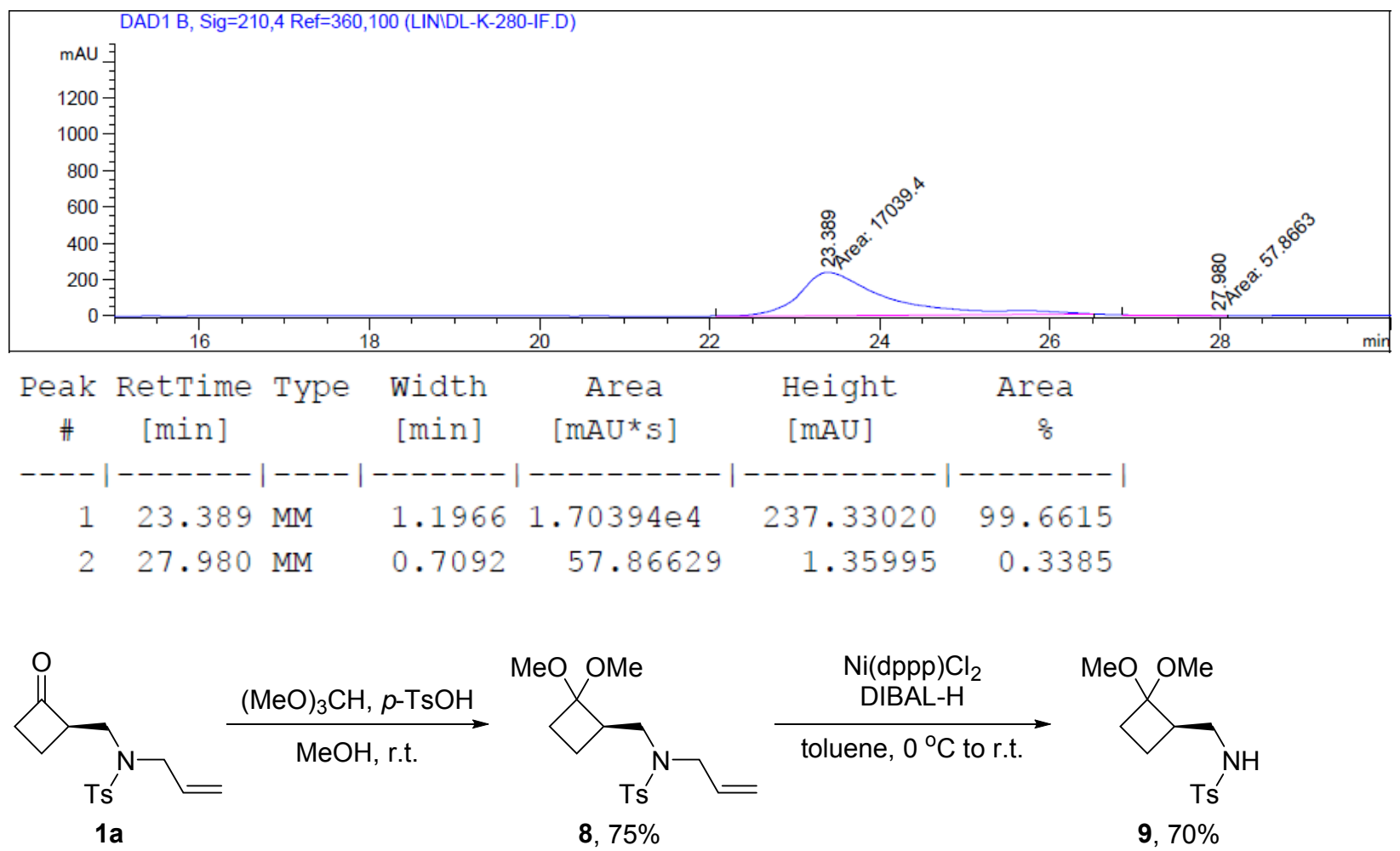

Step 1: A 4-mL vial was charged with 1a (96.5 mg, $0.33 \mathrm{mmol}, 1$ equiv.), $\mathrm{MeOH}$ (0.5 mL), TsOH (11.4 $\mathrm{mg}, 0.066 \mathrm{mmol}, 0.2$ equiv.) and $(\mathrm{MeO})_{3} \mathrm{CH}(104.7 \mathrm{mg}, 0.99 \mathrm{mmol}, 3$ equiv.), and the mixture was stirred at room temperature overnight. The mixture was concentrated, and the residue was purified by column chromatography to give 8 as a colorless oil in $75 \%$ yield $(84 \mathrm{mg}) . \mathrm{R}_{f}=0.7$ (EtOAc/Hexane=1/2). ${ }^{1} \mathbf{H}$ NMR (400 MHz, $\mathbf{C D C l}_{3}$ ): $\delta 7.86-7.60$ (m, 2H), $7.32-7.28$ (m, 2H), 5.54 (dddd, $J=17.0,10.0,6.9,5.9$ $\mathrm{Hz}, 1 \mathrm{H}), 5.31-5.03(\mathrm{~m}, 2 \mathrm{H}), 3.95-3.68(\mathrm{~m}, 2 \mathrm{H}), 3.35$ (ddd, $J=14.3,9.8,0.7 \mathrm{~Hz}, 1 \mathrm{H}), 3.24-3.07$ (m, $7 \mathrm{H}), 2.81-2.65(\mathrm{~m}, 1 \mathrm{H}), 2.43(\mathrm{~s}, 3 \mathrm{H}), 2.23-2.12(\mathrm{~m}, 1 \mathrm{H}), 2.04-1.71(\mathrm{~m}, 2 \mathrm{H}), 1.55-1.42(\mathrm{~m}, 1 \mathrm{H}) .{ }^{13} \mathrm{C}$ NMR (101 MHz, $\left.\mathbf{C D C l}_{3}\right): \delta 143.08,137.06,133.23,129.62,127.20,118.73,102.83,51.19,48.85,48.23$, 47.45, 43.09, 28.50, 21.50, 17.06. IR: v 2949, 1448, 1344, 1263, 1159, 1040, $760 \mathrm{~cm}^{-1}$; HRMS calcd. For $[\mathrm{M}+\mathrm{H}]^{+}: 340.1577$. Found: $340.1569 .[\alpha]_{\mathrm{D}}^{22.7}=-222.2\left(\mathrm{c}=0.99, \mathrm{CH}_{2} \mathrm{Cl}_{2}\right)$ at $98 \%$ ee.

Step 2: A flame-dried Schlenk flask was charged with 8 (10.0 mg, $0.03 \mathrm{mmol}, 1$ equiv.), $\mathrm{Ni}(\mathrm{dppp}) \mathrm{Cl}_{2}$ (1.6 $\mathrm{mg}, 0.003 \mathrm{mmol}, 10 \mathrm{~mol} \%)$ and toluene $(0.5 \mathrm{~mL})$, and the mixture was cooled to $0{ }^{\circ} \mathrm{C}$. DIBAL-H $(1 \mathrm{M}$ in toluene, $0.15 \mathrm{~mL}, 0.15 \mathrm{mmol}, 5$ equiv.) was added dropwise, and the mixture was stirred at room temperature for $1 \mathrm{~h}$. The mixture was quenched with water and concentrated, and the residue was purified by column chromatography to give $\mathbf{9}$ as a colorless oil in $70 \%$ yield $(6.3 \mathrm{mg}) . \mathrm{R}_{f}=0.4$ (EtOAc/Hexane=1/2). ${ }^{1} \mathbf{H}$ NMR (400 $\left.\mathbf{~ M H z , ~} \mathbf{C D C l}_{3}\right): \delta 7.83-7.67(\mathrm{~m}, 2 \mathrm{H}), 7.37-7.29(\mathrm{~m}, 2 \mathrm{H}), 5.08(\mathrm{dd}$, $J=8.0,5.0 \mathrm{~Hz}, 1 \mathrm{H}), 3.17-3.05(\mathrm{~m}, 7 \mathrm{H}), 2.98(\mathrm{ddd}, J=12.9,9.2,5.0 \mathrm{~Hz}, 1 \mathrm{H}), 2.69-2.51(\mathrm{~m}, 1 \mathrm{H}), 2.43$ (s, 3H), 2.13 (dddd, $J=12.3,10.4,7.0,1.1 \mathrm{~Hz}, 1 \mathrm{H}), 1.95$ (tdd, $J=12.3,5.6,2.3 \mathrm{~Hz}, 1 \mathrm{H}), 1.80(\mathrm{dtd}, J=$ 11.7, 9.5, 7.0 Hz, 1H), 1.35 (ddt, $J=11.7,10.8,5.5 \mathrm{~Hz}, 1 \mathrm{H}) .{ }^{13} \mathbf{C}$ NMR (101 MHz, $\mathbf{C D C l}_{3}$ ): $\delta 143.19$, $137.24,129.65,127.00,103.40,48.60,48.15,44.08,42.26,29.42,21.52,14.86$. IR: v 3284, 2948, 1449, $1328,1160,1040,815 \mathrm{~cm}^{-1}$; HRMS calcd. For $[\mathrm{M}+\mathrm{Na}]^{+}: 322.1083$. Found: 322.1091. 
Chiral HPLC for 9 (Chiralpak IA, hexane:isopropanol $=98: 2,1 \mathrm{~mL} / \mathrm{min}, 210 \mathrm{~nm}$ ), $\mathrm{t}_{\text {minor }}=25.3 \mathrm{~min}$, $\mathrm{t}_{\text {major }}=26.9 \mathrm{~min} .[\alpha]_{\mathrm{D}}^{22.7}=-54.9\left(\mathrm{c}=0.71, \mathrm{CH}_{2} \mathrm{Cl}_{2}\right)$ at $98 \%$ ee.

\section{Racemic Sample 9}

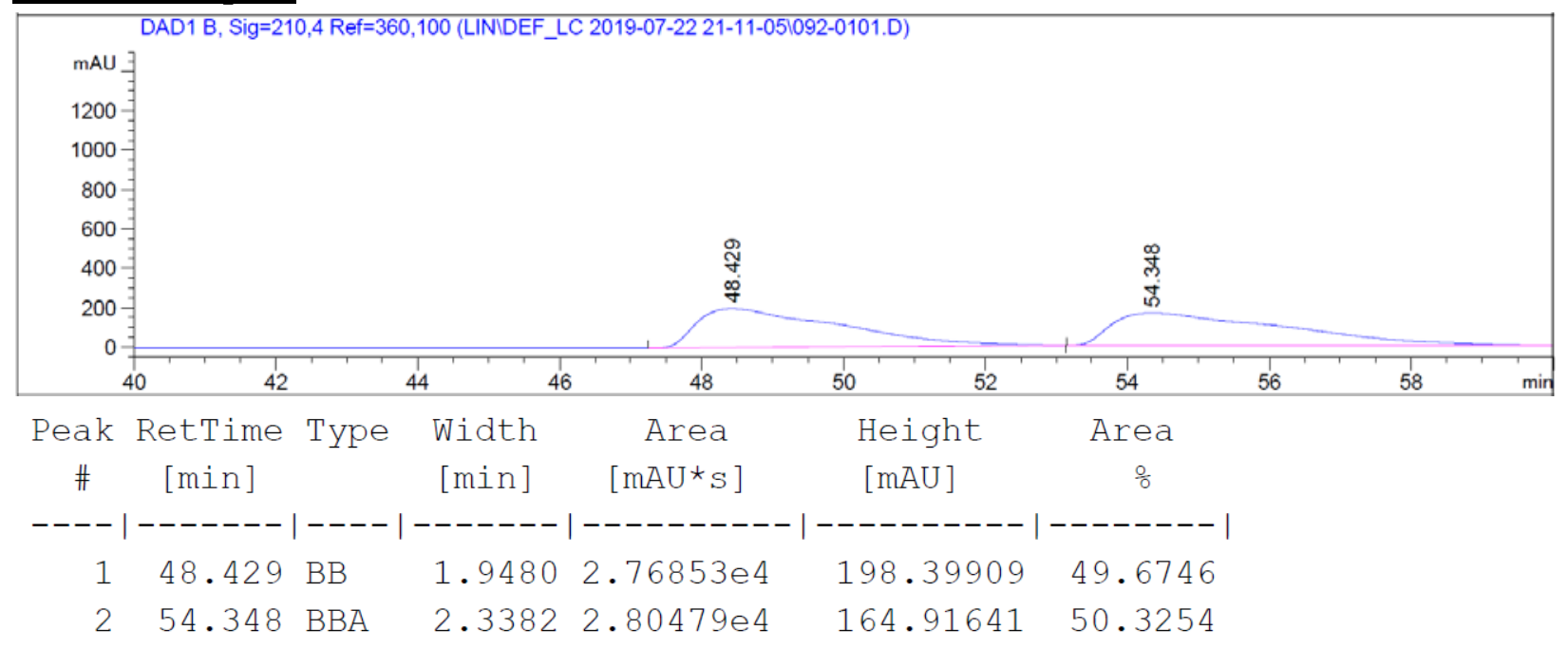

\section{Enantiomeric Sample 9}

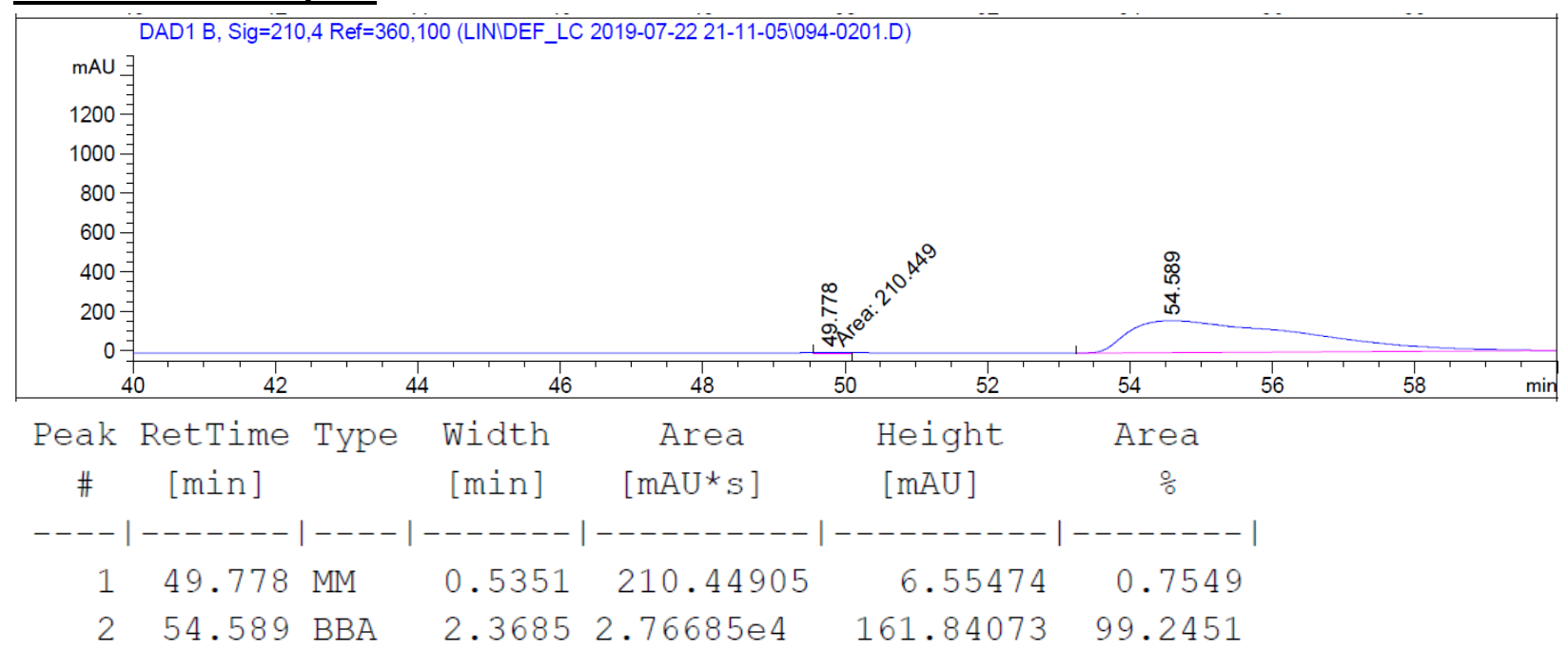

\section{Computational Studies}

\section{$\underline{5.1 \text { Computational details }}$}

Geometry optimizations and single-point energy calculations were carried out using Gaussian $16 .^{8}$ Geometries of intermediates and transition states were optimized using the B3LYP functional ${ }^{9}$ with a mixed basis set of LANL2DZ for Rh and 6-31G(d) for other atoms in the gas phase. Vibrational frequency calculations were performed for all the stationary points to confirm if each optimized structure is a local minimum or a transition state structure. All optimized transition state structures had only one imaginary (negative) frequency, and all minima (reactants, products, and intermediates) had no imaginary 
frequencies. The M06 functiona ${ }^{10}$ with a mixed basis set of $\mathrm{SDD}^{11}$ for $\mathrm{Rh}$ and $6-311+\mathrm{G}(\mathrm{d}, \mathrm{p})$ for other atoms was used for single-point energy calculations in solution. Solvation energy corrections were calculated in 1,4-dioxane solvent with the SMD continuum solvation model ${ }^{12}$ based on the gas-phase optimized geometries. The reported Gibbs free energies and enthalpies include zero-point vibrational energies and thermal corrections at $298 \mathrm{~K}$. The ligand-substrate dispersion energy ${ }^{13}\left(\Delta E_{\text {disp }}\right)$ was calculated from the difference of the dispersion energy of a supramolecular complex of the phosphine ligand and the cyclobutanone substrate at the optimized geometry of the oxidative addition transition state in the absence of the Rh metal center $\left(E_{\text {disp(lig+sub) }}\right)$ and the sum of the dispersion energies of the phosphine ligand and the cyclobutanone substrate at their geometries in the transition state $\left(E_{\text {disp(lig) }}\right.$ and $E_{\text {disp(sub), }}$ respectively):

$$
\Delta E_{\text {disp }}=E_{\text {disp(lig }+ \text { sub })}-\left(E_{\text {disp(lig) }}+E_{\text {disp(sub) })}\right)
$$

The dispersion energies were calculated with Grimme's DFT-D3 method ${ }^{14}$ (Version 3.1) using HF as the reference "functional".

The 3D images of structures were prepared using CYLView ${ }^{15}$ and POV-Ray. ${ }^{16}$

\subsection{Isomers of Rh-substrate $\pi$ Complexes and $\mathrm{C}-\mathrm{C}$ Oxidative Addition Transition States with the $(R)$ -} DTBM-segphos Ligand

Systematic conformational searches were performed for the substrate complexes and oxidative addition transition states with $(S)$-1a and $(R)$-1a using $(R)$-DTBM-segphos as the ligand. Substrate complexes (3 and $\mathbf{4}$ ) with the carbonyl oxygen and the alkenyl $\pi$ bond coordinated with the Rh center are the most stable (Figure S1a-b). In the most stable oxidative addition transition state with (S)-1a (TS1), the sulfonyl oxygen coordinates to the Rh center. Other transition states without the $N$-tosyl oxygen are at least $6 \mathrm{kcal} / \mathrm{mol} \mathrm{less} \mathrm{stable} \mathrm{(Figure} \mathrm{S} 1 \mathrm{c}$ ). In the oxidative addition with $(R)-\mathbf{1 a}$, the most favorable transition state is TS2, in which the $N$-tosyl group does not coordinate to the Rh center. There are two possible isomers of TS2 that can have $N$-Ts coordination to the Rh. We have located one of these isomers, which is higher in energy (TS10 in Figure S1d). In TS10, the Rh approaches from the more substituted face of the chiral carbon (i.e. the tether in TS10 points towards the Rh). Therefore, TS10 is destabilized due to repulsions between the alkyl tether and the Rh catalyst. We also attempted to locate the other isomer of TS2 with $\mathrm{N}$-Ts coordination there the Rh approaches from the less substituted face of the chiral carbon center. Attempts to locate this TS structure were unsuccessful because the $N$-Ts coordination site in this isomer is in the more occupied quadrant (quadrant IV, see Figure 3 ) and thus the $N$-Ts coordination is disfavored due to steric repulsions with the $(R)$-DTBM-SEPHOS ligand. Similar to the reaction with the (S)-enantiomer of 1a, the oxidative addition transition states with the alkene coordinated to the $\mathrm{Rh}$ (TS12 and TS13) are less favorable.

Under the Curtin-Hammett conditions, although the substrate complex with $(R)-\mathbf{1 a}$ is more stable than the substrate complex with $(S)-\mathbf{1 a}$, the oxidative addition with $(S)$-1a is faster because TS1 is more stable than TS2 by $5.1 \mathrm{kcal} / \mathrm{mol}$. 
(a) Substrate complex with (S)-1a

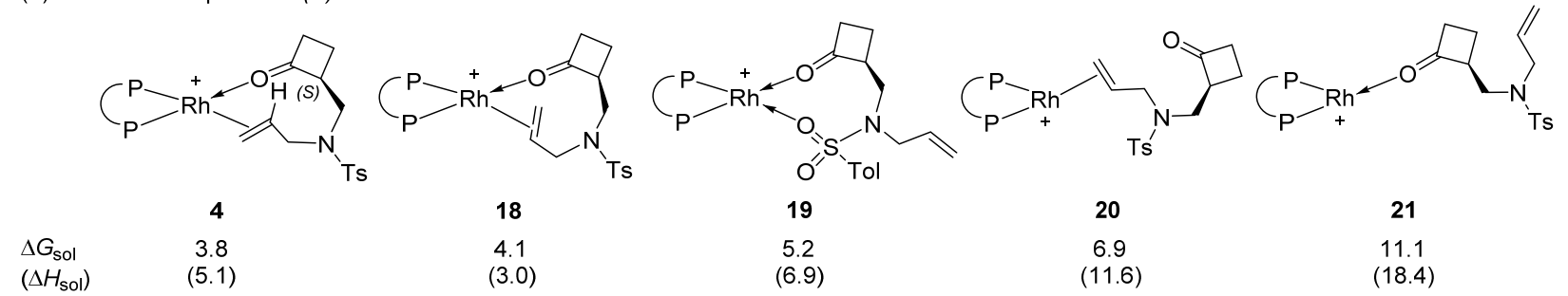

(b) Substrate complex with (R)-1a

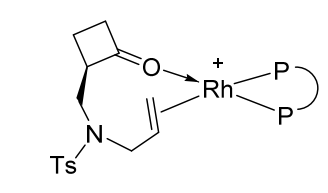

$\begin{array}{lc} & 3 \\ \Delta G_{\text {sol }} & 0.0 \\ \left(\Delta H_{\text {sol }}\right) & (0.0)\end{array}$

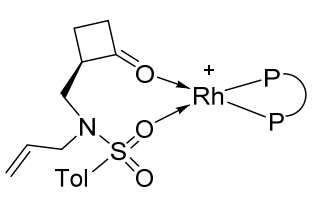

22
8.0

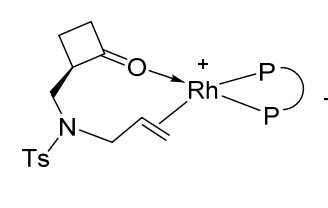

23
10.4

(11.8)

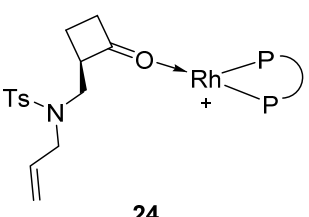

14.7
$(21.8)$

(c) Oxidative addition transition state with (S)-1a

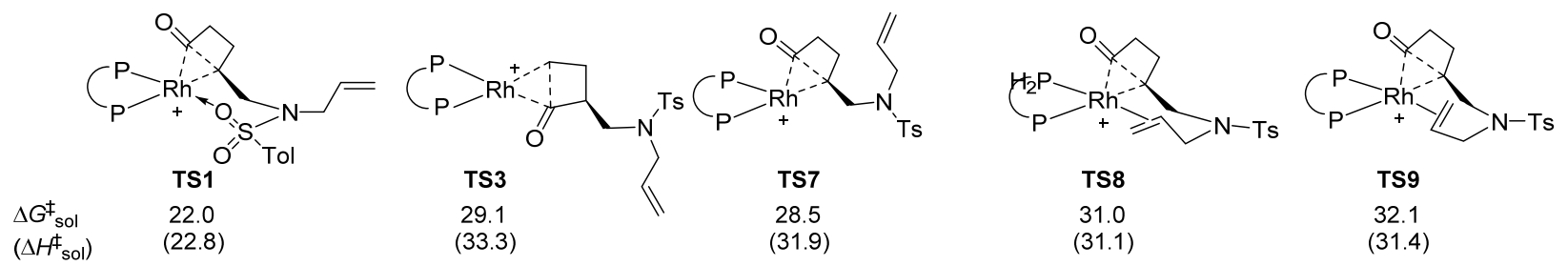

(d) Oxidative addition transition state with $(R)-1 \mathrm{a}$
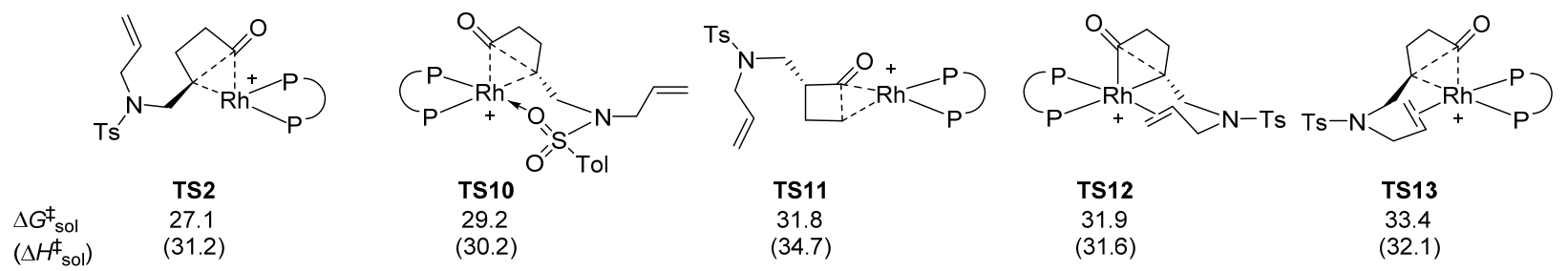

Figure S1. Isomers of the substrate complexes and $\mathrm{C}-\mathrm{C}$ oxidative addition transition states with $(R)$ DTBM-segphos as ligand. All energies are with respect to 3.

To investigate the conformations of the four OMe groups on the DTBM-segphos ligand, we have performed a careful conformer search to study the 16 possible conformers of TS1 with different OMe conformations. The calculated activation free energies are summarized in Table S1. One of the sixteen possible conformers (TS1_8) cannot be located because of the steric clash between the "proximal" OMe2 group and the substrate (see later).

One of the OMe groups, "OMe2", has a strong preference to point away from the substrate (i.e. "distal"). This OMe 2 group is on a $P$-aryl that is involved in the $\pi / \pi$ stacking with the $N$-Ts group on the substrate. The "distal" conformation will avoid steric repulsions that disrupt the $\pi / \pi$ stacking. By contract, the other three OMe groups do not have a clear preference for either of the two possible conformations. Indeed, the activation energies of the eight possible conformers with "distal" OMe 2 are all within $1 \mathrm{kcal} / \mathrm{mol}$, well within the expected error range of DFT calculations. This indicates the conformations of the OMe groups at these three positions have negligible impact on the stability of the TS. This is consistent with the 
different OMe conformations in X-ray crystal structures of DTBM-segphos supported transition metal complexes. ${ }^{17}$ To simplify the calculations, we used the all-“distal" conformation (as in TS1) consistently for all of the DTBM-segphos-supported intermediates and transition states in the calculations.

Table S1. Conformations of the $p$-OMe groups on the $(R)$-DTBM-segphos ligand in the oxidative addition transition state ${ }^{a}$

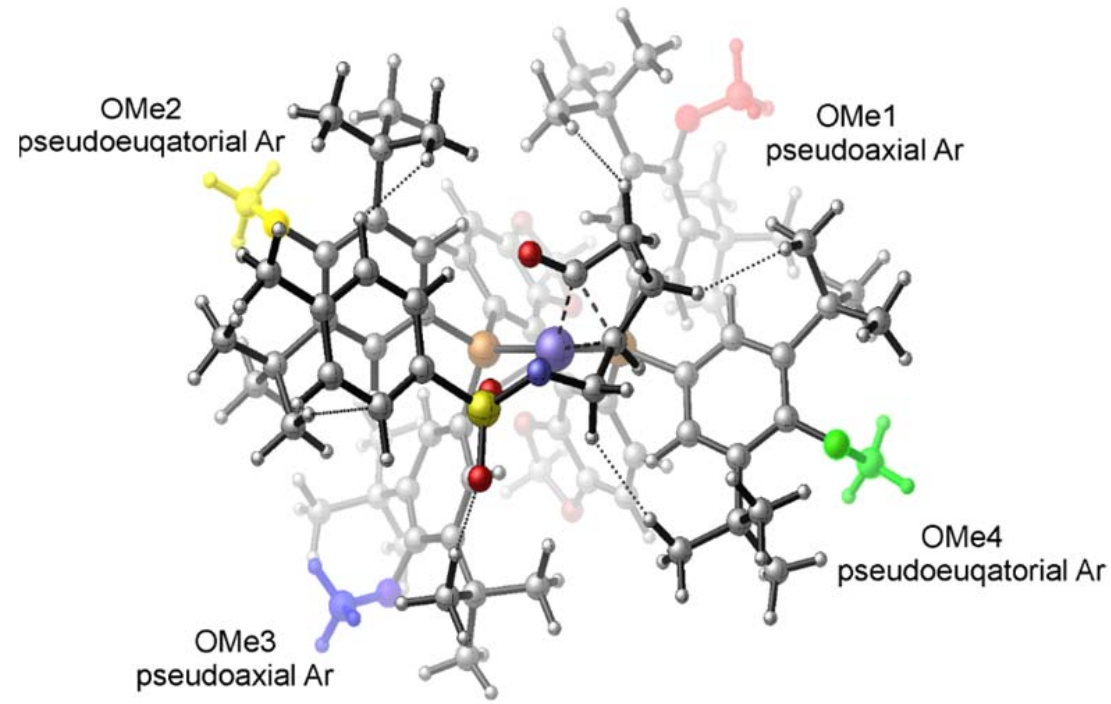

TS1

\begin{tabular}{cccccccc} 
Isomer & OMe1 & OMe2 & OMe3 & OMe4 & $\Delta H_{\text {sol }}^{\ddagger}$ & $\Delta G_{\text {sol }}^{\ddagger}$ & $\Delta G_{\text {sol(quasiharmonic) }}^{\ddagger}$ \\
\hline TS1 & distal & distal & distal & distal & 22.8 & 22.0 & 21.3 \\
TS1_2 & distal & distal & distal & proximal & 22.6 & 21.3 & 20.9 \\
TS1_3 & distal & distal & proximal & distal & 23.0 & 22.4 & 21.7 \\
TS1_4 & distal & distal & proximal & proximal & 23.0 & 22.1 & 21.5 \\
TS1_5 & distal & proximal & distal & distal & 25.6 & 25.2 & 24.6 \\
TS1_6 & distal & proximal & distal & proximal & 24.6 & 23.5 & 23.2 \\
TS1_7 & distal & proximal & proximal & distal & 27.5 & 27.0 & 26.5 \\
TS1_8 & distal & proximal & proximal & proximal & & cannot locate & \\
TS1_9 & proximal & distal & distal & distal & 21.9 & 21.6 & 21.0 \\
TS1_10 & proximal & distal & distal & proximal & 22.5 & 21.9 & 21.4 \\
TS1_11 & proximal & distal & proximal & distal & 22.1 & 21.6 & 20.9 \\
TS1_12 & proximal & distal & proximal & proximal & 22.1 & 21.7 & 21.0 \\
TS1_13 & proximal & proximal & distal & distal & 23.9 & 24.6 & 23.6 \\
TS1_14 & proximal & proximal & distal & proximal & 24.1 & 24.6 & 23.7 \\
TS1_15 & proximal & proximal & proximal & distal & 26.1 & 26.7 & 26.0 \\
TS1_16 & proximal & proximal & proximal & proximal & 26.2 & 27.3 & 26.4
\end{tabular}


${ }^{a}$ The N-allyl group is not shown for clarity. For OMe2 and OMe4, "proximal" represents the OMe pointing towards the substrate, and "distal" represents the OMe pointing away from the substrate. For OMe1 and OMe3, "proximal" and "distal" represent the OMe pointing towards and away from the biaryl backbone. $\Delta G_{\text {sol(quasiharmonic) }}^{\ddagger}$ are the activation Gibbs free energies calculated using Truhlar's quasiharmonic approximations. ${ }^{18}$

\section{$\underline{5.3 \text { Computed Reaction Energy Profiles of Reactions with }(R) \text {-segphos }}$}

We performed density functional theory (DFT) calculations to probe the origin of the low reactivity of the reaction using $(R)$-segphos as the ligand. The computed reaction energy profiles are shown in Figure $\mathrm{S} 2$. The oxidative addition activation barrier is $25.3 \mathrm{kcal} / \mathrm{mol}$, which is $3.3 \mathrm{kcal} / \mathrm{mol}$ higher in energy than TS1. The migratory insertion activation energy barrier TS16 $(13.7 \mathrm{kcal} / \mathrm{mol})$ and reductive elimination activation energy barrier TS17 $(12.7 \mathrm{kcal} / \mathrm{mol})$ are comparable with the corresponding activation energy barriers using $(R)$-DTBM-segphos. The rate-determining step is still the oxidative addition. The higher energy of the oxidative addition transition state with $(R)$-segphos as the ligand is mainly due to the weaker ligand-substrate dispersion interactions, especially the $\pi / \pi$ stabilizing interactions with the $N$-tosyl group. The DFT calculations also suggest the reaction with the $(R)$-segphos ligand is less enantioselective. The difference between oxidative addition activation barriers with (S)-1a (TS6) and (R)-1a (TS14) is only $1.0 \mathrm{kcal} / \mathrm{mol}$. 


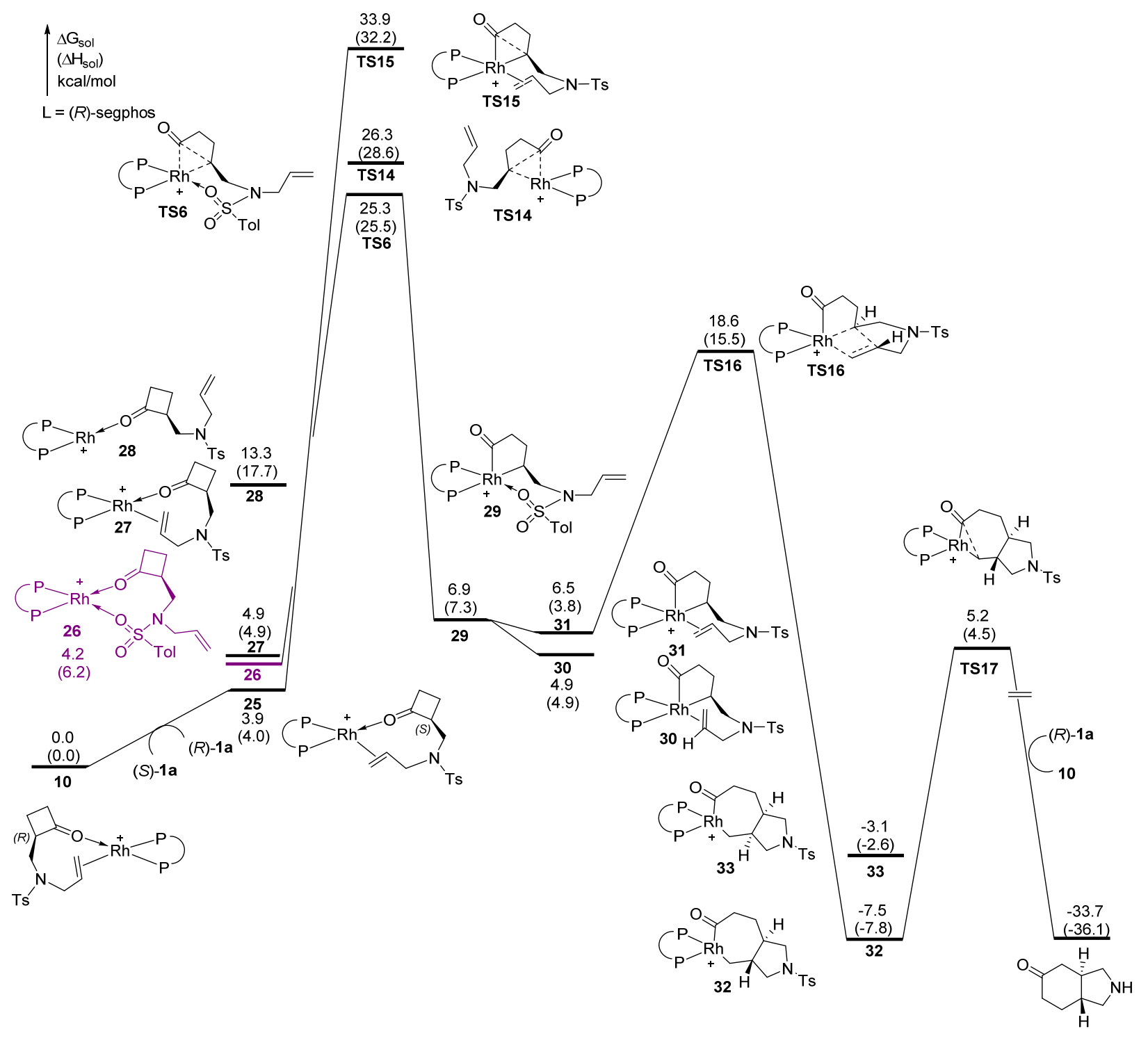

Figure S2. Computed reaction energy profiles of kinetic resolution of 1a via $\mathrm{Rh}$-catalyzed $\mathrm{C}-\mathrm{C}$ activation with the $(R)$-segphos ligand.

\subsection{Catalyst Resting State Analysis}

Computed ligand exchange energies to bind the cyclobutanone substrate $(R)$-1a to different precatalysts are summarized in Table S2. Substrate coordination to the Rh-diethylene precatalyst replacing the two ethylene molecules is slightly exergonic, while corresponding substrate coordination to the RhCOD precatalyst is endergonic. The higher substrate binding energy leads to slower oxidative addition, consistent with the experimentally observed low conversion with the Rh-COD precatalyst. The substrate binding energy is similar with segphos and DTBM-segphos as ligand, which suggests the ligand effects on reactivity is mainly due to the stabilization of the corresponding transition state, rather than promoting substrate coordination. 
Table S2. Substrate coordination to the Rh-diethylene and Rh-1,5-cyclooctadiene precatalysts.

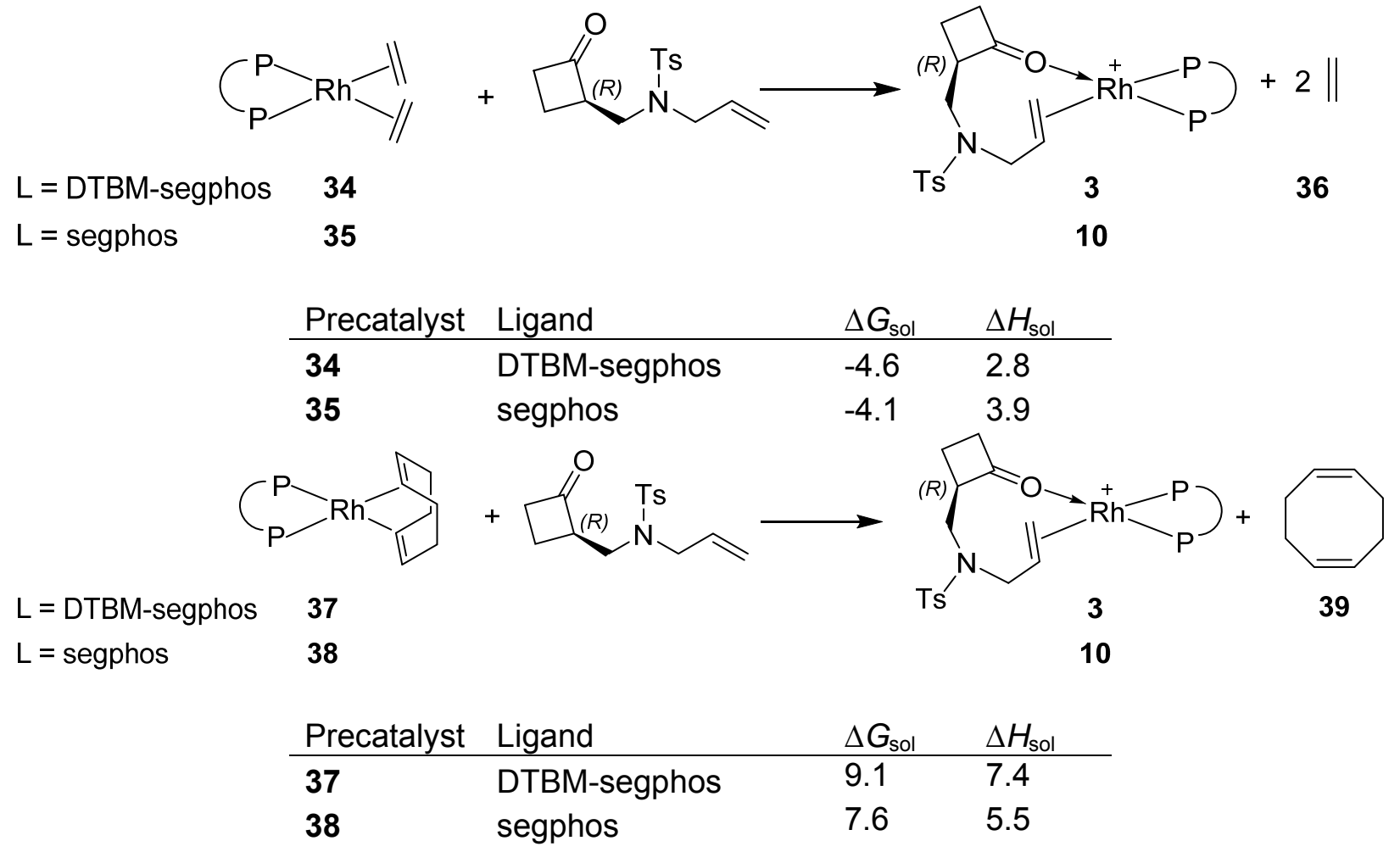

\subsection{Ligand-Substrate Dispersion Interactions with DTBM-segphos}

The oxidative addition of $(S)$-1a with the $(R)$-DTBM-segphos ligand requires a barrier that is 3.3 $\mathrm{kcal} / \mathrm{mol}$ lower than the reaction with the $(R)$-segphos ligand. As described in the main manuscript, the greater reactivity with DTBM-segphos is due to the stabilizing ligand-substrate dispersion interactions $\Delta E_{\text {disp }}$ in the oxidative addition transition state (TS1). Visual examination of TS1 revealed a $\pi / \pi$ stacking interaction between the toluene moiety on the $N$-tosyl group of $(S)$-1a and one of the P-aryl arms of the DTBM-segphos ligand. Similar $\pi / \pi$ stacking interaction was observed between the toluene moiety on $(S)$ 1a and one of the P-phenyl arms of segphos in TS6 (Figure S3). To quantitatively compare the strengths of the $\pi / \pi$ stacking interactions in TS1 and TS6, we calculated the $\pi / \pi$ stacking interaction energy $\left(\Delta E_{\text {int- }}\right.$ $\pi / \pi)$ using the following procedure.

We first constructed a $\pi / \pi$ stacking complex between the toluene moiety on (S)-1a and the P-aryl group on the ligand using the optimized geometries of TS1 and TS6. The $p$-tolyl unit on the substrate was taken from corresponding optimized TS geometry; an additional $\mathrm{H}$ atom was added to the dangling carbon along the direction of previous $\mathrm{C}-\mathrm{S}$ bond and the $\mathrm{C}-\mathrm{H}$ distance was set to $1.07 \AA$. The 3,5-di-tertbutyl-4-methoxyphenyl unit on (R)-DTBM-segphos was taken from corresponding optimized geometry of TS1, an additional $\mathrm{H}$ atom was added to the dangling carbon along the direction of previous $\mathrm{C}-\mathrm{P}$ bond and the $\mathrm{C}-\mathrm{H}$ distance was set to $1.07 \AA$. The phenyl unit on $(R)$-segphos was taken from corresponding optimized geometry of TS6, an additional $\mathrm{H}$ atom was added to the dangling carbon along the direction of previous C-P bond and the $\mathrm{C}-\mathrm{H}$ distance was set to $1.07 \AA$. Then, the interaction energy was calculated from

$$
\begin{gathered}
\Delta E_{\text {int }-\pi / \pi}=\underset{\text { dimer }}{ }-E_{\text {sub- } \pi}-E_{\text {lig- } \pi} \\
\text { S46 }
\end{gathered}
$$


where $E_{\text {dimer }}, E_{\text {sub- } \pi}$, and $E_{\text {lig- } \pi}$ are the electronic energies of the constructed $\pi / \pi$ stacking complex, the toluene moiety on the substrate, and the $P$-aryl moiety on the ligand, respectively. Here, the electronic energies were calculated at the M06/SDD-6-311+G(d,p) level of theory with the SMD(1,4-dioxane) solvation model.

The calculations indicated a stabilizing $\pi / \pi$ stacking interaction in TS1 $\left(\Delta E_{\text {int- } \pi / \pi}=-4.5 \mathrm{kcal} / \mathrm{mol}\right)$ and a much weaker $\pi / \pi$ stacking interaction in TS6 $\left(\Delta E_{\text {int }-\pi / \pi}=-1.0 \mathrm{kcal} / \mathrm{mol}\right)$. These results are in agreement with the ligand-substrate dispersion energy $\left(\Delta E_{\text {disp }}\right)$ calculations, which indicated greater Londondispersion interactions in TS1 than in TS6.

The $\pi / \pi$ stacking interactions in the substrate $\pi$ complexes (3 and 10) were also calculated. Because the $N$-tosyl group does not coordinate to the Rh center in these complexes, no $\pi / \pi$ stacking interaction with the ligand was observed. 

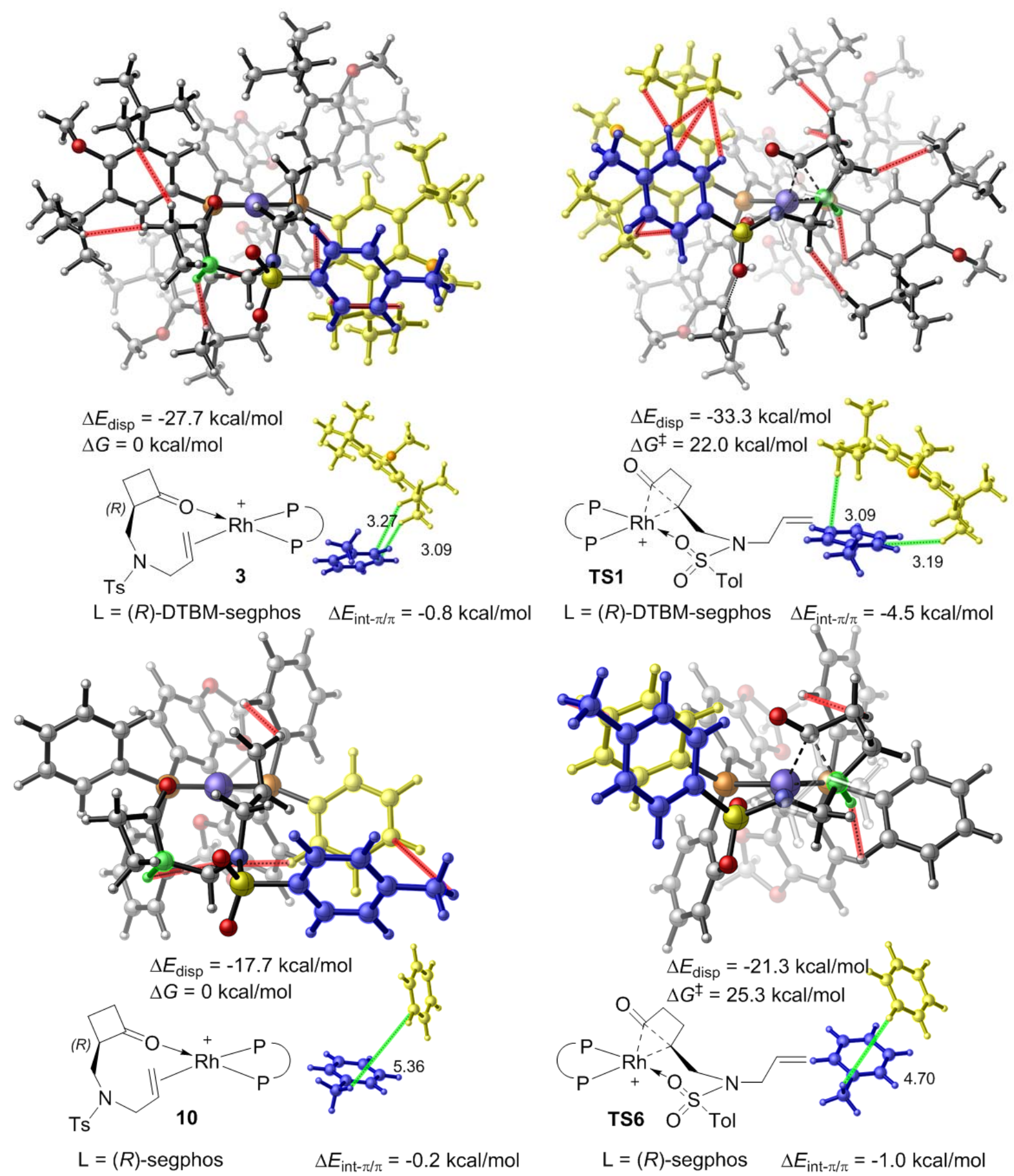

$\mathrm{L}=(R)$-segphos $\quad \Delta E_{\mathrm{int}-\pi / \pi}=-1.0 \mathrm{kcal} / \mathrm{mol}$

Figure S3. Computed $\pi / \pi$ stacking interaction energies $\left(\Delta E_{\mathrm{int}-\pi / \pi}\right)$ in the oxidative addition transition states TS1 and TS6 and the substrate complexes $\mathbf{3}$ and $\mathbf{1 0 .}$ 
To study which of the t-Bu substituents on DTBM-segphos more significantly contribute to the stabilizing ligand-substrate dispersion interactions, we calculated the pair-wise dispersion interactions between the cyclobutanone unit and tosylate unit in (S)-1a and the 6 proximal $t$-Bu substituents in DTBM-segphos. Here, the dispersion energies were calculated using the Grimme's DFT-D3 method with $\mathrm{HF}$ as the reference "functional". The geometry of each pairwise unit was taken from the geometry of TS1. An additional $\mathrm{H}$ atom was added to the dangling sulfur atom in the tosylate group, the carbon atom in the cyclobutanone, and the $t$-Bu groups along the direction of previous $\mathrm{S}-\mathrm{C}$ and $\mathrm{C}-\mathrm{C}$ bonds with the $\mathrm{C}-$ $\mathrm{H}$ distance set to $1.07 \AA$ (Figure S4). The sum of the computed dispersion energies of the 12 pairwise interactions is $-13.3 \mathrm{kcal} / \mathrm{mol}$. Among these, $-5.7 \mathrm{kcal} / \mathrm{mol}$ of the dispersion energies is associated with two pair-wise interactions between the tosyl group and two $t$-Bu groups in the occupied quadrant II. This indicates that the dispersion interactions between the $t$-Bu groups on the ligand and the tosylate on the (S)-1a are an important factor that promotes the reactivity of oxidative addition with the DTBM-segphos ligand. The coordination of sulfonyl oxygen is critical to stabilize TS1 because it not only stabilizes the $\mathrm{Rh}$ center via the oxygen coordination but also enhances the ligand-substrate dispersion interactions with the tosylate group. It should be noted that the dispersion interactions with the cyclobutanone moiety (Cyb) also contribute to the overall highly favorable ligand-substrate dispersion interactions, albeit to a lesser degree compared to the interactions with the tosylate group.

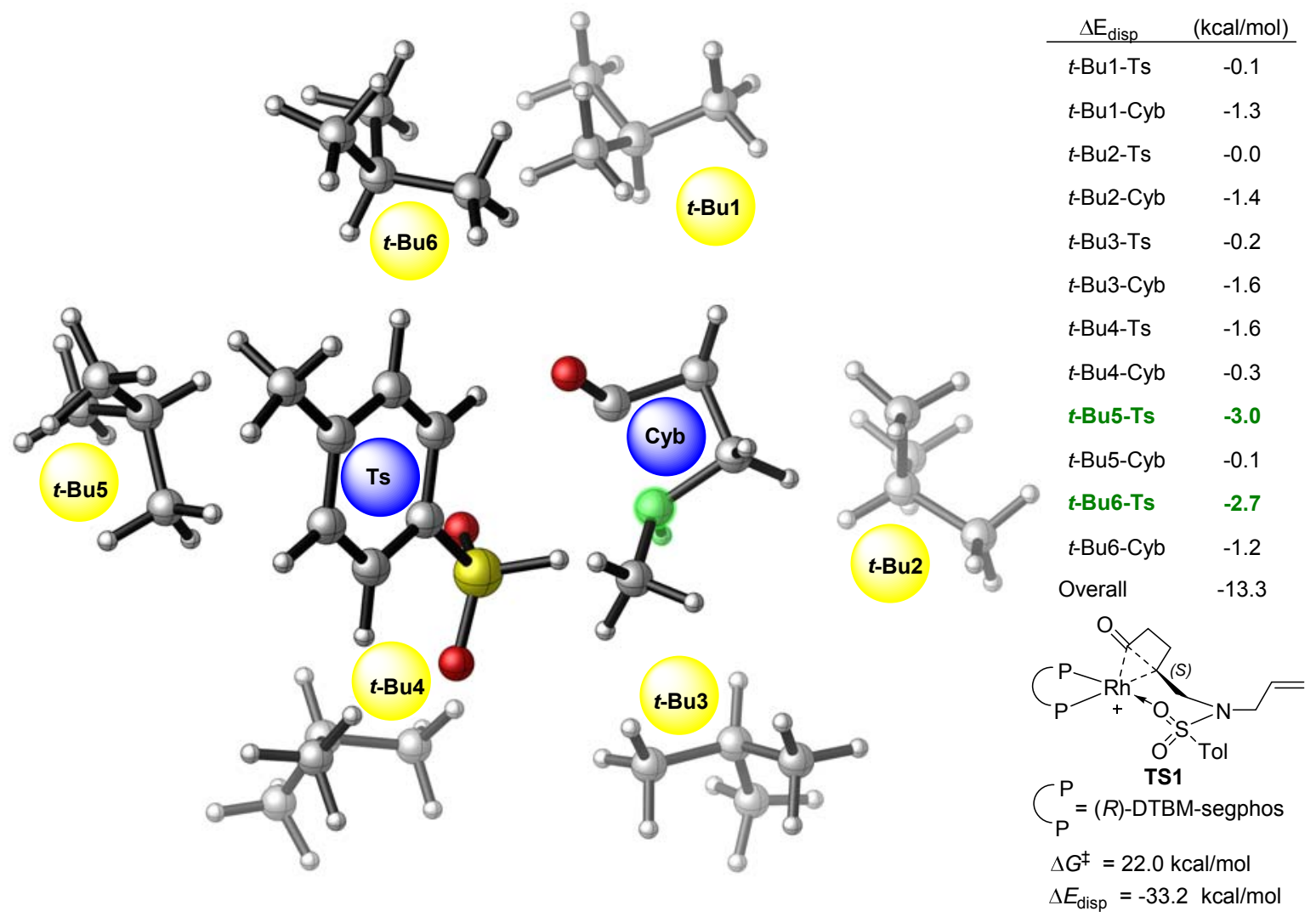

Figure S4. Pair-wise dispersion interactions between each $t$-Bu group on the DTBM-segphos ligand and the tosyl or cyclobutanone moiety in TS1. 
5.6 Cartesian Coordinates $(\AA)$ and $\quad$ Energies of Optimized Structures.

(R) $-1 \mathrm{a}$

B3LYP SCF energy: $\quad-1261.50942127$ a.u.

B3LYP enthalpy: $\quad-1261.169648$ a.u

B3LYP free energy: $\quad-1261.243864$ a.u.

M06 SCF energy in solution: $\quad-1261.17677972$

a.u.

M06 enthalpy in solution:

a.u.

M06 free energy in solution: -1260.911222

a.u.

Cartesian coordinates

\begin{tabular}{lrrr} 
ATOM & \multicolumn{1}{c}{ X } & Y & \multicolumn{1}{c}{$Z$} \\
C & -3.750438 & -2.393071 & -0.140786 \\
C & -4.328588 & -0.969173 & 0.123010 \\
C & -2.406310 & -1.893303 & 0.403257 \\
O & -1.382247 & -2.450714 & 0.713054 \\
C & -2.312875 & 0.567225 & -0.478390 \\
N & -0.899583 & 0.882735 & -0.207469 \\
C & -0.637201 & 2.143996 & 0.512948 \\
C & -1.103190 & 2.074066 & 1.942305 \\
C & -1.949366 & 2.942508 & 2.497942 \\
S & 0.189978 & 0.562241 & -1.481800 \\
C & 5.268798 & -1.216694 & 1.383461 \\
O & -0.367776 & -0.588674 & -2.201971 \\
O & 0.512051 & 1.796356 & -2.215154 \\
H & -3.693321 & -2.671562 & -1.199651 \\
H & -4.190672 & -3.229752 & 0.411759 \\
H & -5.049906 & -0.933341 & 0.943139 \\
H & -4.769426 & -0.481604 & -0.751429 \\
H & -2.901606 & 1.493938 & -0.438459 \\
H & -2.400603 & 0.168237 & -1.491626 \\
H & 0.446536 & 2.308498 & 0.500228 \\
H & -1.098997 & 2.997508 & -0.004813 \\
H & -0.681321 & 1.261025 & 2.532546 \\
H & -2.231213 & 2.876625 & 3.545250 \\
H & -2.383898 & 3.761264 & 1.927691 \\
H & 5.107850 & -1.371692 & 2.456545 \\
H & 5.613127 & -2.172903 & 0.968542 \\
H & 6.080350 & -0.492468 & 1.262853 \\
C & -2.909541 & -0.440287 & 0.517482 \\
H & -2.825455 & -0.044585 & 1.535365 \\
C & 1.662527 & 0.068924 & -0.587464 \\
C & 2.859403 & 0.734449 & -0.851283 \\
H & 2.870796 & 1.563478 & -1.550532 \\
C & 4.023269 & 0.318116 & -0.203008 \\
H & 4.958327 & 0.835599 & -0.402433 \\
C & 4.005152 & -0.753253 & 0.698131 \\
C & 2.783097 & -1.402282 & 0.942444 \\
H & 2.750192 & -2.233884 & 1.642468 \\
C & 1.610181 & -1.006530 & 0.306336 \\
H & 0.670929 & -1.513272 & 0.507904 \\
& & & \\
& & &
\end{tabular}

(S) $-1 \mathrm{a}$

B3LYP SCF energy: $\quad-1261.50744516$ a.u.

B3LYP enthalpy: $\quad-1261.167755 \mathrm{a} \cdot \mathrm{u}$

B3LYP free energy: $\quad-1261.243193$ a.u.

M06 SCF energy in solution: $\quad-1261.17660287$

a.u.

M06 enthalpy in solution:

a.u

M06 free energy in solution:

$-1260.836913$

a.u

$-1260.912351$

Cartesian coordinates

$\begin{array}{lccc}\text { ATOM } & X & Y & Z \\ \text { C } & 3.893517 & -2.378359 & -\odot .608379 \\ \text { C } & 2.381000 & -1.995915 & -0.594083 \\ \text { C } & 4.209477 & -0.903019 & -0.321951 \\ 0 & 5.190058 & -0.309062 & 0.049618\end{array}$

2.135248

$\odot .702685$

0.415416

$\odot .914362$

1.764893

$-0.367092$

$-5.508572$

0.167586

$-0.660719$

4. 267489

4. 249135

1.867420

1.804367

2.690317

2. 237161

$-0.672964$

0.842233

0.515187

2. 073808

2.179965

$-5.336326$

$-5.925683$

$-6.270839$

2.745326

2.645025

$-1.862859$

$-3.016047$

$-2.985830$

$-4.191863$

$-5.092405$

$-4.231080$

$-3.053746$

$-3.066404$

$-1.873743$

0.423487

0.516447

0.715636

0.263606

2. 061082

2. 200959

$-0.279491$

$-1.692238$

$0.226901 \quad 1.498254$

$-1.024533 \quad-1.539501$

$-1.039386 \quad 2.015066$

$1.332454 \quad 2.422249$

$-2.697300-1.588855$

$-3.089802 \quad 0.144135$

$-2.317182 \quad 0.315493$

$-2.320332$

1.368203

$-0.056755$

2. 193373

2. 844325

1. 478958

3. 230059

3.874813

$-1.125547$

$-1.977504$

$-0.254259$

$-0.477355$

$-0.004310$

$-0.106710$

0.625229

1. 409726

$\odot .328722$

$\odot .899535$

$-0.691038$

$-1.415415$

$-2.211863$

$-1.134642$

$-1.701687$

.557803

1. 492291

$-0.272246$

0.363043

$-2.404310$

$-3.134830$

$-1.404478$

$-2.617231$

$-1.189198$

$-1.388390$

$-0.556205$

$-1.541256$

0.566751

0.844070

1.592583

0.151648

0.363273

$-0.806358$

$-1.061630$

$-1.801930$

$-0.381548$

$-0.580140$

cis-2a

B3LYP SCF energy: $\quad-1261.57122689$ a.u.

B3LYP enthalpy: $\quad-1261.227686$ a.u.

B3LYP free energy: $\quad-1261.296157$ a.u.

M06 SCF energy in solution: -1261.24679123

a.u.

M06 enthalpy in solution:

$-1260.903250$

M06 free energy in solution:

$-1260.971721$

a.u.

Cartesian coordinates

\begin{tabular}{lrrr} 
ATOM & $\mathrm{X}$ & $\mathrm{Y}$ & \multicolumn{1}{c}{$\mathrm{Z}$} \\
$\mathrm{C}$ & 4.215853 & -1.058794 & 1.005663 \\
$\mathrm{C}$ & 3.035448 & -2.012697 & 0.726080 \\
$\mathrm{C}$ & 1.837643 & -1.320318 & 0.057684 \\
$\mathrm{C}$ & 4.596503 & -0.289287 & -0.251478 \\
$\mathrm{O}$ & 5.726862 & -0.277532 & -0.699538 \\
$\mathrm{C}$ & 1.010662 & -0.384993 & 0.973860 \\
$\mathrm{~N}$ & 0.577810 & 0.699002 & 0.066514 \\
$\mathrm{C}$ & 0.934191 & 0.409396 & -1.333984 \\
$\mathrm{C}$ & 2.206721 & -0.432292 & -1.159249 \\
$\mathrm{C}$ & 3.444855 & 0.461667 & -0.904147 \\
$\mathrm{~S}$ & -0.760355 & 1.625549 & 0.437325 \\
$\mathrm{C}$ & -2.191734 & 0.575693 & 0.166335 \\
$\mathrm{C}$ & -2.708857 & -0.177659 & 1.223845 \\
$\mathrm{C}$ & -3.790982 & -1.026072 & 0.991953 \\
$\mathrm{C}$ & -4.371472 & -1.132560 & -0.279771 \\
$\mathrm{C}$ & -3.838609 & -0.359813 & -1.322619 \\
$\mathrm{C}$ & -2.757810 & 0.493580 & -1.109841 \\
$\mathrm{C}$ & -5.565783 & -2.026996 & -0.514306 \\
0 & -0.680708 & 1.892337 & 1.875095 \\
0 & -0.803508 & 2.694353 & -0.562951 \\
$\mathrm{H}$ & 5.091877 & -1.605717 & 1.365116
\end{tabular}




$\begin{array}{rrrr}H & 3.933227 & -0.331229 & 1.780187 \\ \mathrm{H} & 2.719806 & -2.499403 & 1.657570 \\ \mathrm{H} & 3.385047 & -2.815627 & 0.063960 \\ \mathrm{H} & 1.593524 & 0.040506 & 1.796997 \\ \mathrm{H} & 0.159835 & -0.920840 & 1.413059 \\ \mathrm{H} & 0.151906 & -0.171500 & -1.848314 \\ \mathrm{H} & 1.092325 & 1.342670 & -1.878296 \\ \mathrm{H} & 2.389005 & -1.052529 & -2.044236 \\ \mathrm{H} & 3.153862 & 1.276922 & -0.225488 \\ \mathrm{H} & 3.800668 & -.913290 & -1.834820 \\ \mathrm{H} & -2.283122 & -0.074986 & 2.216336 \\ \mathrm{H} & -4.197253 & -1.608474 & 1.815396 \\ \mathrm{H} & -4.282533 & -0.420464 & -2.313426 \\ \mathrm{H} & -2.370367 & 1.111279 & -1.913190 \\ \mathrm{H} & -5.591847 & -2.857246 & 0.198839 \\ \mathrm{H} & -6.503655 & -1.467610 & -0.398952 \\ \mathrm{H} & -5.560841 & -2.445960 & -1.526140 \\ \mathrm{H} & 1.158606 & -2.101267 & -0.308395\end{array}$

trans-2a

B3LYP SCF energy: $\quad-1261.56505167$ a.u.

B3LYP enthalpy: $\quad-1261.221917$ a.u.

B3LYP free energy: -1261.293472 a.u.

M06 SCF energy in solution: -1261.23760692

a.u.

M06 enthalpy in solution:

$-1260.894472$

a.u.

M06 free energy in solution:

$-1260.966027$

Cartesian coordinates

\begin{tabular}{|c|c|c|c|}
\hline ATOM & $\mathrm{X}$ & $Y$ & z \\
\hline $\mathrm{c}$ & 5.032346 & $\odot .009885$ & $-\odot .208771$ \\
\hline c & 3.981476 & -0.824019 & $-\odot .999277$ \\
\hline C & 2.553102 & $-\odot .296012$ & $-\odot .779584$ \\
\hline c & 4.593819 & 1.436458 & 0.110479 \\
\hline 0 & 5.303269 & 2.400790 & $-\odot .096288$ \\
\hline c & 1.412544 & -1.312701 & -0.858645 \\
\hline $\mathrm{N}$ & ๑. 313313 & $-\odot .598253$ & $-\odot .156299$ \\
\hline c & $\odot .847179$ & 0.449081 & $\odot .755918$ \\
\hline C & 2.372046 & $\odot .312948$ & $\odot .621898$ \\
\hline C & 3.205749 & 1.590571 & $\odot .750857$ \\
\hline $\mathrm{s}$ & -0.992809 & -1.524592 & 0.365912 \\
\hline C & -2.377516 & -0.417525 & 0.103884 \\
\hline C & -2.799800 & $-\odot .145624$ & -1.201442 \\
\hline C & -3.884160 & 0.701917 & -1.400078 \\
\hline c & -4.561992 & 1.282745 & -0.314303 \\
\hline c & -4.121292 & $\odot .988131$ & $\odot .981150$ \\
\hline C & -3.034708 & 0.138552 & 1.199543 \\
\hline c & -5.748863 & 2.186888 & -0.548883 \\
\hline 0 & -1.081317 & -2.639214 & -0.583451 \\
\hline 0 & -0.909367 & -1.780529 & 1.812384 \\
\hline $\mathrm{H}$ & 5.994436 & 0.053343 & -0.724956 \\
\hline $\mathrm{H}$ & 5.209448 & -0.473986 & 0.763539 \\
\hline $\mathrm{H}$ & 4.048499 & -1.871453 & -0.681432 \\
\hline $\mathrm{H}$ & 4.224142 & -0.806465 & -2.067013 \\
\hline $\mathrm{H}$ & 1.691014 & -2.245100 & -0.348452 \\
\hline H & 1.107403 & -1.562028 & -1.877316 \\
\hline $\mathrm{H}$ & $\odot .501107$ & 1.435027 & 0.419010 \\
\hline H & $\odot .501923$ & $\odot .290952$ & 1.781463 \\
\hline $\mathrm{H}$ & 2.713351 & $-\odot .427705$ & 1.359710 \\
\hline $\mathrm{H}$ & 3.349247 & 1.889634 & 1.798138 \\
\hline H & 2.713463 & 2.436979 & $\odot .254740$ \\
\hline H & -2.288251 & -0.600129 & -2.043568 \\
\hline $\mathrm{H}$ & -4.215846 & 0.915585 & -2.413461 \\
\hline H & -4.636161 & 1.423980 & 1.833651 \\
\hline $\mathrm{H}$ & -2.702353 & -0.101435 & 2.203728 \\
\hline H & -5.522765 & 2.957145 & -1.295477 \\
\hline H & -6.609711 & 1.618420 & -0.923698 \\
\hline H & -6.059073 & 2.689131 & $\odot .372437$ \\
\hline H & 2.351626 & $\odot .503206$ & -1.509167 \\
\hline
\end{tabular}

3

B3LYP SCF energy: $\quad-5535.36368660$ a.u. B3LYP enthalpy: $\quad-5533.327467$ a.u. B3LYP free energy: $\quad-5533.596580$ a.u. M06 SCF energy in solution: $\quad-5534.59354359$ a.u.

M06 enthalpy in solution: $\quad-5532.557324$ a.u. M06 free energy in solution: $\quad-5532.826437$ a.u.

Cartesian coordinates

\begin{tabular}{|c|c|c|c|}
\hline ATOM & x & $\mathrm{Y}$ & Z \\
\hline$P$ & $\odot . ๑ \odot \odot \odot \odot \odot$ & -5.328659 & -5.148935 \\
\hline$P$ & $\odot . ๑ \odot \odot \odot \odot \odot$ & -5.317604 & -5.223714 \\
\hline 0 & $\odot .0 \odot \odot \odot \odot \odot$ & -0.213766 & -6.012821 \\
\hline 0 & $\odot . ๑ \odot \odot \odot \odot \odot$ & 1.281835 & -6.278507 \\
\hline 0 & $\odot . \odot \odot \odot \odot \odot \odot$ & -1.207179 & -4.661078 \\
\hline 0 & $\odot . ๑ \odot \odot \odot \odot \odot$ & $-\odot .990519$ & -2.948692 \\
\hline 0 & $\odot .0 \odot \odot \odot \odot \odot$ & -2.281659 & -1.575008 \\
\hline 0 & $\odot . \odot \odot \odot \odot \odot \odot ~$ & -3.308298 & -3.168338 \\
\hline 0 & $\odot . ๑ \odot \odot \odot \odot \odot$ & -1.593655 & -3.223525 \\
\hline 0 & $\odot . \odot \odot \odot \odot \odot \odot$ & -4.790529 & -5.222537 \\
\hline c & $\odot . \odot \odot \odot \odot \odot \odot$ & -6.712456 & -4.971710 \\
\hline$c$ & $\odot . ๑ \odot \odot \odot \odot \odot$ & -6.698220 & -5.050924 \\
\hline$c$ & 1.618229 & -4.772714 & -5.348577 \\
\hline c & 0.969517 & -1.928154 & $-\odot .569114$ \\
\hline$c$ & $-\odot .273064$ & -2.909776 & -1.337991 \\
\hline c & $-\odot .841208$ & -7.252582 & -4.884686 \\
\hline c & 2.790597 & -7.417748 & -4.919959 \\
\hline c & 2.435689 & -7.230619 & -5.027821 \\
\hline$c$ & 3.260158 & -2.361694 & 0.420603 \\
\hline c & 3.577495 & -1.508126 & -0.813094 \\
\hline c & 3.030427 & -8.918965 & -4.764180 \\
\hline c & 2.203576 & -9.240745 & -4.086599 \\
\hline c & 1.875039 & -9.408406 & -5.730312 \\
\hline c & 2.608877 & -9.295227 & -4.375837 \\
\hline c & -1.171715 & 2.781493 & -0.697347 \\
\hline c & -0.920013 & 2.767830 & -2.067589 \\
\hline c & -1.126949 & 3.895038 & -2.883605 \\
\hline c & -1.694012 & 5.030449 & -2.258608 \\
\hline c & -1.802322 & 5.141439 & -0.842774 \\
\hline c & -1.550153 & 3.989798 & -0.094143 \\
\hline$c$ & -2.111713 & 6.484814 & -0.131834 \\
\hline$c$ & -3.553128 & 6.978784 & -0.398813 \\
\hline$c$ & -1.104058 & 7.559532 & -0.610163 \\
\hline$c$ & -1.949415 & 6.357902 & 1.399438 \\
\hline$c$ & $-\odot .682298$ & 3.787558 & -4.370687 \\
\hline$c$ & $-\odot .58009 \odot$ & 5.130868 & -5.130725 \\
\hline c & 0.748092 & 3.184458 & -4.410427 \\
\hline$c$ & -1.633251 & 2.840313 & -5.141073 \\
\hline$c$ & -3.401055 & 5.924632 & -3.630903 \\
\hline c & -2.805210 & $\odot .937177$ & 0.831960 \\
\hline c & -3.867895 & 1.647619 & $\odot .264707$ \\
\hline$c$ & -3.106919 & -๑.๑959๑7 & 1.727270 \\
\hline$c$ & -4.410580 & -0.355514 & 2.179979 \\
\hline$c$ & -5.433813 & $\odot .497031$ & 1.688445 \\
\hline c & -5.198631 & 1.426670 & 0.641475 \\
\hline$c$ & -6.347653 & 2.136200 & $-\odot .122869$ \\
\hline c & -5.810554 & 2.956186 & -1.317356 \\
\hline$c$ & -7.139497 & 3.118854 & 0.772839 \\
\hline$c$ & -6.982333 & 1.083392 & 3.401602 \\
\hline$c$ & -7.310347 & 1.068854 & $-\odot .701670$ \\
\hline$c$ & -4.612956 & -1.546204 & 3.161618 \\
\hline$c$ & -4.249800 & -1.109969 & 4.602711 \\
\hline c & -6.037172 & -2.151539 & 3.138616 \\
\hline c & -3.661940 & -2.711069 & 2.777689 \\
\hline c & 2.695688 & -0.439736 & 1.462797 \\
\hline$c$ & 3.881123 & 0.123232 & 1.956233 \\
\hline & 4.465867 & -0.314287 & 3.147306 \\
\hline
\end{tabular}




\begin{tabular}{|c|c|c|c|c|c|c|c|}
\hline C & 3.819324 & -1.380968 & 3.833086 & $\mathrm{H}$ & -6.032952 & -3.073693 & 3.731489 \\
\hline C & 2.529868 & -1.839247 & 3.471578 & H & -6.799265 & -1.498000 & 3.558604 \\
\hline C & 2.023245 & -1.378933 & 2. 242271 & H & -6.342228 & -2.408174 & 2.118767 \\
\hline c & 1.611924 & -2.753512 & 4.333423 & $\mathrm{H}$ & -3.787832 & -3.006967 & 1.730925 \\
\hline C & 2.016680 & -2.875530 & 5.821873 & H & -2.607118 & -2.474492 & 2.943217 \\
\hline C & 1.525820 & -4.168743 & 3.714887 & H & -3.886552 & -3.580147 & 3.405847 \\
\hline C & ๑.185351 & -2.140134 & 4.346008 & H & 4.355194 & $\odot .911576$ & 1.386681 \\
\hline C & 5.401508 & ๑.908947 & 5.147561 & H & 1.064637 & -1.743390 & 1.887932 \\
\hline C & 5.100685 & -3.191448 & 4.661692 & H & 2.914028 & -3.471938 & 5.983179 \\
\hline C & 5.716860 & 0.404413 & 3.716860 & H & 2.177349 & -1.894994 & 6.278929 \\
\hline C & 6.092620 & 1.637342 & 2.864872 & H & 1.199880 & -3.369069 & 6.361240 \\
\hline C & 5.073357 & -1.837210 & -3.191386 & $\mathrm{H}$ & 0.847815 & -4.800285 & 4.301112 \\
\hline C & 3.879886 & -1.250562 & -3.693420 & H & 1.142595 & -4.133013 & 2.688314 \\
\hline C & 3.509720 & -2.809360 & -5.642280 & H & 2.501629 & -4.664789 & 3.686981 \\
\hline C & $3.00 \odot 5 \odot 8$ & $-\odot .710964$ & -2.747403 & H & $-\odot .467768$ & -2.745455 & 4.984321 \\
\hline C & 3.244548 & $-\odot .784288$ & -1.369194 & H & $\odot .200202$ & -1.118658 & 4.741238 \\
\hline c & 4.330113 & -1.543458 & $-\odot .925169$ & $\mathrm{H}$ & -0.272376 & -2.108573 & 3.353082 \\
\hline C & 5.249074 & -2.123257 & -1.814476 & H & 6.253594 & 1.477119 & 5.539790 \\
\hline C & 7.038136 & -1.176512 & -4.328515 & H & 4.525842 & 1.568053 & 5.140375 \\
\hline C & 4.353983 & $-\odot .486163$ & -6.132808 & H & 5.197051 & ๑. 082165 & 5.829779 \\
\hline c & 7.770415 & -2.664315 & -1.478686 & H & 4. 362681 & -3.976192 & 4.466203 \\
\hline C & 6.308019 & -3.131393 & -1.287017 & H & 5.788123 & -3.130903 & 3.810446 \\
\hline c & 6.121908 & -3.401851 & 0.223072 & H & 5.660291 & -3.442241 & 5.565555 \\
\hline C & 2.010854 & $-\odot .838264$ & -5.389530 & H & 6.412316 & 1.361564 & 1.852974 \\
\hline c & 6.962208 & -0.512534 & 3.759526 & H & 5.265890 & 2.351064 & 2.784588 \\
\hline C & 3.462937 & -1.328059 & -5.189308 & H & 6.934820 & 2.152618 & 3.339074 \\
\hline C & 6.112004 & -4.481710 & -2.021654 & H & 2.822345 & -3.421775 & -5.046555 \\
\hline H & $-\odot .796902$ & 2. 015169 & 5.256638 & H & 3.201966 & -2.887594 & -6.691851 \\
\hline H & -1.834531 & 1.270337 & 3.145438 & H & 4.514710 & -3.225251 & -5.548854 \\
\hline H & 3.743897 & 3.731376 & 5.061717 & H & 2. 090160 & -0.231329 & -3.081727 \\
\hline H & 2.551623 & 5.007594 & 4.579612 & H & 4.465586 & -1.685080 & 0.136757 \\
\hline $\mathrm{H}$ & 3.696207 & 1.557795 & -2.073118 & H & 7.743212 & -1.593047 & -5.051294 \\
\hline H & 4.231005 & 3.955378 & -2.390041 & $\mathrm{H}$ & 6.574393 & -0.278538 & -4.745234 \\
\hline $\mathrm{H}$ & 1.990321 & 7.163612 & 0.472124 & H & 7.569978 & -0.904544 & -3.412909 \\
\hline H & 3.417218 & 6.455910 & 1.333166 & H & 3.885133 & $-\odot .428493$ & -7.122069 \\
\hline H & $-\odot .538502$ & 1.853461 & -2.506491 & H & 4.475753 & $\odot .539752$ & -5.765279 \\
\hline H & -1.658593 & 4. 019196 & $\odot .982024$ & H & 5.339728 & $-\odot .931435$ & -6.271255 \\
\hline $\mathrm{H}$ & -4.291445 & 6.205097 & -0.155231 & H & 8.080375 & -2.711115 & -2.522911 \\
\hline H & -3.766519 & 7.849302 & 0.232830 & H & 7.920134 & -1.643389 & -1.108079 \\
\hline $\mathrm{H}$ & -3.695779 & 7.285561 & -1.435313 & H & 8.440995 & -3.322219 & -0.913657 \\
\hline H & $-1.1900 \odot 8$ & 7.745928 & -1.681702 & H & 6.830572 & -4.176769 & 0.533820 \\
\hline H & -1.283284 & 8.502148 & -0.079271 & H & $6.3206 \odot 4$ & -2.512933 & 0.833274 \\
\hline H & $-\odot .077279$ & 7.240888 & $-\odot .3979 \odot 2$ & H & 5.114859 & -3.763277 & $\odot .459898$ \\
\hline H & -0.949491 & 6.007344 & 1.677787 & H & 1.301638 & -1.385617 & -4.758942 \\
\hline H & -2.097203 & 7.342802 & 1.855255 & H & $1.90 \odot 873$ & $\odot .233660$ & -5.187623 \\
\hline H & -2.690729 & 5.682983 & 1.843271 & H & 1.722718 & -1.001896 & -6.433336 \\
\hline H & $-\odot .080 \odot 58$ & 4.946230 & -6.088654 & H & 7.160555 & $-\odot .961592$ & 2.778726 \\
\hline $\mathrm{H}$ & -1.547066 & 5.579767 & -5.356486 & $\mathrm{H}$ & 7.843504 & 0.078496 & 4.035474 \\
\hline H & $\odot .012670$ & 5.865444 & -4.578516 & H & 6.860855 & -1.309619 & 4.496155 \\
\hline $\mathrm{H}$ & 1.088423 & 3.122146 & -5.450317 & $\mathrm{H}$ & 6.841429 & -5.215476 & -1.658198 \\
\hline $\mathrm{H}$ & 1.454243 & 3.812879 & -3.857503 & H & 5.110348 & -4.888309 & -1.833491 \\
\hline $\mathrm{H}$ & 0.799663 & 2.176057 & -3.990609 & $\mathrm{H}$ & 6.245162 & -4.372302 & -3.099806 \\
\hline H & -1.659268 & 1.841876 & -4.689280 & $\mathrm{Rh}$ & $-\odot .185598$ & $-\odot .581007$ & -0.786484 \\
\hline $\mathrm{H}$ & -2.659962 & 3.221658 & -5.161989 & 0 & 0.663075 & -2.265978 & -1.946351 \\
\hline H & -1.298187 & 2.729191 & -6.179229 & 0 & -2.504981 & -6.382653 & $\odot .123356$ \\
\hline $\mathrm{H}$ & -4.175499 & 5.713364 & -2.884806 & C & 0.805820 & -3.437104 & -1.620470 \\
\hline H & -3.629286 & 6.865034 & -4.137449 & $\mathrm{~s}$ & -2.873072 & -5.532420 & -1.012991 \\
\hline $\mathrm{H}$ & -3.387494 & 5.112741 & -4.365330 & C & 1.397831 & -4.634550 & -2.336092 \\
\hline H & -3.651815 & 2.394794 & $-\odot .484 \odot 45$ & C & 0.332507 & -4.259128 & -0.435621 \\
\hline $\mathrm{H}$ & -2.296119 & -0.716406 & 2.089911 & $\mathrm{~N}$ & -2.226322 & -3.986876 & -0.654130 \\
\hline H & -6.658837 & 3.380978 & -1.864431 & 0 & -2.499214 & -5.911233 & -2.385466 \\
\hline $\mathrm{H}$ & -5.175805 & 3.791570 & -0.999046 & C & -4.639797 & -5.260218 & -0.999696 \\
\hline $\mathrm{H}$ & -5.241696 & 2.339008 & -2.022004 & C & 0.667320 & -5.532513 & -1.288046 \\
\hline H & -7.739708 & 2.601458 & 1.520948 & H & 2.492153 & -4.646004 & -2.264204 \\
\hline $\mathrm{H}$ & -6.469817 & 3.821472 & 1.283187 & H & 1.117965 & -4.743366 & -3.388700 \\
\hline $\mathrm{H}$ & -7.826914 & 3. 706911 & 0.153429 & C & -1.035718 & -3.960326 & 0.204833 \\
\hline $\mathrm{H}$ & -6.607456 & 0.518422 & 4.260758 & H & 1.083277 & -4.145407 & 0.361614 \\
\hline $\mathrm{H}$ & -6.534907 & 2.082996 & 3.406271 & C & -2.318703 & -3.023069 & -1.762902 \\
\hline $\mathrm{H}$ & -8.068328 & 1.170571 & 3.482851 & C & -5.328659 & -5.148935 & 8.760100 \\
\hline $\mathrm{H}$ & -7.753333 & 0.458029 & $\odot .086707$ & C & -5.317604 & -5.223714 & 9.970300 \\
\hline $\mathrm{H}$ & -8.120729 & 1.559959 & -1.253760 & H & -0.213766 & -6.012821 & 13.791900 \\
\hline $\mathrm{H}$ & -6.782987 & 0.407692 & -1.399768 & $\mathrm{H}$ & 1.281835 & -6.278507 & -0.782178 \\
\hline $\mathrm{H}$ & -3.208473 & -0.771703 & 4.660349 & H & -1.207179 & -4.661078 & 1.023578 \\
\hline $\mathrm{H}$ & -4.883143 & -0.294954 & 4.964955 & $\mathrm{H}$ & -0.990519 & -2.948692 & 0.628484 \\
\hline $\mathrm{H}$ & -4.365844 & -1.953957 & 5.293244 & C & -2.281659 & -1.575008 & -1.328385 \\
\hline
\end{tabular}




$\begin{array}{lrrr}\mathrm{H} & -3.308298 & -3.168338 & -2.215607 \\ \mathrm{H} & -1.593655 & -3.223525 & -2.560121 \\ \mathrm{H} & -4.790529 & -5.222537 & -3.147606 \\ \mathrm{C} & -6.712456 & -4.971710 & -2.185618 \\ \mathrm{C} & -6.698220 & -5.050924 & 0.223912 \\ \mathrm{H} & -4.772714 & -5.348577 & 1.152172 \\ \mathrm{C} & -1.928154 & -0.569114 & -2.206922 \\ \mathrm{H} & -2.909776 & -1.337991 & -0.475303 \\ \mathrm{H} & -7.252582 & -4.884686 & -3.124621 \\ \mathrm{C} & -7.417748 & -4.919959 & -0.976241 \\ \mathrm{H} & -7.230619 & -5.027821 & 1.171304 \\ \mathrm{H} & -2.361694 & 0.420603 & -2.118463 \\ \mathrm{H} & -1.508126 & -0.813094 & -3.181813 \\ \mathrm{C} & -8.918965 & -4.764180 & -0.956997 \\ \mathrm{H} & -9.240745 & -4.086599 & -0.158806 \\ \mathrm{H} & -9.408406 & -5.730312 & -0.778279 \\ \mathrm{H} & -9.295227 & -4.375837 & -1.908069\end{array}$

B3LYP SCF energy:

B3LYP enthalpy:

B3LYP free energy

Mo6 SCF energy in solution:

a.u.

M06 enthalpy in solution:

M06 free energy in solution:

-5535.35702349 a.u. -5533.320701 a.u. -5533.591930 a.u. a.u

Cartesian coordinates

\begin{tabular}{|c|c|c|c|}
\hline АTOM & $\mathrm{X}$ & $\mathrm{Y}$ & Z \\
\hline $\mathrm{P}$ & $\odot . \odot \odot \odot \odot \odot \odot$ & 5.536479 & -5.372700 \\
\hline$P$ & ๑. .००००० & 5.337890 & -5.707836 \\
\hline 0 & $\odot . ๑ \odot \odot \odot \odot \diamond$ & 5.030089 & -2.216912 \\
\hline 0 & ๑. .०००००० & 6.220868 & -1.068989 \\
\hline 0 & $\odot . \diamond \odot \odot \odot \odot \diamond$ & 4.392227 & -2.650217 \\
\hline 0 & $\odot . \diamond \odot \odot \odot \odot \odot$ & 2.841728 & -1.819171 \\
\hline 0 & ๑. .०००००० & ๑. 613108 & -2.668388 \\
\hline 0 & $\odot . \diamond \odot \odot \odot \odot \diamond$ & 1.845269 & -3.991804 \\
\hline 0 & 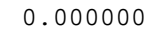 & 2.348992 & -2.334064 \\
\hline 0 & $\odot . \diamond \odot \odot \odot \odot \diamond$ & 5.079557 & -5.100564 \\
\hline C & ๑. .๑०००० & 6.867762 & -5.788654 \\
\hline C & $\odot .0 \odot \odot \odot \odot \odot$ & 6.664820 & -6.120446 \\
\hline C & -2.698674 & 4.733020 & -5.693854 \\
\hline C & -2.167761 & 0.195743 & -2.734988 \\
\hline C & -0.825299 & -0.143292 & -2.685480 \\
\hline C & -0.025406 & 7.457543 & -5.826316 \\
\hline C & -4.307408 & 7.451604 & -6.168256 \\
\hline C & -2.730378 & 7.099032 & -6.419991 \\
\hline C & -3.449297 & -0.846903 & -2.921229 \\
\hline C & -4.062716 & ๑. 892433 & -2.963969 \\
\hline C & -3.929706 & 8.882500 & -6.644871 \\
\hline C & -3.217890 & 9.421164 & -6.184285 \\
\hline C & -2.600877 & 8.925756 & -7.731363 \\
\hline C & -4.181764 & 9.423842 & -6.415555 \\
\hline C & 0.540653 & 3.127383 & ๑. 630186 \\
\hline C & ๑. 608941 & 3. .01220 & 2.015662 \\
\hline C & 0.695428 & 4.116190 & 2.868073 \\
\hline C & ๑. 810112 & 5.380723 & 2.244568 \\
\hline C & 0.588381 & 5.562244 & $\odot .849410$ \\
\hline C & 0.474816 & 4.409756 & $\odot .068086$ \\
\hline C & ๑. 394012 & 6.962029 & $\odot .210569$ \\
\hline C & 1.684463 & 7.814421 & $\odot .246451$ \\
\hline C & -0.737466 & 7.710510 & ○. 958460 \\
\hline C & -0.034869 & 6.848385 & -1.269476 \\
\hline C & 0.607773 & 3.841247 & 4.396423 \\
\hline C & ๑. 357312 & 5.081383 & 5.285422 \\
\hline C & -0.604442 & 2.902564 & 4.648722 \\
\hline C & 1.891541 & 3.123057 & 4.877284 \\
\hline C & 2.483093 & 6.646540 & 3.334553 \\
\hline C & 2.277324 & 1.598712 & -1.128882 \\
\hline C & 3. 261915 & 2.455481 & -0.631086 \\
\hline C & 2.643874 & $\odot .658662$ & -2.100148 \\
\hline C & 3.927764 & 0.614105 & -2.669149 \\
\hline
\end{tabular}

\begin{tabular}{|c|c|c|c|}
\hline C & 4.872116 & 1.570104 & -2.194843 \\
\hline C & 4.577338 & 2.445199 & -1.113158 \\
\hline C & 5.640950 & 3.355043 & -0.440558 \\
\hline C & 5.076001 & 4.061820 & 0.813310 \\
\hline C & 6.119698 & 4.475617 & -1.394446 \\
\hline C & 6.402067 & 2.301397 & -3.935827 \\
\hline C & 6.850660 & 2.509870 & ๑. 030251 \\
\hline C & 4.213903 & -0.441764 & -3.776486 \\
\hline C & 3.993006 & $\odot .195495$ & -5.173089 \\
\hline C & 5.630548 & -1.056832 & -3.666969 \\
\hline C & 3.235288 & -1.640172 & -3.697011 \\
\hline C & -2.760778 & -0.935321 & -1.281916 \\
\hline C & -4.134437 & -0.713127 & -1.463344 \\
\hline C & -4.826305 & -1.227071 & -2.561778 \\
\hline C & -4.080899 & -2.015295 & -3.483850 \\
\hline C & -2.669048 & -2.085400 & -3.445086 \\
\hline C & -2.045529 & -1.573241 & -2.292186 \\
\hline C & -1.741022 & -2.621382 & -4.571997 \\
\hline C & -2.424034 & -2.911042 & -5.928780 \\
\hline C & -1.011276 & -3.900570 & -4.097344 \\
\hline C & -0.686477 & -1.522342 & -4.873454 \\
\hline C & -6.491843 & -0.236262 & -4.182318 \\
\hline C & -4.937685 & $-4.0905 \odot 4$ & -4.215461 \\
\hline C & -6.320482 & -0.875609 & -2.781905 \\
\hline C & -6.811778 & $\odot .158772$ & -1.744849 \\
\hline C & -3.805131 & -3.200937 & 3.519279 \\
\hline C & -2.799219 & -2.273333 & 3.905001 \\
\hline C & -1.644522 & -3.669046 & 5.653770 \\
\hline C & -2.274030 & -1.459827 & 2.893327 \\
\hline C & -2.681851 & -1.570164 & 1.554923 \\
\hline C & -3.530570 & -2.622826 & 1.204096 \\
\hline C & -4.076594 & -3.494146 & 2.159971 \\
\hline C & -5.707569 & -3.252502 & 4.920361 \\
\hline C & -3.164931 & -1.818681 & 6.438774 \\
\hline C & -6.301613 & -4.842213 & 2.106482 \\
\hline C & -4.810050 & -4.785348 & 1.700370 \\
\hline C & -4.760348 & -4.939913 & ๑.163561 \\
\hline C & -0.976947 & -1.280044 & 5.404289 \\
\hline C & -7.242955 & -2.111973 & -2.667184 \\
\hline C & -2.177082 & -2.250145 & 5.329083 \\
\hline C & -4.073840 & -6.006860 & 2.307425 \\
\hline $\mathrm{H}$ & -0.403713 & 2.631270 & -5.241269 \\
\hline $\mathrm{H}$ & 1.036493 & 2. 071878 & -3.303519 \\
\hline $\mathrm{H}$ & -5.183712 & 2.820173 & -4.504240 \\
\hline $\mathrm{H}$ & -4.446809 & 4.405547 & -4.035453 \\
\hline $\mathrm{H}$ & -3.566457 & $\odot .567302$ & 2.526473 \\
\hline $\mathrm{H}$ & -4.623200 & 2.725956 & 3.115256 \\
\hline $\mathrm{H}$ & -3.771697 & 6.508725 & ๑. 212920 \\
\hline $\mathrm{H}$ & -5.121981 & 5.519024 & -0.475671 \\
\hline $\mathrm{H}$ & 0.594587 & 2.005276 & 2.441309 \\
\hline $\mathrm{H}$ & $\odot .336726$ & 4.499941 & -1.001164 \\
\hline $\mathrm{H}$ & 2.532055 & 7.271570 & -0.189417 \\
\hline $\mathrm{H}$ & 1.539020 & 8.727854 & -0.342403 \\
\hline $\mathrm{H}$ & 1.946094 & 8.118381 & 1. 260299 \\
\hline $\mathrm{H}$ & $-\odot .487886$ & 7.874663 & 2.007941 \\
\hline $\mathrm{H}$ & -0.914932 & 8.685412 & $\odot .488659$ \\
\hline $\mathrm{H}$ & -1.671241 & 7.137845 & ๑. 913549 \\
\hline $\mathrm{H}$ & -0.948727 & 6.256045 & -1.388355 \\
\hline $\mathrm{H}$ & $-\odot .234195$ & 7.852292 & -1.659573 \\
\hline $\mathrm{H}$ & ๑. 748014 & 6.407757 & -1.897958 \\
\hline $\mathrm{H}$ & $\odot .128046$ & 4.735632 & $6.30 \odot 223$ \\
\hline $\mathrm{H}$ & 1. 218358 & 5.745321 & 5.361764 \\
\hline $\mathrm{H}$ & -0.493144 & 5.670229 & 4.931088 \\
\hline $\mathrm{H}$ & -0.702830 & 2.713164 & 5.723757 \\
\hline $\mathrm{H}$ & -1.534716 & 3.362078 & 4.297233 \\
\hline $\mathrm{H}$ & -0.502438 & 1.932737 & 4.154168 \\
\hline $\mathrm{H}$ & 2.056050 & 2.188819 & 4.328026 \\
\hline $\mathrm{H}$ & 2.780044 & 3.750834 & 4.744719 \\
\hline $\mathrm{H}$ & 1.812758 & 2.878712 & 5.943517 \\
\hline $\mathrm{H}$ & 3. 109979 & 6.694204 & 2.436621 \\
\hline $\mathrm{H}$ & 2.566464 & 7.585103 & 3.887135 \\
\hline $\mathrm{H}$ & 2.833579 & 5.823112 & 3.965805 \\
\hline $\mathrm{H}$ & 2.992089 & 3.148178 & ๑. 151944 \\
\hline $\mathrm{H}$ & 1.898375 & -0.052994 & -2.431378 \\
\hline $\mathrm{H}$ & 5.880144 & $4.634 \odot 93$ & 1.287864 \\
\hline
\end{tabular}




\begin{tabular}{|c|c|c|c|}
\hline $\mathrm{H}$ & 4.272876 & 4.767248 & $\odot .571725$ \\
\hline $\mathrm{H}$ & 4.694421 & 3.351948 & 1.556589 \\
\hline $\mathrm{H}$ & 6.697119 & 4.090602 & -2.233766 \\
\hline $\mathrm{H}$ & 5.270552 & 5.045241 & -1.789767 \\
\hline $\mathrm{H}$ & 6.764779 & 5.174423 & $-\odot .848847$ \\
\hline $\mathrm{H}$ & 6.501462 & 1.587953 & -4.759558 \\
\hline $\mathrm{H}$ & 5.607603 & 3.018616 & -4.164865 \\
\hline $\mathrm{H}$ & 7.350412 & 2.834544 & -3.827219 \\
\hline $\mathrm{H}$ & 7.325591 & 1.982554 & $-\odot .797477$ \\
\hline $\mathrm{H}$ & 7.596723 & 3.159259 & 0.503487 \\
\hline $\mathrm{H}$ & 6.538294 & 1.768586 & $\odot .777633$ \\
\hline $\mathrm{H}$ & 2.952884 & $\odot .523842$ & -5.282958 \\
\hline $\mathrm{H}$ & 4.631916 & 1.059611 & -5.359313 \\
\hline $\mathrm{H}$ & 4.193897 & -0.543699 & -5.957748 \\
\hline $\mathrm{H}$ & 5.745051 & -1.833452 & -4.431491 \\
\hline $\mathrm{H}$ & 6.434825 & -0.335203 & -3.790698 \\
\hline $\mathrm{H}$ & 5.768247 & -1.533465 & -2.688513 \\
\hline $\mathrm{H}$ & 3.257380 & -2.142909 & -2.725329 \\
\hline $\mathrm{H}$ & 2.201526 & -1.361742 & -3.919441 \\
\hline $\mathrm{H}$ & 3.525625 & -2.379729 & -4.450680 \\
\hline $\mathrm{H}$ & -4.668446 & -0.137989 & -0.718573 \\
\hline $\mathrm{H}$ & -0.970849 & -1.663135 & -2.182082 \\
\hline $\mathrm{H}$ & -3.059911 & -3.796525 & -5.915771 \\
\hline $\mathrm{H}$ & -3.025330 & -2.065754 & -6.273535 \\
\hline $\mathrm{H}$ & -1.639398 & -3.092595 & -6.672748 \\
\hline $\mathrm{H}$ & $-\odot .327158$ & -4.256937 & -4.876498 \\
\hline H & -0.419868 & -3.725646 & -3.191901 \\
\hline $\mathrm{H}$ & -1.717550 & -4.709796 & -3.880205 \\
\hline $\mathrm{H}$ & $-\odot .0 \odot \odot 659$ & -1.874260 & -5.652600 \\
\hline $\mathrm{H}$ & -1.169040 & -0.606331 & -5.231750 \\
\hline $\mathrm{H}$ & $-\odot .085910$ & -1.259494 & -3.998769 \\
\hline $\mathrm{H}$ & -7.538655 & ๑. 052010 & -4.336077 \\
\hline $\mathrm{H}$ & -5.876278 & $\odot .666678$ & -4.267862 \\
\hline $\mathrm{H}$ & -6.204012 & -0.924789 & -4.978458 \\
\hline $\mathrm{H}$ & -3.970226 & -4.599839 & -4.156162 \\
\hline $\mathrm{H}$ & -5.488265 & -4.270283 & -3.284992 \\
\hline $\mathrm{H}$ & $-5.50590 \odot$ & -4.488509 & -5.059029 \\
\hline $\mathrm{H}$ & -6.811377 & -0.241360 & $-\odot .724100$ \\
\hline $\mathrm{H}$ & -6.208364 & 1.073113 & -1.759683 \\
\hline $\mathrm{H}$ & -7.844491 & ๑. 436538 & -1.981618 \\
\hline $\mathrm{H}$ & $-\odot .883864$ & -3.979673 & 4.927476 \\
\hline $\mathrm{H}$ & -1.181947 & -3.675607 & 6.648169 \\
\hline $\mathrm{H}$ & -2.448928 & -4.407304 & 5.646404 \\
\hline $\mathrm{H}$ & -1.522658 & $-\odot .719629$ & 3.140183 \\
\hline $\mathrm{H}$ & -3.768021 & -2.772363 & 0.161326 \\
\hline $\mathrm{H}$ & -6.150162 & -3.908118 & 5.673560 \\
\hline $\mathrm{H}$ & -5.513979 & -2.269810 & 5.359058 \\
\hline $\mathrm{H}$ & -6.401767 & -3.133746 & 4.084533 \\
\hline $\mathrm{H}$ & -2.613173 & -1.641665 & 7.369363 \\
\hline $\mathrm{H}$ & -3.682267 & -0.887065 & 6.180288 \\
\hline $\mathrm{H}$ & -3.906437 & -2.589995 & 6.647027 \\
\hline $\mathrm{H}$ & -6.428497 & -5.013486 & 3.175829 \\
\hline $\mathrm{H}$ & -6.833142 & -3.924690 & 1.826846 \\
\hline $\mathrm{H}$ & -6.788279 & -5.675982 & 1.587223 \\
\hline $\mathrm{H}$ & -5.212823 & -5.899077 & -0.109178 \\
\hline $\mathrm{H}$ & -5.323869 & -4.153792 & -0.353303 \\
\hline $\mathrm{H}$ & -3.734499 & -4.940499 & -0.220865 \\
\hline $\mathrm{H}$ & $-\odot .196586$ & -1.523314 & 4.674051 \\
\hline $\mathrm{H}$ & -1.273912 & -0.235322 & 5.256110 \\
\hline $\mathrm{H}$ & -0.525849 & -1.351743 & 6.399572 \\
\hline $\mathrm{H}$ & -7.080667 & -2.644085 & -1.721952 \\
\hline $\mathrm{H}$ & - 8.291559 & -1.792221 & -2.690443 \\
\hline $\mathrm{H}$ & -7.095713 & -2.809428 & -3.492119 \\
\hline H & -4.559163 & -6.934017 & 1.979627 \\
\hline $\mathrm{H}$ & -3.029561 & -6.039772 & 1.975874 \\
\hline $\mathrm{H}$ & -4.091244 & -5.980375 & 3.398995 \\
\hline $\mathrm{Rh}$ & ๑. 291798 & -0.461376 & ๑. 585955 \\
\hline 0 & 2.136049 & $\odot .056746$ & 1.858452 \\
\hline 0 & 2.276078 & -5.719568 & 1.115304 \\
\hline C & 3.320709 & -0.236547 & 1.888899 \\
\hline S & 3.064440 & -4.837512 & 0.250766 \\
\hline C & 4.396480 & -0.101596 & 2.957293 \\
\hline C & 4. 210148 & -1.065128 & $\odot .981370$ \\
\hline $\mathrm{N}$ & 3. 080328 & -3.313389 & 1.032542 \\
\hline 0 & 2.714092 & -4.656948 & -1.167223 \\
\hline
\end{tabular}

$\begin{array}{lrrr}\mathrm{C} & 4.784048 & -5.327768 & 0.319905 \\ \mathrm{C} & 5.160518 & -1.243334 & 2.209265 \\ \mathrm{H} & 4.906679 & 0.867279 & 2.900376 \\ \mathrm{H} & 4.074200 & -0.267677 & 3.989685 \\ \mathrm{C} & 3.607131 & -2.227175 & 0.196815 \\ \mathrm{H} & 4.662923 & -0.380413 & 0.249607 \\ \mathrm{C} & 1.987273 & -3.058184 & 1.981356 \\ \mathrm{C} & 5.536479 & -5.372700 & 8.273800 \\ \mathrm{C} & 5.337890 & -5.707836 & 11.533600 \\ \mathrm{H} & 5.030089 & -2.216912 & 12.941400 \\ \mathrm{H} & 6.220868 & -1.068989 & 2.020062 \\ \mathrm{H} & 4.392227 & -2.650217 & -0.435884 \\ \mathrm{H} & 2.841728 & -1.819171 & -0.474974 \\ \mathrm{C} & 0.613108 & -2.668388 & 1.460790 \\ \mathrm{H} & 1.845269 & -3.991804 & 2.535298 \\ \mathrm{H} & 2.348992 & -2.334064 & 2.718865 \\ \mathrm{H} & 5.079557 & -5.100564 & -1.798607 \\ \mathrm{C} & 6.867762 & -5.788654 & -\odot .788741 \\ \mathrm{C} & 6.664820 & -6.120446 & 1.590586 \\ \mathrm{H} & 4.733020 & -5.693854 & 2.448324 \\ \mathrm{C} & \odot .195743 & -2.734988 & 0.151941 \\ \mathrm{H} & -0.143292 & -2.685480 & 2.242187 \\ \mathrm{H} & 7.457543 & -5.826316 & -1.700780 \\ \mathrm{C} & 7.451604 & -6.168256 & 0.426026 \\ \mathrm{H} & 7.099032 & -6.419991 & 2.541167 \\ \mathrm{H} & -\odot .846903 & -2.921229 & -0.074727 \\ \mathrm{H} & 0.892433 & -2.963969 & -\odot .649356 \\ \mathrm{C} & 8.882500 & -6.644871 & 0.488825 \\ \mathrm{H} & 9.421164 & -6.184285 & 1.324522 \\ \mathrm{H} & 8.925756 & -7.731363 & 0.637438 \\ \mathrm{H} & 9.423842 & -6.415555 & -\odot .433679\end{array}$

5

B3LYP SCF energy:

B3LYP enthalpy:

B3LYP free energy:

M06 SCF energy in solution: a.u.

M06 enthalpy in solution:

M06 free energy in solution:

a.u.

Cartesian coordinates

\begin{tabular}{|c|c|c|}
\hline ATOM & X & $\mathrm{Y}$ \\
\hline$P$ & $\odot . \diamond \odot \odot \odot \odot \odot$ & -5.019105 \\
\hline$P$ & $\odot . \odot \odot \odot \odot \odot \odot$ & -6.276125 \\
\hline 0 & $\odot .000 \odot \odot \odot$ & -5.181494 \\
\hline 0 & $\odot . \odot \odot \odot \odot \odot \odot$ & -3.077364 \\
\hline 0 & $\odot .0 \odot ० \odot ० \odot$ & -0.407844 \\
\hline 0 & $\odot . ๑ \odot \odot \odot \odot \odot$ & -1.324767 \\
\hline 0 & $\odot . \odot \odot \odot \odot \odot \odot$ & -7.168827 \\
\hline 0 & $\odot .0 \odot \odot \odot \odot \odot$ & -6.351364 \\
\hline 0 & $\odot . \diamond \odot \odot \odot \odot \odot$ & -5.221733 \\
\hline 0 & $\odot . ๑ \odot \odot \odot \odot \odot$ & $-\odot .0 \odot 9410$ \\
\hline$c$ & $\odot .000 \odot \odot \odot$ & -0.200769 \\
\hline c & $\odot .0 \odot \odot \odot \odot \odot$ & -7.659515 \\
\hline c & 1.328628 & -7.520784 \\
\hline C & 1.773699 & -8.101476 \\
\hline C & 2.225889 & -8.386394 \\
\hline$c$ & 2.238886 & 1.090693 \\
\hline c & 1.412514 & $\odot .880625$ \\
\hline$c$ & $-0.4146 \odot 9$ & 2.166985 \\
\hline c & -0.824942 & 0.664737 \\
\hline C & $-\odot .065882$ & $-\odot .362736$ \\
\hline$c$ & 1.123533 & 1.296915 \\
\hline C & 1.538327 & $\odot .760411$ \\
\hline C & $\odot .817233$ & 1.630129 \\
\hline $\mathrm{c}$ & 3. 081661 & 1.809633 \\
\hline C & 3.273314 & -0.748756 \\
\hline$c$ & 3.137507 & -1.028482 \\
\hline$c$ & 4.145622 & -1.684380 \\
\hline$c$ & 5.332623 & -2.029988 \\
\hline$c$ & 5.431766 & -1.927023 \\
\hline
\end{tabular}

-5535.36359833 a.u. -5533.328288 a.u. -5533.599693 a.u. $-5534.58494543$

-5532.549635 a.u. $-5532.821040$

7

3. 561956

4.213490

4.448163

3.978813

7.817423

6.970251

4.278528

4.533818

4.696335

8.680763

7.758214

4.940834

5.758133

4.100032

5.262252

2. 035536

2. 036107

1.856293

3.302033

3.555755

4.151496

3. 023302

2. 097296

3. 031157

$-0.921842$

$-2.284823$

$-3.006886$

$-2.305911$

$-0.894571$ 


\begin{tabular}{|c|c|c|c|c|c|c|c|}
\hline C & 4.397876 & -1.237280 & $-\odot .248625$ & $\mathrm{H}$ & 5.106343 & -3.920034 & -4.385835 \\
\hline C & 6.543218 & -2.545553 & $\odot .004003$ & $\mathrm{H}$ & 3.372503 & -4.154500 & -4.065582 \\
\hline C & 7.521863 & -1.448013 & $\odot .485770$ & H & 2.352833 & -2.084130 & -5.983417 \\
\hline C & 7.340713 & -3.691415 & -0.662065 & $\mathrm{H}$ & 1.720969 & -2.221412 & -4.339659 \\
\hline C & 5.879870 & -3.172780 & 1.262732 & H & 2.284444 & $-\odot .655562$ & -4.953220 \\
\hline C & 3.918294 & -2.070734 & -4.493282 & $\mathrm{H}$ & 4.857210 & $-\odot .241507$ & -5.272352 \\
\hline C & 4.101621 & -3.599998 & -4.667739 & H & 5.913552 & -1.669643 & $-5.34 \odot 2 \odot 7$ \\
\hline C & 2.483958 & -1.732953 & -4.954315 & $\mathrm{H}$ & 4.584721 & -1.501464 & -6.484097 \\
\hline C & 4.884830 & -1.324426 & -5.444607 & H & 8.016945 & -1.402490 & -2.375008 \\
\hline C & 7.453967 & -1.620245 & -3.288054 & H & 8.114900 & -2.108553 & -4.007689 \\
\hline C & 3.048822 & 1.558661 & 0.799344 & $\mathrm{H}$ & 7.083920 & -0.680110 & -3.712080 \\
\hline C & 4.199228 & 1.979534 & 0.132951 & H & 4.523021 & 1.432501 & -0.742430 \\
\hline C & 2.583947 & 2.341111 & 1.870942 & $\mathrm{H}$ & 6.282362 & 3.985164 & -2.547696 \\
\hline C & 3.325161 & 3.408883 & 2.387481 & H & 5.174953 & 2.652753 & -2.231329 \\
\hline C & 4.565183 & 3.708293 & 1.753612 & H & 4.661882 & 4.320499 & -1.915823 \\
\hline C & 4.949926 & 3.097113 & $\odot .539005$ & H & 7.717115 & 2.480433 & ๑.586941 \\
\hline C & 6.084146 & 3.567121 & -0.417186 & $\mathrm{H}$ & 6.917873 & 1.549080 & -0.685709 \\
\hline C & 5.508368 & 3.626259 & -1.860416 & H & 8.028799 & 2.862337 & -1.112248 \\
\hline C & 7.252361 & 2.553076 & -0.402358 & $\mathrm{H}$ & 6.205964 & 3.460287 & 3.832604 \\
\hline C & 6.508096 & 4.144620 & 3.032049 & H & 7.188610 & 3.619020 & 2.355124 \\
\hline C & 6.635976 & 4.984863 & -0.139239 & $\mathrm{H}$ & 7.021592 & 5.006699 & 3.463128 \\
\hline C & 2.768356 & 4.268530 & 3.552880 & H & 7.245641 & 5.047436 & $\odot .761858$ \\
\hline C & 3.667748 & 4.205953 & 4.811988 & H & 7.275701 & 5.275532 & -0.980485 \\
\hline C & 2.640034 & 5.740628 & 3.086818 & $\mathrm{H}$ & 5.834153 & 5.724064 & $-\odot .056383$ \\
\hline C & 1.365612 & 3.797925 & 3.991745 & H & 3.881547 & 3.169371 & 5.098730 \\
\hline C & -1.906819 & -1.491997 & 1.532892 & H & 4.610227 & 4.732911 & 4.667783 \\
\hline C & -2.224929 & -2.802231 & 1.915167 & $\mathrm{H}$ & 3.151655 & 4.681653 & 5.654059 \\
\hline C & -2.811217 & -3.088601 & 3.151688 & H & 2.245112 & 6.356515 & 3.903891 \\
\hline C & -3.097783 & -1.986446 & 4.006363 & H & 3.605547 & 6.151147 & 2.785545 \\
\hline C & -2.642055 & $-\odot .675471$ & 3.728246 & H & 1.948184 & 5.823757 & 2.239566 \\
\hline C & -2.091525 & $-\odot .459831$ & 2.450782 & H & 0.633672 & 3.837609 & 3.177321 \\
\hline C & -2.664827 & $\odot .527022$ & 4.714767 & $\mathrm{H}$ & 1.373687 & 2.779719 & 4.396572 \\
\hline C & -2.767466 & $\odot .132317$ & 6.207396 & H & $1.00 \odot 8 \odot 4$ & 4.459026 & 4.784782 \\
\hline C & -3.811328 & 1.501743 & 4.350854 & H & -2.021578 & -3.606170 & 1.220224 \\
\hline C & -1.326874 & 1.305107 & 4.584315 & H & -1.815880 & ๑.547915 & 2.162243 \\
\hline C & -2.279203 & -4.830841 & 4.897379 & H & -3.744777 & $-\odot .253881$ & 6.493028 \\
\hline C & -5.186794 & -1.899684 & 5.132744 & H & -2.019686 & $-\odot .620408$ & 6.476381 \\
\hline C & -3.053410 & -4.559696 & 3.582629 & H & -2.580299 & 1.024810 & 6.815937 \\
\hline C & -2.534044 & -5.551997 & 2.518272 & H & -3.798703 & 2.365356 & 5.027499 \\
\hline C & -5.411438 & -1.166016 & -2.495850 & $\mathrm{H}$ & -3.702021 & 1.872930 & 3.326696 \\
\hline C & -4.219153 & $-\odot .896978$ & -3.215186 & H & -4.795405 & 1.029497 & 4.436017 \\
\hline C & -5.443960 & -0.364261 & -5.485791 & $\mathrm{H}$ & -1.290739 & 2. 094238 & 5.343543 \\
\hline C & -3.021095 & $-\odot .889580$ & -2.487423 & H & $-\odot .468009$ & $\odot .642278$ & 4.741036 \\
\hline C & -2.975896 & -1.119389 & -1.107057 & $\mathrm{H}$ & -1.214931 & 1.793546 & 3.612777 \\
\hline C & -4.188808 & -1.255887 & -0.427404 & H & -2.382418 & -5.884998 & 5.182965 \\
\hline C & -5.428942 & -1.249369 & -1.082872 & $\mathrm{H}$ & -1.212111 & -4.611480 & 4.775975 \\
\hline C & -6.862950 & -2.684994 & -3.553835 & H & -2.656453 & -4.215556 & 5.716247 \\
\hline C & -3.357839 & -1.751476 & -5.436650 & $\mathrm{H}$ & -5.328227 & -0.815237 & 5.174889 \\
\hline C & -6.745540 & -2.706835 & 0.516021 & H & -5.677367 & -2.288199 & 4.233344 \\
\hline C & -6.691455 & -1.323595 & -0.176866 & $\mathrm{H}$ & -5.639609 & -2.352333 & 6.018171 \\
\hline C & -6.576088 & $-\odot .219649$ & 0.909970 & $\mathrm{H}$ & -3.092446 & -5.477619 & 1.577617 \\
\hline C & -3.294800 & 0.715421 & -4.921656 & $\mathrm{H}$ & -1.471686 & -5.409241 & 2.297432 \\
\hline C & -4.551024 & -4.885685 & 3.797066 & H & -2.665648 & -6.573495 & 2.891557 \\
\hline C & -4.110952 & $-\odot .593183$ & -4.737770 & $\mathrm{H}$ & -6.066024 & 0.391283 & -4.999000 \\
\hline C & -8.048433 & -1.081513 & -0.875863 & $\mathrm{H}$ & -5.210201 & -0.011903 & -6.497890 \\
\hline H & 2.580719 & -1.358362 & 4.841400 & H & -6.038364 & -1.272735 & -5.591232 \\
\hline H & 2.655587 & 0.261347 & 2.980714 & $\mathrm{H}$ & -2.093276 & -0.713867 & -3.012598 \\
\hline H & 0.646754 & -5.728832 & 3.961605 & H & -4.174929 & -1.378087 & 0.646485 \\
\hline H & 2.347990 & -5.638182 & 3.343111 & H & -7.812584 & -2.677620 & -4.094016 \\
\hline H & -1.765224 & -3.049431 & -2.339260 & H & -6.077875 & -3.091778 & -4.204222 \\
\hline $\mathrm{H}$ & -0.403120 & -4.898130 & -3.287249 & H & -6.952772 & -3.328089 & -2.668922 \\
\hline H & 4.024989 & -5.252901 & -1.624669 & H & -3.244683 & -1.539569 & -6.506846 \\
\hline $\mathrm{H}$ & 3.161667 & -6.463080 & $-\odot .595619$ & $\mathrm{H}$ & -2.356555 & -1.897659 & -5.017182 \\
\hline H & 2.225147 & $-\odot .759862$ & -2.791292 & H & -3.905554 & -2.696286 & -5.339752 \\
\hline H & 4.465704 & -1.087176 & 0.821315 & $\mathrm{H}$ & -6.845237 & -3.514828 & -0.217941 \\
\hline H & 6.992686 & $-\odot .654709$ & 1.025912 & H & -5.843186 & -2.902573 & 1.105614 \\
\hline $\mathrm{H}$ & 8.266161 & -1.878725 & 1.166314 & $\mathrm{H}$ & -7.608807 & -2.760207 & 1.190780 \\
\hline H & 8.063084 & $-\odot .981203$ & $-\odot .343694$ & H & -7.455530 & $-\odot .252561$ & 1.563904 \\
\hline $\mathrm{H}$ & 8.004198 & -3.358884 & -1.459122 & $\mathrm{H}$ & -5.691445 & -0.330113 & 1.543502 \\
\hline H & 7.963008 & -4.171998 & 0.101373 & $\mathrm{H}$ & -6.535471 & 0.773993 & 0.448264 \\
\hline H & 6.676005 & -4.454638 & -1.079987 & H & -3.793843 & 1.554311 & -4.422284 \\
\hline H & 5.089425 & -3.877553 & 0.986233 & H & -2.278088 & $\odot .641022$ & -4.528220 \\
\hline H & 6.638602 & -3.711385 & 1.840824 & H & -3.217184 & $\odot .952813$ & -5.989197 \\
\hline H & 5.441741 & -2.427445 & 1.933085 & $\mathrm{H}$ & -5.149454 & -4.606326 & 2.921712 \\
\hline \multirow[t]{2}{*}{$\mathrm{H}$} & 3.938741 & -3.873229 & -5.717286 & H & -4.671889 & -5.965132 & 3.947432 \\
\hline & & & & & & & \\
\hline
\end{tabular}




\begin{tabular}{|c|c|c|c|}
\hline $\mathrm{H}$ & -4.959848 & -4.387666 & 4.675759 \\
\hline H & - 8.822151 & $-1 . \odot \odot 4942$ & $-\odot .102092$ \\
\hline $\mathrm{H}$ & -8.054287 & $-\odot .153910$ & -1.453995 \\
\hline H & -8.338535 & - 1.895199 & -1.541826 \\
\hline $\mathrm{Rh}$ & $-\odot .008196$ & 1.058739 & -0.657872 \\
\hline 0 & -1.931037 & 2.043777 & -1.096078 \\
\hline C & ๑. 378561 & $\odot .850874$ & -2.595239 \\
\hline S & -2.418449 & 3.167240 & -0.230555 \\
\hline 0 & -0.014294 & $-\odot .116051$ & -3.192610 \\
\hline C & ๑. 086015 & 4.079244 & -0.137547 \\
\hline $\mathrm{N}$ & -1.330449 & 4.434247 & -0.472301 \\
\hline 0 & -2.633042 & 2.866187 & 1.194333 \\
\hline C & -3.933371 & 3.736800 & -0.966659 \\
\hline $\mathrm{H}$ & ๑. 615638 & 5.033220 & -0.256571 \\
\hline H & ๑. 158865 & 3.814111 & ๑. 927504 \\
\hline C & -1.754193 & 5.704110 & $\odot .190275$ \\
\hline C & -5.074659 & 3.812630 & -0.166844 \\
\hline C & -3.972366 & $4 . \odot 47961$ & -2.330592 \\
\hline C & -1.129078 & 6.888519 & -0.494154 \\
\hline $\mathrm{H}$ & -2.844128 & 5.775704 & ๑. 108548 \\
\hline $\mathrm{H}$ & -1.502672 & 5.686884 & 1.259717 \\
\hline $\mathrm{H}$ & -5.019105 & 3.561956 & 10.102500 \\
\hline C & -6.276125 & 4.213490 & 13.047700 \\
\hline C & -5.181494 & 4.448163 & 15.031700 \\
\hline $\mathrm{H}$ & -3.077364 & 3.978813 & -2.939126 \\
\hline C & -0.407844 & 7.817423 & 0.134569 \\
\hline $\mathrm{H}$ & -1.324767 & 6.970251 & -1.562500 \\
\hline $\mathrm{H}$ & -7.168827 & 4.278528 & -0.135039 \\
\hline C & -6.351364 & 4.533818 & -2.113289 \\
\hline $\mathrm{H}$ & -5.221733 & 4.696335 & -3.945982 \\
\hline $\mathrm{H}$ & $-\odot .0 \odot 9410$ & 8.680763 & -0.390372 \\
\hline H & $-\odot .2 \odot \odot 769$ & 7.758214 & 1. 201160 \\
\hline C & -7.659515 & 4.940834 & -2.744731 \\
\hline H & -7.520784 & 5.758133 & -3.460293 \\
\hline $\mathrm{H}$ & -8.101476 & 4.100032 & -3.294774 \\
\hline H & -8.386394 & 5.262252 & -1.993294 \\
\hline C & 1.090693 & 2.035536 & -3.242647 \\
\hline H & 0.880625 & 2.036107 & -4.318260 \\
\hline $\mathrm{H}$ & 2.166985 & 1.856293 & -3.109067 \\
\hline C & 0.664737 & 3.302033 & -2.507429 \\
\hline $\mathrm{H}$ & -0.362736 & 3.555755 & -2.787795 \\
\hline H & 1.296915 & 4.151496 & -2.804731 \\
\hline C & 0.760411 & 3.023302 & -1.007303 \\
\hline $\mathrm{H}$ & 1.630129 & 2.097296 & 2.321903 \\
\hline H & 1.809633 & 3.031157 & -0.707725 \\
\hline
\end{tabular}

B3LYP SCF energy:

B3LYP enthalpy:

B3LYP free energy:

M०6 SCF energy in solution:

a.u.

M06 enthalpy in solution:

M06 free energy in solution:

a.u.

Cartesian coordinates

\begin{tabular}{|c|c|c|c|}
\hline АТОM & $X$ & Y & Z \\
\hline $\mathrm{P}$ & $\odot . \diamond \odot \odot \odot \odot \odot$ & -1.026646 & 7.326274 \\
\hline$P$ & $\odot . \diamond \odot \odot \odot \odot \diamond$ & -1.432943 & 1.898402 \\
\hline 0 & ๑. . & -2.301664 & 2.181311 \\
\hline 0 & $\odot .0 \odot \odot \odot \odot \diamond$ & 3.823795 & 8.292129 \\
\hline 0 & 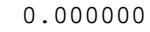 & 1.848902 & 8.798416 \\
\hline 0 & $\odot . \diamond \odot \odot \odot \odot \diamond$ & $-\odot .242177$ & 9.070272 \\
\hline 0 & $\odot . \diamond \odot \odot \odot \odot \odot$ & -2.124637 & 1.114291 \\
\hline 0 & $\odot . \diamond \odot \odot \odot \odot \diamond$ & $-\odot .826559$ & 2.346042 \\
\hline 0 & ०. . 000000 & 2.319301 & 9.899559 \\
\hline 0 & $\odot .0 \odot \odot \odot \odot \odot$ & 2.321995 & 10.866334 \\
\hline C & ०. . 000000 & 1.664983 & 10.002395 \\
\hline C & 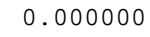 & 3.338718 & 9.718712 \\
\hline C & -0.354545 & 1.512871 & $\odot .273235$ \\
\hline C & ๑. 169113 & 1.312107 & ๑. 070316 \\
\hline C & 1.081679 & 2.365428 & -0.354728 \\
\hline
\end{tabular}

1.462914

$-1.018572$

$-1.648496$

$-2.294303$

$-1.984268$

$-0.995279$

$-0.360578$

$-0.642322$

$\odot .519300$

2. 393371

2. 126291

2. 785228

3. 766504

3.933066

3. 257040

4. 753394

6.103683

5. 008108

3. 959971

2. 391556

1.983513

1.177035

3. 543073

5.855006

3.127808

4. 341963

3.033512

4. 150640

5. 397165

5.483776

6.691878

6.156103

7.359675

7.376732

7.768340

4.007657

4. 813336

4.490577

2.539672

$-2.645655$

$-3.502301$

$-4.147622$

$-3.937278$

$-2.948163$

$-2.351263$

$-2.440287$

$-2.801296$

$-2.956303$

$-\odot .888578$

$-4.398909$

$-5.798144$

$-4.980779$

$-4.900405$

$-5.819512$

$-4.651852$

$-5.656323$

$-3.523648$

$-3.516130$

$-4.650176$

$-5.806411$

$-7.810891$

$-4.322403$

$-7.557029$

$-6.951948$

$-6.350919$

$-3.228993$

$-6.480997$

$-4.499231$

$-8.102488$

1. 508904

2. 228055

$-1.977684$

$-0.367758$

- 3.076791

$-2.504043$
1. 784154

1. 101814

2. 793733

1. 644882

2. 068086

2. 547459

$-0.853942$

$-1.596401$

$-0.596074$

- 2.271342

$-2.885647$

$-4.060166$

$-4.587083$

$-4.097215$

$-2.910402$

$-4.770642$

$-4.041329$

$-6.282273$

$-4.655784$

$-4.774239$

$-6.239614$

$-4.096588$

$-4.760156$

$-5.331060$

๑. 347951

๑. 071928

1. 530689

2. 322511

1. 913362

๑. 876856

$\odot .576772$

0.442698

$-0.760953$

1. 899863

1. 685446

3. 625952

3. 567155

4. 831996

3. 896527

$-0.310407$

$-1.184642$

$-0.813608$

$\odot .516024$

1. 361180

$\odot .922110$

2. 700573

2. 929431

3. 894585

2. 707734

$-1.958676$

1. 797880

$-1.850054$

- 3.251373

$-0.195683$

$-0.732842$

$-0.767596$

$-0.885254$

$-0.520251$

๑. 121878

๑. 336432

$-1.324480$

$-2.699461$

๑. 298800

1. 141041

2. 444172

$-0.486854$

- 1.481686

$-1.162612$

1.597450

$-0.683780$

๑ . 089810

$-4.067224$

$-4.809724$

- 3.042096

$-5.448794$
1. 736279

2. 388379

2. 014937

1. 928263

1.829380

1. 544151

3. 810231

4. 597954

3. 838508

$-0.404880$

$-1.632095$

$-2.025607$

$-1.141717$

0.179864

๑. 490786

1. 320033

1.519193

1.112370

2. 652243

$-3.346897$

- 3.048311

$-4.017288$

$-4.381276$

$-1.998582$

๑. 555006

$-0.074939$

1. 309318

1. 594455

1.040420

0.083462

$-0.850700$

$-2.304440$

$-0.454792$

2. 338658

$-0.912413$

2. 423509

3. 744704

1. 579402

2. 815629

1. 470882

2. 154264

3. 337317

3. 802161

3. 246237

2. 049973

3. 852272

5.339271

3. 013707

3. 801844

5.568963

4. 508577

4.136520

3. 491046

$-2.828105$

$-3.430402$

$-5.864976$

$-2.611202$

$-1.256250$

$-0.749883$

$-1.515890$

$-3.378221$

$-4.986863$

๑. 312178

$-0.837066$

$-0.242146$

$-5.504005$

4.223383

$-4.919111$

$-1.763124$

5. 179549

2. 952858

5.384372

5.008420

$-1.664417$

$-1.772818$ 


\begin{tabular}{|c|c|c|c|}
\hline $\mathrm{H}$ & 1.487290 & -6.648507 & $\odot .412337$ \\
\hline $\mathrm{H}$ & ๑. 302493 & -7.145579 & 1.685237 \\
\hline $\mathrm{H}$ & 1.375409 & -2.464280 & -2.281345 \\
\hline $\mathrm{H}$ & 3.402081 & -2.473147 & 1.470345 \\
\hline $\mathrm{H}$ & 5.949884 & -2.979318 & 1.741490 \\
\hline $\mathrm{H}$ & 6.650380 & -4.484753 & 2.360060 \\
\hline $\mathrm{H}$ & 6.746776 & -4.105899 & 0.635834 \\
\hline $\mathrm{H}$ & 5.707225 & -6.502952 & 0.307243 \\
\hline $\mathrm{H}$ & 5.430394 & -6.695198 & 2.035406 \\
\hline $\mathrm{H}$ & 4.078736 & -6.821729 & $\odot .900573$ \\
\hline $\mathrm{H}$ & 2.941410 & -5.041905 & 2.546650 \\
\hline $\mathrm{H}$ & 4.471863 & -5.235375 & 3. 427969 \\
\hline $\mathrm{H}$ & 3.890401 & -3.628130 & 3.021367 \\
\hline $\mathrm{H}$ & 1.695698 & -6.738574 & -3.981408 \\
\hline $\mathrm{H}$ & 2.803296 & -6.803803 & -2.600555 \\
\hline $\mathrm{H}$ & 1.120566 & -6.278273 & -2.373359 \\
\hline $\mathrm{H}$ & $\odot .896280$ & -4.674640 & -4.904105 \\
\hline $\mathrm{H}$ & ๑. 303669 & -4.059300 & -3.357930 \\
\hline $\mathrm{H}$ & 1.394860 & -3.076955 & -4.353274 \\
\hline $\mathrm{H}$ & 3.923647 & -3.743929 & -4.540835 \\
\hline $\mathrm{H}$ & 4.372529 & -5.401753 & -4.083540 \\
\hline $\mathrm{H}$ & 3.173992 & -5.129846 & -5.345070 \\
\hline $\mathrm{H}$ & 6.496530 & -5.108648 & -1.140305 \\
\hline $\mathrm{H}$ & 6.238105 & -6.216777 & -2.510215 \\
\hline $\mathrm{H}$ & 5.868072 & -4.478300 & -2.686232 \\
\hline $\mathrm{H}$ & 4.406704 & -0.787646 & -0.728488 \\
\hline $\mathrm{H}$ & 6.993912 & $\odot .263300$ & -2.987018 \\
\hline $\mathrm{H}$ & 5.455436 & -0.388025 & -2.429704 \\
\hline $\mathrm{H}$ & 5.654523 & 1.363499 & -2.623718 \\
\hline $\mathrm{H}$ & 7.776640 & -0.725723 & $\odot .557631$ \\
\hline $\mathrm{H}$ & 6.648029 & -1.592976 & -0.491493 \\
\hline $\mathrm{H}$ & 8.180933 & -0.990001 & -1.144249 \\
\hline $\mathrm{H}$ & 6.844773 & 1.651896 & 3. 263984 \\
\hline $\mathrm{H}$ & 7.769392 & $\odot .978559$ & 1.897844 \\
\hline $\mathrm{H}$ & 8.205232 & 2.574791 & 2.563497 \\
\hline $\mathrm{H}$ & 8.371238 & 1.759834 & -0.007560 \\
\hline $\mathrm{H}$ & 8.453113 & 1.453462 & -1.736077 \\
\hline $\mathrm{H}$ & 7.329476 & 2.667866 & -1.108175 \\
\hline $\mathrm{H}$ & 4.582060 & 2.658847 & 4.314020 \\
\hline $\mathrm{H}$ & 5.887740 & 3.608363 & 3.569573 \\
\hline $\mathrm{H}$ & 4.550328 & 4.426679 & 4.372266 \\
\hline H & 4.400269 & 5.754242 & 2.165956 \\
\hline $\mathrm{H}$ & 5.533903 & 4.718576 & 1.279446 \\
\hline $\mathrm{H}$ & 3.877941 & 4.951582 & $\odot .678173$ \\
\hline $\mathrm{H}$ & 1.883389 & 3. 991081 & 1.942941 \\
\hline $\mathrm{H}$ & 2.132929 & 3.119836 & 3.473276 \\
\hline $\mathrm{H}$ & 2.486771 & 4.844505 & 3.361024 \\
\hline $\mathrm{H}$ & -3.669679 & -2.170071 & 1.739690 \\
\hline $\mathrm{H}$ & -1.626799 & 1.564195 & 1.559951 \\
\hline $\mathrm{H}$ & - 3.856339 & 3.144438 & 5.504273 \\
\hline $\mathrm{H}$ & -2.532927 & 2.068186 & 5.957676 \\
\hline $\mathrm{H}$ & -2.234878 & 3.794968 & 5.702292 \\
\hline $\mathrm{H}$ & -2.563159 & 4.837358 & 3.412770 \\
\hline $\mathrm{H}$ & -2.639275 & 3.815626 & 1.966789 \\
\hline $\mathrm{H}$ & -4.049300 & 3.959521 & 3.022383 \\
\hline $\mathrm{H}$ & -0.511385 & 3. 619550 & 4.277564 \\
\hline H & -0.473410 & 1.845897 & 4.335771 \\
\hline $\mathrm{H}$ & -0.493381 & 2.693385 & 2.781723 \\
\hline $\mathrm{H}$ & -4.925700 & -2.741390 & 6.128106 \\
\hline $\mathrm{H}$ & -3.333820 & -2.216046 & 5.540327 \\
\hline $\mathrm{H}$ & -4.502639 & -1.019409 & 6.115205 \\
\hline $\mathrm{H}$ & -5.451398 & 2.759832 & 4.116866 \\
\hline $\mathrm{H}$ & -6.444531 & 1.323203 & 3. 762092 \\
\hline $\mathrm{H}$ & -6.364947 & 1.967492 & 5.426728 \\
\hline $\mathrm{H}$ & -5.372897 & -3.279111 & 2.502111 \\
\hline $\mathrm{H}$ & -3.868665 & -3.603184 & 3.388544 \\
\hline $\mathrm{H}$ & -5.437612 & -3.965311 & 4.124577 \\
\hline $\mathrm{H}$ & -5.891992 & ๑. 297918 & -5.807461 \\
\hline $\mathrm{H}$ & -5.345288 & -0.990524 & -6.892476 \\
\hline H & -6.573450 & -1.327657 & -5.678095 \\
\hline $\mathrm{H}$ & -2.625144 & -1.309391 & -3.038793 \\
\hline $\mathrm{H}$ & -4.646363 & $\odot .456822$ & ๑. 278180 \\
\hline $\mathrm{H}$ & -8.692449 & -1.173587 & -4.005051 \\
\hline $\mathrm{H}$ & -7.289340 & -2.235987 & -3.696866 \\
\hline $\mathrm{H}$ & -8.123157 & -1.444705 & -2.333050 \\
\hline
\end{tabular}

\begin{tabular}{|c|c|c|c|}
\hline $\mathrm{H}$ & -4.189358 & -3.018744 & -6.027346 \\
\hline $\mathrm{H}$ & -3.446145 & -3.035438 & -4.421082 \\
\hline $\mathrm{H}$ & -5.201390 & -3.219285 & -4.587985 \\
\hline $\mathrm{H}$ & -8.011956 & -0.623473 & -0.066791 \\
\hline $\mathrm{H}$ & -6.799789 & ๑. 016763 & 1.052338 \\
\hline $\mathrm{H}$ & -8.339267 & ๑. 868959 & ๑. 827941 \\
\hline $\mathrm{H}$ & -7.144470 & 3.029249 & $\odot .236382$ \\
\hline $\mathrm{H}$ & -5.583200 & 2.257839 & ๑. 514917 \\
\hline $\mathrm{H}$ & -5.907693 & 3. 063339 & -1.030660 \\
\hline $\mathrm{H}$ & -3.318881 & 0.605521 & -5.469017 \\
\hline $\mathrm{H}$ & -2.310668 & -0.774736 & -4.985385 \\
\hline $\mathrm{H}$ & -3.111873 & -0.776634 & -6.554266 \\
\hline $\mathrm{H}$ & -6.906054 & -1.298728 & 3. 229436 \\
\hline $\mathrm{H}$ & -7.035804 & -2.315102 & 4.670252 \\
\hline $\mathrm{H}$ & -6.653841 & $-\odot .604840$ & 4.847284 \\
\hline $\mathrm{H}$ & -8.742703 & 2.285374 & -1.197968 \\
\hline $\mathrm{H}$ & -7.735423 & 2.126446 & -2.646279 \\
\hline $\mathrm{H}$ & -8.732920 & $\odot .774160$ & -2.100718 \\
\hline $\mathrm{Rh}$ & ๑. 048906 & $\odot .673586$ & -1.116514 \\
\hline 0 & 1.457093 & 5.289296 & -3.730317 \\
\hline C & 0. 338685 & -0.246197 & -2.866129 \\
\hline S & 0.404414 & 5.510275 & -2.736923 \\
\hline 0 & -0.386768 & -1.145403 & -3.194928 \\
\hline C & 1.554618 & 3.395594 & -1.434976 \\
\hline $\mathrm{N}$ & ๑. 317306 & 4.069596 & -1.852096 \\
\hline 0 & -0.980582 & 5.810434 & -3.110297 \\
\hline C & ๑. 967534 & 6.802027 & -1.629966 \\
\hline $\mathrm{H}$ & 2.395266 & 3. 933918 & -1.888065 \\
\hline $\mathrm{H}$ & 1.662027 & 3.489401 & -0.344637 \\
\hline C & 2.333806 & 7.078060 & -1.527952 \\
\hline C & ๑. .034256 & 7.518521 & -0.874819 \\
\hline C & -1.525157 & 2.501661 & -1.379329 \\
\hline $\mathrm{H}$ & 3.043232 & 6.545352 & -2.151938 \\
\hline C & 2.762305 & 8.070260 & -0.647511 \\
\hline C & ๑. 482538 & 8.506124 & -0.000573 \\
\hline $\mathrm{H}$ & -1.026646 & 7.326274 & 8.267200 \\
\hline C & -1.432943 & 1.898402 & 11.535700 \\
\hline $\mathrm{H}$ & -2.301664 & 2.181311 & 13.829300 \\
\hline $\mathrm{H}$ & 3.823795 & 8.292129 & -0.573042 \\
\hline C & 1.848902 & 8.798416 & 0.128892 \\
\hline $\mathrm{H}$ & -0.242177 & 9.070272 & ๑. 581088 \\
\hline $\mathrm{H}$ & -2.124637 & 1.114291 & -2.879259 \\
\hline $\mathrm{H}$ & -0.826559 & 2.346042 & -3.375470 \\
\hline C & 2. 319301 & 9.899559 & 1.048357 \\
\hline $\mathrm{H}$ & 2.321995 & 10.866334 & $\odot .528168$ \\
\hline $\mathrm{H}$ & 1.664983 & 10.002395 & 1. 920102 \\
\hline $\mathrm{H}$ & 3.338718 & 9.718712 & 1.403648 \\
\hline C & 1.512871 & ๑. 273235 & -3.694233 \\
\hline $\mathrm{H}$ & 1.312107 & ๑. 070316 & -4.752655 \\
\hline $\mathrm{H}$ & 2.365428 & -0.354728 & -3.396787 \\
\hline C & 1.784154 & 1.736279 & -3.363490 \\
\hline $\mathrm{H}$ & 1. 101814 & 2.388379 & -3.916154 \\
\hline $\mathrm{H}$ & 2.793733 & 2. 014937 & -3.696754 \\
\hline C & 1.644882 & 1.928263 & -1.851537 \\
\hline $\mathrm{H}$ & 2.068086 & 1.829380 & 1.697495 \\
\hline $\mathrm{H}$ & 2.547459 & 1.544151 & -1.377829 \\
\hline C & -0.853942 & 3.810231 & -1.034918 \\
\hline $\mathrm{H}$ & -1.596401 & 4.597954 & -1.209132 \\
\hline $\mathrm{H}$ & -0.596074 & 3.838508 & 0.036364 \\
\hline
\end{tabular}

7

B3LYP SCF energy:

B3LYP enthalpy:

B3LYP free energy:

-5533.577669 a.u.

M06 SCF energy in solution: $\quad-5534.58486295$

M06 enthalpy in solution: $\quad-5532.548426$ a.u. M06 free energy in solution: -5532.817150 a.u.

Cartesian coordinates

ATOM $\mathrm{X} \quad \mathrm{Y} \quad \mathrm{Z}$

$\begin{array}{llll}P & \odot .0 \odot \odot \odot \odot \odot ~ & \odot .259286 & 7.308250\end{array}$ 


\begin{tabular}{|c|c|c|c|c|c|c|c|}
\hline P & $\odot . ๑ \odot \odot \odot \odot \odot$ & -2.338271 & 1.777078 & C & -3.660543 & -2.281796 & -5.460661 \\
\hline 0 & $\odot .0 \odot \odot \odot \odot \odot$ & -1.314242 & 3.135304 & C & -7.253584 & -2.379964 & 0.418758 \\
\hline 0 & $\odot . ๑ \odot \odot \odot \odot \odot$ & -4.189928 & 8.781567 & C & -7.075532 & -1.064214 & -0.376457 \\
\hline 0 & $\odot .0 \odot \odot ० \odot \odot$ & -2.794186 & 8.853596 & C & -6.906264 & 0.109244 & 0.627370 \\
\hline 0 & $\odot . ๑ \odot \odot \odot \odot \odot$ & -1.184342 & 8.730460 & C & -3.488734 & $\odot .224565$ & -5.232058 \\
\hline 0 & $\odot .000 \odot \odot \odot$ & -2.717915 & 1.192404 & C & -5.488481 & -3.768686 & 4.145911 \\
\hline 0 & $\odot .0000 \odot \odot$ & -2.841289 & 1.636788 & C & -4.369621 & -1.016146 & -4.919558 \\
\hline 0 & $\odot .0 \odot \odot \odot \odot \odot$ & -3.649687 & 9.750107 & C & -8.388407 & -0.773253 & -1.139294 \\
\hline 0 & $\odot . ๑ \odot \odot \odot \odot \odot$ & -3.276196 & 10.782028 & $\mathrm{H}$ & 1.956328 & -1.509411 & 5.026578 \\
\hline C & $\odot .000 \odot \odot \odot$ & -3.642078 & 9.425526 & $\mathrm{H}$ & 2.325033 & -0.079209 & 3.059354 \\
\hline C & $\odot . ๑ \odot \odot \odot \odot \odot$ & -4.687770 & 9.768556 & $\mathrm{H}$ & $-\odot .568634$ & -5.617828 & 4.399137 \\
\hline C & 0.526350 & 1.255942 & 1.611789 & $\mathrm{H}$ & 1.147693 & -5.817389 & 3.850950 \\
\hline C & $\odot .969953$ & 1.048348 & 1.682387 & $\mathrm{H}$ & -2.225955 & -3.178607 & -2.281173 \\
\hline C & 1.593954 & 2.209177 & 1.075454 & $\mathrm{H}$ & -1.130796 & -5.293811 & -2.941330 \\
\hline C & 1.773669 & 1.311246 & 2.951337 & $\mathrm{H}$ & 3.061021 & -6.074011 & $-\odot .9660 \odot \odot$ \\
\hline C & 0.295199 & $\odot .523013$ & 3.618600 & $\mathrm{H}$ & 2.046480 & -7.108311 & 0.116133 \\
\hline C & -0.926655 & 2.261039 & 3.458708 & $\mathrm{H}$ & 1.684164 & -1.392008 & -2.652539 \\
\hline C & -1.368673 & 1.198876 & 2.673019 & $\mathrm{H}$ & 3.970208 & -1.928890 & 0.905735 \\
\hline C & -0.762216 & 1.842371 & 1.802383 & $\mathrm{H}$ & 6.629936 & -1.875291 & 0.911986 \\
\hline C & $\odot .314017$ & 2.141190 & 2.233906 & $\mathrm{H}$ & 7.676832 & -3.294972 & 1.079361 \\
\hline C & 0.759142 & -1.410280 & $4.00 \odot 486$ & $\mathrm{H}$ & 7.517578 & -2.491236 & -0.487125 \\
\hline C & 0.183560 & -2.234389 & 4.704337 & $\mathrm{H}$ & 6.939053 & -4.908898 & -1.381751 \\
\hline C & 2.081403 & -1.516563 & 3.631920 & $\mathrm{H}$ & 6.895725 & -5.569396 & 0.250621 \\
\hline C & 2.790617 & -1.476979 & $-\odot .819417$ & $\mathrm{H}$ & 5.481528 & -5.710108 & -0.803074 \\
\hline C & 2.559100 & -1.790513 & -2.161793 & $\mathrm{H}$ & 4.190978 & -4.663194 & 1.309019 \\
\hline C & 3.415821 & -2.641709 & -2.876494 & $\mathrm{H}$ & 5.816973 & -4.739054 & 2.020727 \\
\hline C & 4.557592 & -3.144407 & -2.193429 & $\mathrm{H}$ & 4.902495 & -3.241055 & 2. 081294 \\
\hline C & 4.727759 & -2.991843 & $-\odot .792219$ & $\mathrm{H}$ & 2.760158 & -4.885903 & -5.470093 \\
\hline C & 3.840633 & -2.116581 & -0.152279 & $\mathrm{H}$ & 3.987896 & -5.052631 & -4.203583 \\
\hline C & 5.777264 & -3.724558 & $\odot .096236$ & $\mathrm{H}$ & 2.265126 & -5.018598 & -3.776178 \\
\hline C & 6.969169 & -2.789121 & 0.411586 & $\mathrm{H}$ & 1.461743 & -2.902816 & -5.765200 \\
\hline C & 6.303057 & -5.047616 & $-\odot .5 \odot 9111$ & $\mathrm{H}$ & 0.888825 & -2.871951 & -4.094136 \\
\hline C & 5.123493 & -4.109338 & 1.453146 & $\mathrm{H}$ & 1.644338 & -1.434870 & -4.808258 \\
\hline C & 3.074244 & -3.054087 & -4.334015 & $\mathrm{H}$ & 4.225730 & -1.411875 & -5.234131 \\
\hline C & 3.025363 & -4.599501 & -4.445330 & $\mathrm{H}$ & 5.074477 & -2.972646 & -5.258645 \\
\hline C & 1.686562 & -2.528342 & -4.760843 & $\mathrm{H}$ & 3.740735 & -2.678440 & -6.370464 \\
\hline C & 4.098917 & -2.495144 & -5.350640 & $\mathrm{H}$ & 7.356196 & -3.073062 & -2.431227 \\
\hline C & 6.674728 & -3.154178 & -3.283550 & $\mathrm{H}$ & 7.156393 & -3.741502 & -4.068615 \\
\hline C & 3.063287 & $\odot .899229$ & 0.782084 & $\mathrm{H}$ & 6.453266 & -2.150623 & -3.662970 \\
\hline C & 4.251805 & 1.050827 & $\odot .063252$ & $\mathrm{H}$ & 4.421513 & $\odot .426287$ & $-\odot .803615$ \\
\hline C & 2.812723 & 1.805404 & 1.829033 & $\mathrm{H}$ & 6.548836 & 2.569407 & -2.763749 \\
\hline C & 3.782852 & 2.704154 & 2.286816 & $\mathrm{H}$ & 5.206518 & 1.501243 & -2.361777 \\
\hline C & 5.035805 & 2.706107 & 1.610410 & $\mathrm{H}$ & 5.074006 & 3.243192 & -2.051198 \\
\hline C & 5.231841 & 1.999545 & 0.401823 & $\mathrm{H}$ & 7.810653 & 0.820034 & 0.317929 \\
\hline C & 6.387713 & 2.217104 & $-\odot .617597$ & $\mathrm{H}$ & 6.775750 & $\odot .071303$ & -0.904865 \\
\hline C & 5.757182 & 2.386216 & -2.028911 & $\mathrm{H}$ & 8.109107 & 1.128699 & -1.398339 \\
\hline C & 7.321946 & $\odot .985465$ & $-\odot .648448$ & $\mathrm{H}$ & 6.676099 & 2.140268 & 3.630961 \\
\hline C & 7.081463 & 2.725375 & 2.797806 & $\mathrm{H}$ & 7.596359 & 2.049984 & 2.107195 \\
\hline C & 7.234117 & 3.491544 & $-\odot .391929$ & $\mathrm{H}$ & 7.791271 & 3.461697 & 3.180397 \\
\hline C & 3.470591 & 3.693708 & 3.440810 & $\mathrm{H}$ & 7.891169 & 3.430009 & 0.475440 \\
\hline C & 4.364082 & 3.430019 & 4.678259 & $\mathrm{H}$ & 7.873973 & 3.638014 & -1.269782 \\
\hline C & 3.679443 & 5.150540 & 2.954128 & $\mathrm{H}$ & 6.608850 & 4.381547 & -0.279737 \\
\hline C & 2. $0 \odot 76 \odot 2$ & 3.575118 & 3.918159 & $\mathrm{H}$ & 4.328648 & 2.376560 & 4.982353 \\
\hline C & -2.309060 & -1.210296 & 1.473877 & $\mathrm{H}$ & 5.401901 & 3.709939 & 4.499735 \\
\hline C & -2.857031 & -2.383769 & 2.012722 & $\mathrm{H}$ & $4.0 \odot 4104$ & 4.030207 & 5.521823 \\
\hline C & -3.475174 & -2.401439 & 3.266896 & $\mathrm{H}$ & 3.470882 & 5.844114 & 3.777286 \\
\hline C & -3.558869 & -1.164111 & 3.968429 & $\mathrm{H}$ & 4.704913 & 5.319127 & 2.620628 \\
\hline C & -2.863137 & $-\odot .007493$ & 3.544591 & $\mathrm{H}$ & 2.998206 & 5.402025 & 2.133872 \\
\hline C & -2.285259 & -0.062625 & 2.263337 & $\mathrm{H}$ & 1.285840 & 3.790603 & 3.122854 \\
\hline C & -2.649705 & 1.293397 & 4.370223 & $\mathrm{H}$ & 1.782984 & 2.588473 & 4.340700 \\
\hline C & -2.926353 & 1.163547 & 5.886934 & $\mathrm{H}$ & 1.834123 & 4.312846 & 4.708103 \\
\hline C & -3.511899 & 2.440569 & 3.790668 & $\mathrm{H}$ & -2.808850 & -3.292264 & 1.426589 \\
\hline C & -1.157105 & 1.706157 & 4.260802 & $\mathrm{H}$ & -1.793703 & 0.823687 & 1.875091 \\
\hline C & -3.218915 & -3.962452 & 5.227253 & $\mathrm{H}$ & -3.983727 & 1.070664 & 6.131939 \\
\hline C & -5.603118 & -0.541744 & 4.986953 & $\mathrm{H}$ & -2.403233 & 0.307183 & 6.322038 \\
\hline C & -3.963069 & -3.738186 & 3.886150 & $\mathrm{H}$ & -2.559585 & 2. 069989 & 6.382019 \\
\hline C & -3.645831 & -4.935021 & 2.962394 & $\mathrm{H}$ & -3.324574 & 3.370577 & 4.339882 \\
\hline C & -5.741579 & -1.255209 & -2.658471 & $\mathrm{H}$ & -3.277465 & 2.625102 & 2.735416 \\
\hline C & -4.522186 & -1.137087 & -3.375105 & $\mathrm{H}$ & -4.582965 & 2.222216 & 3.857725 \\
\hline C & -5.675237 & -0.816261 & -5.722346 & $\mathrm{H}$ & $-\odot .974970$ & 2.589106 & 4.882112 \\
\hline C & -3.339659 & -1.110161 & -2.622379 & $\mathrm{H}$ & -0.500858 & 0.900518 & 4.608495 \\
\hline C & -3.332074 & -1.174805 & -1.221119 & $\mathrm{H}$ & $-\odot .858379$ & 1.970569 & 3.242019 \\
\hline C & -4.563471 & -1.157688 & -0.562283 & $\mathrm{H}$ & -3.502643 & -4.932304 & 5.653925 \\
\hline C & -5.789657 & -1.162573 & -1.246756 & $\mathrm{H}$ & -2.132643 & -3.956184 & 5.082916 \\
\hline C & -7.264452 & -2.803167 & -3.569139 & $\mathrm{H}$ & -3.466009 & -3.185713 & 5.953752 \\
\hline
\end{tabular}




\begin{tabular}{|c|c|c|c|}
\hline $\mathrm{H}$ & -5.532836 & $\odot .525948$ & 4.755975 \\
\hline $\mathrm{H}$ & -6.187565 & -1.036853 & 4.203542 \\
\hline $\mathrm{H}$ & -6.102405 & -0.669233 & 5.949967 \\
\hline H & -4 . 201911 & -4.886518 & 2.018508 \\
\hline $\mathrm{H}$ & -2.579404 & -5.010933 & 2.728175 \\
\hline $\mathrm{H}$ & -3.945823 & -5.860566 & 3.465529 \\
\hline $\mathrm{H}$ & -6.269796 & ๑. 019275 & -5.344886 \\
\hline H & -5.403528 & $-\odot .596794$ & -6.761792 \\
\hline $\mathrm{H}$ & -6.309590 & -1.703544 & -5.736725 \\
\hline $\mathrm{H}$ & -2.393900 & -1.038161 & -3.142025 \\
\hline $\mathrm{H}$ & -4.579374 & -1.149668 & $\odot .518126$ \\
\hline H & -8.192274 & -2.798497 & -4.145362 \\
\hline $\mathrm{H}$ & -6.487819 & -3.334508 & -4.133960 \\
\hline $\mathrm{H}$ & -7.428729 & -3.326533 & -2.618771 \\
\hline H & -3.521258 & -2.202263 & -6.545373 \\
\hline $\mathrm{H}$ & -2.672984 & -2.423062 & -5.007746 \\
\hline H & -4.253719 & -3.182995 & -5.265677 \\
\hline $\mathrm{H}$ & -7.402173 & -3.234594 & $-\odot .250927$ \\
\hline H & -6.380590 & -2.595458 & 1. 044558 \\
\hline $\mathrm{H}$ & -8.131237 & -2.312327 & 1.073053 \\
\hline $\mathrm{H}$ & -7.808941 & $\odot .197309$ & 1.242531 \\
\hline $\mathrm{H}$ & -6.060676 & $-\odot .022737$ & 1.308805 \\
\hline $\mathrm{H}$ & -6.768636 & 1.058783 & ๑. 097197 \\
\hline $\mathrm{H}$ & -3.962093 & 1.139989 & -4.856735 \\
\hline $\mathrm{H}$ & -2.484558 & $\odot .155508$ & -4.805980 \\
\hline $\mathrm{H}$ & -3.375361 & 0.329049 & -6.317106 \\
\hline $\mathrm{H}$ & -6.054982 & -3.502295 & 3.246078 \\
\hline H & -5.787347 & -4.783581 & 4. 433301 \\
\hline $\mathrm{H}$ & -5.780604 & -3.102051 & 4.956784 \\
\hline H & -9.172039 & -0.561535 & -0.402031 \\
\hline $\mathrm{H}$ & - 8.298821 & $\odot .096325$ & -1.795733 \\
\hline H & -8.730497 & -1.615588 & -1.741186 \\
\hline $\mathrm{Rh}$ & $-\odot .177302$ & $\odot .998098$ & -0.768414 \\
\hline 0 & -0.763210 & 6.114682 & 1.602833 \\
\hline C & ๑. 209734 & $\odot .691536$ & -2.689272 \\
\hline S & $-\odot .121155$ & 6.240580 & ๑. 285010 \\
\hline 0 & -0.374776 & $-\odot .170627$ & -3.290953 \\
\hline C & 1.030306 & 3.889839 & $-\odot .2 \odot 4451$ \\
\hline $\mathrm{N}$ & -0.150144 & 4.715929 & -0.521076 \\
\hline 0 & 1.260886 & 6.717303 & $\odot .174001$ \\
\hline C & -1.177223 & 7.232105 & -0.761206 \\
\hline H & 1.906277 & 4.537172 & -0.316209 \\
\hline $\mathrm{H}$ & $1.0 \odot 2424$ & 3.566316 & ๑. 847065 \\
\hline C & -2.420410 & 7.647663 & -0.283754 \\
\hline C & -0.720143 & 7.620974 & -2.024938 \\
\hline C & -1.560956 & 2.883266 & -1.307972 \\
\hline H & -2.742769 & 7.354212 & ๑. 709629 \\
\hline C & -3.221181 & 8.453432 & -1.094585 \\
\hline C & -1.533598 & 8.423414 & -2.817471 \\
\hline $\mathrm{H}$ & ๑. 259286 & 7.308250 & 7.619400 \\
\hline C & -2.338271 & 1.777078 & $9.04340 \odot$ \\
\hline $\mathrm{H}$ & -1.314242 & 3.135304 & 13.116900 \\
\hline H & -4.189928 & 8.781567 & -0.727262 \\
\hline C & -2.794186 & 8.853596 & -2.367269 \\
\hline $\mathrm{H}$ & -1.184342 & 8.730460 & -3.800077 \\
\hline $\mathrm{H}$ & -2.717915 & 1.192404 & -1.905802 \\
\hline H & -2.841289 & 1.636788 & -0.125062 \\
\hline C & -3.649687 & 9.750107 & -3.229160 \\
\hline $\mathrm{H}$ & -3.276196 & 10.782028 & -3.208565 \\
\hline $\mathrm{H}$ & -3.642078 & 9.425526 & -4.275435 \\
\hline $\mathrm{H}$ & -4.687770 & 9.768556 & -2.884370 \\
\hline C & 1. 255942 & 1.611789 & -3.324795 \\
\hline $\mathrm{H}$ & 1.048348 & 1.682387 & -4.398538 \\
\hline H & 2. 209177 & 1.075454 & -3.211764 \\
\hline C & 1.311246 & 2.951337 & -2.596842 \\
\hline H & ๑. 523013 & $3.61860 \odot$ & -2.956423 \\
\hline $\mathrm{H}$ & 2.261039 & 3.458708 & -2.818751 \\
\hline C & 1.198876 & 2.673019 & -1.099663 \\
\hline $\mathrm{H}$ & 1.842371 & 1.802383 & 2.308670 \\
\hline $\mathrm{H}$ & 2.141190 & 2.233906 & -0.786577 \\
\hline C & -1.410280 & $4.0 \odot \odot 486$ & -0.315374 \\
\hline H & -2.234389 & 4.704337 & -0.491948 \\
\hline $\mathrm{H}$ & -1.516563 & 3.631920 & $\odot .717805$ \\
\hline
\end{tabular}

8

B3LYP SCF energy: $\quad-5535.37820820$ a.u. B3LYP enthalpy: $\quad-5533.341651$ a.u.

B3LYP free energy: $\quad-5533.614863$ a.u. M06 SCF energy in solution: $\quad-5534.60754828$ a.u.

M06 enthalpy in solution: $\quad-5532.570991 \mathrm{a} . \mathrm{u}$. M06 free energy in solution: -5532.844203 a.u.

Cartesian coordinates

ATOM $X \quad Y \quad Z$

$\begin{array}{llll}P & 0.000000 & -4.528694 & -3.401904 \\ P & 0.000000 & -4.212686 & -1.408942\end{array}$

$\odot .000000$

$0 \quad 0.000000-3.633571-4.312313$

$0 \quad 0.000000 \quad-1.879631-3.545052$

$\begin{array}{llll}0.000000 & -6.105910 & -3.850775\end{array}$

$0.0000000-3.986116-4.528906$

$0.000000 \quad-6.630960 \quad-2.795766$

$0.000000-6.038854-5.254985$

$0.000000 \quad-7.008299 \quad-3.773054$

$\begin{array}{lll}0.000000 & -7.685340 & -2.600324\end{array}$

$\begin{array}{lll}2.687444 & -7.012300 & -4.878912\end{array}$

$\begin{array}{lll}2.299980 & -7.715776 & -1.769723\end{array}$

$\begin{array}{lll}1.059305 & -8.354412 & -2.536774\end{array}$

$\begin{array}{lll}0.210038 & -7.685563 & -4.795947\end{array}$

$\begin{array}{lll}4.292632 & -6.527020 & -5.800285\end{array}$

$\begin{array}{lll}2.839828 & -8.896545 & -1.631907\end{array}$

$\begin{array}{lll}3.463906 & -8.359123 & -3.626113\end{array}$

$\begin{array}{lll}3.737211 & -7.701884 & -5.660372\end{array}$

$\begin{array}{lll}3.366802 & -9.067624 & -3.537424\end{array}$

$2.745336 \quad-9.359885-4.526622$

$\begin{array}{lll}2.439119 & -8.417552 & -3.084865\end{array}$

$3.156175 \quad-9.968735 \quad-2.918916$

$\begin{array}{lll}-0.843955 & 2.595258 & -0.811003\end{array}$

$\begin{array}{lll}-0.795874 & 2.303820 & -2.175491\end{array}$

$\begin{array}{lll}-0.991524 & 3.289207 & -3.149795\end{array}$

$\begin{array}{lll}-1.305355 & 4.601019 & -2.695491\end{array}$

$\begin{array}{lll}-1.243667 & 4.962254 & -1.320674\end{array}$

$\begin{array}{lll}-1.029049 & 3.920420 & -0.407180\end{array}$

$\begin{array}{lll}-1.372913 & 6.408814 & -0.760806\end{array}$

$\begin{array}{lll}-2.853885 & 6.741996 & -0.453505\end{array}$

$\begin{array}{lll}-0.766794 & 7.473535 & -1.706867\end{array}$

$\begin{array}{lll}-0.601671 & 6.543557 & 0.579579\end{array}$

$\begin{array}{lll}-0.814431 & 2.938180 & -4.650265\end{array}$

$0.252143 \quad 3.866266 \quad-5.284131$

$\begin{array}{lll}-0.311955 & 1.488710 & -4.836620\end{array}$

$\begin{array}{lll}-2.146487 & 3.049224 & -5.429403\end{array}$

$\begin{array}{lll}-2.949920 & 5.922398 & -3.869961\end{array}$

$\begin{array}{lll}-2.284970 & 1.185700 & 1.304689\end{array}$

$\begin{array}{lll}-3.389903 & 1.833908 & 0.748375\end{array}$

$\begin{array}{lll}-2.486712 & 0.353227 & 2.419457\end{array}$

$\begin{array}{lll}-3.725290 & 0.276047 & 3.064631\end{array}$

$\begin{array}{lll}-4.789118 & 1.061531 & 2.536322\end{array}$

$\begin{array}{lll}-4.678318 & 1.743126 & 1.301596\end{array}$

$\begin{array}{lll}-5.848327 & 2.361932 & 0.481537\end{array}$

$\begin{array}{lll}-5.679103 & 1.964507 & -1.011589\end{array}$

$\begin{array}{lll}-5.807805 & 3.905836 & 0.570822\end{array}$

$\begin{array}{lll}-6.200217 & 2.295067 & 3.987114\end{array}$

$\begin{array}{lll}-7.255810 & 1.858133 & 0.880414\end{array}$

$\begin{array}{lll}-3.930684 & -0.699390 & 4.253892\end{array}$

$\begin{array}{lll}-4.331783 & 0.031308 & 5.558768\end{array}$

$\begin{array}{lll}-5.029301 & -1.726017 & 3.878938\end{array}$

$\begin{array}{lll}-2.645372 & -1.494355 & 4.569113\end{array}$

$\begin{array}{lll}3.541929 & -0.584403 & 1.219784\end{array}$

$\begin{array}{lll}4.849270 & -0.076640 & 1.214381\end{array}$

$\begin{array}{lll}5.739619 & -0.331056 & 2.260283\end{array}$

$5.272070 \quad-1.155696 \quad 3.323872$

$3.909592 \quad-1.513915 \quad 3.458095$

$\begin{array}{lll}3.080826 & -1.253569 & 2.352104\end{array}$

$\begin{array}{lll}3.230688 & -2.119589 & 4.720009\end{array}$

$\begin{array}{lll}4.074906 & -2.071202 & 6.014530\end{array}$ 


\begin{tabular}{|c|c|c|c|c|c|c|c|}
\hline C & 2.807295 & -3.582338 & 4.444044 & H & 5.172085 & $\odot .515753$ & 0.368368 \\
\hline C & 1.952551 & -1.289347 & 5.026572 & H & 2.046665 & -1.569280 & 2.389809 \\
\hline c & 7.337615 & 1.116488 & 3.585276 & H & 4.913027 & -2.768114 & 6.016848 \\
\hline c & 6.621715 & -2.924825 & 4.122733 & $\mathrm{H}$ & 4.468052 & -1.068953 & 6.206583 \\
\hline c & 7.145201 & $\odot .324526$ & 2.268099 & H & 3.427680 & -2.345296 & 6.855581 \\
\hline c & 7.300637 & 1.329330 & 1.104721 & $\mathrm{H}$ & 2.299680 & -3.999848 & 5.321588 \\
\hline C & 4.031573 & -3.561872 & -3.296990 & H & 2.114278 & -3.646900 & 3.597531 \\
\hline C & 2.906230 & -2.764106 & -3.642093 & H & 3.669077 & -4.221682 & 4.222159 \\
\hline C & 2.321522 & -4.132999 & -5.807651 & H & 1.477713 & -1.678067 & 5.934492 \\
\hline c & 2.501748 & -1.805525 & -2.703097 & $\mathrm{H}$ & 2.203544 & -0.236841 & 5.200178 \\
\hline C & 3.139552 & -1.643574 & -1.464281 & H & 1.208530 & -1.334400 & 4.227323 \\
\hline C & 4.161871 & -2.530461 & -1.126586 & $\mathrm{H}$ & 8.318183 & 1.607055 & 3.585391 \\
\hline C & 4.613351 & -3.527595 & $-2.0 \odot 4618$ & H & 6.572406 & 1.895900 & 3.682304 \\
\hline C & 5.594211 & -3.853167 & -5.039336 & $\mathrm{H}$ & 7.277272 & $\odot .467407$ & 4.460084 \\
\hline C & 2.408631 & -1.621795 & -5.844272 & H & 5.792883 & -3.625680 & 4.265372 \\
\hline C & 7.018081 & -3.703480 & -1.239772 & H & 7.068471 & -3.099916 & 3.137329 \\
\hline c & 5.703178 & -4.492364 & -1.455002 & H & 7.372433 & -3.090542 & 4.898410 \\
\hline C & 5.219408 & -5.047730 & $-\odot .087006$ & H & 7.280080 & $\odot .839320$ & 0.124236 \\
\hline c & $\odot .575366$ & -2.847904 & -4.629255 & H & 6.523222 & 2.101500 & 1.121505 \\
\hline c & 8.281281 & -0.716304 & 2.127675 & $\mathrm{H}$ & 8.270447 & 1.830150 & 1.194103 \\
\hline c & 2.091603 & -2.856272 & -4.965247 & H & 2.215392 & -5.043455 & -5.211624 \\
\hline C & 6.017766 & -5.732451 & -2.322243 & H & 1.566326 & -4.159086 & -6.601760 \\
\hline $\mathrm{H}$ & 0.746763 & 2.787738 & 4.989117 & H & 3.298678 & -4.158873 & -6.289693 \\
\hline H & $-\odot .781942$ & 1.824753 & 3.298011 & H & 1.679213 & -1.142999 & -2.957340 \\
\hline $\mathrm{H}$ & 5.277790 & 3.723208 & 3.614131 & $\mathrm{H}$ & 4.625722 & -2.441088 & -0.153134 \\
\hline H & 4.256981 & 5.146367 & 3.160717 & H & 5.929993 & -4.646650 & -5.710208 \\
\hline $\mathrm{H}$ & 3.770983 & $\odot .505539$ & -2.729125 & H & 5.229129 & -3.005624 & -5.632691 \\
\hline H & 4.231662 & 2.744965 & -3.717016 & H & 6.436730 & -3.515952 & -4.422892 \\
\hline $\mathrm{H}$ & 2.464371 & 6.595301 & -1.415012 & H & 1.818875 & -1.654034 & -6.767995 \\
\hline H & 4.058559 & 6.187974 & $-\odot .664631$ & H & 2.172909 & $-\odot .684017$ & -5.328289 \\
\hline $\mathrm{H}$ & -0.600276 & 1.285208 & -2.484032 & H & 3.467104 & -1.591883 & -6.125789 \\
\hline $\mathrm{H}$ & -0.999647 & 4.145930 & 0.650739 & H & 7.403114 & -3.304549 & -2.185226 \\
\hline H & -3.253876 & 6.057896 & 0.303332 & H & 6.881894 & -2.860503 & $-\odot .552881$ \\
\hline $\mathrm{H}$ & -2.933897 & 7.761101 & -0.056526 & H & 7.787120 & -4.360923 & -0.817238 \\
\hline H & -3.498571 & 6.676642 & -1.332474 & H & 5.975904 & -5.728022 & ๑.319845 \\
\hline H & -1.300960 & 7.579879 & -2.648631 & H & 5.047692 & -4.267333 & $\odot .660143$ \\
\hline H & -0.773769 & 8.446063 & -1.202426 & H & 4.288489 & $-5.61450 \odot$ & -0.204306 \\
\hline $\mathrm{H}$ & 0.273858 & 7.230813 & -1.952139 & H & 0.311469 & -3.697175 & -3.987711 \\
\hline H & 0.430537 & 6.190060 & 0.493851 & H & 0.245976 & -1.928877 & -4.135014 \\
\hline $\mathrm{H}$ & $-\odot .582259$ & 7.597942 & 0.874695 & H & -0.005209 & -2.936265 & -5.553605 \\
\hline H & -1.080890 & $6.0 \odot \odot \odot 78$ & $1.40 \odot 482$ & H & 8.125900 & -1.360915 & 1.254139 \\
\hline H & 0.391000 & 3.602504 & -6.339351 & H & 9.238656 & $-\odot .199676$ & 1.991205 \\
\hline H & $-\odot .03909 \odot$ & 4.915647 & -5.227642 & H & 8.372606 & -1.343792 & 3. 014612 \\
\hline H & 1.217972 & 3.747793 & -4.778616 & H & 6.671291 & -6.395332 & -1.742971 \\
\hline H & -0.141045 & 1.307409 & -5.902673 & H & 5.116754 & -6.292286 & -2.585007 \\
\hline H & 0.638060 & 1.314405 & -4.317520 & H & 6.545471 & -5.487637 & -3.244843 \\
\hline H & -1.042205 & 0.744287 & -4.496972 & $\mathrm{H}$ & -1.662603 & $-\odot .242754$ & 2.782747 \\
\hline $\mathrm{H}$ & -2.934287 & 2.451098 & -4.956234 & $\mathrm{Rh}$ & 0.030440 & -0.683484 & -0.494440 \\
\hline H & -2.493930 & 4.078444 & -5.510575 & $C$ & $-\odot .157136$ & -2.014703 & 0.871373 \\
\hline $\mathrm{H}$ & -2.009135 & 2.670186 & -6.448805 & C & -1.914878 & -0.926160 & -1.304162 \\
\hline $\mathrm{H}$ & -3.186777 & 6.839460 & -3.322429 & 0 & $-\odot .349553$ & -1.960492 & 2.054325 \\
\hline $\mathrm{H}$ & -3.068407 & 6.117248 & -4.938771 & C & 0.002736 & -3.313727 & 0.056553 \\
\hline H & -3.638534 & 5.126611 & -3.567906 & C & -2.816790 & -2.047700 & -0.780702 \\
\hline $\mathrm{H}$ & -3.255452 & 2.418907 & -0.150730 & H & -2.471283 & 0.014192 & -1.282732 \\
\hline H & -6.520092 & 2.362056 & -1.589917 & H & -1.653516 & -1.126143 & -2.359689 \\
\hline H & -4.765108 & 2.359692 & -1.464470 & H & 0.773731 & -3.894915 & 0.576748 \\
\hline H & -5.675398 & $\odot .875824$ & -1.133987 & H & $\odot .389023$ & -3.124071 & -0.957491 \\
\hline $\mathrm{H}$ & -5.935193 & 4.263975 & 1.597700 & C & -1.330616 & -4.081244 & -0.019229 \\
\hline H & -4.856458 & $4.30 \odot 432$ & 0.197193 & C & -4.220052 & -1.999224 & -1.464484 \\
\hline H & -6.611910 & 4.340271 & $-\odot .034961$ & H & -2.970837 & -1.912104 & 0.298520 \\
\hline H & -5.369408 & 2.505652 & 4.669376 & C & -2.329064 & -3.503020 & -1.028428 \\
\hline H & -6.345525 & 3.155138 & 3.326620 & H & -1.775213 & -4.119302 & ๑.983152 \\
\hline $\mathrm{H}$ & -7.111983 & 2.127239 & 4.564451 & H & -1.101112 & -5.116443 & -0.301423 \\
\hline H & -7.603215 & 2.242482 & 1.838829 & $\mathrm{~N}$ & -4.528694 & -3.401904 & 8.202600 \\
\hline $\mathrm{H}$ & -7.970481 & 2.192223 & 0.119667 & H & -4.212686 & -1.408942 & 9.301100 \\
\hline $\mathrm{H}$ & -7.294583 & 0.765224 & 0.921819 & $\mathrm{H}$ & -4.959372 & -1.554991 & 10.646900 \\
\hline $\mathrm{H}$ & -3.640838 & 0.852266 & 5.785676 & C & -3.633571 & -4.312313 & -1.072661 \\
\hline H & -5.345880 & 0.427081 & 5.514520 & H & -1.879631 & -3.545052 & -2.031666 \\
\hline H & -4.291537 & $-\odot .673439$ & 6.397432 & $\mathrm{~s}$ & -6.105910 & -3.850775 & -2.137460 \\
\hline H & -5.184929 & -2.423144 & 4.711303 & H & -3.986116 & -4.528906 & $-\odot .048734$ \\
\hline $\mathrm{H}$ & -5.979867 & -1.235607 & 3.659112 & H & -3.543619 & -5.255680 & -1.614999 \\
\hline H & -4.732627 & -2.313905 & $3.0 \odot 2261$ & 0 & -6.630960 & -2.795766 & -3.006302 \\
\hline $\mathrm{H}$ & -2.289644 & -2.077909 & 3.715289 & 0 & -6.038854 & -5.254985 & -2.544768 \\
\hline $\mathrm{H}$ & -1.826599 & -0.845793 & 4.903367 & C & -7.008299 & -3.773054 & -0.588546 \\
\hline H & -2.855695 & -2.196014 & 5.383250 & C & -7.685340 & -2.600324 & $-\odot .239867$ \\
\hline
\end{tabular}




$\begin{array}{lrrr}\mathrm{C} & -7.012300 & -4.878912 & 0.266794 \\ \mathrm{H} & -7.715776 & -1.769723 & -0.936994 \\ \mathrm{C} & -8.354412 & -2.536774 & 0.981347 \\ \mathrm{C} & -7.685563 & -4.795947 & 1.484703 \\ \mathrm{H} & -6.527020 & -5.800285 & -0.037731 \\ \mathrm{H} & -8.896545 & -1.631907 & 1.245261 \\ \mathrm{C} & -8.359123 & -3.626113 & 1.865625 \\ \mathrm{H} & -7.701884 & -5.660372 & 2.143773 \\ \mathrm{C} & -9.067624 & -3.537424 & 3.196270 \\ \mathrm{H} & -9.359885 & -4.526622 & 3.562204 \\ \mathrm{H} & -8.417552 & -3.084865 & 3.956973 \\ \mathrm{H} & -9.968735 & -2.918916 & 3.129810\end{array}$

B3LYP SCF energy:

B3LYP enthalpy:

B3LYP free energy:

M06 SCF energy in solution:

a.u.

M06 enthalpy in solution:

M06 free energy in solution:

a.u.

Cartesian coordinates

\begin{tabular}{|c|c|c|c|}
\hline АТОМ & $\mathrm{X}$ & $\mathrm{Y}$ & Z \\
\hline $\mathrm{P}$ & $\odot . ๑ \odot \odot \odot \odot \odot ~$ & 4.128813 & 4.135086 \\
\hline$P$ & 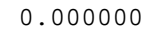 & 4.166397 & 2.432481 \\
\hline 0 & 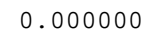 & 4.564466 & 2. 097497 \\
\hline 0 & $\odot . ๑ \odot \odot \odot \odot \odot$ & 3.431462 & 4.477063 \\
\hline 0 & 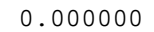 & 1.399485 & 4.359999 \\
\hline 0 & $\odot .0000 \odot \odot$ & 5.633224 & 4.826744 \\
\hline 0 & $\odot . ๑ \odot \odot \odot \odot \odot$ & 3.988318 & 4.126960 \\
\hline 0 & $\odot . ๑ \odot \odot \odot \odot \odot$ & 3.299230 & 5.557662 \\
\hline 0 & $\odot .0000 \odot \odot$ & 6.023026 & 4.297740 \\
\hline 0 & ๑. . ०००००० & 5.471406 & 6.257736 \\
\hline C & $\odot .0 \odot \odot \odot \odot \odot$ & 6.779521 & 4.176921 \\
\hline C & ๑. .००००० & 7.475795 & 2.994437 \\
\hline C & -2.988779 & 6.952986 & 4.849032 \\
\hline C & -2.865695 & 7.359426 & 2.504627 \\
\hline C & -1.736159 & 8.343488 & 2.483224 \\
\hline C & -0.722255 & 7.824756 & 4.322434 \\
\hline C & -4.755329 & 6.433639 & 5.784704 \\
\hline C & -2.569707 & 8.893627 & 1.569096 \\
\hline C & -2.962765 & 8.532729 & 3.135340 \\
\hline C & -3.108762 & 7.967236 & 4.848535 \\
\hline C & -2.851498 & 9.505284 & 2.596331 \\
\hline C & -2.465513 & 10.498545 & 3.044963 \\
\hline C & -2.293585 & 9.181420 & 2.822095 \\
\hline C & -2.750557 & 9.623374 & 1.511724 \\
\hline C & 1.045095 & -2.164168 & -1.420179 \\
\hline C & 1. 226364 & -1.293941 & -2.497011 \\
\hline C & 1.617308 & -1.751417 & -3.760569 \\
\hline C & 1.884059 & -3.143081 & -3.895041 \\
\hline C & 1.591775 & -4.074497 & -2.860173 \\
\hline C & 1.193427 & -3.537937 & -1.627596 \\
\hline C & 1.657926 & -5.623859 & -2.991923 \\
\hline C & 3.065620 & -6.141069 & -2.604908 \\
\hline C & 1.269660 & -6.123857 & -4.404367 \\
\hline C & ๑. 654612 & -6.293627 & -2.014607 \\
\hline C & 1.695205 & -0.761205 & -4.951962 \\
\hline C & $\odot .779878$ & -1.251648 & -6.101995 \\
\hline C & 1.195178 & ๑. 645396 & -4.552670 \\
\hline C & 3.144712 & -0.587496 & -5.464512 \\
\hline C & 3.748457 & -3.889523 & -5.232661 \\
\hline C & 2.043358 & -1.962987 & 1.314832 \\
\hline C & 3. 256311 & -2.312731 & 0.717611 \\
\hline C & 2.011711 & -1.778656 & 2.708481 \\
\hline C & 3.109522 & -2.088583 & 3.516418 \\
\hline C & 4.280258 & -2.576809 & 2.869318 \\
\hline C & 4. 420607 & -2.563261 & 1.462370 \\
\hline C & 5.743603 & -2.743607 & $\odot .662130$ \\
\hline C & 5.841907 & -1.596157 & $-\odot .382237$ \\
\hline C & 5.730901 & -4.091521 & $-\odot .096436$ \\
\hline
\end{tabular}

-5535.37335585 a.u -5533.336457 a.u. -5533.610121 a.u. $-5534.60066996$ -5532.563771 a.u. $-5532.837435$

5086 


\begin{tabular}{|c|c|c|c|}
\hline $\mathrm{H}$ & 5.670187 & -4.879030 & 2.757401 \\
\hline $\mathrm{H}$ & 6.275550 & -4.640041 & 4.418270 \\
\hline $\mathrm{H}$ & 7.208582 & -3.504107 & 2.147729 \\
\hline $\mathrm{H}$ & 7.890369 & -2.590469 & 0.810143 \\
\hline $\mathrm{H}$ & 7.053346 & -1.744757 & 2.121820 \\
\hline $\mathrm{H}$ & 2.548732 & -3.899793 & 5.552489 \\
\hline $\mathrm{H}$ & 4.260292 & -3.498494 & 5.821622 \\
\hline H & 3. 028818 & -2.901426 & 6.930636 \\
\hline $\mathrm{H}$ & 4.145778 & $-\odot .605456$ & 6.491892 \\
\hline $\mathrm{H}$ & 5.163258 & -1.190873 & 5.163140 \\
\hline $\mathrm{H}$ & 4.021230 & ๑.142936 & 4.891042 \\
\hline H & 1.497107 & $-\odot .261792$ & 4.958053 \\
\hline $\mathrm{H}$ & ๑. 868812 & -1.885636 & $5.30 \odot 412$ \\
\hline $\mathrm{H}$ & 1.744127 & -0.994086 & 6.545629 \\
\hline $\mathrm{H}$ & -5.092989 & $-\odot .399938$ & $-\odot .482122$ \\
\hline $\mathrm{H}$ & -2.368643 & 0.374370 & 2.707529 \\
\hline $\mathrm{H}$ & -5.814647 & ๑. 098031 & 6.000484 \\
\hline $\mathrm{H}$ & -5.414105 & -1.547632 & 5.497548 \\
\hline $\mathrm{H}$ & -4.497749 & -0.757446 & 6.789334 \\
\hline $\mathrm{H}$ & -3.118294 & 1.298799 & 6.327534 \\
\hline $\mathrm{H}$ & -2.637188 & 1.709004 & 4.674947 \\
\hline $\mathrm{H}$ & -4.275935 & 2.077861 & 5.238216 \\
\hline $\mathrm{H}$ & -2.419187 & -1.117436 & 5.980383 \\
\hline $\mathrm{H}$ & -3.014415 & -2.041214 & 4.588988 \\
\hline $\mathrm{H}$ & -1.862545 & $-\odot .713974$ & 4.357985 \\
\hline $\mathrm{H}$ & -8.711741 & -2.587413 & 1.398098 \\
\hline $\mathrm{H}$ & -7.001292 & -2.987354 & 1.638485 \\
\hline $\mathrm{H}$ & -7.855215 & -1.999310 & 2.836115 \\
\hline $\mathrm{H}$ & -6.390212 & 1.674983 & 4.677506 \\
\hline $\mathrm{H}$ & -7.458212 & 1. 771226 & 3.254158 \\
\hline $\mathrm{H}$ & -8.050076 & 1.027392 & 4.767643 \\
\hline $\mathrm{H}$ & -7.129720 & -0.449337 & -1.164519 \\
\hline $\mathrm{H}$ & -6.535345 & -2.064996 & $-\odot .721894$ \\
\hline $\mathrm{H}$ & -8.273476 & -1.741994 & -0.806439 \\
\hline $\mathrm{H}$ & -1.471678 & 6.775722 & -2.744816 \\
\hline $\mathrm{H}$ & -0.743533 & 6.515600 & -4.337469 \\
\hline $\mathrm{H}$ & -2.494887 & 6.500585 & -4.157036 \\
\hline $\mathrm{H}$ & -1.217071 & 2.292087 & -2.256746 \\
\hline $\mathrm{H}$ & -4.429310 & 2.459703 & ๑. 550414 \\
\hline $\mathrm{H}$ & -5.172091 & 6.832572 & -3.640851 \\
\hline $\mathrm{H}$ & -4.510778 & 5.275384 & -4.212200 \\
\hline H & -5.804081 & 5.296038 & -2.983782 \\
\hline $\mathrm{H}$ & -1.090713 & 4.327680 & -5.565048 \\
\hline $\mathrm{H}$ & -1.584866 & 2.871672 & -4.684093 \\
\hline $\mathrm{H}$ & -2.782600 & 4.110353 & -5.093063 \\
\hline $\mathrm{H}$ & -6.975026 & 4.262408 & -1.141423 \\
\hline $\mathrm{H}$ & -6.624217 & 3.145098 & 0.185906 \\
\hline $\mathrm{H}$ & -7.465899 & 4.671003 & $\odot .509808$ \\
\hline $\mathrm{H}$ & -5.746171 & 5.326662 & 2.255112 \\
\hline $\mathrm{H}$ & -4.890369 & 3.802118 & 2.022249 \\
\hline $\mathrm{H}$ & -4.018216 & 5.336697 & 1.869081 \\
\hline $\mathrm{H}$ & ๑. 290819 & 4.907270 & -2.113127 \\
\hline $\mathrm{H}$ & ๑. 292335 & 3.370020 & $-3.0 \odot 3498$ \\
\hline $\mathrm{H}$ & 0.687216 & 4.865717 & -3.835606 \\
\hline $\mathrm{H}$ & -8.180572 & 1.085262 & ๑. 668712 \\
\hline $\mathrm{H}$ & -9.384456 & -0.210758 & 0.645727 \\
\hline $\mathrm{H}$ & -8.710316 & ๑. 322014 & 2.184521 \\
\hline $\mathrm{H}$ & -6.215019 & 6.836059 & 0.618420 \\
\hline $\mathrm{H}$ & -4.589469 & 6.994312 & $-\odot .066304$ \\
\hline $\mathrm{H}$ & -5.967510 & 6.630451 & -1.111275 \\
\hline $\mathrm{H}$ & 1.117522 & -1.379573 & 3.163246 \\
\hline $\mathrm{Rh}$ & 0.105286 & 0.682952 & $\odot .167263$ \\
\hline $\mathrm{C}$ & $\odot .063180$ & 1. 270288 & 1.996964 \\
\hline C & 2.187346 & 1.066708 & $\odot .108748$ \\
\hline 0 & ๑. . 061895 & ๑. 669767 & 3.035274 \\
\hline C & $\odot .052581$ & 2.809044 & 1.866481 \\
\hline C & 2.431335 & 2.495076 & -0.383659 \\
\hline $\mathrm{H}$ & 2.644065 & $\odot .366226$ & -0.599872 \\
\hline $\mathrm{H}$ & 2.654698 & ๑.876805 & 1.080688 \\
\hline $\mathrm{H}$ & -0.579377 & 3.156056 & 2.692027 \\
\hline $\mathrm{H}$ & -0.442155 & 3.134448 & ๑. 940059 \\
\hline C & 1.478964 & 3.404482 & 1.946269 \\
\hline C & 3.932327 & 2.700021 & -0.742665 \\
\hline H & 1.866868 & 2.653385 & -1.311463 \\
\hline C & 2.099798 & 3.705238 & $\odot .575453$ \\
\hline
\end{tabular}

$\begin{array}{rrrr}\mathrm{H} & 2.127905 & 2.751771 & 2.542160 \\ \mathrm{H} & 1.412079 & 4.348894 & 2.499893 \\ \mathrm{~N} & 4.128813 & 4.135086 & 4.540000 \\ \mathrm{H} & 4.166397 & 2.432481 & 6.996200 \\ \mathrm{H} & 4.564466 & 2.097497 & 8.875700 \\ \mathrm{C} & 3.431462 & 4.477063 & 0.713816 \\ \mathrm{H} & 1.399485 & 4.359999 & 0.042617 \\ \mathrm{~S} & 5.633224 & 4.826744 & -0.859245 \\ \mathrm{H} & 3.988318 & 4.126960 & 1.601417 \\ \mathrm{H} & 3.299230 & 5.557662 & 0.789602 \\ \mathrm{O} & 6.023026 & 4.297740 & -2.167358 \\ \mathrm{O} & 5.471406 & 6.257736 & -0.600626 \\ \mathrm{C} & 6.779521 & 4.176921 & 0.358097 \\ \mathrm{C} & 7.475795 & 2.994437 & 0.091270 \\ \mathrm{C} & 6.952986 & 4.849032 & 1.571799 \\ \mathrm{H} & 7.359426 & 2.504627 & -0.869741 \\ \mathrm{C} & 8.343488 & 2.483224 & 1.055153 \\ \mathrm{C} & 7.824756 & 4.322434 & 2.523048 \\ \mathrm{H} & 6.433639 & 5.784704 & 1.750188 \\ \mathrm{H} & 8.893627 & 1.569096 & 0.845825 \\ \mathrm{C} & 8.532729 & 3.135340 & 2.282568 \\ \mathrm{H} & 7.967236 & 4.848535 & 3.463736 \\ \mathrm{C} & 9.505284 & 2.596331 & 3.304166 \\ \mathrm{H} & 10.498545 & 3.044963 & 3.171800 \\ \mathrm{H} & 9.181420 & 2.822095 & 4.325424 \\ \mathrm{H} & 9.623374 & 1.511724 & 3.214136\end{array}$

10

B3LYP SCF energy:

B3LYP enthalpy:

B3LYP free energy: M06 SCF energy in solution: a.u.

M06 enthalpy in solution: M06 free energy in solution: a.u.

\begin{tabular}{lrrr}
\multicolumn{4}{l}{ Cartesian coordinates } \\
ATOM & X & Y & Z \\
P & -1.763559 & 1.585409 & -0.168153 \\
P & -0.611669 & -1.587675 & -0.331874 \\
O & -5.030845 & -0.680497 & 2.471616 \\
O & -4.265202 & -1.708100 & 4.401921 \\
O & -5.384581 & -2.273191 & -0.204965 \\
O & -6.608496 & -1.391565 & -1.963277 \\
C & -1.733099 & -1.700904 & 1.138085 \\
C & -3.033514 & -1.104995 & 1.091615 \\
C & -3.775064 & -1.182462 & 2.260158 \\
C & -3.318433 & -1.797152 & 3.425230 \\
C & -2.078062 & -2.392344 & 3.480230 \\
C & -1.298139 & -2.328930 & 2.312350 \\
C & -5.384423 & -1.027293 & 3.817329 \\
C & -3.309334 & 0.744771 & -0.714944 \\
C & -4.069664 & 1.269059 & -1.767150 \\
C & -5.211981 & 0.622537 & -2.271211 \\
C & -5.556471 & -0.571706 & -1.677629 \\
C & -4.816919 & -1.098712 & -0.618865 \\
C & -3.687031 & -0.487534 & -0.099195 \\
C & -6.489191 & -2.519406 & -1.086110 \\
C & -1.615976 & -2.323791 & -1.691779 \\
C & 0.703266 & -2.850091 & -0.002081 \\
C & -2.036211 & 1.930211 & 1.619679 \\
C & -3.290396 & 2.344980 & 2.098506 \\
C & -1.826112 & 3.242008 & -0.982787 \\
H & -1.721739 & -2.890970 & 4.374462 \\
H & -0.330757 & -2.811390 & 2.336215 \\
H & -5.592303 & -0.115110 & 4.385584 \\
H & -6.252888 & -1.694930 & 3.808070 \\
H & -3.785602 & 2.212664 & -2.214858 \\
H & -5.793676 & 1.046332 & -3.081961 \\
H & -6.289497 & -3.420707 & -1.676877 \\
H & -7.407516 & -2.624334 & -0.500627 \\
H & -4.131721 & 2.429320 & 1.416929
\end{tabular}

-3819.35025755 a.u. -3818.405319 a.u. -3818.566462 a.u. $-3819.31812116$ -3818.373183 a.u. $-3818.534326$ 


\begin{tabular}{|c|c|c|c|}
\hline $\mathrm{Rh}$ & 0.276725 & $\odot .488523$ & -0.779234 \\
\hline 0 & 1.082079 & 2.492871 & -1.254612 \\
\hline 0 & 5.719363 & 1.836127 & 1.891850 \\
\hline C & 1.789432 & 3. 208680 & -0.556288 \\
\hline S & 5.628870 & 1.392186 & 0.497463 \\
\hline C & 2.431965 & 4.558995 & -0.797691 \\
\hline C & 2.496417 & 3.003344 & 0.771073 \\
\hline $\mathrm{N}$ & 3.987654 & ๑. 969435 & ๑. 262028 \\
\hline 0 & 6.071435 & 2.265125 & -0.600617 \\
\hline C & 6.477903 & $-\odot .170719$ & ๑. 318369 \\
\hline C & 3.411115 & 4.212565 & $\odot .370217$ \\
\hline H & 1.738131 & 5.377419 & -0.570545 \\
\hline $\mathrm{H}$ & 2.846552 & 4.720676 & -1.797368 \\
\hline C & 3.009268 & 1.602587 & 1.150752 \\
\hline $\mathrm{H}$ & 1.802107 & 3. 316637 & 1.566002 \\
\hline C & 3.611165 & $\odot .703009$ & -1.134767 \\
\hline C & 7.226437 & $-\odot .420658$ & -0.832609 \\
\hline C & 6.425149 & -1.100387 & 1. 361744 \\
\hline $\mathrm{H}$ & 4.388861 & 3.901135 & $\odot .000860$ \\
\hline $\mathrm{H}$ & 3.538027 & 4.977018 & 1.137675 \\
\hline $\mathrm{H}$ & 3.450385 & 1.641192 & 2.147987 \\
\hline $\mathrm{H}$ & 2.140596 & $\odot .929660$ & 1.186567 \\
\hline C & 2.467457 & -0.276163 & -1.272376 \\
\hline $\mathrm{H}$ & 4.476181 & $\odot .218855$ & -1.605814 \\
\hline $\mathrm{H}$ & 3.436355 & 1. 620409 & -1.709417 \\
\hline $\mathrm{H}$ & 7.281558 & ๑. 328219 & -1.615382 \\
\hline C & 7.913829 & -1.629803 & -0.943216 \\
\hline C & 7.121435 & -2.297882 & 1.233833 \\
\hline $\mathrm{H}$ & 5.865351 & $-\odot .876985$ & 2.264100 \\
\hline C & 1.637257 & -0.313123 & -2.376421 \\
\hline $\mathrm{H}$ & 2.584662 & -1.172500 & -0.673752 \\
\hline $\mathrm{H}$ & 8.498537 & -1.828434 & -1.837502 \\
\hline C & 7.875981 & -2.583075 & ๑. . 082868 \\
\hline $\mathrm{H}$ & 7.092391 & -3.020899 & 2.045229 \\
\hline $\mathrm{H}$ & 1.189625 & -1.247738 & -2.698912 \\
\hline $\mathrm{H}$ & 1.678612 & 0.473782 & -3.128131 \\
\hline C & 8.657906 & -3.869696 & -0.024991 \\
\hline $\mathrm{H}$ & 8.136086 & -4.699725 & 0.462706 \\
\hline $\mathrm{H}$ & 9.636638 & -3.771186 & ๑. 462382 \\
\hline H & 8.839020 & -4.143989 & -1.068723 \\
\hline C & 1.858983 & -4.932446 & -0.497363 \\
\hline H & 1.939012 & -5.835224 & -1.096142 \\
\hline C & 2.774529 & -4.686084 & 0.524277 \\
\hline $\mathrm{H}$ & $3.5702 \odot 2$ & -5.396595 & $\odot .728532$ \\
\hline C & 2.668063 & -3.514732 & 1.279280 \\
\hline $\mathrm{H}$ & 3.384253 & -3.305855 & 2.068728 \\
\hline C & 1. 650052 & -2.601107 & 1. 010884 \\
\hline $\mathrm{H}$ & 1.594770 & -1.682146 & 1.588665 \\
\hline C & ๑. 829593 & -4.023453 & $-\odot .759916$ \\
\hline $\mathrm{H}$ & ๑. 127839 & -4.238997 & -1.557112 \\
\hline C & -3.463059 & 2.642707 & 3.450649 \\
\hline $\mathrm{H}$ & -4.434750 & 2.970967 & 3.809346 \\
\hline C & -2.391639 & 2.521007 & 4.340153 \\
\hline $\mathrm{H}$ & -2.529501 & 2.751595 & 5.392688 \\
\hline C & -1.145898 & 2.096257 & 3.874859 \\
\hline $\mathrm{H}$ & -0.314138 & 1.987747 & 4.565292 \\
\hline C & -0.970361 & 1.802226 & 2.521265 \\
\hline $\mathrm{H}$ & $-\odot .008049$ & 1.448517 & 2.161197 \\
\hline C & -1.650050 & -1.717868 & -2.955602 \\
\hline $\mathrm{H}$ & -1.111266 & $-\odot .788926$ & -3.115771 \\
\hline C & -2.392138 & -2.286422 & -3.993155 \\
\hline $\mathrm{H}$ & -2.414715 & -1.805408 & -4.966899 \\
\hline C & -3.104704 & -3.467065 & -3.778377 \\
\hline $\mathrm{H}$ & -3.677457 & -3.911957 & -4.587206 \\
\hline C & -3.083270 & -4.074157 & -2.518989 \\
\hline $\mathrm{H}$ & -3.636803 & -4.993150 & -2.346350 \\
\hline C & -2.349271 & -3.504230 & -1.478964 \\
\hline $\mathrm{H}$ & -2.350616 & -3.975225 & $-0.50 \odot 229$ \\
\hline C & -2.121803 & 4.420913 & -0.283064 \\
\hline H & -2.341421 & 4.388569 & ๑. 778187 \\
\hline C & -2.140878 & 5.651641 & -0.945992 \\
\hline $\mathrm{H}$ & -2.374058 & 6.555230 & -0.389690 \\
\hline C & -1.871748 & 5.719912 & -2.312901 \\
\hline $\mathrm{H}$ & -1.893760 & 6.676690 & -2.826800 \\
\hline C & -1.569534 & 4.551537 & -3.018163 \\
\hline
\end{tabular}

$\begin{array}{llll}\mathrm{H} & -1.351520 & 4.596291 & -4.081492 \\ \mathrm{C} & -1.534465 & 3.325418 & -2.356635 \\ \mathrm{H} & -1.271905 & 2.426909 & -2.907910\end{array}$

18

B3LYP SCF energy:

B3LYP enthalpy:

B3LYP free energy:

-5535.35521110 a.u. -5533.318656 a.u M06 SCF energy in solution: $\quad-5534.58911556$ a.u. M06 enthalpy in solution: $\quad-5532.552560 \mathrm{a} \cdot \mathrm{u}$. M06 free energy in solution: $\quad-5532.819927$ a.u.

Cartesian coordinates

\begin{tabular}{|c|c|c|c|}
\hline АTOM & $X$ & $\mathrm{Y}$ & Z \\
\hline $\mathrm{P}$ & $\odot . \odot \odot \odot \odot \odot \odot$ & -5.365343 & 4.614679 \\
\hline$P$ & ๑. .०००००० & -5.283415 & 4.111229 \\
\hline 0 & 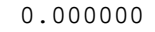 & -0.283455 & 5.582587 \\
\hline 0 & $\odot . \diamond \odot \odot \diamond \odot \odot$ & 1.035576 & 6.263905 \\
\hline 0 & $\odot . ๑ \odot \odot \diamond \odot \odot$ & -1.152648 & 4.467047 \\
\hline 0 & ๑. ๑९९९९९ & $-\odot .763988$ & 2.773748 \\
\hline 0 & $\odot ., ० \odot \odot \odot \odot \odot ~$ & -1.863963 & 1.324802 \\
\hline 0 & $\odot . \odot \odot \odot \odot \odot \odot$ & -2.914360 & 2.926261 \\
\hline 0 & $\odot . \diamond \odot \odot \odot \odot \odot$ & -1.183282 & 3.140987 \\
\hline 0 & $\odot . ๑ \odot \odot \odot \odot \odot$ & -4.873723 & 4.987913 \\
\hline C & $\odot . ๑ \odot \odot \odot \odot \odot$ & -6.704218 & 4.220842 \\
\hline C & $\odot . \odot \odot \odot \odot \odot \odot$ & -6.618683 & 3.725063 \\
\hline C & 1. 448648 & -4.733884 & 4. . 096011 \\
\hline C & 1.883812 & -1.130178 & ๑. 511969 \\
\hline C & 2.404737 & -2.677693 & $\odot .900106$ \\
\hline C & 2.458114 & -7.256963 & 4.278220 \\
\hline C & 1.424745 & -7.350298 & 3.770320 \\
\hline C & ๑ . ๑๑९13० & -7.109755 & 3.395830 \\
\hline C & -0.277387 & -1.404760 & -0.517266 \\
\hline C & $\odot .503547$ & -0.489016 & $\odot .959975$ \\
\hline C & 1.590786 & -8.796012 & 3.337618 \\
\hline C & 1.873181 & -8.876968 & 2. 242947 \\
\hline C & 1.117076 & -9.345463 & 3.715469 \\
\hline C & 3.241360 & -9.300920 & 3.693545 \\
\hline C & 3.223029 & $-\odot .903274$ & -0.747169 \\
\hline C & 2.980538 & -1.107901 & -2.103538 \\
\hline C & 3.881707 & -1.795369 & -2.934473 \\
\hline C & 5.109390 & -2.182625 & -2.347287 \\
\hline C & 5.331067 & -2.123575 & -0.940941 \\
\hline C & 4.371683 & -1.461386 & -0.170707 \\
\hline C & 6.535554 & -2.822158 & $-\odot .257670$ \\
\hline C & 7.888730 & -2.174856 & -0.636820 \\
\hline C & 6.551523 & -4.318509 & $-\odot .658651$ \\
\hline C & 6.420329 & -2.760551 & 1.281870 \\
\hline C & 3. 414491 & -2.091424 & -4.388294 \\
\hline C & 4.237054 & -3.162921 & -5.141260 \\
\hline C & 1.965256 & -2.650588 & -4.329890 \\
\hline C & 3.397406 & -0.784194 & -5.216668 \\
\hline C & 6.880493 & -1.694590 & -3.840076 \\
\hline C & 3.020888 & 1.695530 & 0.509219 \\
\hline C & 4.163180 & 1.941849 & -0.263596 \\
\hline C & 2.630449 & 2.676224 & 1.430084 \\
\hline C & 3.428146 & 3.799085 & 1.728873 \\
\hline C & 4.666379 & 3.895628 & 1.045875 \\
\hline C & 4.987407 & 3.051537 & -0.050505 \\
\hline C & 6.162736 & 3.346510 & -1.019725 \\
\hline C & 6.163781 & 2.370225 & -2.217164 \\
\hline C & 7.547371 & 3. 223199 & -0.339220 \\
\hline C & 6.410238 & 4.494546 & 2.527019 \\
\hline C & $6.0 \odot 1841$ & 4.776640 & -1.593197 \\
\hline C & 2.892407 & 4.808393 & 2.790087 \\
\hline C & 2.951601 & 4.155792 & 4.193453 \\
\hline C & 3. 615958 & 6.176060 & 2.837850 \\
\hline C & 1.412570 & 5.155713 & 2.480710 \\
\hline C & -1.671938 & -1.118642 & 1.622865 \\
\hline C & -2.081814 & -2.238070 & 2.361527 \\
\hline C & -2.746151 & -2.112940 & 3.583351 \\
\hline
\end{tabular}




\begin{tabular}{|c|c|c|c|c|c|c|c|}
\hline C & -3.015888 & $-\odot .791731$ & 4.042302 & H & 3.023366 & 6.853638 & 3.463352 \\
\hline C & -2.461424 & $\odot .350643$ & 3.419997 & H & 4.612668 & 6.129923 & 3.274775 \\
\hline c & -1.837878 & 0.148034 & 2.174137 & H & 3.702654 & 6.626218 & 1.845192 \\
\hline c & -2.434976 & 1.792730 & $4.00076 \odot$ & $\mathrm{H}$ & 1. 328061 & 5.707078 & 1.537206 \\
\hline c & -2.792904 & 1.918916 & 5.500285 & H & $\odot .758951$ & 4.281583 & 2.433202 \\
\hline c & -3.366792 & 2.714814 & 3.178134 & $\mathrm{H}$ & 1. 019682 & 5.805335 & 3.270374 \\
\hline C & -0.982770 & 2.328284 & 3.882550 & H & -1.887267 & -3.222988 & 1.958160 \\
\hline c & -2.424315 & -3.249436 & 5.812630 & H & -1.468044 & 1.000240 & 1.614296 \\
\hline C & -5.157420 & $-\odot .25607 \odot$ & 4.897822 & H & -3.850754 & 1.764541 & 5.711338 \\
\hline C & -3.093177 & -3.371750 & 4.420207 & H & -2.219748 & 1.219816 & 6.115279 \\
\hline C & -2.552693 & -4.656802 & 3.754488 & H & -2.546725 & 2.936069 & 5.826843 \\
\hline C & -4.973269 & -2.666059 & -2.218821 & $\mathrm{H}$ & -3.303499 & 3.745736 & 3.546497 \\
\hline C & -3.759590 & -2.773914 & -2.946934 & H & -3.093638 & 2.719276 & 2.116589 \\
\hline C & -4.622542 & -2.207153 & -5.239806 & $\mathrm{H}$ & -4.413286 & 2. 398117 & 3.250947 \\
\hline C & -2.593143 & -2.362196 & -2.288996 & H & -0.926148 & 3.335769 & 4.308992 \\
\hline C & -2.599838 & -1.830229 & $-\odot .989547$ & H & $-\odot .282951$ & 1.685139 & 4.427774 \\
\hline c & -3.844784 & -1.560781 & -0.408749 & H & -0.641402 & 2.392985 & 2.845713 \\
\hline C & -5.056889 & -1.926453 & -1.013541 & H & -2.608786 & -4.158627 & 6.397759 \\
\hline c & -6.349545 & -4.587306 & -2.288005 & H & -1.340340 & -3.120468 & 5.713671 \\
\hline C & -4.157238 & -4.630368 & -4.725373 & H & -2.815839 & -2.397629 & 6.371676 \\
\hline c & -7.311015 & -2.546190 & 0.119767 & H & -5.202208 & 0.727046 & 4.418104 \\
\hline C & -6.401466 & -1.418070 & $-\odot .422292$ & H & -5.669249 & $-\odot .984415$ & 4.258711 \\
\hline c & -6.163306 & -0.437051 & 0.747671 & H & -5.658410 & -0.207822 & 5.867278 \\
\hline C & -2.281606 & -3.037358 & $-5.026 \odot 27$ & H & -3.039507 & -4.861429 & 2.793488 \\
\hline c & -4.617693 & -3.567094 & 4.600109 & $\mathrm{H}$ & -1.470913 & -4.615877 & 3.591913 \\
\hline C & -3.707967 & -3.176216 & -4.447968 & H & -2.761371 & -5.509745 & 4.409323 \\
\hline C & -7.165436 & -0.638723 & -1.522678 & H & -4.287359 & -1.169648 & -5.124457 \\
\hline H & 2.773778 & $-\odot .063588$ & 5.199520 & H & -4.591649 & -2.453624 & -6.307896 \\
\hline H & 2.904562 & $\odot .945963$ & 2.951149 & H & -5.659390 & -2.276203 & -4.903643 \\
\hline H & $\odot .617521$ & -4.401329 & 5.603624 & H & -1.650530 & -2.472136 & -2.806875 \\
\hline H & 2.334789 & -4.570794 & 5.051379 & H & -3.872470 & -1.054234 & $\odot .544027$ \\
\hline $\mathrm{H}$ & -1.156009 & -3.925986 & -1.475430 & H & -7.291589 & -4.900733 & -2.743281 \\
\hline H & 0.248663 & -5.940848 & -1.563133 & H & -5.547590 & -5.257159 & -2.608833 \\
\hline $\mathrm{H}$ & 4.307566 & -5.966128 & 1.101116 & H & -6.433665 & -4.636658 & -1.199400 \\
\hline H & 2.881565 & -6.423332 & 2.118776 & H & -3.915857 & -4.894140 & -5.761504 \\
\hline H & 2.054569 & -0.725229 & -2.518742 & H & -3.639840 & -5.345649 & -4.074938 \\
\hline H & 4.515113 & -1.365480 & $\odot .897435$ & H & -5.233330 & -4.755060 & -4.604882 \\
\hline $\mathrm{H}$ & 7.875272 & -1.092402 & -0.460405 & H & -7.759354 & -3.132076 & -0.683065 \\
\hline H & 8.686898 & -2.600923 & $-\odot .017412$ & H & -6.763355 & -3.220749 & $\odot .788708$ \\
\hline $\mathrm{H}$ & 8.152625 & -2.357328 & -1.678707 & H & -8.134191 & -2.106783 & 0.695354 \\
\hline H & 6.690397 & -4.446058 & -1.733375 & H & -7.130079 & $-\odot .041393$ & 1.077601 \\
\hline H & 7.368303 & -4.835817 & -0.140964 & H & -5.698420 & -0.923878 & 1.612714 \\
\hline H & 5.609501 & -4.803381 & $-\odot .377022$ & H & -5.541478 & $\odot .416228$ & $\odot .454832$ \\
\hline H & 5.482945 & -3.197380 & 1.642757 & H & -1.887635 & -2.019494 & -4.929460 \\
\hline H & 7.246540 & -3.328480 & 1.722959 & H & -1.570553 & -3.732072 & -4.562869 \\
\hline H & 6.493648 & -1.735401 & 1.663785 & H & -2.309868 & -3.270235 & -6.095394 \\
\hline H & 3.704815 & -3.419967 & -6.064514 & H & -5.139354 & -3.540780 & 3.635606 \\
\hline $\mathrm{H}$ & 5.232598 & -2.826392 & -5.429598 & $\mathrm{H}$ & -4.810658 & -4.546792 & 5.052856 \\
\hline H & 4.347801 & -4.077273 & -4.551404 & H & -5.053395 & -2.814211 & 5.256847 \\
\hline $\mathrm{H}$ & 1.631739 & -2.904972 & -5.342270 & $\mathrm{H}$ & -8.117922 & -0.271216 & -1.120416 \\
\hline $\mathrm{H}$ & 1.919781 & -3.558244 & -3.717758 & H & -6.586395 & $\odot .227671$ & -1.863152 \\
\hline $\mathrm{H}$ & 1.247141 & -1.933385 & -3.922124 & $\mathrm{H}$ & -7.382767 & -1.272953 & -2.384218 \\
\hline H & 2.754992 & $-\odot . \odot 255 \odot 5$ & -4.754821 & $\mathrm{Rh}$ & ๑. 051405 & $\odot .486496$ & -1.042878 \\
\hline $\mathrm{H}$ & 4.399250 & -0.352844 & -5.318008 & 0 & 1.176574 & 2.064145 & -2.131556 \\
\hline H & 3. 016367 & $-\odot .980355$ & -6.226143 & 0 & -2.761733 & 5.919770 & -0.225387 \\
\hline $\mathrm{H}$ & 7.327720 & $-\odot .967602$ & -3.152562 & C & 1.113219 & 3.277705 & -1.972569 \\
\hline H & 7.671993 & -2.232069 & -4.367110 & $\mathrm{~s}$ & -2.969429 & 5.118957 & -1.435822 \\
\hline $\mathrm{H}$ & 6.261743 & -1.158618 & -4.566862 & C & 1.611639 & 4.446785 & -2.803009 \\
\hline H & 4.421625 & 1.238979 & -1.041188 & C & 0.416328 & 4.175767 & $-\odot .972331$ \\
\hline H & 1.697755 & 2.547445 & 1.968746 & $\mathrm{~N}$ & -2.098051 & 3.663165 & -1.175009 \\
\hline H & 6.956073 & 2.664749 & -2.913527 & 0 & -2.647383 & 5.669994 & -2.762136 \\
\hline H & 6.364467 & 1.336648 & -1.911746 & C & -4.663043 & 4.547521 & -1.494434 \\
\hline $\mathrm{H}$ & 5.217320 & 2.387337 & -2.769325 & C & 0.620302 & 5.345138 & -1.994505 \\
\hline H & 7.730714 & 4.034020 & $\odot .365810$ & H & 2.667842 & 4.661254 & -2.601764 \\
\hline $\mathrm{H}$ & 7.650281 & 2.266469 & 0.186686 & H & 1.473315 & 4.357845 & -3.885050 \\
\hline H & 8.334725 & 3.268907 & -1.100723 & C & $-\odot .913072$ & 3.767422 & $-\odot .315858$ \\
\hline $\mathrm{H}$ & 5.821794 & 4.410582 & 3.446410 & H & 1.136183 & 4.297328 & -0.151540 \\
\hline H & 6.930626 & 3.546921 & 2.352365 & C & -1.986229 & 2.807334 & -2.360748 \\
\hline $\mathrm{H}$ & 7.141240 & 5.298083 & 2.639882 & C & -5.365343 & 4.614679 & $9.32580 \odot$ \\
\hline H & 6.002123 & 5.529428 & $-\odot .802799$ & C & -5.283415 & 4.111229 & 10.339300 \\
\hline H & 6.827399 & 4.998518 & -2.279805 & H & $-\odot .283455$ & 5.582587 & 15.269300 \\
\hline H & 5.067353 & 4.867394 & -2.161293 & H & 1.035576 & 6.263905 & -1.577326 \\
\hline $\mathrm{H}$ & 2.336138 & 3.250127 & 4.243463 & H & -1.152648 & 4.467047 & ๑.485975 \\
\hline H & 3.974794 & 3.879474 & 4.470014 & H & $-\odot .763988$ & 2.773748 & $\odot .129802$ \\
\hline H & 2.578612 & 4.854436 & 4.951607 & C & -1.863963 & 1.324802 & -2.077596 \\
\hline
\end{tabular}




$\begin{array}{rrrr}\mathrm{H} & -2.914360 & 2.926261 & -2.933689 \\ \mathrm{H} & -1.183282 & 3.140987 & -3.032668 \\ \mathrm{H} & -4.873723 & 4.987913 & -3.589584 \\ \mathrm{C} & -6.704218 & 4.220842 & -2.723310 \\ \mathrm{C} & -6.618683 & 3.725063 & -0.365258 \\ \mathrm{H} & -4.733884 & 4.096011 & 0.616384 \\ \mathrm{C} & -1.130178 & 0.511969 & -2.924800 \\ \mathrm{H} & -2.677693 & 0.900106 & -1.494995 \\ \mathrm{H} & -7.256963 & 4.278220 & -3.657157 \\ \mathrm{C} & -7.350298 & 3.770320 & -1.565393 \\ \mathrm{H} & -7.109755 & 3.395830 & 0.547004 \\ \mathrm{H} & -1.404760 & -0.517266 & -3.093341 \\ \mathrm{H} & -0.489016 & 0.959975 & -3.681494 \\ \mathrm{C} & -8.796012 & 3.337618 & -1.598636 \\ \mathrm{H} & -8.876968 & 2.242947 & -1.583629 \\ \mathrm{H} & -9.345463 & 3.715469 & -0.729538 \\ \mathrm{H} & -9.300920 & 3.693545 & -2.501396\end{array}$

19

B3LYP SCF energy:

B3LYP enthalpy:

B3LYP free energy:

M06 SCF energy in solution: -5534.58221083

a.u.

M06 enthalpy in solution:

M06 free energy in solution:

-5532.546368 a.u. a.u.

Cartesian coordinates

\begin{tabular}{|c|c|c|c|}
\hline АТОМ & X & $\mathrm{Y}$ & Z \\
\hline P & $\odot .0000 \odot \odot$ & -5.377935 & 3.307295 \\
\hline$P$ & $\odot . \odot \odot \odot \odot \odot \odot$ & -3.684158 & 4.506361 \\
\hline 0 & $\odot . ๑ \odot \odot \odot \odot \odot$ & $-\odot .59734 \odot$ & 6.097814 \\
\hline 0 & $\odot . \odot \odot \odot \odot \odot \odot$ & 0.915827 & 6.673616 \\
\hline 0 & $\odot . ๑ \odot \odot \odot \odot \odot$ & $-\odot .639729$ & 4.370490 \\
\hline 0 & $\odot . \odot \odot \odot \odot \odot \odot$ & $-\odot .520713$ & 2.835056 \\
\hline 0 & $\odot .0 \odot \odot ० \odot \odot$ & -2.473269 & 6.317509 \\
\hline 0 & $\odot . \odot \odot \odot \odot \odot \odot$ & -3.931531 & 4.917887 \\
\hline 0 & $\odot . \diamond \odot \odot \odot \odot \odot$ & -2.995679 & 4.352745 \\
\hline 0 & $\odot . ๑ \odot \odot \odot \odot \odot$ & -5.630373 & 2.615433 \\
\hline c & $\odot .0 \odot \odot \odot \odot \odot$ & -6.368814 & 3.901994 \\
\hline C & $\odot . ๑ \odot \odot \odot \odot \odot$ & -4.688935 & 5.090274 \\
\hline C & 1.590388 & -2.641243 & 4.735701 \\
\hline C & 2.204056 & -2.171499 & 6.814112 \\
\hline C & 2.776259 & -2.425862 & 6.951649 \\
\hline C & 2.701466 & -7.410879 & 3.664193 \\
\hline C & 1.681928 & -6.044569 & 4.798277 \\
\hline C & -0.268799 & -4.419813 & 5.783598 \\
\hline C & -0.746476 & -1.883295 & 7.853070 \\
\hline C & $-\odot .123972$ & -2.220264 & 6.205384 \\
\hline C & 1.004108 & -7.119936 & 5.417304 \\
\hline C & 1.494975 & -6.877568 & 6.452369 \\
\hline C & $\odot .903217$ & -7.231386 & 4.861028 \\
\hline C & 2.682101 & -8.090902 & 5.408922 \\
\hline C & 3.016657 & -0.622821 & -1.249130 \\
\hline C & 2.667280 & -0.188285 & -2.525113 \\
\hline C & 3.460332 & -0.458232 & -3.654197 \\
\hline C & 4.692628 & -1.111246 & -3.418157 \\
\hline C & 5.012398 & -1.699691 & -2.161203 \\
\hline C & 4.158432 & -1.419529 & -1.091581 \\
\hline C & 6.200705 & -2.679133 & -1.977237 \\
\hline C & 7.573473 & -1.988715 & -2.156961 \\
\hline C & 6.069065 & -3.835154 & $-3.00 \odot \odot 41$ \\
\hline C & 6.192562 & -3.312846 & -0.568103 \\
\hline C & 2.875176 & $-\odot .053073$ & -5.036927 \\
\hline C & 3.586442 & -0.661395 & -6.267828 \\
\hline C & 1.411621 & $-\odot .569857$ & -5.110340 \\
\hline C & 2.858163 & 1.488680 & -5.170802 \\
\hline C & 6.391938 & -0.059335 & -4.680376 \\
\hline C & 3.060381 & 1.197030 & 1. 001831 \\
\hline C & 4.153795 & 1.737999 & 0.314794 \\
\hline C & 2.752426 & 1. 718879 & 2.263424 \\
\hline C & 3.585815 & 2.638781 & 2.927917 \\
\hline
\end{tabular}

\begin{tabular}{|c|c|c|c|}
\hline C & 4.771410 & 3.022500 & 2.252689 \\
\hline c & 5.006380 & 2.683985 & 0.893378 \\
\hline c & 6.106162 & 3.367526 & 0.038563 \\
\hline C & 6.026920 & 2.926175 & -1.439828 \\
\hline C & 7.540081 & 3.047209 & $\odot .524648$ \\
\hline C & 6.625505 & 3. 025775 & 3.719259 \\
\hline C & 5.885984 & 4.900791 & 0.073775 \\
\hline C & 3.138597 & 3.129511 & 4.337584 \\
\hline c & 3.291942 & 1.974567 & 5.357734 \\
\hline C & 3.872844 & 4.381125 & 4.876035 \\
\hline C & 1.642621 & 3.536209 & 4.290474 \\
\hline C & -1.673915 & -1.774759 & 1.462806 \\
\hline C & -2.045422 & -3.117133 & 1.620225 \\
\hline C & -2.605228 & -3.591181 & 2.810140 \\
\hline C & -2.803652 & -2.645692 & 3.855900 \\
\hline C & -2.282611 & -1.332006 & 3.795795 \\
\hline C & -1.768150 & -0.917323 & 2.555126 \\
\hline C & -2.187637 & -0.324740 & 4.976824 \\
\hline C & -2.349830 & -0.942811 & 6.385446 \\
\hline C & -3.221053 & 0.812521 & 4.791606 \\
\hline C & -0.766746 & 0.303288 & 4.967607 \\
\hline C & -2.095331 & -5.631798 & 4.200924 \\
\hline C & -4.853301 & -2.633084 & 5.039203 \\
\hline C & -2.902668 & -5.103312 & 2.988526 \\
\hline c & -2.464718 & -5.914413 & 1.748713 \\
\hline C & -4.953379 & $-\odot .939073$ & -2.756344 \\
\hline C & -3.781793 & $-\odot .287976$ & -3.226487 \\
\hline C & -4.915688 & 1.541616 & -4.555163 \\
\hline C & -2.663202 & -0.324482 & -2.383398 \\
\hline C & -2.697301 & $-\odot .938271$ & -1.124605 \\
\hline C & -3.926753 & -1.379907 & -0.631002 \\
\hline C & -5.094137 & -1.353153 & -1.407868 \\
\hline C & -5.988429 & -2.377447 & -4.321226 \\
\hline C & -3.810069 & $-\odot .337306$ & -5.819687 \\
\hline C & -7.155850 & -2.931928 & -1.271117 \\
\hline C & -6.470022 & -1.647796 & -0.747765 \\
\hline C & -6.328626 & -1.807579 & $\odot .783220$ \\
\hline C & -2.435060 & 1.360948 & -4.639595 \\
\hline C & -4.405597 & -5.399321 & 3.207733 \\
\hline C & -3.738274 & 0.535246 & -4.543996 \\
\hline C & -7.408889 & -0.440337 & $-\odot .994875$ \\
\hline $\mathrm{H}$ & 3.273745 & -2.433314 & 4.449480 \\
\hline $\mathrm{H}$ & 3.181314 & $-\odot .537316$ & 2.866145 \\
\hline H & $\odot .920738$ & -6.502870 & 3.235247 \\
\hline H & 2.541476 & -6.439371 & 2.428015 \\
\hline $\mathrm{H}$ & -1.642925 & -2.575226 & -2.636144 \\
\hline H & -0.519412 & -4.399490 & -3.876806 \\
\hline $\mathrm{H}$ & 3.700020 & -6.030933 & -2.139193 \\
\hline H & 2.333802 & -6.844992 & -1.274466 \\
\hline $\mathrm{H}$ & 1.745043 & 0.371254 & -2.635606 \\
\hline $\mathrm{H}$ & 4.379849 & -1.819270 & -0.110877 \\
\hline $\mathrm{H}$ & 7.658387 & -1.100104 & -1.519625 \\
\hline $\mathrm{H}$ & 8.374170 & -2.680230 & -1.868613 \\
\hline $\mathrm{H}$ & 7.752275 & -1.696716 & -3.192071 \\
\hline $\mathrm{H}$ & 6.115413 & -3.471066 & -4.027736 \\
\hline H & 6.879433 & -4.559440 & -2.852885 \\
\hline H & 5.116110 & -4.359959 & -2.864927 \\
\hline $\mathrm{H}$ & 5.250610 & -3.829839 & $-\odot .355241$ \\
\hline $\mathrm{H}$ & 7.000330 & -4.050101 & -0.505510 \\
\hline $\mathrm{H}$ & 6.368435 & -2.573318 & 0.222026 \\
\hline H & 2.973262 & -0.458300 & -7.153769 \\
\hline $\mathrm{H}$ & 4.571774 & $-\odot .235627$ & -6.457573 \\
\hline $\mathrm{H}$ & 3.698301 & -1.745401 & -6.177897 \\
\hline H & $\odot .983107$ & $-\odot .317443$ & -6.087130 \\
\hline $\mathrm{H}$ & 1.374685 & -1.658570 & -4.993259 \\
\hline $\mathrm{H}$ & $\odot .765731$ & -0.129541 & -4.345933 \\
\hline $\mathrm{H}$ & 2.286965 & 1.955468 & -4.360178 \\
\hline $\mathrm{H}$ & 3.870489 & 1.907934 & -5.149453 \\
\hline $\mathrm{H}$ & 2.396472 & 1.781564 & -6.121634 \\
\hline $\mathrm{H}$ & 6.948422 & ๑.243986 & -3.786000 \\
\hline $\mathrm{H}$ & 7.094604 & -0.313966 & -5.477176 \\
\hline $\mathrm{H}$ & 5.764475 & 0.777300 & -5.005223 \\
\hline $\mathrm{H}$ & 4.344314 & 1. $4 \odot 4 \odot 98$ & -0.694264 \\
\hline H & 1.854623 & 1.370313 & 2.762265 \\
\hline H & 6.768976 & 3. 487494 & -2.017653 \\
\hline
\end{tabular}




\begin{tabular}{|c|c|c|c|}
\hline $\mathrm{H}$ & 6.249936 & 1.860585 & -1.567979 \\
\hline $\mathrm{H}$ & 5.044594 & 3.125947 & -1.882491 \\
\hline $\mathrm{H}$ & 7.768446 & 3.531922 & 1.473805 \\
\hline $\mathrm{H}$ & 7.692871 & 1.966817 & 0.632490 \\
\hline $\mathrm{H}$ & 8.266316 & 3.411986 & -0.211289 \\
\hline $\mathrm{H}$ & 6.107191 & 2.568863 & 4.568762 \\
\hline $\mathrm{H}$ & 7.123350 & 2.237709 & 3.144510 \\
\hline $\mathrm{H}$ & 7.370108 & 3.733463 & 4.090577 \\
\hline $\mathrm{H}$ & 5.947459 & 5.290945 & 1.091700 \\
\hline $\mathrm{H}$ & 6.648382 & 5.402998 & -0.533759 \\
\hline $\mathrm{H}$ & 4.904385 & 5.162829 & $-\odot .341568$ \\
\hline H & 2.681048 & 1.108257 & 5.079643 \\
\hline $\mathrm{H}$ & 4.331472 & 1.638307 & 5.434947 \\
\hline $\mathrm{H}$ & 2.973001 & 2.302961 & 6.354161 \\
\hline $\mathrm{H}$ & 3. 335691 & 4.738395 & 5.762595 \\
\hline $\mathrm{H}$ & 4.899755 & 4.187513 & 5.183107 \\
\hline $\mathrm{H}$ & 3.888127 & 5.191932 & 4.142271 \\
\hline $\mathrm{H}$ & 1.498487 & 4.400008 & 3.630558 \\
\hline $\mathrm{H}$ & ๑. 982536 & 2.731401 & 3.958609 \\
\hline $\mathrm{H}$ & 1. 313371 & 3.829947 & 5.293388 \\
\hline $\mathrm{H}$ & -1.900890 & -3.795119 & 0.789423 \\
\hline $\mathrm{H}$ & -1.428444 & 0.103494 & 2.426421 \\
\hline $\mathrm{H}$ & -3.368451 & -1.257546 & 6.609370 \\
\hline $\mathrm{H}$ & -1.692826 & -1.806079 & 6.526471 \\
\hline $\mathrm{H}$ & -2.075194 & -0.185349 & 7.129275 \\
\hline $\mathrm{H}$ & -3.098133 & 1.566277 & 5.579723 \\
\hline $\mathrm{H}$ & -3.107065 & 1.305089 & 3.819884 \\
\hline $\mathrm{H}$ & -4.249146 & $\odot .439401$ & 4.850032 \\
\hline $\mathrm{H}$ & -0.658118 & ๑. 977361 & 5.824851 \\
\hline $\mathrm{H}$ & 0.005767 & -0.470544 & 5.041547 \\
\hline $\mathrm{H}$ & -0.570072 & ๑. 890059 & 4.065803 \\
\hline $\mathrm{H}$ & -2.249536 & -6.712196 & 4.312528 \\
\hline $\mathrm{H}$ & -1.023021 & -5.452207 & 4.060544 \\
\hline $\mathrm{H}$ & -2.401807 & -5.144414 & 5.128428 \\
\hline $\mathrm{H}$ & -4.923758 & -1.547543 & 5.162305 \\
\hline $\mathrm{H}$ & -5.407980 & -2.922934 & 4.139730 \\
\hline $\mathrm{H}$ & -5.291946 & -3.122965 & 5.911724 \\
\hline H & -3.043556 & -5.649590 & $\odot .855832$ \\
\hline $\mathrm{H}$ & -1.401645 & -5.784739 & 1.522300 \\
\hline $\mathrm{H}$ & -2.638443 & -6.978705 & 1.941895 \\
\hline $\mathrm{H}$ & -4.849586 & 2.227617 & -3.703869 \\
\hline H & -4.881898 & 2.139852 & -5.474361 \\
\hline $\mathrm{H}$ & -5.878514 & 1.029199 & -4.514785 \\
\hline $\mathrm{H}$ & -1.733048 & $\odot .125514$ & -2.706655 \\
\hline $\mathrm{H}$ & -3.974209 & -1.750558 & ๑. 382068 \\
\hline $\mathrm{H}$ & -6.871921 & -2.388982 & -4.963820 \\
\hline $\mathrm{H}$ & -5.088923 & -2.475977 & -4.935109 \\
\hline $\mathrm{H}$ & -6.030983 & -3.220048 & -3.625925 \\
\hline $\mathrm{H}$ & -3.567169 & ๑. 274741 & -6.696525 \\
\hline $\mathrm{H}$ & -3.090483 & -1.163873 & -5.784248 \\
\hline $\mathrm{H}$ & -4.808352 & $-\odot .745954$ & -5.981341 \\
\hline $\mathrm{H}$ & -7.543719 & -2.805975 & -2.282684 \\
\hline $\mathrm{H}$ & -6.471642 & -3.788529 & -1.257451 \\
\hline $\mathrm{H}$ & -8.008336 & -3.179889 & -0.627690 \\
\hline $\mathrm{H}$ & -7.326905 & -1.912544 & 1. 221872 \\
\hline $\mathrm{H}$ & -5.756630 & -2.701727 & 1.057606 \\
\hline $\mathrm{H}$ & -5.850125 & -0.938048 & 1.246994 \\
\hline $\mathrm{H}$ & -2.287859 & 2.001055 & -3.763009 \\
\hline $\mathrm{H}$ & -1.547909 & ๑. 728538 & -4.758233 \\
\hline $\mathrm{H}$ & -2.490027 & 2.007796 & -5.522121 \\
\hline $\mathrm{H}$ & -5.020452 & -4.944158 & 2.421666 \\
\hline $\mathrm{H}$ & -4.574640 & -6.482165 & 3.172266 \\
\hline $\mathrm{H}$ & -4.757020 & -5.046423 & 4.177076 \\
\hline $\mathrm{H}$ & -8.379687 & -0.617618 & -0.516109 \\
\hline $\mathrm{H}$ & -6.983980 & ๑. 475108 & -0.566113 \\
\hline $\mathrm{H}$ & -7.576175 & -0.277408 & -2.061444 \\
\hline $\mathrm{Rh}$ & ๑. 024872 & ๑. 833862 & -0.309169 \\
\hline 0 & $1.0 \odot 4738$ & 2.688880 & -1.088209 \\
\hline 0 & -1.748940 & 2.282630 & -0.711524 \\
\hline C & ๑. 926886 & 3.831025 & -0.672450 \\
\hline S & -2.778227 & 2.854373 & ๑. 198140 \\
\hline C & 1.186593 & 5.181091 & -1.324123 \\
\hline C & $\odot .484233$ & 4.467517 & ๑. 641459 \\
\hline $\mathrm{N}$ & -2.029901 & 4. 199021 & $\odot .927398$ \\
\hline 0 & -3.413016 & 1.972390 & 1.185753 \\
\hline
\end{tabular}

$\begin{array}{lrrr}\mathrm{C} & -4.041873 & 3.618987 & -0.801512 \\ \mathrm{C} & 0.427364 & 5.827656 & -0.123295 \\ \mathrm{H} & 2.256281 & 5.420297 & -1.355890 \\ \mathrm{H} & 0.772579 & 5.307403 & -2.329538 \\ \mathrm{C} & -0.676306 & 3.914077 & 1.471011 \\ \mathrm{H} & 1.375485 & 4.443268 & 1.286955 \\ \mathrm{C} & -2.929885 & 4.904565 & 1.887305 \\ \mathrm{C} & -5.377935 & 3.307295 & 9.585200 \\ \mathrm{C} & -3.684158 & 4.506361 & 12.092700 \\ \mathrm{H} & -0.597340 & 6.097814 & 13.875400 \\ \mathrm{H} & 0.915827 & 6.673616 & 0.362935 \\ \mathrm{H} & -0.639729 & 4.370490 & 2.462237 \\ \mathrm{H} & -0.520713 & 2.835056 & 1.609412 \\ \mathrm{C} & -2.473269 & 6.317509 & 2.118793 \\ \mathrm{H} & -3.931531 & 4.917887 & 1.444151 \\ \mathrm{H} & -2.995679 & 4.352745 & 2.834545 \\ \mathrm{H} & -5.630373 & 2.615433 & 0.249744 \\ \mathrm{C} & -6.368814 & 3.901994 & -1.327356 \\ \mathrm{C} & -4.688935 & 5.090274 & -2.586397 \\ \mathrm{H} & -2.641243 & 4.735701 & -2.012191 \\ \mathrm{C} & -2.171499 & 6.814112 & 3.319286 \\ \mathrm{H} & -2.425862 & 6.951649 & 1.234698 \\ \mathrm{H} & -7.410879 & 3.664193 & -1.132755 \\ \mathrm{C} & -6.044569 & 4.798277 & -2.354984 \\ \mathrm{H} & -4.419813 & 5.783598 & -3.378867 \\ \mathrm{H} & -1.883295 & 7.853070 & 3.450866 \\ \mathrm{H} & -2.220264 & 6.205384 & 4.219951 \\ \mathrm{C} & -7.119936 & 5.417304 & -3.213175 \\ \mathrm{H} & -6.877568 & 6.452369 & -3.475399 \\ \mathrm{H} & -7.231386 & 4.861028 & -4.152899 \\ \mathrm{H} & -8.090902 & 5.408922 & -2.709368 \\ & & & \end{array}$

20

B3LYP SCF energy:

B3LYP enthalpy:

B3LYP free energy:

M06 SCF energy in solution: a.u.

M06 enthalpy in solution:

M06 free energy in solution:

a.u.

Cartesian coordinates

\begin{tabular}{|c|c|c|c|}
\hline ATOM & X & Y & Z \\
\hline & $\odot .00 \odot \odot \odot \odot$ & 3.559912 & 6.720343 \\
\hline$P$ & $\odot . ๑ \odot \odot \odot \odot \odot$ & 1.736403 & 6.083038 \\
\hline 0 & $\odot .0 \odot \odot \odot \odot \odot$ & 3.600051 & 5.710186 \\
\hline 0 & $\odot . ๑ \odot \odot \odot \odot \odot$ & 6.004715 & 3.174610 \\
\hline 0 & $\odot . \odot \odot \odot \odot \odot \odot$ & 7.015282 & 2.572353 \\
\hline 0 & $\odot . ๑ \odot \odot \odot \odot \odot$ & 2.179201 & 7.393013 \\
\hline 0 & $\odot .0 \odot \odot \odot \odot \odot$ & 4.443615 & 7.297643 \\
\hline 0 & $\odot .0 \odot \odot \odot \odot \odot$ & 3.666543 & 6.384982 \\
\hline 0 & $\odot . \odot \odot \odot \odot \odot \odot$ & $\odot .658933$ & 5.603066 \\
\hline 0 & $\odot . ๑ \odot \odot \odot \odot \odot$ & 5.143294 & 3.179030 \\
\hline c & $\odot .0 \odot \odot \odot \odot \odot$ & 7.210738 & 3.738383 \\
\hline c & $\odot .0 \odot \odot \odot \odot \odot$ & 8.217039 & 3.142815 \\
\hline c & -2.917121 & 6.925711 & 2.109962 \\
\hline c & -2.943517 & 1.588789 & 7.720072 \\
\hline c & -2.622717 & 2.221376 & 8.214439 \\
\hline c & -2.269863 & 7.285813 & 4.190237 \\
\hline c & -3.735205 & 8.335843 & 3.730889 \\
\hline c & -1.486941 & 9.075723 & 3.127323 \\
\hline c & -1.613267 & 9.643276 & 4.326348 \\
\hline c & -2.840154 & 10.131154 & 3.673404 \\
\hline c & -3.923833 & 9.491171 & 5.297772 \\
\hline C & -3.809743 & 10.339410 & 4.464899 \\
\hline c & -2.615184 & 1.241043 & 2. 014613 \\
\hline c & -5.898632 & 1.920444 & 1. 441381 \\
\hline C & -3.294357 & $\odot .633361$ & $\odot .600090$ \\
\hline c & -3.257643 & 0.647744 & 1.991328 \\
\hline c & -4.418537 & 0.788807 & 2.773173 \\
\hline c & -5.626399 & 1.021132 & 2.073961 \\
\hline c & -5.729017 & 0.862432 & 0.662296 \\
\hline
\end{tabular}
-5533.319124 a.u. -5533.595761 a.u. $-5532.815462$

7

-5535.35473394 a.u. $-5534.57443511$ -5532.538825 a.u.

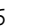

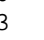

3

2




\begin{tabular}{|c|c|c|c|c|c|c|c|}
\hline C & -4.539079 & 0.681060 & $-\odot .044558$ & $\mathrm{H}$ & -6.199415 & 1.307916 & 5.159163 \\
\hline C & -7.091671 & 0.805214 & -0.076258 & $\mathrm{H}$ & -6.178862 & $-\odot .387180$ & 4.663527 \\
\hline c & -7.855188 & 2.149595 & $-\odot .020516$ & H & -3.302410 & $-\odot .776958$ & 5.671070 \\
\hline C & -7.963258 & -0.309585 & 0.553464 & $\mathrm{H}$ & -3.886778 & -1.529739 & 4.175385 \\
\hline C & -6.908394 & $\odot .448706$ & -1.568199 & H & -2.398971 & $-\odot .574431$ & 4.170667 \\
\hline C & -4.260243 & 0.632313 & 4.313025 & $\mathrm{H}$ & -2.535142 & 1.993036 & 4.431961 \\
\hline C & -5.576264 & $\odot .416116$ & 5.096613 & H & -4.083247 & 2.784904 & 4.750161 \\
\hline c & -3.407140 & -0.636149 & 4.589280 & $\mathrm{H}$ & -3.363753 & 1.729496 & 5.975566 \\
\hline C & -3.518322 & 1.858066 & 4.897577 & H & -6.755111 & 3.405129 & 2.274647 \\
\hline C & -6.841533 & 2.744478 & 3.144637 & $\mathrm{H}$ & -7.818879 & 2.882680 & 3.612399 \\
\hline C & -1.679569 & 2.173444 & -1.275157 & H & -6.057082 & 3.002816 & 3.863316 \\
\hline C & -2.523576 & 3.222631 & $-\odot .889742$ & $\mathrm{H}$ & -3.213851 & 3.060854 & -0.075132 \\
\hline C & -0.711020 & 2.426233 & -2.253463 & H & ๑. $00799 \odot$ & 1.648390 & -2.486810 \\
\hline C & -0.651242 & 3.639945 & -2.959895 & $\mathrm{H}$ & -4.569522 & 6.136773 & 0.740593 \\
\hline C & -1.646425 & 4.601805 & -2.652944 & H & -4.788177 & 4.463528 & $\odot .221627$ \\
\hline C & -2.507889 & 4.464679 & -1.532615 & H & -3.324781 & 4.940724 & 1.113413 \\
\hline C & -3.353322 & 5.640814 & $-\odot .977901$ & H & -4.065402 & 6.579223 & -2.837683 \\
\hline C & -4.043907 & 5.258825 & $\odot .350349$ & H & -5.085535 & 5.236837 & -2.272003 \\
\hline C & -4.469006 & 6.086175 & -1.953162 & $\mathrm{H}$ & -5.128694 & 6.804290 & -1.451835 \\
\hline C & -2.519677 & 5.553935 & -4.632261 & H & -2.000587 & 4.885550 & -5.327388 \\
\hline C & -2.416613 & 6.839036 & -0.678877 & $\mathrm{H}$ & -3.516500 & 5.147622 & -4.428678 \\
\hline C & $\odot .501156$ & 3.816054 & -3.992593 & H & -2.614744 & 6.542394 & -5.087303 \\
\hline C & 0.232370 & 2.925963 & -5.230421 & $\mathrm{H}$ & -1.931294 & 7.199488 & -1.588116 \\
\hline C & 0.756067 & 5.269083 & -4.459868 & H & -2.999513 & 7.664721 & $-\odot .252296$ \\
\hline C & 1.836790 & 3.359992 & -3.346058 & $\mathrm{H}$ & -1.640171 & 6.560238 & ๑. 041939 \\
\hline C & 0.710958 & -2.898725 & -1.072050 & H & 0.155023 & 1.867809 & -4.956218 \\
\hline C & ๑. 391191 & -4.235406 & -1.341849 & $\mathrm{H}$ & -0.695816 & 3.207266 & -5.739601 \\
\hline C & $\odot .973442$ & -4.925284 & -2.407890 & H & 1.051051 & 3.024709 & -5.953349 \\
\hline C & 1.933195 & -4.221413 & -3.189094 & $\mathrm{H}$ & 1.699957 & 5.288855 & -5.017432 \\
\hline C & 2.147515 & -2.828001 & -3.054482 & H & $-\odot .015174$ & 5.656462 & -5.124656 \\
\hline C & 1.551368 & -2.207384 & -1.943346 & $\mathrm{H}$ & ๑.848815 & 5.956910 & -3.614705 \\
\hline C & 2.933936 & -1.916636 & -4.039345 & H & 2. 076917 & 3.971515 & -2.469335 \\
\hline C & 3.240550 & -2.542538 & -5.420185 & $\mathrm{H}$ & 1.838012 & 2.311321 & -3.039167 \\
\hline C & 4.255024 & -1.440530 & -3.388122 & $\mathrm{H}$ & 2.648186 & 3.479370 & -4.073160 \\
\hline C & 2.059945 & -0.668284 & -4.340237 & H & -0.319366 & -4.739242 & -0.700146 \\
\hline C & -0.039232 & -6.369801 & -4.206878 & $\mathrm{H}$ & 1.749024 & -1.158949 & -1.745830 \\
\hline C & 3.972514 & -5.274405 & -3.755980 & $\mathrm{H}$ & 4.007954 & -3.315679 & -5.388219 \\
\hline C & 0.507756 & -6.362665 & -2.756975 & $\mathrm{H}$ & 2.346783 & -2.972449 & -5.881263 \\
\hline C & $-\odot .635542$ & -6.825861 & -1.826832 & $\mathrm{H}$ & 3.611910 & -1.749800 & -6.080216 \\
\hline c & 3.254539 & -2.844272 & 3.716318 & $\mathrm{H}$ & 4.794140 & $-\odot .778999$ & -4.077446 \\
\hline C & 2.130410 & -2.037684 & 4.032259 & $\mathrm{H}$ & 4.078742 & $-\odot .883931$ & -2.461530 \\
\hline C & 3.286317 & -0.366579 & 5.512112 & $\mathrm{H}$ & 4.916686 & -2.282024 & -3.153869 \\
\hline C & 1.175732 & -1.868889 & 3.019996 & $\mathrm{H}$ & 2.576589 & $-\odot .02506 \odot$ & -5.061231 \\
\hline C & 1.347712 & -2.418505 & 1.735872 & $\mathrm{H}$ & 1.095161 & -0.958476 & -4.772025 \\
\hline C & 2.568724 & -3.029530 & 1.423473 & $\mathrm{H}$ & 1.866365 & $-\odot .064597$ & -3.449661 \\
\hline C & 3.568255 & -3.221012 & 2.383136 & $\mathrm{H}$ & -0.412836 & -7.369213 & -4.461344 \\
\hline C & 3.787081 & -4.484956 & 5.328157 & $\mathrm{H}$ & -0.867464 & -5.658799 & -4.314859 \\
\hline C & 1.861660 & -2.133565 & 6.616197 & $\mathrm{H}$ & 0.735231 & -6.100673 & -4.927832 \\
\hline C & 5.425977 & -5.036662 & 2.525541 & $\mathrm{H}$ & 4.587192 & -4.379237 & -3.618484 \\
\hline C & 4.988845 & -3.671378 & 1.945349 & $\mathrm{H}$ & 3.988898 & -5.860887 & -2.830303 \\
\hline C & 5.086421 & -3.784388 & 0.407183 & $\mathrm{H}$ & 4.381785 & -5.872249 & -4.573415 \\
\hline C & $\odot .800036$ & -0.290409 & 5.340914 & $\mathrm{H}$ & -0.308680 & -6.920761 & -0.784454 \\
\hline C & 1.640586 & -7.409107 & -2.630119 & $\mathrm{H}$ & -1.496660 & -6.149915 & -1.857000 \\
\hline C & 2. 015839 & -1.242508 & 5.361270 & $\mathrm{H}$ & -0.976182 & -7.815679 & -2.149429 \\
\hline c & 5.997146 & -2.581057 & 2.391830 & $\mathrm{H}$ & 3.409554 & 0.303054 & 4.653020 \\
\hline H & -2.650213 & $-\odot .855587$ & -5.131187 & $\mathrm{H}$ & 3.208456 & 0.247920 & 6.417360 \\
\hline H & -2.046972 & $\odot .714578$ & -3.317115 & $\mathrm{H}$ & 4.183944 & $-\odot .982817$ & 5.594455 \\
\hline H & -3.301481 & -5.505627 & -3.938725 & $\mathrm{H}$ & $\odot .268786$ & -1.312877 & 3.225539 \\
\hline $\mathrm{H}$ & -4.832820 & -4.590273 & -3.627658 & $\mathrm{H}$ & 2.740224 & -3.347818 & 0.405625 \\
\hline H & $-\odot .738552$ & -3.743940 & 2.686975 & $\mathrm{H}$ & 4.547225 & -4.663932 & 6.091679 \\
\hline $\mathrm{H}$ & -2.927982 & -4.698115 & 3.406952 & $\mathrm{H}$ & 2.797803 & -4.464412 & 5.793930 \\
\hline H & -6.823058 & -3.449904 & $\odot .865231$ & $\mathrm{H}$ & 3.812919 & -5.290267 & 4.589277 \\
\hline $\mathrm{H}$ & -6.098467 & -4.897388 & 0.058295 & $\mathrm{H}$ & 1.608054 & -1.506914 & 7.479205 \\
\hline H & -2.295418 & 0.527564 & 2.473857 & $\mathrm{H}$ & 1.056848 & -2.868475 & 6.494706 \\
\hline $\mathrm{H}$ & -4.568522 & 0.583950 & -1.121440 & $\mathrm{H}$ & 2.786031 & -2.657341 & 6.860068 \\
\hline H & -7.225565 & 2.980196 & $-\odot .362069$ & $\mathrm{H}$ & 5.637634 & -4.979200 & 3.593730 \\
\hline $\mathrm{H}$ & -8.728742 & 2.103712 & $-\odot .681586$ & $\mathrm{H}$ & 4.669321 & -5.811581 & 2.353157 \\
\hline H & -8.217322 & 2.373340 & $\odot .983032$ & $\mathrm{H}$ & 6.348723 & -5.362293 & 2.031364 \\
\hline $\mathrm{H}$ & -8.156415 & -0.121824 & 1.611130 & $\mathrm{H}$ & 6.120194 & -4.024941 & 0.137301 \\
\hline $\mathrm{H}$ & -8.925939 & $-\odot .371510$ & ๑. 031898 & $\mathrm{H}$ & 4.451002 & -4.583535 & $\odot .006281$ \\
\hline H & -7.464292 & -1.280749 & 0.454814 & $\mathrm{H}$ & 4.824725 & -2.846688 & -0.094484 \\
\hline H & -6.382999 & $-\odot .503482$ & -1.701726 & $\mathrm{H}$ & 0.839739 & $\odot .410886$ & 4.499632 \\
\hline H & -7.894838 & $\odot .350569$ & -2.033815 & $\mathrm{H}$ & -0.153922 & $-\odot .829169$ & 5.300328 \\
\hline H & -6.365929 & 1.225793 & -2.119450 & $\mathrm{H}$ & ๑.798572 & $\odot .303578$ & 6.260739 \\
\hline H & -5.321389 & $\odot .129253$ & 6.123531 & $\mathrm{H}$ & 2.128022 & -7.351234 & -1.649493 \\
\hline
\end{tabular}




\begin{tabular}{|c|c|c|c|}
\hline $\mathrm{H}$ & 1.221020 & -8.417030 & -2.731079 \\
\hline H & 2. 396265 & -7.291142 & -3.406529 \\
\hline $\mathrm{H}$ & $7.0 \odot 3479$ & -2.843696 & 2.043337 \\
\hline H & 5.732926 & -1.604556 & 1.970786 \\
\hline $\mathrm{H}$ & 6.026410 & -2.489844 & 3.479794 \\
\hline $\mathrm{Rh}$ & $\odot .131836$ & 0.170702 & $\odot .748474$ \\
\hline C & 1.893942 & 2.717855 & $\odot .304008$ \\
\hline C & $-\odot .035689$ & 2.238823 & 1.925433 \\
\hline $\mathrm{N}$ & 3.293857 & 3.121898 & $\odot .556854$ \\
\hline $\mathrm{H}$ & 1.333228 & 3.620135 & ๑. 051388 \\
\hline $\mathrm{H}$ & 1.909114 & 2.069251 & -0.573028 \\
\hline H & -0.359292 & 1.885759 & 2.899902 \\
\hline H & -0.684377 & 2.949732 & 1.423150 \\
\hline C & 3.512604 & 4.116828 & 1.624592 \\
\hline S & 4.392865 & 1.813986 & $\odot .565333$ \\
\hline $\mathrm{H}$ & 2.947942 & 3.857275 & 2.531106 \\
\hline H & 4.572346 & 4.094065 & 1.895932 \\
\hline C & 3.183820 & 5.544225 & 1.175621 \\
\hline 0 & 4.587521 & 1.288423 & 1. 928732 \\
\hline 0 & 3.926611 & $\odot .903926$ & -0.492553 \\
\hline C & 5.916276 & 2.598739 & ๑. 055233 \\
\hline C & 3. 559912 & 6.720343 & 6.109000 \\
\hline C & 1.736403 & 6.083038 & 7.903500 \\
\hline $\mathrm{H}$ & 3.600051 & 5.710186 & 8.903100 \\
\hline C & $6.0 \odot 4715$ & 3.174610 & -1.216994 \\
\hline C & 7.015282 & 2.572353 & 0.913426 \\
\hline C & 2.179201 & 7.393013 & 1.878700 \\
\hline $\mathrm{H}$ & 4.443615 & 7.297643 & 1.858276 \\
\hline $\mathrm{H}$ & 3.666543 & 6.384982 & 3.173111 \\
\hline 0 & ๑. 658933 & 5.603066 & ๑. 962479 \\
\hline $\mathrm{H}$ & 5.143294 & 3.179030 & -1.876831 \\
\hline C & 7.210738 & 3.738383 & -1.618464 \\
\hline C & 8.217039 & 3.142815 & ○. 490621 \\
\hline H & 6.925711 & 2.109962 & 1.890516 \\
\hline $\mathrm{H}$ & 1.588789 & 7.720072 & 2.739925 \\
\hline H & 2.221376 & 8.214439 & 1.153615 \\
\hline $\mathrm{H}$ & 7.285813 & 4.190237 & -2.604420 \\
\hline C & 8.335843 & 3.730889 & -0.774915 \\
\hline $\mathrm{H}$ & 9.075723 & 3.127323 & 1.156476 \\
\hline C & 9.643276 & 4.326348 & -1.238123 \\
\hline $\mathrm{H}$ & 10.131154 & 3.673404 & -1.973175 \\
\hline H & 9.491171 & 5.297772 & -1.721493 \\
\hline $\mathrm{H}$ & 10.339410 & 4.464899 & $-\odot .405814$ \\
\hline C & 1.241043 & 2.014613 & 1. 482553 \\
\hline $\mathrm{H}$ & 1.920444 & 1.441381 & 2.121386 \\
\hline
\end{tabular}

21

B3LYP SCF energy: B3LYP enthalpy:

B3LYP free energy:

M06 SCF energy in solution: $\quad-5534.56315851$

a.u.

M06 enthalpy in solution:

M06 free energy in solution:

a.u.

Cartesian coordinates

\begin{tabular}{|c|c|c|c|}
\hline АТОM & $X$ & $Y$ & Z \\
\hline $\mathrm{P}$ & $\odot . \diamond \odot \odot \odot \odot \diamond$ & 5.616352 & 4. 301811 \\
\hline$P$ & $\odot . \diamond \odot \odot \odot \odot \diamond$ & 7.339636 & 2.595901 \\
\hline 0 & 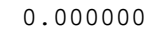 & 2.310787 & 4.677160 \\
\hline 0 & $\odot . \diamond \odot \odot \odot \odot \odot$ & 2.138399 & 5.623326 \\
\hline 0 & 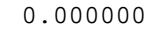 & 2.537472 & 1.320952 \\
\hline 0 & $\odot . \diamond \odot \odot \odot \odot \diamond$ & 2.825350 & 2.197263 \\
\hline 0 & $\odot .0 \odot \odot \odot \odot \odot$ & 5.328491 & 1.363977 \\
\hline 0 & 0.000000 & 6.239112 & 2.808920 \\
\hline 0 & ๑. . $૦ \odot \odot ० ० \odot$ & 5.011055 & 3.475942 \\
\hline 0 & $\odot .0 \odot \odot \odot \odot \odot$ & 4.581963 & 4.607191 \\
\hline C & ०. . 000000 & 6.561377 & 5.186919 \\
\hline C & ๑. . 000000 & 8.273448 & 3.499655 \\
\hline C & -2.494160 & 7.625277 & 1.584161 \\
\hline C & -2.562231 & 5.143815 & 1.379230 \\
\hline C & -2.474851 & 5.558924 & $\odot .434678$ \\
\hline
\end{tabular}

\begin{tabular}{|c|c|c|c|}
\hline C & -2.321322 & 6.255947 & 6.193213 \\
\hline C & -2.956427 & 7.904379 & 4.803213 \\
\hline C & -1.219264 & 9.305091 & 3.182566 \\
\hline C & -1.279571 & 5.231399 & 0.477968 \\
\hline C & -2.397447 & 4.921499 & 2.299377 \\
\hline C & -3.448062 & 8.917860 & 5.783290 \\
\hline C & -3.401184 & 9.877285 & 5.298928 \\
\hline C & -2.316569 & 8.567775 & 6.245719 \\
\hline C & -5.257916 & $9 . \odot 97946$ & 6.595515 \\
\hline C & -3.533044 & ๑. 252409 & 0.623343 \\
\hline C & -3.457621 & ๑. 257816 & 2.014246 \\
\hline C & -4.591645 & $\odot .102845$ & 2.828969 \\
\hline C & -5.841317 & $\odot .045755$ & 2.167094 \\
\hline C & -5.947317 & -0.108299 & 0.755732 \\
\hline C & -4.770904 & ๑. 014435 & 0.010761 \\
\hline C & -7.280337 & -0.482626 & 0.056488 \\
\hline C & -8.349274 & ๑. 629790 & 0.173259 \\
\hline C & -7.827777 & -1.788738 & 0.684583 \\
\hline C & -7.069898 & -0.749624 & -1.450654 \\
\hline C & -4.348243 & -0.036246 & 4.358828 \\
\hline C & -5.544214 & -0.581543 & 5.173781 \\
\hline C & -3.200715 & -1.061235 & 4.576758 \\
\hline C & -3.904975 & 1. 323168 & 4.950802 \\
\hline C & -7.401220 & 1.403351 & 3.314103 \\
\hline C & -2.216489 & 2.379351 & -0.866040 \\
\hline C & -3.350484 & 3.121022 & -0.517301 \\
\hline C & -1.176502 & 3. 024613 & -1.542018 \\
\hline C & -1.276267 & 4.354309 & -1.985014 \\
\hline C & -2.512897 & 5.008404 & -1.752911 \\
\hline C & -3.518094 & 4.450683 & -0.918250 \\
\hline C & -4.719979 & 5.277500 & -0.390361 \\
\hline C & -5.562745 & 4.466297 & ๑. 619066 \\
\hline C & -5.680848 & 5.728491 & -1.516468 \\
\hline C & -3.177991 & 6.223312 & -3.671229 \\
\hline C & -4.183672 & 6.523703 & ๑. 358136 \\
\hline C & $-\odot .022194$ & 4.980783 & -2.662042 \\
\hline C & $\odot .163573$ & 4.381336 & -4.076815 \\
\hline C & -0.024906 & 6.525067 & -2.760755 \\
\hline C & 1. 233555 & 4.631761 & -1.817637 \\
\hline C & 1.019087 & -2.157009 & -1.521342 \\
\hline C & $\odot .944748$ & -3.462059 & -2.025087 \\
\hline C & 1.644478 & -3.846572 & -3.171127 \\
\hline C & 2.461260 & -2.860728 & -3.793426 \\
\hline C & 2.424367 & -1.495928 & -3.416217 \\
\hline C & 1.728592 & -1.190940 & -2.232739 \\
\hline C & 3.041031 & -0.311622 & -4.213927 \\
\hline C & 3.342115 & -0.620009 & -5.699941 \\
\hline C & 4.325131 & ๑. 198236 & -3.516774 \\
\hline C & 2. 016211 & $\odot .855609$ & -4.234592 \\
\hline C & 0.950477 & -5.142296 & -5.224058 \\
\hline C & 4.650542 & -3.433301 & -4.501680 \\
\hline C & 1.453059 & -5.266967 & -3.763775 \\
\hline C & ๑. 389502 & -6.066285 & -2.978061 \\
\hline C & 3.226036 & -3.088923 & 3.345944 \\
\hline C & 2.047630 & -2.408941 & 3.740283 \\
\hline C & 2.768016 & -2.137176 & 6.249491 \\
\hline C & 1.168638 & -2.032803 & 2.710848 \\
\hline C & 1.459087 & -2.251927 & 1.357957 \\
\hline C & 2.719463 & -2.759538 & 1.016076 \\
\hline C & 3. 638513 & -3.168142 & 1.985903 \\
\hline C & 3.587680 & -4.946242 & 4.761510 \\
\hline C & $\odot .431861$ & -2.860848 & 5.648201 \\
\hline C & 5.322494 & -5.114832 & 1.885142 \\
\hline C & 5.060992 & -3.623192 & 1.567549 \\
\hline C & 5.286549 & -3.433920 & ๑. 050601 \\
\hline C & 1.237886 & -0.517633 & 5.200082 \\
\hline C & 2.754703 & -6.103156 & -3.736553 \\
\hline C & 1.646297 & -2.017010 & 5.191648 \\
\hline C & 6.112313 & -2.745418 & 2.292684 \\
\hline $\mathrm{H}$ & -2.532118 & $\odot .193797$ & -5.312596 \\
\hline $\mathrm{H}$ & -2.273014 & 1.390235 & -3.156740 \\
\hline H & -2.347970 & -4.643057 & -5.225686 \\
\hline $\mathrm{H}$ & -4.028122 & -4.106034 & -4.815870 \\
\hline H & -0.431340 & -3.909346 & 1.914475 \\
\hline $\mathrm{H}$ & -2.425494 & -5.357523 & 2.245438 \\
\hline
\end{tabular}




\begin{tabular}{|c|c|c|c|}
\hline $\mathrm{H}$ & -6.309359 & -4.497983 & -0.516575 \\
\hline $\mathrm{H}$ & -5.139022 & -5.539869 & -1.423797 \\
\hline $\mathrm{H}$ & -2.480113 & $\odot .375760$ & 2. 468114 \\
\hline $\mathrm{H}$ & -4.810075 & -0.065502 & -1.067382 \\
\hline $\mathrm{H}$ & -7.957523 & 1.596263 & -0.166629 \\
\hline $\mathrm{H}$ & -9.208680 & ๑. 380984 & -0.460611 \\
\hline $\mathrm{H}$ & -8.717545 & 0.739323 & 1.193308 \\
\hline $\mathrm{H}$ & -8.051372 & -1.661835 & 1.745335 \\
\hline $\mathrm{H}$ & -8.748204 & -2.093824 & 0.172021 \\
\hline $\mathrm{H}$ & -7.096846 & -2.599463 & 0.582971 \\
\hline $\mathrm{H}$ & -6.332158 & -1.538533 & -1.631224 \\
\hline $\mathrm{H}$ & -8.019808 & -1.074022 & -1.889235 \\
\hline $\mathrm{H}$ & -6.755535 & ๑. 150641 & -1.991996 \\
\hline $\mathrm{H}$ & -5.193817 & -0.813073 & 6.186489 \\
\hline $\mathrm{H}$ & -6.364630 & ๑.128698 & 5.273857 \\
\hline $\mathrm{H}$ & -5.945515 & -1.501156 & 4.738682 \\
\hline $\mathrm{H}$ & -3.038242 & -1.205935 & 5.650738 \\
\hline $\mathrm{H}$ & -3.453443 & -2.032493 & 4.136784 \\
\hline $\mathrm{H}$ & $-2.250 \odot 81$ & $-\odot .733751$ & 4.146428 \\
\hline $\mathrm{H}$ & -3.006023 & 1.702863 & 4.451572 \\
\hline $\mathrm{H}$ & -4.686396 & 2. 084451 & 4.851028 \\
\hline $\mathrm{H}$ & -3.679136 & 1.216044 & 6.018720 \\
\hline $\mathrm{H}$ & -7.505174 & 2.081079 & 2.459236 \\
\hline $\mathrm{H}$ & -8.367356 & 1.292564 & 3.811673 \\
\hline $\mathrm{H}$ & -6.679698 & 1.831117 & 4.017869 \\
\hline $\mathrm{H}$ & -4.117601 & 2.645833 & 0.075739 \\
\hline $\mathrm{H}$ & -0.264914 & 2.467690 & -1.731365 \\
\hline $\mathrm{H}$ & -6.356067 & 5.109266 & 1.015069 \\
\hline $\mathrm{H}$ & -6.046300 & 3.598333 & $\odot .155411$ \\
\hline $\mathrm{H}$ & -4.967501 & 4.115220 & 1.469400 \\
\hline $\mathrm{H}$ & -5.235442 & 6.493607 & -2.152258 \\
\hline $\mathrm{H}$ & -5.987272 & 4.882142 & -2.142722 \\
\hline $\mathrm{H}$ & -6.587578 & 6.158178 & -1.074543 \\
\hline $\mathrm{H}$ & -2.383544 & 5.859132 & -4.330785 \\
\hline $\mathrm{H}$ & -4.063956 & 5.592679 & -3.802639 \\
\hline $\mathrm{H}$ & -3.421504 & 7.254417 & -3.937116 \\
\hline $\mathrm{H}$ & -3.592868 & 7.162977 & -0.300785 \\
\hline $\mathrm{H}$ & -5.021362 & 7.114014 & ๑. 748722 \\
\hline $\mathrm{H}$ & -3.559184 & 6.226726 & 1.209605 \\
\hline $\mathrm{H}$ & ๑. 268532 & 3.291844 & -4.040376 \\
\hline $\mathrm{H}$ & -0.684473 & 4.612784 & -4.730149 \\
\hline $\mathrm{H}$ & 1.066266 & 4.790149 & -4.546660 \\
\hline $\mathrm{H}$ & ๑. 976684 & 6.851846 & -3.064458 \\
\hline $\mathrm{H}$ & -0.725045 & 6.914824 & -3.498619 \\
\hline $\mathrm{H}$ & $-\odot .255010$ & 6.993917 & -1.799511 \\
\hline $\mathrm{H}$ & 1.144045 & 5.052546 & -0.808403 \\
\hline $\mathrm{H}$ & 1.422743 & 3.559237 & -1.727241 \\
\hline $\mathrm{H}$ & 2.120260 & 5.073510 & -2.286135 \\
\hline $\mathrm{H}$ & ๑. 332118 & -4.185256 & -1.503543 \\
\hline $\mathrm{H}$ & 1.734632 & -0.175790 & -1.851237 \\
\hline $\mathrm{H}$ & 4.192646 & -1.284883 & -5.845502 \\
\hline $\mathrm{H}$ & 2.478591 & -1.065209 & -6.203797 \\
\hline $\mathrm{H}$ & 3.577978 & $\odot .322863$ & -6.207181 \\
\hline $\mathrm{H}$ & 4.738410 & 1.049801 & -4.071661 \\
\hline $\mathrm{H}$ & 4.118380 & $\odot .530748$ & -2.494731 \\
\hline $\mathrm{H}$ & 5.100644 & $-\odot .573528$ & -3.469808 \\
\hline $\mathrm{H}$ & 2.406283 & 1.669584 & -4.855662 \\
\hline $\mathrm{H}$ & 1.057341 & ๑. 533619 & -4.656862 \\
\hline $\mathrm{H}$ & 1.830833 & 1. 272131 & -3.240809 \\
\hline $\mathrm{H}$ & $\odot .764446$ & -6.138952 & -5.642628 \\
\hline $\mathrm{H}$ & 0. . 012006 & -4.576322 & -5.262612 \\
\hline $\mathrm{H}$ & 1.679239 & -4.633952 & -5.857683 \\
\hline $\mathrm{H}$ & 5.132340 & -2.469067 & -4.313255 \\
\hline $\mathrm{H}$ & 4.779058 & -4.072562 & -3.620925 \\
\hline $\mathrm{H}$ & 5.120744 & -3.906304 & -5.367015 \\
\hline $\mathrm{H}$ & 0. 701801 & -6.271263 & -1.947203 \\
\hline $\mathrm{H}$ & -0.577597 & -5.552944 & -2.950462 \\
\hline $\mathrm{H}$ & ๑. 239922 & -7.034528 & -3.468064 \\
\hline $\mathrm{H}$ & 3.685116 & -1.636829 & 5.926738 \\
\hline $\mathrm{H}$ & 2.424019 & -1.652654 & 7.170919 \\
\hline $\mathrm{H}$ & 3. 018242 & -3.166675 & 6.504660 \\
\hline $\mathrm{H}$ & $\odot .217000$ & -1.576914 & 2.965767 \\
\hline $\mathrm{H}$ & 2.975877 & -2.842740 & -0.029876 \\
\hline $\mathrm{H}$ & 4.347845 & -5.303842 & 5.459526 \\
\hline $\mathrm{H}$ & 2.626606 & -4.870013 & 5. 281001 \\
\hline
\end{tabular}

\begin{tabular}{|c|c|c|c|}
\hline H & 3.487029 & -5.657223 & 3.934291 \\
\hline H & $\odot .122047$ & -2.563719 & 6.657282 \\
\hline $\mathrm{H}$ & $-\odot .427422$ & -2.727986 & 4.981560 \\
\hline H & $\odot .669969$ & -3.929926 & 5.675137 \\
\hline H & 5.412628 & -5.295737 & 2.956640 \\
\hline $\mathrm{H}$ & 4.528157 & -5.755716 & 1.483628 \\
\hline H & 6.265558 & -5.427295 & 1.421374 \\
\hline H & 6.321692 & -3.702025 & $-\odot .186897$ \\
\hline H & 4.636859 & -4.079866 & -0.552438 \\
\hline H & 5.135516 & -2.394422 & $-\odot .259409$ \\
\hline H & 2. 084792 & 0.112322 & 4.903881 \\
\hline H & ๑. 395808 & -0.295441 & 4.538447 \\
\hline H & $\odot .939539$ & -0.226164 & 6.213537 \\
\hline H & 3.193182 & -6.123126 & -2.731511 \\
\hline H & 2.532283 & -7.138617 & -4.020750 \\
\hline $\mathrm{H}$ & 3.499900 & -5.725876 & -4.436754 \\
\hline H & 7.121027 & -3.068538 & 2.007149 \\
\hline H & 6.001553 & -1.695403 & 2.001348 \\
\hline H & 6.024440 & -2.828547 & 3.377960 \\
\hline $\mathrm{Rh}$ & -0.114727 & 0.371557 & $\odot .740591$ \\
\hline 0 & -0.344028 & 2.296011 & 1.846620 \\
\hline 0 & 5.502254 & $\odot .562722$ & 0.822887 \\
\hline C & $\odot .441230$ & 3.194681 & 2.108351 \\
\hline S & 4.805655 & 1.835445 & 0.579841 \\
\hline C & $\odot .264695$ & 4.533978 & 2.802349 \\
\hline C & 1.935778 & 3.394957 & 1.887766 \\
\hline $\mathrm{N}$ & 4.278742 & 2.552276 & 2.024678 \\
\hline 0 & 3.619850 & 1.849154 & -0.294510 \\
\hline C & 6.016867 & 3.008227 & $-\odot .016022$ \\
\hline C & 1.812218 & 4.722274 & 2.706460 \\
\hline $\mathrm{H}$ & $-\odot .334531$ & 5.236577 & 2.211176 \\
\hline $\mathrm{H}$ & -0.170447 & 4.476654 & 3.806317 \\
\hline C & 2.871231 & 2.283862 & 2.377124 \\
\hline $\mathrm{H}$ & 2.124703 & 3.559194 & ๑. 820695 \\
\hline C & 5.261986 & 2.601086 & 3.134913 \\
\hline C & 5.616352 & 4.301811 & 5.218700 \\
\hline C & 7.339636 & 2.595901 & 7.274600 \\
\hline $\mathrm{H}$ & 2.310787 & 4.677160 & 8.182300 \\
\hline H & 2.138399 & 5.623326 & 2.184070 \\
\hline H & 2.537472 & 1.320952 & 1.972108 \\
\hline H & 2.825350 & 2.197263 & 3.467004 \\
\hline C & 5.328491 & 1.363977 & 3.993468 \\
\hline $\mathrm{H}$ & 6.239112 & 2.808920 & 2.683577 \\
\hline H & 5.011055 & 3.475942 & 3.746042 \\
\hline $\mathrm{H}$ & 4.581963 & 4.607191 & -0.250039 \\
\hline C & 6.561377 & 5.186919 & $-\odot .874195$ \\
\hline C & 8.273448 & 3.499655 & -0.685619 \\
\hline H & 7.625277 & 1.584161 & $\odot .090311$ \\
\hline C & 5.143815 & 1.379230 & 5.315165 \\
\hline H & 5.558924 & $\odot .434678$ & 3.476756 \\
\hline H & 6.255947 & 6.193213 & -1.149861 \\
\hline C & $7.9 \odot 4379$ & 4.803213 & -1.039363 \\
\hline $\mathrm{H}$ & 9.305091 & 3.182566 & -0.811515 \\
\hline H & 5.231399 & $\odot .477968$ & 5.915331 \\
\hline H & 4.921499 & 2.299377 & 5.853483 \\
\hline C & 8.917860 & 5.783290 & -1.578212 \\
\hline H & 9.877285 & 5.298928 & -1.780604 \\
\hline H & 8.567775 & 6.245719 & -2.508221 \\
\hline H & 9.097946 & 6.595515 & $-\odot .862745$ \\
\hline
\end{tabular}

22

B3LYP SCF energy:

B3LYP enthalpy:

B3LYP free energy: M06 SCF energy in solution:

a.u.

M06 enthalpy in solution: M06 free energy in solution: a.u.
3.934291
6.657282
4.981560
5.675137
2.956640
1.483628
1.421374
-.186897
-0.552438
-0.259409
4.903881
4.538447
6.213537
-2.731511
-4.020750
-4.436754
2.007149
2.001348
3.377960
0.740591
1.846620
0.822887
2.108351
0.579841
2.802349
1.887766
2.024678
-0.294510
-0.016022
2.706460
2.211176
3.806317
2.377124
0.820695
3.134913
5.218700
7.274600
8.182300
2.184070
1.972108
3.467004
3.993468
2.683577
3.746042
-0.250039
-0.874195
-0.685619
0.090311
5.315165
3.476756
-1.149861
-1.039363
-0.811515
5.915331
5.853483
-1.578212
-1.780604
-2.508221
-0.862745

5535.36000765 a.u. -5533.324355 a.u. -5533.599953 a.u $-5534.57374870$

-5532.538096 a.u. $-5532.813694$ 


\begin{tabular}{|c|c|c|c|}
\hline ATOM & $x$ & Y & Z \\
\hline $\mathrm{P}$ & $\odot . \odot \odot \odot \odot \odot \odot$ & $-\odot .867411$ & 5.766099 \\
\hline$P$ & ๑. . ०००००० & -0.661994 & 3. 367193 \\
\hline 0 & $\odot .0 \odot \odot ० \odot ०$ & 1.653723 & 3.403659 \\
\hline 0 & $\odot . \odot \odot \odot \odot \odot \odot$ & 1.359694 & 2.132885 \\
\hline 0 & $\odot .0 \odot \odot ० \odot ०$ & 3.153995 & 5.721127 \\
\hline 0 & $\odot . \diamond \odot \odot \odot \odot \odot$ & 4.441334 & 4.647498 \\
\hline 0 & $\odot . ๑ \odot \odot \odot \odot \odot$ & 3. 993352 & 3.744650 \\
\hline 0 & $\odot . ๑ \odot \odot \odot \odot \odot$ & 5.902783 & 3. 228557 \\
\hline 0 & $\odot . ๑ \odot \odot \odot \odot \odot ~$ & 5.772313 & 4.637290 \\
\hline 0 & $\odot . \diamond \odot \odot \odot \odot \odot$ & 3.542797 & 5.286799 \\
\hline C & $\odot . ๑ \odot \odot \odot \odot \odot$ & 1.926738 & 4.397002 \\
\hline C & ๑. . 000000 & ๑. 719868 & 5.906526 \\
\hline C & -1.531185 & -0.674235 & 5.814734 \\
\hline C & -1.897316 & 3.131697 & 5.942937 \\
\hline C & -2.294230 & 2.870026 & 6.511735 \\
\hline C & -2.313330 & 6.849500 & 4. 698102 \\
\hline C & -1.581927 & 4.932966 & 5.383602 \\
\hline C & -0.093568 & 2.876507 & 5.856377 \\
\hline C & $\odot .157358$ & 2.835154 & 6.903226 \\
\hline C & -0.623683 & 3.428452 & 5.178027 \\
\hline C & -1.668198 & 5.501549 & 6.252840 \\
\hline C & -1.929925 & 4.942163 & 7.189046 \\
\hline C & -1.184338 & 5.450339 & 5.740484 \\
\hline C & -3.266199 & 6.550941 & 6.499109 \\
\hline C & -3.161476 & -0.601116 & $\odot .823208$ \\
\hline C & -2.981541 & -0.293099 & 2.168974 \\
\hline C & -3.928213 & -0.631676 & 3. 151818 \\
\hline C & -5.130463 & -1.219880 & 2.694386 \\
\hline C & -5.288094 & -1.684990 & 1.358200 \\
\hline C & -4.288488 & -1.342749 & 0.443080 \\
\hline C & -6.458752 & -2.608941 & 0.932058 \\
\hline C & -7.839022 & -1.916896 & 1.030294 \\
\hline C & -6.452322 & -3.868225 & 1.835066 \\
\hline C & -6.297481 & -3.084336 & -0.528968 \\
\hline C & -3.533846 & -0.372510 & 4.633469 \\
\hline C & -4.420179 & -1.066048 & 5.694282 \\
\hline C & -2.105627 & -0.939625 & 4.856538 \\
\hline C & -3.507086 & 1.148707 & 4.916151 \\
\hline C & -6.966775 & -0.227593 & 3.802515 \\
\hline C & -2.862347 & 1.468503 & -1.183269 \\
\hline C & -4.152908 & 1.835488 & -0.788363 \\
\hline C & -2.204759 & 2.264038 & -2.124982 \\
\hline C & -2.821776 & 3.350807 & -2.763016 \\
\hline C & -4.185652 & 3.577325 & -2.450215 \\
\hline C & -4.836317 & 2.902567 & -1.383541 \\
\hline C & -6.211283 & 3.355483 & -0.825561 \\
\hline C & -6.607770 & 2.544298 & ๑. 428081 \\
\hline C & -7.359048 & 3.185528 & -1.849652 \\
\hline C & -5.404114 & 4.056891 & -4.421102 \\
\hline C & -6.119509 & 4.841900 & -0.398028 \\
\hline C & -1.941632 & 4.216106 & -3.711411 \\
\hline C & -1.668082 & 3. 448101 & -5.026862 \\
\hline C & -2.512902 & 5.614241 & -4.047049 \\
\hline C & -0.577870 & 4.488599 & -3.018066 \\
\hline C & 1.720649 & -1.788955 & -1.371821 \\
\hline C & 2.057488 & -3.119020 & -1.654911 \\
\hline C & 2.770236 & -3.468349 & -2.805514 \\
\hline C & 3.168247 & -2.408918 & -3.669556 \\
\hline C & 2.696003 & -1.085078 & -3.508445 \\
\hline C & 2.013393 & -0.804214 & -2.312264 \\
\hline C & 2.819654 & ๑. 071106 & -4.541191 \\
\hline C & 3.204644 & -0.361291 & -5.975462 \\
\hline C & 3.831442 & 1.128042 & -4.037255 \\
\hline C & 1.430455 & $\odot .753586$ & -4.676712 \\
\hline C & 2. 414501 & -5.271164 & -4.533945 \\
\hline C & 5.377561 & -2.362145 & -4.514097 \\
\hline C & 3. 026938 & -4.959925 & -3.145077 \\
\hline C & 2.348431 & -5.895481 & -2.119676 \\
\hline C & 4.501877 & -1.648739 & 3.211983 \\
\hline C & 3.255644 & -1.174246 & 3.700483 \\
\hline C & 4.098275 & 0.462448 & 5.409858 \\
\hline C & 2.218959 & -1.043538 & 2.765646 \\
\hline
\end{tabular}

\begin{tabular}{|c|c|c|}
\hline 2.397091 & -1.355227 & 1. 409498 \\
\hline 3.688311 & -1.636015 & ๑. 957247 \\
\hline 4.782472 & -1.736263 & 1.827418 \\
\hline 5.519456 & -3.360933 & 4.481547 \\
\hline 3.267648 & -1.767993 & 6.235406 \\
\hline 6.984939 & -3.102029 & 1.554887 \\
\hline 6.225950 & -1.788551 & 1.253389 \\
\hline 6.218232 & -1.621247 & -0.283121 \\
\hline 1.662453 & $-\odot .090664$ & $5.3860 \odot 1$ \\
\hline 4.531404 & -5.321227 & -3.155781 \\
\hline 3.073489 & -0.672841 & 5.159598 \\
\hline 7.023282 & $-\odot .595530$ & 1.839377 \\
\hline-2.590622 & -1.517137 & -5.128833 \\
\hline-2.647718 & $\odot .107098$ & -3.259946 \\
\hline-0.799483 & -5.935649 & -4.223043 \\
\hline-2.530584 & -5.838856 & -3.697851 \\
\hline ๑. 999976 & -3.127222 & 2.520554 \\
\hline-0.404291 & -5.018278 & 3.283579 \\
\hline-4.336093 & -6.113942 & ๑. 655771 \\
\hline-2.855380 & -6.851271 & -0.082879 \\
\hline-2.065205 & $\odot .215012$ & 2.451449 \\
\hline-4.384074 & -1.645586 & -0.591263 \\
\hline-7.846454 & -0.962606 & 0.489677 \\
\hline-8.603576 & -2.558419 & 0.576243 \\
\hline - 8.135665 & -1.737942 & 2.063692 \\
\hline-6.626177 & -3.611312 & 2.881767 \\
\hline-7.239309 & -4.562477 & 1.515925 \\
\hline-5.489680 & -4.388283 & 1.767124 \\
\hline-5.348340 & -3.604876 & -0.692526 \\
\hline-7.107441 & -3.782800 & -0.765904 \\
\hline-6.366277 & -2.255861 & -1.244023 \\
\hline-3.930621 & -0.964676 & 6.670120 \\
\hline-5.413431 & -0.627317 & 5.787998 \\
\hline-4.540327 & -2.132872 & 5.487572 \\
\hline-1.811883 & -0.794559 & 5.902432 \\
\hline-2.072935 & -2.012829 & 4.638599 \\
\hline-1.351909 & -0.449472 & 4.234680 \\
\hline-2.818192 & 1.668996 & 4.239378 \\
\hline-4.496773 & 1.602310 & 4.793348 \\
\hline - 3.177753 & 1.338997 & 5.944873 \\
\hline-7.380177 & $\odot .171953$ & 2.869657 \\
\hline-7.782539 & -0.529383 & 3429 \\
\hline-6.377491 & ๑.555936 & 4.290840 \\
\hline - 4.635191 & 1. 271122 & $-0.0 \odot 4305$ \\
\hline-1.172064 & 2. 029905 & -2.357571 \\
\hline-7.549232 & 2.940627 & ๑. 823479 \\
\hline-6.767482 & 1.483176 & ๑. 203815 \\
\hline-5.858632 & 2.617956 & 1.224513 \\
\hline-7.281236 & 3.896309 & -2.672250 \\
\hline-7.378945 & 2.169321 & -2.261071 \\
\hline-8.321920 & 3. 361614 & -1.355493 \\
\hline-4.587076 & 3. 871281 & -5.126108 \\
\hline-5.993221 & 3.139534 & -4.313322 \\
\hline-6.040106 & 4.855057 & -4.810806 \\
\hline-5.864291 & 5.486020 & -1.241605 \\
\hline-7.081939 & 5.174232 & $\odot .0 \odot 9710$ \\
\hline-5.361748 & 4.974983 & ๑. 384053 \\
\hline-1.176078 & 2.488102 & -4.835422 \\
\hline-2.592182 & 3.243959 & -5.578031 \\
\hline-1.014198 & 4.036514 & -5.682131 \\
\hline-1.731310 & 6.195009 & -4.551254 \\
\hline-3.371906 & 5.585630 & -4.716220 \\
\hline-2.808656 & 6.158767 & -3.145438 \\
\hline-0.725446 & 5. 010796 & -2.065408 \\
\hline-0.003779 & 3.579822 & -2.819872 \\
\hline$\odot .035524$ & 5.126409 & -3.665124 \\
\hline 1.762875 & -3.888490 & -0.953558 \\
\hline 1.689883 & ๑. 209316 & -2.100907 \\
\hline 4.241485 & -0.682477 & -6.071518 \\
\hline 2.566301 & -1.171457 & -6.339176 \\
\hline 3. 070519 & ๑. 498560 & -6.642556 \\
\hline 3.883398 & 1.967772 & -4.741350 \\
\hline 3.541464 & 1.526499 & -3.058463 \\
\hline 4.840217 & ๑. 711975 & -3.940749 \\
\hline 1. 483201 & 1.543720 & -5.434383 \\
\hline
\end{tabular}




\begin{tabular}{|c|c|c|c|}
\hline $\mathrm{H}$ & ๑. 667834 & ๑. . 031534 & -4.989175 \\
\hline $\mathrm{H}$ & 1. 092442 & 1.217684 & -3.745902 \\
\hline $\mathrm{H}$ & 2.535643 & -6.336145 & -4.767221 \\
\hline $\mathrm{H}$ & 1. 343224 & -5.038643 & -4.545160 \\
\hline $\mathrm{H}$ & 2.895254 & -4.690397 & -5.323253 \\
\hline $\mathrm{H}$ & 5.503646 & -1.276275 & -4.450912 \\
\hline $\mathrm{H}$ & 5.778072 & -2.817051 & -3.601097 \\
\hline H & 5.927153 & -2.738355 & -5.380149 \\
\hline $\mathrm{H}$ & 2.781550 & -5.795266 & -1.117362 \\
\hline $\mathrm{H}$ & 1. 269997 & -5.719837 & -2.049442 \\
\hline $\mathrm{H}$ & 2.498921 & -6.934204 & -2.433560 \\
\hline H & 3.969937 & 1.270306 & 4.679904 \\
\hline $\mathrm{H}$ & 3.962274 & ๑. 878141 & 6.416158 \\
\hline $\mathrm{H}$ & 5.122698 & ๑. 092818 & 5.327509 \\
\hline $\mathrm{H}$ & 1. 240480 & $-\odot .71290 \odot$ & 3.093088 \\
\hline $\mathrm{H}$ & 3.846766 & -1.769763 & -0.101902 \\
\hline $\mathrm{H}$ & 6.377063 & -3.488673 & 5.146223 \\
\hline $\mathrm{H}$ & 4.604827 & -3.651500 & 5.006218 \\
\hline $\mathrm{H}$ & 5.637989 & -4.001137 & 3.603753 \\
\hline $\mathrm{H}$ & 2.943532 & -1.383500 & 7.209722 \\
\hline $\mathrm{H}$ & 2.666930 & -2.659028 & 6.017583 \\
\hline $\mathrm{H}$ & 4.312518 & -2.060024 & 6.335856 \\
\hline $\mathrm{H}$ & 7.276065 & -3.176181 & 2.603223 \\
\hline $\mathrm{H}$ & 6.390877 & -3.984215 & 1.287430 \\
\hline $\mathrm{H}$ & 7.906980 & -3.134107 & ๑. 962416 \\
\hline $\mathrm{H}$ & 7.253415 & -1.571602 & -0.637983 \\
\hline $\mathrm{H}$ & 5.738611 & -2.465706 & $-\odot .792516$ \\
\hline $\mathrm{H}$ & 5.715551 & $-\odot .699481$ & -0.595078 \\
\hline $\mathrm{H}$ & 1.436451 & 0.734330 & 4.702288 \\
\hline $\mathrm{H}$ & $\odot .879661$ & -0.849402 & 5.278647 \\
\hline $\mathrm{H}$ & 1.598056 & $\odot .303651$ & 6.405873 \\
\hline $\mathrm{H}$ & 5.021064 & -5.014996 & -2.223343 \\
\hline $\mathrm{H}$ & 4.647257 & -6.407759 & -3.247640 \\
\hline $\mathrm{H}$ & 5.057128 & -4.865731 & -3.994962 \\
\hline $\mathrm{H}$ & 8.032852 & $-\odot .575786$ & 1.410313 \\
\hline H & 6.527899 & $\odot .352484$ & 1.603881 \\
\hline $\mathrm{H}$ & 7.115149 & $-\odot .675948$ & 2.924660 \\
\hline $\mathrm{Rh}$ & $\odot .042779$ & $\odot .765380$ & ๑. 279451 \\
\hline 0 & 2.043341 & 1.959325 & ๑. 480479 \\
\hline 0 & -0.687337 & 2.868397 & 0.457724 \\
\hline S & 3.197346 & 2.674249 & 1.066805 \\
\hline C & -0.601391 & 3.724573 & 1.318327 \\
\hline $\mathrm{N}$ & 2.536718 & 3.778408 & 2.197486 \\
\hline 0 & 4. 295247 & 1.901164 & 1.657168 \\
\hline C & 3.866892 & 3.745027 & -0.194346 \\
\hline C & -1.088983 & 5.164191 & 1.363558 \\
\hline C & ๑. . 046194 & 3.820554 & 2.703858 \\
\hline C & 1.420469 & 3.217732 & $3.0 \odot 4259$ \\
\hline C & 3.613977 & 4.433782 & 3. $0 \odot \odot 791$ \\
\hline C & 5.251287 & 3.813664 & -0.357217 \\
\hline C & 3. 001409 & 4.473240 & -1.017607 \\
\hline C & $-\odot .195561$ & 5.362763 & 2.623486 \\
\hline $\mathrm{H}$ & -2.167276 & 5.220395 & 1.555620 \\
\hline $\mathrm{H}$ & -0.867411 & 5.766099 & 6.646500 \\
\hline $\mathrm{H}$ & -0.661994 & 3.367193 & $9.50570 \odot$ \\
\hline $\mathrm{H}$ & 1.653723 & 3.403659 & 11.222600 \\
\hline $\mathrm{H}$ & 1. 359694 & 2.132885 & 2.877155 \\
\hline C & 3.153995 & 5.721127 & 3.623327 \\
\hline $\mathrm{H}$ & 4.441334 & 4.647498 & 2.315303 \\
\hline $\mathrm{H}$ & 3.993352 & 3.744650 & 3.766465 \\
\hline $\mathrm{H}$ & 5.902783 & 3.228557 & ๑. 282837 \\
\hline C & 5.772313 & 4.637290 & -1.355376 \\
\hline C & 3.542797 & 5.286799 & -2.007145 \\
\hline $\mathrm{H}$ & 1.926738 & 4.397002 & -0.891157 \\
\hline $\mathrm{H}$ & $\odot .719868$ & 5.906526 & 2. 390699 \\
\hline $\mathrm{H}$ & -0.674235 & 5.814734 & 3.493708 \\
\hline C & 3.131697 & 5.942937 & 4.938497 \\
\hline $\mathrm{H}$ & $2.870 \odot 26$ & 6.511735 & 2.930399 \\
\hline $\mathrm{H}$ & 6.849500 & 4.698102 & -1.484256 \\
\hline C & 4.932966 & 5.383602 & -2.193183 \\
\hline $\mathrm{H}$ & 2.876507 & 5.856377 & -2.649829 \\
\hline $\mathrm{H}$ & 2.835154 & 6.903226 & 5.350568 \\
\hline $\mathrm{H}$ & 3.428452 & 5.178027 & 5.653281 \\
\hline C & 5.501549 & 6.252840 & -3.287915 \\
\hline $\mathrm{H}$ & 4.942163 & 7.189046 & -3.386911 \\
\hline
\end{tabular}

$\begin{array}{llll}\mathrm{H} & 5.450339 & 5.740484 & -4.257441 \\ \mathrm{H} & 6.550941 & 6.499109 & -3.101518\end{array}$

23

B3LYP SCF energy: $\quad-5535.35009313$ a.u. B3LYP enthalpy: $\quad-5533.313857 \mathrm{a} \cdot \mathrm{u}$ B3LYP free energy: $\quad-5533.585187 \mathrm{a} . \mathrm{u}$. M06 SCF energy in solution: $\quad-5534.57480453$ a.u.

M06 enthalpy in solution: $\quad-5532.538568 \mathrm{a} \cdot \mathrm{u}$ M06 free energy in solution: $\quad-5532.809898$ a.u.

Cartesian coordinates

\begin{tabular}{|c|c|c|c|}
\hline ATOM & $\mathrm{X}$ & Y & Z \\
\hline $\mathrm{P}$ & $\odot . ๑ \odot \odot \odot \odot \odot$ & - 7.139329 & -3.648651 \\
\hline$P$ & 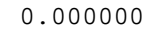 & -6.593579 & -4.640594 \\
\hline 0 & $\odot . \odot \odot \odot \odot \odot \odot$ & -3.107982 & $-5.2750 \odot 2$ \\
\hline 0 & $\odot .0000 \odot \odot$ & -2.569333 & -6.081163 \\
\hline 0 & $\odot . \diamond \odot \odot \odot \odot \odot$ & -4.196343 & -3.124344 \\
\hline 0 & $\odot . \odot \odot \odot \odot \odot \odot$ & -3.014427 & -1.850789 \\
\hline 0 & $\odot . \diamond \diamond \diamond \diamond \diamond \diamond$ & -2.461200 & -0.843512 \\
\hline 0 & $\odot . \diamond \odot \odot \odot \odot \odot ~$ & -3.760862 & -2.184266 \\
\hline 0 & $\odot . \diamond \odot \odot \odot \odot \odot$ & -2.374950 & -2.975945 \\
\hline 0 & $\odot .0 \odot \odot \odot \odot \odot$ & -7.077414 & -2.808942 \\
\hline C & $\odot . \diamond \odot \odot \odot \odot \odot$ & -7.868359 & -4.793127 \\
\hline C & $\odot .0 \odot \odot ० \odot \odot$ & -7.325290 & -5.771165 \\
\hline C & 3.049560 & -6.114248 & -4.567147 \\
\hline C & 2.562160 & -2.799249 & 0.176636 \\
\hline C & 1. 216701 & -1.765681 & -0.658043 \\
\hline C & ๑. 372439 & -8.369694 & -4.845492 \\
\hline C & 4.728718 & -7.974597 & -5.867276 \\
\hline C & 2.894542 & -7.404399 & -6.591699 \\
\hline C & 3. 626159 & -2.443838 & 1.178114 \\
\hline C & 4.387222 & -3.559750 & ๑. . 078408 \\
\hline C & 4.388316 & -8.789430 & - 7.089992 \\
\hline C & 3.662690 & -8.269140 & -8.011226 \\
\hline C & 2.900868 & $-9.7480 \odot 4$ & - $7.08290 \odot$ \\
\hline C & 4.889011 & - $9.0 \odot 9101$ & -7.138071 \\
\hline C & -0.104541 & 3.013340 & -0.914247 \\
\hline C & -0.174803 & 2.724908 & -2.275437 \\
\hline C & $-\odot .109176$ & 3.723298 & -3.263621 \\
\hline C & $-\odot .060709$ & 5.060476 & -2.804630 \\
\hline C & ๑. 180165 & 5.385503 & -1.438893 \\
\hline C & ๑.132943 & 4.336795 & -0.516968 \\
\hline C & 0.560632 & 6.817069 & -0.978968 \\
\hline C & $-\odot .599657$ & 7.825809 & -1.149615 \\
\hline C & 1.785561 & 7.301879 & -1.794275 \\
\hline C & 0.964749 & 6.837862 & 0.512133 \\
\hline C & $-\odot .052615$ & 3.253678 & -4.745411 \\
\hline C & ๑. 385152 & 4.329907 & -5.766427 \\
\hline C & 1. 010180 & 2.126362 & -4.857487 \\
\hline C & -1.424347 & 2.679069 & -5.173092 \\
\hline C & -1.551538 & 6.386657 & -4.073583 \\
\hline C & -2.010917 & 2.072101 & 1.069041 \\
\hline C & -2.783164 & 3.142600 & 0.603904 \\
\hline C & -2.545184 & 1.258542 & 2.074068 \\
\hline C & -3.755180 & 1.555936 & 2.725582 \\
\hline C & -4.410908 & 2.748565 & 2.330591 \\
\hline C & $-4.0 \odot 8854$ & 3.484898 & 1.185263 \\
\hline C & -4.902634 & $4.57460 \odot$ & $\odot .537584$ \\
\hline C & -4.301495 & 5.077997 & -0.793619 \\
\hline C & -5.087778 & 5.814455 & 1.444771 \\
\hline C & -5.192015 & 3.943410 & 4.217366 \\
\hline C & -6.287543 & 3.961127 & $\odot .2 \odot 9856$ \\
\hline C & -4.265507 & $\odot .549946$ & 3.798028 \\
\hline C & -3.410617 & $\odot .672417$ & 5.082410 \\
\hline C & -5.763032 & $\odot .683320$ & 4.164946 \\
\hline C & -4.113695 & $-\odot .892194$ & 3.245969 \\
\hline C & 2.567413 & -1.152165 & 1.412657 \\
\hline C & 3.919468 & $-\odot .998287$ & 1.748778 \\
\hline C & 4.447160 & -1.521077 & 2.933395 \\
\hline C & 3.557186 & -2.245345 & 3.776467 \\
\hline
\end{tabular}




\begin{tabular}{|c|c|c|c|c|c|c|c|}
\hline C & 2.159152 & -2.263018 & 3.560504 & H & -5.991286 & 1.569535 & 4.755633 \\
\hline C & 1.708127 & -1.744372 & 2.334695 & H & -6.396567 & $\odot .698308$ & 3.273647 \\
\hline c & 1.076376 & -2.750419 & $4.56510 \odot$ & H & -4.685361 & -1.010347 & 2.319671 \\
\hline c & 1.560570 & -2.953409 & 6.019956 & $\mathrm{H}$ & -3.072818 & -1.168809 & 3. 051782 \\
\hline c & $\odot .435156$ & -4.064345 & 4.058187 & H & -4.500069 & -1.604812 & 3.983819 \\
\hline c & -0.031810 & -1.665111 & 4.648221 & $\mathrm{H}$ & 4.566306 & -0.466614 & 1.063215 \\
\hline C & 5.924179 & $-\odot .499445$ & $4.70 \odot 454$ & H & $\odot .652436$ & -1.782932 & 2.087215 \\
\hline c & 4.244945 & -4.315943 & 4.687487 & H & 2.204268 & -3.823234 & 6.148450 \\
\hline C & 5.917305 & -1.229646 & 3.333511 & H & 2.098552 & -2.077773 & 6.393230 \\
\hline c & 6.607553 & -0.304132 & 2.307065 & $\mathrm{H}$ & 0.681688 & -3.106110 & 6.657330 \\
\hline C & 3.506087 & -3.830972 & -3.200210 & H & $-\odot .367375$ & -4.382421 & 4.734652 \\
\hline C & 2.468516 & -2.956175 & -3.622649 & $\mathrm{H}$ & 0.003219 & -3.936227 & 3.057836 \\
\hline C & 1.218080 & -4.564368 & -5.105242 & H & 1.165459 & -4.878779 & 4.000798 \\
\hline C & 2.014013 & -2.022319 & -2.684503 & $\mathrm{H}$ & -0.787987 & -1.966816 & 5.381607 \\
\hline C & 2.508474 & -1.976571 & -1.374655 & H & $\odot .386304$ & $-\odot .703328$ & 4.964973 \\
\hline C & 3.385742 & -2.979222 & -0.957458 & $\mathrm{H}$ & -0.546654 & -1.507808 & 3.695978 \\
\hline c & 3.877833 & -3.954787 & -1.838756 & H & 6.951150 & -0.230474 & 4.976398 \\
\hline C & 5.292804 & -4.047480 & -4.732430 & H & 5.330385 & $\odot .420773$ & 4.653002 \\
\hline c & 2.629619 & -2.797020 & -6.215323 & H & 5.511228 & -1.128147 & 5.491693 \\
\hline c & 6.121860 & -5.271992 & -1.793397 & $\mathrm{H}$ & 3.274457 & -4.811847 & 4.578872 \\
\hline c & 4.664911 & -5.174393 & -1.282774 & H & 4.860130 & -4.547639 & 3.810795 \\
\hline C & 4.736644 & -5.138814 & 0.260526 & H & 4.739249 & -4.686610 & 5.588313 \\
\hline c & $\odot .514534$ & -2.176336 & -5.078318 & H & 6.708322 & -0.778813 & 1.323669 \\
\hline C & 6.780253 & -2.510000 & 3.434407 & H & 6.076058 & $\odot .644479$ & 2.180253 \\
\hline c & 1.741652 & -3.110489 & -4.987859 & $\mathrm{H}$ & 7.619243 & -0.076130 & 2. 659781 \\
\hline C & 3.906303 & -6.467147 & -1.676624 & H & $\odot .519716$ & -4.794060 & -4.290630 \\
\hline $\mathrm{H}$ & 0.824241 & 2.832769 & 5.047328 & H & 0.681797 & -4.694305 & -6.053029 \\
\hline $\mathrm{H}$ & $-\odot .69 \odot 381$ & 2.294115 & 3.170974 & H & 2.036052 & -5.286638 & -5.072611 \\
\hline $\mathrm{H}$ & 5.604857 & 2.733833 & 4.193843 & $\mathrm{H}$ & 1.243635 & -1.313728 & -2.961243 \\
\hline $\mathrm{H}$ & 4.889334 & 4.302588 & 3.636569 & H & 3.690528 & -3.003927 & ๑. $07816 \odot$ \\
\hline $\mathrm{H}$ & 3.629044 & -0.105139 & -2.514812 & H & 5.675401 & -4.781502 & -5.445214 \\
\hline $\mathrm{H}$ & 4.954915 & 1.832212 & -3.303499 & H & 5.049562 & -3.119899 & -5.257544 \\
\hline $\mathrm{H}$ & 4.632297 & 5.962291 & $-\odot .783732$ & H & 6.056885 & -3.834727 & -3.980238 \\
\hline $\mathrm{H}$ & 5.820963 & 4.848051 & 0.007228 & H & 2.003852 & -2.736361 & -7.113448 \\
\hline $\mathrm{H}$ & $-\odot .264161$ & 1.684388 & -2.568630 & H & 3.144047 & -1.834985 & -6.106186 \\
\hline $\mathrm{H}$ & 0.272625 & 4.543983 & 0.535868 & H & 3.370741 & -3.576566 & -6.392959 \\
\hline H & -1.513490 & 7.469234 & $-\odot .659210$ & H & 6.168730 & -5.573578 & -2.840096 \\
\hline $\mathrm{H}$ & -0.327139 & 8.780975 & -0.685125 & H & 6.657001 & -4.322848 & -1.671714 \\
\hline H & $-\odot .819309$ & 8.024334 & -2.198820 & H & 6.661452 & -6.030989 & -1.214930 \\
\hline $\mathrm{H}$ & 1.558502 & 7.371887 & -2.859293 & H & 5.226044 & -6.052968 & 0.613095 \\
\hline H & 2.100781 & 8.291606 & -1.442414 & H & 5.323397 & -4.289915 & $\odot .630620$ \\
\hline H & 2.628014 & 6.612022 & -1.668816 & H & 3.743691 & -5.097138 & 0.721958 \\
\hline H & 1.789438 & $6.14890 \odot$ & $\odot .724372$ & H & $-\odot .189562$ & -2.343668 & -4.255055 \\
\hline H & 1.296262 & 7.847955 & 0.775967 & H & $\odot .795771$ & -1.116977 & -5.084293 \\
\hline H & $\odot .126392$ & 6.590787 & 1.174093 & H & $-\odot .017862$ & -2.376987 & -6.014017 \\
\hline H & 0.570498 & 3.836282 & -6.727628 & H & 6.711915 & -3.111187 & 2.519586 \\
\hline H & $-\odot .36844 \odot$ & 5.097341 & -5.941484 & H & 7.832460 & -2.232187 & 3.567644 \\
\hline $\mathrm{H}$ & 1.309467 & 4.827700 & -5.460325 & $\mathrm{H}$ & 6.497164 & -3.130212 & 4.284666 \\
\hline H & 1.085197 & 1.797686 & -5.900363 & H & 4.429815 & -7.344694 & -1.278265 \\
\hline $\mathrm{H}$ & 1.995925 & 2.483977 & -4.540789 & $\mathrm{H}$ & 2.890846 & -6.465626 & -1.262005 \\
\hline $\mathrm{H}$ & 0.766439 & 1.246696 & -4.255023 & $\mathrm{H}$ & 3.837501 & -6.573401 & -2.761283 \\
\hline $\mathrm{H}$ & -1.733483 & 1.848821 & -4.527355 & $\mathrm{Rh}$ & -0.358062 & -0.472772 & -0.472555 \\
\hline H & -2.213702 & 3.437514 & -5.134491 & 0 & -0.341165 & -2.674161 & $-\odot .895828$ \\
\hline $\mathrm{H}$ & -1.374154 & 2.303136 & -6.202016 & 0 & -5.889538 & -1.777020 & -2.578481 \\
\hline H & -2.172803 & 6.608353 & -3.198520 & C & $-\odot .990715$ & -3.646827 & $-\odot .549983$ \\
\hline $\mathrm{H}$ & -1.510361 & 7.263251 & -4.723923 & $\mathrm{~s}$ & -5.598413 & -2.110636 & -1.181715 \\
\hline H & -1.998948 & 5.550829 & -4.621344 & C & $-\odot .908016$ & -5.119943 & -0.921616 \\
\hline $\mathrm{H}$ & -2.417613 & 3.724105 & -0.229087 & C & -2.272807 & -3.838141 & 0.250281 \\
\hline H & -1.988319 & $\odot .377174$ & 2.373885 & $\mathrm{~N}$ & -3.970959 & -2.647815 & -1.168995 \\
\hline H & -4.997792 & 5.790143 & -1.249035 & 0 & -5.794154 & -1.120718 & $-\odot .111401$ \\
\hline H & -3.347372 & 5.599202 & $-\odot .650771$ & C & -6.499440 & -3.586479 & $-0.72500 \odot$ \\
\hline H & -4.147163 & 4.264650 & -1.511584 & C & -2.375144 & -5.254857 & $-\odot .4 \odot 2114$ \\
\hline $\mathrm{H}$ & -5.707680 & 5.595792 & 2.314278 & H & -0.173328 & -5.652846 & -0.306029 \\
\hline H & -4.122916 & 6.207107 & 1.787962 & H & $-\odot .698289$ & -5.337162 & -1.973494 \\
\hline $\mathrm{H}$ & -5.587132 & 6.610506 & $\odot .879896$ & C & -3.393408 & -2.801467 & 0.172352 \\
\hline $\mathrm{H}$ & -4.742441 & 3.302232 & 4.982953 & H & -1.990904 & -3.928839 & 1.310166 \\
\hline $\mathrm{H}$ & -4.508430 & 4.772289 & 4.002896 & C & -3.124847 & -2.200871 & -2.279575 \\
\hline H & -6.138569 & 4.339261 & 4.592288 & C & -7.139329 & -3.648651 & 8.855600 \\
\hline H & -6.792897 & 3.608126 & 1.110562 & C & -6.593579 & -4.640594 & 10.080900 \\
\hline H & -6.923874 & 4.716016 & $-\odot .267693$ & H & -3.107982 & -5.275002 & 11.348200 \\
\hline $\mathrm{H}$ & -6.187479 & 3.118400 & -0.483869 & H & -2.569333 & -6.081163 & 0.283450 \\
\hline H & -2.350535 & $\odot .482889$ & 4.878164 & H & -4.196343 & -3.124344 & 0.840317 \\
\hline $\mathrm{H}$ & -3.489685 & 1.667614 & 5.532783 & H & -3.014427 & -1.850789 & 0.566455 \\
\hline H & -3.743912 & -0.057351 & 5.830283 & C & -2.461200 & $-\odot .843512$ & -2.200235 \\
\hline $\mathrm{H}$ & -6.047485 & -0.187198 & 4.768001 & H & -3.760862 & -2.184266 & -3.171586 \\
\hline
\end{tabular}




$\begin{array}{rrrr}\mathrm{H} & -2.374950 & -2.975945 & -2.470804 \\ \mathrm{H} & -7.077414 & -2.808942 & 1.196248 \\ \mathrm{C} & -7.868359 & -4.793127 & 0.840878 \\ \mathrm{C} & -7.325290 & -5.771165 & -1.294984 \\ \mathrm{H} & -6.114248 & -4.567147 & -2.611367 \\ \mathrm{C} & -2.799249 & 0.176636 & -1.385107 \\ \mathrm{H} & -1.765681 & -0.658043 & -3.019116 \\ \mathrm{H} & -8.369694 & -4.845492 & 1.803662 \\ \mathrm{C} & -7.974597 & -5.867276 & -0.051327 \\ \mathrm{H} & -7.404399 & -6.591699 & -2.003658 \\ \mathrm{H} & -2.443838 & 1.178114 & -1.591532 \\ \mathrm{H} & -3.559750 & 0.078408 & -0.616880 \\ \mathrm{C} & -8.789430 & -7.089992 & 0.294015 \\ \mathrm{H} & -8.269140 & -8.011226 & 0.009575 \\ \mathrm{H} & -9.748004 & -7.082900 & -0.240484 \\ \mathrm{H} & -9.009101 & -7.138071 & 1.364590\end{array}$

24

B3LYP SCF energy:

B3LYP enthalpy:

B3LYP free energy:

-5535.35529402 a.u.

M06 SCF energy in solution:

a.u.

M06 enthalpy in solution:

M06 free energy in solution:

-5533.319739 a.u. -5533.600157 a.u

$-5534.55815975$ a.u

Cartesian coordinates

\begin{tabular}{|c|c|c|c|}
\hline ATOM & X & $\mathrm{Y}$ & Z \\
\hline $\mathrm{P}$ & $\odot . ๑ \odot \odot \odot \odot \odot$ & -6.596128 & -0.903653 \\
\hline$P$ & $\odot .00000 \odot$ & -7.662672 & ๑. 183311 \\
\hline 0 & $\odot . \odot \odot \odot \odot \odot \diamond$ & -11.711183 & -3.169437 \\
\hline 0 & $\odot .0 \odot \odot \odot \odot \odot$ & -11.393624 & -1.063481 \\
\hline 0 & $\odot .00000 \odot$ & -10.729810 & ०. 969726 \\
\hline 0 & $\odot . \odot \odot \odot \odot \odot \diamond$ & -6.680453 & -0.144113 \\
\hline 0 & 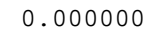 & -6.069452 & -1.816692 \\
\hline 0 & $\odot .0 \odot \odot ० \odot \odot$ & -12.854327 & -0.741101 \\
\hline 0 & $\odot .00000 \odot$ & -13.121700 & 0.214077 \\
\hline 0 & $\odot . \diamond \odot \odot \odot \odot \odot$ & -13.090935 & -0.659071 \\
\hline C & $\odot .0 \odot \odot \odot \odot \odot$ & -13.499283 & -1.518360 \\
\hline C & ๑. .००००० & -2.081632 & -0.064626 \\
\hline C & 5.127299 & -3.088130 & 0.631533 \\
\hline C & 5.561675 & -3.364066 & 2.100631 \\
\hline C & 4.670604 & -4.852866 & 1.693007 \\
\hline C & 3.308558 & -2.800443 & 2.545652 \\
\hline C & 7.358671 & -3.210607 & 2.714783 \\
\hline C & 2.722628 & -5.605106 & 2.283128 \\
\hline C & 2.569021 & -5.103890 & 1.653948 \\
\hline C & 3.144484 & -4.561218 & 0.275440 \\
\hline C & 3.880504 & -4.853472 & ๑. 222196 \\
\hline C & 4.037513 & -5.025763 & -0.997617 \\
\hline C & 3.479658 & -4.466383 & -1.859605 \\
\hline C & 5.327004 & -4.800554 & -0.920911 \\
\hline C & $\odot .784742$ & 2.626077 & -1.296695 \\
\hline C & -0.232694 & 2.260771 & -2.175930 \\
\hline C & -0.539277 & 3.008698 & -3.325296 \\
\hline C & 0.167446 & 4.222962 & -3.493853 \\
\hline C & 1. 308569 & 4.552736 & -2.709465 \\
\hline C & 1.582757 & 3.735982 & -1.608289 \\
\hline C & 2.274805 & 5.704240 & -3.091856 \\
\hline C & 1.610726 & 7.098625 & -3.000183 \\
\hline C & 2.782147 & 5.472332 & -4.537350 \\
\hline C & 3.512509 & 5.731045 & -2.167724 \\
\hline C & -1.572946 & 2.394663 & -4.312453 \\
\hline C & -1.635263 & 3. 054227 & -5.709786 \\
\hline C & -1.170850 & $\odot .916208$ & -4.571968 \\
\hline C & -2.986156 & 2.416391 & -3.682629 \\
\hline C & -1.342231 & 5.921819 & -4.137501 \\
\hline C & ๑. 268786 & 2.701713 & 1.576350 \\
\hline C & $-\odot . ๑ 8 \odot \odot 87$ & 4.042396 & 1.372809 \\
\hline C & -0.051257 & 2.104607 & 2.798506 \\
\hline C & -0.637141 & 2.816282 & 3.859149 \\
\hline C & -0.820133 & 4.208830 & 3.659918 \\
\hline
\end{tabular}

5532.522605 a.u

$-5532.803023$

\begin{tabular}{|c|c|c|}
\hline$-\odot .647524$ & 4.821165 & 2.387254 \\
\hline-1.132055 & 6.263749 & 2.083965 \\
\hline-0.955990 & 6.616279 & 0.590215 \\
\hline-0.357296 & 7.334083 & 2.889975 \\
\hline$-\odot .195327$ & 5.399689 & 5.612197 \\
\hline-2.644300 & 6.371476 & 2.404124 \\
\hline-1.057097 & $2.00776 \odot$ & 5.121718 \\
\hline 0.193898 & 1.641199 & 5.955691 \\
\hline-2.093082 & 2.710566 & 6.031382 \\
\hline-1.738738 & 0.685197 & 4.673671 \\
\hline 3.198353 & -1.936759 & 0.651014 \\
\hline 4.463147 & -2.313070 & 0.180549 \\
\hline 5.419185 & -2.885428 & 1.024785 \\
\hline 5.042871 & -3.098636 & 2.381039 \\
\hline 3.848833 & -2.569496 & 2.927299 \\
\hline 2.924410 & -2.036995 & 2.013077 \\
\hline 3.485041 & -2.477961 & 4.436525 \\
\hline 4.654627 & -2.719495 & 5.419317 \\
\hline 2.333817 & -3.458516 & 4.765038 \\
\hline 2.996649 & -1.032353 & 4.727748 \\
\hline 7.866031 & -2.385187 & 1.358895 \\
\hline 5.528671 & -5.170135 & 3.411616 \\
\hline 6.849053 & -3.180702 & 0.501984 \\
\hline 7.018867 & -2.724145 & -0.964123 \\
\hline-0.193734 & -5.263477 & -1.954850 \\
\hline-0.884347 & -4.027490 & -2.060107 \\
\hline-3.172708 & -4.749515 & -1.349335 \\
\hline-0.180617 & -2.884958 & -1.655348 \\
\hline 1.100865 & -2.956296 & -1.085867 \\
\hline 1.655548 & -4.214782 & $-\odot .830358$ \\
\hline 1.020640 & -5.397935 & -1.228992 \\
\hline$-\odot .367343$ & -6.622820 & -3.878927 \\
\hline-2.773723 & -4.435574 & -3.817612 \\
\hline 2.004493 & -7.712952 & -1.888317 \\
\hline 1.566980 & -6.769386 & -0.743196 \\
\hline 2.794771 & -6.589864 & 0.178190 \\
\hline-2.895970 & -2.466142 & -2.319554 \\
\hline 7.206821 & -4.685518 & 0.547222 \\
\hline-2.396016 & -3.924333 & -2.408274 \\
\hline 0.463119 & -7.466234 & ๑. 092515 \\
\hline 5.001577 & 2.844776 & 3. 023917 \\
\hline 2.587220 & 2.711751 & 2.458536 \\
\hline 8.127110 & $\odot .675933$ & ๑. 029213 \\
\hline 7.726356 & 2.320181 & -0.616210 \\
\hline 1.995456 & -2.057090 & -3.313934 \\
\hline 3.025980 & -0.995059 & -5.320035 \\
\hline 5.247787 & 3.267629 & -4.720114 \\
\hline 6.368614 & 1.921310 & -4.260450 \\
\hline$-\odot .783142$ & 1.350193 & -1.963943 \\
\hline 2.427999 & 3.963037 & -0.972063 \\
\hline 1.146239 & 7.256070 & -2.019298 \\
\hline 2.371786 & 7.876495 & -3.133836 \\
\hline$\odot .856154$ & 7.244462 & -3.773164 \\
\hline 1.963865 & 5.497306 & -5.259281 \\
\hline 3.505676 & 6.250362 & -4.809553 \\
\hline 3.280803 & 4.499566 & -4.618298 \\
\hline 4.059836 & 4.782663 & -2.182742 \\
\hline 4.195100 & 6.515484 & -2.511982 \\
\hline 3.250500 & 5.965106 & -1.128915 \\
\hline-2.250882 & 2.421721 & -6.359904 \\
\hline-2.089507 & 4. 045157 & -5.705152 \\
\hline-0.644240 & 3.138693 & -6.163765 \\
\hline-1.871023 & $\odot .466087$ & -5.284709 \\
\hline$-\odot .163753$ & 0.854453 & -4.998968 \\
\hline-1.188643 & $\odot .302946$ & -3.666890 \\
\hline-3.010357 & 1.869356 & -2.732676 \\
\hline-3.328388 & 3.439573 & -3.489820 \\
\hline-3.710313 & 1.943384 & -4.356309 \\
\hline-1.154147 & 6.507963 & -3.230595 \\
\hline-1.501556 & 6.596674 & -4.981466 \\
\hline-2.240870 & 5.314327 & -3.985929 \\
\hline ๑.096964 & 4.482275 & 0.402119 \\
\hline 0.152127 & 1.045495 & 2.913692 \\
\hline-1.367930 & 7.615030 & 0.411128 \\
\hline ๑. 097782 & 6.639125 & 0.289140 \\
\hline
\end{tabular}

(1)

C

C

C

C

$c$
$c$
$c$

c

c

C

C

c

$c$
$c$

c

C

H

$\mathrm{H}$
$\mathrm{H}$
$\mathrm{H}$
$\mathrm{H}$

H



H

H

H

H

H

H

H

H

H

H

$\mathrm{H}$
$\mathrm{H}$
$\mathrm{H}$

H

H

H

H

H

$\mathrm{H}$
$\mathrm{H}$ 


\begin{tabular}{|c|c|c|c|}
\hline $\mathrm{H}$ & -1.486699 & 5.918496 & -0.067432 \\
\hline $\mathrm{H}$ & -0.606869 & 7.308330 & 3.950546 \\
\hline $\mathrm{H}$ & ๑. 726515 & 7.210806 & 2.778338 \\
\hline $\mathrm{H}$ & -0.616644 & 8.332114 & 2.517729 \\
\hline $\mathrm{H}$ & ๑. 168196 & 4.547067 & 6.194044 \\
\hline $\mathrm{H}$ & 0.648931 & 5.856360 & 5.084447 \\
\hline $\mathrm{H}$ & -0.641486 & 6.131739 & 6.288940 \\
\hline $\mathrm{H}$ & -2.848464 & 6.155481 & 3.454399 \\
\hline $\mathrm{H}$ & -2.999672 & 7.385883 & 2.186658 \\
\hline $\mathrm{H}$ & -3.224014 & 5.675565 & 1.785427 \\
\hline $\mathrm{H}$ & ๑. 910922 & 1. 060819 & 5.365435 \\
\hline $\mathrm{H}$ & ๑. 713723 & 2.530043 & 6.327804 \\
\hline $\mathrm{H}$ & -0.094396 & 1. 037026 & 6.824274 \\
\hline $\mathrm{H}$ & -2.459229 & 1.982274 & 6.764278 \\
\hline $\mathrm{H}$ & -1.685441 & 3.551505 & 6.590771 \\
\hline $\mathrm{H}$ & -2.953009 & 3.073715 & 5.460229 \\
\hline $\mathrm{H}$ & -2.630333 & ๑. 888557 & 4.068640 \\
\hline $\mathrm{H}$ & -1.081346 & $\odot .028388$ & 4.097888 \\
\hline $\mathrm{H}$ & -2.057631 & $\odot .124797$ & 5.559251 \\
\hline $\mathrm{H}$ & 4.694096 & -2.161388 & -0.865619 \\
\hline $\mathrm{H}$ & 1.962395 & -1.679832 & 2.366162 \\
\hline $\mathrm{H}$ & 4.976595 & -3.759447 & 5.467724 \\
\hline $\mathrm{H}$ & 5.526329 & -2.107330 & 5.171823 \\
\hline $\mathrm{H}$ & 4.320869 & -2.439661 & 6.425421 \\
\hline $\mathrm{H}$ & 2.039762 & -3.359058 & 5.816864 \\
\hline $\mathrm{H}$ & 1.449070 & -3.262174 & 4.148530 \\
\hline $\mathrm{H}$ & 2.627791 & -4.500668 & 4.599217 \\
\hline $\mathrm{H}$ & 2.765670 & $-\odot .932038$ & 5.794205 \\
\hline $\mathrm{H}$ & 3.770516 & $-\odot .298670$ & 4.475867 \\
\hline $\mathrm{H}$ & 2.091636 & -0.769585 & 4.172917 \\
\hline $\mathrm{H}$ & 8.882081 & -2.541916 & ๑. 976511 \\
\hline $\mathrm{H}$ & 7.649438 & -1.310734 & 1. 321942 \\
\hline $\mathrm{H}$ & 7.841383 & -2.700219 & 2.403899 \\
\hline $\mathrm{H}$ & 4.588791 & -5.233595 & 3.969598 \\
\hline $\mathrm{H}$ & 5.418188 & -5.718243 & 2.469270 \\
\hline $\mathrm{H}$ & 6.330056 & -5.618467 & 4.003161 \\
\hline $\mathrm{H}$ & 6.383216 & -3.294638 & -1.651809 \\
\hline $\mathrm{H}$ & 6.800910 & -1.659195 & -1.096274 \\
\hline $\mathrm{H}$ & 8.057047 & -2.893075 & -1.269920 \\
\hline $\mathrm{H}$ & -2.954968 & -4.387264 & -0.337249 \\
\hline $\mathrm{H}$ & -4.252471 & -4.654032 & -1.511136 \\
\hline $\mathrm{H}$ & -2.906469 & -5.808472 & -1.399449 \\
\hline $\mathrm{H}$ & -0.633083 & -1.906496 & -1.780060 \\
\hline $\mathrm{H}$ & 2.596519 & -4.266616 & -0.302146 \\
\hline $\mathrm{H}$ & -0.867068 & -7.544128 & -4.186219 \\
\hline $\mathrm{H}$ & -0.681966 & -5.802146 & -4.528743 \\
\hline $\mathrm{H}$ & $\odot .715514$ & -6.746963 & -3.965362 \\
\hline $\mathrm{H}$ & -3.816949 & -4.172890 & -4.026261 \\
\hline $\mathrm{H}$ & -2.156116 & -3.971769 & -4.596256 \\
\hline $\mathrm{H}$ & -2.691717 & -5.519781 & -3.895890 \\
\hline $\mathrm{H}$ & 1.151551 & -8.114596 & -2.435611 \\
\hline $\mathrm{H}$ & 2.675902 & -7.209116 & -2.593810 \\
\hline $\mathrm{H}$ & 2.549089 & -8.566558 & -1.468214 \\
\hline $\mathrm{H}$ & 3.101276 & -7.572437 & 0.552234 \\
\hline $\mathrm{H}$ & 3.653369 & -6.159275 & -0.351068 \\
\hline $\mathrm{H}$ & 2.573860 & -5.961286 & 1.047760 \\
\hline $\mathrm{H}$ & -2.718065 & -2.025017 & -1.332879 \\
\hline $\mathrm{H}$ & -2.426731 & -1.823458 & -3.075225 \\
\hline $\mathrm{H}$ & -3.976200 & -2.442962 & -2.489204 \\
\hline $\mathrm{H}$ & 6.442458 & -5.292075 & 0.046511 \\
\hline $\mathrm{H}$ & 8.155521 & -4.852487 & $\odot .023558$ \\
\hline $\mathrm{H}$ & 7. 329811 & -5.047389 & 1.567836 \\
\hline $\mathrm{H}$ & ๑. 831311 & -8.429430 & $\odot .466115$ \\
\hline $\mathrm{H}$ & 0.183085 & -6.854601 & ๑. 958123 \\
\hline $\mathrm{H}$ & -0.431673 & -7.651435 & -0.505377 \\
\hline $\mathrm{Rh}$ & ๑. 058581 & $-\odot .327300$ & ๑. 256635 \\
\hline 0 & -6.200134 & -1.406072 & -1.343651 \\
\hline S & -6.943759 & -2.054685 & -0.252161 \\
\hline $\mathrm{N}$ & -6.491723 & -1.204615 & 1.168405 \\
\hline 0 & -6.815172 & -3.506428 & -0.058976 \\
\hline C & -8.680046 & -1.657250 & -0.395923 \\
\hline C & -7.096731 & -1.770996 & 2.402995 \\
\hline C & -9.621879 & -2.682540 & $-\odot .3 \odot 4259$ \\
\hline C & -9.068320 & -0.339183 & -0.656630 \\
\hline C & -7.134948 & -0.737559 & 3. 494587 \\
\hline
\end{tabular}

\begin{tabular}{|c|c|c|c|}
\hline $\mathrm{H}$ & -8.122040 & -2.067488 & 2.155295 \\
\hline $\mathrm{H}$ & -6.565914 & -2.675753 & 2.729812 \\
\hline $\mathrm{H}$ & -9.295357 & -3.699354 & -0.114219 \\
\hline C & -10.973145 & -2.375107 & -0.465934 \\
\hline C & -10.420433 & -0.053220 & -0.812469 \\
\hline $\mathrm{H}$ & -8.322867 & $\odot .444959$ & -0.736501 \\
\hline C & -6.596128 & -0.903653 & 3.569100 \\
\hline $\mathrm{H}$ & -7.662672 & ๑. 183311 & 7.306400 \\
\hline $\mathrm{H}$ & -11.711183 & -3.169437 & 8.656300 \\
\hline C & -11.393624 & -1.063481 & -0.722463 \\
\hline $\mathrm{H}$ & -10.729810 & ๑. 969726 & -1.011509 \\
\hline $\mathrm{H}$ & -6.680453 & -0.144113 & 5.475899 \\
\hline $\mathrm{H}$ & -6.069452 & -1.816692 & 4.974341 \\
\hline C & -12.854327 & -0.741101 & -0.924330 \\
\hline $\mathrm{H}$ & -13.121700 & $\odot .214077$ & -0.459876 \\
\hline $\mathrm{H}$ & -13.090935 & -0.659071 & -1.993184 \\
\hline $\mathrm{H}$ & -13.499283 & -1.518360 & -0.503840 \\
\hline 0 & -2.081632 & $-\odot .064626$ & ๑. 612426 \\
\hline C & -3.088130 & $\odot .631533$ & 0.637574 \\
\hline C & -3.364066 & 2.100631 & ๑. 906495 \\
\hline C & -4.852866 & 1.693007 & 1.148666 \\
\hline $\mathrm{H}$ & -2.800443 & 2.545652 & 1.731323 \\
\hline $\mathrm{H}$ & -3.210607 & 2.714783 & ๑. 010946 \\
\hline $\mathrm{H}$ & -5.605106 & 2.283128 & 0.623071 \\
\hline $\mathrm{H}$ & -5.103890 & 1.653948 & 2. 212273 \\
\hline C & -4.561218 & $\odot .275440$ & 0.557530 \\
\hline $\mathrm{H}$ & -4.853472 & ๑. 222196 & -0.498962 \\
\hline C & -5.025763 & -0.997617 & 1.263256 \\
\hline $\mathrm{H}$ & -4.466383 & -1.859605 & ๑. 876124 \\
\hline $\mathrm{H}$ & -4.800554 & -0.920911 & 2.330930 \\
\hline
\end{tabular}

25

B3LYP SCF energy:

B3LYP enthalpy:

B3LYP free energy:

M०6 SCF energy in solution

a.u.

M06 enthalpy in solution:

M०6 free energy in solution:

a.u.

Cartesian coordinates

$\begin{array}{lrrr}\text { ATOM } & X & Y & Z \\ \text { P } & -1.490753 & 1.691610 & -0.378148 \\ \text { P } & -1.154058 & -1.578223 & 0.129646 \\ 0 & -5.524835 & 0.661455 & 2.074764 \\ 0 & -5.240924 & -0.147363 & 4.226708 \\ 0 & -5.887214 & -1.283132 & -0.360686 \\ \text { C } & -6.638455 & -0.548099 & -2.425040 \\ \text { C } & -2.411883 & -1.227430 & 1.427430 \\ \text { C } & -3.522629 & -0.392959 & 1.095222 \\ \text { C } & -4.394409 & -0.106943 & 2.132982 \\ \text { C } & -4.226729 & -0.591621 & 3.430504 \\ \text { C } & -3.172930 & -1.415032 & 3.760242 \\ \text { C } & -2.266535 & -1.721133 & 2.729436 \\ \text { C } & -6.038554 & 0.722987 & 3.412276 \\ \text { C } & -3.113345 & 1.077164 & -1.017071 \\ \text { C } & -3.594460 & 1.517626 & -2.256794 \\ \text { C } & -4.783172 & 1.032142 & -2.829550 \\ \text { C } & -5.470146 & 0.080243 & -2.109705 \\ \text { C } & -5.014208 & -0.358371 & -0.866619 \\ \text { C } & -3.851716 & 0.104717 & -0.271426 \\ \text { C } & -6.892217 & -1.479723 & -1.364550 \\ \text { C } & -2.063760 & -2.564271 & -1.131536 \\ \text { C } & -1.721381 & -2.435062 & -2.486199 \\ \text { C } & -3.084223 & -3.456496 & -0.762013\end{array}$

-3819.34404382 a.u. -3818.398942 a.u. -3818.560333 a.u $-3819.31187705$

-3818.366775 a.u. $-3818.528166$ 


\begin{tabular}{|c|c|c|c|}
\hline C & ๑. .0०7679 & -2.777928 & ๑. 922421 \\
\hline C & ๑. 092163 & -4.120346 & ๑. 524974 \\
\hline C & 0.866890 & -2.316657 & 1. 937091 \\
\hline C & -1.901349 & 2.382672 & 1.278932 \\
\hline C & -3.067575 & 3.144834 & 1.467187 \\
\hline C & -1.043381 & 2.165929 & 2.365872 \\
\hline C & -0.701036 & 2.956319 & -2.759901 \\
\hline C & -1.110416 & 3.166503 & -1.428608 \\
\hline C & -1.170276 & 4.481173 & -0.943286 \\
\hline $\mathrm{H}$ & -3.048968 & -1.810222 & 4.762108 \\
\hline $\mathrm{H}$ & -1.434888 & -2.371339 & 2.967712 \\
\hline $\mathrm{H}$ & -5.955739 & 1.749758 & 3.787228 \\
\hline $\mathrm{H}$ & -7.076955 & ๑. 379379 & 3.421117 \\
\hline $\mathrm{H}$ & -3.048772 & 2.273369 & -2.805572 \\
\hline $\mathrm{H}$ & -5.140997 & 1.393755 & -3.786867 \\
\hline $\mathrm{H}$ & -6.823067 & -2.502186 & -1.753707 \\
\hline $\mathrm{H}$ & -7.879772 & -1.283708 & -0.936681 \\
\hline $\mathrm{H}$ & -0.935012 & -1.746010 & -2.777855 \\
\hline $\mathrm{H}$ & -3.368450 & -3.556347 & 0. 281347 \\
\hline $\mathrm{H}$ & -0.559657 & -4.499258 & -0.254818 \\
\hline $\mathrm{H}$ & ๑. 820414 & -1.276883 & 2. 249541 \\
\hline $\mathrm{H}$ & -3.752875 & 3.305436 & ๑. 639907 \\
\hline $\mathrm{H}$ & -0.153930 & 1.558881 & 2.229411 \\
\hline $\mathrm{H}$ & -0.631805 & 1.943085 & -3.148104 \\
\hline $\mathrm{H}$ & -1.470557 & 4.674417 & ๑. 080333 \\
\hline $\mathrm{Rh}$ & 0.202076 & ๑.190835 & -0.601321 \\
\hline 0 & 1.382466 & -1.387733 & -1.730272 \\
\hline 0 & 5.251527 & 2.750282 & -0.440168 \\
\hline C & 2.447321 & -1.978853 & -1.634787 \\
\hline S & 5.139129 & 1.559658 & $\odot .406141$ \\
\hline C & 3.266058 & -2.794809 & -2.623497 \\
\hline C & 3.547419 & -1.988788 & -0.589289 \\
\hline $\mathrm{N}$ & 4. 227978 & ๑. 429489 & -0.507902 \\
\hline 0 & 4.584558 & 1.644742 & 1.765620 \\
\hline C & 6.737603 & 0.765469 & $\odot .525495$ \\
\hline C & 4.505540 & -2.477617 & -1.723953 \\
\hline $\mathrm{H}$ & 2.982893 & -3.853872 & -2.608567 \\
\hline $\mathrm{H}$ & 3. 258914 & -2.444520 & -3.659944 \\
\hline C & 3. 801762 & -0.746276 & 0. 260927 \\
\hline $\mathrm{H}$ & 3.334059 & -2.816906 & 0.101636 \\
\hline C & 3.368500 & ๑. 990710 & -1.558947 \\
\hline C & 7.181772 & ๑. 294504 & 1.760847 \\
\hline C & 7.544790 & 0.676013 & -0.614021 \\
\hline $\mathrm{H}$ & 5.113088 & -1.661960 & -2.121669 \\
\hline $\mathrm{H}$ & 5.148660 & -3.322806 & -1.474158 \\
\hline $\mathrm{H}$ & 4. 601081 & -0.981622 & $\odot .969589$ \\
\hline $\mathrm{H}$ & $2.90 \odot 452$ & -0.547320 & $\odot .854352$ \\
\hline C & 2.036033 & 1.620743 & -1.185174 \\
\hline $\mathrm{H}$ & 3.951773 & 1.788814 & -2.029620 \\
\hline $\mathrm{H}$ & 3.241572 & ๑. 228191 & -2.334347 \\
\hline $\mathrm{H}$ & 6.551428 & $\odot .400129$ & 2.637201 \\
\hline C & 8.446884 & -0.289542 & 1.847784 \\
\hline C & 8.801767 & ๑. 091757 & -0.506429 \\
\hline $\mathrm{H}$ & 7.197967 & 1.071591 & -1.563188 \\
\hline C & 1.614516 & 1.907419 & ๑. 091263 \\
\hline $\mathrm{H}$ & 1.593857 & 2.173576 & -2.011192 \\
\hline $\mathrm{H}$ & 8.798070 & $-\odot .656022$ & 2.808733 \\
\hline C & 9.274457 & -0.399718 & 0.723470 \\
\hline $\mathrm{H}$ & 9.434367 & ๑. 022596 & -1.387805 \\
\hline $\mathrm{H}$ & $\odot .951295$ & 2.745506 & ๑. 267721 \\
\hline $\mathrm{H}$ & 2.180573 & 1.575746 & ๑. 956764 \\
\hline C & 10.654001 & -1.003484 & $\odot .826672$ \\
\hline $\mathrm{H}$ & 10.854440 & -1.688274 & $-0.0 \odot 4779$ \\
\hline $\mathrm{H}$ & 11.424468 & -0.222577 & 0.793840 \\
\hline $\mathrm{H}$ & 10.783001 & -1.555194 & 1.762427 \\
\hline C & -0.840043 & 5.557866 & -1.771282 \\
\hline $\mathrm{H}$ & -0.887095 & 6.568810 & -1.376855 \\
\hline C & $-\odot .453421$ & 5.339256 & -3.092979 \\
\hline $\mathrm{H}$ & -0.200755 & 6.178653 & -3.734201 \\
\hline C & $-\odot .386846$ & 4.033723 & -3.587626 \\
\hline $\mathrm{H}$ & -0.084090 & 3.853614 & -4.615362 \\
\hline C & -1.336478 & 2.710748 & 3. 617607 \\
\hline $\mathrm{H}$ & -0.664471 & 2.535812 & 4.452884 \\
\hline C & -2.489307 & 3.477594 & 3.793990 \\
\hline $\mathrm{H}$ & -2.714402 & 3.905707 & 4.766744 \\
\hline
\end{tabular}

$\begin{array}{lrrr}\mathrm{C} & -3.354940 & 3.692764 & 2.717329 \\ \mathrm{H} & -4.253106 & 4.289874 & 2.849691 \\ \mathrm{C} & -3.743496 & -4.210730 & -1.733377 \\ \mathrm{H} & -4.526899 & -4.903135 & -1.437350 \\ \mathrm{C} & -3.396511 & -4.077373 & -3.080796 \\ \mathrm{H} & -3.913152 & -4.663340 & -3.835837 \\ \mathrm{C} & -2.386202 & -3.189298 & -3.455161 \\ \mathrm{H} & -2.117348 & -3.079676 & -4.502052 \\ \mathrm{C} & 1.007868 & -4.983334 & 1.134762 \\ \mathrm{H} & 1.057287 & -6.021720 & 0.818873 \\ \mathrm{C} & 1.842101 & -4.521191 & 2.154148 \\ \mathrm{H} & 2.541885 & -5.197963 & 2.636321 \\ \mathrm{C} & 1.767204 & -3.183939 & 2.557087 \\ \mathrm{H} & 2.408548 & -2.816099 & 3.353413\end{array}$

26

B3LYP SCF energy: $\quad-3819.35251902 \mathrm{a} . \mathrm{u}$ B3LYP enthalpy: $\quad-3818.408097$ a.u. B3LYP free energy: $\quad-3818.572454 \mathrm{a} . \mathrm{u}$. M06 SCF energy in solution: $\quad-3819.30776988$ a.u. M06 enthalpy in solution: $\quad-3818.363348$ a.u. M06 free energy in solution: $\quad-3818.527705$ a.u.

Cartesian coordinates

\begin{tabular}{|c|c|c|c|}
\hline ATOM & $\mathrm{X}$ & Y & Z \\
\hline$P$ & $\odot .489389$ & -1.458742 & $\odot .169023$ \\
\hline$P$ & 1.670542 & 1.581925 & $-\odot .337184$ \\
\hline 0 & 5.034893 & -1.877435 & 1.842918 \\
\hline 0 & 5.604259 & -0.516415 & 3.628170 \\
\hline 0 & 5.630320 & -1.037799 & -1.103645 \\
\hline 0 & 5.526927 & -2.550516 & -2.853028 \\
\hline C & 2.922409 & 1. $0 \odot 4838$ & 0.886255 \\
\hline C & 3. 490754 & $-\odot .298569$ & 0.743744 \\
\hline C & 4.380055 & $-\odot .678963$ & 1.736082 \\
\hline C & 4.722187 & 0.134775 & 2.815320 \\
\hline C & 4.193443 & 1.398535 & 2.958473 \\
\hline C & 3.285240 & 1.815230 & 1.969520 \\
\hline C & 5.894284 & -1.764455 & 2.984143 \\
\hline C & 2.049672 & -1.899891 & -0.720954 \\
\hline C & 2.014178 & -2.822612 & -1.773773 \\
\hline C & 3.138157 & -3.120149 & -2.564535 \\
\hline C & 4.300694 & -2.448034 & -2.259842 \\
\hline C & 4.358255 & -1.538103 & -1.204972 \\
\hline C & 3.271249 & -1.228064 & -0.402420 \\
\hline C & 6.353328 & -1.562992 & -2.224569 \\
\hline C & 2.590698 & 1.667261 & -1.930240 \\
\hline C & 1.897431 & 1.444139 & -3.129453 \\
\hline C & 3.957694 & 1.986450 & -1.975189 \\
\hline C & 1.365346 & 3.350847 & ๑.114665 \\
\hline C & 1.842183 & 4.422060 & -0.653413 \\
\hline C & ๑. 592287 & 3.628740 & 1.256904 \\
\hline C & ๑. 838686 & -1.835330 & 1.936667 \\
\hline C & 1.724354 & -2.859554 & 2. 309881 \\
\hline C & $\odot .164080$ & -1.116574 & 2.933516 \\
\hline C & -1.330022 & -2.648583 & -1.606691 \\
\hline C & $-\odot .708807$ & -2.766563 & -0.350636 \\
\hline C & -1.018679 & -3.869740 & $\odot .457521$ \\
\hline $\mathrm{H}$ & 4.464958 & 2.040825 & 3.788563 \\
\hline $\mathrm{H}$ & 2.867010 & 2.809503 & 2.057892 \\
\hline $\mathrm{H}$ & 5.690533 & -2.586667 & 3.676240 \\
\hline $\mathrm{H}$ & 6.941204 & -1.772065 & 2.657777 \\
\hline $\mathrm{H}$ & 1.089964 & -3.338601 & -1.999614 \\
\hline $\mathrm{H}$ & 3.090321 & -3.842777 & -3.371342 \\
\hline $\mathrm{H}$ & 6.560157 & -0.753566 & -2.935987 \\
\hline $\mathrm{H}$ & 7.277960 & -2.030788 & -1.875820 \\
\hline $\mathrm{H}$ & ๑. 842179 & 1.188360 & -3.094687 \\
\hline $\mathrm{H}$ & 4.509132 & 2.150933 & -1.054189 \\
\hline $\mathrm{H}$ & 2.436770 & 4.233920 & -1.540647 \\
\hline $\mathrm{H}$ & ๑. 212922 & 2.805902 & 1.857656 \\
\hline $\mathrm{H}$ & 2.262534 & -3.418067 & 1.549854 \\
\hline $\mathrm{H}$ & $-\odot .525608$ & $-\odot .329264$ & 2.646433 \\
\hline
\end{tabular}




\begin{tabular}{|c|c|c|c|}
\hline $\mathrm{H}$ & -1.115046 & -1.787210 & -2.232853 \\
\hline $\mathrm{H}$ & $-\odot .562839$ & -3.975460 & 1.435540 \\
\hline $\mathrm{Rh}$ & -0.337395 & $\odot .568329$ & -0.415837 \\
\hline 0 & -1.266726 & 2.294437 & -1.476733 \\
\hline 0 & -2.552203 & ๑ . 017587 & -0.516847 \\
\hline C & -2.222277 & 2.985342 & -1.170838 \\
\hline S & -3.557986 & -0.276238 & ๑. 541594 \\
\hline C & -3.172253 & 3.836243 & -2.000000 \\
\hline C & -2.961321 & 3.302055 & ๑. 129597 \\
\hline $\mathrm{N}$ & -4.225944 & 1.241166 & $\odot .934594$ \\
\hline 0 & -3.126051 & -1.013444 & 1.734689 \\
\hline C & -4.918581 & -1.132340 & -0.226408 \\
\hline C & -4.108726 & 3.886291 & -0.752900 \\
\hline $\mathrm{H}$ & -2.730045 & 4.803147 & -2.267408 \\
\hline $\mathrm{H}$ & -3.549618 & 3.363764 & -2.912420 \\
\hline C & -3.195512 & 2. 271895 & 1. 236285 \\
\hline $\mathrm{H}$ & -2.391135 & 4.127111 & 0.584325 \\
\hline C & $-5.30 \odot 844$ & 1.143436 & 1.967418 \\
\hline C & -5.362398 & -2.325707 & 0.344218 \\
\hline C & -5.484043 & $-\odot .632281$ & -1.404680 \\
\hline $\mathrm{H}$ & -4.942846 & 3.187388 & -0.839770 \\
\hline $\mathrm{H}$ & -4.483798 & 4.869515 & -0.464386 \\
\hline $\mathrm{H}$ & -3.545124 & 2.802440 & 2.125447 \\
\hline H & -2.234869 & 1.808089 & 1.496763 \\
\hline C & -6.183545 & 2.359339 & 1.944160 \\
\hline $\mathrm{H}$ & -5.899643 & ๑. 257993 & 1.727762 \\
\hline H & -4.868826 & ๑. 989803 & 2.965353 \\
\hline H & -4.894390 & -2.702376 & 1.247036 \\
\hline C & -6.402071 & -3.019715 & -0.274898 \\
\hline C & -6.519726 & -1.340621 & -2.003465 \\
\hline $\mathrm{H}$ & -5.116761 & ๑. 288562 & -1.844726 \\
\hline C & -6.392387 & 3.146336 & 3. $0006 \odot 4$ \\
\hline $\mathrm{H}$ & -6.688233 & 2.561219 & 1.000466 \\
\hline $\mathrm{H}$ & -6.752792 & -3.950107 & 0.163043 \\
\hline C & -6.998876 & -2.540867 & -1.448746 \\
\hline $\mathrm{H}$ & -6.964570 & $-\odot .960487$ & -2.919387 \\
\hline H & - $7.07 \odot \odot 86$ & 3. 993933 & 2. 955229 \\
\hline $\mathrm{H}$ & -5.909760 & 2.960742 & 3.958110 \\
\hline C & -8.140915 & -3.281799 & -2.099538 \\
\hline $\mathrm{H}$ & -9.097621 & -2.788308 & -1.885057 \\
\hline H & -8.029778 & -3.309708 & -3.188816 \\
\hline $\mathrm{H}$ & -8.211991 & -4.310920 & -1.736072 \\
\hline C & 1.557529 & 5.740416 & -0.285042 \\
\hline H & 1.934416 & 6.558998 & -0.891844 \\
\hline C & 0.803344 & 6.006315 & $\odot .857827$ \\
\hline $\mathrm{H}$ & 0.593247 & 7.032107 & 1.147437 \\
\hline C & ๑. 323764 & 4.945332 & 1.632574 \\
\hline $\mathrm{H}$ & -0.253612 & 5.144206 & 2.531965 \\
\hline C & ๑. 369943 & -1.415837 & 4.281167 \\
\hline $\mathrm{H}$ & $-\odot .158181$ & -0.853280 & 5.046089 \\
\hline C & 1.251319 & -2.435449 & 4.645499 \\
\hline $\mathrm{H}$ & 1.411976 & -2.667424 & 5.694731 \\
\hline C & 1.927048 & -3.157079 & 3.658107 \\
\hline $\mathrm{H}$ & 2.611726 & -3.953678 & 3.936588 \\
\hline C & -1.917557 & -4.841680 & ๑. 011669 \\
\hline $\mathrm{H}$ & -2.144842 & -5.692676 & ๑. 647913 \\
\hline C & -2.515380 & -4.725283 & -1.243818 \\
\hline $\mathrm{H}$ & -3.208611 & -5.486580 & -1.590899 \\
\hline C & -2.219474 & -3.625365 & -2.053243 \\
\hline $\mathrm{H}$ & -2.684178 & -3.524630 & -3.030182 \\
\hline C & 2.559669 & 1.541590 & -4.354541 \\
\hline $\mathrm{H}$ & 2.015403 & 1.363761 & -5.277841 \\
\hline C & 3.918259 & 1.862176 & -4.392651 \\
\hline $\mathrm{H}$ & 4.433038 & 1.937464 & -5.346512 \\
\hline C & 4.615700 & 2.084918 & -3.202141 \\
\hline $\mathrm{H}$ & 5.672126 & 2.338560 & -3.227866 \\
\hline
\end{tabular}

27

B3LYP SCF energy: $\quad-3819.34422860$ a.u. B3LYP enthalpy: B3LYP free energy: -3818.398997 a.u. -3818.398997 a.u.
-3818.560037 a.u M06 SCF energy in solution: $\quad-3819.31067244$ a.u.
M०6 enthalpy in solution: M06 free energy in solution:

-3818.365441 a.u. $-3818.526481$

a.u.

Cartesian coordinates

\begin{tabular}{|c|c|c|c|}
\hline ATOM & X & Y & Z \\
\hline$P$ & $\odot .485914$ & -1.622856 & -0.314178 \\
\hline$P$ & 1.785707 & 1.514726 & ๑. 066390 \\
\hline 0 & 4.876697 & -2.452230 & 1.698064 \\
\hline 0 & 5.381072 & -1.504624 & 3.747277 \\
\hline 0 & 5.722515 & -0.910688 & -0.892574 \\
\hline 0 & 5.878492 & -2.164811 & -2.829919 \\
\hline C & 2.912180 & $\odot .645902$ & 1.235294 \\
\hline C & 3.452840 & $-\odot .619409$ & ๑. 859067 \\
\hline C & 4.269585 & -1.227504 & 1.800358 \\
\hline C & 4.572946 & $-\odot .664025$ & 3.038769 \\
\hline C & 4.076721 & ๑. 567032 & 3.406056 \\
\hline C & 3. 240198 & 1.208190 & 2.476622 \\
\hline C & 5.718630 & -2.572920 & 2.852147 \\
\hline C & 2.174362 & -1.969719 & $-\odot .997434$ \\
\hline C & 2.313082 & -2.767570 & -2.140784 \\
\hline C & 3.522199 & -2.908851 & -2.843505 \\
\hline C & 4.598742 & -2.200373 & -2.361112 \\
\hline C & 4.494898 & -1.436810 & -1.198536 \\
\hline C & 3.325296 & -1.299266 & -0.465824 \\
\hline C & 6.572763 & -1.219804 & $-2.0 \odot 4924$ \\
\hline C & 2.890602 & 2.048045 & -1.307582 \\
\hline C & 2. 378281 & 2.161003 & -2.609694 \\
\hline C & 4.238575 & 2.363708 & -1.069665 \\
\hline C & 1.344683 & 3.072011 & ๑. 959410 \\
\hline C & 1.705532 & 4.336477 & ๑. 472947 \\
\hline C & $\odot .596375$ & 2.992065 & 2.148438 \\
\hline C & $\odot .606070$ & -1.977371 & 1. 489186 \\
\hline C & 1.401038 & -3.030283 & 1.969525 \\
\hline C & $-\odot .182042$ & -1.249170 & 2.390838 \\
\hline C & $-\odot .865919$ & -3.260531 & -2.259179 \\
\hline C & -0.621641 & -3.006136 & -0.894844 \\
\hline C & -1.313718 & -3.787467 & ๑. 048080 \\
\hline H & 4.320039 & 1. 021324 & 4.359803 \\
\hline H & 2.853600 & 2.182582 & 2.742789 \\
\hline H & 5.530395 & -3.531570 & 3. 341631 \\
\hline $\mathrm{H}$ & 6.769832 & -2.479488 & 2.551932 \\
\hline $\mathrm{H}$ & 1.467896 & -3.330842 & -2.505784 \\
\hline $\mathrm{H}$ & 3. 599631 & -3.546284 & -3.716911 \\
\hline $\mathrm{H}$ & 6.766956 & -0.305731 & -2.580079 \\
\hline $\mathrm{H}$ & 7.501012 & -1.667654 & -1.642048 \\
\hline $\mathrm{H}$ & 1.338988 & 1.913444 & -2.801503 \\
\hline $\mathrm{H}$ & 4.651286 & 2.271796 & -0.069818 \\
\hline $\mathrm{H}$ & 2.282148 & 4.423242 & -0.441680 \\
\hline H & $\odot .305201$ & 2. 021562 & 2.542138 \\
\hline $\mathrm{H}$ & 2.018106 & -3.603550 & 1.283795 \\
\hline H & -0.807726 & -0.442136 & 2. 019508 \\
\hline H & $-\odot .365492$ & -2.684432 & -3.028455 \\
\hline $\mathrm{H}$ & -1.167208 & -3.622197 & 1.108058 \\
\hline $\mathrm{Rh}$ & $-\odot .225757$ & $\odot .517409$ & -0.810835 \\
\hline 0 & $-\odot .817264$ & 2.585356 & -1.389262 \\
\hline 0 & -5.647535 & 1.943824 & 1.764582 \\
\hline C & -1.632289 & 3.323660 & -0.845045 \\
\hline S & -5.554279 & 1.455476 & ๑. 385336 \\
\hline C & -2.293120 & 4.614499 & -1.291640 \\
\hline C & -2.476571 & 3.226745 & ๑. 410822 \\
\hline $\mathrm{N}$ & -3.893524 & 1.097991 & $\odot .140996$ \\
\hline 0 & -6.061276 & 2.266677 & -0.732717 \\
\hline C & -6.315761 & $-\odot .157603$ & ๑. 266790 \\
\hline C & -3.397224 & 4.324849 & -0.223240 \\
\hline $\mathrm{H}$ & -1.670700 & 5.485173 & -1.053748 \\
\hline H & -2.580877 & 4.667091 & -2.346084 \\
\hline C & -2.941047 & 1.863343 & ๑. 950798 \\
\hline H & -1.877811 & 3.688507 & 1.208667 \\
\hline C & -3.529840 & $\odot .825311$ & -1.253220 \\
\hline C & -7.175049 & -0.432261 & -0.797478 \\
\hline C & -6.091758 & -1.099068 & 1.276563 \\
\hline $\mathrm{H}$ & -4.306870 & 3. 914238 & $-\odot .664551$ \\
\hline $\mathrm{H}$ & -3.659261 & 5.153617 & 0.435807 \\
\hline $\mathrm{H}$ & -3.387186 & 1.996232 & 1.937326 \\
\hline
\end{tabular}




\begin{tabular}{|c|c|c|c|}
\hline $\mathrm{H}$ & -2.046337 & 1.234981 & 1.061059 \\
\hline C & -2.370937 & -0.132299 & -1.435023 \\
\hline $\mathrm{H}$ & -4.395135 & $\odot .341607$ & -1.723280 \\
\hline $\mathrm{H}$ & -3.366224 & 1.749442 & -1.824617 \\
\hline $\mathrm{H}$ & -7.353181 & ๑. 321949 & -1.556297 \\
\hline C & -7.804702 & -1.676306 & -0.854550 \\
\hline C & -6.730001 & -2.332422 & 1. 201950 \\
\hline $\mathrm{H}$ & -5.438035 & -0.863012 & 2.109853 \\
\hline C & -1.520341 & -0.012102 & -2.522994 \\
\hline $\mathrm{H}$ & -2.504438 & -1.100364 & -0.959551 \\
\hline $\mathrm{H}$ & -8.475246 & -1.894676 & -1.681456 \\
\hline C & -7.597093 & -2.641212 & 0.139478 \\
\hline $\mathrm{H}$ & -6.562483 & $-3 . \odot 67385$ & 1.985236 \\
\hline $\mathrm{H}$ & -1.051809 & -0.871008 & -2.980280 \\
\hline $\mathrm{H}$ & -1.567375 & ๑. 877373 & -3.148836 \\
\hline C & -8.312627 & -3.969543 & ๑. 093438 \\
\hline $\mathrm{H}$ & -7.678940 & -4.780627 & $\odot .467620$ \\
\hline $\mathrm{H}$ & -9.213592 & -3.947445 & 0.720247 \\
\hline $\mathrm{H}$ & -8.628044 & -4.221631 & -0.923554 \\
\hline C & -0.174098 & -1.561625 & 3.752114 \\
\hline $\mathrm{H}$ & -0.790440 & -0.992018 & 4.442119 \\
\hline C & 0.622338 & -2.606861 & 4. 222832 \\
\hline $\mathrm{H}$ & ๑. 629801 & -2.851634 & 5.281138 \\
\hline C & 1.408600 & -3.339981 & 3.329458 \\
\hline $\mathrm{H}$ & 2.026038 & -4.157070 & 3. 692031 \\
\hline C & 3.199665 & 2.587020 & -3.655103 \\
\hline $\mathrm{H}$ & 2.793936 & 2.670001 & -4.659394 \\
\hline C & 4.537900 & 2.902068 & -3.411031 \\
\hline $\mathrm{H}$ & 5.176022 & 3.233835 & -4.225309 \\
\hline C & 5.055203 & 2.790464 & -2.117627 \\
\hline $\mathrm{H}$ & 6. 094901 & 3. 038362 & -1.922251 \\
\hline C & 1.333990 & 5.494147 & 1.163934 \\
\hline $\mathrm{H}$ & 1.624783 & 6.465948 & $\odot .774931$ \\
\hline C & $\odot .611001$ & 5.404091 & 2.353953 \\
\hline $\mathrm{H}$ & $\odot .336474$ & 6.304043 & 2.896631 \\
\hline C & $\odot .246244$ & 4.147545 & 2.848180 \\
\hline $\mathrm{H}$ & -0.311352 & 4.067438 & 3.777449 \\
\hline C & -2.196022 & -4.791289 & -0.357056 \\
\hline $\mathrm{H}$ & -2.710565 & -5.381869 & ๑. 395618 \\
\hline C & -2.410445 & -5.040683 & -1.711725 \\
\hline $\mathrm{H}$ & -3.092226 & -5.825842 & -2.025173 \\
\hline C & -1.740760 & -4.269417 & -2.662257 \\
\hline $\mathrm{H}$ & -1.897444 & -4.448115 & -3.722174 \\
\hline
\end{tabular}

28

B3LYP SCF energy: $\quad-3819.34307259$ a.u.

B3LYP enthalpy:

B3LYP free energy:

Mo6 SCF energy in solution:

a.u.

M06 enthalpy in solution:

M06 free energy in solution:

a.u.

Cartesian coordinates

\begin{tabular}{|c|c|c|c|}
\hline ATOM & $\mathrm{x}$ & $\mathrm{Y}$ & Z \\
\hline $\mathrm{P}$ & 1.376045 & -1.526392 & -0.683361 \\
\hline$P$ & 1.471479 & 1.668640 & ๑. 063063 \\
\hline 0 & 4.353602 & -1.599624 & 3.102693 \\
\hline 0 & 3.434053 & $-\odot .860797$ & 5.095740 \\
\hline 0 & 5.993885 & ๑. 223873 & 1.170995 \\
\hline 0 & 7.259940 & -0.708336 & -0.529306 \\
\hline C & 2.098500 & ๑. 936989 & 1.630856 \\
\hline C & 3. 096201 & $-\odot .090728$ & 1.612617 \\
\hline C & 3.450818 & $-\odot .601021$ & 2.851600 \\
\hline C & 2.895490 & $-\odot .164285$ & 4.054596 \\
\hline C & 1.940210 & ๑.827239 & $4.08722 \odot$ \\
\hline C & 1.554112 & 1.366705 & 2.848501 \\
\hline C & 4.420335 & -1.733709 & 4.528521 \\
\hline C & 3.203786 & -1.413919 & -0.618995 \\
\hline C & 3.984408 & -1.979883 & -1.632564 \\
\hline C & 5.378188 & -1.803173 & -1.695546 \\
\hline C & 5.945152 & -1.028908 & -0.706848 \\
\hline C & 5.178670 & -0.469175 & 0.316905 \\
\hline C & 3.806234 & -0.631049 & $\odot .414585$ \\
\hline
\end{tabular}

7.307917

2.938655

2. 963248

4. 009350

$\odot .387713$

0.818164

$-0.923070$

$\odot .855677$

1. 688774

$-0.435740$

0.988414

$\odot .943516$

0.493394

1.507210

0.807095

4.195380

5. 415475

3. 509145

5.975339

7. 596436

8. 014102

2. 138765

4. 009103

1. 823499

$-1.280765$

2. 694778

$-1.084283$

1. 349561

๑. 445554

0.334100

$-0.694515$

$-3.896948$

$-1.884926$

$-4.384725$

$-2.664053$

- 3.140065

$-4.444913$

$-3.654810$

$-6.105951$

- 3.948919

$-2.634521$

$-2.371823$

- 3.201306

- 3.282995

$-5.098339$

$-6.807867$

$-6.709140$

$-4.287004$

$-4.792586$

$-2.301575$

- 3.164493

$-4.214121$

$-5.975377$

$-5.477266$

$-6.324764$

- 8.132330

$-8.039013$

$-6.143014$

$-3.945253$

$-3.797486$

$-8.684707$

$-8.769289$

$-8.514513$

- 3.314792

$-4.358616$

$-10.200181$

$-10.632142$

$-10.264790$

$-10.827055$

$-0.063374$

$-0.417765$

1.227190

1.874686

$-0.895342$

$-1.901063$
๑. 175332

2. 458334

2. 634694

2. 936879

3. 068857

4. 403891

2. 780383

$-2.444206$

- 3.397654

$-2.233507$

$-2.071904$

$-2.622471$

$-3.942002$

1. 174418

2. 150016

$-2.767261$

$-1.436310$

$-2.576441$

- 2.252597

1. 179016

$-0.214587$

2. 262037

2. 800951

4.647902

1.755354

$-3.562685$

$-1.491743$

$-1.054295$

$-4.379645$

๑. 342243

1. 954221

$-1.554430$

2. 231655

$-\odot .272230$

3. 156117

1. 679228

$-\odot .284115$

๑. 958663

$-\odot .106259$

2. 380449

4.194740

3. 137267

๑.166614

2. 183821

$-1.476987$

1. 047126

$-1.105130$

1. 677289

2. 993378

$-0.148406$

$-0.347702$

$-2.689633$

$-1.706079$

$-1.161712$

1.818487

1. 185679

$-0.945908$

$-1.986161$

- 3.265136

$-3.089838$

2. 077106

$\odot .195350$

$-1.722414$

- 4.146639

$-2.886263$

$\odot .374714$

$-0.565567$

1. 099752

$\odot .753637$

$-3.917368$

$-4.490539$

$-4.129270$

$-4.867414$

-2.969659

$-2.795073$
๑. 597865

$\odot .718002$

$-2.110399$

$\odot .054479$

570372

$\odot .538240$

$\odot .989338$

.814814

1. 423063

1. 319906

$-3.396740$

- 2.099440

$-1.934310$

5. 018337

2. 851004

4.808056

4.879183

$-2.402452$

$-2.480830$

๑. 262959

1. 336307

$-2.711559$

1. 131501

0.212118

1.009478

1. 048229

$\odot .864268$

$-3.536739$

$-0.942767$

$-1.326596$

$-2.467362$

1. 237056

$-2.534440$

$\odot .699702$

$-3.451497$

$-1.876024$

$-1.002376$

1. 045993

1. 144520

- 3.016215

- 3.100913

$-4.506192$

$-1.662609$

$-0.913438$

$-1.607812$

๑. 777092

1. 909366

$-3.782683$

$-2.695818$

$-1.108156$

$-2.627851$

$-1.752834$

$-0.992172$

$-2.586753$

$\odot .186607$

1. 175998

2. 300481

2. 191097

$-2.926343$

- 0.830942

$\odot .890018$

1. 944714

2.893423

$-2.999318$

$-3.859876$

2. 389371

2. 743798

3. 210863

1. 574821

3. 012303

3. 864653

2. 518209

2. 983760

2. 412442

2. 781853 


$\begin{array}{lrrr}\mathrm{C} & -0.047135 & 5.429629 & 0.926756 \\ \mathrm{H} & 0.296647 & 6.459827 & 0.897761 \\ \mathrm{C} & -1.343120 & 5.134735 & 1.353960 \\ \mathrm{H} & -2.009816 & 5.936125 & 1.660485 \\ \mathrm{C} & -1.779767 & 3.807236 & 1.386758 \\ \mathrm{H} & -2.783737 & 3.558581 & 1.718010 \\ \mathrm{C} & 0.599985 & -2.830743 & -4.502344 \\ \mathrm{H} & 0.647425 & -2.398202 & -5.497782 \\ \mathrm{C} & 0.161861 & -4.144984 & -4.326645 \\ \mathrm{H} & -0.135196 & -4.738847 & -5.186443 \\ \mathrm{C} & 0.111407 & -4.697461 & -3.043907 \\ \mathrm{H} & -0.221808 & -5.722366 & -2.906018 \\ \mathrm{C} & 4.040028 & 3.279842 & -2.719796 \\ \mathrm{H} & 4.050640 & 3.409910 & -3.798284 \\ \mathrm{C} & 5.101703 & 3.754156 & -1.945893 \\ \mathrm{H} & 5.939994 & 4.255618 & -2.421415 \\ \mathrm{C} & 5.084005 & 3.582673 & -0.559724 \\ \mathrm{H} & 5.905303 & 3.954616 & 0.046881\end{array}$

29

B3LYP SCF energy:

B3LYP enthalpy:

B3LYP free energy:

M06 SCF energy in solution:

a.u.

M०6 enthalpy in solution:

M०6 free energy in solution:

-3819.35073681 a.u.

-3818.406128 a.u.

-3818.567895 a.u

a.u.

$-3819.30610112$

-3818.361492 a.u.

$-3818.523259$

Cartesian coordinates

\begin{tabular}{|c|c|c|c|}
\hline ATOM & $\mathrm{X}$ & $\mathrm{Y}$ & Z \\
\hline $\mathrm{P}$ & $\odot .277846$ & -1.710526 & -0.096666 \\
\hline$P$ & 1.533237 & 1.685176 & -0.044177 \\
\hline 0 & 4.567070 & -2.271856 & 1.781142 \\
\hline 0 & 4. 903192 & -1.328737 & 3.868582 \\
\hline 0 & 5.417721 & -0.764354 & -0.917700 \\
\hline 0 & 5.545223 & -1.995456 & -2.874224 \\
\hline C & 2.516650 & ๑. 753869 & 1. 220515 \\
\hline C & 3.114297 & -0.500785 & 0.875517 \\
\hline C & 3.900501 & -1.078404 & 1.859803 \\
\hline C & 4.104790 & -0.518014 & 3. 120091 \\
\hline C & 3.535740 & $\odot .686757$ & 3.466499 \\
\hline C & 2.744026 & 1. 310442 & 2.487729 \\
\hline C & 5.289985 & -2.411873 & 3. 010125 \\
\hline C & 1.907780 & -1.942017 & -0.923490 \\
\hline C & 2.010547 & -2.699678 & -2.094103 \\
\hline C & 3.199830 & -2.783081 & -2.840487 \\
\hline C & 4.278258 & -2.070259 & -2.365117 \\
\hline C & 4.196499 & -1.322855 & -1.190514 \\
\hline C & 3.039396 & -1.215858 & -0.435225 \\
\hline C & 6.248104 & -1.059232 & -2.048961 \\
\hline C & 2.825186 & 2.017883 & -1.327440 \\
\hline C & 2.633573 & 1.815512 & -2.700436 \\
\hline C & 4.068052 & 2.508474 & -0.882824 \\
\hline C & 1.240537 & 3.343306 & ๑. 723792 \\
\hline C & 1.709794 & 4.530458 & 0.144086 \\
\hline C & $\odot .434947$ & 3.430040 & 1.875609 \\
\hline C & $\odot .534502$ & -2.274292 & 1.639869 \\
\hline C & 1.427907 & -3.310554 & 1.957950 \\
\hline C & -0.234459 & -1.698276 & 2.662033 \\
\hline C & -1.386352 & -2.868750 & -2.072577 \\
\hline C & -0.843492 & -3.017013 & -0.783305 \\
\hline C & -1.208137 & -4.144115 & -0.028401 \\
\hline $\mathrm{H}$ & 3. 697237 & 1.138821 & 4.438418 \\
\hline $\mathrm{H}$ & 2.325015 & 2.275332 & 2.735260 \\
\hline $\mathrm{H}$ & 5.025244 & -3.361492 & 3.483291 \\
\hline $\mathrm{H}$ & 6.366652 & -2.353034 & 2.812109 \\
\hline $\mathrm{H}$ & 1.145868 & -3.245329 & -2.451424 \\
\hline $\mathrm{H}$ & 3.263956 & -3.381725 & -3.742150 \\
\hline $\mathrm{H}$ & 6.431018 & -0.136703 & -2.614358 \\
\hline $\mathrm{H}$ & 7.184523 & -1.507557 & -1.706079 \\
\hline $\mathrm{H}$ & 1.715396 & 1. 388702 & -3.075480 \\
\hline
\end{tabular}

\begin{tabular}{|c|c|c|c|}
\hline $\mathrm{H}$ & 4.248362 & 2.651471 & ๑.178001 \\
\hline $\mathrm{H}$ & 2. 319322 & 4.498952 & $-\odot .751664$ \\
\hline $\mathrm{H}$ & 2.030623 & -3.765745 & 1.177876 \\
\hline $\mathrm{H}$ & -0.952568 & -0.915970 & 2.432567 \\
\hline $\mathrm{H}$ & -1.127852 & $-2.0 \odot 5314$ & -2.672379 \\
\hline $\mathrm{H}$ & -0.816934 & -4.284167 & ๑. 972114 \\
\hline $\mathrm{Rh}$ & -0.581275 & $\odot .701764$ & -0.457833 \\
\hline 0 & -2.619626 & $-\odot .119856$ & -0.523034 \\
\hline C & -0.520837 & $\odot .821052$ & -2.442775 \\
\hline S & -3.592254 & ๑. 258230 & ๑. 558055 \\
\hline 0 & -0.125028 & -0.106792 & -3.095569 \\
\hline C & -2.687741 & 2.763094 & 0.417373 \\
\hline $\mathrm{N}$ & -3.908969 & 1.899908 & 0.330705 \\
\hline 0 & -3.201893 & -0.061208 & 1.940678 \\
\hline C & -5.121015 & -0.550823 & 0.150729 \\
\hline $\mathrm{H}$ & -3.094387 & 3.778941 & 0.331364 \\
\hline $\mathrm{H}$ & -2.252732 & 2.687349 & 1. 424712 \\
\hline C & -5.010676 & 2.413893 & 1.198993 \\
\hline C & -5.731748 & -1.343387 & 1.123603 \\
\hline C & -5.656424 & -0.425863 & -1.136676 \\
\hline C & -5.639867 & 3.632756 & ๑. 581980 \\
\hline $\mathrm{H}$ & -5.764820 & 1.624451 & 1.284722 \\
\hline $\mathrm{H}$ & -4.639526 & 2.628741 & 2.210693 \\
\hline $\mathrm{H}$ & -5.288703 & -1.433792 & 2.109472 \\
\hline C & -6.908922 & -2.015608 & ๑. 796356 \\
\hline C & -6.832261 & -1.103437 & -1.437081 \\
\hline $\mathrm{H}$ & -5.161306 & ๑.183786 & -1.884680 \\
\hline C & -5.775950 & 4.800680 & 1.210858 \\
\hline $\mathrm{H}$ & -6.020281 & 3.502558 & -0.430253 \\
\hline $\mathrm{H}$ & -7.390030 & -2.637205 & 1.546189 \\
\hline C & -7.478547 & -1.905590 & -0.479364 \\
\hline $\mathrm{H}$ & -7.256413 & -1.014622 & -2.433706 \\
\hline $\mathrm{H}$ & -6.276648 & 5.643810 & 0.743928 \\
\hline $\mathrm{H}$ & -5.406814 & 4.953522 & 2.222963 \\
\hline C & -1.096022 & 2.109881 & -3.027092 \\
\hline $\mathrm{H}$ & -1.433780 & 1.916842 & -4.051626 \\
\hline $\mathrm{H}$ & -0.260511 & 2.822491 & -3.078317 \\
\hline C & -2.176642 & 2.624112 & -2.083110 \\
\hline $\mathrm{H}$ & -3.069272 & 1.997738 & -2.177743 \\
\hline $\mathrm{H}$ & -2.472307 & 3.647015 & -2.357885 \\
\hline C & -1.624615 & 2.560061 & -0.658511 \\
\hline $\mathrm{H}$ & ๑. 039689 & 2.526241 & 2.332207 \\
\hline $\mathrm{H}$ & -0.907583 & 3.371122 & -0.515909 \\
\hline C & 3.650327 & 2.117339 & -3.610722 \\
\hline $\mathrm{H}$ & 3.484256 & 1.948170 & -4.670664 \\
\hline C & 4.869319 & 2.625107 & -3.162528 \\
\hline $\mathrm{H}$ & 5.654500 & 2.868157 & -3.873050 \\
\hline C & 5.077061 & 2.815934 & -1.793316 \\
\hline $\mathrm{H}$ & 6.025658 & 3.202662 & -1.431758 \\
\hline C & 1.401081 & 5.769664 & 0.713873 \\
\hline $\mathrm{H}$ & 1.776063 & 6.677928 & ๑. 250871 \\
\hline C & ๑. 624793 & 5.841462 & 1.869491 \\
\hline $\mathrm{H}$ & ๑. 393991 & 6.804912 & 2.314592 \\
\hline C & 0.141569 & 4.665351 & 2.450439 \\
\hline $\mathrm{H}$ & -0.466310 & 4.709641 & 3.349816 \\
\hline C & -0.107484 & -2.145316 & 3.979374 \\
\hline $\mathrm{H}$ & $-\odot .710581$ & -1.692373 & 4.761387 \\
\hline C & ๑. 785519 & -3.172364 & 4.288372 \\
\hline $\mathrm{H}$ & ๑. 882883 & -3.520577 & 5.312910 \\
\hline C & 1.550715 & -3.755744 & 3. 274598 \\
\hline $\mathrm{H}$ & 2.238629 & -4.564344 & 3.507713 \\
\hline C & -2.077336 & -5.102292 & $-\odot .554278$ \\
\hline $\mathrm{H}$ & -2.343236 & -5.968110 & ๑. 046002 \\
\hline C & -2.595809 & -4.954025 & -1.840431 \\
\hline $\mathrm{H}$ & -3.267233 & -5.703826 & -2.249764 \\
\hline C & -2.245458 & -3.834465 & -2.597563 \\
\hline $\mathrm{H}$ & -2.642886 & -3.707353 & -3.600994 \\
\hline C & -8.763923 & -2.617127 & -0.821888 \\
\hline $\mathrm{H}$ & -9.014277 & -3.377152 & -0.076529 \\
\hline $\mathrm{H}$ & -9.600615 & -1.908371 & -0.868478 \\
\hline $\mathrm{H}$ & -8.699973 & -3.104884 & -1.800620 \\
\hline
\end{tabular}

$-8.699973$ 
B3LYP SCF energy: $\quad-3819.33553643$ a.u. B3LYP enthalpy:

B3LYP free energy: -3818.390067 a.u. M06 SCF energy in solution: a.u.

M06 enthalpy in solution:

M०6 free energy in solution:

a.u.

Cartesian coordinates

\begin{tabular}{|c|c|c|c|}
\hline ATOM & $\mathrm{X}$ & $\mathrm{Y}$ & Z \\
\hline $\mathrm{P}$ & $\odot .761971$ & -1.821446 & -0.192642 \\
\hline$P$ & 1.386466 & 1.743539 & -0.127174 \\
\hline 0 & 4.793532 & -1.660231 & 2.123966 \\
\hline 0 & 4.751994 & -0.647070 & 4.206621 \\
\hline 0 & 5.734728 & $-\odot .066761$ & -0.474280 \\
\hline 0 & 6.288182 & -1.276807 & -2.370194 \\
\hline C & 2.409004 & 1.026704 & 1.244950 \\
\hline C & 5.325214 & -1.726622 & 3. 453654 \\
\hline C & 2.469674 & -1.757778 & -0.883010 \\
\hline C & 4.995509 & -1.537650 & -2.018180 \\
\hline C & 4.656369 & $-\odot .805127$ & -0.880980 \\
\hline C & 3.409670 & -0.865885 & -0.278817 \\
\hline C & 6.756043 & -0.272881 & -1.460529 \\
\hline C & 2.658331 & 2.058891 & -1.430234 \\
\hline C & 0.943143 & 3.448909 & ○. 445930 \\
\hline $\mathrm{H}$ & 5.041243 & -2.678980 & 3. 912407 \\
\hline $\mathrm{H}$ & 6.413271 & -1.609267 & 3.420049 \\
\hline $\mathrm{H}$ & 6.927464 & ๑. 662531 & -2.006024 \\
\hline $\mathrm{H}$ & 7.669826 & -0.621629 & $-\odot .971057$ \\
\hline $\mathrm{Rh}$ & -0.637136 & ๑. 379981 & -0.502452 \\
\hline 0 & -5.449083 & 1.404388 & 2.648999 \\
\hline C & $-\odot .699323$ & $\odot .576951$ & -2.493885 \\
\hline S & -5.456161 & 1.572477 & 1.189230 \\
\hline 0 & -0.185252 & -0.254159 & -3.191885 \\
\hline C & -2.941449 & 2.217149 & ๑. 513233 \\
\hline $\mathrm{N}$ & -3.917936 & 1.103651 & ๑. 541719 \\
\hline 0 & -5.749228 & 2.873358 & ๑.583155 \\
\hline C & -6.539821 & ๑. 335925 & 0.488702 \\
\hline $\mathrm{H}$ & -3.514972 & 3.139464 & 0.367284 \\
\hline $\mathrm{H}$ & -2.449866 & 2.297732 & 1.495218 \\
\hline C & -7.112363 & -0.624866 & 1. 323028 \\
\hline C & -6.848010 & 0.385503 & -0.874985 \\
\hline C & -2.524778 & $-\odot .924201$ & ๑. 250836 \\
\hline $\mathrm{H}$ & -6.884378 & $-\odot .624217$ & 2.383744 \\
\hline C & -7.990013 & -1.560830 & $\odot .774157$ \\
\hline C & -7.724964 & $-\odot .556388$ & -1.402468 \\
\hline $\mathrm{H}$ & -6.421097 & 1.160353 & -1.503324 \\
\hline C & -2.591878 & $-\odot .899764$ & -1.112120 \\
\hline $\mathrm{H}$ & -2.005737 & -1.748835 & 0.735260 \\
\hline $\mathrm{H}$ & -8.440046 & -2.310147 & 1.419937 \\
\hline C & -8.310373 & -1.542873 & -0.589571 \\
\hline $\mathrm{H}$ & -7.971389 & -0.521582 & -2.460680 \\
\hline $\mathrm{H}$ & -2.140708 & -1.689723 & -1.700417 \\
\hline $\mathrm{H}$ & -3.286162 & -0.231944 & -1.604887 \\
\hline C & -9.287847 & -2.534770 & -1.171677 \\
\hline $\mathrm{H}$ & -10.280343 & -2.080216 & -1.286681 \\
\hline $\mathrm{H}$ & -8.971691 & -2.874476 & -2.163797 \\
\hline $\mathrm{H}$ & -9.401352 & -3.412644 & $-\odot .528702$ \\
\hline C & -1.414314 & 1.826264 & -3.007482 \\
\hline $\mathrm{H}$ & -1.784947 & 1.629206 & $-4.02 \odot \odot 42$ \\
\hline $\mathrm{H}$ & -0.627194 & 2.589990 & -3.090763 \\
\hline C & -2.476799 & 2.265549 & -2.005926 \\
\hline $\mathrm{H}$ & -3.387049 & 1.671271 & -2.132039 \\
\hline $\mathrm{H}$ & -2.766837 & 3.307889 & -2.197642 \\
\hline C & -1.899309 & 2.119486 & -0.596435 \\
\hline $\mathrm{H}$ & -1.222809 & 2.957145 & -0.431501 \\
\hline C & -3.388971 & -0.104174 & 1.172395 \\
\hline $\mathrm{H}$ & -4.220785 & -0.756699 & 1. 469982 \\
\hline $\mathrm{H}$ & -2.848702 & $\odot .132434$ & 2.103696 \\
\hline C & 1.015401 & -2.346618 & 1.560014 \\
\hline C & 2.415993 & 1.632176 & 2.510017 \\
\hline $\mathrm{H}$ & 1.848925 & 2.536163 & 2.677299 \\
\hline C & 3.172039 & 1.145827 & 3.589691 \\
\hline $\mathrm{H}$ & 3.165444 & 1.640145 & 4.554418 \\
\hline
\end{tabular}

$-3819.30872267$

-3818.363253 a.u. $-3818.522059$ 


\begin{tabular}{|c|c|c|c|}
\hline C & -3.454193 & ๑. 626455 & 4.888952 \\
\hline C & -3.078153 & 1.119992 & $-\odot .319972$ \\
\hline C & -4.118023 & 1.585398 & -1.134494 \\
\hline C & -5.427716 & 1.081484 & -1.056013 \\
\hline C & -5.653749 & ๑. . 094928 & -0.121754 \\
\hline C & -4.631811 & -0.371685 & ๑. 703124 \\
\hline C & -3.326642 & ๑. .090096 & 0.638278 \\
\hline C & -6.529447 & -1.425807 & 1.267691 \\
\hline C & -2.328847 & -2.297965 & -1.080281 \\
\hline C & -2.780937 & -1.909640 & -2.347674 \\
\hline C & -3.090902 & -3.219008 & $-\odot .336622$ \\
\hline C & ๑. 239529 & -3.287282 & -0.218458 \\
\hline C & -0.200301 & -4.473504 & -0.829368 \\
\hline C & 1.482662 & -3.301553 & ๑. 444771 \\
\hline C & -0.921068 & 2.349464 & 1.198840 \\
\hline C & -1.823329 & 3.073231 & 1.998464 \\
\hline C & ๑. 352845 & 2.059540 & 1.708073 \\
\hline C & -1.901368 & 3.324533 & -2.818986 \\
\hline C & -1.600695 & 3.374946 & -1.444192 \\
\hline C & -1.426247 & 4.632281 & -0.843824 \\
\hline $\mathrm{H}$ & -0.065598 & -1.750304 & 4.594518 \\
\hline $\mathrm{H}$ & 0.431537 & -2.348062 & 2.267995 \\
\hline $\mathrm{H}$ & -3.310169 & 1.658321 & 5.225007 \\
\hline $\mathrm{H}$ & -4.328663 & $\odot .171475$ & 5.365918 \\
\hline $\mathrm{H}$ & -3.928608 & 2.380005 & -1.843773 \\
\hline $\mathrm{H}$ & -6.220353 & 1.459533 & -1.691695 \\
\hline $\mathrm{H}$ & -6.788383 & -2.454876 & 1. 001892 \\
\hline $\mathrm{H}$ & -7.090097 & -1.090941 & 2.148288 \\
\hline $\mathrm{H}$ & -2.238458 & -1.175547 & -2.927009 \\
\hline $\mathrm{H}$ & -2.764385 & -3.522678 & 0.653073 \\
\hline $\mathrm{H}$ & -1.135191 & -4.499187 & -1.374983 \\
\hline $\mathrm{H}$ & -2.822968 & 3.288219 & 1.632390 \\
\hline $\mathrm{H}$ & 1.058501 & 1.468184 & 1.130842 \\
\hline $\mathrm{H}$ & -1.999865 & 2.367867 & -3.317508 \\
\hline $\mathrm{H}$ & -1.177210 & 4.710613 & ๑. 207592 \\
\hline $\mathrm{Rh}$ & $\odot .534611$ & $\odot .171951$ & -1.378324 \\
\hline 0 & 3.726398 & 1.060433 & 2.087769 \\
\hline C & -0.013203 & -0.154774 & -3.263318 \\
\hline S & 4.170626 & -0.073073 & 1.269194 \\
\hline 0 & $-\odot .864084$ & $\odot .513430$ & -3.789485 \\
\hline C & 3.477655 & $-\odot .951002$ & -1.230871 \\
\hline $\mathrm{N}$ & 3.349259 & $\odot .114013$ & -0.212956 \\
\hline 0 & 3. 918861 & -1.459971 & 1.677931 \\
\hline C & 5.922309 & ๑. 112111 & 0.962702 \\
\hline $\mathrm{H}$ & 4.262968 & $-\odot .674507$ & -1.953745 \\
\hline $\mathrm{H}$ & 3.807124 & -1.857816 & -0.720685 \\
\hline C & 3.385431 & 1.464709 & -0.797353 \\
\hline C & 6.505837 & 1.379930 & 1.049532 \\
\hline C & 6.687147 & -1.009886 & ๑. 626791 \\
\hline C & 2.401601 & 1.558000 & -1.941156 \\
\hline $\mathrm{H}$ & 3.136917 & 2.186249 & -0.017490 \\
\hline $\mathrm{H}$ & 4.392757 & 1.699746 & -1.182812 \\
\hline $\mathrm{H}$ & 5.910325 & 2.233891 & 1.354110 \\
\hline C & 7.866877 & 1.518272 & ๑. 783936 \\
\hline C & 8.045501 & $-\odot .849124$ & $\odot .365869$ \\
\hline $\mathrm{H}$ & 6.231525 & -1.993906 & 0.604838 \\
\hline C & 1. 301222 & 2.368950 & -1.899082 \\
\hline $\mathrm{H}$ & 2.727713 & 1.174899 & -2.900528 \\
\hline $\mathrm{H}$ & 8.326429 & 2.499814 & ๑. 864262 \\
\hline C & 8.656348 & $\odot .413165$ & 0.432549 \\
\hline $\mathrm{H}$ & 8.645805 & -1.720914 & ๑. 119102 \\
\hline $\mathrm{H}$ & 0.738108 & 2.610200 & -2.793282 \\
\hline $\mathrm{H}$ & 1.108447 & 2.989152 & -1.029954 \\
\hline C & 10.124697 & $\odot .576524$ & ๑. 124761 \\
\hline $\mathrm{H}$ & 10.698241 & -0.304586 & ○. 429482 \\
\hline H & 10.284343 & $\odot .712166$ & -0.953082 \\
\hline $\mathrm{H}$ & 10.545931 & 1.450872 & $\odot .630080$ \\
\hline C & $\odot .756523$ & -1.268169 & -3.979616 \\
\hline $\mathrm{H}$ & 0.671173 & -1.117263 & -5.061387 \\
\hline $\mathrm{H}$ & $\odot .231789$ & -2.201851 & -3.731822 \\
\hline C & 2.189551 & -1.314428 & -3.455541 \\
\hline H & 2.775238 & $-\odot .505331$ & -3.910678 \\
\hline $\mathrm{H}$ & 2.673393 & -2.250171 & -3.770277 \\
\hline C & 2.131673 & -1.208745 & -1.933182 \\
\hline H & 1.903577 & -2.397364 & $\odot .870564$ \\
\hline
\end{tabular}

$\begin{array}{lrrr}\mathrm{H} & 1.745793 & -2.152964 & -1.566816 \\ \mathrm{C} & 2.225476 & -4.476640 & 0.548464 \\ \mathrm{H} & 3.171193 & -4.455217 & 1.082024 \\ \mathrm{C} & 1.761964 & -5.656295 & -0.038189 \\ \mathrm{H} & 2.341476 & -6.571724 & 0.039340 \\ \mathrm{C} & 0.555364 & -5.64560 \odot & -0.736532 \\ \mathrm{H} & -.190392 & -6.551799 & -1.211532 \\ \mathrm{C} & -4.707243 & -3.361405 & -2.131519 \\ \mathrm{H} & -5.624425 & -3.775199 & -2.540842 \\ \mathrm{C} & -4.266761 & -3.749856 & -0.862682 \\ \mathrm{H} & -4.837319 & -4.468815 & -0.281262 \\ \mathrm{C} & -3.966014 & -2.437803 & -2.867527 \\ \mathrm{H} & -4.306731 & -2.120883 & -3.848908 \\ \mathrm{C} & -2.053407 & 4.498469 & -3.557842 \\ \mathrm{H} & -2.293410 & 4.436117 & -4.615637 \\ \mathrm{C} & -1.889146 & 5.743551 & -2.946825 \\ \mathrm{H} & -2.003175 & 6.656337 & -3.524454 \\ \mathrm{C} & -1.569632 & 5.804555 & -1.591152 \\ \mathrm{H} & -1.430991 & 6.766214 & -1.105062 \\ \mathrm{C} & -.732642 & 2.492797 & 2.980345 \\ \mathrm{H} & 1.723822 & 2.246245 & 3.347562 \\ \mathrm{C} & -0.164041 & 3.227035 & 3.757561 \\ \mathrm{H} & -.127609 & 3.571381 & 4.745829 \\ \mathrm{C} & -1.441941 & 3.514002 & 3.265746 \\ \mathrm{H} & -2.142746 & 4.085198 & 3.868969 \\ & & & \end{array}$

32

B3LYP SCF energy:

B3LYP enthalpy:

B3LYP free energy:

M06 SCF energy in solution

a.u.

M06 enthalpy in solution:

M06 free energy in solution:

-3819.36376301 a.u. -3818.417576 a.u. -3818.578240 a.u. $-3819.33173776$ a.u.

Cartesian coordinates

\begin{tabular}{lrrr} 
ATOM & X & $Y$ & \multicolumn{1}{c}{ Z } \\
P & -2.326356 & 1.729217 & 0.048200 \\
P & -0.731146 & -1.521788 & -0.039214 \\
O & -5.764410 & -0.903440 & 1.544485 \\
O & -5.426166 & -1.916355 & 3.597769 \\
O & -5.235514 & -2.456300 & -1.275253 \\
O & -6.051838 & -1.542746 & -3.239595 \\
C & -2.188521 & -1.707927 & 1.056137 \\
C & -3.467883 & -1.163424 & 0.695636 \\
C & -4.462805 & -1.310161 & 1.647972 \\
C & -4.266539 & -1.924466 & 2.887459 \\
C & -3.045791 & -2.455779 & 3.241755 \\
C & -2.011964 & -2.330976 & 2.299870 \\
C & -6.369658 & -1.166376 & 2.817845 \\
C & -3.499903 & 0.796471 & -1.011623 \\
C & -4.008640 & 1.338612 & -2.197048 \\
C & -4.885794 & 0.624621 & -3.033313 \\
C & -5.231856 & -0.644394 & -2.622393 \\
C & -4.737322 & -1.192085 & -1.436952 \\
C & -3.856495 & -0.525981 & -0.601624 \\
C & -6.174755 & -2.654071 & -2.342820 \\
C & -1.243235 & -2.135030 & -1.691658 \\
C & -0.760990 & -1.525902 & -2.860025 \\
C & -2.078918 & -3.260093 & -1.798603 \\
C & 0.552915 & -2.710386 & 0.537971 \\
C & 0.845328 & -3.868411 & -0.200865 \\
C & 1.281940 & -2.446715 & 1.712610 \\
C & -3.276686 & 2.087074 & 1.580819 \\
C & -4.636645 & 2.435616 & 1.507315 \\
C & -2.649186 & 2.032967 & 2.834579 \\
C & -1.313418 & 3.399448 & -1.954796 \\
C & -2.074617 & 3.361247 & -0.769209 \\
C & -2.514686 & 4.570525 & -0.210903 \\
H & -2.889956 & -2.947280 & 4.195128 \\
H & -1.046462 & -2.747339 & 2.555517 \\
H & -6.584227 & -0.216953 & 3.321995
\end{tabular}

3818.385551 a.u. $-3818.546215$ 


\begin{tabular}{|c|c|c|c|}
\hline $\mathrm{H}$ & -7.276341 & -1.759992 & 2.676267 \\
\hline $\mathrm{H}$ & -3.734692 & 2.347104 & -2.484295 \\
\hline $\mathrm{H}$ & -5.277239 & 1.054307 & -3.948425 \\
\hline $\mathrm{H}$ & -5.932413 & -3.578217 & -2.873738 \\
\hline $\mathrm{H}$ & -7.191780 & -2.684902 & -1.934079 \\
\hline $\mathrm{H}$ & -0.104310 & -0.663578 & -2.796836 \\
\hline $\mathrm{H}$ & -2.471385 & -3.736155 & -0.905462 \\
\hline H & ๑. 310287 & - 4.088719 & -1.116835 \\
\hline $\mathrm{H}$ & -5.139213 & 2.465169 & 0.545013 \\
\hline $\mathrm{H}$ & -1.609958 & 1.734759 & $2.91640 \odot$ \\
\hline $\mathrm{H}$ & -0.970575 & 2.473378 & -2.413419 \\
\hline H & -3.097690 & 4.568656 & 0.703714 \\
\hline $\mathrm{H}$ & 1.082144 & -1.552105 & 2.290690 \\
\hline $\mathrm{Rh}$ & -0.077753 & ๑. 669246 & -0.187879 \\
\hline $\mathrm{C}$ & ๑. 600895 & 1.134202 & 1.548533 \\
\hline C & 1.792810 & $\odot .046389$ & -0.967756 \\
\hline 0 & $\odot .624103$ & $\odot .567014$ & 2.604170 \\
\hline C & 1.177568 & 2.537863 & 1.267789 \\
\hline C & 3.070941 & ๑. 398532 & -0.202220 \\
\hline $\mathrm{H}$ & 1.801281 & -1.022047 & -1.197815 \\
\hline $\mathrm{H}$ & 1.778126 & ๑. 592813 & -1.929053 \\
\hline $\mathrm{H}$ & $\odot .766265$ & 3.186579 & 2.050637 \\
\hline $\mathrm{H}$ & 0.818840 & 2.947141 & ๑. 308980 \\
\hline C & 2.717921 & 2.531127 & 1.282061 \\
\hline C & 4.342860 & -0.109308 & -0.962262 \\
\hline $\mathrm{H}$ & 3.048886 & -0.101312 & 0.775027 \\
\hline C & 3.333297 & 1.917428 & ๑. 020481 \\
\hline $\mathrm{H}$ & 3.068730 & 2.007478 & 2.180057 \\
\hline $\mathrm{H}$ & 3. 048592 & 3.573383 & 1.372734 \\
\hline $\mathrm{N}$ & 5. 200107 & 1.080179 & -1.069822 \\
\hline $\mathrm{H}$ & 4.116319 & -0.483741 & -1.963390 \\
\hline $\mathrm{H}$ & 4.823805 & -0.920844 & -0.395748 \\
\hline C & 4.864310 & 2.032117 & -0.011845 \\
\hline $\mathrm{H}$ & 2.951725 & 2.462215 & -0.855830 \\
\hline$S$ & 6.781803 & ๑. 928567 & -1.618607 \\
\hline $\mathrm{H}$ & 5.297440 & 1.747986 & 0.963938 \\
\hline $\mathrm{H}$ & 5.220753 & 3. 029682 & -0.275288 \\
\hline 0 & 6.710277 & $\odot .0 \odot 8071$ & -2.754382 \\
\hline 0 & 7.289479 & 2.296154 & -1.736040 \\
\hline C & 7.702939 & ๑. 114180 & -0.313226 \\
\hline C & 7.809184 & -1.279456 & -0.306764 \\
\hline C & 8.291466 & ๑.879925 & ๑. 698428 \\
\hline $\mathrm{H}$ & 7.380869 & -1.856881 & -1.119184 \\
\hline C & 8.503391 & -1.904244 & 0.728576 \\
\hline C & 8.980464 & ๑. 237347 & 1.724733 \\
\hline $\mathrm{H}$ & 8.234283 & 1.962683 & 0.660042 \\
\hline H & 8.596232 & -2.987505 & $\odot .729780$ \\
\hline C & 9.098763 & -1.160688 & 1.757671 \\
\hline $\mathrm{H}$ & 9.446437 & ๑. 831601 & 2.506918 \\
\hline C & 9.884111 & -1.842254 & 2.852573 \\
\hline $\mathrm{H}$ & 10.953131 & -1.872923 & 2.604557 \\
\hline $\mathrm{H}$ & 9.788431 & -1.311727 & 3.805745 \\
\hline $\mathrm{H}$ & 9.554498 & -2.875491 & 3.000920 \\
\hline C & -5.347212 & 2.742765 & 2.667838 \\
\hline $\mathrm{H}$ & -6.394747 & 3. 022967 & 2.599205 \\
\hline C & -4.712415 & 2.694770 & 3.912881 \\
\hline $\mathrm{H}$ & -5.266774 & 2.935746 & 4.815554 \\
\hline C & -3.365840 & 2.336210 & 3.994340 \\
\hline $\mathrm{H}$ & -2.870994 & 2. 287804 & 4.959972 \\
\hline C & -2.206615 & 5.785988 & -0.827630 \\
\hline $\mathrm{H}$ & -2.555757 & 6.714114 & -0.384283 \\
\hline C & -1.458965 & 5.811844 & -2.005631 \\
\hline $\mathrm{H}$ & -1.223173 & 6.759034 & -2.481655 \\
\hline C & -1.014416 & 4.613880 & -2.571937 \\
\hline $\mathrm{H}$ & -0.436043 & 4.624658 & -3.491341 \\
\hline C & -1.108725 & -2.029286 & -4.114667 \\
\hline $\mathrm{H}$ & -0.730305 & -1.548540 & -5.011906 \\
\hline C & -1.932829 & -3.151521 & -4.213120 \\
\hline $\mathrm{H}$ & -2.197636 & -3.547268 & -5.189264 \\
\hline C & -2.414737 & -3.766064 & -3.054223 \\
\hline $\mathrm{H}$ & -3.051610 & -4.643220 & -3.126140 \\
\hline C & 1.836101 & -4.750789 & 0.235057 \\
\hline $\mathrm{H}$ & 2.050491 & -5.641838 & -0.347778 \\
\hline C & 2.545106 & -4.490890 & 1.407564 \\
\hline $\mathrm{H}$ & 3. 313291 & -5.180720 & 1.744846 \\
\hline
\end{tabular}

$\begin{array}{llll}\mathrm{C} & 2.264698 & -3.337627 & 2.144194 \\ \mathrm{H} & 2.813162 & -3.126024 & 3.057693\end{array}$

33

B3LYP SCF energy: $\quad-3819.35980589$ a.u. B3LYP enthalpy: $\quad-3818.413447$ a.u. B3LYP free energy: $\quad-3818.575319 \mathrm{a} . \mathrm{u}$. M06 SCF energy in solution: $\quad-3819.32369466$ a.u. M๑6 enthalpy in solution: $\quad-3818.377336$ a.u. Mo6 free energy in solution: $\quad-3818.539208$ a.u.

Cartesian coordinates

\begin{tabular}{|c|c|c|c|}
\hline ATOM & X & $\mathrm{Y}$ & Z \\
\hline$P$ & 2.009137 & 1.730566 & $\odot .223437$ \\
\hline $\mathrm{P}$ & $\odot .9617 \odot 2$ & -1.722488 & $-\odot .271430$ \\
\hline 0 & 5.979482 & -0.150786 & -1.034345 \\
\hline 0 & 6.085356 & -0.965327 & -3.197404 \\
\hline 0 & 5.367923 & -2.119429 & 1.504742 \\
\hline 0 & 5.789974 & -1.341666 & 3.642778 \\
\hline C & 2.567412 & -1.572620 & -1.138386 \\
\hline C & 3.674858 & -0.870713 & -0.550074 \\
\hline C & 4.791198 & -0.745524 & -1.360346 \\
\hline C & 4.864278 & -1.240530 & -2.664955 \\
\hline C & 2.662743 & -2.074132 & -2.444454 \\
\hline C & 6.769824 & -0.154536 & -2.230719 \\
\hline C & 3.180327 & ๑. 855805 & 1.336258 \\
\hline C & 3.451900 & 1.318143 & 2.628649 \\
\hline C & 4.326879 & 0.644410 & 3. 499895 \\
\hline C & 4.918250 & $-\odot .501019$ & 3.013160 \\
\hline C & 4.661353 & $-\odot .966738$ & 1.722454 \\
\hline C & 3.790648 & -0.340578 & ๑.846133 \\
\hline C & 6.212104 & -2.288143 & 2.652919 \\
\hline C & 1.327953 & -2.381353 & 1.402874 \\
\hline C & ๑. 588267 & -1.949477 & 2.514270 \\
\hline C & 2.314920 & -3.366385 & 1.577456 \\
\hline C & -0.014546 & -3.046264 & -1.100040 \\
\hline C & -0.213155 & -4.288075 & -0.473573 \\
\hline C & -0.611305 & -2.811694 & -2.353128 \\
\hline C & 3.087640 & 2.431517 & -1.093178 \\
\hline C & 4.347994 & 2.963288 & -0.768356 \\
\hline C & 2.657580 & 2.457936 & -2.428295 \\
\hline C & $\odot .513179$ & 2.959854 & 2.245669 \\
\hline C & 1.389495 & 3. 189031 & 1.166183 \\
\hline C & 1.663808 & 4.512549 & 0.790417 \\
\hline $\mathrm{H}$ & 1.824733 & -2.609199 & -2.871555 \\
\hline $\mathrm{H}$ & 6.857938 & ๑. 869551 & -2.612329 \\
\hline $\mathrm{H}$ & 7.749513 & $-\odot .590657$ & -2.020396 \\
\hline $\mathrm{H}$ & 2.987680 & 2.233614 & 2.976585 \\
\hline $\mathrm{H}$ & 4.532988 & 1.012735 & 4.498509 \\
\hline $\mathrm{H}$ & 6.094793 & -3.301302 & 3.044970 \\
\hline $\mathrm{H}$ & 7.253900 & -2.087668 & 2.373908 \\
\hline $\mathrm{H}$ & $-\odot .185892$ & -1.195890 & 2.395557 \\
\hline $\mathrm{H}$ & 2.905917 & -3.702572 & 0.731212 \\
\hline $\mathrm{H}$ & 0.220820 & -4.492773 & ๑. 497660 \\
\hline $\mathrm{H}$ & 4.701645 & 2.935736 & 0.258074 \\
\hline $\mathrm{H}$ & 1.704264 & 2.024261 & -2.706868 \\
\hline $\mathrm{H}$ & ๑. 291546 & 1.941244 & 2.559440 \\
\hline $\mathrm{H}$ & 2.330928 & 4.716086 & -0.040199 \\
\hline $\mathrm{H}$ & $-\odot .485662$ & -1.855809 & -2.846998 \\
\hline $\mathrm{Rh}$ & -0.040180 & ๑. 317954 & -0.035063 \\
\hline $\mathrm{C}$ & -0.479982 & ๑. 831359 & -1.857762 \\
\hline C & -1.905923 & -0.637465 & 0.139401 \\
\hline 0 & $-\odot .160162$ & ๑. 291879 & -2.880541 \\
\hline C & -1.339347 & 2.118290 & -1.789509 \\
\hline C & -2.853249 & ๑. 354752 & ๑. 812834 \\
\hline $\mathrm{H}$ & -1.831982 & -1.554539 & 0.734510 \\
\hline $\mathrm{H}$ & -2.266937 & -0.935348 & -0.850297 \\
\hline $\mathrm{H}$ & -1.333877 & 2.496145 & -2.818210 \\
\hline $\mathrm{H}$ & -0.837545 & 2.867918 & -1.164828 \\
\hline C & -2.792063 & 1.925381 & -1.304521 \\
\hline C & -4.337060 & -0.166017 & 0.801157 \\
\hline
\end{tabular}




\begin{tabular}{|c|c|c|c|}
\hline $\mathrm{H}$ & -2.558525 & $\odot .463835$ & 1.866178 \\
\hline C & -2.948178 & 1.800739 & ๑. 227617 \\
\hline $\mathrm{H}$ & -3.263211 & 1. 085711 & -1.825990 \\
\hline $\mathrm{H}$ & -3.336210 & 2.817907 & -1.634295 \\
\hline $\mathrm{N}$ & -5.192965 & 1.011755 & $\odot .547370$ \\
\hline $\mathrm{H}$ & -4.594167 & $-\odot .615140$ & 1.766701 \\
\hline $\mathrm{H}$ & -4.487377 & -0.916094 & ๑. 021462 \\
\hline C & -4.355368 & 2.216566 & 0.705251 \\
\hline $\mathrm{H}$ & -2.203962 & 2.455299 & ๑. 704816 \\
\hline S & -6.199213 & ๑.943763 & -0.837611 \\
\hline $\mathrm{H}$ & -4.789870 & 3.057514 & $\odot .164517$ \\
\hline $\mathrm{H}$ & -4.331145 & 2.469988 & 1.773280 \\
\hline 0 & -6.490259 & 2.339061 & -1.186193 \\
\hline 0 & -5.622820 & ๑ . 032912 & -1.842629 \\
\hline C & -7.676331 & ๑.170308 & $-\odot .196146$ \\
\hline C & -8.040045 & -1.096103 & -0.650324 \\
\hline $\mathrm{C}$ & -8.471649 & ๑. 865828 & ๑. 720672 \\
\hline $\mathrm{H}$ & -7.417493 & -1.609373 & -1.374837 \\
\hline C & -9.214095 & -1.676569 & -0.166806 \\
\hline C & -9.636909 & ๑. 270800 & 1.189979 \\
\hline $\mathrm{H}$ & -8.183032 & 1.857206 & 1.054157 \\
\hline $\mathrm{H}$ & -9.502893 & -2.663585 & -0.518363 \\
\hline C & -10.028372 & -1.007718 & ๑.754955 \\
\hline $\mathrm{H}$ & -10.259087 & ๑. 806681 & 1.902460 \\
\hline C & -11.308945 & -1.627755 & 1. 259712 \\
\hline $\mathrm{H}$ & -11.383507 & -1.557277 & 2.350874 \\
\hline $\mathrm{H}$ & -12.184159 & -1.112964 & $\odot .843408$ \\
\hline $\mathrm{H}$ & -11.381740 & -2.683194 & ๑. 981375 \\
\hline C & 3. 466799 & 3. 018306 & -3.419224 \\
\hline $\mathrm{H}$ & 3.123931 & 3. 029887 & -4.449733 \\
\hline C & 4.711347 & 3.555843 & -3.087073 \\
\hline $\mathrm{H}$ & 5.337192 & 3.996365 & -3.858128 \\
\hline C & 5.151396 & 3.526156 & -1.760106 \\
\hline $\mathrm{H}$ & 6.118690 & 3.944600 & -1.495819 \\
\hline C & 1.082658 & 5.578620 & 1.482482 \\
\hline $\mathrm{H}$ & 1.305705 & 6.597670 & 1.179723 \\
\hline C & ๑. 225988 & 5.340296 & 2.557664 \\
\hline $\mathrm{H}$ & -0.220267 & 6.172054 & 3.094778 \\
\hline C & -0.055687 & 4.026137 & 2.941637 \\
\hline $\mathrm{H}$ & -0.717148 & 3.832025 & 3.781309 \\
\hline C & -1.369608 & -3.807203 & -2.968682 \\
\hline $\mathrm{H}$ & -1.820514 & -3.611741 & -3.937263 \\
\hline C & -1.554879 & -5.041972 & -2.343011 \\
\hline $\mathrm{H}$ & -2.149159 & -5.813620 & -2.823748 \\
\hline C & -0.977195 & -5.277319 & -1.095578 \\
\hline $\mathrm{H}$ & -1.119542 & -6.232421 & $-\odot .598274$ \\
\hline C & ๑. 830441 & -2.488823 & 3.778778 \\
\hline $\mathrm{H}$ & 0.253617 & -2.144437 & 4.632170 \\
\hline C & 1.806693 & -3.472802 & 3.942521 \\
\hline $\mathrm{H}$ & 1.991200 & -3.897349 & 4.925037 \\
\hline C & 2.545862 & -3.911050 & 2.840513 \\
\hline $\mathrm{H}$ & 3.302999 & -4.680411 & 2.962821 \\
\hline C & 3.812553 & -1.923427 & -3.235571 \\
\hline $\mathrm{H}$ & 3.867873 & -2.324870 & -4.240827 \\
\hline
\end{tabular}

34

B3LYP SCF energy: $\quad-4431.03020311$ a.u.

B3LYP enthalpy:

$\begin{array}{ll}\text { B3LYP free energy: } & -4429.454332 \mathrm{a} . \mathrm{u} . \\ \text { MO6 SCF energy in solution: } & -4430.49988353\end{array}$

-4429.220230 a.u.

a.u.

M06 enthalpy in solution:

.4430 .49988353

M०6 free energy in solution:

-4428.689910 a.u.

$-4428.924012$

a.u.

Cartesian coordinates

$\begin{array}{lccc}\text { ATOM } & \mathrm{X} & \mathrm{Y} & \mathrm{Z} \\ \text { C } & -1.399067 & -0.497078 & -3.734079 \\ \text { H } & -2.035723 & -1.308072 & -3.405075 \\ \text { C } & -1.652480 & 0.796089 & -3.346572 \\ \text { H } & -2.513762 & 1.025545 & -2.727997 \\ \text { C } & 1.432212 & 0.543970 & -3.724581 \\ \text { H } & 2.076748 & 1.350958 & -3.400567\end{array}$

\begin{tabular}{|c|c|c|c|}
\hline C & 1.678142 & -0.748934 & -3.328055 \\
\hline $\mathrm{H}$ & 2.537328 & $-\odot .977562$ & -2.706211 \\
\hline $\mathrm{Rh}$ & 0. 012663 & ๑. . 030844 & -1.945254 \\
\hline $\mathrm{P}$ & 1.690715 & ๑. 302494 & -0.250519 \\
\hline$P$ & -1.684587 & $-\odot .254121$ & $-\odot .265921$ \\
\hline C & 1.191000 & 1.286022 & 1.241087 \\
\hline C & 2. 397435 & -1.255445 & 0.417701 \\
\hline C & 3.163984 & 1.204896 & -0.916262 \\
\hline C & -1.205762 & -1.195663 & 1.259522 \\
\hline C & -2.450367 & 1.290744 & 0.367643 \\
\hline C & -3.114038 & -1.205871 & -0.958656 \\
\hline C & 1.721035 & 2.569378 & 1. 433112 \\
\hline C & ๑. 265153 & 0.753418 & 2.193542 \\
\hline C & 3.215090 & -1.245846 & 1.556739 \\
\hline C & 2.166255 & -2.465240 & -0.233764 \\
\hline C & 3.006629 & 2.466622 & -1.511832 \\
\hline C & 4.430702 & ๑. 615790 & -0.942001 \\
\hline C & -0.305473 & -0.624316 & 2.213341 \\
\hline C & -1.735807 & -2.473341 & 1. 486117 \\
\hline C & -2.222667 & 2.507162 & -0.272620 \\
\hline C & -3.306144 & 1.260592 & 1.478254 \\
\hline C & -4.390794 & -0.641050 & -1.055377 \\
\hline C & -2.915107 & -2.483038 & -1.496817 \\
\hline C & 1.385766 & 3.384568 & 2.527654 \\
\hline $\mathrm{H}$ & 2.445454 & 2.956957 & 0.730863 \\
\hline C & -0.042485 & 1.582387 & 3.263412 \\
\hline C & 3.893859 & -2.389768 & 1.984190 \\
\hline $\mathrm{H}$ & 3.325352 & $-\odot .320999$ & 2.107720 \\
\hline C & 2.793414 & -3.662432 & 0.153870 \\
\hline $\mathrm{H}$ & 1. 466348 & -2.473704 & -1.061668 \\
\hline C & 4.091984 & 3. 208751 & -1.994889 \\
\hline $\mathrm{H}$ & 2.007070 & 2.875137 & -1.599908 \\
\hline C & 5.562231 & 1. 291059 & -1.427018 \\
\hline $\mathrm{H}$ & 4.541548 & -0.393341 & -0.574586 \\
\hline C & -0.021937 & -1.411353 & 3. 320749 \\
\hline C & -1.421224 & -3.247880 & 2.616025 \\
\hline $\mathrm{H}$ & -2.445816 & -2.887545 & 0.784438 \\
\hline C & -2.891498 & 3.688411 & 0.094830 \\
\hline $\mathrm{H}$ & -1.492519 & 2.535420 & -1.073814 \\
\hline C & -4.026954 & 2.386733 & 1.882354 \\
\hline $\mathrm{H}$ & -3.413001 & ๑. 332863 & 2.024892 \\
\hline C & -5.484111 & -1.354181 & -1.562931 \\
\hline $\mathrm{H}$ & -4.537478 & ๑. 373195 & -0.715286 \\
\hline C & -3.960923 & -3.259162 & -2.022128 \\
\hline $\mathrm{H}$ & -1.909125 & -2.889002 & -1.508623 \\
\hline C & $\odot .494349$ & 2.857985 & 3.434807 \\
\hline $\mathrm{H}$ & 1.822447 & 4.368288 & 2.658186 \\
\hline 0 & -0.899903 & 1.315712 & 4.303453 \\
\hline C & 3.751290 & -3.564443 & 1.190826 \\
\hline C & 4.696148 & -2.375207 & 3.311574 \\
\hline C & 2.346607 & -4.966672 & -0.566564 \\
\hline C & 5.383174 & 2.642034 & -1.812371 \\
\hline C & 3.851025 & 4.499577 & -2.826815 \\
\hline C & 6.890935 & $\odot .515320$ & -1.647859 \\
\hline 0 & $\odot .807224$ & -1.101137 & 4.371717 \\
\hline C & -0.554036 & -2.684033 & 3.524265 \\
\hline $\mathrm{H}$ & -1.855922 & -4.228902 & 2.771153 \\
\hline C & -3.885612 & 3.564737 & 1.094013 \\
\hline C & -2.444414 & 5.005466 & -0.601488 \\
\hline C & -4.875197 & 2.350436 & 3. 180377 \\
\hline C & -5.264390 & -2.707608 & -1.930893 \\
\hline C & -6.847506 & -0.644816 & -1.772860 \\
\hline C & -3.582193 & -4.618106 & -2.681759 \\
\hline 0 & $\odot .002411$ & 3.420095 & 4.578360 \\
\hline C & -0.664174 & 2.353125 & 5.263391 \\
\hline 0 & 4.554600 & -4.641591 & 1.504255 \\
\hline C & 4.154378 & -3.491073 & 4.240282 \\
\hline C & 4.526465 & -1.031402 & 4.054894 \\
\hline C & 6.213708 & -2.582141 & 3. 093127 \\
\hline C & 2.739705 & -6.281289 & 0.147787 \\
\hline C & 2.892563 & -4.988643 & -2.014547 \\
\hline C & $\odot .794902$ & -4.983408 & -0.632886 \\
\hline 0 & 6.495952 & 3.413638 & -2.091224 \\
\hline C & 4.471607 & 4.297283 & -4.232784 \\
\hline C & 4.450078 & 5.776934 & -2.191935 \\
\hline
\end{tabular}




\begin{tabular}{|c|c|c|c|}
\hline C & 2.343162 & 4.770292 & -3.024712 \\
\hline C & 8.067502 & 1.021409 & $-\odot .780295$ \\
\hline C & 6.720591 & $-\odot .988119$ & -1.333778 \\
\hline C & 7.280396 & 0.630303 & -3.143694 \\
\hline C & 0.549843 & -2.103100 & 5.363165 \\
\hline 0 & - ๑. . 83059 & -3.202452 & 4.697639 \\
\hline 0 & -4.730252 & 4.619501 & 1.372835 \\
\hline C & -2.917581 & 6.309628 & $\odot .082422$ \\
\hline C & $-\odot .892769$ & 5.066995 & -0.574370 \\
\hline C & -2.906894 & $5.0 \odot 9374$ & -2.078307 \\
\hline C & -6.389809 & 2.503477 & 2. 905401 \\
\hline C & -4.407552 & 3.487449 & 4.123130 \\
\hline C & -4.683704 & 1.017200 & 3.937309 \\
\hline 0 & -6.368518 & -3.468846 & -2.252636 \\
\hline C & -6.757583 & ๑. 861520 & -1.442417 \\
\hline C & -7.968546 & -1.234129 & -0.883477 \\
\hline C & -7.254454 & $-\odot .762208$ & -3.263509 \\
\hline C & -3.185028 & -5.640318 & -1.589537 \\
\hline C & -4.673186 & -5.238843 & -3.586312 \\
\hline C & -2.358200 & -4.399566 & -3.613597 \\
\hline $\mathrm{H}$ & -1.617310 & 2.709732 & 5.654462 \\
\hline $\mathrm{H}$ & $-\odot .014615$ & 1.971251 & 6.062813 \\
\hline C & 5.669573 & -4.833468 & 0.627601 \\
\hline $\mathrm{H}$ & 4.666197 & -3.451932 & 5. 209391 \\
\hline $\mathrm{H}$ & 3. 080144 & -3.361874 & 4.415786 \\
\hline $\mathrm{H}$ & 4.309329 & -4.482048 & 3.810453 \\
\hline $\mathrm{H}$ & 4.965547 & -0.192509 & 3.501956 \\
\hline $\mathrm{H}$ & 3.475059 & -0.800617 & 4.256489 \\
\hline $\mathrm{H}$ & 5.047154 & -1.089938 & 5.016714 \\
\hline $\mathrm{H}$ & 6.612801 & -1.866832 & 2.363691 \\
\hline $\mathrm{H}$ & 6.745567 & -2.420337 & 4.038132 \\
\hline $\mathrm{H}$ & 6.446423 & -3.592728 & 2.757441 \\
\hline $\mathrm{H}$ & 3.804620 & -6.505237 & ๑. 095449 \\
\hline $\mathrm{H}$ & 2.448813 & -6.271712 & 1. 202117 \\
\hline $\mathrm{H}$ & 2.212317 & -7.109371 & -0.339728 \\
\hline $\mathrm{H}$ & 2.538946 & -5.883967 & -2.539594 \\
\hline $\mathrm{H}$ & 2.557554 & -4.112753 & -2.582618 \\
\hline $\mathrm{H}$ & 3.987342 & -4.999752 & -2.039262 \\
\hline $\mathrm{H}$ & ๑. 459485 & -5.919855 & -1.091897 \\
\hline $\mathrm{H}$ & ๑. 357239 & -4.917325 & ๑. 369240 \\
\hline $\mathrm{H}$ & ๑. 385711 & -4.165863 & -1.233919 \\
\hline C & 7.007720 & 4.128883 & -0.953848 \\
\hline $\mathrm{H}$ & 4.015192 & 3.442008 & -4.745095 \\
\hline $\mathrm{H}$ & 4.297646 & 5.188142 & -4.848012 \\
\hline $\mathrm{H}$ & 5.548802 & 4.129414 & -4.172211 \\
\hline $\mathrm{H}$ & 4.078286 & 6.657938 & -2.727672 \\
\hline $\mathrm{H}$ & 4.156808 & 5.882969 & -1.140657 \\
\hline $\mathrm{H}$ & 5.537731 & 5.795232 & -2.260391 \\
\hline $\mathrm{H}$ & 1.827578 & 3.933146 & -3.509417 \\
\hline $\mathrm{H}$ & 1.831565 & 4.993949 & -2.081625 \\
\hline $\mathrm{H}$ & 2.222477 & 5.643183 & -3.674478 \\
\hline $\mathrm{H}$ & 8.441449 & 1.986957 & -1.121672 \\
\hline $\mathrm{H}$ & 7.786721 & 1.101140 & $\odot .276673$ \\
\hline $\mathrm{H}$ & 8.900352 & ๑. 311892 & -0.847372 \\
\hline $\mathrm{H}$ & 7.652175 & -1.507914 & -1.580812 \\
\hline $\mathrm{H}$ & 6.514935 & -1.171803 & -0.272374 \\
\hline $\mathrm{H}$ & 5.921662 & -1.448568 & -1.925557 \\
\hline $\mathrm{H}$ & 8.205676 & $\odot .072507$ & -3.331280 \\
\hline $\mathrm{H}$ & 6.498390 & ๑. 208718 & -3.786032 \\
\hline $\mathrm{H}$ & 7.444360 & 1.670187 & -3.433750 \\
\hline $\mathrm{H}$ & 1.492861 & -2.434063 & 5.798381 \\
\hline $\mathrm{H}$ & -0.130122 & -1.697856 & 6.124975 \\
\hline C & -5.812561 & 4.777081 & 0.449480 \\
\hline $\mathrm{H}$ & -2.387762 & 7.151111 & -0.378887 \\
\hline $\mathrm{H}$ & -3.983959 & 6.501441 & -0.033209 \\
\hline $\mathrm{H}$ & -2.687907 & 6.313443 & 1.151646 \\
\hline $\mathrm{H}$ & -0.557505 & 6.006313 & -1.028185 \\
\hline $\mathrm{H}$ & $-\odot .515874$ & 5.030241 & 0.453487 \\
\hline $\mathrm{H}$ & $-\odot .423854$ & 4. 252404 & -1.133496 \\
\hline $\mathrm{H}$ & -2.513010 & 4.144812 & -2.625475 \\
\hline $\mathrm{H}$ & -3.998582 & 4.986165 & -2.164280 \\
\hline $\mathrm{H}$ & -2.552494 & 5.915350 & -2.584114 \\
\hline $\mathrm{H}$ & -6.737721 & 1.769040 & 2.168924 \\
\hline $\mathrm{H}$ & -6.950160 & 2.332132 & 3.832106 \\
\hline $\mathrm{H}$ & -6.643506 & 3.502798 & 2.551228 \\
\hline
\end{tabular}

\begin{tabular}{|c|c|c|c|}
\hline $\mathrm{H}$ & -4.572959 & 4.471185 & 3.681010 \\
\hline $\mathrm{H}$ & -4.958005 & 3.437519 & 5.070220 \\
\hline $\mathrm{H}$ & -3.338706 & 3.388948 & 4.344969 \\
\hline $\mathrm{H}$ & -3.631521 & $\odot .825596$ & 4.174074 \\
\hline $\mathrm{H}$ & -5.236532 & 1.062573 & 4.881711 \\
\hline $\mathrm{H}$ & -5.074838 & ๑. 160199 & 3.376329 \\
\hline C & -6.797201 & -4.363845 & -1.220635 \\
\hline $\mathrm{H}$ & -7.720915 & 1.331024 & -1.667654 \\
\hline $\mathrm{H}$ & -6.544444 & 1.044318 & -0.382473 \\
\hline $\mathrm{H}$ & -5.995680 & 1. 372887 & -2.041653 \\
\hline $\mathrm{H}$ & -8.258268 & -2.235540 & -1.201510 \\
\hline $\mathrm{H}$ & -7.665580 & -1.271111 & 0.169859 \\
\hline $\mathrm{H}$ & -8.860195 & -0.599706 & -0.949547 \\
\hline $\mathrm{H}$ & -7.364836 & -1.804425 & -3.568407 \\
\hline $\mathrm{H}$ & -8.211638 & -0.253252 & -3.428499 \\
\hline $\mathrm{H}$ & -6.507314 & -0.287209 & -3.910418 \\
\hline $\mathrm{H}$ & -2.342721 & -5.280412 & -0.987685 \\
\hline $\mathrm{H}$ & -4.015758 & -5.850909 & -0.907447 \\
\hline $\mathrm{H}$ & -2.885596 & -6.589099 & -2.050476 \\
\hline $\mathrm{H}$ & -4.226615 & -6.073193 & -4.139333 \\
\hline $\mathrm{H}$ & -5.522059 & -5.639743 & -3.033565 \\
\hline $\mathrm{H}$ & -5.054982 & -4.518749 & -4.315480 \\
\hline $\mathrm{H}$ & -2.591435 & -3.676019 & -4.403381 \\
\hline $\mathrm{H}$ & -1.465541 & -4.054399 & -3.083984 \\
\hline $\mathrm{H}$ & -2.095809 & -5.346885 & -4.096862 \\
\hline $\mathrm{H}$ & 5.348994 & -5.093306 & -0.386276 \\
\hline $\mathrm{H}$ & 6.296928 & -3.936270 & 0.581883 \\
\hline $\mathrm{H}$ & 6.246269 & -5.661233 & 1.046023 \\
\hline $\mathrm{H}$ & 7.880995 & 4.678756 & -1.311329 \\
\hline $\mathrm{H}$ & 6.269022 & 4.831556 & -0.559129 \\
\hline $\mathrm{H}$ & 7.306335 & 3.445914 & -0.154489 \\
\hline $\mathrm{H}$ & -6.419539 & 3.866600 & ๑. 391558 \\
\hline $\mathrm{H}$ & -6.421824 & 5.598619 & ๑. 832452 \\
\hline $\mathrm{H}$ & -5.456094 & 5.029040 & -0.554491 \\
\hline $\mathrm{H}$ & -6.048775 & -5.136688 & -1.017603 \\
\hline $\mathrm{H}$ & - 7.010965 & -3.823918 & -0.291664 \\
\hline $\mathrm{H}$ & -7.711221 & -4.835573 & -1.587665 \\
\hline $\mathrm{H}$ & -0.741637 & -0.719860 & -4.567791 \\
\hline $\mathrm{H}$ & -1.191992 & 1.644090 & -3.845977 \\
\hline $\mathrm{H}$ & ๑. 781687 & $\odot .765024$ & -4.564181 \\
\hline $\mathrm{H}$ & 1.217751 & -1.597645 & -3.826271 \\
\hline
\end{tabular}

35

B3LYP SCF energy:

B3LYP enthalpy:

B3LYP free energy: M06 SCF energy in solution:

a.u.

M06 enthalpy in solution:

M06 free energy in solution:

a.u.

Cartesian coordinates

\begin{tabular}{lrrr} 
ATOM & X & $Y$ & \multicolumn{1}{c}{$\mathrm{Z}$} \\
C & -1.473790 & -4.090327 & 0.299727 \\
H & -2.226070 & -3.773364 & 1.010671 \\
C & -1.535498 & -3.692937 & -1.014206 \\
H & -2.356141 & -3.074759 & -1.363845 \\
C & 1.476069 & -4.088551 & -0.306678 \\
H & 2.228878 & -3.770008 & -1.016328 \\
C & 1.536868 & -3.693819 & 1.008206 \\
H & 2.357276 & -3.076307 & 1.359590 \\
Rh & 0.001019 & -2.303764 & -0.002123 \\
P & 1.709890 & -0.613324 & -0.015990 \\
P & -1.709008 & -0.614080 & 0.015753 \\
C & 1.373518 & 0.878012 & -1.054656 \\
C & 2.153622 & 0.066274 & 1.635202 \\
C & -1.373678 & 0.876905 & 1.055151 \\
C & -2.155432 & 0.066517 & -1.634327 \\
C & 0.365176 & 1.806728 & -0.649528 \\
C & -0.366108 & 1.806476 & 0.650165 \\
C & 0.123503 & 2.855886 & -1.522149 \\
C & -0.125483 & 2.855866 & 1.522779
\end{tabular}

-2715.01400721 a.u. -2714.295150 a.u. -2714.420209 a.u. $-2715.22638830$

-2714.507531 a.u.

$-2714.632590$ 


\begin{tabular}{|c|c|c|c|}
\hline 0 & $-\odot .784761$ & 3.866421 & -1.362319 \\
\hline 0 & ๑. 782090 & 3.867057 & 1. 363198 \\
\hline C & $-\odot .807065$ & 3.028425 & 2.727094 \\
\hline 0 & ๑. 352034 & 4.146085 & -3.361778 \\
\hline C & -0.682099 & 4.692120 & -2.530980 \\
\hline C & 0.677427 & 4.693574 & 2.531111 \\
\hline 0 & -0.355358 & 4.145792 & 3.362430 \\
\hline $\mathrm{H}$ & -1.632948 & 4.673711 & -3.074311 \\
\hline $\mathrm{H}$ & -0.412825 & 5.711414 & -2.236795 \\
\hline $\mathrm{H}$ & 1.628308 & 4.677994 & 3.074430 \\
\hline $\mathrm{H}$ & 0.405683 & 5.711970 & 2.235997 \\
\hline $\mathrm{H}$ & -0.856625 & -4.927003 & 0.609023 \\
\hline $\mathrm{H}$ & -0.955967 & -4.187202 & -1.789014 \\
\hline $\mathrm{H}$ & ๑. 859768 & -4.925080 & -0.618133 \\
\hline $\mathrm{H}$ & ๑. 957191 & -4.189975 & 1.781695 \\
\hline C & 2. 019327 & -0.053420 & 4.057196 \\
\hline $\mathrm{H}$ & 1.632936 & -0.532082 & 4.952435 \\
\hline C & 2.865983 & 1.051236 & 4.162918 \\
\hline $\mathrm{H}$ & 3.144923 & 1.430818 & 5.141747 \\
\hline C & 3.352542 & 1.670352 & 3.007545 \\
\hline $\mathrm{H}$ & 4.012945 & 2.529601 & 3. 085901 \\
\hline C & 2.995912 & 1.185711 & 1.749286 \\
\hline $\mathrm{H}$ & 3.367980 & 1.680213 & 0.856757 \\
\hline C & 1.664989 & $-\odot .544519$ & 2.799037 \\
\hline $\mathrm{H}$ & $\odot .994728$ & -1.395704 & 2.716237 \\
\hline C & 4.485479 & -2.396869 & -2.502587 \\
\hline $\mathrm{H}$ & 4.476846 & -2.831931 & -3.497907 \\
\hline C & 5.666305 & -2.374478 & -1.754919 \\
\hline $\mathrm{H}$ & 6.580466 & -2.791246 & -2.167227 \\
\hline C & 5. 661701 & -1.819895 & -0.476113 \\
\hline $\mathrm{H}$ & 6.572755 & -1.804074 & 0.115060 \\
\hline C & 4.488445 & -1.276351 & ๑. 055965 \\
\hline $\mathrm{H}$ & 4.511282 & $-\odot .843235$ & 1.049397 \\
\hline C & 3. 310508 & -1.869060 & -1.968841 \\
\hline $\mathrm{H}$ & 2.395673 & -1.909827 & -2.554959 \\
\hline C & 3.298150 & -1.285801 & -0.686166 \\
\hline C & -4.478103 & -2.404025 & 2.504552 \\
\hline $\mathrm{H}$ & -4.467360 & -2.839775 & 3.499552 \\
\hline C & -5.659938 & -2.383442 & 1.758401 \\
\hline $\mathrm{H}$ & -6.572743 & -2.802327 & 2.171566 \\
\hline C & -5.658114 & -1.827888 & $\odot .480009$ \\
\hline $\mathrm{H}$ & -6.569984 & -1.813424 & -0.109940 \\
\hline C & -4.486616 & -1.281657 & -0.053206 \\
\hline $\mathrm{H}$ & -4.511529 & -0.847849 & -1.046298 \\
\hline C & -3.295374 & -1.289342 & 0.687383 \\
\hline C & -3.304875 & -1.873491 & 1.969679 \\
\hline $\mathrm{H}$ & -2.389215 & -1.912725 & 2.554611 \\
\hline C & -2.999838 & 1.184558 & -1.746448 \\
\hline $\mathrm{H}$ & -3.372229 & 1.677238 & -0.853044 \\
\hline C & -3.358192 & 1.670143 & -3.003858 \\
\hline $\mathrm{H}$ & -4.020252 & 2.528252 & -3.080694 \\
\hline C & -2.871203 & 1.053420 & -4.160332 \\
\hline $\mathrm{H}$ & -3.151493 & 1.433722 & -5.138497 \\
\hline C & -2.022385 & $-\odot . \odot 49767$ & -4.056566 \\
\hline $\mathrm{H}$ & -1.635624 & -0.526515 & -4.952666 \\
\hline C & -1.666354 & -0.541829 & -2.799267 \\
\hline $\mathrm{H}$ & -0.994379 & -1.391814 & -2.717992 \\
\hline C & 2. 061899 & 1.078445 & -2.258416 \\
\hline $\mathrm{H}$ & 2.849766 & ๑. 396974 & -2.547298 \\
\hline C & 1.795255 & 2.156900 & -3.119500 \\
\hline $\mathrm{H}$ & 2.347725 & 2.296303 & -4.041720 \\
\hline C & ๑. 804732 & 3. 029048 & -2.726580 \\
\hline C & -1.796815 & 2.155360 & 3.119935 \\
\hline $\mathrm{H}$ & -2.349472 & 2.294265 & 4.042118 \\
\hline C & -2.062402 & 1.076660 & 2.258830 \\
\hline $\mathrm{H}$ & -2.849662 & ๑. 394438 & 2.547610 \\
\hline
\end{tabular}

36

B3LYP SCF energy:

B3LYP enthalpy:

B3LYP free energy:

M06 SCF energy in solution:

a.u.
M06 enthalpy in solution: M06 free energy in solution: a.u.

Cartesian coordinates

\begin{tabular}{|c|c|c|c|}
\hline ATOM & $x$ & Y & Z \\
\hline C & ๑. .0००००० & 0.665433 & ๑. 000000 \\
\hline H & $\odot .923689$ & 1.239527 & $\odot . ๑ \odot \odot \odot \odot \odot$ \\
\hline $\mathrm{H}$ & $-\odot .923651$ & 1.239571 & $\odot . ๑ \odot \odot \odot \odot \odot$ \\
\hline C & $\odot .0000 \odot \odot$ & -0.665433 & $\odot .0 \odot \odot \odot \odot \odot$ \\
\hline $\mathrm{H}$ & -0.923689 & -1.239527 & $\odot .0000 \odot \odot$ \\
\hline $\mathrm{H}$ & ๑. 923651 & -1.239571 & $\odot .000000$ \\
\hline
\end{tabular}

37

B3LYP SCF energy:

B3LYP enthalpy:

B3LYP free energy:

M06 SCF energy in solution

a.u.

M06 enthalpy in solution:

M06 free energy in solution:

a.u.

Cartesian coordinates

\begin{tabular}{|c|c|c|c|}
\hline ATOM & $\mathrm{X}$ & Y & Z \\
\hline C & $\odot . ๑ \odot \odot \odot \odot \odot$ & -6.330612 & 5.672472 \\
\hline $\mathrm{H}$ & $\odot .0 \odot \odot \odot \odot \odot$ & -5.398924 & 5.169209 \\
\hline C & -1.535916 & -6.298038 & -4.934435 \\
\hline $\mathrm{H}$ & -2.417433 & -7.209237 & -3.545660 \\
\hline C & -0.965976 & -7.948983 & -4.605636 \\
\hline $\mathrm{H}$ & -1.046710 & 2.641903 & -3.585129 \\
\hline $\mathrm{H}$ & -1.588365 & 1.900233 & -5.077756 \\
\hline C & ๑. 507891 & 1.526848 & -4.596417 \\
\hline $\mathrm{H}$ & 0.574420 & 0.948025 & -5.521531 \\
\hline $\mathrm{H}$ & $\odot .943426$ & 2.505783 & -4.826101 \\
\hline C & 1.344730 & 0.875900 & -3.507016 \\
\hline $\mathrm{H}$ & 2.011044 & 1.547643 & -2.980508 \\
\hline C & 1.603715 & -0.476293 & -3.356480 \\
\hline $\mathrm{H}$ & 2.475466 & -0.742776 & -2.760805 \\
\hline C & 1.048978 & -1.601135 & -4.199128 \\
\hline $\mathrm{H}$ & 1.114961 & -2.526272 & -3.614784 \\
\hline H & 1.690165 & -1.757149 & -5.080840 \\
\hline C & -0.414726 & -1.386376 & -4.636967 \\
\hline $\mathrm{H}$ & -0.847216 & -2.357912 & -4.899337 \\
\hline $\mathrm{H}$ & -0.459766 & -0.783669 & -5.547927 \\
\hline Rh & ๑. 021927 & ๑. . 043124 & -1.794749 \\
\hline$P$ & 1.705733 & 0.324075 & -0.084974 \\
\hline$P$ & -1.697116 & -0.274844 & -0.121677 \\
\hline C & 1.206329 & 1.273892 & 1.431972 \\
\hline C & 2.379672 & -1.263038 & ๑. 552991 \\
\hline C & 3.222448 & 1.200813 & -0.697763 \\
\hline C & -1.231035 & -1.208821 & 1. 416468 \\
\hline C & -2.430261 & 1. 292853 & $\odot .500092$ \\
\hline C & -3.170928 & -1.190414 & -0.782172 \\
\hline C & 1.745270 & 2.547132 & 1.663924 \\
\hline C & ○. 262454 & ๑. 729056 & 2.359767 \\
\hline C & 3.182243 & -1.297663 & 1.701674 \\
\hline C & 2.141806 & -2.449092 & -0.138146 \\
\hline C & 3.142822 & 2.509810 & -1.200233 \\
\hline C & 4.450119 & $\odot .538445$ & -0.785477 \\
\hline C & $-\odot .315419$ & -0.647626 & $2.36244 \odot$ \\
\hline C & -1.775347 & -2.478740 & 1.653895 \\
\hline C & -2.204025 & 2.489965 & -0.175905 \\
\hline C & -3.263596 & 1.298785 & 1.627365 \\
\hline C & -4.416046 & -0.561982 & -0.904225 \\
\hline C & -3.047550 & -2.499316 & -1.264763 \\
\hline C & 1. 399522 & 3.343213 & 2.769121 \\
\hline $\mathrm{H}$ & 2.490096 & 2.941227 & ๑. 987941 \\
\hline C & -0.052006 & 1.538335 & 3.443498 \\
\hline C & 3.836043 & -2.463802 & 2.107460 \\
\hline $\mathrm{H}$ & 3.302073 & -0.389043 & 2.276953 \\
\hline C & 2.736173 & -3.669233 & $\odot .2310 \odot 9$ \\
\hline $\mathrm{H}$ & 1.460631 & -2.417674 & -0.981422 \\
\hline
\end{tabular}

-78.482564 a.u.

$-78.508080$

๑ . $000 \odot \odot \odot$ 


\begin{tabular}{|c|c|c|c|}
\hline C & 4.269815 & 3. 217371 & -1.638538 \\
\hline $\mathrm{H}$ & 2.172457 & 2.988752 & -1.247263 \\
\hline C & 5.618269 & 1.174713 & -1.234526 \\
\hline $\mathrm{H}$ & 4.500688 & $-\odot .499944$ & $-\odot .495103$ \\
\hline C & $-\odot .030747$ & -1.439274 & 3.467120 \\
\hline C & -1.459307 & -3.257393 & 2.780397 \\
\hline $\mathrm{H}$ & -2.502257 & -2.883493 & ๑. 964891 \\
\hline C & -2.841581 & 3.691062 & 0.184244 \\
\hline $\mathrm{H}$ & -1.497530 & 2.484202 & -0.998460 \\
\hline C & -3.958562 & 2.444248 & 2.022879 \\
\hline $\mathrm{H}$ & -3.374095 & ○. 383812 & 2.194229 \\
\hline C & -5.550630 & -1.236302 & -1.372112 \\
\hline $\mathrm{H}$ & -4.505363 & $\odot .473687$ & $-\odot .613117$ \\
\hline C & -4.139032 & -3.240827 & -1.746490 \\
\hline $\mathrm{H}$ & -2.067610 & -2.964988 & -1.261189 \\
\hline C & ๑. 489120 & 2.806054 & 3. 650311 \\
\hline $\mathrm{H}$ & 1.844460 & 4.319105 & 2.927722 \\
\hline 0 & -0.921481 & 1. 252961 & 4.469752 \\
\hline C & 3.677659 & -3.617313 & 1.286471 \\
\hline C & 4.630050 & -2.493469 & 3.439738 \\
\hline C & 2.275509 & -4.948967 & -0.524055 \\
\hline C & 5.523456 & 2.559282 & -1.516230 \\
\hline C & 4.104420 & 4.582137 & -2.363959 \\
\hline C & 6.892066 & $\odot .334415$ & -1.530086 \\
\hline 0 & ๑. 809272 & -1.135928 & 4.512574 \\
\hline C & -0.574130 & -2.705280 & 3.677812 \\
\hline $\mathrm{H}$ & -1.907195 & -4.231549 & 2.941301 \\
\hline C & -3.810405 & 3.606402 & 1.212515 \\
\hline C & -2.395104 & 4.988445 & -0.548707 \\
\hline C & -4.786239 & 2.444252 & 3. 334899 \\
\hline C & -5.409133 & -2.615979 & -1.673994 \\
\hline C & -6.874505 & -0.463696 & -1.609226 \\
\hline C & -3.839294 & -4.647678 & -2.342949 \\
\hline 0 & -0.016611 & 3.346613 & 4.799627 \\
\hline C & -0.679925 & 2.261512 & 5.457930 \\
\hline 0 & 4.446873 & -4.721316 & 1.594366 \\
\hline C & 4.058356 & -3.613941 & 4.344714 \\
\hline C & 4.488757 & -1.160088 & 4.207490 \\
\hline C & 6.143961 & -2.729839 & 3.224674 \\
\hline C & 2.587098 & -6.280849 & ๑. 199173 \\
\hline C & 2.885538 & -4.981796 & -1.945849 \\
\hline C & ๑. 728804 & -4.911130 & -0.663691 \\
\hline 0 & 6.681380 & 3.277840 & -1.751966 \\
\hline C & 4.694418 & 4.450913 & -3.791270 \\
\hline C & 4.793133 & 5.764927 & -1.642489 \\
\hline C & 2.615787 & 4.965177 & -2.515149 \\
\hline C & 8.107930 & $\odot .699094$ & -0.645904 \\
\hline C & 6.628792 & -1.174213 & -1.323360 \\
\hline C & 7.272998 & $\odot .534230$ & -3.019152 \\
\hline C & 0.542997 & -2.130273 & 5.508719 \\
\hline 0 & -0.097263 & -3.227787 & 4.847994 \\
\hline 0 & -4.618940 & 4.686224 & 1.504687 \\
\hline C & -2.764374 & 6.305710 & ๑.174144 \\
\hline C & -0.844756 & 4.993867 & $-\odot .644703$ \\
\hline C & -2.965865 & 5.015455 & -1.986875 \\
\hline C & $-6.30 \odot 9 \odot 6$ & 2.633157 & 3. 082349 \\
\hline C & -4.273621 & 3.578824 & 4.257295 \\
\hline C & -4.620409 & 1.113842 & 4.102920 \\
\hline 0 & -6.555865 & -3.332790 & -1.947208 \\
\hline C & -6.699389 & 1. 049518 & -1.353024 \\
\hline C & -8.019571 & $-\odot .946238$ & -0.686695 \\
\hline C & -7.298989 & -0.631115 & -3.090098 \\
\hline C & -3.458774 & -5.630445 & -1.209402 \\
\hline C & -4.978338 & -5.266380 & -3.187536 \\
\hline C & -2.632383 & -4.529768 & -3.314010 \\
\hline $\mathrm{H}$ & -1.630492 & 2.607149 & 5.864181 \\
\hline $\mathrm{H}$ & $-\odot .025491$ & 1.856004 & 6.241947 \\
\hline C & 5.572098 & -4.934593 & $\odot .736879$ \\
\hline $\mathrm{H}$ & 4.563034 & -3.601891 & 5.318359 \\
\hline $\mathrm{H}$ & 2.985429 & -3.467073 & 4.513682 \\
\hline $\mathrm{H}$ & 4.196964 & -4.600226 & 3.898902 \\
\hline $\mathrm{H}$ & 4.949247 & -0.321789 & 3.671308 \\
\hline $\mathrm{H}$ & 3.442586 & $-\odot .908155$ & 4. 409951 \\
\hline $\mathrm{H}$ & 5.004818 & -1.248616 & 5.169575 \\
\hline $\mathrm{H}$ & 6.562049 & -2.010127 & 2.510335 \\
\hline
\end{tabular}

\begin{tabular}{|c|c|c|}
\hline 6.674333 & -2.596768 & 4.175006 \\
\hline 6.357035 & -3.738850 & 2.871899 \\
\hline 3.644225 & -6.543229 & $\odot .195987$ \\
\hline 2.248527 & -6.263425 & 1.239307 \\
\hline 2.052317 & -7.087393 & $-\odot .315705$ \\
\hline 2.519529 & -5.857042 & -2.495861 \\
\hline 2.612969 & -4.087208 & -2.517923 \\
\hline 3.978714 & -5.037143 & -1.923424 \\
\hline ๑. 383231 & -5.835186 & -1.140006 \\
\hline$\odot .247176$ & -4.830488 & 0.317058 \\
\hline$\odot .37675 \odot$ & -4.079548 & -1.281659 \\
\hline 7.249491 & & $-\odot .570780$ \\
\hline 4.177997 & 3.668105 & -4.359504 \\
\hline 4.571805 & 5.396040 & -4.333706 \\
\hline 5.759107 & 4.210478 & -3.761001 \\
\hline 4.473644 & 6.707330 & -2.102129 \\
\hline 4.519794 & 5.807032 & $-\odot .581408$ \\
\hline 5.878 & & -1.727468 \\
\hline 2.041 & & -3.057492 \\
\hline 2.12 & & -1.549277 \\
\hline 2.545536 & 319 & -3.090070 \\
\hline 8.5 & & $-\odot$. \\
\hline 7.843940 & & 0.418219 \\
\hline 8.892581 & $-\odot$. & 7601 \\
\hline 7.522 & -1 & -1. \\
\hline 6.421423 & -1. & -0.275211 \\
\hline 5.7 & -1 & 203 \\
\hline & -0. & -3 \\
\hline 6.4 & & 8542 \\
\hline $7.50 \odot 282$ & & -3. \\
\hline 1.482761 & -2.4 & 47004 \\
\hline 0.13 & -1 & 7328 \\
\hline 5.725873 & & 0.617374 \\
\hline 2.238122 & & $-\odot .320165$ \\
\hline 3.82 & & 813 \\
\hline 2.45 & & \\
\hline 0.512 & & -1 \\
\hline 0.3 & & 504 \\
\hline 0.45 & & -1 \\
\hline 2.65 & & -2 . \\
\hline 4.060 & & -1 \\
\hline 2.607301 & & -2.520676 \\
\hline 6.67 & & \\
\hline 6.85 & 69 & 394 \\
\hline 6.536368 & & \\
\hline 4.42 & & 3.8 \\
\hline 4.803147 & & 5.2 \\
\hline 3.20 & & \\
\hline 3.5 & & 559 \\
\hline 5.161457 & 41 & 52780 \\
\hline 5.040679 & & \\
\hline 7.0 & -4.1 & -0. \\
\hline 7.637002 & & $-1.5 c$ \\
\hline 6.468422 & & -0. \\
\hline 5.91 & & -1. \\
\hline 8.36 & & $-\odot .9$ \\
\hline .7 .71 & $-\odot . \subseteq$ & 5612 \\
\hline 8.875416 & -0.267271 & -0.778925 \\
\hline 7.468444 & & \\
\hline 8.22 & $-\odot$. & -3.2 \\
\hline 6.531858 & -0.2 & -3.763344 \\
\hline 2.584000 & & \\
\hline 4.27 & -5.7 & -0.4 \\
\hline 3.216 & -6.6 & -1.627314 \\
\hline 4.58 & & \\
\hline 5.8 & -5.6004 & -2.594178 \\
\hline 5.32 & -4.5 & \\
\hline 2.858387 & & \\
\hline 1.713670 & -4.195241 & -2.823636 \\
\hline 2.421212 & & \\
\hline & & -0.274677 \\
\hline 6.202434 & -4.040285 & 0.678812 \\
\hline & & \\
\hline & & -0.89678 \\
\hline 6.564021 & 4.588165 & -0.11530 \\
\hline
\end{tabular}




$\begin{array}{rrrr}\mathrm{H} & 7.505890 & 3.108636 & 0.171681 \\ \mathrm{H} & -6.328495 & 3.960346 & 0.537925 \\ \mathrm{H} & -6.330612 & 5.672472 & 13.189300 \\ \mathrm{H} & -5.398924 & 5.169209 & 14.542800 \\ \mathrm{H} & -6.298038 & -4.934435 & 17.867200 \\ \mathrm{H} & -7.209237 & -3.545660 & 0.030672 \\ \mathrm{H} & -7.948983 & -4.605636 & -1.203406\end{array}$

38

B3LYP SCF energy:

B3LYP enthalpy:

-2869.87083207 a.u.

B3LYP free energy:

-2869.075769 a.u.

M06 SCF energy in solution:

a.u.

M06 enthalpy in solution:

M06 free energy in solution:

2869.205899 a.u

a.u.

$-2870.00828120$

-2869.213218 a.u.

$-2869.343348$

Cartesian coordinates

\begin{tabular}{|c|c|c|c|}
\hline ATOM & $\mathrm{X}$ & Y & Z \\
\hline C & -3.706859 & 1.395921 & $\odot .650973$ \\
\hline $\mathrm{H}$ & -3.195668 & 2.144593 & 1.243224 \\
\hline C & -3.546323 & 1.493907 & -0.721411 \\
\hline $\mathrm{H}$ & -2.960726 & 2.337071 & -1.084910 \\
\hline C & -4.368698 & $\odot .797341$ & -1.780338 \\
\hline H & -3.774350 & $\odot .759912$ & -2.701030 \\
\hline $\mathrm{H}$ & -5.255093 & 1.404410 & -2.021167 \\
\hline C & -4.793736 & -0.634239 & -1.391980 \\
\hline $\mathrm{H}$ & -5.712158 & -0.615360 & -0.799492 \\
\hline $\mathrm{H}$ & -5.039431 & -1.186873 & -2.305406 \\
\hline C & -3.706766 & -1.396898 & $-\odot .65146$ \\
\hline $\mathrm{H}$ & -3.195287 & -2.145174 & -1.243977 \\
\hline C & -3.546000 & -1.494920 & 0.720870 \\
\hline $\mathrm{H}$ & -2.960080 & -2.337888 & 1.084288 \\
\hline C & -4.368444 & -0.798550 & 1.779880 \\
\hline $\mathrm{H}$ & -3.774116 & -0.761266 & 2.700586 \\
\hline $\mathrm{H}$ & -5.254828 & -1.405683 & 2.020586 \\
\hline C & -4.793552 & $\odot .633097$ & 1.39178 \\
\hline H & -5.038945 & 1.185627 & 2.305349 \\
\hline H & -5.712176 & $\odot .614309$ & ๑. 799597 \\
\hline $\mathrm{Rh}$ & -1.975342 & $-\odot . \odot \odot \odot 247$ & $-\odot .0 \odot \odot 23$ \\
\hline$P$ & $-\odot .279621$ & -1.726379 & -0.036736 \\
\hline$P$ & -0.280092 & 1.726209 & ๑. 036936 \\
\hline C & 1.236411 & -1.401105 & -1.04679 \\
\hline C & ๑. 363732 & -2.131503 & 1.639722 \\
\hline C & -0.912395 & -3.350815 & -0.668133 \\
\hline C & 1.236309 & 1.401325 & 1.04658 \\
\hline C & ๑. 362889 & 2.132095 & -1.639487 \\
\hline C & -0.913417 & 3.350178 & ๑. 669009 \\
\hline C & 1.469157 & -2.110130 & -2.232698 \\
\hline C & 2.144781 & -0.371724 & $-\odot .64756$ \\
\hline C & 1.508485 & -2.929809 & 1.803385 \\
\hline C & -0.317728 & -1.670437 & 2.774990 \\
\hline C & -1.425465 & -3.441984 & -1.977628 \\
\hline C & -0.948367 & -4.496454 & ๑. 141384 \\
\hline C & 2.144868 & ๑. 372219 & $\odot .647070$ \\
\hline C & 1.469292 & 2.110494 & 2.232355 \\
\hline C & -0.318579 & 1. 671161 & -2.774810 \\
\hline C & 1.507402 & 2.930756 & -1.803060 \\
\hline C & -0.950293 & 4.495913 & -0.140332 \\
\hline C & -1.425925 & 3.440909 & 1.978760 \\
\hline C & 2.554280 & -1.842582 & -3.084900 \\
\hline $\mathrm{H}$ & $\odot .809587$ & -2.918661 & -2.513598 \\
\hline C & 3.199970 & -0.128234 & -1.513419 \\
\hline C & 1.952458 & -3.265699 & 3.08216 \\
\hline $\mathrm{H}$ & 2.053785 & -3.283915 & $\odot .933509$ \\
\hline C & ๑.131934 & -2.004474 & 4.054239 \\
\hline $\mathrm{H}$ & -1.193370 & -1.038562 & 2.65112 \\
\hline C & -1.926411 & -4.647035 & -2.46920 \\
\hline $\mathrm{H}$ & -1.428527 & -2.566945 & -2.622735 \\
\hline C & -1.464855 & -5.699574 & -0.348842 \\
\hline $\mathrm{H}$ & -0.569797 & -4.461103 & 1.156305 \\
\hline C & 3.200412 & ๑. 129056 & 1.512582 \\
\hline
\end{tabular}

2.554801

0.809585

0.130844

$-1.194023$

1.951151

2.052715

$-1.467155$

$-0.572117$

$-1.927215$

$-1.428362$

3. 401695

2. 716884

4.193515

1. 265332

$-1.948671$

4.194422

3. 402342

2. 717592

1. 264019

$-1.950404$

4. 518850

5. 048591

5.048235

4.520049

5. 050954

6. 058761

5. 047385

6. 059502

$-0.401248$

1. 613957

2. 833119

$-2.304944$

$-2.345739$

$-1.485344$

$-0.402339$

1. 612475

2. 831669

$-1.482321$

$-2.304636$

$-2.343733$
1. 843322

2. 918836

2. 005684

1. 038997

3. 267103

3. 284763

5.698714

4. 460886

4.645660

2. 565756

$-0.827428$

$-2.412968$

0.800609

$-2.804170$

$-5.781334$

$-0.799217$

0.828357

2. 413854

2. 805716

5.780060

$-0.365866$

0.676442

$-0.676691$

0.367396

1.619495

0.405907

$-1.619506$

$-0.408936$

$-1.639627$

$-3.066882$

$-3.891367$

4.695662

6. 717404

6.573207

1. 640934

3. 068783

3. 892984

$-6.573989$

$-4.697338$

$-6.718922$
3. 084180

2. 513459

$-4.054015$

$-2.651033$

$-3.081796$

$-0.933153$

0.350283

$-1.155414$

2. 470719

2. 623717

$-2.702128$

$-3.992317$

$-1.356913$

4. 209044

$-1.653578$

1. 355573

2. 701197

3. 991472

$-4.208723$

1.655256

$-3.332297$

$-2.501856$

2. 501637

3. 330823

$-3.058479$

$-2.177167$

3. 058758

2.178216

4. 927583

5.203775

3. 199116

3. 488028

2. 035436

$-\odot .293786$

$-4.927400$

$-5.203419$

$-3.198666$

0.295352

$-3.486314$

$-2.033440$
39

B3LYP SCF energy:

B3LYP enthalpy:

B3LYP free energy:

M06 SCF energy in solution:

a.u.

M06 enthalpy in solution:

M06 free energy in solution: a.u.

Cartesian coordinates

$\begin{array}{lrrr}\text { ATOM } & \mathrm{X} & \mathrm{Y} & \mathrm{Z} \\ \text { C } & -0.008767 & -1.706598 & -0.212819 \\ \text { H } & -0.289886 & -2.640746 & -0.700395 \\ \text { C } & 1.214141 & -1.239255 & -0.492157 \\ \text { H } & 1.821024 & -1.849310 & -1.163641 \\ \text { C } & 1.923095 & 0.010409 & -0.030506 \\ \text { H } & 2.415759 & 0.454602 & -0.908324 \\ \text { H } & 2.748051 & -0.280602 & 0.639995 \\ \text { C } & 1.086969 & 1.106325 & 0.664712 \\ \text { H } & 0.674241 & 0.721504 & 1.600589 \\ \text { H } & 1.777238 & 1.907497 & 0.957173 \\ \text { C } & 0.008837 & 1.706577 & -0.212791 \\ \text { H } & 0.290175 & 2.640600 & -0.700480 \\ \text { C } & -1.214062 & 1.239292 & -0.492230 \\ \text { H } & -1.820818 & 1.849257 & -1.163907 \\ \text { C } & -1.923110 & -0.010276 & -0.030461 \\ \text { H } & -2.416063 & -0.454318 & -0.908186 \\ \text { H } & -2.747846 & 0.280796 & 0.640283 \\ \text { C } & -1.087085 & -1.106430 & 0.664526 \\ \text { H } & -1.777470 & -1.907628 & 0.956640 \\ \text { H } & -0.674521 & -0.721915 & 1.600610\end{array}$

-312.03040747 a.u. -311.840911 a.u. -311.880766 a.u. $-311.85778335$

-311.668287 a.u. $-311.708142$ 
TS1

B3LYP SCF energy:

B3LYP enthalpy:

B3LYP free energy:

-5535.32123709 a.u. -5533.287472 a.u. -5533.557879 a.u. SCF energy in solution: $\quad-5534.55478195$ a.u.

M06 enthalpy in solution: $\quad-5532.521017 \mathrm{a} . \mathrm{u}$ M06 free energy in solution: $\quad-5532.791424$ a.u.

Imaginary frequency:

$-261.7134 \mathrm{~cm}-1$

Cartesian coordinates

\begin{tabular}{|c|c|c|c|}
\hline ATOM & $\mathrm{X}$ & $\mathrm{Y}$ & Z \\
\hline $\mathrm{P}$ & $\odot .0 \odot \odot \odot \odot \odot$ & -5.390211 & 4.180330 \\
\hline$P$ & $\odot . \diamond \odot \odot \odot \odot \odot$ & -6.455569 & 3.959069 \\
\hline 0 & 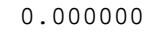 & -5.136359 & 3.190890 \\
\hline 0 & $\odot . \diamond \odot \odot \odot \odot \odot$ & -3.046779 & 2.790855 \\
\hline 0 & $\odot .0 \odot \odot \odot \odot \odot$ & -0.704165 & 7.808746 \\
\hline 0 & 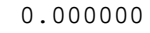 & -1.485376 & 6.291044 \\
\hline 0 & $\odot .0 \odot \odot \odot \odot \odot$ & -7.405156 & 4.285382 \\
\hline 0 & $\odot . \diamond \odot \odot \odot \odot \odot$ & -6.376667 & 3.607271 \\
\hline 0 & $\odot . \diamond \odot \odot \odot \odot \odot$ & -5.061475 & 2.915766 \\
\hline 0 & 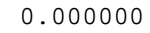 & $-\odot .242516$ & 8.368220 \\
\hline C & ๑. .०००००० & -0.597929 & 8.223361 \\
\hline C & ๑. .००००० & -7.592729 & 3. 661041 \\
\hline C & 1.433638 & -7.393671 & 4.243808 \\
\hline C & 1.965766 & -7.885338 & 2.653966 \\
\hline C & 2. 515191 & -8.448356 & 4.111074 \\
\hline C & 2.512803 & 1.589979 & 2.704789 \\
\hline C & 1.438616 & 1.548570 & 2.828668 \\
\hline C & - ๑. 265555 & 2.412729 & 2.043946 \\
\hline C & -0.724772 & 1.567946 & 3.979378 \\
\hline C & -0.108628 & 1.025277 & 4.782728 \\
\hline C & ๑. 990529 & 2.548170 & 4.345323 \\
\hline C & 1.456343 & $\odot .711626$ & 3. 396722 \\
\hline C & ๑. 872382 & 1.883831 & 1.945607 \\
\hline C & 2.655230 & 1.406824 & 2.977335 \\
\hline C & 2.974538 & $-\odot .758964$ & -1.047120 \\
\hline C & 2.536973 & -0.731090 & -2.372014 \\
\hline C & 3.280525 & -1.312245 & -3.407498 \\
\hline C & 4.524918 & -1.908807 & -3.064018 \\
\hline C & 4.935691 & -2.079367 & -1.712740 \\
\hline C & 4.144355 & -1.458682 & -0.736027 \\
\hline C & 6.155605 & -2.923576 & -1.241455 \\
\hline C & 7.437427 & -2.057028 & -1.181608 \\
\hline C & 6.388609 & -4.172216 & -2.126410 \\
\hline C & 5.919910 & -3.462691 & ๑.194854 \\
\hline C & 2.714543 & -1.331196 & -4.851398 \\
\hline C & 2.632696 & -2.788844 & -5.370195 \\
\hline C & 1.281123 & -0.758875 & -4.903149 \\
\hline C & 3.570481 & -0.473147 & -5.813187 \\
\hline C & 6.446825 & -1.683749 & -4.510117 \\
\hline C & 3.196798 & 1.366315 & ○. 932671 \\
\hline C & 4.359950 & 1.709335 & $\odot .244834$ \\
\hline C & 2.839092 & 2.135945 & 2.054855 \\
\hline C & 3.675678 & 3.124742 & 2.578201 \\
\hline C & 4.919304 & 3.339860 & 1.916440 \\
\hline C & 5.219846 & 2.738903 & ๑. 672494 \\
\hline C & 6.382786 & 3.135305 & $-\odot .283350$ \\
\hline C & 5.820941 & 3.246405 & -1.728237 \\
\hline C & 7.475779 & $2 . \odot 40753$ & $-\odot .276991$ \\
\hline C & 6.910920 & 3.580618 & 3.175627 \\
\hline C & 7.031277 & 4.509084 & $\odot .009020$ \\
\hline C & 3.212178 & 3.995848 & 3.775025 \\
\hline C & 4.118074 & 3.820765 & 5.018223 \\
\hline C & 3.204510 & 5.485400 & 3.348393 \\
\hline C & 1.777358 & 3.637384 & 4.218201 \\
\hline C & -1.774409 & -1.442320 & 1.618383 \\
\hline C & -2.186525 & -2.756538 & 1.879298 \\
\hline C & -2.814931 & -3.103861 & 3.077921 \\
\hline C & -3.039635 & -2.060491 & 4.020567 \\
\hline C & -2.480764 & $-\odot .768404$ & 3.867639 \\
\hline
\end{tabular}

$-1.899874$

$-2.414028$

$-2.594575$

$-3.450028$

$-1.000481$

$-2.453890$

$-5.137911$

$-3.165058$

$-2.670187$

$-4.934142$

$-3.680096$

$-4.699491$

$-2.555339$

$-2.644865$

$-3.910806$

$-5.085606$

$-6.329523$

$-2.751976$

$-6.580397$

$-6.427132$

$-6.391269$

$-2.502850$

$-4.687553$

$-3.440630$

$-7.700981$

2.943194

2. 977053

0.641660

2. 310826

$-1.606626$

$-0.488543$

3.680266

2. 344827

1.588959

4. 435977

7. 314907

8.286456

7.703141

6.686069

7.174408

5.477802

4.960940

6.719441

5.950865

2. 211614

3. 613575

1.977330

$\odot .899297$

0.598355

1. 240442

3. 692213

4. 558841

3. 073600

7. 349766

6. 515294

6. 380685

4. 609798

6. 622866

5.423912

5. 025713

7. 926112

7. 070022

8. 278186

6.561241

7.563936

7.472872

7.645648

7.686125

6. 281217

4.238890

5. 103647

3. 659456

2. 870293

4. 197663

2. 512257
$-0.480647$ $\odot .335983$

$-0.180852$

1. 449401

0.980266

$-5.016962$

$-1.895393$

$-4.585281$

$-5.519924$

$-0.817645$

$-0.537846$

0.021437

$-0.534137$

$-0.814394$

$-0.968730$

$-0.930924$

$-2.297515$

$-1.538999$

$-2.479108$

$-1.041029$

$-0.052110$

$\odot .930760$

$-4.821845$

$-0.289196$

$-0.680507$

$-1.664187$

$-0.000142$

$-5.857442$

$-5.914046$

$-2.740228$

$-4.741861$

$-6.091046$

$-6.860393$

$-0.256951$

$-1.535555$

$-1.238126$

$-2.666102$

$-1.616537$

$-3.938616$

$-4.789456$

$-4.779684$

$-3.982897$

$-4.168027$

$-2.674278$

$-2.797980$

$-3.264343$

$-3.389916$

$-0.848673$

$-1.309460$

0.303152

$\odot .547544$

$-0.899490$

$-0.405721$

$-2.121594$

$-1.765849$

$-0.626946$

1.167700

3. 557162

2. 302402

3. 998658

1.915718

1. 070385

2. 303988

2. 872075

3. 054445

4. 383664

4.520216

4. 766309

5.299725

2. 762386

4. 261620

4. 322168

6.110273

5.821734

5.648228
2. 619740

4. 961458

6.408798

4.674010

4.927940

4.684457

5.114604

3. 377367

2. 251023

$-2.687378$

$-3.294861$

$-5.659176$

$-2.456304$

$-1.086671$

$-0.520406$

$-1.283170$

$-3.885642$

$-5.417903$

0.050823

$-0.502281$

0.694959

$-5.006739$

3. 520066

$-4.814789$

$-1.300607$

4. 903867

3. 078182

4. 092968

3. 391030

$-2.315598$

$-3.250637$

$-1.235349$

$-0.288714$

$-2.586452$

0.303351

$-0.463903$

$-0.848452$

$-2.144690$

$-3.146639$

$-1.676718$

$-2.187008$

0.282033

0.445741

0.953980

$-6.382811$

$-5.401123$

$-4.728899$

$-5.925454$

$-4.246591$

$-4.634821$

$-5.430888$

$-5.981079$

$-6.788275$

$-4.073663$

$-5.598167$

$-4.230864$

$-0.657716$

$-2.406768$

$-2.112184$

$-1.784840$

0.713393

$-0.582602$

$-0.976414$

3. 934556

2. 472626

3. 657293

0.908679

$-0.831415$

0.103825

5.278658

4.871743

5.878368

4. 185149

3. 045323

2. 513182 


\begin{tabular}{|c|c|c|c|}
\hline $\mathrm{H}$ & 1.046739 & 3.760656 & 3.410487 \\
\hline $\mathrm{H}$ & 1.699865 & 2.612779 & 4.599420 \\
\hline $\mathrm{H}$ & 1.477303 & 4.308908 & 5.029170 \\
\hline $\mathrm{H}$ & -2.020949 & -3.512806 & 1.123843 \\
\hline $\mathrm{H}$ & -1.541533 & ๑. 521612 & 2.420991 \\
\hline $\mathrm{H}$ & -3.614288 & $-\odot .485602$ & 6.640931 \\
\hline $\mathrm{H}$ & -1.935038 & -1.028186 & 6.620855 \\
\hline H & -2.334021 & $\odot .629227$ & 7.099752 \\
\hline $\mathrm{H}$ & -3.376029 & 2.234096 & 5.436818 \\
\hline $\mathrm{H}$ & -3.283627 & 1.918283 & 3.698768 \\
\hline $\mathrm{H}$ & -4.476653 & 1.068624 & 4.691898 \\
\hline H & -0.914399 & 1.706705 & 5.743456 \\
\hline $\mathrm{H}$ & -0.217667 & ๑. 224282 & 5.059977 \\
\hline $\mathrm{H}$ & -0.801856 & 1.520585 & 3.998377 \\
\hline $\mathrm{H}$ & -2.642454 & -6.079587 & 4.880346 \\
\hline $\mathrm{H}$ & -1.370512 & -4.872034 & 4.600641 \\
\hline $\mathrm{H}$ & -2.806589 & -4.441615 & 5.542068 \\
\hline $\mathrm{H}$ & -5.183445 & $-\odot .805995$ & 5.205361 \\
\hline $\mathrm{H}$ & -5.651489 & -2.198255 & 4.195090 \\
\hline $\mathrm{H}$ & -5.635416 & -2.347900 & 5.975497 \\
\hline $\mathrm{H}$ & -3.184367 & -5.332512 & 1.300866 \\
\hline $\mathrm{H}$ & -1.591549 & -5.432175 & 2.084318 \\
\hline $\mathrm{H}$ & -2.881659 & -6.557203 & 2.532504 \\
\hline $\mathrm{H}$ & -5.307431 & ๑. 814682 & -5.215650 \\
\hline $\mathrm{H}$ & -4.370829 & ๑. 360124 & -6.648978 \\
\hline $\mathrm{H}$ & -5.342469 & -0.845577 & -5.812260 \\
\hline $\mathrm{H}$ & -1.580887 & -0.334588 & -2.884408 \\
\hline $\mathrm{H}$ & -3.987068 & -1.132079 & ๑. 545311 \\
\hline $\mathrm{H}$ & -7.231625 & -2.254552 & -4.500221 \\
\hline $\mathrm{H}$ & -5.508992 & -2.723705 & -4.476266 \\
\hline $\mathrm{H}$ & -6.511592 & -2.943916 & -3.018552 \\
\hline $\mathrm{H}$ & -2.561891 & -1.388320 & -6.487499 \\
\hline $\mathrm{H}$ & -1.791092 & -1.744725 & -4.933692 \\
\hline $\mathrm{H}$ & -3.379006 & -2.431938 & -5.313666 \\
\hline $\mathrm{H}$ & -6.632596 & -3.218309 & $-\odot .756516$ \\
\hline $\mathrm{H}$ & -5.740559 & -2.753165 & $\odot .699461$ \\
\hline $\mathrm{H}$ & -7.502417 & -2.562280 & $\odot .638948$ \\
\hline $\mathrm{H}$ & -7.334503 & -0.118329 & 1.249368 \\
\hline $\mathrm{H}$ & -5.584601 & -0.258955 & 1.403787 \\
\hline $\mathrm{H}$ & -6.276136 & $\odot .979771$ & ๑. 346290 \\
\hline $\mathrm{H}$ & -2.976901 & 1.850658 & -4.647745 \\
\hline H & -1.544930 & $\odot .840328$ & -4.492230 \\
\hline $\mathrm{H}$ & -2.293474 & 1.059559 & -6.074825 \\
\hline $\mathrm{H}$ & -5.234097 & -4.436125 & 2.651059 \\
\hline $\mathrm{H}$ & -4.886646 & -5.898261 & 3.583578 \\
\hline $\mathrm{H}$ & -5.092519 & -4.362058 & 4.421339 \\
\hline $\mathrm{H}$ & -8.541052 & $-\odot .622737$ & -0.598222 \\
\hline $\mathrm{H}$ & -7.608464 & ๑. 290021 & -1.796092 \\
\hline $\mathrm{H}$ & -7.962209 & -1.421862 & -2.056052 \\
\hline $\mathrm{Rh}$ & ๑. . $\odot \odot 857$ & 1.056562 & -0.090708 \\
\hline 0 & -2.059273 & 2.066349 & 0. 396535 \\
\hline C & ๑. 259276 & 2.305945 & -1.799624 \\
\hline S & -2.693347 & 3.409254 & ๑. 490651 \\
\hline 0 & $-\odot .815416$ & 2.309966 & -2.375248 \\
\hline C & -0.236301 & 4.329083 & ๑. 337323 \\
\hline $\mathrm{N}$ & -1.602303 & 4.489230 & -0.232098 \\
\hline 0 & -3.089506 & 3.846630 & 1.838454 \\
\hline C & -4.117646 & 3.485591 & -0.575670 \\
\hline $\mathrm{H}$ & ๑. 203719 & 5.332243 & ๑. 323668 \\
\hline $\mathrm{H}$ & -0.301987 & 4.038315 & 1.393584 \\
\hline C & -2.088427 & 5.897464 & -0.238825 \\
\hline C & -5.332700 & 3.903272 & -0.028677 \\
\hline C & $-4.0 \odot 2200$ & 3.118915 & -1.920607 \\
\hline C & -1.387080 & 6.685214 & -1.309835 \\
\hline $\mathrm{H}$ & -3.161795 & 5.872733 & -0.455031 \\
\hline H & -1.961473 & 6.365855 & 0.747041 \\
\hline $\mathrm{H}$ & -5.390211 & 4.180330 & -261.713400 \\
\hline C & -6.455569 & 3.959069 & 12.177900 \\
\hline C & -5.136359 & 3.190890 & 12.998500 \\
\hline $\mathrm{H}$ & $-3 . \odot 46779$ & 2.790855 & -2.315672 \\
\hline C & -0.704165 & 7.808746 & -1.084008 \\
\hline H & -1.485376 & 6.291044 & -2.320498 \\
\hline $\mathrm{H}$ & -7.405156 & 4.285382 & -0.439313 \\
\hline C & -6.376667 & 3.607271 & -2.208976 \\
\hline $\mathrm{H}$ & -5.061475 & 2.915766 & -3.771749 \\
\hline
\end{tabular}

$\begin{array}{lrrr}\mathrm{H} & -0.242516 & 8.368220 & -1.892638 \\ \mathrm{H} & -0.597929 & 8.223361 & -0.083467 \\ \mathrm{C} & -7.592729 & 3.661041 & -3.101407 \\ \mathrm{H} & -7.393671 & 4.243808 & -4.008222 \\ \mathrm{H} & -7.885338 & 2.653966 & -3.423905 \\ \mathrm{H} & -8.448356 & 4.111074 & -2.589969 \\ \mathrm{C} & 1.589979 & 2.704789 & -2.432846 \\ \mathrm{H} & 1.548570 & 2.828668 & -3.518397 \\ \mathrm{H} & 2.412729 & 2.043946 & -2.156317 \\ \mathrm{C} & 1.567946 & 3.979378 & -1.566183 \\ \mathrm{H} & 1.025277 & 4.782728 & -2.072666 \\ \mathrm{H} & 2.548170 & 4.345323 & -1.244907 \\ \mathrm{C} & 0.711626 & 3.396722 & -0.408856 \\ \mathrm{H} & 1.883831 & 1.945607 & 2.528806 \\ \mathrm{H} & 1.406824 & 2.977335 & 0.319639\end{array}$

TS1_2

B3LYP SCF energy: $\quad-5535.31759571$ a.u. B3LYP enthalpy: $\quad-5533.284108$ a.u. B3LYP free energy: $\quad-5533.555241 \mathrm{a} . \mathrm{u}$. Mo6 SCF energy in solution: $\quad-5534.55478170$ a.u.

Mo6 enthalpy in solution: M06 free energy in solution: a.u.

Imaginary frequency: $\quad-265.7478 \mathrm{~cm}-1$

Cartesian coordinates

\begin{tabular}{|c|c|c|c|}
\hline АTOM & $X$ & Y & Z \\
\hline $\mathrm{P}$ & 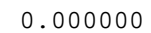 & -5.404372 & 4.118321 \\
\hline $\mathrm{P}$ & $\odot .0 \odot \odot \odot \odot \odot$ & -6.443106 & 3.893770 \\
\hline 0 & 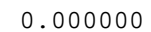 & -5.091779 & 3.147515 \\
\hline 0 & $\odot .0 \odot \odot ० \odot \odot$ & -3.004060 & 2.765132 \\
\hline 0 & $\odot . ๑ \odot \odot \odot \odot \odot$ & -0.717397 & 7.782462 \\
\hline 0 & ๑. . 000000 & -1.450771 & 6.240302 \\
\hline 0 & $\odot . ๑ \odot \odot \odot \odot \odot$ & -7.401210 & 4. 209012 \\
\hline 0 & $\odot .0 \odot \odot \odot \odot \odot$ & -6.342889 & 3.548953 \\
\hline 0 & ๑. . 000000 & -5.000450 & 2.878625 \\
\hline 0 & 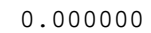 & -0.247048 & 8.339812 \\
\hline C & $\odot .0000 \bullet \odot$ & -0.639546 & 8.210033 \\
\hline C & 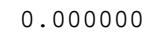 & -7.547692 & 3.593837 \\
\hline C & 1.452795 & -7.340641 & 4.175952 \\
\hline C & 1.995117 & -7.829952 & 2.584271 \\
\hline C & 2.560354 & -8.412547 & 4.039489 \\
\hline C & 2. 554361 & 1.647997 & 2.686107 \\
\hline C & 1.452751 & 1.627971 & 2.814312 \\
\hline C & -0.234347 & 2.465477 & 2.024220 \\
\hline C & -0.682476 & 1.609816 & 3.957403 \\
\hline C & -0.054628 & 1. 081545 & 4.764177 \\
\hline C & 1.045590 & 2.585563 & 4.317429 \\
\hline C & 1.499892 & ๑.727053 & 3.377930 \\
\hline C & ๑. 902633 & 1.876330 & 1.793035 \\
\hline C & 2.718592 & 1.401737 & 2.962680 \\
\hline C & 3.031153 & -0.739370 & -1.005780 \\
\hline C & 2.605532 & -0.719485 & -2.334982 \\
\hline C & 3. 366059 & -1.292472 & -3.362387 \\
\hline C & 4.612680 & -1.876499 & -3.005807 \\
\hline C & 5.010350 & -2.044474 & -1.650162 \\
\hline C & 4. 204872 & -1.427435 & -0.682340 \\
\hline C & 6.223540 & -2.891926 & -1.165781 \\
\hline C & 7.509354 & -2.032897 & -1.087240 \\
\hline C & 6.462519 & -4.138966 & -2.051506 \\
\hline C & 5.966352 & -3.434566 & $\odot .265545$ \\
\hline C & 2.816621 & -1.316468 & -4.812549 \\
\hline C & 2.757021 & -2.774090 & -5.334449 \\
\hline C & 1.377487 & -0.760115 & -4.880749 \\
\hline C & 3.674978 & -0.447515 & -5.762275 \\
\hline C & 6.554943 & -1.638107 & -4.422136 \\
\hline C & 3. 161893 & 1.377891 & 1.010109 \\
\hline C & 4.335234 & 1.760895 & 0.357634 \\
\hline C & 2.786057 & 2.083355 & 2.162840 \\
\hline C & 3.535697 & 3.150093 & 2.675338 \\
\hline C & 4.650487 & 3.577917 & 1.904972 \\
\hline C & 5.116908 & 2.848515 & 0.783423 \\
\hline C & 6.414573 & 3.141355 & -0.024499 \\
\hline
\end{tabular}




\begin{tabular}{|c|c|c|c|c|c|c|c|}
\hline C & 6.029417 & 3.698078 & -1.417159 & $\mathrm{H}$ & 8.118359 & 2.007882 & -0.770451 \\
\hline C & 7.191878 & 1.812442 & -0.219358 & H & 4.876385 & 5.903509 & 0.600581 \\
\hline c & 4.793899 & 5.921895 & 1.694901 & H & 3.742722 & 6.080089 & 1.968253 \\
\hline c & 7.416642 & 4.118870 & 0.632354 & $\mathrm{H}$ & 5.397016 & 6.742912 & 2.088503 \\
\hline C & 3.083203 & 3.735969 & 4.045155 & H & 7.658105 & 3.832452 & 1.659210 \\
\hline C & 4.056868 & 4.733407 & 4.713955 & H & 8.344490 & 4.098094 & 0.048730 \\
\hline C & 1.711521 & 4.434549 & 3.885037 & H & 7.066407 & 5.151410 & 0.643403 \\
\hline c & 2.932459 & 2.560059 & 5.048627 & H & 5.071512 & 4.334577 & 4.786859 \\
\hline C & -1.753406 & -1.463899 & 1.598434 & H & 4.106842 & 5.694736 & 4.200850 \\
\hline C & -2.168398 & -2.775667 & 1.865836 & H & 3.696000 & 4.928476 & 5.730648 \\
\hline C & -2.789434 & -3.116774 & 3. $07 \odot 221$ & H & 1.372773 & 4.827065 & 4.850915 \\
\hline C & -3.003199 & -2.069807 & 4.011702 & H & 1.772714 & 5.277375 & 3.186409 \\
\hline C & -2.441571 & $-\odot .779849$ & 3.850327 & H & 0.940330 & 3.746865 & 3.520135 \\
\hline C & -1.869027 & -0.498747 & 2.597558 & H & 2.187336 & 1.822160 & 4.737347 \\
\hline C & -2.362919 & $\odot .329053$ & 4.938896 & H & 3.888733 & 2.042580 & 5.187689 \\
\hline C & -2.530787 & -0.182170 & 6.389734 & H & 2.616495 & 2.946717 & 6.023807 \\
\hline c & -3.399719 & 1.442977 & 4.656504 & $\mathrm{H}$ & -2.009653 & -3.534916 & 1.111802 \\
\hline C & $-\odot .948431$ & $\odot .971353$ & 4.890767 & H & -1.507860 & $\odot .501275$ & 2.392681 \\
\hline c & -2.420033 & -5.027088 & 4.678045 & H & -3.548650 & -0.485520 & 6.631457 \\
\hline C & -5.091798 & -1.895534 & 5.123027 & H & -1.870042 & -1.029275 & 6.598901 \\
\hline c & -3.142337 & -4.596039 & 3.376805 & H & -2.263848 & 0.630049 & 7.075716 \\
\hline C & -2.662210 & -5.535499 & 2.248064 & H & -3.316669 & 2.231391 & 5.414555 \\
\hline c & -4.887146 & -0.856497 & -2.736945 & H & -3.243196 & 1.906293 & 3.676935 \\
\hline C & -3.629187 & $-\odot .567325$ & -3.332100 & H & -4.426649 & 1.064033 & 4.686983 \\
\hline c & -4.627189 & -0.014855 & -5.707094 & H & -0.853489 & 1.699619 & 5.703571 \\
\hline C & -2.512881 & $-\odot .556604$ & -2.482613 & H & -0.165835 & $\odot .214319$ & 5.017717 \\
\hline C & -2.613557 & -0.841245 & -1.114748 & H & $-\odot .758259$ & 1.508759 & 3.957087 \\
\hline C & -3.883235 & -1.006641 & $-\odot .56 \odot 577$ & H & -2.611719 & -6.088205 & 4.879031 \\
\hline C & -5.051079 & $-\odot .974405$ & -1.334353 & H & -1.336796 & -4.887582 & 4.583363 \\
\hline C & -6.263824 & -2.341741 & -3.950807 & H & -2.761666 & -4.447685 & 5.537434 \\
\hline C & -2.674093 & -1.565254 & -5.444335 & H & -5.133777 & -0.805898 & 5.212759 \\
\hline C & -6.549644 & -2.537347 & -0.021516 & H & -5.613395 & -2.198234 & 4.208018 \\
\hline C & -6.399100 & -1.095802 & $-\odot .566367$ & H & -5.583614 & -2.345622 & 5.988426 \\
\hline C & -6.379802 & -0.112842 & 0.636220 & H & -3.184684 & -5.348275 & 1.302386 \\
\hline C & -2.441007 & $\odot .905422$ & -5.034100 & H & -1.584877 & -5.452909 & 2.070688 \\
\hline C & -4.664355 & -4.826110 & 3.534632 & H & -2.875420 & -6.571146 & 2.534158 \\
\hline C & -3.375396 & $-\odot .318311$ & -4.849716 & H & -5.244002 & $\odot .775153$ & -5.270126 \\
\hline C & -7.667259 & -0.737830 & -1.374846 & H & -4.290425 & 0.325098 & -6.693729 \\
\hline H & 3.002901 & -1.771908 & 4.895591 & H & -5.263805 & $-\odot .885459$ & -5.866200 \\
\hline $\mathrm{H}$ & 3.034024 & $-\odot .078565$ & 3.101525 & H & -1.535594 & $-\odot .349703$ & -2.900621 \\
\hline H & $\odot .653492$ & -5.930033 & 4.030135 & H & -3.967851 & -1.174522 & ๑.5०3901 \\
\hline H & 2.318693 & -5.987963 & 3.318847 & H & -7.160252 & -2.302088 & -4.573843 \\
\hline H & -1.564106 & -2.755569 & -2.345333 & H & -5.435745 & -2.763060 & -4.534319 \\
\hline H & -0.426336 & -4.740392 & -3.290884 & H & -6.450984 & -2.990414 & -3.086562 \\
\hline H & 3.746133 & -6.066178 & $-1.27 \odot 239$ & H & -2.475325 & -1.415376 & -6.512452 \\
\hline H & 2.416886 & -6.862151 & -0.337208 & H & -1.716269 & -1.764172 & -4.951144 \\
\hline H & 1.653240 & $-\odot .258887$ & -2.559525 & H & -3.296623 & -2.461767 & -5.344011 \\
\hline $\mathrm{H}$ & 4.485706 & -1.503054 & 0.360101 & $\mathrm{H}$ & -6.590360 & -3.273098 & -0.832623 \\
\hline H & 7.383338 & -1.213168 & $-\odot .371818$ & H & -5.714369 & -2.809943 & 0.633647 \\
\hline $\mathrm{H}$ & 8.349189 & -2.648760 & -0.743528 & $\mathrm{H}$ & -7.476540 & -2.628267 & 0.557701 \\
\hline $\mathrm{H}$ & 7.791407 & -1.594542 & -2.046684 & H & -7.328254 & $-\odot .18610 \odot$ & 1.180715 \\
\hline $\mathrm{H}$ & 6.775255 & -3.903447 & -3.066788 & $\mathrm{H}$ & -5.579511 & -0.319935 & 1.352184 \\
\hline H & 7.239373 & -4.761006 & -1.593036 & H & -6.265701 & $\odot .921335$ & 0.294013 \\
\hline $\mathrm{H}$ & 5.550027 & -4.742290 & -2.126490 & $\mathrm{H}$ & -2.922667 & 1.824033 & -4.682158 \\
\hline H & 5.005585 & -3.954137 & ๑.337001 & H & -1.487888 & $\odot .820027$ & -4.509829 \\
\hline $\mathrm{H}$ & 6.761189 & -4.141777 & 0.525824 & H & -2.221171 & 1.033078 & -6.100201 \\
\hline H & 5.987718 & -2.648431 & 1.027293 & H & -5.217752 & -4.438419 & 2.670820 \\
\hline $\mathrm{H}$ & 2.347097 & -2.786271 & -6.351606 & $\mathrm{H}$ & -4.867384 & -5.901620 & 3.600599 \\
\hline H & 3.743471 & -3.238389 & -5.355516 & H & -5.058518 & -4.364258 & 4.439617 \\
\hline $\mathrm{H}$ & 2.101551 & -3.383741 & -4.701398 & $\mathrm{H}$ & -8.514171 & -0.687503 & -0.680166 \\
\hline H & 1. 009611 & $-\odot .851761$ & -5.908027 & H & -7.574851 & $\odot .235525$ & -1.864825 \\
\hline $\mathrm{H}$ & 0.692713 & -1.319970 & -4.234091 & $\mathrm{H}$ & -7.917730 & -1.476774 & -2.136237 \\
\hline $\mathrm{H}$ & 1.321357 & 0.300728 & -4.610558 & $\mathrm{Rh}$ & 0.019455 & 1.034157 & -0.089410 \\
\hline H & 3.776376 & 0.575139 & -5.379275 & 0 & -2.052091 & 2.035464 & 0.365347 \\
\hline $\mathrm{H}$ & 4.671878 & -0.859995 & -5.913753 & C & 0.305452 & 2. 280719 & -1.791893 \\
\hline $\mathrm{H}$ & 3.192431 & -0.388118 & -6.745068 & $\mathrm{~s}$ & -2.693787 & 3.375576 & 0.445798 \\
\hline $\mathrm{H}$ & 7.452912 & -2.084934 & -3.984707 & 0 & -0.756925 & 2.283945 & -2.389483 \\
\hline H & 6.632978 & -1.702226 & -5.510794 & C & $-\odot .242155$ & 4.312188 & 0.329429 \\
\hline $\mathrm{H}$ & 6.488021 & -0.586057 & -4.125836 & $\mathrm{~N}$ & -1.598617 & 4.457553 & -0.267718 \\
\hline $\mathrm{H}$ & 4.648069 & 1.204832 & -0.516083 & 0 & -3.109018 & 3.816203 & 1.786836 \\
\hline $\mathrm{H}$ & 6.930334 & 3.873434 & -2.017131 & C & -4.104522 & 3.441775 & -0.638843 \\
\hline $\mathrm{H}$ & 5.388263 & 3. 002471 & -1.970616 & H & $0.19 \odot 440$ & 5.318476 & 0.312204 \\
\hline $\mathrm{H}$ & 5.495958 & 4.652149 & -1.333079 & H & -0.325949 & 4.034304 & 1.387289 \\
\hline $\mathrm{H}$ & 7.462639 & 1.372370 & 0.747202 & C & -2.094268 & 5.861885 & -0.293224 \\
\hline $\mathrm{H}$ & 6.631544 & 1. 064935 & -0.786887 & C & -5.330643 & 3.845508 & -0.106227 \\
\hline
\end{tabular}




$\begin{array}{lrrr}\mathrm{C} & -3.967719 & 3.082381 & -1.983730 \\ \mathrm{C} & -1.379660 & 6.646945 & -1.357486 \\ \mathrm{H} & -3.163334 & 5.828406 & -0.528701 \\ \mathrm{H} & -1.988636 & 6.338666 & 0.691220 \\ \mathrm{H} & -5.404372 & 4.118321 & -265.747800 \\ \mathrm{C} & -6.443106 & 3.893770 & 11.143400 \\ \mathrm{C} & -5.091779 & 3.147515 & 13.271600 \\ \mathrm{H} & -3.004060 & 2.765132 & -2.367589 \\ \mathrm{C} & -0.717397 & 7.782462 & -1.130039 \\ \mathrm{H} & -1.450771 & 6.240302 & -2.365474 \\ \mathrm{H} & -7.401210 & 4.209012 & -0.542426 \\ \mathrm{C} & -6.342889 & 3.548953 & -2.301304 \\ \mathrm{H} & -5.000450 & 2.878625 & -3.84980 \odot \\ \mathrm{H} & -0.247048 & 8.339812 & -1.935143 \\ \mathrm{H} & -0.639546 & 8.210033 & -0.132292 \\ \mathrm{C} & -7.547692 & 3.593837 & -3.209324 \\ \mathrm{H} & -7.340641 & 4.175952 & -4.114728 \\ \mathrm{H} & -7.829952 & 2.584271 & -3.533332 \\ \mathrm{H} & -8.412547 & 4.039489 & -2.709708 \\ \mathrm{C} & 1.647997 & 2.686107 & -2.395602 \\ \mathrm{H} & 1.627971 & 2.814312 & -3.481335 \\ \mathrm{H} & 2.465477 & 2.024220 & -2.106272 \\ \mathrm{C} & 1.609816 & 3.957403 & -1.525287 \\ \mathrm{H} & 1.081545 & 4.764177 & -2.041705 \\ \mathrm{H} & 2.585563 & 4.317429 & -1.184230 \\ \mathrm{C} & 0.727053 & 3.377930 & -0.386656 \\ \mathrm{H} & 1.876330 & 1.793035 & 2.678186 \\ \mathrm{H} & 1.401737 & 2.962680 & 0.363187\end{array}$

TS1 3

B3LYP SCF energy: $\quad-5535.32022585$ a.u. B3LYP enthalpy:

B3LYP free energy:

-5535.32022585 a.u.
-5533.286479 a.u. -5533.556550 a.u

M06 SCF energy in solution: $\quad-5534.55448736$ a.u.

M06 enthalpy in solution: $\quad-5532.520741 \mathrm{a} . \mathrm{u}$. M06 free energy in solution: $\quad-5532.790812$ a.u.

Imaginary frequency:

$-260.1318 \mathrm{~cm}-1$

\begin{tabular}{|c|c|c|c|}
\hline ATOM & $x$ & $Y$ & Z \\
\hline $\mathrm{P}$ & $\odot . \diamond \odot \odot \odot \odot \odot$ & -5.366137 & 4.150532 \\
\hline P & $\odot .0000 \odot \odot$ & -6.516103 & 4.023375 \\
\hline 0 & $\odot .0 \odot \odot \odot \odot \odot$ & -5.288220 & 3.334605 \\
\hline 0 & $\odot . ๑ \odot \odot \odot \odot \odot$ & -3.185312 & 2.906595 \\
\hline 0 & $\odot .0 \odot \odot \odot \odot \odot$ & -0.774602 & 7.871995 \\
\hline 0 & $\odot .0 \odot \odot \odot \odot \odot$ & -1.608061 & 6.415970 \\
\hline 0 & $\odot . ๑ \odot \odot \odot \odot \odot$ & -7.443823 & 4.333830 \\
\hline 0 & $\odot .0000 \odot \odot$ & -6.500739 & 3.734870 \\
\hline 0 & $\odot . \odot \odot \odot \odot \odot \odot$ & -5.263263 & 3.105669 \\
\hline 0 & $\odot .0 \odot \odot \odot \odot \odot$ & -0.346452 & 8.471132 \\
\hline C & $\odot . ๑ \odot \odot \odot \odot \odot$ & $-\odot .626565$ & 8.237045 \\
\hline C & $\odot .0 \odot \odot \odot \odot \odot$ & -7.753529 & 3.843106 \\
\hline c & 1.475556 & -7.606461 & 4.523524 \\
\hline C & 2. 042223 & -8.032845 & 2.867340 \\
\hline C & 2.594226 & -8.598588 & 4.212871 \\
\hline C & 2.559343 & 1.450450 & 2.897667 \\
\hline C & 1.561447 & $1.3600 \odot 1$ & 3.093468 \\
\hline C & -0.295076 & 2.286909 & 2.224972 \\
\hline C & $-\odot .775140$ & 1.463639 & 4.111314 \\
\hline C & -0.165593 & $\odot .895003$ & 4.942771 \\
\hline C & 0.948919 & 2.456492 & 4.462178 \\
\hline C & 1.435179 & $\odot .665236$ & 3.448426 \\
\hline C & $\odot .857725$ & 1.864864 & 1.755298 \\
\hline c & 2.643146 & 1.393151 & 2.986697 \\
\hline C & 2.923214 & $-\odot .617629$ & -1.196516 \\
\hline c & 2. 449279 & -0.498164 & -2.503904 \\
\hline C & 3.173234 & $-\odot .986701$ & -3.599309 \\
\hline c & 4.434837 & -1.586987 & -3.333711 \\
\hline C & 4.882738 & -1.854561 & -2.010552 \\
\hline c & 4.110081 & -1.320882 & -0.969544 \\
\hline c & 6.124266 & -2.714828 & -1.634454 \\
\hline
\end{tabular}

\begin{tabular}{|c|c|c|}
\hline 7.390598 & -1.831876 & -1.514906 \\
\hline 6.368652 & -3.875162 & -2.629524 \\
\hline 5.917668 & -3.384194 & $-\odot .249091$ \\
\hline 2.569614 & -0.906004 & -5.02590 \\
\hline 2.497954 & -2.321183 & -5.652621 \\
\hline 1.126174 & -0.357368 & -4.998007 \\
\hline 3.386147 & $\odot .037648$ & -5.940804 \\
\hline 6.311089 & -1.207517 & -4.802601 \\
\hline 3.164591 & 1.334882 & ๑.956516 \\
\hline 4.312954 & 1.752378 & $\odot .285786$ \\
\hline 2.811883 & 1.999440 & 2.145241 \\
\hline 3.641418 & & 2.74193 \\
\hline 4.871739 & 3.243154 & 2. 084231 \\
\hline 5.163608 & 2.755195 & 0.789465 \\
\hline 6.307726 & 3.249474 & -0.143364 \\
\hline 5.726858 & 3.469288 & -1.568152 \\
\hline 7.418940 & 2.177643 & -0.239445 \\
\hline 6.877731 & 3254 & 3.333155 \\
\hline 6.936333 & & $\odot .2544 \odot 4$ \\
\hline 3.181197 & & 5640 \\
\hline 4.112646 & 622 & 5569 \\
\hline 3.13 & & \\
\hline 1.762270 & 588 & \\
\hline-1.715168 & 978 & 3267 \\
\hline-2.162299 & -2.9 & \\
\hline$-2.58 c$ & -3 & 694 \\
\hline-2.4 & -2 . & 153 \\
\hline-2.214 & & \\
\hline-1.789468 & -0.7 & \\
\hline-2.3991 & $-\odot .2$ & \\
\hline-3.167307 & -0.7 & .2 to 0 \\
\hline-3.24 & & 320 \\
\hline$-1.02 C$ & & \\
\hline-2.374563 & $-5 . \varepsilon$ & 422 \\
\hline-1.47 & -3 & 967 \\
\hline-3.26 & -4.5 & 2.8 \\
\hline-3.34 & & \\
\hline-5.0 & $-\odot$. & -2 . \\
\hline-3.82 & & -3 \\
\hline-4.92 & & \\
\hline-2.66 & -0.3 & -2 \\
\hline-2.76 & -0.7 & 9883 \\
\hline-3.95 & & $-\odot$. \\
\hline-5.155225 & $-\odot . \varepsilon$ & -1 \\
\hline-6.486787 & & \\
\hline-2.9 & -1 & -5 \\
\hline-6.61 & -2.4 & 9327 \\
\hline-6.47 & -0.9 & $-\Theta$. \\
\hline-6.3 & $-\odot$. & \\
\hline-2.721359 & & $-4 . \varepsilon$ \\
\hline-4.635086 & -4.7 & \\
\hline-3.6 & & -4 \\
\hline-7.76 & & -1.1 \\
\hline 3.04 & -2 & \\
\hline 3. 023871 & -0.2 & \\
\hline 0.81 & & \\
\hline 2.45 & & 2.7 \\
\hline-1.669457 & -2.50 & -2.530806 \\
\hline$-\odot .561541$ & & \\
\hline 3.6 & -5.8 & $-1 . \varepsilon$ \\
\hline 2.362549 & -6.7 & -0.964637 \\
\hline 1.488170 & & \\
\hline 4.43 & 32 & \\
\hline 7.26 & -1.0 & -0.725488 \\
\hline & & \\
\hline 7.632082 & -1.3021 & -2.438922 \\
\hline & & \\
\hline & & \\
\hline 5.467430 & -4.490076 & -2.738530 \\
\hline & & \\
\hline & -4.095851 & -0.072335 \\
\hline 5.9 & -2.6666 & 0.577369 \\
\hline & & \\
\hline & -2.775546 & \\
\hline 1. 868071 & -2.980847 & -5.04433 \\
\hline
\end{tabular}




\begin{tabular}{|c|c|c|c|}
\hline $\mathrm{H}$ & ๑. 719150 & -0.376953 & -6.014329 \\
\hline $\mathrm{H}$ & $\odot .469778$ & $-\odot .967811$ & -4.368046 \\
\hline $\mathrm{H}$ & 1.075759 & $\odot .680674$ & -4.649441 \\
\hline $\mathrm{H}$ & 3.504190 & 1.027689 & -5.483985 \\
\hline $\mathrm{H}$ & 4.375246 & $-\odot .358551$ & -6.168116 \\
\hline $\mathrm{H}$ & 2.861052 & 0.172052 & -6.893913 \\
\hline $\mathrm{H}$ & 7.231987 & -1.633503 & -4.392755 \\
\hline $\mathrm{H}$ & 6.373586 & -1.235539 & -5.893641 \\
\hline $\mathrm{H}$ & 6.210480 & -0.168132 & -4.472637 \\
\hline $\mathrm{H}$ & 4.557347 & 1.293401 & -0.663034 \\
\hline $\mathrm{H}$ & 6.515402 & 3.848926 & -2.226998 \\
\hline $\mathrm{H}$ & 5.340997 & 2.553477 & -2.024841 \\
\hline $\mathrm{H}$ & 4.918783 & 4.209751 & -1.554043 \\
\hline $\mathrm{H}$ & 7.884877 & 1. 980412 & ๑. 731764 \\
\hline $\mathrm{H}$ & 7.025337 & 1.228307 & -0.618290 \\
\hline $\mathrm{H}$ & 8.207239 & 2.511097 & -0.924804 \\
\hline $\mathrm{H}$ & 6.551809 & 2.637678 & 4.034944 \\
\hline $\mathrm{H}$ & 7.528835 & 2.959607 & 2.579736 \\
\hline $\mathrm{H}$ & 7.433149 & 4.183469 & 3.872543 \\
\hline $\mathrm{H}$ & 7.561685 & 4.553956 & 1.144957 \\
\hline $\mathrm{H}$ & 7.576034 & 4.943491 & -0.569088 \\
\hline $\mathrm{H}$ & 6.174083 & 5.372367 & $\odot .422649$ \\
\hline $\mathrm{H}$ & 4.262793 & 2.371864 & 5.390358 \\
\hline $\mathrm{H}$ & 5.084830 & 3. 922043 & 5.103071 \\
\hline $\mathrm{H}$ & 3.657538 & 3.858503 & 6.133279 \\
\hline $\mathrm{H}$ & 2.805409 & 5.769973 & 4.612649 \\
\hline $\mathrm{H}$ & 4.116251 & 5.607678 & 3.429436 \\
\hline $\mathrm{H}$ & 2.424492 & 5.445014 & 2.913805 \\
\hline $\mathrm{H}$ & 1. 013661 & 3.454499 & 3.669821 \\
\hline $\mathrm{H}$ & 1.714361 & 2.223141 & 4.743186 \\
\hline $\mathrm{H}$ & 1.463336 & 3.870121 & 5.322119 \\
\hline $\mathrm{H}$ & -2.177038 & -3.548885 & $\odot .723091$ \\
\hline $\mathrm{H}$ & -1.523795 & ๑. 259078 & 2.531121 \\
\hline $\mathrm{H}$ & -4.115658 & -1.186721 & 6.005912 \\
\hline $\mathrm{H}$ & -2.602023 & -1.413843 & 6.888455 \\
\hline $\mathrm{H}$ & -3.392291 & $\odot .156544$ & 6.902200 \\
\hline $\mathrm{H}$ & -3.395777 & 1.706769 & 5.249701 \\
\hline $\mathrm{H}$ & -2.771258 & 1.473386 & 3.622698 \\
\hline $\mathrm{H}$ & -4.227152 & $\odot .609051$ & 4.136610 \\
\hline $\mathrm{H}$ & -1.147874 & 1.096123 & 6.239849 \\
\hline $\mathrm{H}$ & -0.384803 & -0.463039 & 5.883265 \\
\hline $\mathrm{H}$ & -0.481823 & 0.782121 & 4.629023 \\
\hline $\mathrm{H}$ & -2.793242 & -6.904836 & 3.617353 \\
\hline $\mathrm{H}$ & -1.340739 & -5.927380 & 3.357490 \\
\hline $\mathrm{H}$ & -2.374762 & -5.644355 & 4.778645 \\
\hline $\mathrm{H}$ & -0.785344 & -4.123031 & 5.348076 \\
\hline $\mathrm{H}$ & $-\odot .970949$ & -2.588358 & 6.229597 \\
\hline $\mathrm{H}$ & -1.779070 & -4.058834 & 6.834494 \\
\hline $\mathrm{H}$ & -3.943621 & -4.881311 & $\odot .80 \odot 419$ \\
\hline $\mathrm{H}$ & -2.367298 & -5.680560 & 0.997513 \\
\hline $\mathrm{H}$ & -3.844825 & -6.479334 & 1.543324 \\
\hline $\mathrm{H}$ & -5.526116 & 1.198793 & -4.990569 \\
\hline $\mathrm{H}$ & -4.626314 & ๑.863367 & -6.478914 \\
\hline $\mathrm{H}$ & -5.555164 & $-\odot .415134$ & $-5.7 \odot 3891$ \\
\hline $\mathrm{H}$ & -1.708130 & -0.095374 & -2.872022 \\
\hline $\mathrm{H}$ & -3.993318 & -1.152521 & 0.571374 \\
\hline $\mathrm{H}$ & -7.407373 & -1.905006 & -4.430197 \\
\hline $\mathrm{H}$ & -5.684793 & -2.371661 & -4.492805 \\
\hline $\mathrm{H}$ & -6.642181 & -2.696795 & -3.024189 \\
\hline $\mathrm{H}$ & -2.790848 & -0.852277 & -6.503992 \\
\hline $\mathrm{H}$ & -1.962737 & -1.311179 & $-5.0 \odot 7974$ \\
\hline $\mathrm{H}$ & -3.551214 & -1.997710 & -5.388100 \\
\hline $\mathrm{H}$ & -6.692624 & -3.132933 & $-\odot .787509$ \\
\hline $\mathrm{H}$ & -5.756010 & -2.776179 & 0.671830 \\
\hline $\mathrm{H}$ & -7.516479 & -2.578034 & 0.678152 \\
\hline $\mathrm{H}$ & -7.312409 & -0.188572 & 1.463627 \\
\hline $\mathrm{H}$ & -5.559661 & $-\odot .340696$ & 1.545271 \\
\hline $\mathrm{H}$ & -6.284188 & $\odot .972998$ & ๑. 605835 \\
\hline $\mathrm{H}$ & -3.202176 & 2.228622 & -4.407612 \\
\hline $\mathrm{H}$ & -1.749203 & 1. 237419 & -4.364111 \\
\hline $\mathrm{H}$ & -2.542512 & 1.561407 & -5.907188 \\
\hline $\mathrm{H}$ & -5.270136 & -4.146902 & 2.866884 \\
\hline $\mathrm{H}$ & $-5.10 \odot 811$ & -5.789899 & 3.506378 \\
\hline $\mathrm{H}$ & -4.614226 & -4.401859 & 4.497207 \\
\hline $\mathrm{H}$ & -8.583783 & -0.550989 & -0.376299 \\
\hline
\end{tabular}

$\begin{array}{lrrr}\mathrm{H} & -7.687804 & 0.443598 & -1.536298 \\ \mathrm{H} & -8.058338 & -1.244379 & -1.906819 \\ \mathrm{Rh} & -0.041906 & 1.084821 & -0.015458 \\ \mathrm{O} & -2.070436 & 2.062327 & 0.672774 \\ \mathrm{C} & 0.151317 & 2.448165 & -1.636240 \\ \mathrm{~S} & -2.701173 & 3.398331 & 0.859249 \\ \mathrm{O} & -0.949492 & 2.489602 & -2.159393 \\ \mathrm{C} & -0.252996 & 4.324409 & 0.649048 \\ \mathrm{~N} & -1.640438 & 4.513701 & 0.144659 \\ \mathrm{O} & -3.036797 & 3.770733 & 2.241718 \\ \mathrm{C} & -4.171260 & 3.526072 & -0.137987 \\ \mathrm{H} & 0.184437 & 5.328219 & 0.686247 \\ \mathrm{H} & -0.276142 & 3.962419 & 1.684688 \\ \mathrm{C} & -2.124626 & 5.919874 & 0.227558 \\ \mathrm{C} & -5.357537 & 3.923661 & 0.482331 \\ \mathrm{C} & -4.119351 & 3.220627 & -1.502062 \\ \mathrm{C} & -1.467387 & 6.760163 & -0.831398 \\ \mathrm{H} & -3.206016 & 5.905020 & 0.054768 \\ \mathrm{H} & -1.956801 & 6.339310 & 1.229237 \\ \mathrm{H} & -5.366137 & 4.150532 & -260.131800 \\ \mathrm{C} & -6.516103 & 4.023375 & 11.448700 \\ \mathrm{C} & -5.288220 & 3.334605 & 12.883400 \\ \mathrm{H} & -3.185312 & 2.906595 & -1.955266 \\ \mathrm{C} & -0.774602 & 7.871995 & -0.579185 \\ \mathrm{H} & -1.608061 & 6.415970 & -1.855229 \\ \mathrm{H} & -7.443823 & 4.333830 & 0.184520 \\ \mathrm{C} & -6.500739 & 3.734870 & -1.659660 \\ \mathrm{H} & -5.263263 & 3.105669 & -3.309813 \\ \mathrm{H} & -0.346452 & 8.471132 & -1.377783 \\ \mathrm{H} & -0.626565 & 8.237045 & 0.435262 \\ \mathrm{C} & -7.753529 & 3.843106 & -2.494559 \\ \mathrm{H} & -7.606461 & 4.523524 & -3.341699 \\ \mathrm{H} & -8.032845 & 2.867340 & -2.910608 \\ \mathrm{H} & -8.598588 & 4.212871 & -1.906979 \\ \mathrm{H} & 1.450450 & 2.897667 & -2.300059 \\ \mathrm{H} & 1.360001 & 3.093468 & -3.371976 \\ \mathrm{H} & 2.286909 & 2.224972 & -2.105224 \\ \mathrm{H} & 1.463639 & 4.111314 & -1.350231 \\ \mathrm{H} & 0.895003 & 4.942771 & -1.776288 \\ \mathrm{H} & 2.456492 & 4.462178 & -1.051593 \\ \mathrm{H} & 1.364864 & 3.448426 & -0.195174 \\ \mathrm{H} & 1.755298 & 2.610879 \\ \mathrm{H} & 2.986697 & 0.472519\end{array}$

TS1 4

B3LYP SCF energy: $\quad-5535.31865961 \mathrm{a} \cdot \mathrm{u}$. B3LYP enthalpy: $\quad-5533.284871 \mathrm{a} . \mathrm{u}$. B3LYP free energy: $\quad-5533.555463 \mathrm{a.u}$. M06 SCF energy in solution: $\quad-5534.55439575$ a.u.

M06 enthalpy in solution: $\quad-5532.520607$ a.u. Mo6 free energy in solution: $\quad-5532.791199$ a.u.

Imaginary frequency: $\quad-262.2120 \mathrm{~cm}-1$

Cartesian coordinates

\begin{tabular}{|c|c|c|c|}
\hline АТOM & $X$ & $\mathrm{Y}$ & Z \\
\hline $\mathrm{P}$ & ๑. .०००००० & -5.437548 & 3.998057 \\
\hline$P$ & $\odot .00 \odot \odot \odot \odot$ & -6.536239 & 4.007877 \\
\hline 0 & $\odot .0 \odot \odot \odot \odot \odot$ & -5.259149 & 3.442954 \\
\hline 0 & ๑. . & -3.169276 & 2.971908 \\
\hline 0 & $\odot .0000 \odot \odot$ & $-\odot .845107$ & 7.858765 \\
\hline 0 & 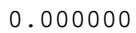 & -1.630251 & 6.485488 \\
\hline 0 & $\odot .00000 \odot$ & -7.473881 & 4. 296717 \\
\hline 0 & $\odot . \diamond \odot \odot \odot \odot \odot$ & -6.484503 & 3.814024 \\
\hline 0 & $\odot . \diamond \odot \odot \odot \odot \odot$ & -5.205142 & 3.290649 \\
\hline 0 & 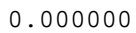 & $-\odot .40920 \odot$ & 8.518143 \\
\hline C & ๑. . $00000 \odot$ & -0.725338 & 8.154187 \\
\hline C & $\odot . \diamond \odot \odot \odot \odot \odot$ & -7.714453 & 3.985143 \\
\hline C & 1.521635 & -7.510454 & 4.629481 \\
\hline C & 2.080836 & -8.053090 & 3.018150 \\
\hline C & 2.642308 & -8.541610 & 4.424634 \\
\hline C & 2.607894 & 1.459980 & 2.961946 \\
\hline
\end{tabular}




\begin{tabular}{|c|c|c|c|c|c|c|c|}
\hline C & 1.572997 & 1.387241 & 3.205989 & $\mathrm{H}$ & 2.543916 & -6.723572 & -1.244131 \\
\hline c & -0.270969 & 2.299463 & 2.288454 & $\mathrm{H}$ & 1.505960 & 0.030707 & -2.668188 \\
\hline c & $-\odot .755612$ & 1.443791 & 4.130880 & $\mathrm{H}$ & 4.478074 & -1.422115 & $\odot .0 \odot 5883$ \\
\hline c & -0.129394 & 0.877907 & 4.977577 & $\mathrm{H}$ & 7.275785 & -0.949375 & -0.702956 \\
\hline c & 1.007221 & 2.428848 & 4.472081 & H & 8.319273 & -2.262091 & -1.268309 \\
\hline C & 1.496469 & 0.627911 & 3.408787 & $\mathrm{H}$ & 7.681476 & -1.079231 & -2.417029 \\
\hline C & $\odot .901253$ & 1.894365 & 1.532609 & H & 6.809223 & -3.292964 & -3.723749 \\
\hline c & 2.758350 & 1.347873 & 2.921569 & H & 7.315037 & -4.312529 & -2.373707 \\
\hline C & 2.954352 & $-\odot .565597$ & -1.222362 & H & 5.627855 & -4.311739 & -2.913184 \\
\hline c & 2.478221 & -0.421436 & -2.526591 & $\mathrm{H}$ & 5.055285 & -3.882321 & -0.354645 \\
\hline C & 3.214697 & -0.862814 & -3.633368 & H & 6.820172 & -4.011204 & $-\odot .202 \odot 41$ \\
\hline C & 4.491457 & -1.436985 & -3.381978 & $\mathrm{H}$ & 5.983400 & -2.637322 & 0.499155 \\
\hline C & 4.943457 & -1.732857 & -2.066675 & H & 2.127279 & -2.097761 & -6.713765 \\
\hline C & 4.156041 & -1.248444 & -1.012826 & $\mathrm{H}$ & 3. 573812 & -2.592723 & -5.814631 \\
\hline C & 6.204484 & -2.572737 & -1.709072 & H & 1.962709 & -2.857216 & -5.121148 \\
\hline c & 7.439936 & -1.658121 & -1.522678 & H & 0.744654 & $-\odot .260553$ & -6.034132 \\
\hline c & 6.506754 & -3.676450 & -2.751459 & H & 0.510652 & -0.897484 & $-4.4 \odot 2772$ \\
\hline C & 5.990702 & -3.313948 & $-\odot .361419$ & H & 1.073683 & $\odot .772647$ & -4.643721 \\
\hline c & 2.608545 & -0.764821 & -5.057855 & $\mathrm{H}$ & 3.496009 & 1.200455 & -5.474405 \\
\hline c & 2.574392 & -2.167688 & -5.714770 & $\mathrm{H}$ & 4. 396314 & -0.149574 & -6.193075 \\
\hline c & 1.151061 & -0.255029 & -5.017345 & H & 2.867367 & 0.362481 & -6.901300 \\
\hline C & 3.399289 & 0.218598 & -5.953364 & H & 7.281870 & -1.315218 & -4.379599 \\
\hline c & 6.354207 & -0.942865 & -4.824744 & H & 6.454724 & -0.975238 & -5.912692 \\
\hline C & 3.125971 & 1.325931 & 1.001630 & H & 6.186600 & ๑. 091509 & -4.506461 \\
\hline c & 4.275214 & 1.794790 & 0.354123 & $\mathrm{H}$ & 4.577682 & 1.324387 & -0.569808 \\
\hline C & 2.779864 & 1.895890 & 2.232832 & H & 7.583760 & 2.727974 & -1.530468 \\
\hline C & 3.534479 & 2.911702 & 2.840819 & H & 6.705601 & 1.356189 & $-\odot .854570$ \\
\hline C & 4.589099 & 3.461098 & 2.068410 & H & 5.860305 & 2.545413 & -1.869438 \\
\hline C & 5.031975 & 2.860785 & 0.858899 & H & 7.409280 & 3.698295 & 2.030963 \\
\hline C & 6.345331 & 3.287677 & $\odot .151642$ & H & 7.615926 & 2. 039163 & 1.432148 \\
\hline C & 6.627041 & 2.421277 & -1.095026 & $\mathrm{H}$ & 8.468403 & 3.378488 & 0.645478 \\
\hline C & 7.528985 & 3.090021 & 1.132099 & H & $4.34 \odot 272$ & 5.977920 & 1.234835 \\
\hline c & 4.556354 & 5.818611 & 2.297440 & $\mathrm{H}$ & 3.617950 & 5.852327 & 2.859823 \\
\hline C & 6.316697 & 4.758862 & $-\odot .329131$ & H & 5.219241 & 6.609147 & 2.655772 \\
\hline C & 3.169826 & 3.285948 & 4.308236 & $\mathrm{H}$ & 6.359345 & 5.462612 & 0.501410 \\
\hline C & 4.245980 & 4.098165 & 5.067134 & $\mathrm{H}$ & 7.188170 & 4.951490 & -0.965545 \\
\hline C & 1.827069 & 4.053668 & 4. 351199 & H & 5.420692 & 4.967723 & -0.926682 \\
\hline C & 3.003007 & 1.971317 & 5.120594 & $\mathrm{H}$ & 5.234398 & 3.637318 & 4.986604 \\
\hline C & -1.656300 & -1.697536 & 1.471151 & $\mathrm{H}$ & 4.328959 & 5.132831 & 4.735596 \\
\hline C & -2.127667 & $-3.0 \odot 8753$ & 1.570457 & H & 3.971818 & 4.122586 & 6.127954 \\
\hline C & -2.542199 & -3.567779 & 2.788095 & $\mathrm{H}$ & 1.557566 & 4.285302 & 5.388238 \\
\hline C & -2.367376 & -2.761683 & 3.947345 & $\mathrm{H}$ & 1.879807 & 5.000982 & 3.803226 \\
\hline C & -1.994638 & -1.390812 & 3.869788 & $\mathrm{H}$ & $1.0 \odot 9885$ & 3.462279 & 3.922123 \\
\hline C & -1.638110 & -0.891816 & 2.612128 & $\mathrm{H}$ & 2.187961 & 1.338844 & 4.757134 \\
\hline C & -2.011000 & $-\odot .446242$ & 5.099174 & $\mathrm{H}$ & 3.927438 & 1.382435 & 5.105507 \\
\hline C & -0.972420 & -0.878029 & 6.162027 & $\mathrm{H}$ & 2.777800 & 2.214007 & 6.164665 \\
\hline C & -3.428789 & $-\odot .421543$ & 5.722766 & $\mathrm{H}$ & -2.187759 & -3.604749 & $\odot .669090$ \\
\hline c & -1.665663 & 1.007705 & 4.706840 & $\mathrm{H}$ & -1.336993 & 0.142081 & 2.507635 \\
\hline C & -2.127071 & -6.087668 & 2.988943 & $\mathrm{H}$ & -1.246164 & -1.811279 & 6.652260 \\
\hline C & -1.702182 & -4.164441 & 5.801408 & $\mathrm{H}$ & 0.022424 & -0.998467 & 5.715724 \\
\hline C & -3.189483 & -4.983823 & 2.764772 & $\mathrm{H}$ & $-\odot .899658$ & $-\odot .108664$ & 6.939935 \\
\hline c & -3.821900 & -5.273618 & 1.378282 & $\mathrm{H}$ & -3.440872 & 0.247962 & 6.591344 \\
\hline C & -5.055708 & $-\odot .527772$ & -2.583483 & $\mathrm{H}$ & -4.161625 & $-\odot . \odot 43 \odot 97$ & $5.0005 \odot 5$ \\
\hline C & -3.821842 & -0.232537 & -3.222551 & $\mathrm{H}$ & -3.744080 & -1.413859 & 6.048899 \\
\hline C & -4.920431 & $\odot .552287$ & -5.487425 & $\mathrm{H}$ & -1.733972 & 1.638520 & 5.599626 \\
\hline C & -2.662033 & $-\odot .341455$ & -2.441689 & $\mathrm{H}$ & $-\odot .641093$ & 1.099381 & 4.323958 \\
\hline C & -2.699197 & $-\odot .750596$ & -1.102776 & $\mathrm{H}$ & -2.351585 & 1.423609 & 3.962098 \\
\hline c & -3.942873 & -0.918742 & -0.490656 & $\mathrm{H}$ & -2.596354 & -7.076703 & 2.922257 \\
\hline C & -5.149033 & $-0.76639 \odot$ & -1.188497 & $\mathrm{H}$ & -1.351750 & -6.025762 & 2.217780 \\
\hline C & -6.563052 & -1.835809 & -3.846649 & $\mathrm{H}$ & -1.642819 & -6.018392 & 3.965137 \\
\hline C & -2.985493 & -1.039034 & -5.462926 & $\mathrm{H}$ & -2.097108 & -5.185133 & 5.782402 \\
\hline C & -6.711025 & -2.374373 & $-\odot . \odot \odot \odot 235$ & $\mathrm{H}$ & -0.731365 & -4.135038 & 5.295589 \\
\hline c & -6.465254 & -0.891174 & -0.366150 & $\mathrm{H}$ & -1.571402 & -3.867118 & 6.845696 \\
\hline C & -6.317492 & $-\odot .084879$ & 0.952959 & $\mathrm{H}$ & -4.520467 & -4.486659 & 1.076250 \\
\hline c & -2.692283 & 1.379938 & -4.831640 & $\mathrm{H}$ & -3.076994 & -5.396178 & 0.585466 \\
\hline c & -4.345124 & -5.117357 & 3.785693 & $\mathrm{H}$ & -4.381511 & -6.213406 & 1.433027 \\
\hline C & -3.636795 & $\odot .154918$ & -4.720961 & $\mathrm{H}$ & -5.500263 & 1.311254 & -4.955713 \\
\hline C & -7.731848 & -0.332449 & -1.055748 & $\mathrm{H}$ & -4.622280 & $\odot .970504$ & -6.456305 \\
\hline $\mathrm{H}$ & 3.101527 & -2.207152 & 4.552109 & $\mathrm{H}$ & -5.580415 & -0.291539 & -5.690719 \\
\hline H & 3.083326 & $-\odot .344552$ & 2.932722 & $\mathrm{H}$ & -1.702260 & $-\odot .124995$ & -2.894356 \\
\hline $\mathrm{H}$ & 0.799296 & -6.303136 & 3.306747 & $\mathrm{H}$ & -3.973915 & -1.176819 & 0.558915 \\
\hline H & 2.455415 & -6.231619 & 2.576889 & $\mathrm{H}$ & -7.469572 & -1.694243 & -4.439560 \\
\hline $\mathrm{H}$ & -1.664532 & -2.517954 & -2.606802 & $\mathrm{H}$ & -5.773185 & -2.250154 & -4.485754 \\
\hline $\mathrm{H}$ & -0.528305 & -4.364991 & -3.804740 & $\mathrm{H}$ & -6.767611 & -2.544517 & -3.035458 \\
\hline $\mathrm{H}$ & 3.766833 & -5.734340 & -2.135471 & $\mathrm{H}$ & -2.837529 & -0.792668 & -6.521313 \\
\hline
\end{tabular}




\begin{tabular}{|c|c|c|c|}
\hline $\mathrm{H}$ & -2.007463 & -1.294542 & -5.040296 \\
\hline $\mathrm{H}$ & -3.617381 & -1.933439 & -5.413826 \\
\hline $\mathrm{H}$ & -6.835599 & -2.999623 & $-\odot .891126$ \\
\hline $\mathrm{H}$ & -5.878422 & -2.783819 & ๑. 581905 \\
\hline $\mathrm{H}$ & -7.621057 & -2.469423 & $\odot .604242$ \\
\hline $\mathrm{H}$ & -7.248809 & -0.154888 & 1.526155 \\
\hline $\mathrm{H}$ & -5.516540 & -0.453829 & 1.599541 \\
\hline $\mathrm{H}$ & -6.126178 & $\odot .973254$ & 0.746626 \\
\hline $\mathrm{H}$ & -3.143127 & 2.265785 & -4.371475 \\
\hline $\mathrm{H}$ & -1.718688 & 1.233262 & -4.361172 \\
\hline $\mathrm{H}$ & -2.517718 & 1.606526 & -5.889704 \\
\hline $\mathrm{H}$ & -5.115095 & -4.360551 & 3. 596196 \\
\hline $\mathrm{H}$ & -4.812021 & -6.102588 & 3.674203 \\
\hline $\mathrm{H}$ & -4.028546 & -5.005865 & 4.820875 \\
\hline $\mathrm{H}$ & -8.539569 & $-\odot .298304$ & -0.315090 \\
\hline $\mathrm{H}$ & -7.573439 & $\odot .683696$ & -1.428520 \\
\hline $\mathrm{H}$ & -8.078806 & -0.946374 & -1.886821 \\
\hline $\mathrm{Rh}$ & $-\odot .047291$ & 1.036918 & -0.012551 \\
\hline 0 & -2.115675 & 1.959055 & ๑. 621424 \\
\hline C & $\odot .152546$ & 2.471030 & -1.579088 \\
\hline S & -2.756143 & 3.274499 & ๑. 891161 \\
\hline 0 & -0.936719 & 2.519094 & -2.123583 \\
\hline C & -0.309239 & 4.233038 & ๑. 790728 \\
\hline $\mathrm{N}$ & -1.689379 & 4.445383 & 0.276893 \\
\hline 0 & -3.126028 & 3.546014 & 2.288494 \\
\hline C & -4.201679 & 3.473392 & -0.130805 \\
\hline $\mathrm{H}$ & 0.122161 & 5.235414 & ๑. 888075 \\
\hline $\mathrm{H}$ & -0.345225 & 3. 816181 & 1.805030 \\
\hline C & -2.185606 & 5.838437 & $\odot .453701$ \\
\hline C & -5.401296 & 3.841933 & ๑. 481937 \\
\hline C & -4.113496 & 3.263387 & -1.510776 \\
\hline C & -1.516441 & 6.758584 & -0.528736 \\
\hline $\mathrm{H}$ & -3.263507 & 5.830345 & ๑. $26 \odot 4 \odot 1$ \\
\hline $\mathrm{H}$ & -2.039069 & 6.184646 & 1.486335 \\
\hline $\mathrm{H}$ & -5.437548 & 3.998057 & $-262.21200 \odot$ \\
\hline C & -6.536239 & $4.0 \odot 7877$ & 10.394900 \\
\hline C & -5.259149 & 3. 442954 & 13. $.080 \odot \odot$ \\
\hline $\mathrm{H}$ & -3.169276 & 2.971908 & -1.957974 \\
\hline C & -0.845107 & 7.858765 & -0.184333 \\
\hline $\mathrm{H}$ & -1.630251 & 6.485488 & -1.577052 \\
\hline $\mathrm{H}$ & -7.473881 & 4.296717 & 0.154949 \\
\hline C & -6.484503 & 3.814024 & -1.699141 \\
\hline $\mathrm{H}$ & -5.205142 & 3.290649 & -3.354265 \\
\hline $\mathrm{H}$ & -0.409200 & 8.518143 & -0.929505 \\
\hline $\mathrm{H}$ & -0.725338 & 8.154187 & ๑. 856233 \\
\hline C & -7.714453 & 3.985143 & -2.557119 \\
\hline $\mathrm{H}$ & -7.510454 & 4.629481 & -3.419876 \\
\hline $\mathrm{H}$ & -8.053090 & 3.018150 & -2.949884 \\
\hline $\mathrm{H}$ & -8.541610 & 4.424634 & -1.992384 \\
\hline C & 1.459980 & 2.961946 & -2.195974 \\
\hline $\mathrm{H}$ & 1.387241 & 3.205989 & -3.259331 \\
\hline $\mathrm{H}$ & 2.299463 & 2.288454 & -2.016829 \\
\hline C & 1.443791 & 4.130880 & -1.191146 \\
\hline $\mathrm{H}$ & $\odot .877907$ & 4.977577 & -1.590282 \\
\hline $\mathrm{H}$ & 2.428848 & 4.472081 & -0.856806 \\
\hline C & ๑. 627911 & 3.408787 & $-\odot .084372$ \\
\hline $\mathrm{H}$ & 1.894365 & 1.532609 & 2.743603 \\
\hline $\mathrm{H}$ & 1.347873 & 2.921569 & 0.573397 \\
\hline
\end{tabular}

TS1_5

B3LYP SCF energy: $\quad-5535.31850295$ a.u. B3LYP enthalpy:

B3LYP free energy: -5533.284351 a.u.
-5533.554163 a.u.

M06 SCF energy in solution: $\quad-5534.55068097$ a.u.

M06 enthalpy in solution: $\quad-5532.516529 \mathrm{a} . \mathrm{u}$. M०6 free energy in solution: $\quad-5532.786341$ a.u.

Imaginary frequency:

$-248.8413 \mathrm{~cm}-1$

Cartesian coordinates

$\begin{array}{lccc}\text { ATOM } & X & Y & Z \\ P & 0.000000 & -5.617087 & 3.669413\end{array}$

\begin{tabular}{|c|c|c|}
\hline$\odot .000000$ & -6.561535 & 4.103866 \\
\hline$\odot .0 \odot \odot \odot \odot \odot$ & -5.112734 & 4.039347 \\
\hline$\odot . ๑ \odot \odot \odot \odot \odot$ & -3.050491 & 3. 492061 \\
\hline$\odot .0 \odot \odot \odot \odot \odot$ & $-\odot .840649$ & 7.845962 \\
\hline ๑. ๑๑९९७९ & -1.628233 & 6.679386 \\
\hline$\odot .0 \odot \odot \odot \odot \odot$ & -7.541887 & 4.265343 \\
\hline ๑. ๑๑९९७९ & -6.387819 & 4.264857 \\
\hline$\odot .00000 \odot$ & -4.963433 & 4.155431 \\
\hline$\odot .0 \odot \odot \odot \odot \odot$ & $-\odot .374799$ & 8.582289 \\
\hline$\odot .000000$ & -0.733579 & $8.0 \odot 4777$ \\
\hline$\odot .0 \odot \odot \odot \odot \odot$ & -7.535880 & 4. 701598 \\
\hline 1.615682 & -7.546837 & 5.794013 \\
\hline 2.133901 & -7.458163 & 4.277743 \\
\hline 2.595235 & -8.501127 & 4.407783 \\
\hline 2.522774 & 1.432282 & 3.026309 \\
\hline 1.766398 & 1.378463 & 3. 285691 \\
\hline - .183217 & 2.288132 & 2.377571 \\
\hline 16958 & 1.347891 & 4.174985 \\
\hline ๑. 056767 & $\odot .746462$ & 5.000683 \\
\hline 1.187519 & 2.307934 & 4.556335 \\
\hline 1.630859 & $\odot .551911$ & 3. 381732 \\
\hline ๑. 990881 & 1.747368 & 1.650492 \\
\hline 2.928472 & 1.293780 & 2.887790 \\
\hline 2.996399 & $-\odot .539605$ & -1.129414 \\
\hline 2.593907 & -0.362876 & -2.453800 \\
\hline 3.397073 & $-\odot .767162$ & -3.528887 \\
\hline 4.662173 & -1.339594 & -3.221883 \\
\hline 5.044466 & -1.662300 & -1.890309 \\
\hline 4.193981 & -1.213376 & -0.870591 \\
\hline 6.296126 & -2.495722 & -1.487635 \\
\hline 7.523609 & -1.577632 & -1.268873 \\
\hline 6.627341 & -3.603462 & -2.516935 \\
\hline 6.049042 & -3.230306 & -0.142794 \\
\hline 2.873450 & $-\odot .629519$ & -4.982183 \\
\hline 2.883790 & -2.011818 & -5.682237 \\
\hline 1.413632 & -0.128018 & -5.008947 \\
\hline $3.7 \odot 8149$ & ๑. 385093 & -5.799296 \\
\hline 6.606695 & -0.830723 & -4.561143 \\
\hline 3. 081321 & 1.340288 & 1.084603 \\
\hline 4.221281 & 1.827353 & 0.446078 \\
\hline 2.690244 & 1. 945231 & 2.293451 \\
\hline 3.479561 & 2.901675 & 2.937436 \\
\hline 4.707035 & 3.261276 & 2.309071 \\
\hline 5.030078 & 2.838110 & ๑. 999527 \\
\hline 6.166218 & 3. 410029 & 0.102012 \\
\hline 5.592648 & 3.672021 & -1.318511 \\
\hline 7.313602 & 2.380816 & -0.027719 \\
\hline 6.691629 & 3.442367 & 3.589394 \\
\hline 6.745068 & 4.768012 & 0.564809 \\
\hline 2.981931 & 3.591381 & 4.234736 \\
\hline 3.905505 & 3.309701 & 5.445065 \\
\hline 2.895047 & 5.120198 & 3.998004 \\
\hline 1.571435 & 3.106700 & 4.632106 \\
\hline-1.698065 & -1.743089 & 1.436901 \\
\hline-1.983500 & -3.106741 & 1.594523 \\
\hline-2.571322 & -3.603391 & 2.761742 \\
\hline-2.883269 & -2.662500 & 3.783998 \\
\hline-2.452970 & -1.314428 & 3.730552 \\
\hline-1.910635 & -0.877471 & 2.508979 \\
\hline-2.489001 & -0.296303 & 4.906454 \\
\hline-2.595639 & -0.939296 & 6.310066 \\
\hline-3.639385 & ๑. 720616 & 4. 710552 \\
\hline-1.152463 & ๑. 495194 & 4.917593 \\
\hline-1.977403 & -5.592883 & 4.189368 \\
\hline-4.973640 & -2.791600 & 4.899505 \\
\hline-2.779605 & -5.130106 & 2.946883 \\
\hline-2.257592 & -5.923749 & 1.728515 \\
\hline-5.006602 & -0.754113 & -2.706058 \\
\hline-3.775573 & -0.363421 & -3.297089 \\
\hline-4.777465 & -0.103202 & -5.726558 \\
\hline-2.635793 & -0.402425 & -2.480086 \\
\hline-2.671418 & $-\odot .909083$ & -1.175186 \\
\hline-3.887348 & -1.376507 & -0.673615 \\
\hline-5.076303 & -1.329574 & -1.414241 \\
\hline-6.801171 & $\odot .672254$ & -3.236064 \\
\hline
\end{tabular}




\begin{tabular}{|c|c|c|c|}
\hline C & -2.475379 & -0.912718 & -5.373232 \\
\hline C & -5.978131 & -3.389126 & $-\odot .274775$ \\
\hline C & -6.333280 & -1.949782 & $-\odot .737614$ \\
\hline C & -6.710462 & -1.099359 & $\odot .500415$ \\
\hline C & -3.052459 & 1.493668 & -4.874922 \\
\hline C & -4.266476 & -5.520649 & 3.126401 \\
\hline C & -3.557434 & ๑. . 034129 & -4.787729 \\
\hline C & -7.592924 & -2.095878 & -1.620860 \\
\hline $\mathrm{H}$ & 3.009690 & -2.174198 & 4.639404 \\
\hline $\mathrm{H}$ & 2.923946 & $-\odot .287605$ & 3.046939 \\
\hline $\mathrm{H}$ & 1.020263 & -6.383384 & 3.265720 \\
\hline H & 2.684202 & -6.211574 & 2.568288 \\
\hline $\mathrm{H}$ & -1.522674 & -2.551947 & -2.652802 \\
\hline $\mathrm{H}$ & -0.304445 & -4.338544 & -3.856376 \\
\hline $\mathrm{H}$ & 3.953810 & -5.713934 & -2.057190 \\
\hline $\mathrm{H}$ & 2.672818 & -6.677781 & -1.219604 \\
\hline $\mathrm{H}$ & 1.627077 & ๑. . 085695 & -2.638132 \\
\hline $\mathrm{H}$ & 4. 461904 & -1.407335 & $\odot .159749$ \\
\hline $\mathrm{H}$ & 7.333569 & $-\odot .868703$ & -0.455254 \\
\hline $\mathrm{H}$ & 8.396485 & -2.179273 & -0.987892 \\
\hline H & 7.790781 & $-1.0 \odot \odot \odot 23$ & -2.156175 \\
\hline $\mathrm{H}$ & 6.939275 & -3.223017 & -3.487396 \\
\hline $\mathrm{H}$ & 7.435047 & -4.230055 & -2.122388 \\
\hline $\mathrm{H}$ & 5.756257 & -4.247149 & -2.687284 \\
\hline $\mathrm{H}$ & 5.119532 & -3.807615 & -0.159836 \\
\hline $\mathrm{H}$ & 6.880070 & -3.918877 & 0.044258 \\
\hline $\mathrm{H}$ & 6.011643 & -2.547799 & ๑. 712561 \\
\hline H & 2.491853 & -1.913006 & -6.701699 \\
\hline $\mathrm{H}$ & 3.889083 & -2.430576 & -5.739219 \\
\hline $\mathrm{H}$ & 2.243694 & -2.721201 & -5.144561 \\
\hline $\mathrm{H}$ & 1.063180 & -0.106907 & -6.046072 \\
\hline H & $\odot .746019$ & $-\odot .791915$ & -4.449544 \\
\hline $\mathrm{H}$ & 1.307805 & $\odot .887694$ & -4.610382 \\
\hline $\mathrm{H}$ & 3. 766927 & 1.353102 & -5.287041 \\
\hline $\mathrm{H}$ & 4.721252 & $\odot .032088$ & -5.988638 \\
\hline H & 3.233223 & $\odot .552987$ & -6.773185 \\
\hline $\mathrm{H}$ & 7.519356 & -1.266893 & -4.143530 \\
\hline H & 6.715630 & $-\odot .778376$ & -5.647701 \\
\hline $\mathrm{H}$ & 6.468686 & $\odot .179970$ & -4.162677 \\
\hline H & 4. 493900 & 1.416357 & -0.516658 \\
\hline $\mathrm{H}$ & 6.375330 & 4.103155 & -1.952201 \\
\hline H & 5.239855 & 2.764908 & -1.817370 \\
\hline $\mathrm{H}$ & 4.762060 & 4.386366 & -1.282353 \\
\hline $\mathrm{H}$ & 7.775021 & 2.155706 & ๑. 939612 \\
\hline $\mathrm{H}$ & 6.956301 & 1.437037 & -0.453275 \\
\hline $\mathrm{H}$ & 8.097968 & 2.770724 & -0.687354 \\
\hline $\mathrm{H}$ & 6.382903 & 2.626952 & 4.252712 \\
\hline $\mathrm{H}$ & 7.365974 & 3.043040 & 2.825728 \\
\hline $\mathrm{H}$ & 7.215184 & 4.205893 & 4.168685 \\
\hline $\mathrm{H}$ & 7.362764 & 4.698096 & 1.459482 \\
\hline $\mathrm{H}$ & 7.381422 & 5.161811 & $-\odot .23600 \odot$ \\
\hline $\mathrm{H}$ & 5.956035 & 5.500977 & 0.757074 \\
\hline H & 4.085181 & 2.235026 & 5.568835 \\
\hline $\mathrm{H}$ & 4.864641 & 3.818755 & 5.355416 \\
\hline H & 3. 426849 & 3.672053 & 6.362209 \\
\hline $\mathrm{H}$ & 2.536866 & 5.617690 & 4.907168 \\
\hline H & 3.867226 & 5.542323 & 3. 737191 \\
\hline $\mathrm{H}$ & 2.188190 & 5.350425 & 3.191221 \\
\hline $\mathrm{H}$ & ๑. 828835 & 3.292449 & 3.847880 \\
\hline $\mathrm{H}$ & 1.549475 & 2.039783 & 4.881175 \\
\hline $\mathrm{H}$ & 1.244424 & 3.654512 & 5.521869 \\
\hline H & -1.754198 & -3.783290 & $\odot .782128$ \\
\hline $\mathrm{H}$ & -1.651499 & $\odot .167155$ & 2. 387491 \\
\hline $\mathrm{H}$ & -3.575240 & -1.364782 & 6.523741 \\
\hline $\mathrm{H}$ & -1.849893 & -1.727907 & 6.451236 \\
\hline $\mathrm{H}$ & -2.409849 & -0.162438 & 7.060771 \\
\hline $\mathrm{H}$ & -3.640694 & 1.445305 & 5.533967 \\
\hline H & -3.530690 & 1.280785 & 3.776348 \\
\hline $\mathrm{H}$ & -4.620015 & $\odot .233526$ & 4.698771 \\
\hline H & -1.132481 & 1.160447 & 5.787702 \\
\hline $\mathrm{H}$ & -0.292865 & $-\odot .181823$ & 4.985975 \\
\hline H & -1.024438 & 1.125193 & 4.033295 \\
\hline H & -2.061876 & -6.680349 & 4.306567 \\
\hline $\mathrm{H}$ & -0.915742 & -5.340757 & 4.084385 \\
\hline $\mathrm{H}$ & -2.347438 & -5.120893 & 5.101583 \\
\hline
\end{tabular}

\begin{tabular}{|c|c|c|c|}
\hline $\mathrm{H}$ & -5.126067 & -1.723507 & 5.080749 \\
\hline H & -5.465830 & -3.068087 & 3.960375 \\
\hline $\mathrm{H}$ & -5.413114 & -3.360638 & 5.721977 \\
\hline H & -2.823333 & -5.699113 & $\odot .816424$ \\
\hline H & -1.197143 & -5.737138 & 1.532538 \\
\hline $\mathrm{H}$ & -2.377171 & -6.994592 & 1.926072 \\
\hline $\mathrm{H}$ & -5.560227 & 0.628949 & -5.521079 \\
\hline $\mathrm{H}$ & -4.433664 & 0.073665 & -6.752939 \\
\hline H & -5.224436 & -1.099014 & -5.686869 \\
\hline H & -1.692903 & -0.044562 & -2.877364 \\
\hline H & -3.914004 & -1.790985 & 0.324318 \\
\hline H & -7.711384 & 0.656770 & -3.840248 \\
\hline H & -7.063025 & $\odot .839678$ & -2.183556 \\
\hline $\mathrm{H}$ & -6.154209 & 1.493871 & -3.565834 \\
\hline H & -2.284657 & $-\odot .655819$ & -6.421605 \\
\hline H & -1.522629 & -0.846360 & -4.842222 \\
\hline H & -2.812728 & -1.955521 & -5.340206 \\
\hline H & -5.713031 & -4.018886 & -1.131700 \\
\hline H & -5.145731 & -3.417946 & 0.434032 \\
\hline $\mathrm{H}$ & -6.845866 & -3.841907 & 0.218784 \\
\hline H & -7.578481 & -1.536756 & 1.008588 \\
\hline H & -5.890444 & -1.041154 & 1.224529 \\
\hline H & -6.971590 & $-\odot .074475$ & 0.211749 \\
\hline H & -3.821175 & 2.189685 & -4.520223 \\
\hline H & -2.151327 & 1.665119 & -4.279121 \\
\hline H & -2.826905 & 1.749361 & -5.917491 \\
\hline H & -4.887171 & -5.113994 & 2.319311 \\
\hline $\mathrm{H}$ & -4.362069 & -6.612498 & 3.096285 \\
\hline H & -4.667785 & -5.185459 & 4.082239 \\
\hline $\mathrm{H}$ & -8.336746 & -2.671768 & -1.057107 \\
\hline H & -8.052877 & -1.138865 & -1.872146 \\
\hline $\mathrm{H}$ & -7.387084 & -2.629362 & -2.551621 \\
\hline $\mathrm{Rh}$ & -0.086046 & 1.002324 & -0.039032 \\
\hline 0 & -2.220047 & 1.914428 & 0.437776 \\
\hline C & 0.125877 & 2.490835 & -1.574647 \\
\hline $\mathrm{s}$ & -2.860364 & 3.209246 & 0.785328 \\
\hline 0 & -0.952803 & 2.524973 & -2.142489 \\
\hline C & -0.393462 & 4.132287 & 0.871178 \\
\hline $\mathrm{N}$ & -1.753124 & 4.422373 & 0.347515 \\
\hline 0 & -3.316473 & 3.355391 & 2.176764 \\
\hline C & -4.234894 & 3.559660 & -0.297351 \\
\hline H & 0.054707 & 5.111013 & 1.076427 \\
\hline $\mathrm{H}$ & $-\odot .461586$ & 3.618058 & 1.838598 \\
\hline C & -2.235871 & 5.794129 & 0.678155 \\
\hline C & -5.491200 & 3.762716 & $\odot .278403$ \\
\hline C & -4.031145 & 3.676759 & -1.675782 \\
\hline C & -1.529087 & 6.814483 & -0.169503 \\
\hline H & -3.308819 & 5.826897 & 0.462583 \\
\hline H & -2.109524 & 6.009438 & 1.748028 \\
\hline $\mathrm{H}$ & -5.617087 & 3.669413 & -248.841300 \\
\hline C & -6.561535 & 4.103866 & 9.347300 \\
\hline C & -5.112734 & 4.039347 & 12.981200 \\
\hline H & $-3.05 \odot 491$ & 3.492061 & -2.100687 \\
\hline C & -0.840649 & 7.845962 & $\odot .322059$ \\
\hline H & -1.628233 & 6.679386 & -1.245866 \\
\hline H & -7.541887 & 4.265343 & -0.106792 \\
\hline C & -6.387819 & 4.264857 & -1.929338 \\
\hline H & -4.963433 & 4.155431 & -3.545531 \\
\hline H & -0.374799 & 8.582289 & -0.326616 \\
\hline H & -0.733579 & $8.0 \odot 4777$ & 1.393295 \\
\hline C & -7.535880 & 4.701598 & -2.806985 \\
\hline H & -7.546837 & 5.794013 & -2.916125 \\
\hline H & -7.458163 & 4.277743 & -3.813461 \\
\hline H & -8.501127 & 4.407783 & -2.383113 \\
\hline C & 1.432282 & 3.026309 & -2.154491 \\
\hline H & 1.378463 & 3.285691 & -3.214980 \\
\hline H & 2.288132 & 2.377571 & -1.961738 \\
\hline C & 1.347891 & 4.174985 & -1.128516 \\
\hline $\mathrm{H}$ & 0.746462 & 5.000683 & -1.519260 \\
\hline $\mathrm{H}$ & 2.307934 & 4.556335 & -0.767085 \\
\hline C & $\odot .551911$ & 3.381732 & -0.056527 \\
\hline H & 1.747368 & 1.650492 & 2.737899 \\
\hline $\mathrm{H}$ & 1.293780 & 2.887790 & 0.571048 \\
\hline
\end{tabular}


TS1 6

B3LYP SCF energy: $\quad-5535.31782437 \mathrm{a} . \mathrm{u}$.

B3LYP enthalpy:

B3LYP free energy:

-5533.283885 a.u

-5533.554784 a.u

Mo6 SCF energy in solution: $\quad-5534.55208164$

a.u

M06 enthalpy in solution:

-5532.518142 a.u

M०6 free energy in solution: $-5532.789041$

Imaginary frequency: $\quad-249.8331 \mathrm{~cm}-1$

Cartesian coordinates

\begin{tabular}{|c|c|c|c|}
\hline ATOM & $\mathrm{X}$ & $\mathrm{Y}$ & Z \\
\hline$P$ & $\odot . ๑ \odot \odot \diamond \odot \odot$ & -5.549587 & 3.737027 \\
\hline$P$ & $\odot . \odot \odot \odot \odot \odot \odot ~$ & -6.537598 & 4.063558 \\
\hline 0 & ๑. .००००० & -5.134328 & 3.884074 \\
\hline 0 & 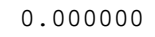 & -3.064526 & 3.353098 \\
\hline 0 & ๑. $0000 \odot ०$ & -0.798550 & 7.831266 \\
\hline 0 & $\odot . \diamond \odot \odot \odot \odot \odot$ & -1.587194 & 6.555265 \\
\hline 0 & $\odot .00000 \odot$ & -7.507373 & 4.252038 \\
\hline 0 & $\odot . \diamond \odot \odot \odot \odot \odot$ & -6.396028 & 4.143478 \\
\hline 0 & $\odot .000000$ & -5.009949 & 3.938579 \\
\hline 0 & $\odot .0 \odot \odot ० \odot \odot$ & -0.342678 & 8.525308 \\
\hline C & $\odot . ๑ \odot \odot \odot \odot \odot ~$ & -0.687803 & 8.064041 \\
\hline C & ๑. . 000000 & -7.564275 & 4.531134 \\
\hline C & 1.642368 & -7.589953 & 5.617957 \\
\hline C & 2.173858 & -7.499633 & 4.066847 \\
\hline C & 2.646431 & -8.518455 & 4.244847 \\
\hline C & 2.562299 & 1.453977 & 2.848734 \\
\hline C & 1.795553 & 1.393917 & 3.047090 \\
\hline C & -0.174593 & 2.305682 & 2.204072 \\
\hline C & -0.607989 & 1.388245 & 4.056090 \\
\hline C & ๑. 086665 & $\odot .791716$ & 4.863273 \\
\hline C & 1. 241091 & 2.356748 & 4.447401 \\
\hline C & 1.679541 & $\odot .596603$ & 3.337789 \\
\hline C & 1.014419 & 1.726290 & 1.536168 \\
\hline C & 3.088284 & 1.337485 & 2.877517 \\
\hline C & 3.071519 & -0.570997 & -1.072730 \\
\hline C & 2.684506 & -0.513395 & -2.413233 \\
\hline C & 3.531249 & $-\odot .947492$ & -3.441957 \\
\hline C & 4.821815 & -1.416780 & -3.071221 \\
\hline C & 5.187057 & -1.640095 & -1.716858 \\
\hline C & 4. 292936 & -1.167240 & -0.746471 \\
\hline C & 6.459100 & -2.389766 & -1.221979 \\
\hline C & 7.572352 & -1.383753 & $-\odot .840965$ \\
\hline C & 7.006083 & -3.419237 & -2.239716 \\
\hline C & 6.121460 & -3.201267 & ๑. 059618 \\
\hline C & 3.028996 & $-\odot .969556$ & -4.909537 \\
\hline C & 3.146550 & -2.406020 & -5.479328 \\
\hline C & 1.540192 & -0.570506 & -4.999206 \\
\hline C & 3.811807 & ๑. 014920 & -5.811100 \\
\hline C & 6.728761 & -0.772446 & -4.358248 \\
\hline C & 3.014784 & 1.379457 & 1.094206 \\
\hline C & 4.151333 & 1.909158 & $\odot .47006 \odot$ \\
\hline C & 2.601552 & 1.944030 & 2.307215 \\
\hline C & 3.279680 & 3. 011556 & 2.918541 \\
\hline C & 4.318664 & 3. 613978 & 2.165124 \\
\hline C & 4.829279 & 3.025335 & $\odot .977160$ \\
\hline C & 6.134010 & 3.519474 & 0.298203 \\
\hline C & 6.501530 & 2.653522 & -0.926498 \\
\hline C & 7.299513 & 3.407095 & 1.313319 \\
\hline C & 4.134329 & 5.967179 & 2. 355971 \\
\hline C & 6.031694 & 4.979353 & -0.206231 \\
\hline C & 2.861700 & 3.380304 & 4.373109 \\
\hline C & 3.863111 & 4.274736 & 5.141847 \\
\hline C & 1.469837 & 4.055860 & 4.379423 \\
\hline C & 2.771187 & 2.067123 & 5.199563 \\
\hline C & -1.682263 & -1.716848 & 1.467793 \\
\hline C & -1.961095 & -3.072940 & 1.687029 \\
\hline C & -2.534818 & -3.519858 & 2.881276 \\
\hline C & -2.839111 & -2.536366 & 3.865298 \\
\hline C & -2.417531 & -1.189385 & 3.747088 \\
\hline C & -1.888974 & -0.805446 & 2.502436 \\
\hline C & -2.450537 & -0.117741 & 4.874692 \\
\hline
\end{tabular}

$-2.533897$

$-3.613529$

$-1.122692$

$-1.909660$

$-4.914430$

$-2.735611$

$-2.234487$

$-4.985265$

$-3.753850$

$-4.728579$

$-2.621506$

$-2.661850$

$-3.875627$

$-5.059374$

$-6.787600$

$-2.390760$

$-5.950451$

$-6.315121$

$-6.708137$

- 3.096603

$-4.218101$

$-3.530163$

$-7.567845$

3.077498

2. 976343

1. 045981

2.706220

$-1.524674$

$-0.272881$

4. 084093

2. 997610

1. 696862

4. 548975

7.233829

8.453830

7.890595

7.439901

7.787850

6. 219256

5. 243637

6. 974448

5. 934533

2. 764752

4. 181271

2. 552484

1. 209486

0.905148

1. 363189

3. 821569

4. 840851

3. 328889

7. 569151

7.078136

6.352080

4. 507073

7.449715

6.637299

5. 750785

7.119116

7. 440002

8. 233441

3. 941988

3. 178413

4. 734244

6. 006781

6. 908702

5. 143222

4.882607

3. 880520

3. 565351

1.164213

1. 471453

๑. 702845

2.013374

3. 736256
$-0.696259$

0.877681

$\odot .688197$

$-5.446825$

$-2.629837$

$-5.037885$

$-5.880921$

$-0.903925$

$-0.518424$

$-0.395666$

$-0.515746$

$-0.982218$

$-1.447903$

$-1.437183$

๑. 485250

$-1.066750$

- 3.469187

$-2.047954$

$-1.163066$

1. 317976

$-5.423354$

$-0.161162$

$-2.231775$

$-2.153156$

$-\odot .281897$

$-6.362878$

$-6.213942$

- 2. 670676

$-4.446593$

$-5.470853$

$-6.632398$

$-0.137350$

$-1.278309$

- $\odot .710564$

$-1.920590$

$-0.765952$

$-2.969091$

$-4.014162$

$-4.106609$

$-3.838662$

$-3.838524$

$-2.564723$

$-2.428937$

$-2.751425$

- 3.110541

$-0.662105$

$-1.225243$

๑. 467465

1. 024752

$-0.304252$

๑. 075993

$-0.890850$

$-\odot .969577$

$\odot .254232$

1. 445918

3. 011708

1. 598661

2. 720228

4.020157

2. 367747

3. 743844

6. 100407

5.947455

6.802399

5. 696036

5.215490

5. 125468

3. 883192

5.308832

4. 292572

4.280990

4. 998602

3. 406824

1. 373197

1. 547532
6. 307621

.644894

4. 834466

4. 379799

013198

3. 133955

1. 940270

2. 727472

- 3.321799

$-5.769688$

$-2.494391$

$-1.174721$

$-0.667032$

$-1.417697$

- 3.327434

$-5.362944$

$-0.220891$

$-0.730226$

0.478382

$-4.951478$

3. 356202

- 4.821971

$-1.616157$

4. 655216

3. 048934

3. 342671

2. 631662

$-2.596376$

$-3.780634$

$-2.098837$

$-1.240202$

$-2.644797$

๑. 299646

$-0.044878$

$-0.470282$

$-1.685331$

$-3.130718$

$-1.754386$

$-2.570862$

$-0.084937$

0.316311

๑. 929992

$-6.507156$

$-5.490619$

$-4.885627$

$-6.039031$

$-4.392889$

$-4.693291$

$-5.383192$

$-5.974751$

$-6.793550$

- 3. 667606

$-5.374559$

$-4.295221$

$-0.438127$

$-1.340864$

$-0.667321$

$-1.723356$

2. 198710

1. 632468

0.847615

1. 285155

2. 889019

2. 723827

0.613928

$-0.819967$

$-0.833199$

5. 087909

4. 798509

6.196437

5. 407891

3. 820850

3. 941650

4.825835

5. 213246 


\begin{tabular}{|c|c|c|c|}
\hline $\mathrm{H}$ & 2.504843 & 2.305077 & 6.235089 \\
\hline $\mathrm{H}$ & -1.736568 & -3.783275 & 0.902435 \\
\hline $\mathrm{H}$ & -1.635833 & $\odot .233706$ & 2.331759 \\
\hline $\mathrm{H}$ & -3.505792 & -1.122938 & 6.551498 \\
\hline $\mathrm{H}$ & -1.777318 & -1.469102 & 6.475843 \\
\hline $\mathrm{H}$ & -2.349306 & $\odot .116144$ & 7.020024 \\
\hline $\mathrm{H}$ & -3.615935 & 1.637709 & 5.435780 \\
\hline H & -3.517444 & 1.397586 & 3.686257 \\
\hline $\mathrm{H}$ & -4.588867 & ๑. 380424 & 4.662392 \\
\hline $\mathrm{H}$ & -1.100334 & 1.391895 & 5.673674 \\
\hline $\mathrm{H}$ & -0.255554 & ๑. . 023428 & 4.924081 \\
\hline H & -1.012913 & 1.279043 & 3.920540 \\
\hline $\mathrm{H}$ & -1.996068 & -6.527383 & 4.548370 \\
\hline $\mathrm{H}$ & -0.848993 & -5.205568 & 4.242735 \\
\hline $\mathrm{H}$ & -2.259871 & -4.932229 & 5.276791 \\
\hline $\mathrm{H}$ & -5.072747 & -1.556484 & 5.153796 \\
\hline $\mathrm{H}$ & -5.415867 & -2.947008 & 4.092087 \\
\hline $\mathrm{H}$ & -5.339663 & -3.168608 & 5.863068 \\
\hline $\mathrm{H}$ & -2.817136 & -5.694446 & 1.030198 \\
\hline $\mathrm{H}$ & -1.178153 & -5.702261 & 1.716719 \\
\hline $\mathrm{H}$ & -2.349037 & -6.942721 & 2.184146 \\
\hline $\mathrm{H}$ & -5.547195 & ๑. 307295 & -5.608093 \\
\hline $\mathrm{H}$ & -4.378358 & -0.250803 & -6.798851 \\
\hline $\mathrm{H}$ & -5.130426 & -1.408468 & -5.689311 \\
\hline $\mathrm{H}$ & -1.679896 & $-\odot .158615$ & -2.894365 \\
\hline $\mathrm{H}$ & -3.904376 & -1.832491 & ๑. 342966 \\
\hline $\mathrm{H}$ & -7.695930 & 0.435582 & -3.932605 \\
\hline $\mathrm{H}$ & -7.054010 & $\odot .694838$ & -2.283601 \\
\hline $\mathrm{H}$ & -6.147413 & 1. 298098 & -3.690843 \\
\hline $\mathrm{H}$ & -2.206596 & -0.838109 & -6.418899 \\
\hline $\mathrm{H}$ & -1.446726 & $-\odot .926579$ & -4.830390 \\
\hline $\mathrm{H}$ & -2.668421 & -2.125301 & -5.293703 \\
\hline $\mathrm{H}$ & -5.673441 & -4.122477 & -1.056142 \\
\hline $\mathrm{H}$ & -5.123052 & -3.468843 & 0.494466 \\
\hline $\mathrm{H}$ & -6.817642 & -3.914600 & ๑. 280214 \\
\hline $\mathrm{H}$ & -7.575038 & -1.592915 & $\odot .994793$ \\
\hline $\mathrm{H}$ & -5.893418 & -1.074159 & 1.205317 \\
\hline H & -6.977410 & $-\odot .150401$ & $\odot .156032$ \\
\hline $\mathrm{H}$ & -3.902215 & 1.987378 & -4.629021 \\
\hline $\mathrm{H}$ & -2.211667 & 1.548311 & -4.350903 \\
\hline $\mathrm{H}$ & -2.869807 & 1.551001 & -5.999139 \\
\hline H & -4.854685 & -5.047124 & 2.546650 \\
\hline $\mathrm{H}$ & -4.312195 & -6.515641 & 3.367638 \\
\hline $\mathrm{H}$ & -4.601834 & -5.054098 & 4.306625 \\
\hline $\mathrm{H}$ & -8.311109 & -2.794853 & -1.038900 \\
\hline $\mathrm{H}$ & -8.032937 & -1.286545 & -1.900666 \\
\hline $\mathrm{H}$ & -7.352174 & -2.793033 & -2.528129 \\
\hline $\mathrm{Rh}$ & -0.069995 & ๑. 963195 & -0.112596 \\
\hline 0 & -2.188325 & 1.912548 & 0. 343238 \\
\hline C & ๑. 148167 & 2.357202 & -1.720521 \\
\hline$S$ & -2.808730 & 3.230026 & ๑. 636959 \\
\hline 0 & -0.936421 & 2.378598 & -2.278337 \\
\hline C & -0.337389 & 4.150072 & ๑. 631891 \\
\hline $\mathrm{N}$ & -1.704407 & 4.409547 & $\odot .107760$ \\
\hline 0 & -3.228780 & 3.454432 & 2. 029533 \\
\hline C & -4.206612 & 3.528657 & -0.430360 \\
\hline $\mathrm{H}$ & $\odot .113207$ & 5.139898 & 0.766216 \\
\hline $\mathrm{H}$ & -0.393511 & 3.702326 & 1.632267 \\
\hline C & -2.179216 & 5.800192 & ๑. 359788 \\
\hline C & -5.448696 & 3.768439 & ๑. 161982 \\
\hline C & -4.034816 & 3.564879 & -1.817504 \\
\hline C & -1.482541 & 6.764069 & $-\odot .559390$ \\
\hline $\mathrm{H}$ & -3.254871 & 5.823758 & $\odot .156964$ \\
\hline $\mathrm{H}$ & -2.037912 & 6.081498 & 1.412448 \\
\hline $\mathrm{H}$ & -5.549587 & 3.737027 & -249.833100 \\
\hline C & -6.537598 & 4.063558 & 8.848900 \\
\hline C & -5.134328 & 3.884074 & 12.182900 \\
\hline $\mathrm{H}$ & -3.064526 & 3. 353098 & -2.253733 \\
\hline C & $-\odot .798550$ & 7.831266 & -0.143743 \\
\hline $\mathrm{H}$ & -1.587194 & 6.555265 & -1.623362 \\
\hline $\mathrm{H}$ & -7.507373 & 4.252038 & -0.203839 \\
\hline C & -6.396028 & 4.143478 & -2.049655 \\
\hline $\mathrm{H}$ & -5.009949 & 3.938579 & -3.689336 \\
\hline $\mathrm{H}$ & -0.342678 & 8.525308 & -0.844101 \\
\hline $\mathrm{H}$ & -0.687803 & 8.064041 & ๑. 913579 \\
\hline
\end{tabular}

$\begin{array}{lrrr}\mathrm{C} & -7.564275 & 4.531134 & -2.923698 \\ \mathrm{H} & -7.589953 & 5.617957 & -3.077265 \\ \mathrm{H} & -7.499633 & 4.066847 & -3.913040 \\ \mathrm{H} & -8.518455 & 4.244847 & -2.470496 \\ \mathrm{C} & 1.453977 & 2.848734 & -2.338733 \\ \mathrm{H} & 1.393917 & 3.047090 & -3.412089 \\ \mathrm{H} & 2.305682 & 2.204072 & -2.115622 \\ \mathrm{C} & 1.388245 & 4.056090 & -1.380844 \\ \mathrm{H} & 0.791716 & 4.863273 & -1.815513 \\ \mathrm{H} & 2.356748 & 4.447401 & -1.053039 \\ \mathrm{C} & 0.596603 & 3.337789 & -0.254345 \\ \mathrm{H} & 1.726290 & 1.536168 & 2.801544 \\ \mathrm{H} & 1.337485 & 2.877517 & 0.398274\end{array}$

$\mathrm{TS}_{1} 7$

B3LYP SCF energy: $\quad-5535.31812153$ a.u B3LYP enthalpy: $\quad-5533.283993$ a.u. B3LYP free energy: $\quad-5533.553980$ a.u. M06 SCF energy in solution: $\quad-5534.54760586$ a.u.

M06 enthalpy in solution: 532.513477 a.u. Mo6 free energy in solution: $\quad-5532.783464$ a.u.

Imaginary frequency: $\quad-237.0308 \mathrm{~cm}-1$

Cartesian coordinates

\begin{tabular}{|c|c|c|c|}
\hline АTOM & $X$ & Y & Z \\
\hline $\mathrm{P}$ & 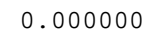 & -5.586916 & 3.762926 \\
\hline $\mathrm{P}$ & $\odot .0 \odot \odot \odot \odot \odot$ & -6.704726 & 3.948624 \\
\hline 0 & 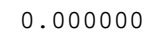 & -5.430277 & 3.668112 \\
\hline 0 & $\odot .0 \odot \odot ० \odot \odot$ & -3.329156 & 3.202455 \\
\hline 0 & $\odot . ๑ \odot \odot \odot \odot \odot$ & -0.966525 & 7.818396 \\
\hline 0 & ๑. . 000000 & -1.854032 & 6.489514 \\
\hline 0 & $\odot . ๑ \odot \odot \odot \odot \odot$ & -7.646772 & 4.146590 \\
\hline 0 & $\odot .0 \odot \odot \odot \odot \odot$ & -6.657917 & 3.937987 \\
\hline 0 & $\odot .000000$ & -5.378844 & 3.655278 \\
\hline 0 & 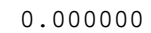 & -0.560122 & 8.484791 \\
\hline C & $\odot .0000 \bullet \odot$ & -0.781857 & 8. 089598 \\
\hline C & 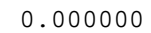 & -7.891727 & 4.241787 \\
\hline C & 1.860072 & -7.980824 & 5.320892 \\
\hline C & 2.397787 & -7.860725 & 3.750446 \\
\hline C & 2.772766 & -8.803038 & 3.924098 \\
\hline C & 2.599009 & 1.177873 & 2.847212 \\
\hline C & 2.200695 & 1.030008 & 2.989538 \\
\hline C & -0.124806 & 2. 078023 & 2.260276 \\
\hline C & -0.581624 & 1.112391 & 4.093671 \\
\hline C & ๑. 132918 & ๑. 430532 & 4.839912 \\
\hline C & 1.333837 & 2.075350 & 4.561270 \\
\hline C & 1.797690 & 0.464386 & 3.381767 \\
\hline C & 1.112807 & 1.481879 & 1.700851 \\
\hline C & 3. 266521 & 1.281770 & 2.982677 \\
\hline C & 3.051564 & -0.416457 & -1.158895 \\
\hline C & 2.630542 & -0.408188 & -2.490975 \\
\hline C & 3.475095 & $-\odot .814006$ & -3.532754 \\
\hline C & 4.796787 & -1.206902 & -3.184292 \\
\hline C & 5.202433 & -1.390885 & -1.835804 \\
\hline C & 4. 309987 & $-\odot .942350$ & -0.851813 \\
\hline C & 6.513994 & -2.083103 & -1.359804 \\
\hline C & 7.581186 & -1.029957 & -0.976892 \\
\hline C & 7.105438 & -3.074795 & -2.390121 \\
\hline C & 6.221651 & -2.922784 & $-\odot .085029$ \\
\hline C & 2.942035 & -0.890389 & -4.987876 \\
\hline C & 3.131262 & -2.326610 & -5.538947 \\
\hline C & 1.430687 & $-\odot .579668$ & -5.049737 \\
\hline C & 3.647138 & ๑. 121809 & -5.922469 \\
\hline C & 6.638137 & -0.471811 & -4.508728 \\
\hline C & 2.922892 & 1.505601 & 1.039753 \\
\hline C & 4.048869 & 2.084886 & ๑. 456329 \\
\hline C & 2. 419328 & 2.074769 & 2.223640 \\
\hline C & 3. 092642 & 3.090600 & 2.905827 \\
\hline C & 4.315027 & 3.553791 & 2.337746 \\
\hline C & 4.738862 & 3.161215 & 1.046882 \\
\hline C & 5.865539 & 3.830751 & ๑. 206457 \\
\hline
\end{tabular}




\begin{tabular}{|c|c|c|c|c|c|c|c|}
\hline C & 5.339989 & 4.053638 & -1.238911 & H & 7.880914 & 3.361510 & $-\odot .486872$ \\
\hline c & 7.099407 & 2.901087 & 0.129231 & $\mathrm{H}$ & 5.942674 & 3. 066731 & 4.367869 \\
\hline c & 6.212845 & 3.901432 & 3.711744 & H & 6.950998 & 3.554855 & 2.982098 \\
\hline c & 6.308123 & 5.229324 & 0.697986 & $\mathrm{H}$ & 6.645758 & 4.708204 & 4.307015 \\
\hline c & 2.475514 & 3.725823 & 4.179338 & H & 6.890196 & 5.205616 & 1.618633 \\
\hline c & 3.364339 & 3.521343 & 5.430504 & $\mathrm{H}$ & 6.944126 & 5.679153 & -0.073071 \\
\hline C & 2.263314 & 5.242076 & 3.941846 & H & 5.453349 & 5.893153 & 0.856881 \\
\hline C & 1.097615 & 3.113127 & 4.510786 & H & 3.634605 & 2.466492 & 5.560680 \\
\hline C & -1.584931 & -1.753733 & 1.506930 & H & 4.276633 & 4.115398 & 5.386484 \\
\hline C & -1.654199 & -3.126302 & 1.784809 & $\mathrm{H}$ & 2.813034 & 3.833596 & 6.324984 \\
\hline C & -2.055382 & -3.602049 & 3.036755 & H & 1.823391 & 5.700768 & 4.835352 \\
\hline C & -2.359782 & -2.631643 & 4.031086 & $\mathrm{H}$ & 3.204763 & 5.749962 & 3.724340 \\
\hline C & -2.485053 & -1.254652 & 3.732470 & H & 1.573946 & 5.413125 & 3.105977 \\
\hline C & -2.032915 & -0.843194 & 2.465946 & $\mathrm{H}$ & 0.375127 & 3.237196 & 3.696340 \\
\hline C & -3.141713 & -0.171165 & 4.635717 & H & 1.161934 & 2. 046272 & 4.754306 \\
\hline c & -3.863614 & $-\odot .689569$ & 5.900941 & H & 0.683339 & 3.621804 & 5.387203 \\
\hline c & -4.239012 & 0.539749 & 3.796672 & H & -1.386753 & -3.829413 & 1.007413 \\
\hline C & -2.085150 & $\odot .875336$ & 5.060895 & H & -2.061033 & $\odot .210288$ & 2.210886 \\
\hline c & -1.320106 & -5.665600 & 4. 396133 & H & -4.571617 & -1.489853 & 5.671026 \\
\hline c & -1.472034 & -2.871159 & 6.202483 & $\mathrm{H}$ & -3.184849 & -1.049313 & 6.675106 \\
\hline c & -2.246129 & -5.121393 & 3.280563 & H & -4.427719 & 0.144907 & 6.333728 \\
\hline C & -1.955615 & -5.939047 & 2.002845 & H & -4.719264 & 1.314701 & 4.404700 \\
\hline c & -5.056731 & -1.159734 & -2.587257 & H & -3.844877 & 1.030501 & 2.905240 \\
\hline C & -3.872800 & $-\odot .68939 \odot$ & -3.215910 & H & -5.011685 & $-\odot .174055$ & 3.487634 \\
\hline c & -4.930956 & -0.623087 & -5.629467 & H & -2.553252 & 1.655507 & 5.672576 \\
\hline C & -2.718314 & -0.617181 & -2.423381 & H & -1.283989 & $\odot .419221$ & 5.654606 \\
\hline C & -2.688290 & -1.093321 & -1.106718 & H & -1.629054 & 1.365280 & 4.194412 \\
\hline C & -3.850064 & -1.650271 & $-\odot .568862$ & H & -1.329839 & -6.762192 & 4.380155 \\
\hline C & -5.053699 & -1.715155 & -1.284236 & H & -0.284986 & -5.333648 & 4.256579 \\
\hline C & -6.961403 & 0.122122 & -3.100762 & H & -1.650704 & -5.351474 & 5.386273 \\
\hline C & -2.538134 & -1.127296 & -5.302472 & H & -0.583174 & -3.415381 & 5.863492 \\
\hline C & -5.764360 & -3.800131 & -0.053043 & H & -1.223406 & -1.808080 & 6.289660 \\
\hline C & -6.245917 & -2.417400 & $-\odot .571599$ & H & -1.783751 & -3.251615 & 7.177757 \\
\hline C & -6.691334 & -1.554764 & $\odot .634416$ & H & -2.602971 & -5.645974 & 1.168711 \\
\hline C & -3.398409 & 1.197941 & -4.852765 & H & $-\odot .912900$ & -5.847268 & 1.679449 \\
\hline C & -3.721339 & -5.385897 & 3.674310 & H & -2.150156 & -6.997562 & 2.206492 \\
\hline C & -3.721809 & $-\odot .308580$ & -4.719325 & H & -5.791009 & ๑. 019891 & -5.436301 \\
\hline C & -7.489995 & -2.708969 & -1.441156 & H & -4.625680 & -0.450373 & -6.668615 \\
\hline H & 3.198318 & -2.007164 & 4.559224 & H & -5.258380 & -1.661859 & -5.542031 \\
\hline $\mathrm{H}$ & 2.932556 & -0.124415 & 2.981079 & H & -1.815049 & $-\odot .196409$ & -2.848010 \\
\hline H & 1.538247 & -6.366955 & 3.189703 & H & -3.820206 & -2.045678 & ๑.436987 \\
\hline H & 3.156150 & -6.021156 & 2.448485 & H & -7.878891 & $\odot .030627$ & -3.686875 \\
\hline H & -1.532199 & -2.717300 & -2.558711 & H & -7.215926 & ๑.288871 & -2.046297 \\
\hline H & -0.246137 & -4.420802 & -3.806945 & H & -6.380928 & 0.981741 & -3.457731 \\
\hline H & 4.228583 & -5.204227 & -2.332578 & H & -2.405522 & $-\odot .881241$ & -6.362233 \\
\hline H & 3.261751 & -6.444469 & -1.441566 & H & -1.589521 & -0.921646 & -4.799579 \\
\hline H & 1.617320 & $-\odot .095214$ & -2.704623 & H & -2.736277 & -2.203467 & -5.231603 \\
\hline $\mathrm{H}$ & 4.595632 & -1.022093 & 0.189695 & $\mathrm{H}$ & -5.438384 & -4.436056 & -0.884102 \\
\hline H & 7.219228 & $-\odot .382272$ & $-\odot .17 \odot 6 \odot 1$ & H & -4.938709 & -3.727955 & $\odot .660724$ \\
\hline $\mathrm{H}$ & 8.491007 & -1.528075 & -0.620896 & $\mathrm{H}$ & -6.590524 & -4.309325 & 0.455944 \\
\hline $\mathrm{H}$ & 7.861814 & $-\odot .389131$ & -1.817976 & H & -7.507502 & -2.050943 & 1.173219 \\
\hline H & 7.522965 & -2.594095 & -3.273182 & $\mathrm{H}$ & -5.872919 & -1.388305 & 1.343275 \\
\hline H & 7.911501 & -3.641099 & -1.910233 & H & -7.052839 & $-\odot .572896$ & 0.307213 \\
\hline $\mathrm{H}$ & 6.350856 & -3.791571 & -2.732968 & $\mathrm{H}$ & -4.237136 & 1.806813 & -4.496664 \\
\hline H & 5.378794 & -3.604193 & $-\odot .235954$ & H & -2.510142 & 1.485956 & -4.283029 \\
\hline $\mathrm{H}$ & 7.107425 & -3.516168 & $\odot .165996$ & $\mathrm{H}$ & -3.227378 & 1.451734 & -5.906208 \\
\hline H & 6.000530 & -2.304937 & $\odot .790691$ & H & -4.401635 & -5.078219 & 2.871538 \\
\hline $\mathrm{H}$ & 2.726077 & -2.388564 & -6.556109 & $\mathrm{H}$ & -3.873844 & -6.458021 & 3.848339 \\
\hline H & 4.184387 & -2.609405 & -5.572727 & H & -3.994296 & -4.850065 & 4.585861 \\
\hline H & 2.596056 & -3.056601 & -4.920736 & H & -8.175447 & -3.331920 & $-\odot .854135$ \\
\hline H & 1. 083962 & -0.706380 & -6.080637 & H & -8.036667 & -1.807596 & -1.722141 \\
\hline H & ๑.846912 & -1.259786 & -4.420250 & $\mathrm{H}$ & -7.238326 & -3.252424 & -2.355087 \\
\hline $\mathrm{H}$ & 1.200812 & 0.451277 & -4.755730 & $\mathrm{Rh}$ & -0.141792 & 0.977544 & -0.172679 \\
\hline H & 3.609877 & 1.137573 & -5.510403 & 0 & -2.263013 & 1.925926 & 0.435897 \\
\hline $\mathrm{H}$ & 4.688529 & -0.142494 & -6.105702 & C & -0.049811 & 2.321847 & -1.816752 \\
\hline $\mathrm{H}$ & 3.139240 & 0.139597 & -6.893963 & $\mathrm{~s}$ & -2.885194 & 3.249557 & 0.700290 \\
\hline $\mathrm{H}$ & 7.466455 & $-\odot .495877$ & -3.794060 & 0 & -1.177139 & 2.285040 & -2.285962 \\
\hline H & 7.025066 & $-\odot .694046$ & -5.505944 & C & $-\odot .431254$ & 4.184415 & $\odot .501148$ \\
\hline $\mathrm{H}$ & 6.189909 & 0.527848 & -4.506484 & $\mathrm{~N}$ & -1.829308 & 4. 411991 & 0.049499 \\
\hline H & 4.402167 & 1.698002 & $-\odot .490629$ & 0 & -3.221872 & 3.540142 & 2.102772 \\
\hline $\mathrm{H}$ & 6.117823 & 4.540097 & -1.837568 & C & -4.352012 & 3.473534 & -0.290443 \\
\hline H & 5.073778 & 3.125100 & -1.751615 & H & ๑. 010640 & 5.182378 & $\odot .6 \odot 3136$ \\
\hline $\mathrm{H}$ & 4.461044 & 4.708647 & -1.239652 & H & -0.422810 & 3.743143 & 1.505494 \\
\hline H & 7.528400 & 2.705212 & 1.117655 & C & -2.304720 & 5.808614 & 0.266984 \\
\hline $\mathrm{H}$ & 6.844709 & 1.935367 & -0.319915 & C & -5.557771 & 3.728380 & 0.367746 \\
\hline
\end{tabular}




$\begin{array}{lrrr}\mathrm{C} & -4.273070 & 3.423256 & -1.685235 \\ \mathrm{C} & -1.676151 & 6.737337 & -0.733644 \\ \mathrm{H} & -3.390889 & 5.812035 & 0.128595 \\ \mathrm{H} & -2.103938 & 6.137726 & 1.295712 \\ \mathrm{H} & -5.586916 & 3.762926 & -237.03080 \odot \\ \mathrm{C} & -6.704726 & 3.948624 & 7.57030 \odot \\ \mathrm{C} & -5.430277 & 3.668112 & 13.33310 \odot \\ \mathrm{H} & -3.329156 & 3.202455 & -2.171871 \\ \mathrm{C} & -0.966525 & 7.818396 & -0.406173 \\ \mathrm{H} & -1.854032 & 6.489514 & -1.779393 \\ \mathrm{H} & -7.646772 & 4.146590 & -.111978 \\ \mathrm{C} & -6.657917 & 3.937987 & -1.794107 \\ \mathrm{H} & -5.378844 & 3.655278 & -3.506906 \\ \mathrm{H} & -0.560122 & 8.484791 & -1.161653 \\ \mathrm{H} & -0.781857 & 8.089598 & 0.631405 \\ \mathrm{C} & -7.891727 & 4.241787 & -2.609205 \\ \mathrm{H} & -7.980824 & 5.320892 & -2.790938 \\ \mathrm{H} & -7.860725 & 3.750446 & -3.586895 \\ \mathrm{H} & -8.803038 & 3.924098 & -2.092756 \\ \mathrm{C} & 1.177873 & 2.847212 & -2.554106 \\ \mathrm{H} & 1.030008 & 2.989538 & -3.627732 \\ \mathrm{H} & 2.078023 & 2.260276 & -2.364173 \\ \mathrm{C} & 1.112391 & 4.093671 & -1.647912 \\ \mathrm{H} & 0.430532 & 4.839912 & -2.064911 \\ \mathrm{H} & 2.075350 & 4.561270 & -1.420128 \\ \mathrm{C} & 0.464386 & 3.381767 & -0.430309 \\ \mathrm{H} & 1.481879 & 1.700851 & 2.616924 \\ \mathrm{H} & 1.281770 & 2.982677 & 0.168466 \\ & & & \end{array}$

TS1_9

B3LYP SCF energy: $\quad-5535.32159819$ a.u. B3LYP enthalpy:

B3LYP free energy:

-5535.32159819 a.u.
-5533.287778 a.u. -5533.287778 a.u.
-5533.557368 a.u. M06 SCF energy in solution: $\quad-5534.55622763$ a.u.

M06 enthalpy in solution: $\quad-5532.522407 \mathrm{a} . \mathrm{u}$. M06 free energy in solution: $\quad-5532.791997$ a.u.

Imaginary frequency:

$-269.9207 \mathrm{~cm}-1$

\begin{tabular}{|c|c|c|c|}
\hline ATOM & $\begin{array}{c}\text { coorc } \\
x\end{array}$ & $\mathrm{Y}$ & Z \\
\hline $\mathrm{P}$ & $\odot .0 \odot \odot \odot \odot \odot$ & -5.481478 & 4.111820 \\
\hline$P$ & $\odot .0 \odot \odot \odot \odot \odot$ & -6.523569 & 3.820224 \\
\hline 0 & $\odot .0 \odot \odot \odot \odot \odot$ & -5.167691 & 3.046586 \\
\hline 0 & $\odot .0 \odot \odot \odot \odot \odot$ & -3.072073 & 2.710926 \\
\hline 0 & $\odot .0 \odot \odot \odot \odot \odot$ & $-\odot .846368$ & 7.797188 \\
\hline 0 & $\odot . ๑ \odot \odot \odot \odot \odot$ & -1.584601 & 6.233526 \\
\hline 0 & $\odot .0 \odot \odot \odot \odot \odot$ & -7.484920 & 4.131371 \\
\hline 0 & $\odot . \odot \odot \odot \odot \odot \odot$ & -6.423227 & 3.440604 \\
\hline 0 & $\odot .0 \odot \odot \odot \odot \odot$ & -5.075712 & 2.752207 \\
\hline 0 & $\odot . ๑ \odot \odot \odot \odot \odot$ & -0.387255 & 8.345091 \\
\hline C & $\odot .0 \odot \odot \odot \odot \odot$ & $-\odot .758790$ & 8.238955 \\
\hline C & $\odot . ๑ \odot \odot \odot \odot \odot$ & -7.633177 & 3.439333 \\
\hline C & 1.508281 & -7.428235 & 3.963672 \\
\hline C & 2.060585 & -7.924435 & 2.413771 \\
\hline C & 2.607233 & -8.492134 & 3.919803 \\
\hline C & 2.579265 & 1.554366 & 2.708849 \\
\hline C & 1.548063 & 1.517882 & 2.819119 \\
\hline C & -0.232598 & 2.381419 & 2.058365 \\
\hline C & $-\odot .696306$ & 1.518304 & 3.993004 \\
\hline C & $-\odot .075757$ & 0.982158 & 4.789831 \\
\hline C & 1.029416 & 2.493819 & 4.363470 \\
\hline C & 1.500963 & 0.646121 & 3.425522 \\
\hline C & 0.912765 & 2.000022 & 2.166518 \\
\hline C & 2.632282 & 1.332879 & 3.029871 \\
\hline c & 2.920729 & $-\odot .797279$ & -1.052965 \\
\hline C & 2.484505 & $-\odot .816569$ & -2.375486 \\
\hline C & 3.165863 & -1.525791 & -3.382434 \\
\hline C & 4.283087 & -2.296808 & -2.971699 \\
\hline C & 4.830119 & -2.202144 & -1.659311 \\
\hline C & 4.107697 & -1.464529 & -0.720346 \\
\hline C & 6.193044 & -2.831616 & -1.267549 \\
\hline
\end{tabular}

\begin{tabular}{|c|c|c|}
\hline 7.299028 & -2.338129 & -2.233332 \\
\hline 6.135165 & -4.376759 & -1.277838 \\
\hline 6.619599 & -2.419337 & 0.15959 \\
\hline 2.658431 & -1.391812 & -4.84700 \\
\hline 1.541556 & -2.426164 & -5.126228 \\
\hline 2.044720 & 0.015125 & -5.073120 \\
\hline 3.786634 & -1.515142 & -5.900166 \\
\hline 4.335378 & -4.391153 & -4.155299 \\
\hline 3.239590 & 1.458467 & 0.762138 \\
\hline 4.385113 & 1.729428 & 0.015513 \\
\hline 2.940011 & 2.304769 & 1.845217 \\
\hline 3.816283 & 3.303523 & 2.276305 \\
\hline 5.041691 & 3.444351 & 1.562552 \\
\hline 5.284901 & 2.759189 & ๑.349866 \\
\hline 6.424005 & 3.059300 & -0.667653 \\
\hline 5.810413 & 8709 & -2.094988 \\
\hline 7.479257 & 1.928101 & -0.635808 \\
\hline 7. 080257 & $07 \odot$ & 2.738062 \\
\hline 7.1299 & 4.4 & -0.478838 \\
\hline 3.412713 & 3798 & 3.2 \\
\hline 4.354873 & 9267 & 2439 \\
\hline 3.42 & & \\
\hline 1.984710 & & \\
\hline-1.73 & -1.3 & 2315 \\
\hline-2.14 & -2.6 & \\
\hline-2.74 & -2.5 & 3.2 \\
\hline-2.95 & $-1 . \varepsilon$ & 4. \\
\hline-2.40 & -0.5 & \\
\hline-1.84 & -0.3 & 4935 \\
\hline-2.31 & & \\
\hline-2.4439 & 0.1 & \\
\hline-3.372306 & 1.7 & 4879 \\
\hline-0.91 & 1.2 & \\
\hline-2.37 & -4.7 & \\
\hline-5.03 & -1.6 & \\
\hline-3.0 & -4.3 & \\
\hline-2.60 & -5.3 & \\
\hline-4.9 & $-\odot . \subseteq$ & -2. \\
\hline-3.64 & -0.7 & -3. \\
\hline-4.66 & -0.2 & -5. \\
\hline-2.52 & -0.6 & -2 \\
\hline-2.62 & -0.8 & -1. \\
\hline-3.88 & $-1 . c$ & $-\odot$. \\
\hline-5.05 & -1.0 & -1.2 \\
\hline-6.26 & -2.5 & -3.7 \\
\hline-2.6 & -1.7 & -5 \\
\hline-6.53 & -2.5 & 0. \\
\hline-6.46 & -1.1 & $-0.2=$ \\
\hline-6.35 & -0.1 & 0. \\
\hline-2.484522 & 0.7 & -4.5 \\
\hline-4.616279 & -4.6 & \\
\hline-3.46 & -0.5 & -4.7 \\
\hline-7.6778 & -0.8 & -1.2 \\
\hline 3.0525 & -1.2 & \\
\hline 3.04 & 0.2 & \\
\hline 0.76 & -5.5 & \\
\hline 2.41 & -5.6 & 3.7 \\
\hline-1.583794 & -2.8 & -2.1 \\
\hline$-\odot .456797$ & -4.9 & \\
\hline 3.6 & -6.2 & -0.7 \\
\hline 2.193753 & -6.7 & 0.236195 \\
\hline 1.58 & -0.2 & -2.6 \\
\hline 4.46 & -1.4 & 0.2 \\
\hline 7.39 & -1.24 & -2.1 \\
\hline 8.26 & & \\
\hline & -2.619460 & -3.26627 \\
\hline & & \\
\hline & & -0.90402 \\
\hline 5.334380 & -4.731294 & $-\odot .619153$ \\
\hline & & \\
\hline 7.61 & -2.8 & 0.366839 \\
\hline 6.690917 & $-1.33<0$ & 0.275010 \\
\hline & & \\
\hline & -3.457489 & -5.026982 \\
\hline$\odot .708073$ & -2.292707 & -4.427558 \\
\hline
\end{tabular}




\begin{tabular}{|c|c|c|c|}
\hline $\mathrm{H}$ & 1.788638 & $\odot .127694$ & -6.131887 \\
\hline $\mathrm{H}$ & 1.122965 & ๑. 182311 & -4.507970 \\
\hline $\mathrm{H}$ & 2.756065 & 0.807427 & -4.814708 \\
\hline $\mathrm{H}$ & 4.596269 & -0.807561 & -5.689611 \\
\hline $\mathrm{H}$ & 4.227802 & -2.507572 & -5.960784 \\
\hline $\mathrm{H}$ & 3.377748 & -1.270120 & -6.886938 \\
\hline $\mathrm{H}$ & 3.737975 & -4.333636 & -5.070480 \\
\hline $\mathrm{H}$ & 5.149921 & -5.101636 & -4.319824 \\
\hline $\mathrm{H}$ & 3.703622 & -4.743105 & -3.333076 \\
\hline $\mathrm{H}$ & 4.589979 & 1.128277 & -0.860187 \\
\hline $\mathrm{H}$ & 6.594619 & 3.354204 & -2.819264 \\
\hline $\mathrm{H}$ & 5.370747 & 2.158304 & -2.410266 \\
\hline $\mathrm{H}$ & 5.037100 & 3.882619 & -2.164054 \\
\hline $\mathrm{H}$ & 7.965021 & 1.847630 & ๑. 342536 \\
\hline $\mathrm{H}$ & 7.030353 & $\odot .955130$ & -0.865133 \\
\hline $\mathrm{H}$ & 8.260752 & 2.119710 & -1.380561 \\
\hline $\mathrm{H}$ & 6.739123 & 3.067833 & 3.561415 \\
\hline $\mathrm{H}$ & 7.683638 & 3.104192 & 2.050851 \\
\hline $\mathrm{H}$ & 7.689385 & 4.519924 & 3.135030 \\
\hline $\mathrm{H}$ & 7.776412 & 4.462850 & ๑. 397316 \\
\hline $\mathrm{H}$ & 7.763087 & 4.606153 & -1.354454 \\
\hline $\mathrm{H}$ & 6.412058 & 5.244829 & -0.405972 \\
\hline $\mathrm{H}$ & 4. 459052 & 3.096705 & 4.976154 \\
\hline $\mathrm{H}$ & 5.345246 & 4.544125 & 4.446355 \\
\hline $\mathrm{H}$ & 3.937525 & 4.705933 & 5.492878 \\
\hline $\mathrm{H}$ & 3.134196 & 6.403223 & 3.714569 \\
\hline $\mathrm{H}$ & 4.416833 & 6.008834 & 2.555353 \\
\hline $\mathrm{H}$ & 2.711699 & 5.845799 & 2.087104 \\
\hline $\mathrm{H}$ & 1.231412 & 4.058065 & 3.148690 \\
\hline $\mathrm{H}$ & 1.894515 & 2.969086 & 4.387019 \\
\hline $\mathrm{H}$ & 1.728006 & 4.693911 & 4.715858 \\
\hline $\mathrm{H}$ & -1.990289 & -3.425792 & 1.351666 \\
\hline $\mathrm{H}$ & -1.493990 & ๑. 670537 & 2.428170 \\
\hline $\mathrm{H}$ & -3.451390 & -0.142424 & 6.744490 \\
\hline $\mathrm{H}$ & -1.765889 & $-\odot .662758$ & 6.697545 \\
\hline $\mathrm{H}$ & -2.174138 & 1.009687 & 7.112010 \\
\hline $\mathrm{H}$ & -3.280495 & 2.526329 & 5.403666 \\
\hline $\mathrm{H}$ & -3.245018 & 2.121782 & 3.681540 \\
\hline $\mathrm{H}$ & -4.393596 & 1.314860 & 4.758104 \\
\hline $\mathrm{H}$ & $-\odot .808649$ & 2.036116 & 5.664391 \\
\hline $\mathrm{H}$ & -0.114387 & $\odot .533822$ & 5.023422 \\
\hline $\mathrm{H}$ & -0.749238 & 1.775582 & 3.926227 \\
\hline $\mathrm{H}$ & -2.561263 & -5.797876 & 5.246237 \\
\hline $\mathrm{H}$ & -1.290389 & -4.608944 & 4.893812 \\
\hline $\mathrm{H}$ & -2.716202 & -4.128331 & 5.826727 \\
\hline $\mathrm{H}$ & -5.077856 & -0.516157 & 5.383202 \\
\hline $\mathrm{H}$ & -5.559722 & -1.934516 & 4.416312 \\
\hline $\mathrm{H}$ & -5.515962 & -2.037739 & 6.199550 \\
\hline $\mathrm{H}$ & -3.130679 & -5.236556 & 1.640254 \\
\hline $\mathrm{H}$ & -1.530608 & -5.289935 & 2.414328 \\
\hline $\mathrm{H}$ & -2.813449 & -6.393342 & 2.932195 \\
\hline $\mathrm{H}$ & -5.284806 & ๑.533823 & -5.215738 \\
\hline $\mathrm{H}$ & -4.331115 & $\odot .0396 \odot 2$ & -6.624614 \\
\hline $\mathrm{H}$ & -5.287203 & -1.149113 & -5.746756 \\
\hline $\mathrm{H}$ & -1.554651 & -0.459333 & -2.819171 \\
\hline $\mathrm{H}$ & -3.968265 & -1.151410 & ๑. 627316 \\
\hline $\mathrm{H}$ & -7.160233 & -2.533583 & -4.392848 \\
\hline $\mathrm{H}$ & -5.430575 & -2.973062 & -4.335287 \\
\hline $\mathrm{H}$ & -6.443264 & -3.152075 & -2.878860 \\
\hline $\mathrm{H}$ & -2.493089 & -1.669310 & -6.382377 \\
\hline $\mathrm{H}$ & -1.724264 & -1.945849 & -4.811356 \\
\hline $\mathrm{H}$ & -3.295674 & -2.680170 & -5.170072 \\
\hline $\mathrm{H}$ & -6.573263 & -3.339698 & -0.616889 \\
\hline $\mathrm{H}$ & -5.697217 & -2.809108 & 0.826378 \\
\hline $\mathrm{H}$ & -7.461453 & -2.649257 & $\odot .750034$ \\
\hline $\mathrm{H}$ & -7.339347 & $-\odot .181929$ & 1.272778 \\
\hline $\mathrm{H}$ & -5.589493 & $-\odot .293207$ & 1.447191 \\
\hline $\mathrm{H}$ & -6.287685 & ๑.899176 & 0.341641 \\
\hline $\mathrm{H}$ & -2.975683 & 1.632512 & -4.675589 \\
\hline $\mathrm{H}$ & -1.528536 & ๑. 654538 & -4.471506 \\
\hline $\mathrm{H}$ & -2.269664 & 0.797250 & -6.066303 \\
\hline $\mathrm{H}$ & -5.168281 & -4.268697 & 2.955590 \\
\hline $\mathrm{H}$ & -4.816269 & -5.678210 & 3.964824 \\
\hline $\mathrm{H}$ & -5.014776 & -4.097671 & 4.717829 \\
\hline $\mathrm{H}$ & -8.523428 & -0.769371 & -0.562163 \\
\hline
\end{tabular}

\begin{tabular}{|c|c|c|c|}
\hline $\mathrm{H}$ & -7.596486 & 0.114101 & -1.786131 \\
\hline $\mathrm{H}$ & -7.922700 & -1.611138 & -1.986774 \\
\hline $\mathrm{Rh}$ & $-\odot .012120$ & 1.090324 & -0.115189 \\
\hline 0 & -2.094634 & 2.070500 & ๑. 326431 \\
\hline C & ๑. 224056 & 2.303372 & -1.862608 \\
\hline S & -2.761415 & 3.400008 & ๑. 376023 \\
\hline 0 & -0.844641 & 2.269896 & -2.446722 \\
\hline C & -0.324251 & 4.364713 & ๑. 220829 \\
\hline $\mathrm{N}$ & -1.687247 & 4.483224 & -0.365359 \\
\hline 0 & -3.181659 & 3.865750 & 1.706858 \\
\hline C & -4.176769 & 3. 414161 & -0.704918 \\
\hline $\mathrm{H}$ & ๑. . 998757 & 5.374193 & ๑.178084 \\
\hline $\mathrm{H}$ & -0.394883 & 4.106845 & 1.285416 \\
\hline C & -2.201465 & 5.880721 & -0.412661 \\
\hline C & -5.407344 & 3.812160 & -0.178024 \\
\hline C & -4.039480 & 3.021662 & -2.040427 \\
\hline C & $-1.5 \odot 4773$ & 6.654996 & -1.496456 \\
\hline $\mathrm{H}$ & -3.271892 & 5.828426 & $-\odot .638538$ \\
\hline $\mathrm{H}$ & -2.094094 & 6.376234 & 0.562186 \\
\hline $\mathrm{H}$ & -5.481478 & 4.111820 & -269.920700 \\
\hline C & -6.523569 & 3.820224 & 10.992300 \\
\hline C & -5.167691 & $3 . \odot 46586$ & 13.875200 \\
\hline $\mathrm{H}$ & -3.072073 & 2.710926 & -2.419839 \\
\hline C & -0.846368 & 7.797188 & -1.292429 \\
\hline $\mathrm{H}$ & -1.584601 & 6.233526 & -2.497678 \\
\hline $\mathrm{H}$ & -7.484920 & 4.131371 & -0.614999 \\
\hline C & -6.423227 & 3.440604 & -2.360083 \\
\hline $\mathrm{H}$ & -5.075712 & 2.752207 & -3.896336 \\
\hline $\mathrm{H}$ & $-\odot .387255$ & 8.345091 & -2.110360 \\
\hline $\mathrm{H}$ & -0.758790 & 8.238955 & -0.301749 \\
\hline C & -7.633177 & 3.439333 & -3.262327 \\
\hline $\mathrm{H}$ & -7.428235 & 3.963672 & -4.202612 \\
\hline $\mathrm{H}$ & -7.924435 & 2.413771 & -3.522318 \\
\hline $\mathrm{H}$ & -8.492134 & 3. 919803 & -2.785237 \\
\hline C & 1.554366 & 2.708849 & -2.491475 \\
\hline $\mathrm{H}$ & 1.517882 & 2.819119 & -3.577983 \\
\hline $\mathrm{H}$ & 2.381419 & 2.058365 & -2.203601 \\
\hline C & 1.518304 & 3. 993004 & -1.640603 \\
\hline $\mathrm{H}$ & ๑. 982158 & 4.789831 & -2.164170 \\
\hline $\mathrm{H}$ & 2.493819 & 4.363470 & -1.310359 \\
\hline C & ๑. 646121 & 3.425522 & -0.487602 \\
\hline $\mathrm{H}$ & $2.0 \odot \odot \odot 22$ & 2.166518 & 2.365413 \\
\hline $\mathrm{H}$ & 1.332879 & 3. 029871 & 0.262268 \\
\hline
\end{tabular}

TS1_10

B3LYP SCF energy: $\quad-5535.31776116$ a.u. B3LYP enthalpy: $\quad-5533.284162$ a.u. B3LYP free energy: $\quad-5533.554342$ a.u. M06 SCF energy in solution: -5534.55499700 a.u.

M06 enthalpy in solution: $-5532.521398 \mathrm{a} . \mathrm{u}$. M06 free energy in solution: $\quad-5532.791578$ a.u.

Imaginary frequency: $\quad-273.5489 \mathrm{~cm}-1$

Cartesian coordinates

\begin{tabular}{|c|c|c|c|}
\hline АТOM & $x$ & $Y$ & Z \\
\hline $\mathrm{P}$ & $\odot . ๑ \odot \odot \odot \odot \odot$ & -5.493539 & 4.045239 \\
\hline$P$ & $\odot . \odot \odot \odot \odot \odot \odot$ & -6.502319 & 3.773611 \\
\hline 0 & $\odot . ๑ \odot \odot \odot \odot \odot$ & -5.109858 & 3.039828 \\
\hline 0 & $\odot . \odot \odot \odot \odot \odot \odot$ & -3.019101 & 2.713300 \\
\hline 0 & $\odot . ๑ \odot \odot \odot \odot \odot$ & -0.849528 & 7.787632 \\
\hline 0 & $\odot . ๑ \odot \odot \odot \odot \odot$ & -1.538870 & 6.218337 \\
\hline 0 & $\odot . ๑ \odot \odot \odot \odot \odot$ & -7.472616 & 4.071004 \\
\hline 0 & $\odot . \odot \odot \odot \odot \odot \odot$ & -6.376633 & 3.415865 \\
\hline 0 & $\odot . ๑ \odot \odot \odot \odot \odot$ & -4.998326 & 2.763058 \\
\hline 0 & $\odot . ๑ \odot \odot \odot \odot \odot$ & $-\odot .378805$ & 8.344093 \\
\hline C & $\odot . ๑ \odot \odot \odot \odot \odot$ & $-\odot .792270$ & 8.227924 \\
\hline C & $\odot . ๑ \odot \odot \odot \odot \odot$ & -7.571434 & 3.418613 \\
\hline C & 1.514129 & -7.354784 & 3.957133 \\
\hline C & 2.075613 & -7.850835 & 2.394624 \\
\hline C & 2.642287 & -8.441623 & 3.886441 \\
\hline C & 2.616911 & 1.619473 & 2.724563 \\
\hline
\end{tabular}




\begin{tabular}{|c|c|c|c|c|c|c|c|}
\hline C & 1.539887 & 1.605335 & 2.854146 & $\mathrm{H}$ & 2.217319 & -6.804802 & 0.162551 \\
\hline C & -0.204296 & 2.441080 & 2.069186 & $\mathrm{H}$ & 1.653287 & $-\odot .234243$ & -2.598385 \\
\hline C & $-\odot .656997$ & 1.565882 & 3.993820 & $\mathrm{H}$ & 4.502475 & -1.407400 & 0.343412 \\
\hline C & $-\odot .030738$ & 1.046515 & 4.801742 & $\mathrm{H}$ & 7.476020 & -1.249608 & $-2.09 \odot 495$ \\
\hline C & 1.068679 & 2.535499 & 4.353048 & $\mathrm{H}$ & 8.317006 & -2.789469 & -1.859733 \\
\hline C & 1.528996 & $\odot .66354 \odot$ & 3.414186 & $\mathrm{H}$ & 7.166702 & -2.600967 & -3.197216 \\
\hline C & 0.934712 & 2.007525 & 2.023180 & $\mathrm{H}$ & 6.013933 & -4.764337 & -2.256940 \\
\hline C & 2.662457 & 1.327335 & 3.012640 & $\mathrm{H}$ & 7.097774 & -4.799778 & $-\odot .867265$ \\
\hline C & 2.972926 & $-\odot .778989$ & -1.018409 & $\mathrm{H}$ & 5.347135 & -4.730928 & -0.607597 \\
\hline C & 2.551275 & $-\odot .783134$ & -2.345901 & $\mathrm{H}$ & 5.947101 & -2.836366 & 0.974544 \\
\hline C & 3.243696 & -1.480260 & -3.353490 & $\mathrm{H}$ & 7.627604 & -2.878629 & $0.44 \odot 459$ \\
\hline C & 4.354228 & -2.259309 & -2.939323 & $\mathrm{H}$ & 6.728400 & -1.362816 & 0.357694 \\
\hline C & 4.885363 & -2.183511 & -1.619018 & $\mathrm{H}$ & 1.269540 & -2.217527 & -6.152272 \\
\hline C & 4.155047 & -1.452688 & $-\odot .680274$ & $\mathrm{H}$ & 1.990551 & -3.390891 & -5.042397 \\
\hline C & 6.236300 & -2.832124 & -1.215693 & $\mathrm{H}$ & $\odot .800366$ & -2.237020 & -4.440981 \\
\hline C & 7.362920 & -2.338058 & -2.157019 & $\mathrm{H}$ & 1.897538 & $\odot .209277$ & -6.096667 \\
\hline C & 6.161385 & -4.376303 & -1.249376 & $\mathrm{H}$ & 1.209083 & $\odot .238313$ & -4.481596 \\
\hline C & 6.644771 & -2.446718 & 0.224233 & $\mathrm{H}$ & 2.844553 & 0.872387 & -4.756449 \\
\hline C & 2.753933 & -1.326376 & -4.822178 & $\mathrm{H}$ & 4.698623 & $-\odot .720727$ & -5.632284 \\
\hline C & 1.643001 & -2.358855 & -5.130814 & $\mathrm{H}$ & 4.344988 & -2.419562 & -5.927910 \\
\hline C & 2.139173 & ๑.๑81994 & -5.036164 & $\mathrm{H}$ & 3.499127 & -1.176786 & -6.851159 \\
\hline C & 3.896413 & -1.430863 & -5.861937 & $\mathrm{H}$ & 3.842603 & -4.262175 & -5.086883 \\
\hline C & 4.415411 & -4.335475 & -4.157247 & $\mathrm{H}$ & 5.229249 & -5.049418 & -4.309969 \\
\hline C & 3.223445 & 1.460608 & 0.847419 & $\mathrm{H}$ & 3.760478 & -4.693894 & -3.356256 \\
\hline C & 4.388093 & 1.760619 & 0.138689 & $\mathrm{H}$ & 4.655204 & 1.142009 & $-\odot .707676$ \\
\hline C & 2.908418 & 2.249730 & 1.963587 & $\mathrm{H}$ & 6.988029 & 3.572124 & -2.446273 \\
\hline C & 3.707760 & 3.320354 & 2.384473 & $\mathrm{H}$ & 5.430081 & 2.745252 & -2.292345 \\
\hline C & 4.813449 & 3.658071 & 1.557793 & $\mathrm{H}$ & 5.586636 & 4.440657 & -1.801380 \\
\hline C & 5.220267 & 2.842766 & 0.472823 & $\mathrm{H}$ & 7.535434 & 1.313815 & $\odot .518229$ \\
\hline C & 6.504314 & 3. 028972 & $-\odot .386920$ & $\mathrm{H}$ & 6.644933 & $\odot .884049$ & -0.952507 \\
\hline C & 6.097856 & 3.470823 & -1.814318 & $\mathrm{H}$ & 8.154543 & 1.783141 & -1.073507 \\
\hline C & 7.242812 & 1.666501 & -0.477345 & $\mathrm{H}$ & 5.073213 & 5.877147 & 0.095992 \\
\hline C & 5.032840 & 5.975798 & 1.188430 & $\mathrm{H}$ & 3.999805 & 6.197059 & 1.486327 \\
\hline C & 7.547046 & 4.033784 & 0.154469 & $\mathrm{H}$ & 5.682456 & 6.796206 & 1.500920 \\
\hline C & 3.313498 & 4. 011465 & 3.722999 & $\mathrm{H}$ & 7.814274 & 3.831028 & 1.194595 \\
\hline C & 4.345183 & 5.005833 & 4.303518 & $\mathrm{H}$ & 8.455248 & 3.940292 & -0.452410 \\
\hline C & 1.969113 & 4.757541 & 3.546348 & $\mathrm{H}$ & 7.219984 & 5.071888 & ๑. 085037 \\
\hline C & 3.135672 & 2.911854 & 4.805472 & $\mathrm{H}$ & 5.343613 & 4.568133 & 4.378067 \\
\hline C & -1.727001 & -1.365408 & 1.702379 & $\mathrm{H}$ & 4.422625 & 5.929150 & 3.728103 \\
\hline c & -2.138045 & -2.664976 & 2. 028656 & $\mathrm{H}$ & 4.019781 & 5.280714 & 5.313707 \\
\hline C & -2.744188 & -2.956498 & 3.253348 & $\mathrm{H}$ & 1.671135 & 5.227738 & 4.490762 \\
\hline C & -2.945637 & -1.872358 & 4.154631 & $\mathrm{H}$ & 2.047843 & 5.548212 & 2.790835 \\
\hline C & -2.385959 & -0.590069 & 3.933597 & $\mathrm{H}$ & 1.161519 & 4. 080401 & 3.245723 \\
\hline C & -1.830730 & $-\odot .360318$ & 2.662797 & $\mathrm{H}$ & 2.349295 & 2.191143 & 4.562199 \\
\hline C & -2.292383 & 0.560976 & 4.976474 & $\mathrm{H}$ & 4.070505 & 2.359798 & 4.956554 \\
\hline C & -2.416042 & 0.099448 & 6.448280 & $\mathrm{H}$ & 2.863358 & 3.375997 & 5.759750 \\
\hline C & -3.349658 & 1.651927 & 4.680800 & $\mathrm{H}$ & -1.987395 & -3.454933 & 1.305220 \\
\hline C & -0.888278 & 1.219210 & 4.868289 & $\mathrm{H}$ & -1.471582 & $\odot .629984$ & 2.413225 \\
\hline C & -2.375155 & -4.803396 & 4.937478 & $\mathrm{H}$ & -3.422449 & -0.210724 & 6.726180 \\
\hline C & -5.017987 & -1.654710 & 5.293154 & $\mathrm{H}$ & -1.737133 & $-\odot .730329$ & 6.669288 \\
\hline C & -3.097187 & -4.421797 & 3.620910 & $\mathrm{H}$ & -2.144105 & 0.938666 & 7.098832 \\
\hline C & -2.617712 & -5.406949 & 2.531344 & $\mathrm{H}$ & -3.253212 & 2.470750 & 5.404293 \\
\hline C & -4.847586 & -0.990413 & -2.683995 & $\mathrm{H}$ & -3.227057 & 2.076505 & 3.679207 \\
\hline C & -3.588845 & $-\odot .701981$ & -3.278159 & $\mathrm{H}$ & -4.371108 & 1.265071 & 4.756563 \\
\hline C & -4.574287 & $-\odot .236256$ & -5.676612 & $\mathrm{H}$ & -0.781579 & 1.973837 & 5.655181 \\
\hline C & -2.480501 & $-\odot .647781$ & -2.420425 & $\mathrm{H}$ & $-\odot .091642$ & $\odot .477289$ & 4.996737 \\
\hline c & -2.589222 & -0.886050 & -1.044679 & $\mathrm{H}$ & $-0.7312 \odot 4$ & 1.729967 & 3.913643 \\
\hline C & -3.860346 & -1.054084 & -0.495412 & $\mathrm{H}$ & -2.569186 & -5.855428 & 5.179505 \\
\hline C & -5.021792 & -1.066537 & -1.279630 & $\mathrm{H}$ & -1.291836 & -4.671237 & 4.834581 \\
\hline C & -6.191627 & -2.532408 & -3.863473 & $\mathrm{H}$ & -2.713067 & -4.189947 & 5.774148 \\
\hline C & -2.600688 & -1.749216 & -5.350920 & $\mathrm{H}$ & -5.059381 & $-\odot .564323$ & 5.372016 \\
\hline C & -6.508942 & -2.615015 & $\odot .062687$ & $\mathrm{H}$ & -5.547148 & -1.967363 & 4.385856 \\
\hline C & -6.374538 & -1.186624 & $-\odot .519722$ & $\mathrm{H}$ & -5.502676 & -2.095318 & 6.167448 \\
\hline C & -6.380314 & -0.171961 & $\odot .656401$ & $\mathrm{H}$ & -3.141370 & -5.260523 & 1.579166 \\
\hline C & -2.407112 & 0.734951 & -5.014892 & $\mathrm{H}$ & -1.540422 & -5.329942 & 2. 350141 \\
\hline C & -4.619717 & -4.641499 & 3.787282 & $\mathrm{H}$ & -2.828945 & -6.430039 & 2.860749 \\
\hline c & -3.325611 & -0.495828 & -4.800499 & $\mathrm{H}$ & -5.205551 & $\odot .557411$ & -5.267946 \\
\hline C & -7.640244 & -0.868827 & -1.348678 & $\mathrm{H}$ & -4.234257 & $\odot .078967$ & -6.670315 \\
\hline H & 3.100951 & -1.399195 & 4.956425 & $\mathrm{H}$ & -5.197082 & -1.120380 & -5.814583 \\
\hline $\mathrm{H}$ & 3.098484 & 0.168685 & 3.053232 & $\mathrm{H}$ & -1.501848 & -0.442736 & -2.836593 \\
\hline H & 0.750113 & -5.610069 & 4.413433 & $\mathrm{H}$ & -3.950765 & -1.187716 & $\odot .573503$ \\
\hline H & 2.403343 & -5.713466 & 3.680234 & $\mathrm{H}$ & -7.082057 & -2.524815 & -4.496240 \\
\hline H & -1.540295 & -2.859017 & -2.159235 & $\mathrm{H}$ & -5.351647 & -2.959179 & -4.425689 \\
\hline $\mathrm{H}$ & -0.403451 & -4.897074 & -2.986898 & $\mathrm{H}$ & -6.378471 & -3.157179 & -2.981744 \\
\hline \multirow[t]{2}{*}{$\mathrm{H}$} & 3.688056 & -6.274988 & $-\odot .753290$ & $\mathrm{H}$ & -2.395679 & -1.629653 & -6.421643 \\
\hline & & & & & & & \\
\hline
\end{tabular}




\begin{tabular}{|c|c|c|c|}
\hline $\mathrm{H}$ & -1.643680 & -1.917185 & -4.844444 \\
\hline $\mathrm{H}$ & -3.209212 & -2.652283 & -5.226682 \\
\hline $\mathrm{H}$ & -6.532424 & -3.373091 & $-\odot .728267$ \\
\hline $\mathrm{H}$ & -5.675215 & -2.857387 & ๑. 731582 \\
\hline $\mathrm{H}$ & -7.439059 & -2.703768 & $\odot .637004$ \\
\hline $\mathrm{H}$ & -7.333691 & -0.242721 & 1.192480 \\
\hline $\mathrm{H}$ & -5.585709 & -0.350188 & 1.386330 \\
\hline $\mathrm{H}$ & -6.275010 & $\odot .854116$ & ๑. 288033 \\
\hline $\mathrm{H}$ & -2.903349 & 1.656842 & -4.693747 \\
\hline $\mathrm{H}$ & -1.456966 & $\odot .679223$ & -4.480751 \\
\hline $\mathrm{H}$ & -2.180019 & $\odot .833655$ & -6.082500 \\
\hline $\mathrm{H}$ & -5.171236 & -4.291585 & 2.906292 \\
\hline $\mathrm{H}$ & -4.825634 & -5.712399 & 3. 901791 \\
\hline $\mathrm{H}$ & -5.013934 & -4.137921 & 4.669794 \\
\hline $\mathrm{H}$ & -8.493982 & $-\odot .811029$ & -0.663037 \\
\hline $\mathrm{H}$ & -7.556636 & ๑ . 091811 & -1.864735 \\
\hline $\mathrm{H}$ & -7.873691 & -1.631913 & -2.091410 \\
\hline $\mathrm{Rh}$ & ๑ . 010732 & 1.072407 & $-\odot .095674$ \\
\hline 0 & -2.085183 & 2.038164 & ๑. 315868 \\
\hline C & $\odot .276340$ & 2.305001 & -1.822942 \\
\hline S & -2.760249 & 3.363418 & ๑. 365791 \\
\hline 0 & $-\odot .778287$ & 2.276239 & -2.431911 \\
\hline C & -0.329124 & 4.345605 & 0.267244 \\
\hline $\mathrm{N}$ & -1.679652 & 4.458116 & -0.349076 \\
\hline 0 & -3.204157 & 3.815491 & 1.693694 \\
\hline C & -4.157759 & 3.380991 & -0.737786 \\
\hline $\mathrm{H}$ & ๑. 087931 & 5.357905 & ๑. 237317 \\
\hline $\mathrm{H}$ & -0.422382 & 4.085184 & 1.328947 \\
\hline C & -2.202133 & 5.852114 & $-\odot .398347$ \\
\hline C & -5.399997 & 3.761489 & $-\odot .225482$ \\
\hline C & -3.995145 & 3. 010582 & -2.076733 \\
\hline C & -1.488306 & 6.637788 & -1.462677 \\
\hline $\mathrm{H}$ & -3.267135 & 5.794481 & -0.647387 \\
\hline $\mathrm{H}$ & -2.118979 & 6.342608 & 0.581464 \\
\hline $\mathrm{H}$ & -5.493539 & $4 . \odot 45239$ & -273.548900 \\
\hline C & -6.502319 & 3.773611 & 11.549800 \\
\hline C & -5.109858 & 3. 039828 & 12.941300 \\
\hline $\mathrm{H}$ & - 3.019101 & 2.713300 & -2.444745 \\
\hline C & -0.849528 & 7.787632 & $-1.2400 \odot 2$ \\
\hline $\mathrm{H}$ & -1.538870 & 6.218337 & -2.466646 \\
\hline $\mathrm{H}$ & -7.472616 & 4.071004 & -0.691944 \\
\hline C & -6.376633 & 3.415865 & -2.429555 \\
\hline $\mathrm{H}$ & -4.998326 & 2.763058 & -3.953760 \\
\hline $\mathrm{H}$ & -0.378805 & 8.344093 & -2.045500 \\
\hline $\mathrm{H}$ & -0.792270 & 8.227924 & -0.246412 \\
\hline C & -7.571434 & 3. 418613 & -3.351723 \\
\hline $\mathrm{H}$ & -7.354784 & 3.957133 & -4.281297 \\
\hline $\mathrm{H}$ & -7.850835 & 2.394624 & -3.630268 \\
\hline $\mathrm{H}$ & -8.441623 & 3.886441 & -2.882494 \\
\hline C & 1.619473 & 2.724563 & -2.414987 \\
\hline $\mathrm{H}$ & 1.605335 & 2.854146 & -3.499928 \\
\hline H & 2.441080 & 2.069186 & -2.122479 \\
\hline C & 1.565882 & 3.993820 & -1.543435 \\
\hline $\mathrm{H}$ & 1.046515 & 4.801742 & -2.067151 \\
\hline $\mathrm{H}$ & 2.535499 & 4.353048 & -1.184792 \\
\hline C & $\odot .663540$ & 3.414186 & -0.420448 \\
\hline $\mathrm{H}$ & 2.007525 & 2.023180 & 2.524598 \\
\hline $\mathrm{H}$ & 1.327335 & 3.012640 & 0.347073 \\
\hline
\end{tabular}

TS1_11

B3LYP SCF energy: $\quad-5535.31992902$ a.u. B3LYP enthalpy:

B3LYP free energy:

-5533.286121 a.u. -5533.286121 a.u.
-5533.555983 a.u.

M०6 SCF energy in solution:

a.u.

M06 enthalpy in solution:

$-5534.55593338$

M०6 free energy in solution:

a.u.

Imaginary frequency:

-5532.522125 a.u $-5532.791987$

$-270.7088 \mathrm{~cm}-1$

Cartesian coordinates

$\begin{array}{lccc}\text { ATOM } & X & Y & Z \\ P & 0 . \odot \odot \odot \odot \odot \odot ~ & -5.592647 & 3.833632\end{array}$

$\odot .00000 \odot-6.634687$

$\odot .00 \odot \odot \odot \odot-5.278042$

$\odot .0 \odot \odot \odot \odot \odot-3.182212$

$\odot .000000-1.030455$

$\odot .00000 \odot-1.777467$

$\odot .000000 \quad-7.596054$

$0.000000-6.534070$

$0.000000-5.184429$

$\odot .0000 \odot \odot-0.59390 \odot$

$\odot .00000 \odot-0.927178$

$\odot .000000-7.745242$

$1.590889-8.076878$

$2.151297-8.583937$

$2.691917-7.523096$

$2.639411 \quad 1.395802$

$1.673388 \quad 1.328910$

$-0.246288$

$-0.741629$

$-0.123910$

1. 010584

1. 515188

๑. 931409

2. 654274

2.831228

2.335138

2. 972643

4.104223

4. 714410

4.036063

6.102394

7.148485

6.072283

6.592479

2. 401224

1.197900

1.886637

3. 449488

4.067708

3. 214408

4. 329279

2. 951754

3. 836780

5. 031615

5. 233004

6. 329053

5.661882

7. 398650

7.113246

7. 024001

3. 470796

4. 466580

3. 442184

2. 070578

$-1.622326$

$-2.079393$

$-2.470459$

$-2.287700$

$-1.927755$

$-1.594159$

$-1.931524$

$-0.866735$

- 3.336914

$-1.610357$

- 2.018508

$-1.582550$

$-3.099779$

- 3.752638

$-5.046224$

$-3.814348$

$-4.919928$

$-2.655150$

$-2.690118$

- 3.931733

$-5.138807$

$-6.551370$
2. 243012

1. 355613

๑. 791855

2. 331156

$\odot .522103$

2. 033646

1. 236773

$-0.576826$

$-0.400656$

$-0.942440$

$-1.760606$

$-1.855821$

$-1.272489$

$-2.512281$

$-1.845244$

- 4.036855

$-2.321617$

$-0.580311$

$-1.492041$

๑. 884324

$-0.634938$

$-3.672529$

1. 404497

1. 787270

2. 092601

3. 033865

3. 287186

2. 774462

3. 224410

3. 449496

2. 117128

3. 421860

4.563671

3. 816936

3. 552650

5. 332975

3. 430290

$-1.619392$

$-2.931053$

$-3.453471$

$-2.607983$

$-1.237523$

$-0.777145$

- -.253367

$-0.639040$

- $\odot .222620$

1. 190499

$-5.959236$

$-3.933577$

$-4.877313$

$-5.218181$

$-0.610419$

- $\odot .309122$

$\odot .514672$

$-0.395202$

$-0.782517$

$-\odot .962949$

$-0.837486$

$-1.925484$
3.882014

3. 455630

3. 048344

7. 892291

6.561917

4. 116002

3. 752346

3. 358057

8. 585004

8.149377

3. 910046

2. 935591

4.359206

4. 537804

3. 108402

3. 392265

2. 439884

4. 236561

5. 095616

4. 570044

3. 467727

1. 874376

2. 968484

$-1.285181$

$-2.574458$

$-3.706525$

- 3.465494

$-2.181191$

$-1.110252$

$-1.958166$

$-2.885799$

$-2.214715$

$-0.505107$

$-5.107704$

$-5.447111$

$-5.099135$

$-6.245489$

$-4.902941$

๑. 815622

๑. 071524

2. 014146

2. 544713

1. 810556

๑. 508242

$-0.501404$

$-1.887278$

$-0.656274$

2. 932114

- 0.160106

3. 832763

4. 988335

3. 514081

4.357504

1. 594038

1. 743653

2. 985146

4. 114620

3. 986842

2. 708249

5. 185153

6. 239759

5.835965

4. 738952

3. 258526

6. 010593

3. 018087

1. 652988

$-2.500797$

$-3.142182$

$-5.384392$

$-2.358334$

$-1.013254$

$-0.401522$

$-1.103662$

$-3.768477$ 


\begin{tabular}{|c|c|c|c|}
\hline C & -3.053912 & -1.164015 & -5.393513 \\
\hline C & -6.714343 & -2.468149 & ๑. . 037499 \\
\hline C & -6.457079 & $-\odot .977107$ & $-\odot .286409$ \\
\hline C & -6.310759 & $-\odot .212170$ & 1.057288 \\
\hline C & -2.639755 & 1.238985 & -4.786294 \\
\hline C & -4.235701 & -4.994421 & 4.062863 \\
\hline C & -3.637919 & $\odot .060545$ & -4.645964 \\
\hline C & -7.715467 & -0.387713 & -0.966427 \\
\hline $\mathrm{H}$ & 3.148375 & -1.825203 & 4.640753 \\
\hline $\mathrm{H}$ & 3.096153 & -0.071710 & 2.903801 \\
\hline $\mathrm{H}$ & ๑. 910079 & -6.029362 & 3.674584 \\
\hline $\mathrm{H}$ & 2.562849 & -5.983420 & 2.935450 \\
\hline $\mathrm{H}$ & -1.653265 & -2.598795 & -2.441439 \\
\hline $\mathrm{H}$ & -0.530095 & -4.510673 & -3.547573 \\
\hline $\mathrm{H}$ & 3.668230 & -6.077582 & -1.660083 \\
\hline $\mathrm{H}$ & 2. 241840 & -6.719645 & $-\odot .746226$ \\
\hline $\mathrm{H}$ & 1.421819 & $\odot .167975$ & -2.697333 \\
\hline $\mathrm{H}$ & 4.444078 & -1.358354 & -0.111728 \\
\hline $\mathrm{H}$ & 7.232561 & $-\odot .773350$ & -2.670352 \\
\hline $\mathrm{H}$ & 8.133817 & -2.296362 & -2.718223 \\
\hline $\mathrm{H}$ & 6.889640 & -1.965566 & -3.938778 \\
\hline $\mathrm{H}$ & 5.888678 & -4.278112 & -3.261391 \\
\hline $\mathrm{H}$ & 7.038358 & -4.476493 & -1.939784 \\
\hline $\mathrm{H}$ & 5.301294 & -4.511788 & -1.597644 \\
\hline $\mathrm{H}$ & 5. 953059 & -2.836487 & ๑. 221289 \\
\hline $\mathrm{H}$ & 7.597788 & -2.745694 & -0.411586 \\
\hline $\mathrm{H}$ & 6.656477 & -1.264983 & -0.222198 \\
\hline $\mathrm{H}$ & $\odot .766745$ & -1.205344 & -6.413826 \\
\hline $\mathrm{H}$ & 1.477235 & -2.547764 & -5.505185 \\
\hline $\mathrm{H}$ & 0.414641 & -1.402213 & -4.686080 \\
\hline $\mathrm{H}$ & 1.570759 & 1.160344 & -6.110780 \\
\hline $\mathrm{H}$ & 1.021730 & 1.036132 & -4.447210 \\
\hline $\mathrm{H}$ & 2.675131 & 1.580845 & -4.792405 \\
\hline $\mathrm{H}$ & 4.337948 & -0.046601 & -5.992816 \\
\hline $\mathrm{H}$ & 3.780646 & -1.641469 & -6.493102 \\
\hline $\mathrm{H}$ & 3. $0 \odot 7801$ & $-\odot .201503$ & -7.149965 \\
\hline $\mathrm{H}$ & 3. 290588 & -3.481314 & -5.649288 \\
\hline $\mathrm{H}$ & 4.843745 & -4.292633 & -5.358628 \\
\hline $\mathrm{H}$ & 3.624410 & -4.203768 & -4.054086 \\
\hline $\mathrm{H}$ & 4.505413 & 1. 312592 & $-\odot .884407$ \\
\hline $\mathrm{H}$ & 6.414622 & 3.805026 & -2.599156 \\
\hline $\mathrm{H}$ & 5. 226361 & 2.540162 & -2.310959 \\
\hline $\mathrm{H}$ & 4.874039 & 4.209234 & -1.825968 \\
\hline $\mathrm{H}$ & 7.922748 & 1.917983 & ○. 284547 \\
\hline $\mathrm{H}$ & 6.953053 & 1.174809 & -0.994367 \\
\hline $\mathrm{H}$ & 8.148484 & 2.417478 & -1.397668 \\
\hline $\mathrm{H}$ & 6.812769 & 2.672721 & 3.672937 \\
\hline $\mathrm{H}$ & 7.697408 & 2.931020 & 2.147828 \\
\hline $\mathrm{H}$ & 7.726661 & 4.186239 & 3.413844 \\
\hline $\mathrm{H}$ & 7.702154 & 4.500622 & 0.690308 \\
\hline $\mathrm{H}$ & 7.621267 & 4.871584 & -1.025903 \\
\hline $\mathrm{H}$ & 6.298495 & 5.356159 & $\odot .045627$ \\
\hline $\mathrm{H}$ & 4.600825 & 2.478241 & 5.162297 \\
\hline $\mathrm{H}$ & 5.440730 & 4.002643 & 4.799519 \\
\hline $\mathrm{H}$ & 4.077024 & 3.991057 & 5.914427 \\
\hline $\mathrm{H}$ & 3.177086 & 5.898396 & 4.415405 \\
\hline $\mathrm{H}$ & 4.412689 & 5.686676 & 3.161197 \\
\hline $\mathrm{H}$ & 2.690891 & 5.556350 & 2.746612 \\
\hline $\mathrm{H}$ & 1.281005 & 3.614225 & 3.619935 \\
\hline $\mathrm{H}$ & 2.014457 & 2.380434 & 4.668654 \\
\hline $\mathrm{H}$ & 1.838956 & 4.040814 & 5.236196 \\
\hline $\mathrm{H}$ & -2.146130 & -3.557603 & $\odot .863736$ \\
\hline $\mathrm{H}$ & -1.302312 & $\odot .254720$ & 2.566117 \\
\hline $\mathrm{H}$ & -1.116874 & -1.560602 & 6.763661 \\
\hline $\mathrm{H}$ & ๑. 120675 & -0.758759 & 5.777162 \\
\hline $\mathrm{H}$ & -0.789880 & $\odot .154648$ & 6.992434 \\
\hline $\mathrm{H}$ & -3.338813 & $\odot .476247$ & 6.681166 \\
\hline $\mathrm{H}$ & -4.087718 & 0.123824 & 5.116155 \\
\hline $\mathrm{H}$ & -3.635374 & -1.206128 & $6.2020 \odot 8$ \\
\hline $\mathrm{H}$ & -1.668870 & 1.848905 & 5.612398 \\
\hline $\mathrm{H}$ & -0.594716 & 1.281610 & 4.333648 \\
\hline $\mathrm{H}$ & -2.317184 & 1.573332 & 3. 996102 \\
\hline $\mathrm{H}$ & -2.473898 & -6.956490 & 3. 226846 \\
\hline $\mathrm{H}$ & -1.252807 & -5.908769 & 2.476878 \\
\hline $\mathrm{H}$ & -1.523730 & -5.854568 & $4.2264 \odot 4$ \\
\hline
\end{tabular}

\begin{tabular}{|c|c|c|c|}
\hline H & -1.947441 & -4.965644 & 6.008559 \\
\hline $\mathrm{H}$ & $-\odot .608501$ & -3.885293 & 5.512233 \\
\hline $\mathrm{H}$ & -1.469757 & -3.612814 & 7.049963 \\
\hline $\mathrm{H}$ & -4.466341 & -4.448988 & 1.340530 \\
\hline H & -3.020837 & -5.355792 & 0.850499 \\
\hline $\mathrm{H}$ & -4.299454 & -6.162419 & 1.746480 \\
\hline H & -5.457056 & 1.291132 & -4.832940 \\
\hline $\mathrm{H}$ & -4.626732 & $\odot .929709$ & -6.356035 \\
\hline H & -5.617993 & $-\odot .29954 \odot$ & -5.578698 \\
\hline H & -1.696149 & -0.174305 & -2.810275 \\
\hline $\mathrm{H}$ & -3.959806 & -1.205565 & $\odot .651559$ \\
\hline H & -7.465053 & -1.784553 & -4.350514 \\
\hline H & -5.766985 & -2.330765 & -4.419542 \\
\hline $\mathrm{H}$ & -6.743649 & -2.641330 & -2.960995 \\
\hline $\mathrm{H}$ & -2.905118 & $-\odot .926208$ & -6.453811 \\
\hline H & -2.084760 & -1.463974 & -4.979086 \\
\hline $\mathrm{H}$ & -3.724787 & -2.028620 & -5.336905 \\
\hline H & -6.853776 & -3.066802 & $-\odot .868949$ \\
\hline $\mathrm{H}$ & -5.879760 & -2.902063 & ๑.598771 \\
\hline $\mathrm{H}$ & -7.619508 & -2.572062 & $\odot .647816$ \\
\hline $\mathrm{H}$ & -7.252914 & -0.275290 & 1.612998 \\
\hline H & -5.531665 & -0.620099 & 1.707165 \\
\hline $\mathrm{H}$ & -6.089787 & 0.846374 & 0.885329 \\
\hline $\mathrm{H}$ & -3.014654 & 2.137503 & -4.286001 \\
\hline $\mathrm{H}$ & -1.649810 & 1.030466 & -4.375698 \\
\hline H & -2.507962 & 1.474731 & -5.848290 \\
\hline $\mathrm{H}$ & -5.018100 & -4.252604 & 3.865356 \\
\hline H & -4.692291 & -5.987971 & 3.988404 \\
\hline H & -3.902503 & -4.849056 & 5.088513 \\
\hline H & -8.526600 & -0.363560 & $-\odot .229194$ \\
\hline $\mathrm{H}$ & -7.543632 & $\odot .636309$ & -1.311532 \\
\hline H & -8.063881 & -0.975108 & -1.815516 \\
\hline $\mathrm{Rh}$ & $-\odot .076111$ & 1.093848 & ๑. 025978 \\
\hline 0 & -2.177912 & 1.951397 & 0.622938 \\
\hline C & ๑. 089801 & 2.576180 & -1.524529 \\
\hline $\mathrm{s}$ & -2.867056 & 3.239723 & $0.9 \odot 4725$ \\
\hline 0 & -0.989715 & 2.599812 & -2.087006 \\
\hline C & -0.442387 & 4.253062 & 0.884093 \\
\hline $\mathrm{N}$ & -1.816699 & 4.454231 & $\odot .353006$ \\
\hline 0 & -3.287984 & 3.463105 & 2.295976 \\
\hline C & -4.287751 & 3.426436 & $-\odot .154651$ \\
\hline H & -0.029410 & 5.259567 & 1. 012263 \\
\hline H & $-\odot .487608$ & 3.809642 & 1.887211 \\
\hline C & -2.347464 & 5.829750 & $\odot .567139$ \\
\hline C & -5.518606 & 3.723555 & 0.434877 \\
\hline C & -4.150300 & 3.283118 & -1.538989 \\
\hline C & -1.681276 & 6.796667 & $-\odot .371339$ \\
\hline $\mathrm{H}$ & -3.421501 & 5.803630 & 0.354531 \\
\hline H & -2.226471 & 6.143155 & 1.613316 \\
\hline $\mathrm{H}$ & -5.592647 & 3.833632 & -270.708800 \\
\hline C & -6.634687 & 3.882014 & 8.531400 \\
\hline c & -5.278042 & 3.455630 & 13.349400 \\
\hline $\mathrm{H}$ & -3.182212 & 3.048344 & -1.967586 \\
\hline c & -1.030455 & 7.892291 & 0.023335 \\
\hline $\mathrm{H}$ & -1.777467 & 6.561917 & -1.430669 \\
\hline $\mathrm{H}$ & -7.596054 & 4.116002 & ๑. 062392 \\
\hline c & $-6.53407 \odot$ & 3.752346 & -1.778796 \\
\hline $\mathrm{H}$ & -5.184429 & 3.358057 & -3.413966 \\
\hline H & -0.593900 & 8.585004 & -0.690514 \\
\hline $\mathrm{H}$ & -0.927178 & 8.149377 & 1.075723 \\
\hline c & -7.745242 & $3.910 \odot 46$ & -2.665436 \\
\hline $\mathrm{H}$ & -8.076878 & 2.935591 & -3.046128 \\
\hline $\mathrm{H}$ & -8.583937 & 4.359206 & -2.125689 \\
\hline H & -7.523096 & 4.537804 & -3.535489 \\
\hline c & 1.395802 & 3.108402 & -2.108506 \\
\hline $\mathrm{H}$ & 1.328910 & 3.392265 & -3.161537 \\
\hline $\mathrm{H}$ & 2.243012 & 2.439884 & -1.949377 \\
\hline C & 1.355613 & 4.236561 & -1.059426 \\
\hline $\mathrm{H}$ & 0.791855 & 5.095616 & -1.434442 \\
\hline $\mathrm{H}$ & 2.331156 & 4.570044 & -0.691912 \\
\hline C & $\odot .522103$ & 3.467727 & ๑. 001887 \\
\hline H & 2.033646 & 1.874376 & 2.545711 \\
\hline $\mathrm{H}$ & 1.236773 & 2.968484 & 0.658046 \\
\hline
\end{tabular}


TS1 12

B3LYP SCF energy: $\quad-5535.31865514$ a.u. B3LYP enthalpy:

B3LYP free energy: -5533.284838 a.u.

M०6 SCF energy in -5533.554713 a.u a.u.

M06 enthalpy in solution: $-5534.55586055$

M०6 free energy in solution

-5532.522043 a.u.

Imaginary frequency: $-5532.791918$

Cartesian coordinates

\begin{tabular}{|c|c|c|c|}
\hline ATOM & $\mathrm{x}$ & $\mathrm{Y}$ & Z \\
\hline $\mathrm{P}$ & $\odot . ๑ \odot \odot \odot \odot \odot$ & -5.589652 & 3.820496 \\
\hline $\mathrm{P}$ & $\odot . ๑ \odot \odot \odot \odot \odot$ & -6.618268 & 3.834750 \\
\hline 0 & ๑. . & -5.244577 & 3.390540 \\
\hline 0 & $\odot . \odot \odot \odot \odot \odot \odot$ & -3.149161 & 3.000394 \\
\hline 0 & ๑. . & -1.034229 & 7.876743 \\
\hline 0 & $\odot . \diamond \odot \odot \odot \odot \odot$ & -1.750875 & 6.500872 \\
\hline 0 & ०. . 000000 & -7.584317 & 4. 069026 \\
\hline 0 & $\odot . \odot \odot \odot \odot \odot \odot$ & -6.506539 & 3.686697 \\
\hline 0 & $\odot .0000 \odot \odot$ & -5.142420 & 3.279263 \\
\hline 0 & ๑. . $૦ \odot \odot ० ० \odot$ & -0.595373 & 8.554259 \\
\hline C & $\odot . ๑ \odot \odot \odot \odot \diamond$ & $-\odot .947825$ & 8.163441 \\
\hline C & ๑. . & -7.712214 & 3.823783 \\
\hline C & 1.608484 & -8.038427 & 2.841013 \\
\hline C & 2.192127 & -8.555781 & 4.280665 \\
\hline C & 2.756936 & -7.486190 & 4.435151 \\
\hline C & 2.697477 & 1.437834 & 3.043488 \\
\hline C & 1.687633 & 1.382837 & 3.308370 \\
\hline C & -0.238071 & 2.282122 & 2.376377 \\
\hline C & $-\odot .736697$ & 1.389621 & 4.191141 \\
\hline C & -0.122382 & $\odot .835491$ & 5.046000 \\
\hline C & 1.012086 & 2.363389 & 4.525461 \\
\hline C & 1.519714 & $\odot .539486$ & 3.448479 \\
\hline C & 0.939323 & 2.070565 & 1.816006 \\
\hline C & 2.653297 & 1. 242018 & 2.961687 \\
\hline C & 2.857532 & -0.612223 & -1.275709 \\
\hline C & 2.361649 & -0.460379 & -2.568280 \\
\hline C & 2.995280 & -1.029524 & -3.688782 \\
\hline C & 4.121993 & -1.849505 & -3.431387 \\
\hline C & 4.732825 & -1.921138 & -2.146004 \\
\hline C & 4.058195 & -1.311760 & -1.087098 \\
\hline C & 6.114870 & -2.585488 & -1.908780 \\
\hline C & 7.166984 & -1.958514 & -2.857326 \\
\hline C & 6.065075 & -4.116396 & -2.120977 \\
\hline C & 6.611672 & -2.359321 & -0.463053 \\
\hline C & 2.424498 & $-\odot .694481$ & -5.096885 \\
\hline C & 1.217398 & -1.608316 & -5.416741 \\
\hline C & 1.915619 & ๑. 772015 & -5.118845 \\
\hline C & 3.472356 & $-\odot .776966$ & -6.233360 \\
\hline C & 4.071727 & -3.791478 & -4.828900 \\
\hline C & 3.210864 & 1.419326 & ๑. 799213 \\
\hline C & 4. 356081 & 1.776411 & $\odot .077093$ \\
\hline C & 2.945447 & 2.098593 & 1.994646 \\
\hline C & 3.768396 & 3.122853 & 2.489318 \\
\hline C & 4.817541 & 3.554266 & 1.638763 \\
\hline C & 5.186211 & 2.834716 & $\odot .470303$ \\
\hline C & 6.495463 & 3.128773 & -0.308585 \\
\hline C & 6.700572 & 2.133075 & -1.470919 \\
\hline C & 7.699551 & 2.969436 & ๑.653923 \\
\hline C & 4.906322 & 5.920902 & 1.663131 \\
\hline C & 6.515623 & 4.546951 & -0.928639 \\
\hline C & 3.477302 & 3.634791 & 3.931370 \\
\hline C & 4.618210 & 4.452587 & 4.581973 \\
\hline C & 2.175528 & 4.470841 & 3.953352 \\
\hline C & 3.278010 & 2.402896 & 4.858036 \\
\hline C & -1.600740 & -1.591157 & 1.623941 \\
\hline C & -2.066680 & -2.894168 & 1.814788 \\
\hline C & -2.444605 & -3.379522 & 3.075519 \\
\hline C & -2.237504 & -2.506406 & 4.179528 \\
\hline C & -1.866928 & -1.143313 & 4. 010158 \\
\hline C & -1.549258 & -0.720318 & 2.715108 \\
\hline C & -1.841374 & -0.127831 & 5.181680 \\
\hline
\end{tabular}

$-\odot .766415$ $-3.236833$

$-1.509218$

$-2.010203$

$-1.516189$

$-3.085996$

$-3.765628$

$-5.030144$

$-3.797581$

$-4.899111$

$-2.639704$

$-2.676280$

$-3.918374$

$-5.124600$

$-6.534359$

$-3.038284$

$-6.712932$

$-6.444960$

$-6.297140$

- 2.616224

$-4.204999$

$-3.618847$

$-7.697350$

3. 236815

3. 175151

$\odot .924475$

2.565926

$-1.648128$

$-0.531017$

3. 666328

2. 239447

1. 450924

4. 465259

7. 264052

8.146840

6.905175

5. 872487

7.027481

5. 291946

5. 970849

7. 613608

6.686076

๑. 786962

1. 492426

๑. 434806

1. 601261

1. 051012

2. 706902

4. 362844

3. 800132

3. 032138

3. 301314

4. 845211

3. 618224

4. 598601

7.657875

6.736478

5. 920162

7.638005

7. 748128

8. 634512

4.659446

3. 991975

5. 619796

6. 611434

7. 376252

5. 612632

5.580106

4.737096

4.385665

1. 956804

2. 253005

1. 315757

2. 419070

4. 171089 $-\odot .498364$

- 0.062240

1. 299808

$-5.881444$

$-3.789274$

$-4.796068$

$-5.164671$

$-0.679888$

$-\odot .390408$

0.388907

$-0.458240$

$-\odot .817989$

$-0.988198$

$-0.878924$

$-2.017316$

$-1.295937$

- 2. 490939

$-1.004326$

- 0.230936

1. 117693

$-4.876323$

$-0.053655$

$-0.411372$

$-1.796893$

$-0.070810$

$-5.985421$

$-5.969417$

$-2.662773$

$-4.598330$

$-6.137215$

$-6.757804$

๑. 109624

$-1.381883$

$-0.882056$

$-2.416750$

$-2.107029$

$-4.385526$

$-4.559547$

$-4.563656$

$-2.847930$

$-2.789248$

- 1.295812

$-1.340276$

$-2.666132$

$-1.499326$

1. 028288

0.940689

1. 471416

$-0.186503$

$-1.789668$

$-0.361437$

- 3.611794

$-4.429188$

$-4.298348$

1. 222788

2. 347871

1. 093947

2. 216599

3. 666258

1. 949133

3. 163160

5. 997691

6. 052498

6. 704317

5. 324192

4.638806

4. 737627

3. 937817

5. 450374

4.579185

4. 802444

5. 364289

3. 887176

1. 785739

1. 767372
6. 231346

5.851039

4.692615

3. 404859

. 098676

3. 155647

1. 811048

$-2.498511$

- 3.144108

$-5.403942$

$-2.356622$

$-1.004012$

$-0.390484$

$-1.097044$

$-3.746082$

$-5.376029$

๑. 054609

$-0.280678$

1. 058005

$-4.821230$

4. 221973

$-4.655415$

$-0.968878$

4.639147

2. 878288

3. 775236

3. 010060

$-2.394404$

$-3.464195$

$-1.552091$

$-0.624153$

$-2.703064$

$-0.086896$

$-2.673944$

$-2.677380$

$-3.905862$

$-3.159333$

$-1.838904$

$-1.486210$

๑. 280030

$-0.361075$

$-0.209550$

$-6.389138$

$-5.452689$

$-4.657473$

$-6.136161$

$-4.470624$

$-4.826172$

$-5.993119$

$-6.459280$

$-7.146928$

$-5.585077$

$-5.264247$

$-3.970615$

$-0.817825$

$-1.956912$

$-1.126595$

$-2.236597$

1. 492153

1. 052084

0.114771

๑. 598004

2. 250505

1. 927681

$-0.170944$

$-1.601224$

$-1.521419$

4. 507698

4.160458

5.645474

4. 975111

3. 323885

3. 604222

4.578714

4. 860905 


\begin{tabular}{|c|c|c|c|}
\hline $\mathrm{H}$ & 3.106010 & 2.742875 & 5.884952 \\
\hline $\mathrm{H}$ & -2.150153 & -3.543658 & $\odot .953018$ \\
\hline $\mathrm{H}$ & $-1.2500 \odot 2$ & $\odot .304471$ & 2.540829 \\
\hline $\mathrm{H}$ & -1.021048 & -1.401850 & 6.783696 \\
\hline $\mathrm{H}$ & ๑. 212706 & -0.643953 & 5.758627 \\
\hline $\mathrm{H}$ & -0.668642 & ๑. 314321 & 6.960984 \\
\hline $\mathrm{H}$ & -3.218274 & ๑. 658163 & 6.677771 \\
\hline $\mathrm{H}$ & -3.993720 & ๑. 274394 & 5.132996 \\
\hline $\mathrm{H}$ & -3.541414 & -1.032302 & 6.246827 \\
\hline $\mathrm{H}$ & -1.545828 & 1.981584 & 5.549133 \\
\hline $\mathrm{H}$ & -0.498387 & 1.365824 & 4.269645 \\
\hline H & - 2.222351 & 1.671822 & 3.950154 \\
\hline $\mathrm{H}$ & -2.474705 & -6.874981 & 3.406063 \\
\hline $\mathrm{H}$ & -1.257584 & -5.857456 & 2.609370 \\
\hline $\mathrm{H}$ & -1.497876 & -5.756975 & 4. 361222 \\
\hline $\mathrm{H}$ & -1.889908 & -4.817769 & 6.126173 \\
\hline $\mathrm{H}$ & $-\odot .548516$ & -3.761680 & 5.586559 \\
\hline $\mathrm{H}$ & -1.386883 & -3.444734 & 7.128443 \\
\hline $\mathrm{H}$ & -4.478090 & -4.396753 & 1.492719 \\
\hline $\mathrm{H}$ & -3.049564 & -5.328492 & $\odot .999383$ \\
\hline $\mathrm{H}$ & -4.318909 & -6.101284 & 1.937313 \\
\hline $\mathrm{H}$ & -5.434663 & 1.177460 & -4.868386 \\
\hline $\mathrm{H}$ & -4.604240 & $\odot .783717$ & -6.383467 \\
\hline $\mathrm{H}$ & -5.599086 & -0.427210 & -5.582562 \\
\hline $\mathrm{H}$ & -1.680220 & $-\odot .245410$ & -2.811347 \\
\hline $\mathrm{H}$ & -3.947258 & -1.209162 & 0.667334 \\
\hline $\mathrm{H}$ & -7.445871 & -1.885023 & -4.333528 \\
\hline $\mathrm{H}$ & -5.748525 & -2.434751 & -4.387527 \\
\hline $\mathrm{H}$ & -6.730482 & -2.719025 & -2.927353 \\
\hline $\mathrm{H}$ & -2.888354 & -1.081495 & -6.441133 \\
\hline $\mathrm{H}$ & -2.070153 & -1.589530 & -4.954722 \\
\hline $\mathrm{H}$ & -3.711527 & -2.157265 & -5.301167 \\
\hline $\mathrm{H}$ & -6.860187 & -3.095368 & $-\odot .846586$ \\
\hline $\mathrm{H}$ & -5.879787 & -2.927258 & 0.616072 \\
\hline $\mathrm{H}$ & -7.616897 & -2.583251 & $\odot .668568$ \\
\hline $\mathrm{H}$ & -7.243462 & $-\odot .277877$ & 1.608110 \\
\hline $\mathrm{H}$ & -5.527922 & -0.644401 & 1.716133 \\
\hline $\mathrm{H}$ & -6.061313 & $\odot .823431$ & ๑. 879915 \\
\hline $\mathrm{H}$ & -2.987100 & 2.028131 & -4.339913 \\
\hline $\mathrm{H}$ & -1.626590 & $\odot .913865$ & -4.407495 \\
\hline $\mathrm{H}$ & -2.484426 & 1.330305 & -5.888080 \\
\hline H & -4.983611 & $-4.13190 \odot$ & 4.019394 \\
\hline $\mathrm{H}$ & -4.672128 & -5.866845 & 4.179530 \\
\hline $\mathrm{H}$ & -3.853403 & -4.709428 & 5. 238127 \\
\hline $\mathrm{H}$ & -8.510653 & $-\odot .375404$ & $-\odot .234546$ \\
\hline $\mathrm{H}$ & -7.516802 & 0.608754 & -1.321304 \\
\hline $\mathrm{H}$ & -8.046808 & -1.002516 & -1.814790 \\
\hline $\mathrm{Rh}$ & -0.056901 & 1.075316 & -0.002373 \\
\hline 0 & -2.161803 & 1.943675 & ○. 561412 \\
\hline C & $\odot .123547$ & 2.524338 & -1.579799 \\
\hline$S$ & -2.855880 & 3.235808 & ๑. 810876 \\
\hline 0 & -0.949111 & 2.540188 & -2.155470 \\
\hline C & -0.435089 & 4.255894 & ๑. 786241 \\
\hline $\mathrm{N}$ & -1.805219 & 4.440228 & $\odot .237961$ \\
\hline 0 & -3.284528 & 3. 488295 & 2.194842 \\
\hline C & -4.270460 & 3.396897 & -0.260395 \\
\hline $\mathrm{H}$ & $-\odot .025723$ & 5.266419 & ๑. 891240 \\
\hline $\mathrm{H}$ & -0.488741 & 3.840416 & 1.800636 \\
\hline C & -2.341596 & 5.818424 & $\odot .415097$ \\
\hline C & -5.507279 & 3.694884 & 0.316040 \\
\hline C & -4.121807 & 3.235402 & -1.641578 \\
\hline C & -1.670350 & 6.764993 & -0.540430 \\
\hline $\mathrm{H}$ & -3.413630 & 5.784322 & ๑. 193622 \\
\hline $\mathrm{H}$ & -2.231028 & 6.157260 & 1.454544 \\
\hline $\mathrm{H}$ & -5.589652 & 3.820496 & -272.572900 \\
\hline C & -6.618268 & 3.834750 & $7.52070 \odot$ \\
\hline C & -5.244577 & 3.390540 & 12.678300 \\
\hline $\mathrm{H}$ & -3.149161 & 3..$\odot \odot 394$ & -2.059792 \\
\hline C & -1.034229 & 7.876743 & -0.167467 \\
\hline $\mathrm{H}$ & -1.750875 & 6.500872 & -1.594124 \\
\hline $\mathrm{H}$ & -7.584317 & 4.069026 & -0.076968 \\
\hline C & -6.506539 & 3.686697 & -1.905166 \\
\hline $\mathrm{H}$ & -5.142420 & 3.279263 & -3.524924 \\
\hline $\mathrm{H}$ & -0.595373 & 8.554259 & -0.894413 \\
\hline $\mathrm{H}$ & -0.947825 & 8.163441 & ๑. 878813 \\
\hline
\end{tabular}

$\begin{array}{lrrr}\mathrm{C} & -7.712214 & 3.823783 & -2.802603 \\ \mathrm{H} & -8.038427 & 2.841013 & -3.166252 \\ \mathrm{H} & -8.555781 & 4.280665 & -2.277108 \\ \mathrm{H} & -7.486190 & 4.435151 & -3.683226 \\ \mathrm{C} & 1.437834 & 3.043488 & -2.158185 \\ \mathrm{H} & 1.382837 & 3.308370 & -3.216871 \\ \mathrm{H} & 2.282122 & 2.376377 & -1.977999 \\ \mathrm{C} & 1.389621 & 4.191141 & -1.130990 \\ \mathrm{H} & 0.835491 & 5.04600 \odot & -1.529512 \\ \mathrm{H} & 2.363389 & 4.525461 & -0.759365 \\ \mathrm{C} & 0.539486 & 3.448479 & -0.064485 \\ \mathrm{H} & 2.070565 & 1.816006 & 2.570957 \\ \mathrm{H} & 1.242018 & 2.961687 & 0.613765\end{array}$

TS1_13

B3LYP SCF energy: $\quad-5535.31953915$ a.u. B3LYP enthalpy: $\quad-5533.285368$ a.u. B3LYP free energy: $\quad-5533.553260$ a.u. M06 SCF energy in solution: $\quad-5534.55344768$ a.u.

M06 enthalpy in solution: 532.519277 a.u. M06 free energy in solution: $\quad-5532.787169$ a.u.

Imaginary frequency: $\quad-258.8602 \mathrm{~cm}-1$

Cartesian coordinates

\begin{tabular}{|c|c|c|c|}
\hline ATOM & $\mathrm{X}$ & Y & Z \\
\hline$P$ & ๑.๑७७९९९ & -5.693761 & 3.607249 \\
\hline$P$ & $\odot . ๑ \odot \odot \odot \odot \odot ~$ & -6.666954 & 3.894719 \\
\hline 0 & $\odot .00 \odot \odot \odot \odot$ & -5.229036 & 3.779567 \\
\hline 0 & $\odot . ๑ \odot \odot \odot \odot \odot ~$ & -3.143836 & 3.337467 \\
\hline 0 & $\odot . ๑ \odot \odot \odot \odot \odot$ & -1.044652 & 7.874010 \\
\hline 0 & $\odot . ๑ \odot \odot \odot \odot \odot ~$ & -1.791542 & 6.588981 \\
\hline 0 & $\odot . ๑ \odot \odot \odot \odot \odot$ & -7.650278 & 4.041183 \\
\hline 0 & $\odot . ๑ \odot \odot \odot \odot \odot ~$ & -6.508676 & 3.983526 \\
\hline 0 & $\odot . ๑ \odot \odot \odot \odot \odot$ & -5.091116 & 3.841626 \\
\hline 0 & $\odot . ๑ \odot \odot \odot \odot \odot ~$ & -0.597827 & 8.581687 \\
\hline c & $\odot . ๑ \odot \odot \odot \odot \odot$ & $-\odot .94649 \odot$ & 8.102488 \\
\hline c & $\odot . \diamond \odot \odot \odot \odot \odot$ & -7.679554 & 4.323376 \\
\hline c & 1.683442 & $-7.7496 \odot 4$ & 5.408602 \\
\hline c & 2.219168 & -7.580462 & 3. 865661 \\
\hline c & 2.679338 & -8.627149 & $3.9950 \odot 4$ \\
\hline c & 2.585713 & 1.373569 & 2.979013 \\
\hline c & 1.865779 & 1.318691 & 3.195838 \\
\hline c & -0.146298 & 2.233420 & 2.343348 \\
\hline c & $-\odot .581946$ & 1.284055 & 4.166857 \\
\hline c & 0.096399 & $\odot .689080$ & 4.978494 \\
\hline c & 1.231354 & 2.242912 & 4.559211 \\
\hline C & 1.676812 & $\odot .475716$ & 3.423850 \\
\hline c & 1.032111 & 1.872324 & 1.979970 \\
\hline c & 2.913155 & 1.210582 & 2.970330 \\
\hline C & 2.929948 & $-\odot .603188$ & -1.132537 \\
\hline c & 2.504039 & $-\odot .52661 \odot$ & -2.456455 \\
\hline c & 3.230962 & -1.103377 & -3.514905 \\
\hline c & 4.391731 & -1.840289 & -3.163142 \\
\hline c & 4.921266 & -1.836652 & $-1.84 \odot 223$ \\
\hline c & 4.149794 & -1.231375 & -0.847321 \\
\hline c & 6.313100 & -2.424338 & -1.486728 \\
\hline c & 7.401781 & -1.789982 & -2.387707 \\
\hline c & 6.335661 & $-3.96330 \odot$ & -1.633554 \\
\hline c & 6.703676 & -2.119617 & $-\odot .022605$ \\
\hline c & 2.728283 & -0.871211 & -4.968798 \\
\hline c & 1.739771 & -1.986677 & -5.387183 \\
\hline c & 1.960640 & 0.472906 & -5.067312 \\
\hline c & 3.883641 & $-\odot .754063$ & -5.993026 \\
\hline c & 4.603838 & -3.792768 & -4.569971 \\
\hline C & 3.130371 & 1.488102 & 0.879244 \\
\hline c & 4.253590 & 1.887312 & 0.156231 \\
\hline c & 2.800877 & 2.210841 & 2.040703 \\
\hline c & 3.633923 & 3.203314 & 2.563099 \\
\hline c & 4.843998 & 3.472322 & 1.859966 \\
\hline c & 5.106208 & 2.922459 & 0.584215 \\
\hline c & 6.218440 & 3.376359 & $-\odot .4 \odot 5724$ \\
\hline
\end{tabular}




\begin{tabular}{|c|c|c|c|c|c|c|c|}
\hline C & 5.588960 & 3.531805 & -1.818436 & H & 8.091774 & 2.603812 & -1.217035 \\
\hline C & 7.328964 & 2.302250 & $-\odot .489508$ & H & 6.582082 & 2.983087 & 3.798860 \\
\hline C & 6.881839 & 3.715587 & 3.041295 & H & 7.505999 & 3.217356 & 2.293356 \\
\hline C & 6.856970 & 4.749384 & $-\odot .089396$ & H & 7.454682 & 4.516271 & 3.513895 \\
\hline C & 3.200036 & 4.020225 & 3.808265 & H & 7.509662 & 4.737899 & 0.782946 \\
\hline C & 4.161681 & 3.826140 & $5.0059 \odot 2$ & H & 7.470782 & 5.048741 & -0.946591 \\
\hline C & 3.138487 & 5.522798 & 3.435074 & H & 6.099307 & 5.522862 & 0.066864 \\
\hline C & 1.794175 & 3.610342 & 4.297897 & H & 4.319209 & 2.762926 & 5.223389 \\
\hline C & -1.669213 & -1.595643 & 1.614131 & H & 5.129034 & 4.296983 & 4.833899 \\
\hline C & -1.957265 & -2.942209 & 1.877463 & H & 3.728028 & 4.285817 & 5.901602 \\
\hline C & -2.514499 & -3.349215 & 3.093152 & H & 2.825134 & 6.110347 & 4.306273 \\
\hline C & -2.792282 & -2.335543 & 4.053719 & H & 4.109741 & 5.894132 & 3.103242 \\
\hline C & -2.354943 & $-\odot .998072$ & 3.892069 & H & 2.408331 & 5.696507 & 2.634922 \\
\hline C & -1.846267 & $-\odot .654187$ & 2.627407 & H & 1.027495 & 3.743413 & 3.526052 \\
\hline C & -2.347470 & 0.099223 & 4.995244 & H & 1.756562 & 2.571450 & 4.645332 \\
\hline C & -2.382313 & $-\odot .451993$ & 6.440966 & $\mathrm{H}$ & 1.513370 & 4.245521 & 5.144291 \\
\hline C & -3.515897 & 1.092514 & 4.785805 & H & -1.750114 & -3.677757 & 1.111684 \\
\hline C & -1.020962 & $\odot .9 \odot 2107$ & 4.892747 & H & -1.580249 & ๑.375836 & 2.425722 \\
\hline C & -1.920788 & -5.237326 & 4.657923 & H & -3.344221 & -0.876764 & 6.724478 \\
\hline C & -4.848333 & -2.372044 & 5.243513 & H & -1.618437 & -1.220341 & 6.597938 \\
\hline C & -2.727927 & -4.857111 & 3. 391038 & H & -2.175613 & 0.373598 & 7.131739 \\
\hline C & -2.215891 & -5.739762 & 2.230895 & H & -3.483462 & 1.873237 & 5.555687 \\
\hline C & -4.918776 & -1.007572 & -2.683336 & H & -3.458130 & 1.585238 & 3.810135 \\
\hline C & -3.682127 & -0.612537 & -3.261380 & H & -4.491559 & $\odot .6 \odot \odot 992$ & 4.856734 \\
\hline C & -4.587262 & $-\odot .638282$ & -5.733775 & H & $-\odot .966470$ & 1.616969 & 5.721026 \\
\hline C & -2.570456 & -0.561911 & -2.408715 & H & -0.150379 & $\odot .238900$ & 4.956410 \\
\hline C & -2.631381 & -0.991817 & -1.077897 & H & $-\odot .945151$ & 1.481520 & 3.968374 \\
\hline C & -3.845460 & -1.468510 & -0.583058 & $\mathrm{H}$ & -2.007799 & -6.314076 & 4.848480 \\
\hline C & -5.012248 & -1.502792 & -1.359795 & H & $-\odot .859245$ & -4.997135 & 4.527812 \\
\hline C & -6.735665 & 0.321273 & -3.376995 & $\mathrm{H}$ & -2.281958 & -4.702821 & 5.538238 \\
\hline C & -2.213084 & -1.094111 & -5.252443 & H & -4.988920 & -1.298315 & 5.398666 \\
\hline C & -5.894585 & -3.519384 & -0.132015 & $\mathrm{H}$ & -5.365464 & -2.671420 & 4.324998 \\
\hline C & -6.271252 & -2.116102 & $-\odot .680575$ & $\mathrm{H}$ & -5.271439 & -2.914000 & 6.092495 \\
\hline C & -6.698117 & -1.208927 & 0.499866 & $\mathrm{H}$ & -2.786907 & -5.582725 & 1. 308056 \\
\hline C & -3.122042 & 1.229100 & -4.919610 & H & -1.155986 & -5.569368 & 2.016111 \\
\hline C & -4.216903 & -5.220613 & 3.604669 & $\mathrm{H}$ & -2.335441 & -6.792828 & 2.507892 \\
\hline C & -3.439039 & $-\odot .27684 \odot$ & -4.763252 & H & -5.460351 & ๑.๑๑6178 & -5.625991 \\
\hline C & -7.506410 & -2.338034 & -1.582080 & $\mathrm{H}$ & -4.217666 & -0.512486 & -6.758585 \\
\hline H & 3.109530 & -1.627884 & 4.795763 & H & -4.915828 & -1.674391 & -5.618592 \\
\hline H & 2.986902 & 0.084764 & 3.022547 & H & -1.627527 & -0.192813 & -2.794069 \\
\hline H & 1.132596 & -5.968483 & 3.890142 & H & -3.888036 & -1.824138 & 0.437283 \\
\hline H & 2.783633 & -5.854867 & 3.151748 & H & -7.642099 & 0.219370 & -3.978475 \\
\hline $\mathrm{H}$ & -1.490448 & -2.759861 & -2.377835 & H & -7.007438 & $\odot .577438$ & -2.345163 \\
\hline H & -0.264121 & -4.647059 & -3.408597 & H & -6.115295 & 1.129532 & -3.782490 \\
\hline H & 3.944768 & -5.989865 & -1.382229 & H & -2.028113 & $-\odot .882520$ & -6.311582 \\
\hline $\mathrm{H}$ & 2.529021 & -6.691681 & $-\odot .496368$ & H & -1.295052 & $-\odot .852967$ & -4.710317 \\
\hline $\mathrm{H}$ & 1.576689 & $-\odot .008988$ & -2.664894 & $\mathrm{H}$ & -2.395028 & -2.170822 & -5.153095 \\
\hline $\mathrm{H}$ & 4.497550 & -1.244734 & 0.177028 & H & -5.595423 & -4.188223 & -0.947116 \\
\hline $\mathrm{H}$ & 7.441103 & -0.703659 & -2.243234 & $\mathrm{H}$ & -5.078263 & -3.489429 & 0.595368 \\
\hline $\mathrm{H}$ & 8.384919 & -2.195952 & -2.121197 & H & -6.762692 & -3.965599 & $\odot .366741$ \\
\hline $\mathrm{H}$ & 7.221129 & -1.988988 & -3.444557 & H & -7.568887 & -1.638459 & 1.009912 \\
\hline H & 6.195022 & -4.284847 & -2.665263 & H & -5.898883 & -1.092253 & 1.239863 \\
\hline $\mathrm{H}$ & 7.303025 & -4.353683 & -1.295776 & H & -6.974234 & -0.207661 & 0.148890 \\
\hline $\mathrm{H}$ & 5.555912 & -4.415290 & -1.010369 & H & -3.984063 & 1.840942 & -4.631184 \\
\hline H & 6.037806 & -2.608119 & 0.698052 & H & -2.271962 & 1.538608 & -4.303879 \\
\hline H & 7.714339 & -2.499529 & $\odot .160509$ & H & -2.888308 & 1.456384 & -5.967085 \\
\hline H & 6.715082 & -1.044772 & 0.189969 & H & -4.836141 & -4.874018 & 2.768891 \\
\hline $\mathrm{H}$ & 1.352072 & -1.786824 & -6.393456 & $\mathrm{H}$ & -4.322131 & -6.310471 & 3.662880 \\
\hline H & 2.199798 & -2.976853 & -5.399582 & H & -4.613215 & -4.805529 & 4.530907 \\
\hline H & $\odot .889060$ & -2.028237 & -4.698491 & H & -8.252324 & -2.895471 & -1.002769 \\
\hline $\mathrm{H}$ & 1.712130 & 0.665139 & -6.116333 & H & -7.977986 & -1.405732 & -1.897340 \\
\hline $\mathrm{H}$ & 1.015637 & 0.474578 & -4.515530 & $\mathrm{H}$ & -7.269083 & -2.919078 & -2.475993 \\
\hline $\mathrm{H}$ & 2.570680 & 1.309807 & -4.709578 & $\mathrm{Rh}$ & -0.101099 & 1.046290 & -0.063329 \\
\hline H & 4.586397 & $\odot .033029$ & -5.696515 & 0 & -2.250982 & 1.923145 & $\odot .356544$ \\
\hline H & 4.455714 & -1.670763 & -6.118164 & C & 0.070445 & 2.456120 & -1.682118 \\
\hline $\mathrm{H}$ & 3.467901 & $-\odot .479105$ & -6.968998 & $\mathrm{~s}$ & -2.926649 & 3.217135 & 0.635185 \\
\hline $\mathrm{H}$ & 4.114701 & -3.681479 & -5.542559 & 0 & -1.005237 & 2.445169 & -2.255104 \\
\hline $\mathrm{H}$ & 5.459949 & -4.462345 & -4.691473 & C & $-\odot .491685$ & 4.216585 & 0.667032 \\
\hline $\mathrm{H}$ & 3.898180 & -4.229197 & -3.855538 & $\mathrm{~N}$ & -1.857201 & 4.433519 & $\odot .121867$ \\
\hline H & 4.479131 & 1.384933 & -0.774759 & 0 & -3.378105 & 3.429257 & 2.019588 \\
\hline H & 6.352612 & 3.885865 & -2.519346 & C & -4.318665 & 3.459583 & -0.453974 \\
\hline H & 5.194825 & 2.595039 & -2.222276 & H & - ๑. 069953 & 5.219060 & 0.799918 \\
\hline H & 4.777053 & 4.268327 & -1.808478 & H & $-\odot .551426$ & 3.773934 & 1.670022 \\
\hline H & 7.828879 & 2.152632 & 0.473422 & C & -2.380783 & 5.808313 & 0.367463 \\
\hline H & 6.926320 & 1.334573 & $-\odot .809017$ & C & -5.578516 & 3.644684 & 0.120448 \\
\hline
\end{tabular}




$\begin{array}{lrrr}\mathrm{C} & -4.128505 & 3.506337 & -1.838362 \\ \mathrm{C} & -1.700867 & 6.794167 & -0.540822 \\ \mathrm{H} & -3.453567 & 5.796261 & 0.148879 \\ \mathrm{H} & -2.263741 & 6.092492 & 1.422216 \\ \mathrm{H} & -5.693761 & 3.607249 & -258.860200 \\ \mathrm{C} & -6.666954 & 3.894719 & 11.827300 \\ \mathrm{C} & -5.229036 & 3.779567 & 15.90890 \odot \\ \mathrm{H} & -3.143836 & 3.337467 & -2.260443 \\ \mathrm{C} & -1.044652 & 7.874010 & -0.113161 \\ \mathrm{H} & -1.791542 & 6.588981 & -1.606785 \\ \mathrm{H} & -7.650278 & 4.041183 & -\odot .274711 \\ \mathrm{C} & -6.508676 & 3.983526 & -2.104375 \\ \mathrm{H} & -5.091116 & 3.841626 & -3.723776 \\ \mathrm{H} & -0.597827 & 8.581687 & -0.805615 \\ \mathrm{H} & -0.946490 & 8.102488 & 0.946273 \\ \mathrm{C} & -7.679554 & 4.323376 & -2.994644 \\ \mathrm{H} & -7.749604 & 5.408602 & -3.145651 \\ \mathrm{H} & -7.580462 & 3.865661 & -3.984204 \\ \mathrm{H} & -8.627149 & 3.995004 & -2.556392 \\ \mathrm{C} & 1.373569 & 2.979013 & -2.279534 \\ \mathrm{H} & 1.318691 & 3.195838 & -3.348958 \\ \mathrm{H} & 2.233420 & 2.343348 & -2.062653 \\ \mathrm{C} & 1.284055 & 4.166857 & -1.300535 \\ \mathrm{H} & 0.689080 & 4.978494 & -1.729057 \\ \mathrm{H} & 2.242912 & 4.559211 & -0.947689 \\ \mathrm{C} & 0.475716 & 3.423850 & -0.201905 \\ \mathrm{H} & 1.872324 & 1.979970 & 2.548550 \\ \mathrm{H} & 1.210582 & 2.970330 & -.463837\end{array}$

TS1 14

B3LYP SCF energy: $\quad-5535.31825166$ a.u. B3LYP enthalpy:

B3LYP free energy:

-5535.31825166 a.u

-5533.284123 a.u.
-5533.552402 a.u.

M06 SCF energy in solution: $\quad-5534.55306478$

a.u.

M06 enthalpy in solution:

M06 free energy in solution:

-5532.518936 a.u.

$-5532.787215$

a.u.

Imaginary frequency:

$-261.4772 \mathrm{~cm}-1$

Cartesian coordinates

\begin{tabular}{|c|c|c|c|}
\hline ATOM & $\mathrm{X}$ & $\mathrm{Y}$ & Z \\
\hline $\mathrm{P}$ & $\odot .0 \odot \odot \odot \odot \odot$ & -5.707025 & 3.568585 \\
\hline$P$ & 0.000000 & -6.655100 & 3.838698 \\
\hline 0 & ๑. . ०००००० & -5.190746 & 3.713634 \\
\hline 0 & 0.000000 & -3.109655 & 3.280693 \\
\hline 0 & 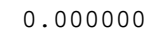 & -1.071515 & 7.851178 \\
\hline 0 & $\odot . \odot \odot \odot \odot \odot \odot$ & -1.770937 & 6.535476 \\
\hline 0 & 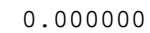 & -7.644673 & 3.985621 \\
\hline 0 & $\odot .00000 \odot$ & -6.478197 & 3.918026 \\
\hline 0 & $\odot .0 \odot \odot \odot \odot \diamond$ & -5.038502 & 3.768139 \\
\hline 0 & $\odot . \odot \odot \odot \odot \odot \diamond$ & $-\odot .619823$ & 8.554830 \\
\hline C & 0.000000 & -0.999873 & 8.096612 \\
\hline C & $\odot .00 \odot \odot \odot \odot$ & -7.637723 & 4.248135 \\
\hline C & 1.718419 & -7.707476 & 5.331872 \\
\hline C & 2.276139 & -7.524794 & 3.781905 \\
\hline C & 2.757158 & -8.590513 & 3. 921992 \\
\hline C & 2.652737 & 1.414408 & 2.935913 \\
\hline C & 1.901893 & 1.374037 & 3.144595 \\
\hline C & -0.122294 & 2.273518 & 2.304984 \\
\hline C & -0.556590 & 1.307649 & 4.131215 \\
\hline C & 0.127295 & 0.720015 & 4.939395 \\
\hline C & 1. 266888 & 2.261940 & 4.525312 \\
\hline C & 1.709943 & ๑. 482494 & 3. 398839 \\
\hline C & 1.059090 & 1.896825 & 1.879450 \\
\hline C & 2.960507 & 1.204952 & 2.954582 \\
\hline C & 2.967144 & -0.606707 & -1.119648 \\
\hline C & 2.540399 & -0.547678 & -2.444330 \\
\hline C & 3.270229 & -1.132221 & -3.496182 \\
\hline C & 4.434816 & -1.859374 & -3.136474 \\
\hline C & 4.964764 & -1.838072 & -1.813992 \\
\hline C & 4.190144 & -1.225717 & -0.827776 \\
\hline C & 6.356986 & -2.420061 & -1.452438 \\
\hline
\end{tabular}

\begin{tabular}{|c|c|c|}
\hline 7.446697 & -1.805811 & -2.365988 \\
\hline 6.374134 & -3.961727 & -1.569945 \\
\hline 6.751018 & -2.088316 & ๑. .๑4925 \\
\hline 2.766198 & $-\odot .919546$ & -4.952623 \\
\hline 1. 787502 & -2.047453 & -5.360535 \\
\hline 1.986734 & $\odot .416513$ & -5.065864 \\
\hline 3.921747 & -0.802775 & -5.976692 \\
\hline 4.655736 & -3.825575 & -4.524544 \\
\hline 3.110793 & 1.506417 & ๑. 893166 \\
\hline 4.254324 & 1.912345 & 0.194326 \\
\hline 2.772966 & 2.195403 & 2.064760 \\
\hline 3.525764 & 3.273299 & 2.558457 \\
\hline 4.573173 & 3.744676 & 1.727233 \\
\hline 5.013761 & 3. 023012 & 0.585528 \\
\hline 6.326865 & 3.371586 & -0.163852 \\
\hline 6.617693 & 2.365477 & -1.298599 \\
\hline 7.509537 & 3. 296814 & $\odot .834856$ \\
\hline 4.528755 & 6.111780 & 1.700414 \\
\hline 6.289473 & 4.776229 & -0.813152 \\
\hline 3.170259 & 3.796128 & 3.982042 \\
\hline 4. 241485 & 4.697546 & 4.640564 \\
\hline 1.819160 & 4.549593 & 3.956823 \\
\hline 3.027347 & 2.572505 & 4. 929617 \\
\hline-1.636028 & -1.596261 & 1.624912 \\
\hline-1.927287 & -2.936395 & 1.914853 \\
\hline-2.470427 & -3.319389 & 3.144764 \\
\hline-2.730388 & -2.287943 & 4.091577 \\
\hline-2.289488 & -0.955405 & 3.900596 \\
\hline-1.795778 & -0.636744 & 2.623874 \\
\hline-2.263445 & 0.162347 & 4.982791 \\
\hline-2.277762 & -0.362193 & 6.438745 \\
\hline-3.432843 & 1.154154 & 4.771863 \\
\hline-0.936842 & 0.960864 & 4.846905 \\
\hline-1.865243 & -5.184625 & 4.732875 \\
\hline-4.769664 & -2.295790 & 5.311320 \\
\hline-2.687492 & -4.820929 & 3.470836 \\
\hline - 2.195884 & -5.725757 & 2. 318917 \\
\hline-4.899080 & -1.083301 & -2.677219 \\
\hline-3.664097 & -0.693882 & -3.262539 \\
\hline-4.572598 & -0.751822 & -5.733976 \\
\hline-2.552060 & -0.626036 & -2.411690 \\
\hline-2.610579 & -1.035599 & -1.074459 \\
\hline-3.822723 & -1.507566 & -0.570675 \\
\hline-4.990136 & -1.557399 & -1.345688 \\
\hline-6.719429 & ๑. 229739 & -3.390025 \\
\hline-2.202186 & -1.221660 & -5.245984 \\
\hline-5.866360 & -3.552389 & -0.077706 \\
\hline-6.247382 & -2.162045 & -0.655561 \\
\hline-6.679109 & -1.232715 & ๑. 505712 \\
\hline-3.091004 & 1.115113 & -4.952058 \\
\hline-4.175508 & -5.172268 & 3. 710108 \\
\hline-3.420564 & -0.385195 & -4.770164 \\
\hline-7.480160 & -2.407197 & -1.554352 \\
\hline 3.21 & -1.654279 & 4.778026 \\
\hline 3.072728 & 0.055577 & 3.006840 \\
\hline 1.168519 & -5.974606 & 3.911752 \\
\hline 2.810163 & -5.879371 & 3. 150236 \\
\hline-1.46 & -2.812373 & -2.360191 \\
\hline$-\odot .232197$ & -4.698695 & -3.380030 \\
\hline 3.992928 & -5.992895 & -1.356936 \\
\hline 2.587650 & -6.706879 & -0.464269 \\
\hline 1.609487 & -0.038914 & -2.658354 \\
\hline 4.537082 & -1.228547 & ๑. 197008 \\
\hline 7.487035 & -0.717030 & -2.243733 \\
\hline 8.428890 & -2.207933 & -2.090344 \\
\hline 7.266569 & -2.026031 & -3.418595 \\
\hline 6.227575 & -4.301953 & $-2.5950 \odot \odot$ \\
\hline 7.341877 & -4.348891 & -1.229566 \\
\hline 5.595608 & -4.399483 & -0.935037 \\
\hline 6.087378 & -2.562616 & $\odot .736997$ \\
\hline 7.762103 & -2.464602 & ๑. 192774 \\
\hline 6.763414 & -1.009462 & 0.197322 \\
\hline 1.398370 & -1.860468 & -6.368744 \\
\hline 2.255743 & -3.033767 & -5.363554 \\
\hline$\odot .937006$ & -2.089587 & -4.671538 \\
\hline
\end{tabular}




\begin{tabular}{|c|c|c|c|}
\hline $\mathrm{H}$ & 1.737479 & ๑. 595368 & -6.117092 \\
\hline $\mathrm{H}$ & 1.041262 & $\odot .415723$ & -4.514787 \\
\hline $\mathrm{H}$ & 2.589217 & 1.262403 & -4.716647 \\
\hline $\mathrm{H}$ & 4.616068 & -0.005295 & -5.688213 \\
\hline $\mathrm{H}$ & 4.503314 & -1.715042 & -6.089906 \\
\hline $\mathrm{H}$ & 3.504838 & $-\odot .543496$ & -6.956471 \\
\hline $\mathrm{H}$ & 4.179004 & -3.727268 & -5.504729 \\
\hline $\mathrm{H}$ & 5.513110 & -4.496935 & -4.626237 \\
\hline $\mathrm{H}$ & 3.940838 & -4.252437 & -3.813566 \\
\hline $\mathrm{H}$ & 4.552924 & 1.353798 & -0.680257 \\
\hline H & 7.575546 & 2.621915 & -1.762829 \\
\hline $\mathrm{H}$ & 6.698999 & 1.336603 & -0.932021 \\
\hline $\mathrm{H}$ & 5.855988 & 2.391990 & -2.086894 \\
\hline $\mathrm{H}$ & 7.386657 & 4.005807 & 1.655995 \\
\hline $\mathrm{H}$ & 7.601077 & 2. 288774 & 1. 255968 \\
\hline $\mathrm{H}$ & 8.447990 & 3.530050 & 0. 317887 \\
\hline $\mathrm{H}$ & 4.314662 & 6.155024 & $\odot .626285$ \\
\hline $\mathrm{H}$ & 3.588415 & 6.199853 & 2.254123 \\
\hline $\mathrm{H}$ & 5.185936 & 6.940369 & 1.973062 \\
\hline $\mathrm{H}$ & 6.320508 & 5.572392 & -0.069805 \\
\hline $\mathrm{H}$ & 7.163711 & 4.901322 & -1.462407 \\
\hline $\mathrm{H}$ & 5.395911 & 4.905857 & -1.436131 \\
\hline $\mathrm{H}$ & 5.235311 & 4.243450 & 4.600000 \\
\hline $\mathrm{H}$ & 4.307481 & 5.692401 & 4.200665 \\
\hline $\mathrm{H}$ & 3.975294 & 4.830366 & 5.695395 \\
\hline $\mathrm{H}$ & 1.556304 & 4.887589 & 4.966032 \\
\hline $\mathrm{H}$ & 1.856827 & 5.433522 & 3. 310246 \\
\hline $\mathrm{H}$ & 1.005531 & 3.906860 & 3.601120 \\
\hline $\mathrm{H}$ & 2.217633 & 1.895791 & 4.642353 \\
\hline $\mathrm{H}$ & 3.959364 & 1.996864 & 4.967150 \\
\hline $\mathrm{H}$ & 2.808270 & 2.920554 & 5.944876 \\
\hline $\mathrm{H}$ & -1.732577 & -3.686024 & 1.159480 \\
\hline $\mathrm{H}$ & -1.526319 & ๑. 387907 & 2.400333 \\
\hline $\mathrm{H}$ & -3.235883 & -0.780767 & 6.743316 \\
\hline $\mathrm{H}$ & -1.512491 & -1.128577 & 6.598486 \\
\hline $\mathrm{H}$ & -2.060534 & 0.475365 & 7.111666 \\
\hline $\mathrm{H}$ & -3.387165 & 1.949871 & 5.525592 \\
\hline $\mathrm{H}$ & -3.390148 & 1.626819 & 3.785484 \\
\hline $\mathrm{H}$ & -4.408102 & ๑. 666233 & 4.867656 \\
\hline $\mathrm{H}$ & $-\odot .869361$ & 1.690237 & 5.661436 \\
\hline $\mathrm{H}$ & -0.066878 & ๑. 296821 & 4.910496 \\
\hline $\mathrm{H}$ & -0.873860 & 1.522960 & 3.910386 \\
\hline $\mathrm{H}$ & -1.957217 & -6.257211 & 4.943483 \\
\hline $\mathrm{H}$ & -0.803848 & -4.954760 & 4.583951 \\
\hline $\mathrm{H}$ & -2.210287 & -4.632351 & 5.608680 \\
\hline $\mathrm{H}$ & -4.904429 & -1.219729 & 5.455306 \\
\hline $\mathrm{H}$ & -5.299905 & -2.604386 & 4.403453 \\
\hline $\mathrm{H}$ & -5.183450 & -2.825748 & 6.172373 \\
\hline $\mathrm{H}$ & -2.778711 & -5.581619 & 1.401363 \\
\hline $\mathrm{H}$ & -1.138200 & -5.564644 & 2.086717 \\
\hline $\mathrm{H}$ & -2.316877 & -6.773290 & 2.615516 \\
\hline $\mathrm{H}$ & $-5.44090 \odot$ & $-\odot .099583$ & -5.633581 \\
\hline $\mathrm{H}$ & -4.203594 & $-\odot .642118$ & -6.760852 \\
\hline $\mathrm{H}$ & -4.908259 & -1.783882 & -5.604622 \\
\hline $\mathrm{H}$ & -1.610285 & $-\odot .261140$ & -2.804076 \\
\hline $\mathrm{H}$ & -3.863106 & -1.846696 & ๑. 455398 \\
\hline $\mathrm{H}$ & -7.622780 & ๑. 118597 & -3.994492 \\
\hline $\mathrm{H}$ & -6.996735 & ๑. 497998 & -2.362717 \\
\hline $\mathrm{H}$ & -6.098588 & 1.034206 & -3.802281 \\
\hline $\mathrm{H}$ & -2.014837 & -1.028735 & -6.308285 \\
\hline $\mathrm{H}$ & -1.282193 & $-\odot .980804$ & -4.706926 \\
\hline $\mathrm{H}$ & -2.394616 & -2.294847 & -5.129705 \\
\hline $\mathrm{H}$ & -5.561816 & -4.236270 & -0.878189 \\
\hline $\mathrm{H}$ & -5.052648 & -3.504488 & ๑. 651662 \\
\hline $\mathrm{H}$ & -6.734154 & -3.992101 & ๑. 427275 \\
\hline $\mathrm{H}$ & -7.548275 & -1.655444 & 1.024102 \\
\hline $\mathrm{H}$ & -5.880637 & -1.097459 & 1.243415 \\
\hline $\mathrm{H}$ & -6.959384 & $-\odot .24006 \odot$ & 0.134166 \\
\hline $\mathrm{H}$ & -3.948475 & 1.738349 & -4.674939 \\
\hline $\mathrm{H}$ & -2.238908 & 1.428935 & -4.341252 \\
\hline $\mathrm{H}$ & -2.854544 & 1.322338 & -6.003051 \\
\hline $\mathrm{H}$ & -4.803961 & -4.835313 & 2.877213 \\
\hline $\mathrm{H}$ & -4.286143 & -6.260409 & 3.787071 \\
\hline $\mathrm{H}$ & -4.557036 & -4.740284 & 4.634816 \\
\hline $\mathrm{H}$ & -8.224268 & -2.957145 & -0.965664 \\
\hline
\end{tabular}

\begin{tabular}{|c|c|c|c|}
\hline $\mathrm{H}$ & -7.955748 & -1.483494 & -1.888218 \\
\hline $\mathrm{H}$ & -7.238513 & -3.004077 & -2.436637 \\
\hline $\mathrm{Rh}$ & -0.083572 & 1.019960 & -0.082178 \\
\hline 0 & -2.244787 & 1.893715 & ๑. 299376 \\
\hline C & $\odot .104008$ & 2.411671 & -1.712720 \\
\hline $\mathrm{S}$ & -2.930801 & 3.186751 & 0.554556 \\
\hline 0 & $-\odot .962191$ & 2.390513 & -2.302589 \\
\hline C & $-\odot .502001$ & 4.198343 & 0.610026 \\
\hline $\mathrm{N}$ & -1.860179 & 4.402667 & 0.041578 \\
\hline 0 & -3.401322 & 3.412607 & 1.930437 \\
\hline C & -4.309274 & 3.412385 & -0.555254 \\
\hline $\mathrm{H}$ & -0.087255 & 5.204349 & 0.737209 \\
\hline $\mathrm{H}$ & -0.574823 & 3.768586 & 1.617523 \\
\hline C & -2.393924 & 5.776889 & $\odot .264648$ \\
\hline C & -5.577343 & 3.597998 & ๑. . 000638 \\
\hline C & -4.100515 & 3.449482 & -1.937263 \\
\hline C & -1.705213 & 6.757036 & -0.643257 \\
\hline $\mathrm{H}$ & -3.463021 & 5.757613 & ๑. 029071 \\
\hline $\mathrm{H}$ & -2.295435 & 6.073078 & 1. 318057 \\
\hline $\mathrm{H}$ & -5.707025 & 3.568585 & -261.477200 \\
\hline C & -6.655100 & 3.838698 & 11.349400 \\
\hline C & -5.190746 & 3.713634 & 15.540300 \\
\hline $\mathrm{H}$ & -3.109655 & 3.280693 & -2.344736 \\
\hline C & -1.071515 & 7.851178 & -0.217978 \\
\hline $\mathrm{H}$ & -1.770937 & 6.535476 & -1.707767 \\
\hline $\mathrm{H}$ & -7.644673 & 3.985621 & -0.425434 \\
\hline C & -6.478197 & 3. 918026 & -2.239005 \\
\hline $\mathrm{H}$ & -5.038502 & 3.768139 & -3.837988 \\
\hline $\mathrm{H}$ & -0.619823 & 8.554830 & -0.911427 \\
\hline $\mathrm{H}$ & $-\odot .999873$ & 8. 096612 & 0.839838 \\
\hline C & -7.637723 & 4.248135 & -3.147592 \\
\hline $\mathrm{H}$ & -7.707476 & 5.331872 & -3.309015 \\
\hline $\mathrm{H}$ & -7.524794 & 3.781905 & -4.131663 \\
\hline $\mathrm{H}$ & -8.590513 & 3.921992 & -2.719060 \\
\hline C & 1.414408 & 2.935913 & -2.293755 \\
\hline $\mathrm{H}$ & 1.374037 & 3.144595 & -3.365465 \\
\hline $\mathrm{H}$ & 2.273518 & 2.304984 & -2.060136 \\
\hline C & 1.307649 & 4.131215 & -1.325928 \\
\hline $\mathrm{H}$ & ๑. 720015 & 4.939395 & -1.771019 \\
\hline $\mathrm{H}$ & 2.261940 & 4.525312 & -0.962444 \\
\hline C & ๑. 482494 & 3. 398839 & -0.232972 \\
\hline $\mathrm{H}$ & 1.896825 & 1.879450 & 2.621602 \\
\hline $\mathrm{H}$ & 1. 204952 & 2.954582 & 0.452192 \\
\hline
\end{tabular}

TS1 15

B3LYP SCF energy: $\quad-5535.31822347$ a.u. B3LYP enthalpy: $\quad-5533.283918$ a.u. B3LYP free energy: $\quad-5533.552009$ a.u. M06 SCF energy in solution: $\quad-5534.55002772$ a.u.

M06 enthalpy in solution: $\quad-5532.515722$ a.u. M06 free energy in solution: $\quad-5532.783813$ a.u.

Imaginary frequency: $\quad-256.2080 \mathrm{~cm}-1$

Cartesian coordinates

\begin{tabular}{|c|c|c|c|}
\hline ATOM & X & $\mathrm{Y}$ & Z \\
\hline$P$ & $\odot . ๑ \odot \odot \odot \odot \odot ~$ & -5.811555 & 3.457084 \\
\hline$P$ & 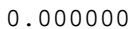 & -6.821860 & 3.732789 \\
\hline 0 & $\odot . \odot \odot \odot \odot \odot \odot$ & -5.411440 & 3.676883 \\
\hline 0 & $\odot . ๑ \odot \odot \odot \odot \odot ~$ & -3.308456 & 3.292892 \\
\hline 0 & $\odot . ๑ \odot \odot \odot \odot \odot$ & -1.269067 & 7.849569 \\
\hline 0 & $\odot . ๑ \odot \odot \odot \odot \odot$ & -2.004478 & 6.565709 \\
\hline 0 & 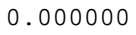 & -7.802086 & 3.846036 \\
\hline 0 & 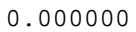 & -6.688032 & 3.837886 \\
\hline 0 & $\odot . ๑ \odot \odot \odot \odot \odot ~$ & -5.292262 & 3.752985 \\
\hline 0 & $\odot . ๑ \odot \odot \odot \odot \odot$ & -0.843161 & 8.573283 \\
\hline c & $\odot . ๑ \odot \odot \odot \odot \odot$ & -1.165450 & 8.068963 \\
\hline c & $\odot . \odot \odot \odot \odot \odot \odot$ & -7.882157 & 4.149752 \\
\hline c & 1.943081 & -7.782756 & 3.706759 \\
\hline c & 2.510972 & -8.812033 & 3.785580 \\
\hline c & 2.895837 & -7.989652 & 5.233798 \\
\hline c & 2.697599 & 1.203531 & 3.067215 \\
\hline
\end{tabular}




\begin{tabular}{|c|c|c|c|c|c|c|c|}
\hline C & 2.332247 & 1.114760 & 3.297049 & H & 2.844541 & -6.520429 & $-\odot .951675$ \\
\hline C & -0.074799 & 2. 092938 & 2.463608 & H & 1.485303 & $\odot .138455$ & -2.698684 \\
\hline C & $-\odot .532397$ & 1.089770 & 4.235961 & H & 4.564068 & $-\odot .994861$ & ๑. 017025 \\
\hline C & 0.172915 & 0.452203 & 5.028498 & $\mathrm{H}$ & 7.403958 & $-\odot .209769$ & -2.445991 \\
\hline C & 1.361795 & 2.040238 & 4.662599 & H & 8.429719 & -1.651943 & -2.411588 \\
\hline C & 1.829926 & $\odot .340017$ & 3.446874 & $\mathrm{H}$ & 7.219351 & -1.455733 & -3.694136 \\
\hline C & 1.156849 & 1.731155 & 1.899005 & $\mathrm{H}$ & 6.350056 & -3.835011 & -2.982419 \\
\hline C & 3.147599 & 1.105223 & 3.012756 & $\mathrm{H}$ & 7.486657 & -3.897630 & -1.636467 \\
\hline C & 2.921820 & -0.408795 & -1.225666 & $\mathrm{H}$ & 5.750963 & -4.065192 & -1.323332 \\
\hline C & 2.450630 & -0.322085 & -2.533984 & $\mathrm{H}$ & 6.184881 & -2.300490 & 0.448547 \\
\hline C & 3.178813 & -0.819049 & -3.631224 & H & 7.838241 & -2.079873 & -0.125470 \\
\hline C & 4.393416 & -1.491984 & -3.336729 & H & 6.763890 & -0.684249 & -0.013297 \\
\hline C & 4.960714 & -1.497243 & -2.029556 & H & 1.271051 & -1.534797 & -6.484509 \\
\hline C & 4.185738 & $-\odot .968992$ & $-\odot .996183$ & H & 2.213693 & -2.697595 & -5.545581 \\
\hline C & 6.391573 & -2.018431 & -1.733312 & H & 0.863637 & -1.853134 & -4.788060 \\
\hline C & 7.419017 & -1.289941 & -2.634911 & H & 1.466859 & 0.920435 & -6.131631 \\
\hline C & 6.493334 & -3.547321 & -1.941181 & $\mathrm{H}$ & 0.837142 & 0.639956 & -4.516416 \\
\hline C & 6.804101 & -1.749089 & -0.268350 & H & 2.326968 & 1.581808 & -4.734160 \\
\hline C & 2.619283 & -0.574906 & -5.062736 & $\mathrm{H}$ & 4.388574 & 0.480333 & -5.807258 \\
\hline C & 1.693922 & -1.738932 & -5.493376 & H & 4.367489 & -1.214410 & -6.280587 \\
\hline C & 1.761631 & $\odot .716483$ & -5.096886 & H & 3.275056 & $-\odot .071321$ & -7.067419 \\
\hline C & 3.735417 & $-\odot .345589$ & -6.111514 & H & 4.185761 & -3.250356 & -5.798933 \\
\hline C & 4.676302 & -3.373496 & -4.828527 & H & 5.560498 & -4.001744 & -4.968906 \\
\hline C & 3.030568 & 1.581308 & ๑.9๑8288 & H & 3.987353 & -3.863369 & -4.132892 \\
\hline C & 4.133656 & 2.084343 & 0.221212 & $\mathrm{H}$ & 4.394844 & 1.648961 & -0.734136 \\
\hline C & 2.649544 & 2.214097 & 2.105506 & H & 6.141456 & 4.343837 & -2.312406 \\
\hline C & 3.417531 & 3.221277 & 2.693654 & H & 5.053673 & 2.977502 & -2.100631 \\
\hline C & 4.614188 & 3.602332 & 2.019210 & H & 4.539972 & 4.600680 & -1.603683 \\
\hline C & 4.919910 & 3.141785 & 0.717588 & H & 7.682457 & 2.537081 & ๑. 601552 \\
\hline C & 6.013419 & 3.714259 & $-\odot .231118$ & H & 6.845290 & 1.743527 & -0.739328 \\
\hline C & 5.391241 & 3.911041 & -1.641857 & H & 7.938630 & 3.099860 & -1.056120 \\
\hline C & 7.186218 & 2.712940 & $-\odot .358818$ & H & 6.361815 & 3.113566 & 3.950435 \\
\hline C & 6.622983 & 3.902876 & 3.236934 & $\mathrm{H}$ & 7.282649 & 3.484466 & 2.470799 \\
\hline C & 6.566791 & 5.102531 & $\odot .168398$ & H & 7.142122 & 4.709655 & 3.758661 \\
\hline C & 2.923957 & 3.934212 & 3.979825 & H & 7.211520 & 5.080526 & 1.046426 \\
\hline C & 3.890091 & 3.735803 & 5.173144 & H & 7.169255 & 5.484867 & $-\odot .663469$ \\
\hline C & 2.765533 & 5.448403 & 3.692503 & H & 5.763381 & 5.820304 & ๑. 358189 \\
\hline C & 1.545720 & 3.404070 & 4.433006 & H & 4.118473 & 2.674768 & 5.330241 \\
\hline C & -1.544547 & -1.722890 & 1.609829 & H & 4.824094 & 4.280271 & 5.037926 \\
\hline C & -1.602491 & -3.096098 & 1.888886 & H & 3.421237 & 4.111986 & 6.089797 \\
\hline C & -1.973626 & -3.574462 & 3.148834 & H & 2.410402 & 5.963578 & 4.592965 \\
\hline C & -2.255161 & -2.606113 & 4.151628 & H & 3.712150 & 5.900270 & 3.390360 \\
\hline C & -2.393059 & -1.229924 & 3.857273 & H & 2.029353 & 5.619813 & 2.897468 \\
\hline C & -1.976809 & $-\odot .815756$ & 2.579174 & H & $\odot .774400$ & 3.531857 & 3.664955 \\
\hline C & -3.036541 & $-\odot .149286$ & 4.773037 & H & 1.576374 & 2.345762 & 4.717676 \\
\hline C & -3.672683 & -0.655229 & 6.088004 & H & 1.220133 & 3.967419 & 5.313433 \\
\hline C & -4.199650 & ๑.499801 & 3.973888 & H & -1.346857 & -3.798076 & 1.106735 \\
\hline C & -1.993827 & 0.939604 & 5.120848 & H & -2.017687 & 0.237821 & 2.326212 \\
\hline C & -1.229211 & -5.639826 & $4.5040 \odot \odot$ & H & -4.373198 & -1.476244 & 5.917723 \\
\hline C & -1.301550 & -2.846794 & 6.293160 & H & -2.941575 & $-\odot .979757$ & 6.829783 \\
\hline C & -2.160294 & -5.093948 & 3.393668 & H & -4.230528 & $\odot .176702$ & 6.533629 \\
\hline C & -1.875863 & -5.911277 & 2.114518 & H & -4.674186 & 1.278022 & 4.582406 \\
\hline C & -4.982950 & -1.187596 & -2.540701 & H & -3.869488 & 0.973850 & 3.048446 \\
\hline C & -3.794660 & -0.703288 & -3.151187 & H & -4.962209 & $-\odot .249165$ & 3.729008 \\
\hline C & -4.805704 & $-\odot .66705 \odot$ & -5.584555 & H & -2.455872 & 1.718048 & 5.739186 \\
\hline C & -2.655855 & -0.612613 & -2.339038 & H & -1.150754 & 0.520323 & 5.683172 \\
\hline C & -2.640931 & -1.088361 & -1.022685 & H & -1.595554 & 1.425093 & 4.223676 \\
\hline C & -3.802873 & -1.658443 & -0.501347 & H & -1.231940 & -6.736249 & 4.481207 \\
\hline C & -4.995027 & -1.736929 & -1.234907 & H & $-\odot .197261$ & -5.300293 & 4.361900 \\
\hline C & -6.891982 & $\odot .068906$ & -3.100043 & H & -1.558456 & -5.332555 & 5.496613 \\
\hline C & -2.409418 & -1.118080 & -5.210123 & H & -0.431572 & -3.412205 & 5.940225 \\
\hline c & -5.709140 & -3.819970 & $-\odot . \odot \odot 1227$ & H & -1.033232 & -1.786391 & 6.350659 \\
\hline C & -6.192070 & -2.443467 & -0.534889 & H & -1.591243 & -3.202241 & 7.284486 \\
\hline C & -6.658637 & -1.575865 & 0.659805 & $\mathrm{H}$ & -2.529235 & -5.621220 & 1.284067 \\
\hline C & -3.326357 & 1.188795 & -4.783847 & H & -0.834867 & -5.815348 & 1.786322 \\
\hline C & -3.633256 & -5.355846 & 3.797186 & H & -2.065211 & -6.970410 & 2.319748 \\
\hline C & -3.621508 & $-\odot .323599$ & -4.652327 & H & -5.682098 & $-\odot . \odot 4 \odot 765$ & -5.411713 \\
\hline C & -7.422603 & -2.747738 & -1.419106 & $\mathrm{H}$ & -4.483876 & -0.493861 & -6.618608 \\
\hline H & 3.348314 & -1.747086 & 4.603729 & H & -5.113849 & -1.711782 & -5.497236 \\
\hline H & 3.041701 & $\odot .059690$ & 2.951194 & H & -1.750763 & $-\odot .178797$ & -2.747558 \\
\hline H & 1.699532 & -6.181365 & 3. 461841 & H & -3.782546 & -2.050902 & 0.506202 \\
\hline $\mathrm{H}$ & 3.287816 & -5.825946 & 2.663006 & $\mathrm{H}$ & -7.797896 & -0.038343 & -3.701304 \\
\hline H & -1.479515 & -2.736601 & -2.431223 & H & -7.167088 & 0.242831 & -2.051953 \\
\hline H & $-\odot .207276$ & -4.497111 & -3.612302 & H & -6.314677 & 0.931388 & -3.454714 \\
\hline \multirow[t]{2}{*}{ H } & 4.169750 & -5.685652 & -1.862125 & H & -2.262634 & $-\odot .873702$ & -6.268308 \\
\hline & & & & & & & \\
\hline
\end{tabular}




\begin{tabular}{|c|c|c|c|}
\hline H & -1.474677 & $-\odot .888403$ & -4.691163 \\
\hline $\mathrm{H}$ & -2.583218 & -2.198271 & -5.137138 \\
\hline $\mathrm{H}$ & -5.367749 & -4.458905 & $-\odot .823763$ \\
\hline $\mathrm{H}$ & -4.894019 & -3.738443 & ๑. 723619 \\
\hline $\mathrm{H}$ & -6.539109 & -4.331260 & $\odot .499342$ \\
\hline $\mathrm{H}$ & -7.480494 & -2.072498 & 1.189455 \\
\hline $\mathrm{H}$ & -5.851621 & -1.401465 & 1.379809 \\
\hline $\mathrm{H}$ & -7.019502 & -0.597504 & ○. 321474 \\
\hline $\mathrm{H}$ & -4.183532 & 1.781412 & -4.445965 \\
\hline $\mathrm{H}$ & -2.455828 & 1.496545 & -4.196745 \\
\hline $\mathrm{H}$ & -3.138200 & 1.443300 & -5.834202 \\
\hline $\mathrm{H}$ & -4.318700 & -5.046259 & 2.999544 \\
\hline $\mathrm{H}$ & -3.787320 & -6.427464 & 3.973043 \\
\hline $\mathrm{H}$ & -3.897972 & -4.818870 & 4.710804 \\
\hline $\mathrm{H}$ & -8.112523 & -3.370625 & $-\odot .837293$ \\
\hline $\mathrm{H}$ & -7.970354 & -1.851179 & -1.713132 \\
\hline $\mathrm{H}$ & -7.155715 & -3.295591 & -2.326042 \\
\hline $\mathrm{Rh}$ & -0.162106 & 1.037021 & $-\odot .050095$ \\
\hline 0 & -2.341216 & 1.872000 & ๑. 442563 \\
\hline C & -0.063352 & 2.482052 & -1.639392 \\
\hline S & -3.045386 & 3.149817 & 0.730080 \\
\hline 0 & -1.151205 & 2.439165 & -2.188307 \\
\hline C & -0.635745 & 4.194214 & ๑.744305 \\
\hline $\mathrm{N}$ & -2.008959 & 4.391411 & ๑. 211687 \\
\hline 0 & -3.483867 & 3. 349192 & 2.120246 \\
\hline C & -4.457910 & 3.363981 & -0.339132 \\
\hline $\mathrm{H}$ & -0.234896 & 5.202311 & ๑. 898918 \\
\hline $\mathrm{H}$ & -0.677384 & 3.725621 & 1.736063 \\
\hline C & $-2.5590 \odot 9$ & 5.752642 & $\odot .474065$ \\
\hline C & -5.713854 & 3.507653 & ๑. 255852 \\
\hline C & -4.290783 & 3.428866 & -1.725676 \\
\hline C & -1.907541 & 6.761207 & -0.430020 \\
\hline $\mathrm{H}$ & -3.633033 & 5.719763 & ๑. 263624 \\
\hline $\mathrm{H}$ & -2.439754 & 6.029401 & 1.530476 \\
\hline $\mathrm{H}$ & -5.811555 & 3.457084 & $-256.2080 \odot \odot$ \\
\hline C & -6.821860 & 3.732789 & 6.853700 \\
\hline C & -5.411440 & 3.676883 & 12.505200 \\
\hline $\mathrm{H}$ & -3.308456 & 3. 292892 & -2.164620 \\
\hline C & -1.269067 & 7.849569 & ๑. $0 \odot 2874$ \\
\hline $\mathrm{H}$ & -2.004478 & 6.565709 & -1.497266 \\
\hline $\mathrm{H}$ & -7.802086 & 3.846036 & -0.103689 \\
\hline C & -6.688032 & 3.837886 & -1.951162 \\
\hline $\mathrm{H}$ & -5.292262 & 3.752985 & -3.592970 \\
\hline $\mathrm{H}$ & -0.843161 & 8.573283 & -0.686187 \\
\hline $\mathrm{H}$ & -1.165450 & 8.068963 & 1. 063700 \\
\hline C & -7.882157 & 4.149752 & -2.820501 \\
\hline $\mathrm{H}$ & -7.782756 & 3.706759 & -3.816661 \\
\hline $\mathrm{H}$ & -8.812033 & 3.785580 & -2.372648 \\
\hline $\mathrm{H}$ & -7.989652 & 5.233798 & -2.957269 \\
\hline C & 1.203531 & 3.067215 & -2.257377 \\
\hline $\mathrm{H}$ & 1.114760 & 3.297049 & -3.321588 \\
\hline H & 2.092938 & 2.463608 & -2.070251 \\
\hline C & 1.089770 & 4.235961 & -1.259614 \\
\hline $\mathrm{H}$ & $\odot .452203$ & 5.028498 & -1.661654 \\
\hline $\mathrm{H}$ & 2.040238 & 4.662599 & -0.924288 \\
\hline C & 0.340017 & 3.446874 & -0.152886 \\
\hline $\mathrm{H}$ & 1.731155 & 1.899005 & 2.585967 \\
\hline $\mathrm{H}$ & 1.105223 & 3.012756 & 0. 490232 \\
\hline
\end{tabular}

TS1_16

B3LYP SCF energy: $\quad-5535.31706620$ a.u. B3LYP enthalpy:

B3LYP free energy:

a.u.

M06 enthalpy in solution: $\quad-5532.515622$ a.u.

M06 free energy in solution: $\quad-5532.782918$

a.u.

Imaginary frequency:

$-254.5953 \mathrm{~cm}-1$

Cartesian coordinates

\begin{tabular}{|c|c|c|}
\hline & X & $\mathrm{Y}$ \\
\hline$P$ & $\odot . \diamond \odot \odot \odot \odot \odot$ & -5.827418 \\
\hline
\end{tabular}

\begin{tabular}{|c|c|c|}
\hline 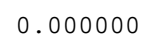 & -6.813334 & 3.649808 \\
\hline$\odot .0 \odot \odot \odot \odot \odot$ & -5.376162 & 3.559248 \\
\hline$\odot . ๑ \odot \odot \odot \odot \odot$ & -3.275763 & 3.196471 \\
\hline$\odot . ๑ \odot \odot \odot \odot \odot$ & -1.316853 & 7.826467 \\
\hline$\odot . ๑ \odot \odot \odot \odot \odot$ & -2.001687 & 6.508134 \\
\hline$\odot . \odot \odot \odot \odot \odot \odot$ & -7.800409 & 3.766488 \\
\hline$\odot . ๑ \odot \odot \odot \odot \odot$ & -6.661233 & 3.724384 \\
\hline$\odot . \diamond \odot \odot \odot \odot \odot$ & -5.242899 & 3.611941 \\
\hline$\odot . ๑ \odot \odot \odot \odot \odot$ & $-\odot .89 \odot \odot \odot 8$ & 8.546955 \\
\hline$\odot . \diamond \odot \odot \odot \odot \odot$ & -1.239194 & 8.064104 \\
\hline$\odot . ๑ \odot \odot \odot \odot \odot$ & -7.845685 & 4.008282 \\
\hline 1.965427 & -7.965887 & \\
\hline 2.530320 & -7.724742 & 3.553538 \\
\hline 2.920232 & -8.777422 & 3.637290 \\
\hline 2.722956 & 1.244399 & 3. 014317 \\
\hline 2.350645 & 1.173044 & 3.220856 \\
\hline$\odot .011112$ & 2.137896 & 2.426752 \\
\hline-0.439063 & 1.097593 & 4.202349 \\
\hline$\odot .285920$ & $\odot .45$ & \\
\hline 1.462954 & 2.0 & 4.6 \\
\hline $1.9 \odot 2405$ & $\odot .343431$ & 3.427847 \\
\hline 1.209859 & 1.6 & 1.8 \\
\hline 3.234177 & 1.1 & \\
\hline 2.996594 & $-\odot .4$ & 212 \\
\hline 2.558295 & -0.3 & -2 . \\
\hline 3.313579 & -0.8 & -3.5 \\
\hline 4.518135 & -1.5 & -3.2 \\
\hline 5.051109 & -1.5 & -1. \\
\hline 4.253355 & -0.9 & -0.87 \\
\hline 6.467855 & -2.0 & -1 \\
\hline 7.528481 & -1.3 & -2.41 \\
\hline 6.562779 & -3.5 & -1 \\
\hline 6.838059 & -1.7 & $-\Theta . c$ \\
\hline 2.789637 & $-\odot .6$ & 88616 \\
\hline 1.869122 & -1.8 & -5 \\
\hline 1.938542 & 0.6 & -5. \\
\hline 3.931400 & $-\odot .4$ & -6. \\
\hline 4.831 & -3.4 & -4. \\
\hline 2.986992 & 1.6 & \\
\hline 4.116856 & 2.1 & \\
\hline 2.5505 & 2.2 & 802 \\
\hline 3.175899 & 3.3 & \\
\hline 4.245690 & 3.9 & \\
\hline 4.8061 & 3.2 & 0.8 \\
\hline 6.1289 & & \\
\hline 5.8 & 4.2 & -1. \\
\hline 6.965200 & 2.3 & -0.1 \\
\hline 4.324548 & 6.2 & \\
\hline 7.057 & 4.6 & \\
\hline 2.760505 & 7745 & 4149 \\
\hline 3.995438 & 3.8 & \\
\hline 2.198 & 5.3 & 3. \\
\hline 1.669554 & 3.050919 & 4.717935 \\
\hline-1.542145 & -1.7 & \\
\hline-1.585946 & -3.0 & \\
\hline-1.983 & -3.5 & \\
\hline-2.30 & -2.5 & 4. \\
\hline-2.460529 & -1.203529 & 3.817831 \\
\hline-2.015920 & $-\odot .8$ & \\
\hline-3.1 & -0.1 & \\
\hline-3.803309 & $-\odot .602931$ & 05130 \\
\hline-4.310954 & 0.4 & \\
\hline-2.14324 & 1.0 & 5.6 \\
\hline-1.237850 & -5.5 & 4.58054 \\
\hline-1.40710 & -2.7 & \\
\hline-2.153248 & -5.071309 & 3.44012 \\
\hline-1.829799 & -5.909061 & \\
\hline & -1.2 & \\
\hline-3.689958 & $-\odot .802639$ & -3.222270 \\
\hline & & \\
\hline-2.571944 & -0.686895 & -2.384739 \\
\hline-2.58 & -1.146053 & -1.06247 \\
\hline & -1.725953 & \\
\hline-4.921382 & -1.830416 & -1.32250 \\
\hline - & -0.073394 & -3.25226 \\
\hline
\end{tabular}




\begin{tabular}{|c|c|c|c|}
\hline C & -2.248377 & -1.216171 & -5.243080 \\
\hline C & -5.634860 & -3.906545 & $-\odot .076288$ \\
\hline C & -6.125045 & -2.545956 & -0.643234 \\
\hline C & -6.636391 & -1.668791 & 0.525792 \\
\hline C & -3.215739 & 1.078368 & -4.865908 \\
\hline C & -3.630437 & -5.348501 & 3.817240 \\
\hline C & -3.487309 & -0.437451 & -4.723324 \\
\hline C & -7.326508 & -2.883155 & -1.555113 \\
\hline $\mathrm{H}$ & 3.375978 & -1.677914 & 4.671669 \\
\hline $\mathrm{H}$ & 3.073276 & 0.101163 & 2.989696 \\
\hline $\mathrm{H}$ & 1.714629 & -6.126040 & 3.602579 \\
\hline $\mathrm{H}$ & 3.306525 & -5.787550 & 2.803564 \\
\hline $\mathrm{H}$ & -1.378302 & -2.803212 & -2.415365 \\
\hline $\mathrm{H}$ & -0.071478 & -4.577013 & -3.537468 \\
\hline $\mathrm{H}$ & 4.264857 & -5.725226 & -1.662777 \\
\hline $\mathrm{H}$ & 2. 916099 & -6.539311 & -0.768125 \\
\hline $\mathrm{H}$ & 1.596554 & $\odot .107171$ & -2.667837 \\
\hline $\mathrm{H}$ & 4.605288 & $-\odot .979795$ & $\odot .145215$ \\
\hline $\mathrm{H}$ & 7.520636 & $-\odot .260334$ & -2.231744 \\
\hline $\mathrm{H}$ & 8.528244 & -1.713486 & -2.159457 \\
\hline $\mathrm{H}$ & 7.357095 & -1.512663 & -3.477119 \\
\hline $\mathrm{H}$ & 6.451743 & -3.879738 & -2.774457 \\
\hline $\mathrm{H}$ & 7.542740 & $-3.9400 \odot 2$ & -1.391648 \\
\hline $\mathrm{H}$ & 5.796787 & -4.091192 & -1.133803 \\
\hline $\mathrm{H}$ & 6.188599 & -2.318874 & ๑.637507 \\
\hline $\mathrm{H}$ & 7.862219 & -2.125087 & $\odot .114873$ \\
\hline $\mathrm{H}$ & 6.806259 & -0.713846 & 0.185725 \\
\hline $\mathrm{H}$ & 1.471264 & -1.624666 & -6.424415 \\
\hline $\mathrm{H}$ & 2.384878 & -2.773852 & -5.441019 \\
\hline $\mathrm{H}$ & 1. 021377 & -1.908864 & -4.732674 \\
\hline $\mathrm{H}$ & 1.671197 & $\odot .836942$ & -6.112192 \\
\hline $\mathrm{H}$ & ○. 999116 & 0.586358 & -4.509184 \\
\hline $\mathrm{H}$ & 2. 498061 & 1.519067 & -4.704565 \\
\hline $\mathrm{H}$ & 4.578747 & ๑. 393025 & -5.710252 \\
\hline $\mathrm{H}$ & 4.565723 & -1.310264 & -6.151241 \\
\hline $\mathrm{H}$ & 3.494979 & $-\odot .181029$ & -6.986261 \\
\hline $\mathrm{H}$ & 4.357289 & -3.327681 & -5.638800 \\
\hline $\mathrm{H}$ & 5.717123 & -4.070155 & -4.777149 \\
\hline $\mathrm{H}$ & 4.130197 & -3.921880 & -3.969534 \\
\hline $\mathrm{H}$ & 4.482150 & 1.644397 & -0.561871 \\
\hline $\mathrm{H}$ & 6.742634 & 4.471358 & -1.856284 \\
\hline $\mathrm{H}$ & 5. 231746 & 3.551473 & -1.934692 \\
\hline $\mathrm{H}$ & 5.242341 & 5.181970 & -1.245756 \\
\hline $\mathrm{H}$ & 7.186511 & 1.876771 & 0.824121 \\
\hline $\mathrm{H}$ & 6.472911 & 1.626005 & -0.779411 \\
\hline $\mathrm{H}$ & 7.918383 & 2.614598 & -0.610058 \\
\hline $\mathrm{H}$ & 4.669944 & 6.229132 & 0.541879 \\
\hline $\mathrm{H}$ & 3.232186 & 6.373084 & 1.586061 \\
\hline $\mathrm{H}$ & 4.765095 & 7.157934 & $2.06 \odot 489$ \\
\hline $\mathrm{H}$ & 7.233438 & 4.277970 & 1.869874 \\
\hline $\mathrm{H}$ & 8.024623 & 4.648649 & ๑. 331012 \\
\hline $\mathrm{H}$ & 6.687168 & 5.641549 & $\odot .888997$ \\
\hline $\mathrm{H}$ & 4.370938 & 2.876708 & 5.120637 \\
\hline $\mathrm{H}$ & 4.804777 & 4.522666 & 4.614968 \\
\hline $\mathrm{H}$ & 3.717549 & 4.272891 & 5.993286 \\
\hline $\mathrm{H}$ & 1.776824 & 5.639993 & 4.974327 \\
\hline $\mathrm{H}$ & 2.971490 & 6.099089 & 3.763794 \\
\hline $\mathrm{H}$ & 1.394025 & 5.451054 & 3.258318 \\
\hline $\mathrm{H}$ & ๑. 725728 & 3.067140 & 4.159345 \\
\hline $\mathrm{H}$ & 1.984914 & 2.007727 & 4.831803 \\
\hline $\mathrm{H}$ & 1.457275 & 3.437383 & 5.720022 \\
\hline $\mathrm{H}$ & -1.298582 & -3.808474 & 1.149762 \\
\hline $\mathrm{H}$ & -2.068342 & $\odot .239239$ & 2.267762 \\
\hline $\mathrm{H}$ & -4.480779 & -1.444453 & 5.842360 \\
\hline $\mathrm{H}$ & -3.080710 & $-\odot .890273$ & 6.770355 \\
\hline $\mathrm{H}$ & -4.389515 & $\odot .227037$ & 6.416751 \\
\hline $\mathrm{H}$ & -4.815567 & 1.266652 & 4.423152 \\
\hline $\mathrm{H}$ & -3.970952 & $\odot .933579$ & 2.916125 \\
\hline $\mathrm{H}$ & -5.051007 & $-\odot .292289$ & 3.611697 \\
\hline $\mathrm{H}$ & -2.637408 & 1.786966 & 5.624239 \\
\hline $\mathrm{H}$ & -1.300809 & $\odot .622878$ & 5.620715 \\
\hline $\mathrm{H}$ & -1.740856 & 1.482986 & 4.132676 \\
\hline $\mathrm{H}$ & -1.225514 & -6.676887 & 4.581758 \\
\hline $\mathrm{H}$ & -0.207752 & -5.229747 & 4.451862 \\
\hline $\mathrm{H}$ & -1.591532 & -5.256277 & 5.559358 \\
\hline
\end{tabular}

\begin{tabular}{|c|c|c|c|}
\hline $\mathrm{H}$ & -0.521426 & -3.314843 & 6.001861 \\
\hline $\mathrm{H}$ & -1.152361 & -1.685437 & 6.348906 \\
\hline $\mathrm{H}$ & -1.720204 & -3.081058 & 7.307282 \\
\hline $\mathrm{H}$ & -2.468697 & -5.643710 & 1.333861 \\
\hline H & $-\odot .783339$ & -5.805524 & 1.876110 \\
\hline $\mathrm{H}$ & -2.009762 & -6.966517 & 2.405375 \\
\hline H & -5.533638 & $-\odot .199891$ & -5.535269 \\
\hline $\mathrm{H}$ & -4.299216 & -0.647632 & -6.707224 \\
\hline H & -4.935268 & -1.861749 & -5.585188 \\
\hline H & -1.663050 & -0.246386 & -2.777157 \\
\hline $\mathrm{H}$ & -3.746652 & -2.106109 & 0.450733 \\
\hline H & -7.688362 & -0.200233 & -3.873855 \\
\hline H & -7.101692 & 0.107504 & -2.213030 \\
\hline $\mathrm{H}$ & -6.225696 & ๑.793718 & -3.602523 \\
\hline $\mathrm{H}$ & -2.081646 & $-\odot .981893$ & -6.300584 \\
\hline H & -1.330107 & -0.963089 & -4.705931 \\
\hline $\mathrm{H}$ & -2.404143 & -2.298416 & -5.160639 \\
\hline H & -5.260011 & -4.551271 & $-\odot .879502$ \\
\hline $\mathrm{H}$ & -4.842086 & -3.801307 & 0.669964 \\
\hline $\mathrm{H}$ & -6.470191 & -4.424452 & 0.408262 \\
\hline $\mathrm{H}$ & -7.463110 & -2.171334 & 1.042149 \\
\hline H & -5.850769 & -1.470171 & 1.262978 \\
\hline $\mathrm{H}$ & -7.004701 & -0.701780 & 0.163734 \\
\hline $\mathrm{H}$ & $-4.09 \odot 483$ & 1.659611 & -4.554092 \\
\hline $\mathrm{H}$ & -2.363818 & 1.407562 & -4.263383 \\
\hline H & -3.008794 & 1.324340 & -5.914773 \\
\hline $\mathrm{H}$ & -4.302959 & -5.065427 & 2.999006 \\
\hline H & -3.771652 & -6.418844 & 4. 010702 \\
\hline H & -3.923469 & -4.798326 & 4.714130 \\
\hline H & -8.021221 & -3.509675 & $-\odot .983011$ \\
\hline $\mathrm{H}$ & -7.881044 & -2.000278 & -1.876451 \\
\hline H & -7.026423 & -3.438882 & -2.446802 \\
\hline $\mathrm{Rh}$ & -0.140741 & 1.014188 & -0.070098 \\
\hline 0 & -2.333081 & 1.839660 & $\odot .374650$ \\
\hline C & $-\odot .026822$ & 2.424744 & -1.682103 \\
\hline $\mathrm{s}$ & -3.051213 & 3.114812 & 0.636943 \\
\hline 0 & -1.104777 & 2.366787 & -2.249111 \\
\hline C & -0.649820 & 4.180394 & 0.668171 \\
\hline $\mathrm{N}$ & -2.018136 & 4.358281 & $\odot .115615$ \\
\hline 0 & -3.509039 & 3.329272 & 2.018619 \\
\hline C & -4.450557 & 3.300856 & $-\odot .454127$ \\
\hline H & -0.260163 & 5.194715 & 0.811521 \\
\hline H & $-\odot .698525$ & 3.726957 & 1.666859 \\
\hline C & -2.583986 & 5.717125 & 0.353223 \\
\hline C & -5.715258 & 3.449769 & 0.120747 \\
\hline C & -4.264794 & 3.336194 & -1.839343 \\
\hline C & -1.928987 & 6.721290 & $-\odot .553256$ \\
\hline $\mathrm{H}$ & -3.654390 & 5.672086 & 0.126887 \\
\hline H & -2.483682 & $6 . \odot \odot 8 \odot 4 \odot$ & 1.407871 \\
\hline $\mathrm{H}$ & -5.827418 & 3.421472 & -254.595300 \\
\hline C & -6.813334 & 3.649808 & $10.62200 \odot$ \\
\hline c & -5.376162 & 3.559248 & 14.098900 \\
\hline $\mathrm{H}$ & -3.275763 & 3.196471 & -2.261900 \\
\hline c & -1.316853 & 7.826467 & -0.124912 \\
\hline $\mathrm{H}$ & -2.001687 & 6.508134 & -1.619040 \\
\hline $\mathrm{H}$ & -7.800409 & 3.766488 & $-\odot .274378$ \\
\hline c & -6.661233 & 3.724384 & $-2.106 \odot 49$ \\
\hline $\mathrm{H}$ & -5.242899 & 3.611941 & -3.726668 \\
\hline H & $-\odot .890008$ & 8.546955 & $-\odot .816827$ \\
\hline $\mathrm{H}$ & -1.239194 & 8.064104 & 0.934245 \\
\hline c & -7.845685 & 4.008282 & -2.997884 \\
\hline $\mathrm{H}$ & -7.965887 & 5.088848 & -3.150693 \\
\hline $\mathrm{H}$ & -7.724742 & 3.553538 & -3.986285 \\
\hline H & -8.777422 & 3.637290 & -2.559525 \\
\hline c & 1.244399 & 3.014317 & -2.287960 \\
\hline $\mathrm{H}$ & 1.173044 & 3.220856 & -3.358223 \\
\hline $\mathrm{H}$ & 2.137896 & 2.426752 & -2.070964 \\
\hline C & 1.097593 & 4.202349 & -1.317804 \\
\hline $\mathrm{H}$ & 0.455672 & 4.976628 & -1.747773 \\
\hline $\mathrm{H}$ & 2.037924 & 4.648806 & -0.979182 \\
\hline C & 0.343431 & 3.427847 & -0.204505 \\
\hline H & 1.687793 & 1.836877 & 2.658054 \\
\hline $\mathrm{H}$ & 1.104073 & 3.016010 & 0.456840 \\
\hline
\end{tabular}


TS2

B3LYP SCF energy:

B3LYP enthalpy:

B3LYP free energy:

M06 SCF energy in solution:

a.u

M06 enthalpy in solution:

Mo6 free energy in solution:

a.u

Imaginary frequency: $-259.2393 \mathrm{~cm}-1$

Cartesian coordinates

\begin{tabular}{|c|c|c|c|}
\hline ATOM & X & $\mathrm{Y}$ & Z \\
\hline $\mathrm{P}$ & $\odot . \diamond \odot \odot \odot \odot \diamond$ & -3.910999 & 3.044835 \\
\hline$P$ & 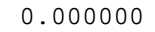 & -7.745704 & 1.924799 \\
\hline 0 & 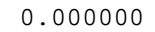 & -8.688797 & 3.472678 \\
\hline 0 & $\odot .000 \odot \odot \odot$ & -7.297533 & 4.493764 \\
\hline 0 & $\odot . \diamond \odot \odot \odot \odot \odot$ & -5.275615 & 3.756392 \\
\hline 0 & $\odot . ๑ \odot \odot \diamond \odot \odot$ & -2.844515 & 5.457803 \\
\hline 0 & $\odot . ๑ \odot \odot \odot \odot \odot$ & -3.918115 & 5.495768 \\
\hline 0 & 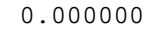 & -9.643639 & 3.387289 \\
\hline 0 & $\odot . \diamond \odot \odot \diamond \odot \odot$ & -8.542724 & 4.402406 \\
\hline 0 & $\odot . ๑ \odot \odot \odot \odot \odot ~$ & -7.167057 & 5.209351 \\
\hline C & $\odot .0 \odot \odot \odot \odot \odot$ & -2.467376 & 6.472747 \\
\hline C & $\odot . \diamond \odot \odot \odot \odot \diamond$ & -2.552280 & 4.905507 \\
\hline C & 1.862993 & -9.695169 & 5.276208 \\
\hline C & 1.866156 & -9.380052 & 6.317697 \\
\hline C & 1.965906 & -10.100956 & 4.940571 \\
\hline C & 2.074782 & -10.510984 & 5.254982 \\
\hline C & 1.932900 & -0.777726 & 0.606339 \\
\hline C & 1.136896 & $-\odot .581188$ & 1.225393 \\
\hline C & 1.332745 & -0.373068 & -0.393978 \\
\hline C & 2.410493 & -2.229798 & $\odot .578354$ \\
\hline C & 3. 286769 & -2.869498 & 1.283598 \\
\hline C & 3.101806 & -2.687054 & -0.409795 \\
\hline C & 2.054313 & -2.006927 & 1. 003156 \\
\hline C & 4.844204 & -2.382908 & 0.247815 \\
\hline C & 3.699370 & ๑. 022633 & -0.344929 \\
\hline C & 3.744142 & -0.398304 & -1.673968 \\
\hline C & 4. 929567 & -0.859486 & -2.258922 \\
\hline C & 6.106367 & -0.841627 & -1.458099 \\
\hline C & 6.077282 & -0.511284 & -0.073393 \\
\hline C & 4.851748 & -0.062644 & ०. 439168 \\
\hline C & 7.280372 & -0.629914 & $\odot .906063$ \\
\hline C & 8.158690 & ๑. 645587 & $\odot .852558$ \\
\hline C & 8.128763 & -1.896432 & $\odot .637140$ \\
\hline C & 6.791391 & $-\odot .752050$ & 2.373114 \\
\hline C & 4. 906395 & -1.412225 & -3.707654 \\
\hline C & 5.398528 & -2.881198 & -3.716343 \\
\hline C & 3.475085 & -1.415048 & -4.287808 \\
\hline C & 5.765986 & $-\odot .555829$ & -4.667435 \\
\hline C & 8.263917 & $-\odot .303648$ & -2.434642 \\
\hline C & 2.487193 & 2.564343 & ๑. 390879 \\
\hline C & 3.655352 & 3.106554 & -0.141919 \\
\hline C & 1.475937 & 3.439378 & ๑. 823420 \\
\hline C & 1.664292 & 4.823041 & $\odot .860104$ \\
\hline C & 2.932095 & 5.317141 & ๑. 438408 \\
\hline C & 3.890583 & 4.490473 & -0.191173 \\
\hline C & 5.148372 & 4.960867 & -0.977015 \\
\hline C & 5.174092 & 4.216258 & -2.341781 \\
\hline C & 6.432220 & 4.593987 & $-\odot .195357$ \\
\hline C & 4.052259 & 6.962419 & 1. 714791 \\
\hline C & 5.181716 & 6.465881 & -1.331285 \\
\hline C & $\odot .501591$ & 5.769734 & 1. 254917 \\
\hline C & ๑. 811553 & 6.591623 & 2.529350 \\
\hline C & ๑. 209882 & 6.732177 & $\odot .075920$ \\
\hline C & $-\odot .797016$ & 4.984587 & 1.535380 \\
\hline C & -1.277526 & -1.616475 & 1.504705 \\
\hline C & -1.425513 & -2.838081 & 2.173510 \\
\hline C & -2.274888 & -2.977056 & 3.276766 \\
\hline C & -3.019223 & -1.826905 & 3.655984 \\
\hline C & -2.728590 & $-\odot .534504$ & 3.149322 \\
\hline C & -1.885417 & -0.474756 & 2.029773 \\
\hline
\end{tabular}

-5535.31983535 a.u. 5533.285328 a.u. $-5532.783270$ $-5534.54203597$ -5532.507529 a.u. 


\begin{tabular}{|c|c|c|c|}
\hline $\mathrm{H}$ & -0.705108 & 4.319702 & 2.401769 \\
\hline $\mathrm{H}$ & -1.600909 & 5.695160 & 1.755445 \\
\hline $\mathrm{H}$ & -0.869509 & -3.695221 & 1.817536 \\
\hline $\mathrm{H}$ & -1.695814 & $\odot .476933$ & 1.550167 \\
\hline $\mathrm{H}$ & -3.170923 & -0.123835 & 5.863372 \\
\hline $\mathrm{H}$ & -1.627042 & ๑. 618624 & 5.394267 \\
\hline $\mathrm{H}$ & -3.003188 & 1.640963 & 5.847245 \\
\hline $\mathrm{H}$ & -4.926183 & 2.031369 & 4.157128 \\
\hline $\mathrm{H}$ & -5.099382 & ๑. 918637 & 2.790380 \\
\hline $\mathrm{H}$ & -5.253771 & $\odot .333315$ & 4.469679 \\
\hline $\mathrm{H}$ & -2.790332 & 2.896571 & 3.790628 \\
\hline H & -1.428295 & 1.933260 & 3.209410 \\
\hline $\mathrm{H}$ & -2.868587 & 2.164774 & 2.183223 \\
\hline $\mathrm{H}$ & -1.909527 & -4.890251 & 6.163691 \\
\hline $\mathrm{H}$ & $-1.0 \odot 5974$ & -3.446658 & 5.662895 \\
\hline $\mathrm{H}$ & -2.738322 & -3.328378 & 6.014120 \\
\hline $\mathrm{H}$ & -5.612848 & -1.401973 & 3.352667 \\
\hline $\mathrm{H}$ & -5.354623 & -3.153780 & 3.501734 \\
\hline $\mathrm{H}$ & -6.013839 & -2.233714 & 4.882392 \\
\hline $\mathrm{H}$ & -1.361239 & -5.627501 & 2.587433 \\
\hline $\mathrm{H}$ & -0.179475 & -4.789324 & 3.619424 \\
\hline $\mathrm{H}$ & -1.138284 & -6.131234 & 4.261428 \\
\hline $\mathrm{H}$ & -2.011833 & -1.970599 & -5.884589 \\
\hline $\mathrm{H}$ & -1.649741 & -3.304219 & -6.991314 \\
\hline $\mathrm{H}$ & -3.086181 & -3.379324 & -5.951644 \\
\hline $\mathrm{H}$ & ๑. 065933 & -2.455436 & -2.852519 \\
\hline $\mathrm{H}$ & -3.080951 & -2.445381 & ๑. 021160 \\
\hline $\mathrm{H}$ & -4.128529 & -6.260627 & -4.840562 \\
\hline $\mathrm{H}$ & -3.533797 & -6.284668 & -3.158923 \\
\hline $\mathrm{H}$ & -5.235722 & -5.859477 & -3.515502 \\
\hline $\mathrm{H}$ & -0.620085 & -5.489006 & -6.090437 \\
\hline $\mathrm{H}$ & -0.612162 & -5.796656 & -4.347618 \\
\hline $\mathrm{H}$ & -2.142931 & -5.713349 & -5.238575 \\
\hline $\mathrm{H}$ & -5.134895 & -5.747011 & -1.495539 \\
\hline $\mathrm{H}$ & -4.643176 & $-5 . \odot 95390$ & $\odot .067607$ \\
\hline $\mathrm{H}$ & -6.341072 & -5.022075 & -0.431859 \\
\hline $\mathrm{H}$ & -6.516401 & -2.584360 & -0.072015 \\
\hline $\mathrm{H}$ & -4.938989 & -2.751240 & 0.685203 \\
\hline $\mathrm{H}$ & -5.209405 & -1.487818 & -0.535316 \\
\hline $\mathrm{H}$ & ๑. 364569 & -2.174411 & -4.985338 \\
\hline $\mathrm{H}$ & ๑. 960984 & -3.739252 & -4.391779 \\
\hline H & 0.599484 & -3.511781 & -6.103083 \\
\hline $\mathrm{H}$ & -3.948215 & -5.197923 & 2.984818 \\
\hline $\mathrm{H}$ & -3.477703 & -6.074497 & 4.447323 \\
\hline $\mathrm{H}$ & -4.401964 & -4.584684 & 4.591988 \\
\hline $\mathrm{H}$ & -7.099898 & -3.365924 & -2.173862 \\
\hline $\mathrm{H}$ & -5.980940 & -2.171280 & -2.844420 \\
\hline $\mathrm{H}$ & -5.933716 & -3.838360 & -3.420179 \\
\hline $\mathrm{Rh}$ & $\odot .130721$ & ๑. 595151 & -0.773392 \\
\hline $\mathrm{H}$ & $\odot .528590$ & 3.021451 & 1.142809 \\
\hline 0 & -4.471936 & 1.093959 & 0.120006 \\
\hline C & $-\odot .242126$ & 1. 220492 & -2.625122 \\
\hline S & -5.058000 & 1.680864 & -1.098084 \\
\hline 0 & ๑. 369071 & 2.267172 & -2.442658 \\
\hline C & -2.472138 & 2.393424 & -1.417264 \\
\hline $\mathrm{N}$ & -3.888133 & 2.745027 & -1.676914 \\
\hline 0 & -5.545833 & $\odot .788810$ & -2.162481 \\
\hline C & -6.405724 & 2.767277 & -0.642442 \\
\hline $\mathrm{H}$ & -2.295892 & 2.480022 & $-\odot .341739$ \\
\hline $\mathrm{H}$ & -1.892635 & 3.183150 & -1.903534 \\
\hline C & -4.226843 & 3.540100 & -2.876942 \\
\hline C & -7.629992 & 2.650335 & -1.300016 \\
\hline C & -6.230698 & 3.683490 & ๑. 400690 \\
\hline C & -3.639477 & 4.923323 & -2.791334 \\
\hline $\mathrm{H}$ & -5.319635 & 3.613545 & -2.916751 \\
\hline $\mathrm{H}$ & -3.910999 & 3.044835 & -259.239300 \\
\hline $\mathrm{H}$ & -7.745704 & 1.924799 & 7.663400 \\
\hline C & -8.688797 & 3.472678 & 8.644500 \\
\hline C & -7.297533 & 4.493764 & 0.773104 \\
\hline H & -5.275615 & 3.756392 & ๑. 910614 \\
\hline C & -2.844515 & 5.457803 & -3.719209 \\
\hline $\mathrm{H}$ & -3.918115 & 5.495768 & -1.907352 \\
\hline $\mathrm{H}$ & -9.643639 & 3.387289 & -1.422700 \\
\hline C & -8.542724 & 4.402406 & ๑. 126122 \\
\hline $\mathrm{H}$ & -7.167057 & 5.209351 & 1.581112 \\
\hline
\end{tabular}

$\begin{array}{lrrr}\mathrm{H} & -2.467376 & 6.472747 & -3.632999 \\ \mathrm{H} & -2.552280 & 4.905507 & -4.610273 \\ \mathrm{C} & -9.695169 & 5.276208 & 0.558426 \\ \mathrm{H} & -9.380052 & 6.317697 & 0.687246 \\ \mathrm{H} & -10.100956 & 4.940571 & 1.521407 \\ \mathrm{H} & -10.510984 & 5.254982 & -0.169896 \\ \mathrm{C} & -0.777726 & 0.606339 & -3.889506 \\ \mathrm{H} & -0.581188 & 1.225393 & -4.770704 \\ \mathrm{H} & -0.373068 & -0.393978 & -4.051928 \\ \mathrm{C} & -2.229798 & 0.578354 & -3.351247 \\ \mathrm{H} & -2.869498 & 1.283598 & -3.883529 \\ \mathrm{H} & -2.687054 & -0.409795 & -3.420144 \\ \mathrm{C} & -2.006927 & 1.003156 & -1.872250 \\ \mathrm{H} & -2.382908 & 0.247815 & -1.184351\end{array}$

TS3

B3LYP SCF energy: $\quad-5535.31541971 \mathrm{a} \cdot \mathrm{u}$ B3LYP enthalpy: $\quad-5533.282057 \mathrm{a} \cdot \mathrm{u}$. B3LYP free energy: $\quad-5533.557914$ a.u. M06 SCF energy in solution: $\quad-5534.53758660$ a.u. M06 enthalpy in solution: $\quad-5532.504224$ a.u. M०6 free energy in solution: $\quad-5532.780081$ a.u.

Imaginary frequency: $\quad-285.4428 \mathrm{~cm}-1$

Cartesian coordinates

ATOM $X$ Y$$
P
$$$$
0
$$

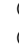

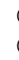$$
\begin{array}{lll}
0 \\
0 \\
0
\end{array}
$$

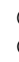$$
\text { C }
$$$$
\text { c }
$$$$
\begin{array}{ll}
c \\
c \\
c
\end{array}
$$$$
\text { c }
$$$$
\text { c }
$$$$
\begin{array}{ll}
c \\
c \\
c
\end{array}
$$$$
\text { c }
$$$$
\text { c }
$$$$
\text { c }
$$$$
\begin{array}{ll}
c \\
c \\
c \\
c
\end{array}
$$$$
\text { c }
$$$$
\text { C }
$$$$
\text { c }
$$$$
\text { c }
$$$$
\text { C }
$$$$
\text { c }
$$$$
\text { c }
$$$$
\begin{aligned}
& c \\
& c \\
& c \\
& c
\end{aligned}
$$

c

c

c

$\mathrm{c}$
$\mathrm{c}$
$\mathrm{c}$

$\mathrm{C}$
$\mathrm{C}$
$\mathrm{C}$
$\mathrm{C}$
$\mathrm{C}$

S112
-3.544170
-2.556592
-4.345672
-1.894648
-6.172656
-5.692275
-3.532510
-1.235453
-7.242798
-5.833780
-3.175774
-4.547313
-0.900166
-0.484882
-1.860766
-3.931426
0.125545
-1.474973
-0.695223
-1.081278
-2.333982
-2.864901
-3.759233
-2.830001
0.643047
2.032314
2.866435
2.228517
0.814421
0.049836
0.130348
0.269474
0.752444
-1.380616
4.385414
5.202424
4.544220
5.017370
3.458711
-0.990398
-0.472305
-1.907992
-2.439888
-2.009280
-0.929607
0.000000

0.000000

$\odot .000000$

0.000000

$\odot .00000 \odot$

$\odot .000000$

0.000000

$\odot .000000$

$\odot .00000 \odot$

0.000000

๑. 000000

0.000000

$-3.315719$

$-2.734279$

$-1.367933$

$-0.602930$

$-4.938143$

$-3.394302$

$-4.259146$

$-5.145772$

5.128162

$-3.376441$

5.637918

$-0.560081$

$-0.597778$

$-0.861956$

$-0.982136$

$-1.105336$

$-0.873929$

$-1.546860$

$-2.883387$

$-1.794232$

$-1.689269$

$-1.986113$

$\odot .330518$

$\odot .231229$

1.629821

2. 289958

2. 313174

3. 565648

4. 109237

3. 550317
6.23709

7.715520

6.371595

5.817750

$.1245 \odot \odot$

4.476392

8.692372

7.916962

6. 059694

6.929952

9.881866

8.517864

. 110069

7.155748

10.111242

10.646575

9.274227

11.416574

11.273177

12.121934

11.894930

3. 660852

3. 104170

. 634901

3. 104402

2. 997243

4. 097512

5.355203

5.481135

4.335403

6.801141

7. 920661

7. 276897

6.593866

3. 809445

4.948222

2. 590644

3. 448040

6. 973512

2. 110271

3. 231317

1. 303609

1. 659581

2. 896713

3. 630676 


\begin{tabular}{|c|c|c|c|c|c|c|c|}
\hline C & 4.306574 & 4.785187 & -0.221503 & $\mathrm{H}$ & 5.253002 & 5.812425 & -1.924103 \\
\hline C & 3.536160 & 5.284289 & 1.021096 & $\mathrm{H}$ & 3.585100 & 6.338453 & -1.601123 \\
\hline C & 4.524453 & 6.012614 & -1.138475 & H & 4.907937 & 6.849770 & -0.543622 \\
\hline C & 5.003714 & 4.104164 & -3.835896 & $\mathrm{H}$ & 4.641325 & 3.430109 & -4.618906 \\
\hline C & 5.678668 & 4.259270 & $\odot .270639$ & H & 4.277331 & 4.912354 & -3.699295 \\
\hline C & 4.235093 & 0.669194 & -3.438532 & $\mathrm{H}$ & 5.964987 & 4.524382 & -4.139372 \\
\hline C & 3.480977 & $\odot .704470$ & $-4.79 \odot 228$ & H & 6.294498 & 3.909608 & $-\odot .560249$ \\
\hline C & 5.744355 & ๑.899573 & -3.692170 & $\mathrm{H}$ & 6.220932 & 5.061060 & 0.785788 \\
\hline C & 4.140000 & $-\odot .770464$ & -2.867918 & H & 5.548476 & 3.434376 & 0.981225 \\
\hline C & -2.562473 & -1.649818 & -1.339389 & $\mathrm{H}$ & 2.426436 & $\odot .430816$ & -4.669542 \\
\hline C & -3.857094 & -1.690520 & -1.874451 & $\mathrm{H}$ & 3.517061 & 1.697445 & -5.250948 \\
\hline C & -4.154906 & -2.443805 & -3.013927 & $\mathrm{H}$ & 3.931690 & -0.005195 & -5.494110 \\
\hline C & -3.089283 & -3.186111 & -3.598767 & H & 6.133948 & $\odot .036646$ & -4.244631 \\
\hline C & -1.742299 & -3.021686 & -3.195721 & $\mathrm{H}$ & 5.957795 & 1.784222 & -4.290598 \\
\hline C & -1.525825 & -2.282615 & -2.020684 & H & 6.305426 & $\odot .980619$ & -2.756896 \\
\hline C & -0.483451 & -3.539615 & -3.947804 & H & 4.668304 & $-\odot .847123$ & -1.910910 \\
\hline C & -0.722553 & -4.004404 & -5.403491 & H & 3.110218 & -1.109942 & -2.726166 \\
\hline C & 0.178444 & -4.687149 & -3.147880 & H & 4.611094 & -1.469928 & -3.567349 \\
\hline C & $\odot .530781$ & -2.367627 & -4.054180 & $\mathrm{H}$ & -4.641555 & -1.133426 & -1.379101 \\
\hline C & -5.448275 & -1.858210 & -5.094782 & H & $-\odot .521316$ & -2.190531 & -1.622044 \\
\hline C & -3.444079 & -5.433834 & -4.244118 & H & -1.264388 & -4.946788 & -5.476954 \\
\hline C & -5.571415 & -2.386419 & -3.643057 & H & -1.267554 & -3.255408 & -5.984818 \\
\hline C & -6.494217 & -1.419279 & -2.868721 & H & $\odot .252800$ & -4.157141 & -5.880160 \\
\hline C & -3.583394 & -3.921955 & 3.483892 & H & 1.097502 & -5.016462 & -3.647449 \\
\hline C & -2.670506 & -2.892359 & 3.850632 & H & 0.440643 & -4.370958 & -2.131586 \\
\hline C & -1.334635 & -4.145148 & 5.585383 & H & $-\odot .482085$ & -5.556366 & -3.061289 \\
\hline C & -2.282592 & -2.002582 & 2.847176 & H & 1.414154 & -2.696620 & -4.612646 \\
\hline C & -2.736933 & -2.127729 & 1.525780 & H & ๑. $09 \odot 621$ & -1.514940 & -4.583553 \\
\hline C & -3.512029 & -3.235108 & 1.185882 & H & 0.875998 & -2.016036 & -3.077454 \\
\hline C & -3.917349 & -4.188277 & 2.134919 & H & -6.443975 & -1.766118 & -5.545498 \\
\hline C & -5.447149 & -4.369704 & 4.865971 & H & -4.972403 & $-\odot .870336$ & -5.113238 \\
\hline C & -3.082651 & -2.490887 & 6.364343 & H & -4.854010 & -2.531845 & -5.715470 \\
\hline C & -6.062621 & -5.070426 & 1.096131 & H & -2.461908 & -5.791662 & -3.919519 \\
\hline C & -4.646670 & -5.450918 & 1.591425 & H & -4.166464 & -5.603212 & -3.437978 \\
\hline C & -3.834696 & -6.001648 & $\odot .385560$ & H & -3.753843 & -5.986123 & -5.133984 \\
\hline C & $-\odot .964806$ & -1.696215 & 5.333700 & H & -6.691721 & -1.765543 & -1.847052 \\
\hline C & -6.280240 & -3.762132 & -3.657436 & H & -6.085686 & $-\odot .405 \odot 47$ & -2.814647 \\
\hline C & -2.040226 & -2.803180 & 5.264509 & H & -7.460192 & $-1.36400 \odot$ & -3.382077 \\
\hline C & -4.757491 & -6.632124 & 2.583407 & H & $-\odot .544859$ & -4.353343 & 4.854288 \\
\hline H & -0.904900 & 2.098019 & -5.291788 & H & -0.868948 & -4.091784 & 6.576778 \\
\hline H & $\odot .469919$ & 1.988552 & -3.241885 & H & -2.038971 & -4.979129 & 5.583388 \\
\hline $\mathrm{H}$ & -5.687014 & 1.440424 & -4.826420 & H & -1.607497 & -1.190808 & 3.087448 \\
\hline H & -5.262038 & 3.160865 & -4.449913 & H & -3.805238 & -3.369653 & 0.153206 \\
\hline H & -4.262326 & -0.290940 & 2.256104 & $\mathrm{H}$ & -5.711030 & -5.039427 & 5.687326 \\
\hline H & -5.818396 & 1.602464 & 2.673709 & H & -5.519918 & -3.329795 & 5.204131 \\
\hline $\mathrm{H}$ & -5.476961 & 5.474110 & $-\odot .215759$ & H & -6.145157 & -4.521757 & 4.036127 \\
\hline H & -6.486419 & 4.203847 & -1.019200 & H & -2.567188 & -2.286649 & 7.310168 \\
\hline $\mathrm{H}$ & -0.429380 & 2.022719 & 2.477889 & H & -3.676343 & -1.603516 & 6.112726 \\
\hline $\mathrm{H}$ & -0.924528 & 4.393164 & -1.029383 & H & -3.758295 & -3.328788 & 6.536350 \\
\hline $\mathrm{H}$ & 0.496677 & 7.582874 & $-\odot .075435$ & H & -6.690654 & -4.692318 & 1.910376 \\
\hline $\mathrm{H}$ & -0.777826 & 8.779186 & -0.347894 & $\mathrm{H}$ & -6.024391 & -4.296510 & 0.320599 \\
\hline $\mathrm{H}$ & $-\odot .397927$ & 8.273858 & 1.296922 & H & -6.563191 & -5.948249 & 0.670315 \\
\hline $\mathrm{H}$ & -2.778485 & 7.497102 & 1.815845 & H & -4.318067 & -6.908974 & $\odot .0 \odot 6 \odot 82$ \\
\hline $\mathrm{H}$ & -3.229487 & 8.184566 & $\odot .243792$ & H & -3.767721 & -5.296653 & -0.448114 \\
\hline $\mathrm{H}$ & -3.655602 & 6.507733 & 0.636272 & $\mathrm{H}$ & -2.814831 & -6.263844 & $\odot .688500$ \\
\hline $\mathrm{H}$ & -2.535769 & 5.810509 & -1.571990 & H & -0.170800 & -1.847367 & 4.593912 \\
\hline H & -2.175760 & 7.526767 & -1.809129 & $\mathrm{H}$ & -1.388851 & -0.694148 & 5.194182 \\
\hline $\mathrm{H}$ & -0.875086 & 6.342614 & -1.923015 & H & $-\odot .499703$ & -1.715431 & 6.324887 \\
\hline $\mathrm{H}$ & -1.931823 & 4.560481 & 6.198479 & H & -6.280875 & -4.221287 & -2.661465 \\
\hline $\mathrm{H}$ & -1.040332 & 5.811890 & 5.345479 & H & -7.325777 & -3.630834 & -3.959918 \\
\hline $\mathrm{H}$ & -2.618365 & 5.296106 & 4.742081 & H & -5.821278 & -4.453324 & -4.363806 \\
\hline $\mathrm{H}$ & -2.153031 & 2.395811 & 5.609393 & H & -5.111324 & -7.511169 & 2.032242 \\
\hline $\mathrm{H}$ & -2.957816 & 2.786326 & 4.077573 & H & -3.790306 & -6.881355 & 3.028456 \\
\hline $\mathrm{H}$ & -1.583152 & 1.672142 & 4.107918 & H & -5.465840 & -6.456670 & 3.392937 \\
\hline $\mathrm{H}$ & 0.787312 & 2.582767 & 4.523096 & $\mathrm{Rh}$ & -0.032721 & -0.269225 & 0.848370 \\
\hline $\mathrm{H}$ & 1.041836 & 4.278762 & 4.955519 & C & 1.552832 & -1.449021 & 1.162809 \\
\hline $\mathrm{H}$ & $\odot .202509$ & 3.198089 & 6.077137 & C & 2.978566 & -1.604680 & 0.682512 \\
\hline $\mathrm{H}$ & $\odot .929296$ & 7.152268 & 2.633053 & 0 & 0.824415 & -2.226978 & 1.769056 \\
\hline $\mathrm{H}$ & 0.034392 & 7.912699 & 3.980136 & C & 3.404739 & -0.234244 & 1.274759 \\
\hline $\mathrm{H}$ & 0.682778 & 6.260550 & 4.155646 & $\mathrm{H}$ & 3.015821 & -1.606053 & -0.411413 \\
\hline $\mathrm{H}$ & 1.803756 & 3.810533 & 0.298253 & H & 4.199791 & $-\odot .344980$ & 2. 016818 \\
\hline H & 1.846670 & 0.382129 & -2.239137 & H & 3.728599 & $\odot .462062$ & ๑.499153 \\
\hline $\mathrm{H}$ & 4.139355 & 6.043522 & 1.529813 & C & 2.065268 & 0.206753 & 1.906380 \\
\hline $\mathrm{H}$ & 2.578460 & 5.749562 & 0.758785 & $\mathrm{H}$ & 1.966642 & $-\odot .047098$ & 2.965478 \\
\hline \multirow[t]{2}{*}{$\mathrm{H}$} & 3.344714 & 4.480991 & 1.741418 & H & 1.823491 & 1.260992 & 1.769738 \\
\hline & & & & & & & \\
\hline
\end{tabular}




$\begin{array}{rrrr}\mathrm{N} & 5.057079 & -2.951066 & 0.771338 \\ \mathrm{C} & 5.289078 & -3.830917 & -0.395333 \\ \mathrm{~S} & 6.211935 & -2.994330 & 2.037422 \\ \mathrm{C} & 5.263577 & -5.319603 & -0.141841 \\ \mathrm{H} & 4.502170 & -3.557143 & -1.111957 \\ \mathrm{H} & 6.237093 & -3.544170 & -285.442800 \\ \mathrm{C} & 7.715520 & -2.556592 & 5.07500 \odot \\ \mathrm{O} & 6.371595 & -4.345672 & 6.89040 \odot \\ \mathrm{O} & 5.817750 & -1.894648 & 2.930751 \\ \mathrm{C} & 6.124500 & -6.172656 & -0.696196 \\ \mathrm{H} & 4.476392 & -5.692275 & 0.512012 \\ \mathrm{C} & 8.692372 & -3.532510 & 0.974505 \\ \mathrm{C} & 7.916962 & -1.235453 & 0.765908 \\ \mathrm{H} & 6.059694 & -7.242798 & -0.522843 \\ \mathrm{H} & 6.929952 & -5.833780 & -1.345316 \\ \mathrm{C} & 9.881866 & -3.175774 & 0.339245 \\ \mathrm{H} & 8.517864 & -4.547313 & 1.315105 \\ \mathrm{C} & 9.110069 & -0.900166 & 0.132893 \\ \mathrm{H} & 7.155748 & -0.484882 & 0.950877 \\ \mathrm{C} & 10.111242 & -1.860766 & -0.088138 \\ \mathrm{H} & 10.646575 & -3.931426 & 0.179442 \\ \mathrm{H} & 9.274227 & 0.125545 & -0.187871 \\ \mathrm{C} & 11.416574 & -1.474973 & -0.741079 \\ \mathrm{H} & 11.273177 & -0.695223 & -1.496414 \\ \mathrm{H} & 12.121934 & -1.081278 & 0.002445 \\ \mathrm{H} & 11.894930 & -2.333982 & -1.221603 \\ \mathrm{C} & 3.660852 & -2.864901 & 1.240255 \\ \mathrm{H} & 3.104170 & -3.759233 & 0.937754 \\ \mathrm{H} & 3.634901 & -2.830001 & 2.331927\end{array}$

TS4-trans

B3LYP SCF energy:

B3LYP enthalpy:

B3LYP free energy:

M06 SCF energy in solution:

a.u.

M06 enthalpy in solution:

M06 free energy in solution:

a.u.

Imaginary frequency:

$-281.4805 \mathrm{~cm}-1$

Cartesian coordinates

\begin{tabular}{|c|c|c|c|}
\hline ATOM & X & Y & Z \\
\hline $\mathrm{P}$ & 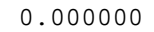 & -3.299778 & 6.849116 \\
\hline$P$ & $\odot .0000 \odot \odot$ & -3.116477 & 8.231925 \\
\hline 0 & $\odot . \odot \odot \odot \odot \odot \odot$ & $-\odot .870358$ & 8.690365 \\
\hline 0 & $\odot .0 \odot \odot \odot \odot \odot$ & ๑. 707691 & 7.671211 \\
\hline 0 & $\odot . \diamond \diamond \odot \odot \odot \diamond$ & -2.180836 & 1.528574 \\
\hline 0 & ๑. . & -1.210474 & 3. 076717 \\
\hline 0 & $\odot . \odot \odot \odot \odot \odot \odot$ & -4.186792 & 8.409547 \\
\hline 0 & ๑. . 000000 & -2.251529 & 8.912762 \\
\hline 0 & $\odot . \diamond \diamond \diamond \diamond \odot \diamond$ & -0.182490 & 9.228684 \\
\hline 0 & ๑. . 000000 & -2.461338 & 1. 010922 \\
\hline C & $\odot .00000 \odot$ & -2.926908 & 1.456460 \\
\hline C & $\odot . ๑ \odot \odot \odot \odot \odot$ & -2.786934 & 9.859913 \\
\hline C & $\odot .449019$ & -3.803768 & 10.186849 \\
\hline C & $\odot .877235$ & -2.155385 & 10.749625 \\
\hline C & 1.526587 & -2.816239 & 9.378252 \\
\hline C & 1.744303 & 1.458656 & 0.997060 \\
\hline C & 0.164790 & 1.584086 & $\odot .763422$ \\
\hline C & -0.880984 & 2. 352427 & 0.638775 \\
\hline C & -1.262695 & 1.274536 & 2.494194 \\
\hline C & -0.636348 & 0.578850 & 2.902682 \\
\hline C & ๑. 398069 & 2.234224 & 3.000518 \\
\hline C & ๑. 783783 & 1.848575 & 2.201325 \\
\hline C & $\odot .187042$ & $\odot .782702$ & 2.806181 \\
\hline C & 2.138634 & 1.456753 & 2.434823 \\
\hline C & 2.804895 & -1.617068 & -0.561178 \\
\hline C & 2.610211 & -2.205713 & -1.814170 \\
\hline C & 3.505230 & -3.155969 & -2.329496 \\
\hline C & 4.643745 & -3.477696 & -1.540558 \\
\hline C & 4.781162 & -3.036133 & -0.198466 \\
\hline C & 3.860318 & -2.073168 & ๑. 234501 \\
\hline
\end{tabular}

$5.828829-3.539581$

$6.984579-2.519485$

$6.406873-4.939910$

$5.151249-3.664591$

$3.207859-3.858783$

$3.193133 \quad-5.395887$

$1.821489-3.461955$

$4.246091-3.489713$

$6.784014-3.649760$

$3.001560 \quad 1.050178$

4.155449

2. 798971

3.805749

5.025762

5.155543

6.271195

5.588423

7.195808

7.127813

7.132570

3.572684

4.498800

3.823269

2. 122326

$-2.361031$

$-2.918527$

$-3.563639$

$-3.652520$

$-2.950646$

$-2.356663$

$-2.753473$

$-2.941275$

$-3.698014$

$-1.291955$

$-3.387093$

$-5.700435$

$-4.083070$

$-3.749780$

$-5.570708$

$-4.319330$

$-5.351423$

$-3.181994$

$-3.254400$

$-4.519024$

$-5.702967$

$-6.972461$

$-3.297348$

$-7.201355$

$-7.038217$

$-6.977771$

$-3.238990$

$-5.617409$

$-4.085805$

$-8.318158$

1. 876492

2. 308862

$-0.713274$

1. 010116

$-2.084376$

$-0.955460$

3. 130194

2. 107092

1. 738953

3. 965709

6. 605067

7.685918

7.550562

7. 057866

6. 997469

5.611426

4. 238982

5.846648

4.887759

2. 960686

4. 158186
1. 080967

2. 113667

3. 044463

2. 927066

2. 055928

2. 098726

2. 056535

0.864651

3. 056439

3. 383144

4. 159771

3.976463

4. 146044

$-0.925779$

$-1.967876$

$-1.731430$

$-0.378825$

0.672099

$\odot .360304$

2. 114546

2. 246881

3. 102729

2. 563675

0.435529

$-2.917510$

$-4.275257$

$-1.861536$

$-1.789726$

$-1.868576$

$-1.597939$

$-1.452059$

$-1.401893$

$-1.568930$

$-3.599243$

$-3.183086$

$-2.612553$

$-1.415393$

$-0.104353$

$-0.664739$

$-2.886601$

$-1.887752$

$-1.304713$

$-0.538696$

0.481759

$-4.654491$

$-4.970859$

$-3.609537$

$-5.807609$

$-6.090316$

$-6.936949$

$-1.939944$

$-1.666829$

$-1.532431$

$-2.849817$

$-2.399154$

$-4.961700$

$-5.275556$

$-5.675093$

$-4.267356$

$-4.144323$

$-2.696107$

$-5.888558$

$-5.766639$
5.552828

$-2.879608$
๑. 838455

0.978198

0.525401

2. 232163

$-3.681871$

$-3.482596$

$-4.234609$

$-4.769106$

$-2.568726$

0.450582

$-0.339010$

1. 349421

1.638941

0.915990

$-0.190601$

$-1.274510$

$-2.670373$

$-1.153097$

1.998128

$-1.278692$

2. 691925

3. 920052

2. 062213

3. 218903

1.412348

2. 167117

3. 384830

3. 824781

3.185957

1. 950055

3.736077

5.266251

3. 009891

3. 461701

5.624511

4. 712411

4. 240278

3. 582613

$-2.819210$

$-3.485040$

$-5.907919$

$-2.688165$

$-1.296214$

$-0.705021$

$-1.439847$

$-3.567959$

$-5.333720$

0.311557

$-0.655993$

0.175103

$-5.468083$

4.441854

$-5.020862$

$-1.516095$

5.162953

2. 957814

5.286578

4.821664

$-1.861555$

$-2.028071$

0.235797

1. 464155

$-2.393153$

1.232193

1.265593

1.753867

0.049447

$-0.346886$

1.385252

๑. 360739

2. 183974

2. 929258

2. 667605

$-4.434374$

$-3.133872$ 


\begin{tabular}{|c|c|c|c|}
\hline $\mathrm{H}$ & 2.424629 & -5.691476 & -2.759128 \\
\hline $\mathrm{H}$ & 1.631791 & -4.031290 & -5.150859 \\
\hline $\mathrm{H}$ & 1.013756 & -3.690204 & -3.531306 \\
\hline $\mathrm{H}$ & 1.756877 & -2.399954 & -4.494602 \\
\hline $\mathrm{H}$ & 4.351557 & -2.402310 & -4.866741 \\
\hline $\mathrm{H}$ & 5.227153 & -3.919070 & -4.565858 \\
\hline $\mathrm{H}$ & 3.914695 & -3.878196 & -5.739290 \\
\hline H & 7.441084 & -3.373262 & -1.738464 \\
\hline $\mathrm{H}$ & 7.300844 & -4.371655 & -3.205278 \\
\hline $\mathrm{H}$ & 6.547284 & -2.753240 & -3.152271 \\
\hline $\mathrm{H}$ & 4.294158 & ๑. 317461 & -1.093588 \\
\hline H & 6.351586 & 2.114362 & -3.454296 \\
\hline $\mathrm{H}$ & 5.019842 & 1.137631 & -2.841772 \\
\hline $\mathrm{H}$ & 4.910906 & 2.908906 & -2.799505 \\
\hline $\mathrm{H}$ & 7.728045 & $\odot .846437$ & $-\odot .196097$ \\
\hline $\mathrm{H}$ & 6.632164 & -0.071178 & -1.236049 \\
\hline $\mathrm{H}$ & 7.947730 & ๑. 877538 & -1.951240 \\
\hline $\mathrm{H}$ & 6.746971 & 2.561395 & 2.898228 \\
\hline $\mathrm{H}$ & 7.626444 & 2.312828 & 1.368445 \\
\hline $\mathrm{H}$ & 7.846311 & 3.827988 & 2.282890 \\
\hline $\mathrm{H}$ & 7.822600 & 3.447863 & -0.437858 \\
\hline $\mathrm{H}$ & 7.737298 & 3.389493 & -2.192962 \\
\hline $\mathrm{H}$ & 6.515323 & 4.286074 & -1.277504 \\
\hline $\mathrm{H}$ & 4.437412 & 2.957649 & 4.322444 \\
\hline $\mathrm{H}$ & 5.539909 & 4.199530 & 3.686295 \\
\hline $\mathrm{H}$ & 4.187931 & 4.664999 & 4.714268 \\
\hline $\mathrm{H}$ & 3.685926 & 6.326982 & 2.826442 \\
\hline $\mathrm{H}$ & 4.836841 & 5.640024 & 1.667340 \\
\hline $\mathrm{H}$ & 3.103599 & 5.756962 & 1.264397 \\
\hline $\mathrm{H}$ & 1.389195 & 4. 271976 & 2.417515 \\
\hline $\mathrm{H}$ & 1.889668 & 3.229976 & 3.775896 \\
\hline $\mathrm{H}$ & 1.988815 & 4.986171 & 3.907788 \\
\hline $\mathrm{H}$ & -2.856298 & -2.977045 & 1.781434 \\
\hline $\mathrm{H}$ & -1.865380 & 1.155263 & 1.398336 \\
\hline $\mathrm{H}$ & -3.974534 & 2.139176 & 5.592718 \\
\hline $\mathrm{H}$ & -2.340641 & 1.509840 & 5.808730 \\
\hline $\mathrm{H}$ & -2.605403 & 3.244368 & 5.570786 \\
\hline $\mathrm{H}$ & -3.473217 & 4.132024 & 3.309404 \\
\hline $\mathrm{H}$ & -3.574005 & 3.043763 & 1.922748 \\
\hline H & -4.751934 & 2.906372 & 3.231003 \\
\hline $\mathrm{H}$ & -1.118931 & 3.538514 & 3.927354 \\
\hline H & -0.575793 & 1.847810 & 3.881016 \\
\hline $\mathrm{H}$ & -1.069685 & 2.692785 & 2.398662 \\
\hline $\mathrm{H}$ & -3.695620 & -3.747066 & 6.220737 \\
\hline $\mathrm{H}$ & -2.296825 & -2.907081 & 5.518395 \\
\hline $\mathrm{H}$ & -3.647906 & -1.975292 & 6.177284 \\
\hline $\mathrm{H}$ & -5.629493 & 1.478678 & 4.391000 \\
\hline $\mathrm{H}$ & -6.252587 & -0.134823 & 3.957262 \\
\hline $\mathrm{H}$ & -6.233616 & ๑. 390555 & 5.664678 \\
\hline $\mathrm{H}$ & -4.274006 & -4.412705 & 2.629203 \\
\hline $\mathrm{H}$ & -2.677005 & -4.401501 & 3.406428 \\
\hline $\mathrm{H}$ & -4.077524 & -5.080951 & 4. 248312 \\
\hline $\mathrm{H}$ & -5.999008 & -1.016496 & -5.687144 \\
\hline $\mathrm{H}$ & -5.031095 & -1.789036 & -6.953636 \\
\hline H & -5.948179 & -2.777811 & -5.823257 \\
\hline $\mathrm{H}$ & -2.210480 & -1.566630 & -3.162086 \\
\hline H & -4.593407 & -1.236756 & ๑. 360295 \\
\hline $\mathrm{H}$ & -7.867181 & -3.723798 & -4.181828 \\
\hline $\mathrm{H}$ & -6.144169 & -4.165505 & -4.012636 \\
\hline $\mathrm{H}$ & -7.164925 & -3.989193 & -2.560508 \\
\hline $\mathrm{H}$ & -3.098695 & -3.253810 & -6.409777 \\
\hline $\mathrm{H}$ & -2.333098 & -3.211118 & -4.814420 \\
\hline $\mathrm{H}$ & -3.865215 & -4.073868 & -5.040264 \\
\hline $\mathrm{H}$ & -7.272465 & -3.560512 & -0.233699 \\
\hline $\mathrm{H}$ & -6.357266 & -2.690635 & 1.005880 \\
\hline H & - 8.117132 & -2.499254 & $\odot .904292$ \\
\hline $\mathrm{H}$ & -7.917203 & $\odot .023760$ & $\odot .724646$ \\
\hline $\mathrm{H}$ & -6.168003 & -0.095228 & ๑. 910612 \\
\hline $\mathrm{H}$ & -6.850514 & ๑. 766231 & -0.478689 \\
\hline H & -3.768077 & $\odot .271730$ & -5.253747 \\
\hline $\mathrm{H}$ & -2.258012 & -0.624823 & -4.987522 \\
\hline $\mathrm{H}$ & -3.069735 & -0.712070 & -6.549866 \\
\hline $\mathrm{H}$ & -6.145828 & -2.800167 & 3. 485109 \\
\hline $\mathrm{H}$ & -5.941073 & -3.822266 & 4.912719 \\
\hline $\mathrm{H}$ & -5.931420 & -2.070464 & 5.091767 \\
\hline
\end{tabular}

\begin{tabular}{|c|c|c|c|}
\hline $\mathrm{H}$ & -9.150411 & -1.031623 & -0.856426 \\
\hline $\mathrm{H}$ & -8.230926 & $-\odot .533672$ & -2.286170 \\
\hline $\mathrm{H}$ & -8.588217 & -2.240679 & $-2.0 \odot 59 \odot 9$ \\
\hline $\mathrm{Rh}$ & -0.346873 & ๑. 678080 & $-\odot .935175$ \\
\hline 0 & -1.641170 & 5.767796 & 1.456813 \\
\hline C & ๑. 289327 & $\odot .148494$ & -2.722382 \\
\hline S & $-\odot .609081$ & 5.977375 & 0.438849 \\
\hline 0 & -0.187349 & -0.761253 & -3.347728 \\
\hline C & 0.583103 & 4.302182 & -1.352223 \\
\hline $\mathrm{N}$ & -0.453325 & 4.473547 & -0.335219 \\
\hline 0 & $\odot .755803$ & 6.386081 & 0.775844 \\
\hline C & -1.249915 & 7.131644 & -0.771102 \\
\hline $\mathrm{H}$ & ๑. 315921 & 4.814341 & -2.294031 \\
\hline $\mathrm{H}$ & 1.507557 & 4.748339 & -0.976549 \\
\hline C & -1.707680 & 3.825545 & -0.734794 \\
\hline C & -2.628477 & 7.343894 & -0.862755 \\
\hline C & -0.362519 & 7.807983 & -1.615958 \\
\hline C & -1.430380 & 2.732386 & -1.740908 \\
\hline $\mathrm{H}$ & -2.213694 & 3.434911 & ๑.149982 \\
\hline $\mathrm{H}$ & -2.393952 & 4.538209 & -1.223132 \\
\hline $\mathrm{H}$ & -3.299778 & 6.849116 & -281.480500 \\
\hline C & -3.116477 & 8.231925 & 10.493500 \\
\hline C & -0.870358 & 8.690365 & 11.973400 \\
\hline $\mathrm{H}$ & ๑. 707691 & 7.671211 & -1.502249 \\
\hline C & -2.180836 & 1.528574 & -1.646815 \\
\hline $\mathrm{H}$ & -1.210474 & 3.076717 & -2.747569 \\
\hline $\mathrm{H}$ & -4.186792 & 8.409547 & -1.884795 \\
\hline C & -2.251529 & 8.912762 & -2.689260 \\
\hline $\mathrm{H}$ & -0.182490 & 9.228684 & -3.212876 \\
\hline $\mathrm{H}$ & -2.461338 & 1. 010922 & -2.559653 \\
\hline $\mathrm{H}$ & -2.926908 & 1.456460 & -0.857068 \\
\hline C & -2.786934 & 9.859913 & -3.735584 \\
\hline $\mathrm{H}$ & -3.803768 & 10.186849 & -3.498904 \\
\hline $\mathrm{H}$ & -2.155385 & 10.749625 & -3.829388 \\
\hline $\mathrm{H}$ & -2.816239 & 9.378252 & -4.721797 \\
\hline C & 1.458656 & ๑. 997060 & -3.244925 \\
\hline $\mathrm{H}$ & 1.584086 & $\odot .763422$ & -4.307799 \\
\hline $\mathrm{H}$ & 2.352427 & $\odot .638775$ & -2.721273 \\
\hline C & 1.274536 & 2.494194 & -3.000347 \\
\hline $\mathrm{H}$ & $\odot .578850$ & 2.902682 & -3.746321 \\
\hline $\mathrm{H}$ & 2.234224 & 3. 000518 & -3.182597 \\
\hline $\mathrm{H}$ & 1.848575 & 2.201325 & 1.859612 \\
\hline C & ๑. 782702 & 2.806181 & -1.598070 \\
\hline $\mathrm{H}$ & 1.456753 & 2.434823 & -0.830852 \\
\hline
\end{tabular}

TS4-cis

B3LYP SCF energy: $\quad-5535.32070924$ a.u. B3LYP enthalpy: $\quad-5533.286247 \mathrm{a} \cdot \mathrm{u}$. B3LYP free energy: $\quad-5533.554878 \mathrm{a} . \mathrm{u}$. M06 SCF energy in solution: $\quad-5534.55656203$ a.u.

M06 enthalpy in solution: $\quad-5532.522100 \mathrm{a} \cdot \mathrm{u}$. M06 free energy in solution: $\quad-5532.790731$ a.u.

Imaginary frequency: $\quad-237.9516 \mathrm{~cm}-1$

Cartesian coordinates

$Y$

\begin{tabular}{|c|c|c|c|}
\hline AIVIT & $\wedge$ & 1 & 2 \\
\hline $\mathrm{P}$ & $\odot .0 \odot \odot \odot \odot \odot$ & -3.790152 & 6.944594 \\
\hline$P$ & ๑. . 000000 & -4.632901 & 8.340155 \\
\hline 0 & ๑. . ०००००० & -3.198845 & 8.959744 \\
\hline 0 & $\odot .0000 \odot \odot$ & -1.241451 & 8.052027 \\
\hline 0 & $\odot .0 \odot \odot \odot \odot \odot$ & -2.455518 & 1.434019 \\
\hline 0 & ๑. . 000000 & -1.802318 & 2.780497 \\
\hline 0 & 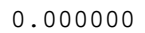 & -5.576626 & 8.426640 \\
\hline 0 & $\odot . \diamond \odot \odot \odot \odot \odot$ & -4.431063 & 9.081349 \\
\hline 0 & 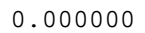 & -3.022165 & 9.533088 \\
\hline 0 & 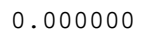 & -3.048247 & $\odot .858937$ \\
\hline C & $\odot .00000 \odot$ & -2.818361 & 1.367986 \\
\hline C & $\odot . \diamond \odot \odot \odot \odot \odot$ & -5.496029 & 10.014501 \\
\hline C & 1.165487 & -5.567420 & 9.968436 \\
\hline C & 1.537878 & -5.267760 & 11.054929 \\
\hline C & 1.942398 & -6.479250 & 9.777175 \\
\hline
\end{tabular}




\begin{tabular}{|c|c|c|c|c|c|c|c|}
\hline C & 1.979760 & 1. 012351 & 1.815673 & $\mathrm{H}$ & 4.219144 & -5.285795 & -1.335370 \\
\hline C & 1.255411 & 1.024569 & 1.832453 & $\mathrm{H}$ & 3.445389 & -6.628573 & $-\odot .402171$ \\
\hline C & $-\odot .350886$ & 2.026254 & 1.564183 & $\mathrm{H}$ & 2.092398 & $-\odot .973649$ & -2.617181 \\
\hline C & -0.612838 & 0.567373 & 3.144044 & $\mathrm{H}$ & 4.315692 & -1.251453 & 1.010488 \\
\hline C & $\odot .227356$ & $-\odot .325508$ & 3.498536 & $\mathrm{H}$ & 6.845253 & $-\odot .651445$ & 1.205073 \\
\hline C & 1.348061 & 1.337184 & 3.903891 & $\mathrm{H}$ & 8.180492 & -1.808863 & 1. 340148 \\
\hline C & 1.617819 & 1.326518 & 1.796258 & $\mathrm{H}$ & 7.928410 & $-\odot .918707$ & -0.166622 \\
\hline C & 0.810874 & 0.342479 & 3.059527 & $\mathrm{H}$ & 7.977196 & -3.296288 & -1.297507 \\
\hline C & 3.287063 & 1.207827 & 2.663064 & $\mathrm{H}$ & 7.995810 & -4.110414 & ๑.263623 \\
\hline C & 3.122794 & -0.947884 & -0.736770 & $\mathrm{H}$ & 6.713691 & -4.460043 & -0.904770 \\
\hline C & 3.014982 & -1.204424 & -2.107358 & $\mathrm{H}$ & 5.106986 & -3.959901 & 1.175660 \\
\hline C & 4.066275 & -1.783383 & -2.833997 & $\mathrm{H}$ & 6.657580 & -3.728145 & 2. 010898 \\
\hline C & 5.265427 & -2.075649 & -2.127898 & $\mathrm{H}$ & 5.407842 & -2.498079 & 2.122355 \\
\hline C & 5.346273 & -1.997867 & -0.713010 & $\mathrm{H}$ & 4.018095 & -3.899698 & -5.610253 \\
\hline C & 4.267193 & -1.384734 & -0.062840 & $\mathrm{H}$ & 5.189743 & -3.906265 & -4.281329 \\
\hline C & 6.490754 & -2.560364 & 0.180981 & $\mathrm{H}$ & 3.479184 & -4.268426 & -3.965908 \\
\hline C & 7.413790 & -1.415030 & $\odot .662162$ & $\mathrm{H}$ & 2.331052 & -2.210085 & -5.840844 \\
\hline C & 7.341594 & -3.662587 & -0.492906 & $\mathrm{H}$ & 1.695516 & -2.439386 & -4.208563 \\
\hline C & 5.869345 & -3.221482 & 1.443691 & $\mathrm{H}$ & 2.157900 & -0.821547 & -4.769316 \\
\hline C & 3.878550 & -2.139727 & -4.333541 & $\mathrm{H}$ & 4.696376 & $-\odot .228001$ & -5.048207 \\
\hline C & 4.165201 & -3.647108 & -4.553306 & $\mathrm{H}$ & 5.850377 & -1.576451 & -5.143118 \\
\hline C & 2.429268 & -1.882466 & -4.800420 & $\mathrm{H}$ & 4.525784 & -1.467722 & -6.298596 \\
\hline C & 4.801269 & -1.300820 & -5.250693 & $\mathrm{H}$ & 7.945138 & -1.330649 & -2.178536 \\
\hline C & 7.369571 & -1.527328 & -3.088305 & $\mathrm{H}$ & 8.034209 & -1.946675 & -3.846960 \\
\hline C & 2.760360 & 1.331277 & $\odot .977936$ & $\mathrm{H}$ & 6.946124 & -0.585684 & -3.454641 \\
\hline C & 3.881596 & 1.820073 & 0.304288 & $\mathrm{H}$ & 4.224952 & 1.301095 & $-\odot .580814$ \\
\hline c & 2.268906 & 2. 082723 & 2. 061549 & $\mathrm{H}$ & 5.835873 & 3.977995 & -2.358568 \\
\hline C & 2.968067 & 3.174189 & 2.588151 & $\mathrm{H}$ & 4.789511 & 2.593757 & -2.058741 \\
\hline C & 4.193053 & 3.530337 & 1.954663 & $\mathrm{H}$ & 4.214151 & 4.233124 & -1.696764 \\
\hline C & 4.587391 & 2.961960 & 0.722546 & $\mathrm{H}$ & 7.379063 & 2.460768 & $\odot .717969$ \\
\hline C & 5.687411 & 3.501822 & -0.236368 & $\mathrm{H}$ & 6.601298 & 1.527510 & -0.566834 \\
\hline C & 5.087769 & 3.571673 & -1.669255 & $\mathrm{H}$ & 7.648940 & 2.897402 & $-\odot .975045$ \\
\hline C & 6.896965 & 2.537716 & -0.262711 & $\mathrm{H}$ & 5.866178 & 3.284426 & 4. 007272 \\
\hline C & 6.133335 & 4.002993 & 3.224200 & $\mathrm{H}$ & 6.826561 & 3.524470 & 2.525609 \\
\hline c & 6.183128 & 4.934196 & $\odot .07034 \odot$ & $\mathrm{H}$ & 6.617059 & 4.871935 & 3.675204 \\
\hline C & 2.388413 & 3.992751 & 3.771451 & $\mathrm{H}$ & 6.802381 & 4.999440 & $\odot .964828$ \\
\hline C & 3.275515 & 3.890831 & 5.036517 & $\mathrm{H}$ & 6.798139 & 5.272257 & -0.771449 \\
\hline C & 2.247712 & 5.479830 & 3.359535 & $\mathrm{H}$ & 5.352627 & 5.636952 & $\odot .181553$ \\
\hline C & 0.984625 & 3.494139 & 4.173080 & $\mathrm{H}$ & 3.486513 & 2.845817 & 5.293625 \\
\hline C & -2.043040 & -1.867710 & 1.357194 & $\mathrm{H}$ & 4.220163 & 4.420318 & 4.915789 \\
\hline C & -2.351292 & -3.178792 & 1.749600 & $\mathrm{H}$ & 2.753241 & 4.342671 & 5.887942 \\
\hline C & -2.986174 & -3.456866 & 2.964044 & $\mathrm{H}$ & 1.835300 & 6.057712 & 4.195274 \\
\hline c & -3.338311 & -2.345743 & 3.782593 & $\mathrm{H}$ & 3.210158 & 5.914612 & 3.085787 \\
\hline C & -2.882395 & -1.034411 & 3.510802 & $\mathrm{H}$ & 1.564220 & 5.588979 & 2.509313 \\
\hline C & -2.275828 & $-\odot .830956$ & 2.258235 & $\mathrm{H}$ & $\odot .271662$ & 3.542946 & 3.341597 \\
\hline C & -2.956529 & $\odot .183246$ & 4.475396 & $\mathrm{H}$ & $\odot .998745$ & 2.467616 & 4.556324 \\
\hline C & -3.247592 & $-\odot .162522$ & 5.955095 & $\mathrm{H}$ & 0.594567 & 4.133622 & 4.971732 \\
\hline C & -4.011000 & 1.198471 & 3.972640 & $\mathrm{H}$ & -2.102993 & -3.990504 & 1.078480 \\
\hline C & -1.571605 & $\odot .884386$ & 4.484720 & $\mathrm{H}$ & -1.974529 & $\odot .174874$ & 1.984330 \\
\hline C & -2.468339 & -5.139013 & 4.764250 & $\mathrm{H}$ & -4.272713 & -0.484147 & 6.134834 \\
\hline c & -5.492491 & -2.257925 & 4.759317 & $\mathrm{H}$ & -2.578963 & -0.943595 & 6.327734 \\
\hline C & -3.208003 & -4.923649 & 3.419264 & $\mathrm{H}$ & -3.082710 & 0.738318 & 6.557505 \\
\hline C & -2.629808 & -5.924549 & 2.394551 & $\mathrm{H}$ & -4.029271 & 2. 080168 & 4.624306 \\
\hline C & -5.356680 & -1.988472 & -2.862504 & $\mathrm{H}$ & -3.788464 & 1.538705 & 2.954131 \\
\hline C & -4.171721 & -1.563861 & -3.516949 & $\mathrm{H}$ & -5.018876 & $\odot .770569$ & 3.963338 \\
\hline C & -5.343943 & -1.109788 & -5.831636 & $\mathrm{H}$ & -1.585587 & 1.713509 & 5.200227 \\
\hline C & -3.016830 & -1.441569 & -2.732254 & $\mathrm{H}$ & -0.780342 & $\odot .187852$ & 4.783463 \\
\hline C & -3.009693 & -1.693226 & -1.353863 & $\mathrm{H}$ & -1.298896 & 1.306798 & 3.513019 \\
\hline C & -4.229783 & -1.984010 & $-\odot .736634$ & $\mathrm{H}$ & -2.555225 & -6.187440 & 5.074589 \\
\hline C & -5.430276 & -2.106164 & -1.453431 & $\mathrm{H}$ & -1.403873 & -4.895581 & 4.672211 \\
\hline C & -6.574748 & -3.631388 & -4.029118 & $\mathrm{H}$ & -2.889306 & -4.514047 & 5.554461 \\
\hline c & -3.112004 & -2.243153 & -5.712298 & $\mathrm{H}$ & -5.634843 & -1.179568 & 4.635399 \\
\hline C & -6.661823 & -3.776899 & $-\odot .0 \odot 6867$ & $\mathrm{H}$ & -5.943405 & -2.775751 & 3.905715 \\
\hline C & -6.718912 & -2.356115 & -0.618077 & $\mathrm{H}$ & -5.979913 & -2.581728 & 5.681512 \\
\hline C & -6.771647 & -1.315176 & $\odot .533755$ & $\mathrm{H}$ & -3.158102 & -5.883333 & 1.434498 \\
\hline C & -3.367262 & $\odot .198406$ & -5.133696 & $\mathrm{H}$ & -1.563856 & -5.761927 & 2. 207781 \\
\hline C & -4.701810 & -5.285314 & 3.601163 & $\mathrm{H}$ & -2.750726 & -6.940776 & 2.784887 \\
\hline C & -4.029245 & -1.202624 & -5.024231 & $\mathrm{H}$ & -6.073878 & -0.447710 & -5.359289 \\
\hline C & -8.057941 & $-2.20600 \odot$ & -1.376101 & $\mathrm{H}$ & -5.106289 & -0.701164 & -6.821014 \\
\hline $\mathrm{H}$ & 2.245682 & -1.681039 & 5.016528 & $\mathrm{H}$ & -5.818999 & -2.078909 & -5.989993 \\
\hline $\mathrm{H}$ & 2.357540 & $-\odot .027803$ & 3.180194 & $\mathrm{H}$ & -2.093168 & -1.149590 & -3.209165 \\
\hline $\mathrm{H}$ & 0.498774 & -6.101236 & 3.985417 & $\mathrm{H}$ & -4.255543 & -2.129135 & ๑. 333615 \\
\hline $\mathrm{H}$ & 2.220746 & -5.926500 & 3.446488 & $\mathrm{H}$ & -7.487145 & -3.707110 & -4.624681 \\
\hline $\mathrm{H}$ & -1.502973 & -3.378422 & -2.554907 & $\mathrm{H}$ & -5.714492 & -3.930135 & -4.641535 \\
\hline $\mathrm{H}$ & $\odot .001767$ & -5.170449 & -3.375433 & $\mathrm{H}$ & -6.645160 & -4.307299 & -3.167420 \\
\hline \\
\hline
\end{tabular}




\begin{tabular}{|c|c|c|c|}
\hline $\mathrm{H}$ & -2.977970 & -1.987501 & -6.770121 \\
\hline $\mathrm{H}$ & -2.119252 & -2.282002 & -5.250539 \\
\hline $\mathrm{H}$ & -3.546357 & -3.248674 & -5.664487 \\
\hline $\mathrm{H}$ & -6.647433 & -4.546440 & $-\odot .786989$ \\
\hline $\mathrm{H}$ & -5.769481 & -3.915586 & ๑. 613542 \\
\hline $\mathrm{H}$ & -7.542678 & -3.955640 & ๑. 621770 \\
\hline $\mathrm{H}$ & -7.677848 & -1.473728 & 1.129506 \\
\hline $\mathrm{H}$ & -5.918420 & -1.380943 & 1.215345 \\
\hline $\mathrm{H}$ & -6.807316 & -0.294820 & 0.134763 \\
\hline $\mathrm{H}$ & -3.989300 & 0.960698 & -4.649329 \\
\hline $\mathrm{H}$ & -2.368984 & ๑. 235184 & -4.689664 \\
\hline $\mathrm{H}$ & -3.263867 & $\odot .474148$ & -6.189203 \\
\hline $\mathrm{H}$ & -5.286688 & -5.039614 & 2.707124 \\
\hline $\mathrm{H}$ & -4.795738 & -6.364712 & 3.768205 \\
\hline $\mathrm{H}$ & -5.144594 & -4.783799 & 4.461262 \\
\hline H & -8.871623 & -2.240152 & -0.641697 \\
\hline $\mathrm{H}$ & -8.124508 & -1.254402 & -1.909855 \\
\hline $\mathrm{H}$ & -8.236786 & $-3 . \odot \odot 7687$ & -2.093249 \\
\hline $\mathrm{Rh}$ & -0.436352 & $\odot .707734$ & $-\odot .496152$ \\
\hline 0 & ๑. 161824 & 6.950494 & -1.396747 \\
\hline C & ๑. 124196 & ๑. 644866 & -2.385055 \\
\hline$S$ & -1.143095 & 6.331326 & -1.651137 \\
\hline 0 & -0.177330 & $-\odot .254022$ & -3.124635 \\
\hline C & ๑. . 023462 & 4.403939 & $-\odot . \odot 9 \odot \odot 87$ \\
\hline $\mathrm{N}$ & -1.226174 & 4.974963 & -0.621525 \\
\hline 0 & -1.535850 & 5.923473 & -3.008164 \\
\hline C & -2.429553 & 7.395841 & -1.015029 \\
\hline $\mathrm{H}$ & 0.833660 & 5.110976 & -0.293946 \\
\hline $\mathrm{H}$ & -0.053860 & 4.317787 & 1.001077 \\
\hline C & -2.198623 & 3.947136 & -0.996396 \\
\hline C & -3.639592 & 7.499713 & -1.701015 \\
\hline C & -2.196728 & 8.126400 & ○. 154464 \\
\hline C & -1.895630 & 2.659924 & $-\odot .278431$ \\
\hline $\mathrm{H}$ & -3.202375 & 4.253897 & -0.667307 \\
\hline H & -2.253335 & 3.792634 & -2.080542 \\
\hline $\mathrm{H}$ & -3.790152 & 6.944594 & -237.951600 \\
\hline C & -4.632901 & 8.340155 & $8.34700 \odot$ \\
\hline C & -3.198845 & 8.959744 & 8.478500 \\
\hline $\mathrm{H}$ & -1.241451 & 8.052027 & 0.663693 \\
\hline C & -2.455518 & 1.434019 & -0.722153 \\
\hline $\mathrm{H}$ & -1.802318 & 2.780497 & ๑. 801329 \\
\hline $\mathrm{H}$ & -5.576626 & 8.426640 & -1.728548 \\
\hline C & -4.431063 & 9.081349 & -0.025063 \\
\hline H & -3.022165 & 9.533088 & 1.546453 \\
\hline $\mathrm{H}$ & -3.048247 & ๑. 858937 & -0.015392 \\
\hline $\mathrm{H}$ & -2.818361 & 1.367986 & -1.746500 \\
\hline C & -5.496029 & 10.014501 & ๑. 498506 \\
\hline H & -5.567420 & 9.968436 & 1.590627 \\
\hline $\mathrm{H}$ & -5.267760 & 11.054929 & ๑. 233205 \\
\hline $\mathrm{H}$ & -6.479250 & 9.777175 & ๑. 081286 \\
\hline C & 1. 012351 & 1.815673 & -2.814572 \\
\hline H & 1. 024569 & 1.832453 & -3.909810 \\
\hline $\mathrm{H}$ & 2.026254 & 1.564183 & -2.477434 \\
\hline C & 0.567373 & 3.144044 & -2.218423 \\
\hline $\mathrm{H}$ & -0.325508 & 3.498536 & -2.742064 \\
\hline $\mathrm{H}$ & 1.337184 & 3. 903891 & -2.422176 \\
\hline $\mathrm{H}$ & 1. 326518 & 1.796258 & 2.511798 \\
\hline C & 0.342479 & 3.059527 & -0.718187 \\
\hline $\mathrm{H}$ & 1.207827 & 2.663064 & -0.194835 \\
\hline
\end{tabular}

TS5-trans

B3LYP SCF energy: B3LYP enthalpy:

B3LYP free energy: M06 SCF energy in solution: $\quad-5534.58860009$ a.u.

M06 enthalpy in solution: M06 free energy in solution: a.u.

Imaginary frequency:

$-301.1922 \mathrm{~cm}-1$

Cartesian coordinates ATOM $\mathrm{X}$ Y
-5535.35555387 a.u. -5533.319623 a.u. -5532.552669 a.u. $-5532.823081$

Z $\odot . ๑ \odot \odot \odot \odot \odot$ $\odot .000000$

$\odot .00000 \odot$

๑. 000000

$\odot .000000$

$\odot .0 \odot \odot \odot \odot \odot$

$\odot .000000$

$\odot .0000 \odot \odot$

$\odot .000000$

$\odot .000000$

$\odot .000000$

0.000000

3. 749918

3. 640954

2. 436849

1. 335728

5. 702386

2. 900668

3. 316002

3. 824213

3. 902644

3. 496598

2. 978769

4. 409891

$-0.237863$

$-0.577570$

$-0.764476$

$-0.651346$

$-\odot .204561$

$-0.026910$

0.121490

$-1.156379$

0.809340

1. 110991

$-1.028586$

0.057563

$-0.953620$

$-2.436395$

$-2.123069$

$-1.537022$

$-2.547599$

$-1.723561$

$-2.804012$

$-3.735799$

$-3.701733$

$-4.833889$

$-5.062684$

$-4.411317$

$-4.529436$

- 6.212619

- 3.013411

$-3.008932$

$-4.362499$

$-1.904456$

3. 243343

4.628736

5.481333

4.886361

3. 483926

2. 690276

2. 744285

3. 623464

2. 063091

1. 636973

7. 359121

5.873271

6.993068

7.334831

2. 742813

1. 758903

๑. 735112

1. 633103

2. 410667

3. 272838

3. 440288
$-5.156265$

$-4.684819$

$-5.583309$

$-4.305630$

$-2.525728$

$-6.717477$

$-4.696868$

$-4.218842$

$-7.161242$

$-6.655840$

$-7.713554$

$-8.367763$

$-7.805804$

$-8.319512$

$-9.107778$

$-8.549913$

$-7.325572$

$-9.623779$

$-9.211128$

$-8.629098$

$-10.046449$

$-11.066813$

$-9.626843$

$-10.125516$

2. 651361

2. 039985

2. 776575

4. 192227

4.841731

4.032623

6.358383

7.169546

6. 914549

6. 616794

2. 037801

2. 422562

$\odot .504711$

2. 348018

5. 630656

2. 210827

2. 983332

1. 728926

2. 138579

3. 040809

3. 363686

4.048634

3. 225727

5. 481357

4. 914506

4.101423

1. 549876

2. 637397

๑. 788331

0.540434

$-0.926021$

$-0.734556$

$-1.030859$

$-1.570269$

$-1.602853$

$-1.316557$

$-1.879461$

$-1.780960$

$-3.268533$

$-0.804094$

0.270097

$-3.444036$

$-0.696804$

0.014061

$-4.715751$

$-3.787530$

$-5.396626$

$-2.603459$

$-2.339967$

$-3.335881$

$-4.554617$
$-2.832436$

. 779109

$-1.198607$

$-3.925838$

2. 961519

- 3.119015

$-4.383061$

$-4.701299$

$-1.859202$

$-4.375881$

$-3.403768$

$-2.330886$

$-4.693631$

$-1.343802$

$-2.556654$

$-4.899273$

$-5.524042$

$-1.724713$

$-3.838636$

$-5.902741$

- 4 . 081091

$-4.381743$

$-4.881677$

- 3.179278

$-1.132756$

$-2.340428$

$-3.516114$

- 3.424046

$-2.237927$

$-1.106767$

$-2.113618$

$-1.783671$

- 3.384162

$-0.947217$

$-4.853935$

$-5.889768$

$-4.678585$

$-5.415283$

$-4.708825$

1. 433949

0.858305

2. 741868

3. 528774

2. 939214

1. 562778

0.743119

$-0.556054$

○. 341182

4. 146441

1. 442088

4. 948716

6. 050489

4. 979909

5. 313744

1.141706

1. 048073

2. 114616

3. 291277

3. 484508

2. 359804

4. 824609

6.093347

4. 780019

5. 001106

3. 179830

4. 347859

2. 025401

0.697007

- 2. 696276

$-3.135992$

$-4.945834$

$-2.396665$

$-1.259444$

$-0.798832$

$-1.472568$ 
$7.201132-0.192941$

$4.932498-3.927859$

$6.213691-3.855700$

$6.623155-3.641001$

$7.178609-0.635125$

$6.751102 \quad 0.929420$

$8.394421 \quad 0.291062$

$0.523019-6.142472$

$-0.084095$

1.641327

0.922942

3. 837614

4.167036

3.797222

4.997078

0.600639

1. 254961

2.285692

6.194318

5.916263

6.493032

4.565909

3.959187

2. 889793

$-0.982570$

$-0.739402$

$-1.302504$

7.586901

8.928354

7.877846

4.936588

3. 359148

4.847535

$-1.017607$

$-0.149312$

$-1.119403$

$-2.477185$

$-1.383110$

$-0.748830$

$-3.485392$

$-2.781133$

$-2.391044$

$-0.021079$

$-0.246701$

$-1.990220$

$-4.808406$

$-3.738577$

$-2.997239$

$-2.446732$

$-1.660480$

$-5.156265$

$-4.684819$

$-5.583309$

$-4.305630$

$-2.525728$

$-6.717477$

$-4.696868$

$-4.218842$

$-7.161242$

$-6.655840$

$-7.713554$

$-8.367763$

$-7.805804$

$-8.319512$

$-9.107778$

$-8.549913$

$-7.325572$

$-9.623779$

$-9.211128$

$-8.629098$

$-10.046449$

$-11.066813$

$-9.626843$

$-10.125516$
$-5.420862$

$-5.701696$

$-1.851695$

$-3.157085$

$-6.601778$

$-4.868456$

$-5.373988$

$-3.093667$

$-1.958038$

$-3.233786$

$-4.940224$

$-4.099638$

$-5.775150$

$-6.501572$

$-4.846838$

$-6.154723$

$-4.257017$

$-2.563362$

$-3.733627$

$-2.709357$

$-1.678431$

$-2.405623$

$-7.676848$

$-7.423481$

$-7.063663$

1. 015869

$-0.592689$

$-1.650588$

$-0.702502$

$-1.511899$

$-3.001997$

$-1.853895$

0.116168

$-0.343467$

$-3.486887$

$-2.862702$

$-3.887115$

$-1.544538$

$-2.059603$

$-3.178448$

$-4.118196$

$-4.835153$

$-2.832436$

$-0.779109$

$-1.198607$

$-3.925838$

$-2.961519$

$-3.119015$

$-4.383061$

$-4.701299$

$-1.859202$

$-4.375881$

$-3.403768$

$-2.330886$

$-4.693631$

$-1.343802$

$-2.556654$

$-4.899273$

$-5.524042$

$-1.724713$

$-3.838636$

$-5.902741$

$-4.081091$

$-4.381743$

$-3.179278$
$-4.881677$
4.155591

4. 628510

3. 390964

5.117034

$-0.172835$

0.552931

0.705405

$-4.175092$

$-5.673736$

$-5.469108$

$-2.726556$

0.106285

$-4.935672$

$-5.157390$

$-3.937469$

$-6.354399$

$-5.163136$

$-5.831167$

$-1.834898$

$-0.302122$

$-0.302505$

1.180598

1. 259754

0.727486

$-3.143863$

$-3.617621$

$-4.803237$

1.369972

1. 884499

3. 097643

$-0.737829$

$-1.501667$

$-2.384550$

3. 143472

$-0.021041$

1.334766

0.109066

2. 502199

0.664277

0.147892

0.755761

$-0.926657$

1.322173

$-0.313880$

0.427539

$-0.604557$

1. 196105

$-0.472845$

1. 398421

- 0.014229

301.192200

5.907600

9.696400

$-0.749641$

$-1.442923$

$-1.770716$

0.175333

$-1.512988$

$-2.370985$

$-2.518784$

$-0.307272$

0.304756

0.225500

$-0.142637$

1.464626

1.385273

$-0.281356$

1.937532

2. 023351

1.796232

3. 257687

2. 985880

3. 875767

3.873080 
TS5-cis

B3LYP SCF energy:

B3LYP enthalpy:

B3LYP free energy:

M06 SCF energy in solution:

a.u

M06 enthalpy in solution:

M06 free energy in solution:

a.u

Imaginary frequency: $-320.6607 \mathrm{~cm}-1$

Cartesian coordinates

\begin{tabular}{|c|c|c|c|}
\hline АTOM & $x$ & Y & Z \\
\hline $\mathrm{P}$ & $\odot . \odot \odot \odot \odot \odot \odot$ & 3.283273 & -4.732726 \\
\hline$P$ & $\odot .0 \odot \odot \odot \odot \odot$ & 1.287204 & -4.041365 \\
\hline 0 & $\odot .0 \odot \odot \odot \odot \odot$ & 5.131243 & -4.176473 \\
\hline 0 & $\odot . \odot \odot \odot \odot \odot \odot$ & 3. 317202 & -5.687190 \\
\hline 0 & $\odot .0 \odot \odot \odot \odot \odot$ & 3. 361332 & -4.936225 \\
\hline 0 & $\odot . ๑ \odot \odot \odot \odot \odot$ & 5.022013 & -5.620544 \\
\hline 0 & $\odot . ๑ \odot \odot \odot \odot \odot$ & 4.589957 & -3.236026 \\
\hline 0 & $\odot . \odot \odot \odot \odot \odot \odot$ & 6.837726 & -3.727885 \\
\hline 0 & $\odot . ๑ \odot \odot \odot \odot \odot$ & 7.408647 & -2.699716 \\
\hline 0 & 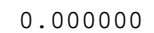 & 7.595106 & -4.455989 \\
\hline C & $\odot .000000$ & 6.807111 & -2.159022 \\
\hline C & ๑. . ०००००० & 8.756736 & -2.391282 \\
\hline C & -2.980995 & 8.935599 & -4.133230 \\
\hline C & -2.662417 & 7.138648 & -5.259119 \\
\hline C & -1.430759 & 9.206974 & -1.590993 \\
\hline C & -0.522027 & 9.539218 & -3.098813 \\
\hline C & -4.653760 & 9.528339 & -4.694360 \\
\hline C & -2.825509 & 11.002600 & -2.779526 \\
\hline C & -3.390915 & 11.633820 & -3.567983 \\
\hline C & -3.760322 & 11.274613 & -1.837152 \\
\hline C & -3.544130 & 11.261043 & -2.703882 \\
\hline C & -2.986247 & 2.211160 & -1.521657 \\
\hline C & -2.596581 & 2.501272 & -0.486972 \\
\hline C & -3.594701 & 2.811553 & -1.826918 \\
\hline C & ๑. 661131 & 2.526558 & ๑.972495 \\
\hline C & ๑. 788398 & 2.020815 & 2.266384 \\
\hline C & 1.032960 & 2.854379 & 3.364673 \\
\hline C & 1. 207192 & 4.242850 & 3.105839 \\
\hline C & $\odot .987445$ & 4.807452 & 1.817122 \\
\hline C & 0.732493 & 3.907619 & $\odot .772658$ \\
\hline C & $\odot .984482$ & 6.329387 & 1.491727 \\
\hline C & 2.422122 & 6.834622 & 1.212054 \\
\hline C & 0.317232 & 7.163181 & 2.612486 \\
\hline C & $\odot .169609$ & 6.619111 & ๑. 204078 \\
\hline C & 1.050090 & 2. 257671 & 4.795868 \\
\hline C & -0.014043 & 2.963845 & 5.673169 \\
\hline C & ๑. 692096 & $\odot .755156$ & 4.785949 \\
\hline C & 2.445787 & 2.373041 & 5.452936 \\
\hline C & 2.828065 & 5.540847 & 4. 363157 \\
\hline C & 2.074889 & 1.516730 & -1.352044 \\
\hline C & 3.161253 & 2.126996 & -0.720326 \\
\hline C & 2.294663 & $\odot .858315$ & -2.575234 \\
\hline C & 3.520040 & ๑. 941097 & -3.245448 \\
\hline C & 4.554946 & 1. 694698 & -2.621992 \\
\hline C & $4.440 \odot 73$ & 2.177289 & -1.297909 \\
\hline C & 5.596220 & 2.710290 & -0.402518 \\
\hline C & 5.500926 & 2.014671 & ๑. 984685 \\
\hline C & 5.449597 & 4.235628 & -0.191155 \\
\hline C & 5.858508 & 3.211050 & -3.884359 \\
\hline C & 7.022833 & 2.402812 & -0.914746 \\
\hline C & 3.752106 & $\odot .157166$ & -4.564478 \\
\hline C & 4.113854 & 1.084352 & -5.750584 \\
\hline C & 4.893380 & -0.868346 & -4.342648 \\
\hline C & 2.494980 & -0.633035 & -4.985511 \\
\hline C & -3.292126 & -0.542734 & -1.190109 \\
\hline C & -4.627477 & -0.114486 & -1.211745 \\
\hline C & -5.457144 & $-\odot .361426$ & -2.307624 \\
\hline C & -4.898059 & -1.097428 & -3.391449 \\
\hline C & -3.512536 & -1.369939 & -3.485846 \\
\hline C & -2.744732 & -1.122939 & -2.333195 \\
\hline
\end{tabular}

-5535.35279153 a.u. -5533.316675 a.u. -5533.585826 a.u. $-5532.815590$ $-5534.58255583$ -5532.546439 a.u. 


\begin{tabular}{|c|c|c|c|}
\hline $\mathrm{H}$ & 1.651322 & ๑. . 028825 & -5.218833 \\
\hline $\mathrm{H}$ & 2.724067 & -1.197305 & -5.895809 \\
\hline $\mathrm{H}$ & - 5.019451 & $\odot .409978$ & -0.350147 \\
\hline $\mathrm{H}$ & -1.691419 & -1.376176 & -2.336912 \\
\hline $\mathrm{H}$ & -4.342276 & -2.539553 & -6.148697 \\
\hline $\mathrm{H}$ & -3.997757 & $-\odot .808271$ & -6.228334 \\
\hline $\mathrm{H}$ & -2.855357 & -1.981422 & -6.900056 \\
\hline $\mathrm{H}$ & -1.699433 & -3.652734 & -5.422119 \\
\hline $\mathrm{H}$ & -1.567827 & -3.377299 & -3.681618 \\
\hline $\mathrm{H}$ & -3.075772 & -4.011859 & -4.368306 \\
\hline $\mathrm{H}$ & -0.992525 & -1.245157 & -5.871859 \\
\hline $\mathrm{H}$ & -1.825688 & ๑. 101558 & -5.072961 \\
\hline $\mathrm{H}$ & $-\odot .798800$ & -1.006379 & -4.139634 \\
\hline $\mathrm{H}$ & - 8.036161 & 1.561694 & -3.640717 \\
\hline $\mathrm{H}$ & -6.293056 & 1.897849 & -3.630443 \\
\hline $\mathrm{H}$ & -6.920825 & $\odot .508746$ & -4.531752 \\
\hline $\mathrm{H}$ & -5.230674 & -3.533649 & -4.473401 \\
\hline $\mathrm{H}$ & -6.597952 & -3.144172 & -3.398834 \\
\hline $\mathrm{H}$ & -6.801213 & -3.056981 & -5.172473 \\
\hline $\mathrm{H}$ & -7.166723 & $\odot .569370$ & -0.188357 \\
\hline $\mathrm{H}$ & -6.439367 & 1.943765 & -1.049746 \\
\hline $\mathrm{H}$ & -8.160791 & 1.578067 & -1.238076 \\
\hline $\mathrm{H}$ & -2.105348 & -5.383727 & 4. 924288 \\
\hline $\mathrm{H}$ & -1.464844 & -4.636082 & 6.395699 \\
\hline $\mathrm{H}$ & -3.192752 & -4.605469 & 6.077112 \\
\hline $\mathrm{H}$ & -1.517077 & -1.334054 & 2.991389 \\
\hline $\mathrm{H}$ & -4.301186 & -2.538960 & $-\odot .008398$ \\
\hline $\mathrm{H}$ & -5.858810 & -4.878610 & 5.468138 \\
\hline $\mathrm{H}$ & -4.894095 & -3.387287 & 5.623830 \\
\hline $\mathrm{H}$ & -6.182860 & -3.485972 & 4. 396661 \\
\hline $\mathrm{H}$ & -1.699966 & -2.138118 & 6.776448 \\
\hline $\mathrm{H}$ & -2.028124 & -1.051952 & 5.416532 \\
\hline $\mathrm{H}$ & -3.341345 & -1.996046 & 6.132606 \\
\hline $\mathrm{H}$ & -6.702222 & -5.087542 & 2.949209 \\
\hline $\mathrm{H}$ & -7.169727 & -3.692009 & 1.950524 \\
\hline $\mathrm{H}$ & -7.456980 & -5.341099 & $1.3786 \odot 5$ \\
\hline $\mathrm{H}$ & -6.276665 & -5.304095 & -0.653139 \\
\hline $\mathrm{H}$ & -6.142452 & -3.553097 & -0.470959 \\
\hline $\mathrm{H}$ & -4.706451 & -4.524449 & -0.865126 \\
\hline $\mathrm{H}$ & $-\odot .191997$ & -3.976556 & 3.872386 \\
\hline $\mathrm{H}$ & -0.113931 & -2.221751 & 4.124822 \\
\hline $\mathrm{H}$ & 0.114555 & -3.313994 & 5.485591 \\
\hline $\mathrm{H}$ & -7.846333 & -1.574220 & -1.519411 \\
\hline $\mathrm{H}$ & -8.970165 & -0.414402 & -2.241301 \\
\hline $\mathrm{H}$ & -7.990514 & -1.441360 & -3.286466 \\
\hline $\mathrm{H}$ & -5.433687 & -6.899632 & $\odot .962876$ \\
\hline $\mathrm{H}$ & -3.805986 & -6.215971 & $\odot .821932$ \\
\hline $\mathrm{H}$ & -4.560014 & -6.395425 & 2.420578 \\
\hline $\mathrm{H}$ & 1.502342 & ๑. 258335 & -2.998335 \\
\hline $\mathrm{Rh}$ & -0.004101 & -0.750385 & 0.188872 \\
\hline $\mathrm{C}$ & $\odot .707874$ & -2.081001 & -1.094823 \\
\hline 0 & $\odot .875624$ & -1.975979 & -2.288911 \\
\hline C & $\odot .170430$ & -3.356688 & $-\odot .415319$ \\
\hline C & 2.517664 & -2.395401 & 1.312716 \\
\hline $\mathrm{H}$ & -0.523673 & -3.813955 & -1.127209 \\
\hline $\mathrm{H}$ & $-\odot .406048$ & -3.143307 & 0.503868 \\
\hline C & 1.344216 & -4.291152 & -0.056149 \\
\hline C & 4.068867 & -2.517189 & 1.523606 \\
\hline $\mathrm{H}$ & 2.094329 & -1.914887 & 2.202379 \\
\hline C & 2.020904 & -3.884854 & 1.260792 \\
\hline $\mathrm{H}$ & 2.059680 & -4.307690 & -0.886011 \\
\hline $\mathrm{H}$ & ๑. 954594 & -5.310869 & 0.047465 \\
\hline N & 4.445382 & -3.902178 & 1.194981 \\
\hline $\mathrm{H}$ & 4.322145 & -2.346914 & 2.576955 \\
\hline $\mathrm{H}$ & 4.638195 & -1.804266 & ๑. 923348 \\
\hline C & 3.283273 & -4.732726 & -320.660700 \\
\hline $\mathrm{H}$ & 1.287204 & -4.041365 & 6.408700 \\
\hline S & 5.131243 & -4.176473 & 10.578100 \\
\hline $\mathrm{H}$ & 3.317202 & -5.687190 & 1. 036425 \\
\hline $\mathrm{H}$ & 3.361332 & -4.936225 & 2.638896 \\
\hline 0 & 5.022013 & -5.620544 & -0.572339 \\
\hline 0 & 4.589957 & -3.236026 & -1.345678 \\
\hline C & 6.837726 & -3.727885 & -0.060563 \\
\hline C & 7.408647 & -2.699716 & -0.809202 \\
\hline C & 7.595106 & -4.455989 & ๑.863272 \\
\hline
\end{tabular}

$\begin{array}{lrrr}\mathrm{H} & 6.807111 & -2.159022 & -1.531780 \\ \mathrm{C} & 8.756736 & -2.391282 & -0.616986 \\ \mathrm{C} & 8.935599 & -4.133230 & 1.040138 \\ \mathrm{H} & 7.138648 & -5.259119 & 1.432256 \\ \mathrm{H} & 9.206974 & -1.590993 & -1.198538 \\ \mathrm{C} & 9.539218 & -3.098813 & 0.303559 \\ \mathrm{H} & 9.528339 & -4.694360 & 1.758238 \\ \mathrm{C} & 11.002600 & -2.779526 & 0.492610 \\ \mathrm{H} & 11.633820 & -3.567983 & 0.063323 \\ \mathrm{H} & 11.274613 & -1.837152 & 0.008023 \\ \mathrm{H} & 11.261043 & -2.703882 & 1.554942 \\ \mathrm{C} & 2.211160 & -1.521657 & 0.090130 \\ \mathrm{H} & 2.501272 & -0.486972 & 0.280456 \\ \mathrm{H} & 2.811553 & -1.826918 & -0.768501\end{array}$

TS6

B3LYP SCF energy: $\quad-3819.30933138 \mathrm{a} \cdot \mathrm{u}$. B3LYP enthalpy: -3818.366147 a.u. B3LYP free energy: $\quad-3818.527568 \mathrm{a} . \mathrm{u}$. Mo6 SCF energy in solution: $\quad-3819.27575142$ a.u. M06 enthalpy in solution: $\quad-3818.332567 \mathrm{a} \cdot \mathrm{u}$. M06 free energy in solution: -3818.493988 a.u. Imaginary frequency: $\quad-277.9560 \mathrm{~cm}-1$

Cartesian coordinates

\begin{tabular}{|c|c|c|c|}
\hline ATOM & $\mathrm{x}$ & $Y$ & Z \\
\hline $\mathrm{P}$ & $\odot .363765$ & -1.465376 & ๑. 090822 \\
\hline$P$ & 1.582073 & 1.628143 & -0.095211 \\
\hline 0 & 4.927193 & -2.137282 & 1.584323 \\
\hline 0 & 5.567382 & -1.006270 & 3.500800 \\
\hline 0 & 5.481470 & -1.009718 & -1.233810 \\
\hline 0 & 5.294554 & -2.286288 & -3.158001 \\
\hline C & 2.846280 & $\odot .872285$ & 1.026414 \\
\hline C & 3.386897 & -0.416626 & 0.720232 \\
\hline C & 4.293057 & -0.924744 & 1.637594 \\
\hline C & 4.678415 & -0.250870 & 2.795682 \\
\hline C & 4.178941 & ๑. 996333 & 3. 097295 \\
\hline C & 3.256106 & 1.540631 & 2.187707 \\
\hline C & 5.820428 & -2.172700 & 2.705896 \\
\hline C & 1.881726 & -1.790577 & -0.895514 \\
\hline C & 1.792334 & -2.565076 & -2.059880 \\
\hline C & 2.892794 & -2.798976 & -2.901790 \\
\hline C & 4.086754 & -2.221816 & -2.529103 \\
\hline C & 4.194748 & -1.456583 & -1.367934 \\
\hline C & 3.129318 & -1.205391 & -0.517626 \\
\hline C & 6.199220 & -1.493728 & -2.377122 \\
\hline C & 2.418235 & 1.628484 & -1.737659 \\
\hline C & 1.730642 & 1.217250 & -2.887580 \\
\hline C & 3.757124 & 2.043107 & -1.850703 \\
\hline C & 1.535681 & 3.413665 & 0.405016 \\
\hline C & 1.990328 & 4.443477 & -0.432202 \\
\hline C & $\odot .974142$ & 3.760228 & 1.650430 \\
\hline C & $\odot .709305$ & -2.088863 & 1.784980 \\
\hline C & 1.658077 & -3.097664 & 2.017428 \\
\hline C & -0.038742 & -1.585127 & 2.861458 \\
\hline C & -1.577035 & -2.287577 & -1.769184 \\
\hline C & -0.880665 & -2.633082 & -0.599544 \\
\hline C & -1.101746 & -3.889257 & -0.014648 \\
\hline $\mathrm{H}$ & 4.484678 & 1.535676 & 3. 986517 \\
\hline $\mathrm{H}$ & 2.876561 & 2.529987 & 2.401399 \\
\hline $\mathrm{H}$ & 5.622290 & -3.068476 & 3.301013 \\
\hline $\mathrm{H}$ & 6.857263 & -2.153743 & 2.349982 \\
\hline $\mathrm{H}$ & $\odot .845214$ & -3.012490 & -2.331556 \\
\hline $\mathrm{H}$ & 2.805462 & -3.405205 & -3.796229 \\
\hline $\mathrm{H}$ & 6.547633 & -0.644172 & -2.974755 \\
\hline $\mathrm{H}$ & 7.036992 & -2.116128 & -2.047177 \\
\hline $\mathrm{H}$ & $\odot .707102$ & ๑. 866244 & -2.803663 \\
\hline $\mathrm{H}$ & 4.311101 & 2.342846 & -0.965763 \\
\hline $\mathrm{H}$ & 2.419673 & 4.212757 & -1.400388 \\
\hline $\mathrm{H}$ & 2.247215 & -3.494199 & 1.196527 \\
\hline $\mathrm{H}$ & -0.790945 & -0.821981 & 2.689257 \\
\hline
\end{tabular}




\begin{tabular}{|c|c|c|c|}
\hline $\mathrm{H}$ & -1.446067 & -1.306567 & -2.212453 \\
\hline $\mathrm{H}$ & -0.580807 & -4.170402 & 0.893838 \\
\hline $\mathrm{Rh}$ & -0.507643 & 0.705180 & $\odot .076086$ \\
\hline 0 & -2.444780 & $-\odot .255974$ & $\odot .876247$ \\
\hline C & -1.637225 & 1.717984 & -1.462588 \\
\hline$S$ & -3.846485 & $\odot .078122$ & 1.260023 \\
\hline 0 & $-2.41800 \odot$ & $\odot .905135$ & -1.923778 \\
\hline C & -3.040517 & 2.570649 & 1. 027108 \\
\hline $\mathrm{N}$ & -4.130663 & 1.633402 & $\odot .645804$ \\
\hline 0 & -4.159281 & $-\odot .024370$ & 2.691660 \\
\hline C & -4.993937 & -0.933776 & ๑. 346756 \\
\hline $\mathrm{H}$ & -3.521720 & 3.549702 & 1.123803 \\
\hline $\mathrm{H}$ & -2.655313 & 2.316691 & 2.023337 \\
\hline C & -5.496337 & 2.143644 & ๑. 959196 \\
\hline C & -5.874534 & -1.742525 & 1.070019 \\
\hline C & -5.000075 & -0.906958 & -1.051273 \\
\hline C & -5.881892 & 3. 226181 & -0.009561 \\
\hline $\mathrm{H}$ & -6.191014 & 1.303037 & 0.857771 \\
\hline $\mathrm{H}$ & -5.557400 & 2.497618 & 1.997406 \\
\hline $\mathrm{H}$ & -5.851695 & -1.736352 & 2.154212 \\
\hline C & -6.781676 & -2.536492 & ๑. 371602 \\
\hline C & -5.925017 & -1.701734 & -1.723492 \\
\hline $\mathrm{H}$ & -4.300778 & $-\odot .276002$ & -1.588297 \\
\hline C & -6.250201 & 4.455921 & 0.353633 \\
\hline $\mathrm{H}$ & -5.866769 & 2.941368 & -1.060823 \\
\hline $\mathrm{H}$ & -7.473920 & -3.164605 & ๑. 925861 \\
\hline C & -6.824183 & -2.528337 & -1.030365 \\
\hline $\mathrm{H}$ & -5.951430 & -1.679167 & -2.810016 \\
\hline $\mathrm{H}$ & -6.557423 & 5.198643 & -0.377072 \\
\hline $\mathrm{H}$ & -6.279593 & 4.760263 & 1.398030 \\
\hline C & -7.800697 & -3.403666 & -1.777429 \\
\hline $\mathrm{H}$ & -8.081138 & -2.962778 & -2.739176 \\
\hline $\mathrm{H}$ & -7.360947 & -4.388184 & -1.984978 \\
\hline $\mathrm{H}$ & -8.713750 & -3.571952 & -1.197971 \\
\hline C & -1.213897 & 3.009527 & -2.157157 \\
\hline $\mathrm{H}$ & -1.556951 & 3.088606 & -3.192085 \\
\hline $\mathrm{H}$ & -0.142261 & 3.204008 & -2.099206 \\
\hline C & -1.994214 & 3.791333 & -1.081393 \\
\hline $\mathrm{H}$ & -3.038148 & 3.924560 & -1.379215 \\
\hline $\mathrm{H}$ & -1.565339 & 4.758954 & -0.803082 \\
\hline C & -1.888611 & 2.718177 & ๑. 037585 \\
\hline $\mathrm{H}$ & ๑.599315 & 2.982334 & 2.311472 \\
\hline $\mathrm{H}$ & -0.990269 & 2.953239 & $\odot .612229$ \\
\hline C & $\odot .164352$ & -2.081101 & 4.150049 \\
\hline $\mathrm{H}$ & -0.421393 & -1.686613 & 4.975368 \\
\hline C & 1.110942 & -3.082559 & 4.375830 \\
\hline $\mathrm{H}$ & 1.266727 & -3.467957 & 5.379526 \\
\hline C & 1.854650 & -3.590757 & 3.308248 \\
\hline $\mathrm{H}$ & 2.586939 & -4.375475 & 3.477656 \\
\hline C & -1.992975 & -4.789946 & -0.600405 \\
\hline $\mathrm{H}$ & -2.156197 & -5.759299 & -0.138001 \\
\hline C & -2.665517 & -4.449794 & -1.774839 \\
\hline $\mathrm{H}$ & -3.352411 & -5.155948 & -2.232940 \\
\hline C & -2.456545 & -3.197280 & -2.356265 \\
\hline $\mathrm{H}$ & -2.983873 & -2.922212 & -3.265201 \\
\hline C & 1.899151 & 5.779498 & -0.030275 \\
\hline $\mathrm{H}$ & 2.257268 & 6.561721 & $-\odot .693489$ \\
\hline C & 1.357674 & 6.108063 & 1. 212020 \\
\hline $\mathrm{H}$ & 1.293253 & 7.146578 & 1.523337 \\
\hline C & ๑. 895388 & 5.092659 & 2.053970 \\
\hline $\mathrm{H}$ & 0.470706 & 5.336892 & 3.023574 \\
\hline C & 4. 383617 & 2.066028 & -3.096411 \\
\hline $\mathrm{H}$ & 5.414818 & 2.399526 & -3.174144 \\
\hline C & 3.687168 & 1.662336 & -4.239707 \\
\hline $\mathrm{H}$ & 4.176947 & 1.679177 & -5.209241 \\
\hline C & 2. 363524 & 1.233123 & -4.132462 \\
\hline $\mathrm{H}$ & 1.822155 & ๑.907241 & -5.015951 \\
\hline
\end{tabular}

TS7

B3LYP SCF energy: B3LYP enthalpy: B3LYP free energy:
M๑6 SCF energy in solution: a.u.

M०6 enthalpy in solution: M06 free energy in solution: a.u .

Imaginary frequency: $-250.9602 \mathrm{~cm}-1$

Cartesian coordinates

\begin{tabular}{|c|c|c|c|}
\hline ATOM & X & $\mathrm{Y}$ & Z \\
\hline$P$ & $\odot . ๑ \odot \odot \diamond \odot \odot$ & -7.168001 & 4.201381 \\
\hline$P$ & 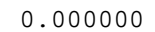 & -8.391210 & 3.976816 \\
\hline 0 & $\odot .0 \odot \odot \odot \odot \odot$ & -7.206647 & 3.843798 \\
\hline 0 & $\odot .0000 \odot \odot$ & -5.054294 & 3.962524 \\
\hline 0 & 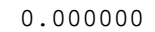 & -5.373568 & -0.577118 \\
\hline 0 & $\odot . \odot \odot \odot \odot \odot \diamond$ & -6.376680 & 1.113050 \\
\hline 0 & $\odot .0 \odot \odot \odot \odot \odot$ & -9.324371 & 4.002589 \\
\hline 0 & $\odot .0 \odot \odot ० \odot \odot$ & -8.424359 & 3.857256 \\
\hline 0 & $\odot .000000$ & -7.212492 & 3.765843 \\
\hline 0 & $\odot . \diamond \odot \odot \odot \odot \diamond$ & -6.093684 & -1.296167 \\
\hline C & $\odot .00000 \odot$ & -4.530720 & -0.979159 \\
\hline C & 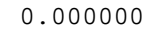 & -9.740182 & 3.785817 \\
\hline C & 2.737319 & -9.629973 & 3.298720 \\
\hline C & 2.510016 & -10.141674 & 4.791611 \\
\hline C & 1.964244 & -10.492395 & 3. 235641 \\
\hline C & 1.655722 & - ๑. 091693 & 2.157894 \\
\hline C & 3.615548 & -0.159619 & 2.090675 \\
\hline C & 1.887522 & ๑. 944909 & 2.314669 \\
\hline C & 2.345298 & -1.060640 & 3.135136 \\
\hline C & 3.694624 & -1.918000 & 3.366549 \\
\hline C & 4.560899 & $-\odot .592053$ & 4.067858 \\
\hline C & 4.118815 & -1.498471 & 2.258032 \\
\hline C & 2.796216 & $-\odot .552419$ & 2.470645 \\
\hline C & 6.294748 & -1.057143 & 2.642025 \\
\hline C & 3. 026164 & 1.026618 & $-\odot .507973$ \\
\hline C & 3.111803 & ๑. 638802 & -1.845709 \\
\hline C & 4.321443 & ๑. 689118 & -2.551529 \\
\hline C & 5.459438 & 1.195659 & -1.864757 \\
\hline C & 5.441154 & 1.471356 & -0.469260 \\
\hline C & 4.193113 & 1.401727 & ๑. 164954 \\
\hline C & 6.688179 & 1.804443 & ๑. 401064 \\
\hline C & 6.946596 & 3.330838 & ๑. 432861 \\
\hline C & 7.957307 & 1.052717 & -0.068445 \\
\hline C & 6.456391 & 1.365298 & 1.871693 \\
\hline C & 4.393334 & $\odot .155327$ & $-4.0 \odot 6 \odot 38$ \\
\hline C & 5.476357 & $-\odot .947739$ & -4.112873 \\
\hline C & 3.058606 & -0.490393 & -4.436984 \\
\hline C & 4.687799 & 1.287600 & -5.018511 \\
\hline C & 7.042394 & 2.627081 & -2.995622 \\
\hline C & 1.180682 & 2.827149 & 0.832109 \\
\hline C & 1.939079 & 3.821811 & ๑. 213091 \\
\hline C & 0.123269 & 3.226280 & 1.670474 \\
\hline C & $-\odot .070547$ & 4.560739 & 2.037897 \\
\hline C & ๑. 842230 & 5.517266 & 1.508006 \\
\hline C & 1.751958 & 5.190657 & ๑. 476719 \\
\hline C & 2.524159 & 6.198674 & -0.422559 \\
\hline C & 2.325503 & 5.774892 & -1.904726 \\
\hline C & 4.036647 & 6.153629 & -0.103662 \\
\hline C & 1.830783 & 7.151872 & 2.902395 \\
\hline C & 2.030963 & 7.662404 & -0.345247 \\
\hline C & -1.268146 & 4.971927 & 2.932307 \\
\hline C & $-\odot .801344$ & 5.497723 & 4.311354 \\
\hline C & -2.110341 & 6.058282 & 2.216393 \\
\hline C & -2.205649 & 3.775435 & 3.200410 \\
\hline C & -0.550240 & -2.577708 & 1.468747 \\
\hline C & -0.075020 & -3.729057 & 2.110118 \\
\hline C & -0.704087 & -4.247981 & 3. 245597 \\
\hline C & -1.867269 & -3.570741 & 3.711578 \\
\hline C & -2.267249 & -2.312613 & 3.199490 \\
\hline C & -1.610156 & -1.874042 & 2.037271 \\
\hline C & -3.326774 & -1.357951 & 3.820207 \\
\hline C & -3.720946 & -1.671724 & 5.282797 \\
\hline C & -4.599873 & -1.331432 & 2.940653 \\
\hline C & -2.729497 & $\odot .075549$ & 3.854956 \\
\hline C & $\odot .233648$ & -5.029711 & 5.449079 \\
\hline C & -3.783800 & -4.820535 & 4.318292 \\
\hline
\end{tabular}




\begin{tabular}{|c|c|c|c|c|c|c|c|}
\hline C & - ๑. 090883 & -5.459293 & 3.995814 & H & -4.311712 & $-\odot .832214$ & 5.667256 \\
\hline C & 1.228106 & -5.921589 & 3.337734 & H & -5.326780 & $-\odot .619369$ & 3. 349418 \\
\hline C & -1.991415 & -4.806012 & -3.205121 & H & -4.372693 & -1.026636 & 1.912539 \\
\hline C & -1.190645 & -3.736009 & -3.697921 & H & -5.085372 & -2.312024 & 2.894520 \\
\hline C & -2.488939 & -3.195178 & -5.791742 & H & -3.439426 & $\odot .755672$ & 4.338065 \\
\hline C & -0.580740 & -2.906578 & -2.752625 & H & -1.794563 & $\odot .09759 \odot$ & 4.426266 \\
\hline C & $-\odot .752772$ & -3.106052 & -1.372107 & H & -2.526904 & 0.479125 & 2.858833 \\
\hline C & -1.659589 & -4.077325 & -0.946155 & H & 0.713231 & -5.857421 & 5.985654 \\
\hline C & -2.344962 & -4.914670 & -1.840507 & H & $\odot .917961$ & -4.172939 & 5.460790 \\
\hline C & -1.760504 & -6.970807 & -4.125557 & H & -0.669923 & -4.748263 & 5.993499 \\
\hline C & $-\odot .373526$ & -4.564949 & $-6.0 \odot \odot 290$ & H & -4.516392 & -4.110658 & 3.921546 \\
\hline C & -2.790336 & -6.951166 & $-\odot .385009$ & H & -3.578563 & -5.581333 & 3.557095 \\
\hline C & -3.443932 & -5.834696 & -1.234290 & H & -4.192388 & -5.298735 & 5.211174 \\
\hline C & -4.341769 & -4.970549 & $-\odot .304863$ & H & 1. 070059 & -6.312689 & 2. 325519 \\
\hline C & $-\odot .255654$ & -2.142131 & -5.467799 & H & 1.971471 & -5.119726 & 3.284668 \\
\hline C & -1.029830 & -6.689232 & 4.023269 & H & 1.655269 & -6.734913 & 3.934147 \\
\hline C & -1.070141 & -3.428571 & -5.213725 & H & -2.973680 & -2.344921 & -5.297480 \\
\hline C & -4.410932 & -6.476182 & -2.256467 & H & -2.421006 & -2.965526 & -6.861949 \\
\hline H & 1.796229 & 0.786468 & 5.388161 & H & -3.122480 & -4.076193 & -5.671685 \\
\hline H & 1.267334 & 1.841729 & 3.217092 & H & 0.040635 & -2.087907 & -3.087835 \\
\hline H & 3.310101 & -3.822336 & 5.339601 & H & -1.845667 & -4.189587 & $\odot .11280 \odot$ \\
\hline H & 4.694252 & -2.708242 & 4.984963 & H & -2.227404 & -7.580218 & -4.902306 \\
\hline H & 1.636527 & -3.804985 & -1.878128 & H & $-0.70 \odot 419$ & -6.825973 & -4.362024 \\
\hline H & 4.032631 & -4.442780 & -2.065642 & H & -1.841364 & -7.486028 & -3.162973 \\
\hline H & 7.157839 & -2.146610 & 0.790560 & H & -0.165657 & -4.226882 & -7.022244 \\
\hline $\mathrm{H}$ & 6.517805 & -3.557134 & 1.724213 & H & 0.584839 & -4.838965 & -5.542407 \\
\hline $\mathrm{H}$ & 2.216589 & 0.272494 & -2.329730 & H & $-\odot .996597$ & -5.456093 & -6.072665 \\
\hline H & 4.126886 & 1.634989 & 1.219479 & H & -2.158198 & -7.607554 & -0.993071 \\
\hline $\mathrm{H}$ & 6.093007 & 3.857281 & ๑.873935 & H & -2.164926 & -6.535874 & 0.413820 \\
\hline $\mathrm{H}$ & 7.826818 & 3.548475 & 1.049780 & H & -3.563439 & -7.573399 & ๑. 081593 \\
\hline $\mathrm{H}$ & 7.122501 & 3.756512 & -0.557169 & $\mathrm{H}$ & -5.139719 & -5.594342 & 0.113641 \\
\hline H & 8.310365 & 1.358007 & -1.051282 & H & -3.797300 & -4.531744 & 0.536210 \\
\hline H & 8.764647 & 1.227762 & 0.651352 & H & -4.811093 & -4.153834 & $-\odot .864969$ \\
\hline H & 7.775105 & $-\odot . \odot 27593$ & $-\odot .112226$ & H & $-\odot .688081$ & -1.273350 & -4.961944 \\
\hline $\mathrm{H}$ & 6.135895 & 0.321017 & 1.939312 & H & $\odot .789614$ & -2.246805 & -5.154153 \\
\hline H & 7.393690 & 1.475337 & 2.427523 & H & -0.251059 & -1.933649 & -6.543076 \\
\hline $\mathrm{H}$ & 5.714878 & 1.984624 & 2.386909 & $\mathrm{H}$ & -1.375971 & -6.950068 & 3. 015764 \\
\hline $\mathrm{H}$ & 5.507558 & -1.336479 & -5.137813 & H & -0.485448 & -7.554974 & 4. 418187 \\
\hline H & 6.467101 & -0.570420 & -3.857905 & H & -1.896945 & -6.530392 & 4.663743 \\
\hline H & 5.242007 & -1.784295 & -3.444020 & H & -5.239918 & -6.934099 & -1.704367 \\
\hline H & 3.174520 & $-\odot .906914$ & -5.442960 & H & -4.831826 & -5.733399 & -2.939699 \\
\hline H & 2.766662 & -1.310727 & -3.771797 & $\mathrm{H}$ & -3.953186 & -7.263392 & -2.855274 \\
\hline H & 2.235179 & 0.231925 & -4.481473 & $\mathrm{Rh}$ & $-\odot .389596$ & 0.192884 & -0.691442 \\
\hline H & 3.966659 & 2.107607 & -4.916954 & 0 & -3.421061 & 4.683759 & $-\odot .78533 \odot$ \\
\hline $\mathrm{H}$ & 5.691857 & 1.694677 & $-4.9 \odot 4836$ & c & -0.719533 & 1. $0 \odot 4398$ & -2.492471 \\
\hline H & 4.606473 & $\odot .898629$ & -6.040335 & $\mathrm{~s}$ & -4.426549 & 4.142253 & -1.709431 \\
\hline H & 7.805263 & 3.016225 & -2.314505 & 0 & -1.467091 & 0.124753 & -2.913257 \\
\hline H & 7.484108 & 2.533332 & -3.991150 & C & -2.992511 & 1.924256 & -1.091513 \\
\hline H & 6.199124 & 3.324284 & -3.038022 & $\mathrm{~N}$ & -3.919935 & 2.573184 & -2.018462 \\
\hline H & 2.698668 & 3.532517 & $-\odot .500889$ & 0 & -4.702882 & 4.762114 & -3.007740 \\
\hline H & 2.848167 & 6.480921 & -2.559422 & C & -5.984980 & 4.051261 & $-\odot .826135$ \\
\hline H & 2.720122 & 4.778293 & -2.124196 & H & -3.261628 & 2.164260 & -0.058323 \\
\hline H & 1.263955 & 5.790700 & -2.176865 & H & -3.160270 & 0.847555 & -1.215840 \\
\hline H & 4.245160 & 6.449546 & 0.930354 & C & -4.567560 & 1.771760 & -3.077537 \\
\hline H & 4.449207 & 5.149938 & $-\odot .252396$ & C & -7.184136 & 4.078618 & -1.544342 \\
\hline $\mathrm{H}$ & 4.581581 & 6.840654 & -0.761949 & C & -5.988780 & 3.942330 & 0.566719 \\
\hline H & 1.849055 & 6.480237 & 3.768330 & C & -5.524120 & $\odot .735344$ & -2.544572 \\
\hline $\mathrm{H}$ & 2.809637 & 7.126396 & 2.413030 & $\mathrm{H}$ & -5.074542 & 2.476571 & -3.743387 \\
\hline H & 1.619481 & 8.170348 & 3.234792 & H & -3.778965 & 1.286058 & -3.663103 \\
\hline $\mathrm{H}$ & 2.291051 & 8.161030 & $\odot .588436$ & $\mathrm{H}$ & -7.168001 & 4.201381 & -250.960200 \\
\hline H & 2.506948 & 8.227584 & -1.154836 & C & -8.391210 & 3.976816 & $6.36 \odot 40 \odot$ \\
\hline $\mathrm{H}$ & 0.947911 & 7.734411 & -0.477246 & c & -7.206647 & 3.843798 & 8.273700 \\
\hline H & $-\odot .124969$ & 4.788519 & 4.804429 & $\mathrm{H}$ & -5.054294 & 3.962524 & 1.116990 \\
\hline $\mathrm{H}$ & -0.299846 & 6.462193 & 4.230657 & c & -5.373568 & -0.577118 & -2.733725 \\
\hline H & -1.671048 & 5.635480 & 4.964553 & H & -6.376680 & 1.113050 & -1.980999 \\
\hline $\mathrm{H}$ & -2.963142 & 6.333320 & 2.849419 & H & -9.324371 & 4.002589 & -1.413049 \\
\hline $\mathrm{H}$ & -1.525678 & 6.960080 & 2.028644 & c & -8.424359 & 3.857256 & $\odot .541744$ \\
\hline $\mathrm{H}$ & -2.501324 & 5.690011 & 1.262507 & H & -7.212492 & 3.765843 & 2.321765 \\
\hline H & -2.590998 & 3.341940 & 2.270060 & H & -6.093684 & -1.296167 & -2.352184 \\
\hline H & -1.723152 & 2.981294 & 3.782218 & H & -4.530720 & $-\odot .979159$ & -3.292785 \\
\hline $\mathrm{H}$ & -3.066005 & 4.123547 & 3.781799 & c & -9.740182 & 3.785817 & 1.278580 \\
\hline $\mathrm{H}$ & 0.791393 & -4.231178 & 1.700431 & H & -9.629973 & 3.298720 & 2.252533 \\
\hline $\mathrm{H}$ & -1.934139 & $-\odot .953743$ & 1.560638 & $\mathrm{H}$ & -10.141674 & 4.791611 & 1.458839 \\
\hline $\mathrm{H}$ & -4.332121 & -2.567392 & 5.388482 & H & -10.492395 & 3.235641 & 0.703756 \\
\hline $\mathrm{H}$ & -2.842245 & -1.785613 & 5.924027 & c & -๑. 091693 & 2.157894 & -3.228005 \\
\hline \multicolumn{8}{|c|}{122} \\
\hline
\end{tabular}




$\begin{array}{rrrr}H & -0.159619 & 2.090675 & -4.317864 \\ \mathrm{H} & 0.944909 & 2.314669 & -2.920632 \\ \mathrm{C} & -1.060640 & 3.135136 & -2.511175 \\ \mathrm{H} & -1.918000 & 3.366549 & -3.144590 \\ \mathrm{H} & -0.592053 & 4.067858 & -2.191758 \\ \mathrm{C} & -1.498471 & 2.258032 & -1.303912 \\ \mathrm{H} & -0.552419 & 2.470645 & 2.052437 \\ \mathrm{H} & -1.057143 & 2.642025 & -0.389301\end{array}$

TS8

B3LYP SCF energy:

B3LYP enthalpy:

B3LYP free energy:

-5535.30368433 a.u. -5533.268736 a.u. -5533.537922 a.u.

M06 SCF energy in solution: $\quad-5534.54278894$

a.u.

M06 enthalpy in solution: $\quad-5532.507841$ a.u

M06 free energy in solution: $\quad-5532.777027$

a.u.

Imaginary frequency:

$-234.3067 \mathrm{~cm}-1$

Cartesian coordinates

\begin{tabular}{|c|c|c|c|}
\hline ATOM & $X$ & $\mathrm{Y}$ & Z \\
\hline $\mathrm{P}$ & $\odot .0 \odot \odot \odot \odot \odot$ & -5.631296 & 5.255818 \\
\hline$P$ & 0.000000 & -7.398452 & 5.412186 \\
\hline 0 & ०. . 000000 & -6.996781 & 5.618297 \\
\hline 0 & ๑. . & -4.928904 & 5.623539 \\
\hline 0 & ๑. .००००० & -2.781355 & $\odot .936030$ \\
\hline 0 & $\odot . \odot \odot \odot \odot \odot \odot$ & -3.329720 & $\odot .598386$ \\
\hline 0 & $\odot . \diamond \odot \odot \odot \odot \odot$ & -8.085505 & 5.362420 \\
\hline 0 & $\odot .0000 \odot \odot$ & -7.905405 & 5.550275 \\
\hline 0 & $\odot .0 \odot \odot \odot \odot \odot$ & -7.373231 & 5.730206 \\
\hline 0 & ๑. .०००००० & -2.879585 & -0.114434 \\
\hline C & $\odot . \odot \odot \odot \odot \odot \diamond$ & -2.658546 & 1.612041 \\
\hline C & $\odot .0 \odot \odot \odot \odot \odot$ & -9.390800 & 5.652865 \\
\hline C & 2.610358 & -9.691862 & 5.065491 \\
\hline C & 3.065759 & -9.682708 & 6.693109 \\
\hline C & 3.134783 & -9.968011 & 5.303960 \\
\hline C & 2.730539 & ๑. 922493 & 3.130422 \\
\hline C & 3.362875 & 1.018235 & 3. 276192 \\
\hline C & $\odot .554445$ & 1.877683 & 2.833598 \\
\hline C & ๑. 300232 & ๑. 255988 & 4.248687 \\
\hline C & 1.248828 & $-\odot .499292$ & 4.775732 \\
\hline C & 2.468286 & $\odot .945576$ & 4.969964 \\
\hline C & 2.738069 & -0.405187 & 3.314433 \\
\hline C & 1.823751 & ๑. 912338 & 1.882587 \\
\hline C & 4.495723 & ๑. 372862 & 3. 069407 \\
\hline C & 2.994792 & ๑. 211718 & -1.081916 \\
\hline C & 2.545762 & $\odot .205979$ & -2.404635 \\
\hline C & 3.423603 & $\odot . \odot 47445$ & -3.484191 \\
\hline C & 4.808095 & $-\odot .080245$ & -3.185370 \\
\hline C & 5.283279 & -0.238258 & -1.854425 \\
\hline C & 4.345338 & $-\odot .050159$ & -0.828867 \\
\hline C & 6.734381 & -0.639833 & -1.459238 \\
\hline C & 7.616779 & ๑. 614257 & -1.248104 \\
\hline C & 7.392745 & -1.588327 & -2.490411 \\
\hline C & 6.730847 & -1.415672 & -0.114678 \\
\hline C & 2.868779 & -0.020018 & -4.931767 \\
\hline C & 3. 352938 & -1.314089 & -5.633165 \\
\hline C & 1. 323928 & $-\odot .059871$ & -4.945933 \\
\hline C & 3.292255 & 1. 218748 & -5.756496 \\
\hline C & 6.467349 & 1.015543 & -4.550716 \\
\hline C & 2.457975 & 1.981585 & 1.203659 \\
\hline C & 3.492804 & 2.766092 & ๑. 695784 \\
\hline C & 1.779964 & 2.436217 & 2.349575 \\
\hline C & 2.188850 & 3.569877 & 3.056546 \\
\hline C & 3. 338016 & 4.259098 & 2.570638 \\
\hline C & 3.924807 & 3.951505 & 1.321104 \\
\hline C & 4.969150 & 4.821688 & $\odot .562323$ \\
\hline C & 4.583371 & 4.874671 & -0.942213 \\
\hline C & 6.375219 & 4.185722 & ๑. 668958 \\
\hline C & 5.010781 & 4.994514 & 4.086285 \\
\hline C & 5.032382 & 6.298330 & 1.020657 \\
\hline C & 1. 362648 & 4.091790 & 4.261448 \\
\hline
\end{tabular}

2.164541

$\odot .899373$

0.092903

$-1.018710$

$-1.023040$

$-1.382865$

$-1.785667$

$-1.600792$

$-1.255652$

$-1.700306$

$-1.762998$

$-2.926365$

$-0.415372$

$-\odot .351758$

$-3.751919$

$-1.263271$

$-0.609288$

$-4.538312$

$-3.593537$

$-5.065206$

$-2.474167$

$-2.309342$

$-3.369423$

$-4.515154$

$-5.147467$

$-2.589745$

$-5.980678$

$-5.719119$

$-5.495060$

$-3.504166$

$-2.634415$

$-3.703356$

$-6.985420$

3. 496505

2.807725

2. 861559

4. 390210

$-0.673144$

1. 026791

5.472936

4.545542

1.486025

4.670244

7. 221426

8.635424

7.684199

7.583065

8. 349562

6. 760201

6.028047

7.735245

6.482261

2. 928562

4. 439452

3. 017252

0.984276

$\odot .934730$

0.866318

2. 996758

4. 366781

2. 797105

7.472596

6.552986

6. 000829

3. 978114

5. 279486

4.632360

3. 573326

6. 730708

6.379809

7. 100589

4.885021

5.879575

5.176189

5.485326
4. 054899

5.542925

3. 244408

$-2.438465$

3. 841807

$-4.576743$

$-3.839109$

$-2.440320$

$-1.766041$

$-1.589600$

$-2.388271$

$-0.648563$

$-0.725481$

$-6.557030$

$-4.460433$

$-6.123121$

$-6.636088$

$-2.622878$

$-1.740747$

$-1.237167$

$-1.410241$

$-1.886637$

$-2.590766$

$-2.946918$

$-4.286215$

$-1.627860$

$-5.054314$

$-3.607052$

$-3.672616$

$\odot .451926$

$-6.827483$

$-1.078561$

$-2.746685$

$-1.457046$

0.350878

$-6.038248$

$-5.367251$

$-2.957565$

$-4.281307$

$-4.390639$

$-5.729437$

$\odot .315653$

$-0.129310$

1. 231127

0.315113

1. 244111

$-1.123422$

$-1.942464$

$-2.465028$

$-2.254747$

$-1.810677$

$-0.780456$

$-1.366225$

$-1.352358$

$-2.200789$

$-0.169435$

$-0.912834$

$\odot .853871$

2. 147991

1.250585

1.197863

0.916122

1.078243

1. 930663

2. 456973

5.534844

3. 900212

5. 277882

4.136346

3.166630

4. 775201

4.086697

4.872656

5.848481

6.433247
5.585287

976371

4. 490135

1.402921

. 406305

2. 537776

3. 687193

3. 786233

2. 599951

5.084869

6.407939

5. 009143

5. 191042

3. 714681

4. 843165

2.539493

1.237180

$-2.653621$

$-3.234772$

$-5.356415$

$-2.448859$

$-1.140695$

$-0.552886$

$-1.271857$

$-4.212866$

$-5.563911$

$-1.032752$

$-0.551432$

$\odot .975296$

$-4.474894$

2. 668977

$-4.640136$

$-0.791081$

4. 633682

3. 090218

3. 173389

2. 470553

$-2.739156$

$-3.956802$

$-2.112490$

$-1.325907$

$-2.588433$

0.200608

$-0.433643$

$-0.973166$

$-2.137768$

$-3.455575$

$-2.090822$

$-2.672886$

$-0.132710$

0.071843

0.741663

$-6.642973$

$-5.714195$

$-5.082733$

$-5.981383$

$-4.378633$

$-4.551643$

$-5.254575$

$-5.936109$

$-6.734492$

$-4.129535$

$-5.638764$

$-4.171111$

$-0.220107$

$-1.470497$

$-1.436862$

$-1.078137$

1. 703030

0.267768

0.095828

4. 686597

3. 432757

4. 746641

2. 001885 


\begin{tabular}{|c|c|c|c|}
\hline $\mathrm{H}$ & 5.642705 & 6.857357 & ๑. 302378 \\
\hline $\mathrm{H}$ & 4.038980 & 6.756651 & 1.043764 \\
\hline $\mathrm{H}$ & 2.601490 & 3.064340 & 5.760522 \\
\hline $\mathrm{H}$ & 2.960853 & 4.798236 & 5.602730 \\
\hline $\mathrm{H}$ & 1.494599 & 4.274090 & 6.424823 \\
\hline $\mathrm{H}$ & ๑. 310023 & 5.916434 & 4.822164 \\
\hline $\mathrm{H}$ & 1.746342 & 6.214437 & 3.827287 \\
\hline $\mathrm{H}$ & ๑. 262809 & 5.583506 & 3.084357 \\
\hline $\mathrm{H}$ & -0.559338 & 3.226004 & 3.609265 \\
\hline $\mathrm{H}$ & ๑. 325423 & 2.211850 & 4.772817 \\
\hline $\mathrm{H}$ & -0.484036 & 3.683947 & 5.310339 \\
\hline $\mathrm{H}$ & -0.752282 & -4.362817 & ๑. 497747 \\
\hline $\mathrm{H}$ & -1.155383 & -0.686844 & 2.612886 \\
\hline $\mathrm{H}$ & -2.714245 & -2.896222 & 6.566307 \\
\hline $\mathrm{H}$ & -0.965649 & -3.133297 & 6.472917 \\
\hline $\mathrm{H}$ & -1.634462 & -1.683767 & 7.237973 \\
\hline $\mathrm{H}$ & -2.976895 & $-\odot .019595$ & 5.905798 \\
\hline $\mathrm{H}$ & -2.875129 & ๑. 015150 & 4.138304 \\
\hline $\mathrm{H}$ & -3.864791 & -1.210175 & 4.943032 \\
\hline $\mathrm{H}$ & $-\odot .460078$ & $-\odot .112302$ & 6.098228 \\
\hline $\mathrm{H}$ & 0.476387 & -1.358466 & $5.25 \odot 286$ \\
\hline $\mathrm{H}$ & -0.286066 & $-\odot . \odot 47219$ & 4.343586 \\
\hline $\mathrm{H}$ & -0.211317 & -7.644401 & 3.696565 \\
\hline $\mathrm{H}$ & ๑. 633857 & -6.085246 & 3.630589 \\
\hline $\mathrm{H}$ & -0.780029 & -6.281935 & 4.679908 \\
\hline $\mathrm{H}$ & -4.084641 & -3.430696 & 5.010100 \\
\hline $\mathrm{H}$ & -4.237964 & -4.846736 & 3.940250 \\
\hline $\mathrm{H}$ & -4.033169 & -5.075063 & 5.700977 \\
\hline $\mathrm{H}$ & -1.233701 & -6.448930 & ๑. 355572 \\
\hline $\mathrm{H}$ & 0.375589 & -6.187720 & 1.067260 \\
\hline $\mathrm{H}$ & -0.474366 & -7.720463 & 1.311119 \\
\hline $\mathrm{H}$ & -5.901747 & -0.947088 & -4.715329 \\
\hline $\mathrm{H}$ & -5.065875 & $-\odot .574667$ & -6.229622 \\
\hline $\mathrm{H}$ & -5.253552 & -2.246325 & -5.723425 \\
\hline $\mathrm{H}$ & -1.714055 & $-\odot .761452$ & -2.869810 \\
\hline $\mathrm{H}$ & -3.291585 & -2.881443 & 0.483638 \\
\hline $\mathrm{H}$ & -6.037288 & -4.608446 & -4.757772 \\
\hline $\mathrm{H}$ & -4.362630 & -4.015923 & -4.927024 \\
\hline $\mathrm{H}$ & -4.785993 & -5.106635 & -3.582725 \\
\hline $\mathrm{H}$ & -2.644169 & -1.145012 & -6.546652 \\
\hline $\mathrm{H}$ & -1.591236 & -1.436588 & -5.155511 \\
\hline $\mathrm{H}$ & -2.688702 & -2.708659 & -5.718668 \\
\hline $\mathrm{H}$ & -6.363971 & -5.081430 & -2.052830 \\
\hline $\mathrm{H}$ & -5.070120 & -5.663391 & $-\odot .980887$ \\
\hline $\mathrm{H}$ & -6.731957 & -5.525481 & -0.388034 \\
\hline $\mathrm{H}$ & -6.396144 & -4.076806 & 1.448680 \\
\hline $\mathrm{H}$ & -4.660318 & -4.329589 & 1. 246430 \\
\hline $\mathrm{H}$ & -5.311207 & -2.683250 & 1.409595 \\
\hline $\mathrm{H}$ & -4.299345 & $\odot .878316$ & -3.851096 \\
\hline $\mathrm{H}$ & -2.545082 & 0.725388 & -4.034033 \\
\hline $\mathrm{H}$ & -3.562750 & 0.933619 & -5.457462 \\
\hline $\mathrm{H}$ & -3.337947 & -6.476243 & 1.904505 \\
\hline H & -2.506626 & -7.907249 & 2.527979 \\
\hline $\mathrm{H}$ & -3.080713 & -6.677916 & 3.652317 \\
\hline $\mathrm{H}$ & -7.842902 & -3.195681 & -0.275643 \\
\hline $\mathrm{H}$ & -6.849473 & -1.733630 & -0.393925 \\
\hline $\mathrm{H}$ & -7.225561 & -2.673838 & -1.853374 \\
\hline $\mathrm{Rh}$ & -0.436524 & $\odot .772276$ & -0.103520 \\
\hline 0 & -2.736915 & 6.397290 & ๑. 105918 \\
\hline C & -0.235327 & 2.219294 & -1.744802 \\
\hline$S$ & -3.385345 & 5.383461 & -0.733174 \\
\hline 0 & -1.087268 & 1.827775 & -2.522295 \\
\hline C & -1.595757 & 3.825514 & 0.541132 \\
\hline $\mathrm{N}$ & -2.934467 & 3.867546 & -0.062414 \\
\hline 0 & -3.167061 & 5.378502 & -2.188245 \\
\hline C & -5.148584 & 5.411243 & -0.440926 \\
\hline $\mathrm{H}$ & -1.367168 & 4.852236 & ๑. 847369 \\
\hline $\mathrm{H}$ & -1.665174 & 3. 250031 & 1.466526 \\
\hline C & -3.368690 & 2.736630 & -0.894571 \\
\hline C & -6.024484 & 5.345882 & -1.524414 \\
\hline C & -5.624370 & 5.554502 & ๑. 866658 \\
\hline C & -3.102531 & 1.358963 & -0.366015 \\
\hline $\mathrm{H}$ & -4.461598 & 2.807698 & $-1.0 \odot 2917$ \\
\hline $\mathrm{H}$ & -2.953805 & 2.796340 & -1.905733 \\
\hline $\mathrm{H}$ & -5.631296 & 5.255818 & -234.306700 \\
\hline
\end{tabular}

$\begin{array}{rrrr}\text { C } & -7.398452 & 5.412186 & 9.505400 \\ \mathrm{C} & -6.996781 & 5.618297 & 11.549400 \\ \mathrm{H} & -4.928904 & 5.623539 & 1.696611 \\ \mathrm{C} & -2.781355 & 0.936030 & 0.882503 \\ \mathrm{H} & -3.329720 & 0.598386 & -1.107625 \\ \mathrm{H} & -8.085505 & 5.362420 & -2.128487 \\ \mathrm{C} & -7.905405 & 5.550275 & 0.010439 \\ \mathrm{H} & -7.373231 & 5.730206 & 2.094749 \\ \mathrm{H} & -2.879585 & -0.114434 & 1.129375 \\ \mathrm{H} & -2.658546 & 1.612041 & 1.720576 \\ \mathrm{C} & -9.390800 & 5.652865 & 0.259288 \\ \mathrm{H} & -9.691862 & 5.065491 & 1.133671 \\ \mathrm{H} & -9.682708 & 6.693109 & 0.453056 \\ \mathrm{H} & -9.968011 & 5.303960 & -0.602124 \\ \mathrm{C} & 0.922493 & 3.130422 & -2.135400 \\ \mathrm{H} & 1.018235 & 3.276192 & -3.213752 \\ \mathrm{H} & 1.877683 & 2.833598 & -1.702795 \\ \mathrm{C} & 0.255988 & 4.248687 & -1.313091 \\ \mathrm{H} & -0.499292 & 4.775732 & -1.902688 \\ \mathrm{H} & 0.945576 & 4.969964 & -0.862575 \\ \mathrm{C} & -0.405187 & 3.314433 & -0.268218 \\ \mathrm{H} & 0.912338 & 1.882587 & 2.687855 \\ \mathrm{H} & 0.372862 & 3.069407 & 0.456248\end{array}$

TS9

B3LYP SCF energy:

B3LYP enthalpy:

B3LYP free energy:

:5533.516792 a.u.

M06 SCF energy in solution: -5534.54209003

a.u

M06 enthalpy in solution: $\quad-5532.506843$ a.u.

M06 free energy in solution: $\quad-5532.774719$

a.u.

Imaginary frequency: $\quad-231.1371 \mathrm{~cm}-1$

Cartesian coordinates

\begin{tabular}{|c|c|c|c|}
\hline ATOM & $\mathrm{X}$ & Y & Z \\
\hline$P$ & $\odot . \odot \odot \odot \odot \odot \odot ~$ & 1.040620 & 7.045137 \\
\hline$P$ & $\odot . ๑ \odot \odot \odot \odot \odot$ & 3.052034 & 7.493605 \\
\hline 0 & $\odot . \odot \odot \odot \odot \odot \odot$ & 4.818184 & 6.766543 \\
\hline 0 & $\odot .0 \odot \odot \odot \odot \odot ~$ & 4.191960 & 5.739096 \\
\hline 0 & $\odot .0 \odot \odot \odot \odot \odot$ & -1.033872 & 1.877152 \\
\hline 0 & $\odot . ๑ \odot \odot \odot \odot \odot$ & -1.697797 & 2.752742 \\
\hline 0 & $\odot . ๑ \odot \odot \odot \odot \odot$ & 2.724972 & 8.005655 \\
\hline 0 & $\odot . ๑ \odot \odot \odot \odot \odot ~$ & 4.423468 & 7.414506 \\
\hline 0 & $\odot .0 \odot \odot \odot \odot \odot$ & 5.874474 & 6.707561 \\
\hline 0 & $\odot .0 \odot \odot \odot \odot \odot ~$ & -1.940709 & 1.330669 \\
\hline c & $\odot . ๑ \odot \odot \odot \odot \odot$ & -0.417852 & 2.051239 \\
\hline c & $\odot .0 \odot \odot \odot \odot \odot$ & 5.442958 & 8.048910 \\
\hline c & -1.093970 & 6.420009 & 7.563829 \\
\hline c & -0.426804 & 5.128820 & 7.999231 \\
\hline C & 0.768905 & 5.580831 & 9.109500 \\
\hline c & 1.266513 & 2.203773 & 1.865768 \\
\hline c & -2.103032 & 1.364821 & 2.380563 \\
\hline c & -2.082168 & 2.834576 & 1.430424 \\
\hline c & -2.861742 & 2.908154 & 2.731260 \\
\hline c & -2.990481 & 2.655739 & 3.792112 \\
\hline$c$ & -2.300485 & 3.996168 & 2.630646 \\
\hline c & -1.534105 & 2.359738 & 2. 018719 \\
\hline c & -1.390475 & 2.714904 & 1.104937 \\
\hline c & -1.540229 & 3.106146 & 1.365007 \\
\hline c & 1.512126 & -2.677200 & -0.334276 \\
\hline c & 1.077318 & -2.997396 & -1.619721 \\
\hline c & 1.300853 & -4.260731 & -2.181022 \\
\hline c & 2.025881 & -5.204401 & -1.402615 \\
\hline c & 2.371373 & -4.958641 & -0.045047 \\
\hline c & 2.118636 & -3.668421 & 0.441262 \\
\hline c & 2.986018 & -5.998273 & $\odot .936676$ \\
\hline c & 4.533136 & -5.951118 & 0.878400 \\
\hline c & 2.479170 & -7.438265 & $\odot .681890$ \\
\hline c & 2.587904 & -5.664606 & 2.399717 \\
\hline c & 0.733297 & -4.588735 & -3.587282 \\
\hline & -0.176538 & -5.840203 & -3.515469 \\
\hline
\end{tabular}




\begin{tabular}{|c|c|c|c|c|c|c|c|}
\hline C & -0.135942 & -3.431193 & -4.129711 & $\mathrm{H}$ & 1.427556 & -4.907240 & -5.625568 \\
\hline C & 1.861020 & -4.813626 & -4.622612 & H & 4.219166 & -7.214420 & -1.703904 \\
\hline C & 3.646542 & -6.654471 & -2.449821 & H & 3.576362 & -7.260941 & -3.356778 \\
\hline C & 3.090723 & -0.492524 & 0.668776 & $\mathrm{H}$ & 4.165284 & -5.717878 & -2.679970 \\
\hline C & 4.085446 & -1.071545 & -0.123388 & H & 3.796200 & -1.801443 & $-\odot .866642$ \\
\hline C & 3.475162 & 0.541086 & 1.543877 & $\mathrm{H}$ & 6.417630 & -1.392911 & -3.280999 \\
\hline C & 4.816656 & $\odot .860988$ & 1.780721 & H & 4.776652 & -1.407367 & -2.628222 \\
\hline C & 5.797055 & 0.132814 & 1.049711 & $\mathrm{H}$ & 5.706122 & ๑. 098252 & -2.645504 \\
\hline C & 5.444548 & $-\odot .730949$ & $-\odot .012412$ & H & 6.971964 & -3.114173 & ๑.0๑5434 \\
\hline C & 6.398026 & -1.300748 & -1.102652 & $\mathrm{H}$ & 5.546100 & -3.329744 & -1.018513 \\
\hline C & 5.775423 & $-\odot .985584$ & -2.491941 & H & 7.157718 & -3.241050 & -1.749845 \\
\hline C & 6.521586 & -2.835599 & $-\odot .953997$ & H & 7.219005 & -0.877754 & 3.079795 \\
\hline C & 7.719058 & $-\odot .739016$ & 2.114899 & H & 7.685864 & -1.682169 & 1.560593 \\
\hline C & 7.815412 & -0.684032 & -1.141331 & H & 8.759590 & -0.452087 & 2.280834 \\
\hline C & 5.192771 & 2. 011993 & 2.752254 & H & 8.448754 & -0.992129 & -0.309214 \\
\hline C & 6.043470 & 1.513156 & 3.946444 & H & 8.309228 & -1.020356 & -2.060433 \\
\hline C & 5.986489 & 3.092874 & 1.976676 & $\mathrm{H}$ & 7.785250 & ๑.408827 & -1.157098 \\
\hline C & 3.944879 & 2.693736 & 3.355678 & H & 5.568916 & $\odot .659641$ & 4.445324 \\
\hline C & -2.543094 & $\odot .503131$ & 1.305646 & H & 7.049852 & 1.228252 & 3.641806 \\
\hline C & -3.627280 & $-\odot .078391$ & 1.981766 & $\mathrm{H}$ & 6.139364 & 2.316087 & 4.686344 \\
\hline C & -4.192044 & $\odot .516 \odot 20$ & 3.112544 & H & 6.254508 & 3.914929 & 2.650951 \\
\hline C & -3.639595 & 1.758306 & 3.538453 & H & 6.905362 & 2.685996 & 1.549344 \\
\hline C & -2.436620 & 2.274836 & 3. 001391 & H & 5.381377 & 3.513121 & 1.163352 \\
\hline C & -1.945356 & 1.640869 & 1.844559 & H & 3.293930 & 3.149015 & 2.602752 \\
\hline C & -1.598544 & 3.444861 & 3.592135 & $\mathrm{H}$ & 3.347409 & 2.003617 & 3.963606 \\
\hline C & $-1.9 \odot 4981$ & 3.778209 & 5.071741 & H & 4.271151 & 3.502973 & 4.016973 \\
\hline C & -1.769916 & 4.717759 & 2.729852 & H & $-4.0374 \odot 4$ & -1.006054 & 1.605090 \\
\hline C & $-\odot .098834$ & 3.041813 & 3.564073 & H & -1.062499 & 2.038135 & 1.358207 \\
\hline C & -4.833857 & $-\odot .408565$ & 5.368647 & H & -2.868290 & 4.264477 & 5.221282 \\
\hline C & -5.082494 & 3.543294 & 4.131721 & H & -1.878117 & 2.884066 & 5.702264 \\
\hline C & -5.310162 & $-\odot .209044$ & 3.907518 & H & -1.135124 & 4.468808 & 5.434007 \\
\hline C & -5.603839 & -1.606095 & 3.317163 & H & -1.128907 & 5.521175 & 3.109134 \\
\hline C & -5.542193 & $\odot .700011$ & -3.169809 & $\mathrm{H}$ & -1.485385 & 4.542813 & 1.687580 \\
\hline C & -4.447752 & $-\odot . \odot 6587 \odot$ & -3.657598 & H & -2.802317 & 5.083355 & 2.736493 \\
\hline C & -5.306200 & $-\odot .118692$ & -6.145145 & H & $\odot .497292$ & 3.825977 & 4.041230 \\
\hline C & -3.406037 & $-\odot .321171$ & -2.758881 & H & ๑. 067351 & 2.102332 & 4.103633 \\
\hline c & -3.404166 & 0.157879 & -1.439239 & H & 0.292568 & 2.933444 & 2.549741 \\
\hline C & -4.436993 & 1.012626 & -1.054874 & H & -5.578069 & $-\odot .987943$ & 5.928568 \\
\hline C & -5.506571 & 1.337458 & -1.906677 & H & -3.883473 & $-\odot .952700$ & 5.396089 \\
\hline C & -7.662313 & $-\odot .136847$ & -3.765245 & H & -4.690035 & $\odot .546473$ & 5.876799 \\
\hline C & -4.380960 & -2.176194 & -5.052155 & H & -4.457046 & 4.358905 & 3.757071 \\
\hline C & -7.345782 & 1.724372 & -0.214634 & H & -5.798245 & 3.255413 & 3.353184 \\
\hline C & -6.538569 & 2.368678 & -1.367260 & H & -5.624614 & 3.883361 & 5.016838 \\
\hline C & -5.765584 & 3.596861 & $-\odot .812508$ & H & -6.019753 & -1.548455 & 2.304142 \\
\hline C & -2.890968 & $-\odot .228336$ & -5.642506 & H & -4.711615 & -2.239933 & 3.290544 \\
\hline C & -6.648172 & $\odot .567765$ & 3.912083 & H & -6.350849 & -2.105843 & 3.943277 \\
\hline C & -4.289314 & $-\odot .631408$ & -5.099606 & H & -5.357069 & $\odot .972640$ & -6.171762 \\
\hline C & -7.534433 & 2.934757 & $-2.4052 \odot 2$ & H & -4.982397 & -0.467240 & -7.132942 \\
\hline $\mathrm{H}$ & 1.314718 & $-\odot .930192$ & 5.401936 & H & -6.315022 & $-\odot .50014 \odot$ & -5.983837 \\
\hline $\mathrm{H}$ & 2.233124 & $-\odot .488605$ & 3.164565 & H & -2.558430 & $-\odot .905181$ & -3.101551 \\
\hline $\mathrm{H}$ & -3.057606 & -3.050791 & 5.684014 & H & -4.421469 & 1.435131 & -0.059816 \\
\hline $\mathrm{H}$ & -1.743230 & -4.250334 & 5.338502 & H & -8.472271 & 0.104902 & -4.456652 \\
\hline $\mathrm{H}$ & -3.433614 & -2.325817 & -1.700294 & H & -7.285062 & -1.143940 & -3.984015 \\
\hline $\mathrm{H}$ & -3.614019 & -4.769155 & -1.518430 & $\mathrm{H}$ & -8.043057 & -0.117999 & -2.736534 \\
\hline $\mathrm{H}$ & -0.741219 & -6.956443 & 1.674728 & H & -4.252196 & -2.593588 & -6.057775 \\
\hline H & -2.242186 & -6.469842 & 2.558316 & H & -3.607646 & -2.613061 & -4.410049 \\
\hline H & $\odot .570208$ & -2.237039 & -2.193814 & H & -5.356538 & -2.505872 & -4.676622 \\
\hline $\mathrm{H}$ & 2.398589 & -3.427038 & 1.458229 & H & -7.926987 & $\odot .863864$ & -0.565213 \\
\hline H & 4.897702 & -4.952599 & 1.145235 & H & -6.691890 & 1.378620 & $\odot .593495$ \\
\hline $\mathrm{H}$ & 4.958462 & -6.666030 & 1.593323 & H & -8.048776 & 2.452365 & 0.207622 \\
\hline $\mathrm{H}$ & 4.930216 & -6.193919 & -0.109630 & H & -6.479300 & 4.339408 & -0.438841 \\
\hline $\mathrm{H}$ & 2.789614 & -7.851180 & $-\odot .275474$ & H & -5.094822 & 3.348935 & ๑.015383 \\
\hline $\mathrm{H}$ & 2.855090 & -8.096793 & 1.473010 & H & -5.170037 & 4.072106 & $-1.60 \odot 438$ \\
\hline $\mathrm{H}$ & 1.383533 & -7.475130 & 0.707734 & $\mathrm{H}$ & -2.790206 & 0.862438 & -5.687070 \\
\hline $\mathrm{H}$ & 1.507117 & -5.528390 & 2.506388 & H & -2.063685 & $-\odot .623838$ & -5.045443 \\
\hline $\mathrm{H}$ & 2.898517 & -6.488591 & 3. 051063 & H & -2.767167 & $-\odot .617310$ & -6.659134 \\
\hline $\mathrm{H}$ & 3.081942 & -4.764212 & 2.778872 & H & -6.975755 & ๑.808067 & 2.893885 \\
\hline $\mathrm{H}$ & -0.588893 & -6.056954 & -4.508515 & $\mathrm{H}$ & -7.427781 & -0.052157 & 4.370478 \\
\hline $\mathrm{H}$ & $\odot .37 \odot 4 \odot 3$ & -6.718498 & -3.170614 & H & -6.585654 & 1.490841 & 4.487850 \\
\hline $\mathrm{H}$ & -1.017187 & -5.667757 & -2.833113 & H & -8.081299 & 3.761569 & -1.936914 \\
\hline $\mathrm{H}$ & $-\odot .559721$ & -3.729250 & -5.094739 & H & -7.027780 & 3.325155 & -3.291750 \\
\hline $\mathrm{H}$ & -0.971295 & -3.199945 & -3.458633 & $\mathrm{H}$ & -8.273885 & 2.203674 & -2.733598 \\
\hline $\mathrm{H}$ & 0.444557 & -2.516053 & -4.294546 & $\mathrm{Rh}$ & ๑. 092379 & $\odot .713598$ & -1.077112 \\
\hline $\mathrm{H}$ & 2.557106 & -3.966605 & -4.638113 & 0 & 0.067844 & 6.317752 & 0.011439 \\
\hline \multirow[t]{2}{*}{$\mathrm{H}$} & 2.426508 & -5.724338 & -4.428356 & C & 1.821195 & $\odot .825839$ & -2.604341 \\
\hline & & & & & & & \\
\hline
\end{tabular}




$\begin{array}{lrrr}\mathrm{S} & 1.307593 & 5.547449 & -.133600 \\ \mathrm{O} & 2.127108 & -0.342573 & -2.644749 \\ \mathrm{C} & 1.870255 & 2.910958 & -0.164700 \\ \mathrm{~N} & 0.953593 & 4.012480 & -0.482562 \\ \mathrm{O} & 1.959477 & 5.310801 & 1.425179 \\ \mathrm{C} & 2.522481 & 6.295156 & -0.952660 \\ \mathrm{H} & 2.775270 & 3.352367 & 0.267232 \\ \mathrm{H} & 1.432895 & 2.295216 & 0.626707 \\ \mathrm{C} & 0.011962 & 3.900112 & -1.590755 \\ \mathrm{C} & 2.099395 & 6.945123 & -2.117125 \\ \mathrm{C} & 3.880643 & 6.208902 & -0.635031 \\ \mathrm{C} & -0.897600 & 2.693427 & -1.525412 \\ \mathrm{H} & -0.624404 & 4.789274 & -1.556677 \\ \mathrm{H} & 0.528303 & 3.908168 & -2.559471 \\ \mathrm{H} & 1.040620 & 7.045137 & -231.137100 \\ \mathrm{C} & 3.052034 & 7.493605 & 8.901500 \\ \mathrm{C} & 4.818184 & 6.766543 & 11.662200 \\ \mathrm{H} & 4.191960 & 5.739096 & 0.291857 \\ \mathrm{C} & -1.033872 & 1.877152 & -2.638713 \\ \mathrm{H} & -1.697797 & 2.752742 & -0.791794 \\ \mathrm{H} & 2.724972 & 8.005655 & -3.874186 \\ \mathrm{C} & 4.423468 & 7.414506 & -2.682896 \\ \mathrm{H} & 5.874474 & 6.707561 & -1.253819 \\ \mathrm{H} & -1.940709 & 1.330669 & -2.832896 \\ \mathrm{H} & -0.417852 & 2.051239 & -3.512502 \\ \mathrm{C} & 5.442958 & 8.048910 & -3.597772 \\ \mathrm{H} & 6.420009 & 7.563829 & -3.509070 \\ \mathrm{H} & 5.128820 & 7.999231 & -4.645434 \\ \mathrm{H} & 5.580831 & 9.109500 & -3.350159 \\ \mathrm{C} & 2.203773 & 1.865768 & -3.650852 \\ \mathrm{H} & 1.364821 & 2.380563 & -4.120518 \\ \mathrm{H} & 2.834576 & 1.430424 & -4.430228 \\ \mathrm{C} & 2.908154 & 2.731260 & -2.579220 \\ \mathrm{H} & 2.655739 & 3.792112 & -2.623419 \\ \mathrm{H} & 3.996168 & 2.630646 & -2.636852 \\ \mathrm{C} & 2.359738 & 2.018719 & -1.316003 \\ \mathrm{H} & 2.714904 & 1.104937 & 2.065326 \\ \mathrm{H} & 3.106146 & 1.365007 & -0.868345 \\ & & & \end{array}$

TS10

B3LYP SCF energy:

B3LYP enthalpy:

B3LYP free energy:

M06 SCF energy in solution:

a.u.

M06 enthalpy in solution:

Mo6 free energy in solution:

a.u

Imaginary frequency:

$-233.8550 \mathrm{~cm}-1$

-5535.31199122 a.u -5533.278200 a.u. -5533.548904 a.u.

$-5534.54299446$

-5532.509203 a.u $-5532.779907$

Cartesian coordinates

\begin{tabular}{|c|c|c|c|}
\hline ATOM & X & $\mathrm{Y}$ & Z \\
\hline $\mathrm{P}$ & ๑. .०००००० & -5.202821 & 3. 480152 \\
\hline$P$ & $\odot .000000$ & -6.521802 & 3.949134 \\
\hline 0 & $\odot .0 \odot \odot ० \odot \odot$ & -5.489102 & 4.031956 \\
\hline 0 & 0.000000 & -3.373806 & 3. 619672 \\
\hline 0 & 0.000000 & -0.648631 & 7.759352 \\
\hline 0 & $\odot .0 \odot \odot ० \odot \odot$ & -1.796978 & 6.855277 \\
\hline 0 & $\odot .0 \odot \odot ० \odot \odot$ & -7.400319 & 4.040348 \\
\hline 0 & $\odot .00000 \odot$ & -6.641280 & 4.150406 \\
\hline 0 & $\odot . \odot \odot \odot \odot \odot \diamond$ & -5.563687 & 4.186021 \\
\hline 0 & $\odot .0 \odot \odot ० \odot \odot$ & -0.347358 & 8.612844 \\
\hline C & $\odot .0 \odot \odot \odot \odot \odot ~$ & $-\odot .283875$ & 7.732365 \\
\hline C & 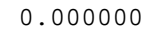 & -7.974619 & 4.472407 \\
\hline C & 1.490213 & -7.893353 & 5.314576 \\
\hline C & 1.922587 & -8.351731 & 3. 615837 \\
\hline C & 2.318746 & -8.725131 & 4.724512 \\
\hline C & 2.282416 & 2.084377 & 2.086612 \\
\hline C & 1.636657 & 2.276359 & 2.087257 \\
\hline C & -0.169938 & 2.858305 & 1.495491 \\
\hline C & -0.550517 & 1.395474 & 2.383861 \\
\hline C & 0.146477 & ๑. 364039 & 3.254734 \\
\hline C & 1.245949 & -0.288496 & 3.558263 \\
\hline
\end{tabular}

\section{636305}

$\odot .967606$

3. 028958

3. 073601

2.752969

3. 643265

4.907905

5.198992

4. 266738

6.429145

7.525172

7.032583

5.999427

3. 217742

3. 380231

1.732094

4.042267

6.886338

2.929055

4.170095

2. 385886

3. 093269

4. 393665

4. 912127

6.208393

6.119935

7. 441489

6. 214986

6.429631

2. 447120

3. 230249

2. 378969

1. 003462

$-1.747156$

$-2.051529$

$-2.688964$

$-3.036117$

$-2.600947$

$-1.999084$

$-2.702601$

$-2.746204$

$-3.927206$

$-1.437670$

$-2.199933$

$-5.160700$

$-2.921969$

$-2.337683$

$-4.939734$

$-3.743994$

$-4.795110$

$-2.622502$

$-2.664926$

$-3.895681$

$-5.066998$

$-6.086175$

$-2.404569$

$-6.339333$

$-6.382979$

$-6.483409$

- 3.218687

$-4.422129$

- 3.569442

$-7.693841$

2. 659693

2. 636060

0.894352

2.589244

$-1.423540$

$-0.166678$

4. 048435

2. 888353

1.786696

4. 470324

7. 140146

8. 375350
1. 848688

2. 461828

2. 008013

$-0.810355$

$-1.141336$

$-1.844936$

$-2.187793$

- 2 . 026242

$-1.296005$

$-2.614534$

$-1.536029$

$-3.868862$

$-3.067752$

$-2.263112$

$-3.794642$

$-1.933550$

$-1.527872$

$-1.901834$

1. 650095

1. 939781

2. 572843

3. 701945

3. 908948

3. 088273

3. 373531

2. 734556

2. 742037

4. 800178

4.885612

4.693726

4. 739198

6.111784

4. 285246

$-1.311263$

$-2.607985$

$-2.842361$

$-1.704048$

$-0.397055$

$-\odot .233356$

$\odot .848346$

$\odot .505861$

1. 714932

1. 727798

$-4.487413$

$-1.626544$

$-4.295280$

$-5.329147$

$-1.517266$

$-1.069919$

$-0.877058$

$-\odot .896600$

$-1.155094$

$-1.467570$

$-1.613646$

$-3.228541$

$-1.502316$

- 3.251180

$-1.853157$

$-0.773516$

0.825409

$-4.638029$

$-0.685725$

$-1.738556$

$-0.817283$

0.599297

$-5.325518$

$-5.250642$

- 3.071086

$-5.106296$

$-5.797458$

$-6.711410$

$-0.847267$

$-1.109966$

$-0.679040$

$-1.947717$
3. 469084

3. 625443

4.308576

$-0.865009$

2.185000

$-3.006787$

$-2.453597$

$-1.073273$

$-0.322015$

$-0.321587$

$-0.146292$

$-0.997444$

1. 101558

$-4.439833$

$-4.612871$

$-4.707237$

$-5.523836$

$-3.756877$

0.719594

$\odot .151408$

1. 627916

2. 057242

1. 512548

0.477460

$-0.335379$

$-1.747180$

๑. 357122

2. 837050

$-0.584134$

3.060863

4. 395744

2. 441252

3. 423272

1. 643622

2. 082269

3. 303433

4. 084392

3. 752673

2. 490401

4. 679141

. 187959

4.295151

4. 489536

5.154524

5. 169556

3. 797051

2. 808486

$-2.684161$

- 3.304304

$-5.722927$

$-2.479056$

$-1.103204$

$-0.521261$

$-1.276536$

- 3.819508

$-5.415241$

0.180136

$-0.482369$

๑. 630481

$-4.855163$

3. 961216

$-4.802850$

$-1.293538$

5.062773

3. 031625

4. 701498

4. 063777

$-2.129265$

$-2.793302$

$-0.664138$

๑. 378256

$-2.571265$

๑. 725213

0.418215

$\odot .410853$ 


\begin{tabular}{|c|c|c|c|}
\hline $\mathrm{H}$ & 7.903968 & -1.161539 & -1.101761 \\
\hline $\mathrm{H}$ & 7.534007 & -3.661523 & -1.941098 \\
\hline $\mathrm{H}$ & 7.771251 & -4.312854 & $-\odot .320606$ \\
\hline $\mathrm{H}$ & 6.263045 & -4.623663 & -1.194688 \\
\hline $\mathrm{H}$ & 5.131081 & -3.732943 & 1.066775 \\
\hline $\mathrm{H}$ & 6.829132 & -3.604150 & 1.574522 \\
\hline $\mathrm{H}$ & 5.753137 & -2.229052 & 1. 759951 \\
\hline $\mathrm{H}$ & 3.044745 & -4.089802 & -5.614390 \\
\hline $\mathrm{H}$ & 4.418338 & -4.107822 & -4.493499 \\
\hline $\mathrm{H}$ & 2.769561 & -4.337802 & -3.882483 \\
\hline $\mathrm{H}$ & 1.464785 & -2.299975 & -5.704303 \\
\hline $\mathrm{H}$ & 1.068598 & -2.424752 & -3.987468 \\
\hline $\mathrm{H}$ & 1.521201 & -0.858946 & -4.688635 \\
\hline $\mathrm{H}$ & 4.012043 & -0.441372 & -5.377177 \\
\hline $\mathrm{H}$ & 5.083978 & -1.848829 & -5.540620 \\
\hline $\mathrm{H}$ & 3. 619313 & -1.740011 & -6.512882 \\
\hline $\mathrm{H}$ & 7.677469 & -1.804941 & -3.006743 \\
\hline $\mathrm{H}$ & 7.303798 & -2.372516 & -4.650235 \\
\hline $\mathrm{H}$ & 6.509342 & $-\odot .905658$ & -4.013154 \\
\hline $\mathrm{H}$ & 4.568994 & 1.260406 & -0.586911 \\
\hline $\mathrm{H}$ & 6.983278 & 3.059535 & -2.337309 \\
\hline $\mathrm{H}$ & 6.146636 & 1.640413 & -1.726494 \\
\hline $\mathrm{H}$ & 5. 215018 & 3. 048927 & -2.279321 \\
\hline $\mathrm{H}$ & 7.622938 & 3.140163 & 1.356758 \\
\hline $\mathrm{H}$ & 7.317810 & 1.657423 & ๑. 450772 \\
\hline $\mathrm{H}$ & 8.341548 & 2.925209 & -0.241966 \\
\hline $\mathrm{H}$ & 6.173243 & 3.822058 & 3. 326798 \\
\hline $\mathrm{H}$ & 7.150932 & 4.892103 & 2.278492 \\
\hline $\mathrm{H}$ & 6.189186 & 5.586004 & 3.596713 \\
\hline $\mathrm{H}$ & 6.586217 & 5.463370 & $\odot .324152$ \\
\hline $\mathrm{H}$ & 7.307015 & 5.018222 & -1.227016 \\
\hline $\mathrm{H}$ & 5.567827 & 5.320465 & -1.103757 \\
\hline $\mathrm{H}$ & 3. 354194 & 3.733745 & 4.815227 \\
\hline $\mathrm{H}$ & 4.214020 & 5.192060 & 4.285597 \\
\hline $\mathrm{H}$ & 2.674822 & 5.337456 & 5.127535 \\
\hline $\mathrm{H}$ & 1.917243 & 6.805765 & 3.154259 \\
\hline $\mathrm{H}$ & 3.368826 & 6.490444 & 2.184208 \\
\hline $\mathrm{H}$ & 1.765484 & 6.109063 & 1.531571 \\
\hline $\mathrm{H}$ & ๑. 345298 & 4.236835 & 2.548653 \\
\hline $\mathrm{H}$ & ๑. 958893 & 3. 319981 & 3.938424 \\
\hline $\mathrm{H}$ & $\odot .583073$ & 5.033973 & 4.102947 \\
\hline $\mathrm{H}$ & -1.797735 & -3.444604 & 1.445781 \\
\hline $\mathrm{H}$ & -1.722810 & 0.759801 & 2.159689 \\
\hline $\mathrm{H}$ & -3.677409 & $\odot .041395$ & 6.507494 \\
\hline $\mathrm{H}$ & -1.927515 & -0.165731 & 6.467622 \\
\hline $\mathrm{H}$ & -2.628929 & 1.433654 & 6.759762 \\
\hline $\mathrm{H}$ & -3.970669 & 2.604205 & 4.936282 \\
\hline $\mathrm{H}$ & -3.855556 & 2. 049810 & 3.255937 \\
\hline $\mathrm{H}$ & -4.871946 & 1.176109 & 4.413454 \\
\hline $\mathrm{H}$ & -1.450165 & 2.539737 & 5.224976 \\
\hline $\mathrm{H}$ & -0.522360 & 1.144264 & 4.643571 \\
\hline $\mathrm{H}$ & -1.393360 & 2.194255 & 3.502499 \\
\hline $\mathrm{H}$ & -2.298923 & -5.527967 & 5.487385 \\
\hline $\mathrm{H}$ & -1.132388 & -4.257422 & 5.061819 \\
\hline $\mathrm{H}$ & -2.618229 & -3.838709 & 5.926020 \\
\hline $\mathrm{H}$ & -5.341252 & $-\odot .555580$ & 5.300933 \\
\hline $\mathrm{H}$ & -5.599575 & -1.951913 & 4. 219872 \\
\hline $\mathrm{H}$ & -5.634653 & -2.167278 & 5.992416 \\
\hline $\mathrm{H}$ & -2.847470 & -5.307569 & 1.837852 \\
\hline $\mathrm{H}$ & -1.265926 & -5.184489 & 2.640500 \\
\hline $\mathrm{H}$ & -2.477972 & -6.333324 & 3. 223168 \\
\hline $\mathrm{H}$ & -5.677063 & -0.344444 & -5.362527 \\
\hline $\mathrm{H}$ & -4.536697 & -0.478927 & -6.711751 \\
\hline $\mathrm{H}$ & -5.062919 & -1.927200 & -5.861815 \\
\hline $\mathrm{H}$ & -1.696680 & -0.542238 & -2.915832 \\
\hline $\mathrm{H}$ & -3.948503 & -1.605620 & 0.549457 \\
\hline $\mathrm{H}$ & -6.948550 & -3.342720 & -4.480107 \\
\hline $\mathrm{H}$ & -5.179278 & -3.553980 & -4.344935 \\
\hline $\mathrm{H}$ & -6.222179 & -3.857984 & -2.930865 \\
\hline $\mathrm{H}$ & $-2.270 \odot \odot 2$ & -1.226763 & -6.467883 \\
\hline $\mathrm{H}$ & -1.453255 & -1.322973 & -4.905818 \\
\hline $\mathrm{H}$ & -2.616110 & -2.577975 & -5.376764 \\
\hline $\mathrm{H}$ & -6.289291 & -4.047806 & -0.570893 \\
\hline $\mathrm{H}$ & -5.471164 & -3.360487 & ๑.839505 \\
\hline $\mathrm{H}$ & -7.242211 & -3.415338 & ๑.780922 \\
\hline
\end{tabular}

\begin{tabular}{|c|c|c|c|}
\hline $\mathrm{H}$ & -7.408914 & $-\odot .918278$ & 1.199947 \\
\hline $\mathrm{H}$ & -5.653020 & $-\odot .808987$ & 1.341588 \\
\hline $\mathrm{H}$ & -6.508318 & ๑. 232250 & $\odot .195029$ \\
\hline H & -4.052785 & 1. 420481 & -4.464244 \\
\hline $\mathrm{H}$ & -2.322655 & 1.066708 & -4.279281 \\
\hline $\mathrm{H}$ & -3.045688 & 1.128380 & -5.895269 \\
\hline $\mathrm{H}$ & -4.984137 & -4.420111 & 3.045261 \\
\hline $\mathrm{H}$ & -4.531194 & -5.709462 & 4.167160 \\
\hline $\mathrm{H}$ & -4.881256 & -4.099882 & 4.789709 \\
\hline $\mathrm{H}$ & -8.534374 & -1.757833 & -0.589268 \\
\hline $\mathrm{H}$ & -7.746765 & -0.803744 & -1.857874 \\
\hline $\mathrm{H}$ & -7.840306 & -2.562389 & -1.992835 \\
\hline $\mathrm{Rh}$ & -0.065320 & ๑. 935010 & -0.599212 \\
\hline 0 & -2.089056 & 1.894644 & -1.000643 \\
\hline C & $\odot .645456$ & 1.619429 & -2.584888 \\
\hline S & -2.613493 & 3.074884 & -0.255935 \\
\hline 0 & $-\odot .091567$ & 1. 081158 & -3.390144 \\
\hline C & -0.144479 & 4. 009601 & -0.635686 \\
\hline $\mathrm{N}$ & -1.591052 & 4.350652 & -0.678352 \\
\hline 0 & -2.783370 & 2.954672 & 1.200585 \\
\hline C & -4.170124 & 3.525819 & -0.990865 \\
\hline $\mathrm{H}$ & ๑. 356016 & 4.985067 & -0.651490 \\
\hline $\mathrm{H}$ & 0.130703 & 3.538759 & ๑. 315366 \\
\hline C & -1.932968 & 5.629791 & $\odot .007945$ \\
\hline C & -5.293097 & 3.635626 & -0.169798 \\
\hline C & -4.254430 & 3.717744 & -2.374606 \\
\hline C & -1.441686 & 6.805074 & -0.790850 \\
\hline $\mathrm{H}$ & -3.025308 & 5.675358 & ๑. . 084368 \\
\hline $\mathrm{H}$ & -1.532285 & 5.647986 & 1.030303 \\
\hline $\mathrm{H}$ & -5.202821 & 3.480152 & -233.855000 \\
\hline C & -6.521802 & 3.949134 & 10.629500 \\
\hline C & -5.489102 & 4.031956 & $10.70910 \odot$ \\
\hline $\mathrm{H}$ & -3.373806 & 3. 619672 & -2.999652 \\
\hline C & -0.648631 & 7.759352 & $-\odot .301554$ \\
\hline $\mathrm{H}$ & -1.796978 & 6.855277 & -1.819287 \\
\hline $\mathrm{H}$ & $-7.4 \odot \odot 319$ & 4.040348 & -0.119136 \\
\hline C & -6.641280 & 4.150406 & -2.133246 \\
\hline $\mathrm{H}$ & -5.563687 & 4.186021 & $-4.0 \odot 4285$ \\
\hline $\mathrm{H}$ & -0.347358 & 8.612844 & -0.902010 \\
\hline $\mathrm{H}$ & $-\odot .283875$ & 7.732365 & $\odot .723200$ \\
\hline C & -7.974619 & 4.472407 & -2.761644 \\
\hline $\mathrm{H}$ & - 7.893353 & 5.314576 & -3.457792 \\
\hline $\mathrm{H}$ & -8.351731 & 3. 615837 & -3.334598 \\
\hline $\mathrm{H}$ & - 8.725131 & 4.724512 & $-2.0 \odot 719 \odot$ \\
\hline C & 2.084377 & 2. 086612 & -2.865413 \\
\hline $\mathrm{H}$ & 2.276359 & 2.087257 & -3.943045 \\
\hline $\mathrm{H}$ & 2.858305 & 1. 495491 & -2.375846 \\
\hline $\mathrm{H}$ & 1.395474 & 2.383861 & 2.023405 \\
\hline C & ๑. 364039 & 3.254734 & -1.873626 \\
\hline $\mathrm{H}$ & $-\odot .288496$ & 3.558263 & -2.698166 \\
\hline C & 1.848688 & 3. 469084 & -2.222319 \\
\hline $\mathrm{H}$ & 2.461828 & 3.625443 & -1.331135 \\
\hline $\mathrm{H}$ & 2.008013 & 4.308576 & -2.907996 \\
\hline
\end{tabular}

TS11

B3LYP SCF energy: $\quad-5535.31447957$ a.u. B3LYP enthalpy: $\quad-5533.280418$ a.u. B3LYP free energy: $\quad-5533.554106$ a.u. M06 SCF energy in solution: $\quad-5534.53610506$ a.u.

M06 enthalpy in solution: $\quad-5532.502043 \mathrm{a} . \mathrm{u}$. M06 free energy in solution: -5532.775731 a.u.

Imaginary frequency: $\quad-282.6530 \mathrm{~cm}-1$

Cartesian coordinates

$\begin{array}{lccc}\text { ATOM } & \mathrm{X} & \mathrm{Y} & \mathrm{Z} \\ \mathrm{P} & 0.0000 \odot \odot & -7.372974 & 3.308894 \\ \mathrm{P} & 0.0000 \odot \odot & -5.075937 & 3.274924 \\ 0 & 0.0000 \odot \odot & -6.474629 & 4.202129 \\ 0 & 0.000000 & -4.971712 & 5.113853 \\ 0 & 0.000000 & -8.448953 & 4.190687 \\ 0 & 0.000000 & -7.570111 & 1.924868\end{array}$




\begin{tabular}{|c|c|c|c|c|c|c|c|}
\hline 0 & $\odot . ๑ \odot \odot \odot \odot \odot$ & -4.430143 & 3.737104 & C & 8.505534 & 0.331438 & -1.087891 \\
\hline 0 & $\odot .00 \odot \odot \odot \odot$ & -5.453962 & 2.252772 & C & $\odot .770953$ & 5.536616 & 2.278177 \\
\hline 0 & $\odot . ๑ \odot \odot \odot \odot \odot$ & -8.272906 & 5.260554 & C & 5.167885 & 6.745241 & -0.611477 \\
\hline 0 & $\odot .00 \odot \odot \odot \odot$ & -9.736878 & 3.673425 & $\mathrm{H}$ & 2.722939 & -5.148573 & -2.235883 \\
\hline C & $\odot . ๑ \odot \odot \odot \odot \odot$ & -8.861862 & 1.428665 & H & $\odot .698119$ & -3.761085 & -1.970324 \\
\hline C & $\odot .00 \odot \odot \odot \odot$ & -6.726010 & 1.250231 & $\mathrm{H}$ & 6.484655 & -4.221346 & 0.684642 \\
\hline C & 3.567116 & -4.272725 & 3.121839 & H & 5.275719 & -5.267222 & 1.537903 \\
\hline C & 3.672803 & -4.052538 & 4.756498 & $\mathrm{H}$ & 2.721891 & 1.743624 & 2.894838 \\
\hline C & 2.658743 & -10.577293 & 4.356934 & H & 3. 019196 & $\odot .660924$ & 5.112451 \\
\hline C & 1.522203 & -9.965131 & 2.291343 & $\mathrm{H}$ & 2.396780 & -4.110504 & 5.338278 \\
\hline C & 5.428272 & -9.021452 & $\odot .353429$ & H & 4.124456 & -3.817162 & 4.891873 \\
\hline C & 2.522016 & -11.364721 & 1.738359 & H & -1.026500 & 0.061834 & 2.084806 \\
\hline C & 2.699251 & -11.776438 & 1.505194 & H & -0.376587 & -4.075265 & 1.332192 \\
\hline C & 2.871001 & -12.041159 & 2.455854 & $\mathrm{H}$ & -2.503367 & -5.920748 & 2.211793 \\
\hline c & 2.858240 & -11.382641 & 0.812490 & H & -2.268871 & -6.989064 & 3.603025 \\
\hline C & 2.687804 & -1.919113 & 0.759054 & H & -3.268095 & -5.539491 & 3.757787 \\
\hline C & 2.511940 & -1.776320 & 1.466860 & $\mathrm{H}$ & -1.622106 & -5.025457 & 5.847941 \\
\hline C & 3.090990 & -1.700379 & -0.244800 & H & $-\odot .588996$ & -6.388972 & 5.410216 \\
\hline c & -0.730246 & -1.979174 & 1.547909 & H & $\odot .08 \odot 437$ & -4.751662 & 5.507039 \\
\hline C & -1.114155 & $-\odot .962621$ & 2.423451 & $\mathrm{H}$ & 1.029066 & -5.272336 & 3.073718 \\
\hline C & -1.574071 & -1.243203 & 3.717177 & H & $\odot .250673$ & -6.840079 & 3.374710 \\
\hline C & -1.699850 & -2.611377 & 4.084926 & H & $-\odot .086971$ & -5.971964 & 1.887559 \\
\hline C & -1.199362 & -3.664442 & 3.271554 & H & -1.208538 & $\odot .55524 \odot$ & 6.688040 \\
\hline C & -0.743867 & -3.303612 & 1.996096 & H & -1.271537 & -1.208349 & 6.511422 \\
\hline C & -1.085266 & -5.158155 & 3.695057 & $\mathrm{H}$ & 0.048604 & -0.287499 & 5.766827 \\
\hline C & -2.361501 & -5.939712 & 3.298050 & H & -1.593759 & 2.053930 & 4.875585 \\
\hline C & -0.798937 & -5.333104 & 5.206210 & H & $-\odot .391955$ & 1.296069 & 3. 825251 \\
\hline C & ๑. 099728 & -5.838495 & 2.958064 & H & -2.054821 & 1.562799 & 3.248451 \\
\hline C & -1.859615 & $-\odot . \odot 86051$ & 4.709688 & H & -3.979028 & ๑.๑88936 & 4.162327 \\
\hline C & -1.022471 & -0.278222 & 5.999926 & H & -3.706787 & $-\odot .841381$ & 5.648130 \\
\hline C & -1.448156 & 1.278500 & 4.115871 & H & -3.539231 & 0.910542 & 5.670026 \\
\hline C & -3.361707 & 0.012460 & 5.065804 & H & -3.641560 & -4.434981 & 5.396579 \\
\hline C & -3.603428 & -3.341786 & 5.367087 & H & -4.043027 & -2.955275 & 6.290279 \\
\hline C & -1.394531 & -2.483946 & -1.240544 & H & -4.182058 & -2.979638 & 4.510544 \\
\hline C & -2.604329 & -2.947108 & -0.724078 & H & -2.791233 & -2.844540 & 0.336488 \\
\hline C & -1.207582 & -2.533336 & -2.634107 & H & -6.374951 & -2.781769 & $\odot .426801$ \\
\hline C & -2.118295 & -3.161750 & -3.486990 & H & -4.704199 & -2.343400 & 0.770634 \\
\hline C & -3.267749 & -3.751584 & -2.886664 & H & -5.499434 & -1.713927 & -0.683274 \\
\hline C & -3.597667 & -3.534897 & -1.529748 & H & -4.537327 & -5.952889 & $-0.4 \odot 4721$ \\
\hline C & -4.967829 & -3.839657 & $-\odot .857053$ & H & -4.085376 & -4.836887 & ๑.890875 \\
\hline C & -5.399822 & -2.592822 & $-\odot .035786$ & H & -5.789998 & -5.228037 & 0.612889 \\
\hline C & -4.832301 & -5.034896 & 0.114873 & H & -2.934996 & -6.247786 & -3.715688 \\
\hline C & -3.963701 & -5.919789 & -3.529548 & H & -4.261909 & -6.239075 & -2.525858 \\
\hline C & -6.135728 & -4.108692 & -1.835042 & H & -4.630261 & -6.373828 & -4.265842 \\
\hline C & -1.916280 & -3.123271 & -5.024098 & H & -6.068965 & -5.072007 & -2.339801 \\
\hline C & -1.736522 & -4.535717 & -5.631593 & H & -7.070032 & -4.110727 & -1.261531 \\
\hline C & -3.137398 & -2.429310 & -5.678981 & H & -6.212302 & -3.331578 & -2.600842 \\
\hline C & -0.664121 & -2.308231 & -5.411767 & H & -0.952555 & -5.098861 & -5.110786 \\
\hline C & 3.582374 & 0.311324 & $-\odot .937237$ & H & -2.660452 & -5.112827 & -5.605537 \\
\hline c & 4.873950 & $\odot .047361$ & -0.469660 & H & -1.436161 & -4.445963 & -6.681964 \\
\hline C & 5.953235 & -0.131929 & -1.346095 & H & -3.000627 & -2.386646 & -6.766024 \\
\hline C & 5.683028 & $\odot .023369$ & -2.732903 & H & -4.064022 & -2.968001 & -5.472620 \\
\hline C & 4.361659 & 0.094843 & -3.247578 & H & -3.243570 & -1.400409 & -5.315406 \\
\hline C & 3.334486 & 0.281775 & -2.311943 & $\mathrm{H}$ & -0.709714 & -1.274800 & -5.050189 \\
\hline C & 4.015784 & -0.178799 & -4.737426 & H & 0.262382 & -2.765158 & -5.044355 \\
\hline C & 4.546424 & -1.590221 & -5.097120 & H & -0.592540 & -2.265577 & -6.503424 \\
\hline C & $4.6 \odot 2542$ & $\odot .847847$ & -5.734444 & H & 5.036514 & $-\odot .010613$ & ๑.598568 \\
\hline C & 2.488087 & $-\odot .187464$ & -4.962305 & H & 2.313706 & 0.410720 & -2.652911 \\
\hline C & 7.626351 & -1.999222 & $-1.45 \odot 284$ & H & 5.632138 & -1.644544 & -4.989362 \\
\hline C & 7.244910 & 1.351577 & -3.911472 & H & 4.094829 & -2.353875 & -4.452886 \\
\hline C & 7.323366 & $-\odot .624249$ & $-\odot .801589$ & H & 4.292987 & -1.831037 & -6.136632 \\
\hline c & 7.280660 & -0.822039 & 0.730326 & H & 4.157154 & ๑.686580 & -6.722907 \\
\hline C & 3.075988 & 5.255776 & 1.104115 & H & 4.375230 & 1.878123 & -5.436542 \\
\hline c & 1.853107 & 4.687912 & 1.562242 & $\mathrm{H}$ & 5.681228 & 0.738944 & -5.847460 \\
\hline C & ๑.385467 & 6.731661 & 1.369903 & H & 2.283159 & $-\odot .456756$ & -6.003540 \\
\hline C & 1.619117 & 3.344411 & 1.258267 & H & 1.981757 & $-\odot .924031$ & -4.329378 \\
\hline C & 2.539260 & 2.581103 & 0.521208 & H & 2.034936 & $\odot .795371$ & -4.784494 \\
\hline C & 3.648429 & 3.223215 & $-\odot .028459$ & H & 8.567183 & -2.401124 & -1.054336 \\
\hline C & 3.923077 & 4.581970 & $\odot .195788$ & H & 6.829079 & -2.721150 & -1.238194 \\
\hline C & 4.387999 & 6.564797 & 2.569144 & H & 7.724437 & -1.911604 & -2.534831 \\
\hline C & 1.238641 & 6.062962 & 3.656077 & H & 6.469850 & 1.986527 & -4.347493 \\
\hline C & 6.445818 & 4.631260 & -0.114131 & $\mathrm{H}$ & 7.634176 & 1.837220 & -3.013109 \\
\hline C & 5.097189 & 5.200575 & -0.616152 & H & 8.053877 & 1.213988 & -4.632332 \\
\hline C & 4.915576 & 4.801179 & -2.107614 & H & 7.135640 & $0.125 \odot 43$ & 1.264036 \\
\hline \multirow[t]{2}{*}{ C } & $-\odot .514072$ & 4.717872 & 2.522043 & H & 6.494988 & -1.516721 & 1.042279 \\
\hline & & & & & & & \\
\hline
\end{tabular}




\begin{tabular}{|c|c|c|c|}
\hline $\mathrm{H}$ & 8.240667 & -1.233694 & 1. 060131 \\
\hline $\mathrm{H}$ & $-\odot .010597$ & 6.378614 & 0.410714 \\
\hline $\mathrm{H}$ & $-\odot .393134$ & 7.332139 & 1.856035 \\
\hline $\mathrm{H}$ & 1. 242011 & 7.379178 & 1.174113 \\
\hline $\mathrm{H}$ & $\odot .708278$ & 2.871184 & 1.599536 \\
\hline $\mathrm{H}$ & 4.322568 & 2.656957 & $-\odot .655729$ \\
\hline $\mathrm{H}$ & 4.525178 & 7.618725 & 2.820630 \\
\hline $\mathrm{H}$ & 4.059279 & 6.015937 & 3.459094 \\
\hline $\mathrm{H}$ & 5.340603 & 6.146630 & 2.227895 \\
\hline $\mathrm{H}$ & ०. 390309 & 6.515886 & 4.182919 \\
\hline $\mathrm{H}$ & 1.623259 & 5.250820 & 4. 285122 \\
\hline $\mathrm{H}$ & 2. 008469 & 6.828412 & 3.560735 \\
\hline $\mathrm{H}$ & 6.636138 & 4.899339 & ๑. 931087 \\
\hline $\mathrm{H}$ & 6.474787 & 3.537793 & -0.186676 \\
\hline $\mathrm{H}$ & 7.271596 & 5.030427 & $-\odot .715250$ \\
\hline $\mathrm{H}$ & 5.720448 & 5. 245319 & -2.704225 \\
\hline $\mathrm{H}$ & 4.946999 & 3.720201 & -2.271816 \\
\hline $\mathrm{H}$ & 3.961757 & 5.173565 & -2.497651 \\
\hline $\mathrm{H}$ & -0.923243 & 4.316582 & 1.588894 \\
\hline $\mathrm{H}$ & -0.354534 & 3.887123 & 3.219032 \\
\hline $\mathrm{H}$ & -1.273236 & 5.371855 & 2.964441 \\
\hline $\mathrm{H}$ & 8.278461 & 1.359531 & $-\odot .781354$ \\
\hline $\mathrm{H}$ & 9.380791 & ๑ . $0 \odot 5182$ & -0.514341 \\
\hline $\mathrm{H}$ & 8.791353 & $\odot .328050$ & -2.139592 \\
\hline $\mathrm{H}$ & 5.906297 & 7.057029 & -1.359293 \\
\hline $\mathrm{H}$ & 4. 208661 & 7.197146 & -0.878073 \\
\hline $\mathrm{H}$ & 5.484872 & 7.164295 & ๑. 343622 \\
\hline $\mathrm{Rh}$ & ๑. . 082453 & 0.592129 & -0.763526 \\
\hline $\mathrm{H}$ & -0.324697 & -2.069540 & -3.057059 \\
\hline C & -1.390141 & 1.941350 & $-\odot .673732$ \\
\hline 0 & -0.579425 & 2.833049 & $-\odot .900367$ \\
\hline C & -2.857507 & 2.006761 & -0.273224 \\
\hline $\mathrm{H}$ & $-3.0 \odot 7597$ & 1.683523 & ๑. 762542 \\
\hline C & -3.264068 & ๑. 927616 & -1.309708 \\
\hline $\mathrm{H}$ & -3.567348 & $-\odot .0 \odot 3449$ & -0.825410 \\
\hline $\mathrm{H}$ & -4.059106 & 1.268931 & -1.976935 \\
\hline C & -3.435222 & 3.420844 & -0.475847 \\
\hline $\mathrm{N}$ & -4.906005 & 3.443472 & $-\odot .313535$ \\
\hline $\mathrm{H}$ & -3.165507 & 3.771164 & -1.475161 \\
\hline $\mathrm{H}$ & -2.982613 & 4.112652 & ๑. 242376 \\
\hline S & -5.731617 & 3.975053 & -1.726595 \\
\hline C & -5.386619 & 4.100830 & 0.923938 \\
\hline 0 & -5.081522 & 3.266790 & -2.838146 \\
\hline 0 & -5.834143 & 5.439775 & -1.750793 \\
\hline C & -7.372974 & 3.308894 & -282.653000 \\
\hline C & -5.075937 & 3. 274924 & 6.357800 \\
\hline $\mathrm{H}$ & -6.474629 & 4.202129 & 8.060400 \\
\hline $\mathrm{H}$ & -4.971712 & 5.113853 & 1.021444 \\
\hline C & -8.448953 & 4.190687 & -1.364202 \\
\hline C & -7.570111 & 1.924868 & -1.455032 \\
\hline C & -4.430143 & 3.737104 & 3.213180 \\
\hline $\mathrm{H}$ & -5.453962 & 2.252772 & 2.124632 \\
\hline $\mathrm{H}$ & -8.272906 & 5.260554 & -1.394411 \\
\hline C & -9.736878 & 3.673425 & -1.223515 \\
\hline C & -8.861862 & 1.428665 & -1.311582 \\
\hline $\mathrm{H}$ & -6.726010 & 1. 250231 & -1.551822 \\
\hline $\mathrm{H}$ & -4.272725 & 3. 121839 & 4.094079 \\
\hline $\mathrm{H}$ & -4.052538 & 4.756498 & 3. 257014 \\
\hline $\mathrm{H}$ & -10.577293 & 4.356934 & -1.136334 \\
\hline C & -9.965131 & 2. 291343 & -1.197059 \\
\hline $\mathrm{H}$ & -9.021452 & $\odot .353429$ & -1.293814 \\
\hline C & -11.364721 & 1.738359 & -1.078268 \\
\hline $\mathrm{H}$ & -11.776438 & 1.505194 & -2.068965 \\
\hline $\mathrm{H}$ & -12.041159 & 2.455854 & -0.603950 \\
\hline $\mathrm{H}$ & -11.382641 & $\odot .812490$ & -0.493724 \\
\hline C & -1.919113 & $\odot .759054$ & -2.038347 \\
\hline $\mathrm{H}$ & -1.776320 & 1.466860 & -2.860486 \\
\hline $\mathrm{H}$ & $-1.70 \odot 379$ & $-\odot .24480 \odot$ & -2.392642 \\
\hline
\end{tabular}

TS12

B3LYP SCF energy:

B3LYP enthalpy:

B3LYP free energy:
-5535.29591990 a.u.

-5533.261090 a.u.

-5533.529665 a.u.
M06 SCF energy in solution:

a.u.

Mo6 enthalpy in solution:

M06 free energy in solution:

a.u.

Imaginary frequency:

$-238.2059 \mathrm{~cm}-1$

Cartesian coordinates

\begin{tabular}{|c|c|c|c|}
\hline ATOM & $x$ & $\mathrm{Y}$ & Z \\
\hline $\mathrm{P}$ & $\odot . ๑ \odot \odot \diamond \odot \odot$ & -3.924304 & -6.467649 \\
\hline$P$ & 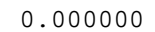 & -6.040590 & -6.592057 \\
\hline 0 & $\odot .0000 \odot \odot$ & -6.784736 & -5.710751 \\
\hline 0 & $\odot .0000 \odot \odot$ & -5.237788 & -4.893524 \\
\hline 0 & 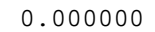 & ๑. 130795 & -3.053195 \\
\hline 0 & $\odot .000000$ & ๑. 888928 & -3.164539 \\
\hline 0 & $\odot .0 \odot \odot \odot \odot \odot$ & -6.257489 & -7.078943 \\
\hline 0 & $\odot .0 \odot \odot ० \odot \odot$ & -7.089543 & -6.331271 \\
\hline 0 & $\odot .000000$ & -7.581087 & -5.506000 \\
\hline 0 & $\odot . \diamond \odot \odot \odot \odot \diamond$ & 1.105252 & -2.880618 \\
\hline C & $\odot .00000 \odot$ & -0.671427 & -3.306452 \\
\hline C & 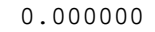 & -8.504020 & -6.736928 \\
\hline C & 2.008145 & -9.227083 & -6.276909 \\
\hline C & 1.389468 & -8.768583 & -6.451186 \\
\hline C & $\odot .105361$ & -8.626147 & -7.825186 \\
\hline C & $-\odot .546722$ & -2.631560 & ๑. 586581 \\
\hline C & 3.332805 & -2.812556 & 0.737600 \\
\hline C & 2.483115 & -2.538692 & 1.564503 \\
\hline C & 3.287006 & -2.337517 & -0.537554 \\
\hline C & 3.798038 & -2.667888 & -1.559758 \\
\hline C & 3. 462816 & -2.615475 & -2.067676 \\
\hline C & 2.667837 & -3.615708 & -0.369308 \\
\hline C & 2.152912 & -3.955319 & $\odot . \odot \odot 8792$ \\
\hline C & 3.289947 & -4.495950 & -0.608860 \\
\hline C & -0.831730 & 2. 906142 & $-\odot .744283$ \\
\hline C & -0.412198 & 2.933828 & -2.078023 \\
\hline C & $-\odot .393800$ & 4.119773 & -2.823682 \\
\hline C & -0.836059 & 5.305333 & -2.173912 \\
\hline C & -1.091567 & 5.351092 & -0.777935 \\
\hline C & -1.118310 & 4.118547 & -0.108925 \\
\hline C & -1.306086 & 6.639577 & ๑. 069730 \\
\hline C & -2.815332 & 6.902995 & ๑. 287868 \\
\hline C & -0.635034 & 7.896810 & -0.533407 \\
\hline C & $-\odot .666782$ & 6.453537 & 1.473676 \\
\hline C & $\odot .131469$ & 4.115316 & -4.284567 \\
\hline C & 1.270414 & 5.154012 & -4.443418 \\
\hline C & $\odot .717579$ & 2.739134 & -4.671726 \\
\hline C & $-\odot .996477$ & 4.421684 & -5.299430 \\
\hline C & -2.258151 & 6.842863 & -3.327465 \\
\hline C & -2.679551 & 1. 258702 & ๑. 839866 \\
\hline C & -3.654540 & 2.160373 & ๑. 411565 \\
\hline C & -3.072730 & $\odot .207767$ & 1.683960 \\
\hline C & -4.370982 & 0.111009 & 2.198504 \\
\hline C & -5.312154 & 1.102998 & 1.795858 \\
\hline C & -4.997939 & 2. 081907 & $\odot .817179$ \\
\hline C & -6.021917 & 3.037883 & 0.138648 \\
\hline C & -5.526419 & 3.459459 & -1.270507 \\
\hline C & -6.185923 & 4.343894 & ๑. 954844 \\
\hline C & -7.009118 & 1.975847 & 3.292334 \\
\hline C & -7.393098 & 2.358125 & -0.096449 \\
\hline C & -4.749527 & -1.057720 & 3.146992 \\
\hline C & -5.140530 & -0.533316 & 4.550527 \\
\hline C & -5.917281 & -1.878141 & 2.545183 \\
\hline C & -3.572339 & -2.032611 & 3.362877 \\
\hline C & 2.546579 & -0.697852 & 1.447523 \\
\hline C & 3.852323 & -0.284342 & 1.758603 \\
\hline C & 4.485270 & $-\odot .665653$ & 2.943839 \\
\hline C & 3.766756 & -1.538280 & 3.809272 \\
\hline C & 2.389964 & -1.808074 & 3.631495 \\
\hline C & 1.821750 & -1.397239 & 2.411022 \\
\hline C & 1.445451 & -2.482297 & 4.667824 \\
\hline C & 2.012822 & -2.617172 & 6.100561 \\
\hline C & 1.017990 & -3.881364 & 4.162774 \\
\hline C & ๑. 178115 & -1.598874 & 4.812986 \\
\hline C & 5.750318 & ๑. 626148 & 4.697600 \\
\hline C & 4.853690 & -3.443675 & 4.685438 \\
\hline
\end{tabular}




\begin{tabular}{|c|c|c|c|c|c|c|c|}
\hline C & 5.869968 & $-\odot .077484$ & 3.322284 & $\mathrm{H}$ & 1.194391 & -2.921134 & 6.763423 \\
\hline C & 6.326284 & 0.985116 & 2.298095 & H & 0.329623 & -4.346830 & 4.877706 \\
\hline C & 4.432475 & -3.009765 & -3.062138 & $\mathrm{H}$ & 0.503843 & -3.828075 & 3.196752 \\
\hline c & 3.387382 & -2.227692 & -3.622825 & H & 1.877582 & -4.550363 & 4.045025 \\
\hline c & 3.799568 & $-3.0404 \odot 5$ & -6.105455 & $\mathrm{H}$ & $-0.5 \odot 4236$ & -2.056104 & 5.538092 \\
\hline C & 2.619910 & -1.457944 & -2.734830 & $\mathrm{H}$ & 0.438699 & -0.597522 & 5.172191 \\
\hline C & 2.861965 & -1.446854 & -1.352759 & $\mathrm{H}$ & -0.373201 & -1.485926 & 3.876065 \\
\hline C & 3.816125 & -2.329870 & $-\odot .840259$ & H & $6.7 \odot 2589$ & 1.104179 & 4.956876 \\
\hline C & 4.589612 & -3.162833 & -1.662208 & $\mathrm{H}$ & 4.976419 & 1.401098 & 4.666544 \\
\hline C & 6.485006 & -2.934391 & -4.215764 & H & 5.494096 & $-\odot . \odot 80184$ & 5.489227 \\
\hline C & 3.002132 & -0.728610 & -5.631886 & $\mathrm{H}$ & 3.990926 & -4.106264 & 4.561695 \\
\hline C & 6.716504 & -3.396318 & -0.308267 & H & 5.507216 & -3.544848 & 3.811460 \\
\hline C & 5.539076 & -4.168704 & -0.949933 & H & 5.402032 & -3.727923 & 5.586092 \\
\hline C & 4.745581 & -4.889255 & 0.174813 & $\mathrm{H}$ & 6.512421 & $\odot .555054$ & 1. 306784 \\
\hline C & 1.511986 & -2.728457 & -5.210595 & H & 5.597636 & 1.796417 & 2.194569 \\
\hline C & 6.983107 & -1.148919 & 3.396567 & $\mathrm{H}$ & 7.269175 & 1.425478 & 2.639544 \\
\hline C & 2.965500 & -2.190492 & -5.121686 & H & 3.843705 & -4.092285 & -5.817949 \\
\hline C & 6.112523 & -5.294342 & -1.841682 & H & 3.320979 & -2.976795 & -7.090009 \\
\hline $\mathrm{H}$ & -0.385515 & 2.280984 & 5.122652 & H & 4.821764 & -2.673567 & -6.223185 \\
\hline $\mathrm{H}$ & -1.569491 & 1.490307 & 3.098998 & H & 1.805506 & -0.860051 & -3.127555 \\
\hline $\mathrm{H}$ & 4.276921 & 3.548717 & 4.670191 & H & 3.971037 & -2.370550 & ๑. 228618 \\
\hline $\mathrm{H}$ & 3.200115 & 4.898425 & 4.120981 & $\mathrm{H}$ & 7.037609 & -3.536706 & -4.939830 \\
\hline $\mathrm{H}$ & 3.552165 & 0.760948 & -2.349684 & H & 6.249828 & -1.957826 & -4.657492 \\
\hline $\mathrm{H}$ & 4.426509 & 3.014407 & -2.898312 & $\mathrm{H}$ & 7.104019 & -2.778497 & -3.323693 \\
\hline $\mathrm{H}$ & 2.667045 & 6.685847 & $-\odot .382610$ & H & 2.691119 & $-\odot .693572$ & -6.682392 \\
\hline $\mathrm{H}$ & 4.122301 & 6.148686 & 0.546272 & $\mathrm{H}$ & 2.332391 & $-\odot .069783$ & -5.070483 \\
\hline $\mathrm{H}$ & -0.091366 & 2.010561 & -2.540150 & H & 4. 016671 & $-\odot .316269$ & -5.570996 \\
\hline $\mathrm{H}$ & -1.356193 & 4.103584 & 0.947161 & $\mathrm{H}$ & 7. 328441 & -2.892870 & -1.064971 \\
\hline $\mathrm{H}$ & -3.284465 & 6.060262 & $\odot .808092$ & H & 6.362097 & -2.633996 & ๑.394449 \\
\hline $\mathrm{H}$ & -2.957327 & 7.797299 & 0.906437 & $\mathrm{H}$ & 7.367712 & -4.086531 & 0.241412 \\
\hline $\mathrm{H}$ & -3.357125 & 7.057610 & -0.649469 & H & 5.395600 & -5.620444 & 0.668032 \\
\hline $\mathrm{H}$ & -1.103854 & 8.248815 & -1.450371 & $\mathrm{H}$ & 4.379221 & -4.209569 & 0.949853 \\
\hline $\mathrm{H}$ & -0.686429 & 8.710174 & 0.199031 & H & 3.885111 & -5.429499 & -0.236017 \\
\hline $\mathrm{H}$ & 0.422191 & 7.713651 & $-\odot .756527$ & $\mathrm{H}$ & 1.470985 & -3.775803 & -4.886562 \\
\hline $\mathrm{H}$ & 0.377307 & 6.133902 & 1.400848 & H & ๑.810931 & -2.147970 & -4.606797 \\
\hline $\mathrm{H}$ & -0.701097 & 7.406298 & 2.012945 & $\mathrm{H}$ & 1.167255 & -2.689878 & -6.250750 \\
\hline $\mathrm{H}$ & -1.200119 & 5.725223 & 2.092387 & H & 7.037259 & -1.736454 & 2.472828 \\
\hline $\mathrm{H}$ & 1.658758 & 5.120774 & -5.468438 & $\mathrm{H}$ & 7.954029 & -0.657922 & 3.531843 \\
\hline $\mathrm{H}$ & $\odot .924370$ & 6.168416 & -4.241162 & $\mathrm{H}$ & 6.841136 & -1.828227 & 4.237175 \\
\hline $\mathrm{H}$ & 2.100898 & 4.931282 & -3.763867 & $\mathrm{H}$ & 6.606912 & -6.026647 & -1.192671 \\
\hline H & 1.126720 & 2.803827 & -5.685666 & $\mathrm{H}$ & 5.328838 & -5.814368 & -2.399446 \\
\hline $\mathrm{H}$ & 1.534215 & 2.440778 & -4.005711 & $\mathrm{H}$ & 6.856203 & -4.941394 & -2.556263 \\
\hline $\mathrm{H}$ & -0.031848 & 1.940266 & -4.674700 & $\mathrm{Rh}$ & -0.384616 & $-\odot .746170$ & $-\odot .7 \odot 6686$ \\
\hline $\mathrm{H}$ & -1.851837 & 3.750038 & -5.157632 & 0 & -1.889288 & -6.317294 & -0.612936 \\
\hline $\mathrm{H}$ & -1.347103 & 5.451492 & -5.231073 & C & -1.401748 & -0.327429 & -2.586411 \\
\hline $\mathrm{H}$ & -0.622976 & 4.271101 & -6.319158 & $\mathrm{~s}$ & -2.764055 & -5.211251 & -0.211498 \\
\hline $\mathrm{H}$ & -2.745702 & 7.424288 & -2.538924 & 0 & -0.579194 & -0.583424 & -3.444016 \\
\hline $\mathrm{H}$ & -2.146719 & 7.474916 & -4.211767 & C & -2.982236 & -2.622312 & -0.879746 \\
\hline $\mathrm{H}$ & -2.881335 & 5.976874 & -3.575615 & $\mathrm{~N}$ & -2.414079 & -3.927251 & -1.266335 \\
\hline $\mathrm{H}$ & -3.370581 & 2.934269 & $-\odot .285466$ & 0 & -2.752072 & -4.708366 & 1.172874 \\
\hline $\mathrm{H}$ & -6.322222 & 4.020267 & -1.771877 & C & -4.455729 & -5.622466 & -0.620187 \\
\hline $\mathrm{H}$ & -4.650276 & 4.115151 & -1.239804 & H & -4.076622 & -2.738037 & -0.855297 \\
\hline $\mathrm{H}$ & -5.287504 & 2.590422 & -1.893992 & $\mathrm{H}$ & -2.680387 & -2.322011 & 0.128316 \\
\hline $\mathrm{H}$ & -6.569849 & 4.176022 & 1.961979 & C & -1.152357 & -3.968541 & -2.006605 \\
\hline $\mathrm{H}$ & -5.225618 & 4.862671 & 1. 049601 & C & -4.728599 & -6.246844 & -1.842335 \\
\hline $\mathrm{H}$ & -6.881405 & 5.019662 & $\odot .442860$ & C & -5.473573 & -5.356664 & ๑. 294974 \\
\hline $\mathrm{H}$ & -6.160388 & 2.494502 & 3.749250 & C & 0.019950 & -3.291492 & -1.354851 \\
\hline $\mathrm{H}$ & -7.679959 & 2.704707 & 2.828389 & $\mathrm{H}$ & -0.890554 & -5.024124 & -2.140221 \\
\hline $\mathrm{H}$ & -7.565061 & 1.441519 & 4.067380 & $\mathrm{H}$ & -1.308405 & -3.567833 & -3.014182 \\
\hline $\mathrm{H}$ & -7.900548 & 2.063022 & 0.819414 & $\mathrm{H}$ & -3.924304 & -6.467649 & -238.205900 \\
\hline $\mathrm{H}$ & -8.046910 & 3.048234 & -0.641429 & C & -6.040590 & -6.592057 & 8.373100 \\
\hline $\mathrm{H}$ & -7.275350 & 1.457018 & $-\odot .709625$ & C & -6.784736 & -5.710751 & 12.266100 \\
\hline $\mathrm{H}$ & -4.353199 & 0.106875 & 4.966464 & H & -5.237788 & -4.893524 & 1.246536 \\
\hline $\mathrm{H}$ & -6.073748 & $\odot .027061$ & 4.542938 & C & $\odot .130795$ & -3.053195 & -0.021429 \\
\hline $\mathrm{H}$ & -5.272972 & -1.380503 & 5.233379 & $\mathrm{H}$ & 0.888928 & -3.164539 & -1.994355 \\
\hline $\mathrm{H}$ & -6.173228 & -2.703328 & 3.220928 & $\mathrm{H}$ & -6.257489 & -7.078943 & -3.093272 \\
\hline $\mathrm{H}$ & -6.808609 & -1.268055 & 2.393591 & C & -7.089543 & -6.331271 & -1.246478 \\
\hline $\mathrm{H}$ & -5.627759 & -2.313896 & 1.581451 & $\mathrm{H}$ & -7.581087 & -5.506000 & 0.681736 \\
\hline $\mathrm{H}$ & -3.231718 & -2.508473 & 2.438306 & $\mathrm{H}$ & 1.105252 & -2.880618 & 0.415724 \\
\hline $\mathrm{H}$ & -2.718241 & -1.547319 & 3.848008 & $\mathrm{H}$ & -0.671427 & -3.306452 & 0.666684 \\
\hline $\mathrm{H}$ & -3.900821 & -2.839674 & 4.026077 & C & -8.504020 & -6.736928 & -1.583154 \\
\hline H & 4.382001 & $\odot .339189$ & 1.050759 & H & -9.227083 & -6.276909 & $-\odot .9 \odot 3239$ \\
\hline $\mathrm{H}$ & 0.780601 & -1.621260 & 2.215118 & H & -8.768583 & -6.451186 & -2.607383 \\
\hline $\mathrm{H}$ & 2.792856 & -3.372943 & 6.191529 & $\mathrm{H}$ & -8.626147 & -7.825186 & -1.510654 \\
\hline $\mathrm{H}$ & 2.412857 & -1.670067 & 6.472706 & C & -2.631560 & $\odot .586581$ & -2.762943 \\
\hline
\end{tabular}




$\begin{array}{rrrr}H & -2.812556 & 0.737600 & -3.832385 \\ H & -2.538692 & 1.564503 & -2.291956 \\ H & -2.337517 & -0.537554 & 1.961125 \\ \mathrm{C} & -2.667888 & -1.559758 & -1.927181 \\ \mathrm{H} & -2.615475 & -2.067676 & -2.894498 \\ \mathrm{C} & -3.615708 & -0.369308 & -2.065499 \\ \mathrm{H} & -3.955319 & 0.008792 & -1.100986 \\ \mathrm{H} & -4.495950 & -0.608860 & -2.674288\end{array}$

TS13

B3LYP SCF energy:

B3LYP enthalpy:

B3LYP free energy:

-5533.263271 a.u.

MO6 SCF energy in solution:

a.u.

M06 enthalpy in solution: $\quad-5532.506176 \mathrm{a} \cdot \mathrm{u}$

M06 free energy in solution: $\quad-5532.773279$

a.u.

Imaginary frequency:

$-176.7715 \mathrm{~cm}-1$

Cartesian coordinates

\begin{tabular}{|c|c|c|c|}
\hline ATOM & X & Y & Z \\
\hline $\mathrm{P}$ & $\odot . ๑ \odot \odot \odot \odot \odot ~$ & $\odot .296877$ & 8.581889 \\
\hline$P$ & $\odot .0 \odot \odot ० \odot \odot$ & 0.453741 & 8.066526 \\
\hline 0 & $\odot .00000 \odot$ & 2.171504 & 2.526475 \\
\hline 0 & $\odot . \odot \odot \odot \odot \odot \odot$ & 0.768499 & 3.225540 \\
\hline 0 & $\odot . ๑ \odot \odot \odot \odot \odot$ & 1.250989 & 9.747731 \\
\hline 0 & $\odot . \odot \odot \odot \odot \odot \odot$ & 2.108684 & 9.513159 \\
\hline 0 & ๑.๑๑९९७९ & 1.627805 & 10.039566 \\
\hline 0 & $\odot . ๑ \odot \odot \odot \odot \odot$ & 0.753085 & 10.623724 \\
\hline 0 & $\odot . ๑ \odot \odot \odot \odot \odot$ & -0.978377 & 3.485113 \\
\hline 0 & $\odot . \odot \odot \odot \odot \odot \odot$ & $-\odot .664980$ & 4.459909 \\
\hline C & $\odot . \odot \odot \odot \odot \odot \odot$ & -1.639047 & 3.042243 \\
\hline C & $\odot . \odot \odot \odot \odot \odot \odot$ & -2.407997 & 2.534811 \\
\hline C & 0.472210 & -1.630404 & 1.744825 \\
\hline C & ๑.918603 & -2.754335 & 1.889132 \\
\hline C & 1.614827 & -3.299788 & 2.897007 \\
\hline C & 1.845817 & -1.048420 & 2.596079 \\
\hline C & 0.118493 & -2.325076 & 0.978935 \\
\hline C & -0.901446 & 0.226677 & 1.762412 \\
\hline C & -1.371225 & $-\odot .83984 \odot$ & 3.616218 \\
\hline C & $-\odot .783777$ & -1.720406 & 2.634774 \\
\hline C & 0.304234 & -0.140032 & $\odot .753620$ \\
\hline C & 0.764393 & 1.165475 & 2.290791 \\
\hline C & $\odot .201705$ & 0.283277 & 1.267586 \\
\hline C & 2.090185 & $-\odot .116865$ & $-\odot .466629$ \\
\hline C & 2.828524 & -1.499668 & -0.606254 \\
\hline C & 2.605231 & -1.900020 & -1.926460 \\
\hline C & 3.462192 & -2.812747 & -2.563120 \\
\hline C & 4.600503 & -3.262172 & -1.838338 \\
\hline C & 4.769645 & -2.998668 & -0.454052 \\
\hline C & 3.873776 & -2.085393 & 0.115022 \\
\hline C & 5.829242 & -3.642595 & ๑. 488592 \\
\hline C & 7.011094 & -2.668304 & 0.713606 \\
\hline C & 6.366654 & -5.007410 & $-\odot . \odot \odot 32 \odot 8$ \\
\hline C & 5.186625 & -3.916572 & 1.877237 \\
\hline C & 3.121101 & -3.355222 & -3.977572 \\
\hline C & 3.058066 & -4.903859 & -3.938093 \\
\hline C & 1.738471 & -2.861818 & -4.453223 \\
\hline C & 4.149524 & -2.906276 & -5.043521 \\
\hline C & 6.712602 & -3.356929 & -2.932919 \\
\hline C & 3.128723 & 1.003360 & 0.763751 \\
\hline C & 4.231481 & 1.131578 & $-\odot .086584$ \\
\hline C & 2.992024 & 1.947338 & 1.795953 \\
\hline C & 3.984356 & 2.889740 & 2.086463 \\
\hline C & 5.154898 & 2.872719 & 1.279735 \\
\hline C & 5.236043 & 2.094548 & 0.101090 \\
\hline C & 6.296246 & 2.234258 & -1.029907 \\
\hline C & 5.549179 & 2.295576 & -2.392168 \\
\hline C & 7.229502 & 1.000552 & -1.042075 \\
\hline C & 7.299220 & 3.002337 & 2.270062 \\
\hline C & 7.153741 & 3.520044 & $-\odot .974828$ \\
\hline C & 3.757540 & 3.953382 & 3.192784 \\
\hline
\end{tabular}

\begin{tabular}{|c|c|c|c|}
\hline C & 4.776247 & 3.825348 & 4.351263 \\
\hline c & 3.861733 & 5.366736 & 2.565908 \\
\hline c & 2.351664 & 3.835518 & 3.820698 \\
\hline C & -2.284290 & -0.980017 & 1.528735 \\
\hline C & -2.929793 & -2.094267 & 2. 082831 \\
\hline C & -3.605383 & -2.023347 & 3.303585 \\
\hline C & -3.646957 & -0.755393 & 3.951124 \\
\hline C & -2.863808 & 0.340941 & 3.516064 \\
\hline C & -2.229518 & $\odot .199655$ & 2.267342 \\
\hline C & -2.616785 & 1.656282 & 4.310050 \\
\hline C & -2.901296 & 1.556026 & 5.828266 \\
\hline C & -3.444648 & 2.818691 & 3.710225 \\
\hline C & -1.112536 & $2 . \odot 28249$ & 4.202782 \\
\hline C & -3.578490 & -3.500912 & 5.348905 \\
\hline C & -5.696120 & 0.018130 & 4.864113 \\
\hline C & -4.202714 & -3.304054 & 3.944126 \\
\hline C & -3.869418 & -4.558903 & 3.106615 \\
\hline C & -5.525702 & -1.037743 & -2.708689 \\
\hline C & -4.301867 & -1.320756 & -3.369857 \\
\hline C & -5.369140 & -1.411949 & -5.776855 \\
\hline C & -3.136217 & -1.310772 & -2.588601 \\
\hline C & -3.152338 & -1.039813 & -1.211267 \\
\hline C & -4.365283 & -0.641538 & $-\odot .640306$ \\
\hline C & -5.564463 & $-\odot .587307$ & -1.365660 \\
\hline c & -7.313757 & -2.465146 & -3.270548 \\
\hline C & -3.705960 & -3.132833 & -5.029936 \\
\hline C & -7.235217 & -1.056755 & 0.477101 \\
\hline C & -6.811276 & $-\odot .041244$ & $-\odot .611887$ \\
\hline C & -6.425363 & 1.297216 & 0.075851 \\
\hline C & -3.010388 & -0.752421 & -5.472130 \\
\hline C & -5.743612 & -3.247776 & 4.072622 \\
\hline C & -4.130809 & -1.650947 & -4.882477 \\
\hline C & -8.043554 & $\odot .279 \odot 46$ & -1.488584 \\
\hline H & 1.989473 & -1.104384 & 5.245277 \\
\hline H & 2.448316 & 0.139056 & 3.171340 \\
\hline $\mathrm{H}$ & $-\odot .784850$ & -5.082208 & 4.923898 \\
\hline $\mathrm{H}$ & 0.923493 & -5.444043 & 4.439134 \\
\hline $\mathrm{H}$ & -2.234975 & -3.376292 & -1.889498 \\
\hline H & -1.172784 & -5.573689 & -2.294783 \\
\hline H & 3.048308 & -6.070266 & -0.343921 \\
\hline $\mathrm{H}$ & 2.109341 & -7.027478 & 0.870378 \\
\hline $\mathrm{H}$ & 1.748381 & -1.510421 & -2.457435 \\
\hline H & 3.995594 & -1.820682 & 1.157327 \\
\hline $\mathrm{H}$ & 6.660931 & -1.715656 & 1.127193 \\
\hline H & 7.725977 & -3.101438 & 1.423577 \\
\hline H & 7.553933 & -2.448567 & -0.210649 \\
\hline H & 6.994278 & -4.938288 & $-\odot .890014$ \\
\hline H & 6.970815 & -5.454664 & 0.794168 \\
\hline $\mathrm{H}$ & 5.550145 & -5.701327 & -0.231474 \\
\hline H & 4.256705 & -4.486268 & 1.785966 \\
\hline $\mathrm{H}$ & 5.887135 & -4.492914 & 2.490977 \\
\hline $\mathrm{H}$ & 4.963593 & -3.000558 & 2.432793 \\
\hline H & 2.792212 & -5.286721 & -4.930752 \\
\hline $\mathrm{H}$ & 4. 015031 & -5.341338 & -3.649539 \\
\hline H & 2.290703 & -5.246846 & -3.234130 \\
\hline H & 1.511731 & -3.328442 & -5.418100 \\
\hline H & $\odot .939336$ & -3.134876 & -3.756475 \\
\hline H & 1.703715 & -1.777521 & -4.597104 \\
\hline H & 4.290005 & -1.818581 & -5.027722 \\
\hline $\mathrm{H}$ & 5.119163 & -3.386402 & -4.911479 \\
\hline H & 3.784659 & -3.177822 & -6.041121 \\
\hline H & 7.397332 & -3.196624 & -2.094356 \\
\hline H & 7.195328 & -4.007897 & -3.665496 \\
\hline H & 6.482983 & -2.392157 & -3.398372 \\
\hline H & 4.312253 & 0.466283 & -0.935722 \\
\hline H & 6.276492 & 2.424431 & -3.201224 \\
\hline $\mathrm{H}$ & 4.979433 & 1.388384 & -2.613238 \\
\hline $\mathrm{H}$ & 4.860010 & 3.147581 & -2.422047 \\
\hline $\mathrm{H}$ & 7.803559 & $\odot .914820$ & -0.113003 \\
\hline H & 6.665776 & ๑. 069891 & -1.170245 \\
\hline $\mathrm{H}$ & 7.945351 & 1.078258 & -1.869079 \\
\hline $\mathrm{H}$ & 6.977819 & 2.476552 & 3.176046 \\
\hline H & 7.762193 & 2.282596 & 1.587715 \\
\hline $\mathrm{H}$ & 8.028019 & 3.771791 & 2.533174 \\
\hline H & 7.881877 & 3.524352 & -0.163976 \\
\hline
\end{tabular}




\begin{tabular}{|c|c|c|c|}
\hline $\mathrm{H}$ & 7.716483 & 3.597647 & -1.912182 \\
\hline $\mathrm{H}$ & 6.535967 & 4.417411 & $-\odot .878987$ \\
\hline $\mathrm{H}$ & 4.808898 & 2.801859 & 4.743685 \\
\hline $\mathrm{H}$ & 5.780631 & 4.116652 & 4.045133 \\
\hline $\mathrm{H}$ & 4.480136 & 4.485905 & 5.174486 \\
\hline $\mathrm{H}$ & 3.694572 & 6.128330 & 3.337008 \\
\hline $\mathrm{H}$ & 4.845167 & 5.537846 & 2.124510 \\
\hline $\mathrm{H}$ & 3. 101741 & 5.505189 & 1.787446 \\
\hline $\mathrm{H}$ & 1.552569 & 3.946768 & 3.078720 \\
\hline $\mathrm{H}$ & 2.204977 & 2.886527 & 4.350069 \\
\hline $\mathrm{H}$ & 2.223170 & 4.637030 & 4.555366 \\
\hline $\mathrm{H}$ & -2.906529 & -3.028720 & 1.538024 \\
\hline $\mathrm{H}$ & -1.667780 & 1.031891 & 1.861245 \\
\hline $\mathrm{H}$ & -3.961198 & 1.494842 & 6.070572 \\
\hline $\mathrm{H}$ & -2.404362 & ๑. 689887 & 6.275814 \\
\hline $\mathrm{H}$ & -2.509871 & 2.457452 & 6.313870 \\
\hline $\mathrm{H}$ & -3.236383 & 3.748817 & 4.252457 \\
\hline $\mathrm{H}$ & -3.208719 & 2.991476 & 2.654309 \\
\hline $\mathrm{H}$ & -4.521294 & 2.633445 & 3.776573 \\
\hline $\mathrm{H}$ & $-\odot .912039$ & 2.913584 & 4.815845 \\
\hline $\mathrm{H}$ & -0.477420 & 1.211243 & 4.563131 \\
\hline $\mathrm{H}$ & -0.803590 & 2.271920 & 3.181987 \\
\hline $\mathrm{H}$ & -3.937594 & -4.439019 & 5.789246 \\
\hline $\mathrm{H}$ & -2.485044 & -3.548730 & 5.287165 \\
\hline $\mathrm{H}$ & -3.841663 & -2.683489 & 6.022383 \\
\hline $\mathrm{H}$ & -5.560439 & 1.088132 & 4.679308 \\
\hline $\mathrm{H}$ & -6.245710 & -0.421527 & 4.024445 \\
\hline $\mathrm{H}$ & -6.268448 & -0.116527 & 5.784690 \\
\hline $\mathrm{H}$ & -4.347655 & -4.537576 & 2.120254 \\
\hline $\mathrm{H}$ & -2.791614 & -4.688683 & 2.963600 \\
\hline $\mathrm{H}$ & -4.247717 & -5.444435 & 3.628606 \\
\hline $\mathrm{H}$ & -5.779976 & -0.406725 & -5.654228 \\
\hline $\mathrm{H}$ & -5.059938 & -1.526730 & -6.822624 \\
\hline $\mathrm{H}$ & -6.171431 & -2.128695 & -5.598363 \\
\hline $\mathrm{H}$ & -2.189832 & -1.532589 & -3.062799 \\
\hline $\mathrm{H}$ & -4.386286 & $-\odot .374896$ & ๑. 407314 \\
\hline $\mathrm{H}$ & -8.228544 & -2.435601 & -3.866405 \\
\hline $\mathrm{H}$ & -6.657429 & -3.258645 & -3.650156 \\
\hline $\mathrm{H}$ & -7.566400 & -2.686399 & -2.226145 \\
\hline $\mathrm{H}$ & -3.566522 & -3.383025 & -6.088359 \\
\hline $\mathrm{H}$ & -2.762819 & -3.340483 & -4.511875 \\
\hline $\mathrm{H}$ & -4.468794 & -3.807703 & -4.624882 \\
\hline $\mathrm{H}$ & -7.539211 & -2.013138 & ๑. 036361 \\
\hline $\mathrm{H}$ & -6.421433 & -1.258895 & 1.182786 \\
\hline $\mathrm{H}$ & -8.087615 & -0.666027 & 1.045898 \\
\hline $\mathrm{H}$ & -7.295618 & 1.700747 & $\odot .605293$ \\
\hline $\mathrm{H}$ & -5.621925 & 1.189923 & ๑. 810564 \\
\hline $\mathrm{H}$ & -6.110880 & 2.042736 & -0.663185 \\
\hline $\mathrm{H}$ & -3.287536 & 0.306745 & -5.403012 \\
\hline $\mathrm{H}$ & -2.042869 & $-\odot .890569$ & -4.981870 \\
\hline $\mathrm{H}$ & -2.872708 & $-\odot .989431$ & -6.533035 \\
\hline $\mathrm{H}$ & -6.215769 & -3.013660 & 3.111123 \\
\hline $\mathrm{H}$ & -6.119253 & -4.226191 & 4.394805 \\
\hline $\mathrm{H}$ & -6.068693 & -2.515408 & 4.811355 \\
\hline $\mathrm{H}$ & -8.774815 & $\odot .810246$ & -0.867989 \\
\hline $\mathrm{H}$ & -7.789838 & $\odot .922831$ & -2.334799 \\
\hline $\mathrm{H}$ & -8.537157 & $-\odot .611977$ & -1.878065 \\
\hline $\mathrm{H}$ & 2.099985 & 1.943023 & 2.405501 \\
\hline $\mathrm{Rh}$ & ๑. . 044726 & 0.744177 & $-\odot .928066$ \\
\hline 0 & -3.369789 & 5.187679 & ๑. 296241 \\
\hline S & -2.706706 & 5.125069 & -1.013957 \\
\hline $\mathrm{N}$ & -1.702819 & 3.716021 & -1.090985 \\
\hline 0 & -3.491704 & 5.110439 & -2.252197 \\
\hline C & -1.510718 & 6.450994 & -1.097696 \\
\hline C & -1.234315 & 7.198171 & 0.047882 \\
\hline C & -0.912743 & 6.760962 & -2.323536 \\
\hline C & 0.284520 & 2.667636 & ๑. 018135 \\
\hline $\mathrm{H}$ & -1.736692 & 6.963164 & 0.980256 \\
\hline C & -0.327048 & 8.255087 & -0.035873 \\
\hline C & $-\odot .011397$ & 7.818542 & -2.386253 \\
\hline $\mathrm{H}$ & -1.168770 & 6.196882 & -3.214228 \\
\hline C & 1.100766 & 2.664898 & -1.127203 \\
\hline $\mathrm{H}$ & 0.747373 & 2.493643 & ๑. 982875 \\
\hline $\mathrm{H}$ & -0.109892 & 8.841082 & 0.853246 \\
\hline C & ๑. 296877 & 8.581889 & -176.771500 \\
\hline
\end{tabular}

$\begin{array}{rrrr}\text { H } & 0.453741 & 8.066526 & 8.15950 \odot \\ \mathrm{H} & 2.171504 & 2.526475 & 12.883300 \\ \mathrm{H} & 0.768499 & 3.225540 & -1.992332 \\ \mathrm{C} & 1.250989 & 9.747731 & -1.340762 \\ \mathrm{H} & 2.108684 & 9.513159 & -1.980388 \\ \mathrm{H} & 1.627805 & 10.039566 & -0.355980 \\ \mathrm{H} & 0.753085 & 10.623724 & -1.776180 \\ \mathrm{C} & -0.978377 & 3.485113 & 0.160859 \\ \mathrm{H} & -0.664980 & 4.459909 & 0.557870 \\ \mathrm{H} & -1.639047 & 3.042243 & 0.919265 \\ \mathrm{C} & -2.407997 & 2.534811 & -1.620806 \\ \mathrm{C} & -1.630404 & 1.744825 & -2.686395 \\ \mathrm{H} & -2.754335 & 1.889132 & -0.806371 \\ \mathrm{H} & -3.299788 & 2.897007 & -2.143621 \\ \mathrm{C} & -1.048420 & 2.596079 & -3.838026 \\ \mathrm{H} & -2.325076 & 0.978935 & -3.041380 \\ \mathrm{C} & 0.226677 & 1.762412 & -4.095562 \\ \mathrm{H} & -0.839840 & 3.616218 & -3.511006 \\ \mathrm{H} & -1.720406 & 2.634774 & -4.70 \odot 869 \\ \mathrm{C} & -0.140032 & 0.753620 & -3.017137 \\ \mathrm{H} & 1.165475 & 2.290791 & -3.915490 \\ \mathrm{H} & \odot .283277 & 1.267586 & -5.069259 \\ \mathrm{O} & -0.116865 & -0.466629 & -3.123442\end{array}$

TS14

B3LYP SCF energy: $\quad-3819.30961918$ a.u. B3LYP enthalpy: $\quad-3818.366716 \mathrm{a} . \mathrm{u}$. B3LYP free energy: $\quad-3818.531489$ a.u. M06 SCF energy in solution: $\quad-3819.27055844$ a.u.

M๑6 enthalpy in solution: $\quad-3818.327655$ a.u. M06 free energy in solution: -3818.492428 a.u.

Imaginary frequency: $\quad-281.2868 \mathrm{~cm}-1$

Cartesian coordinates

$\begin{array}{lrrr}\text { ATOM } & \mathrm{X} & \mathrm{Y} & \mathrm{Z} \\ \text { P } & -1.103387 & 1.443631 & 0.681918 \\ \text { P } & -1.513340 & -1.215124 & -1.386420 \\ \text { O } & -3.876652 & -1.457068 & 3.330931 \\ \text { O } & -3.008526 & -3.532484 & 3.874902 \\ \text { O } & -5.753156 & -1.114403 & 0.838744 \\ \text { C } & -7.046170 & 0.789868 & 0.590850 \\ \text { C } & -2.042254 & -2.015869 & 0.170938 \\ \text { C } & -2.868266 & -1.292458 & 1.085646 \\ \text { C } & -3.111218 & -1.921198 & 2.295936 \\ \text { C } & -2.591776 & -3.174264 & 2.627346 \\ \text { C } & -1.806726 & -3.887404 & 1.747633 \\ \text { C } & -1.538581 & -3.277963 & 0.510301 \\ \text { C } & -3.717796 & -2.401823 & 4.398372 \\ \text { C } & -2.946298 & 1.293011 & 0.674695 \\ \text { C } & -3.751872 & 2.431815 & 0.540825 \\ \text { C } & -5.156016 & 2.377733 & 0.503963 \\ \text { C } & -5.726347 & 1.129184 & 0.608167 \\ \text { C } & -4.941843 & -0.013601 & 0.761475 \\ \text { C } & -3.556784 & 0.003533 & 0.808806 \\ \text { C } & -7.099290 & -0.619749 & \odot .850780 \\ \text { C } & -3.005719 & -0.903736 & -2.397637 \\ \text { C } & -2.941590 & 0.059309 & -3.417122 \\ \text { C } & -4.179469 & -1.653273 & -2.224712 \\ \text { C } & -0.532589 & -2.461540 & -2.309108 \\ \text { C } & -1.059713 & -3.161155 & -3.405265 \\ \text { C } & 0.800130 & -2.689651 & -1.918424 \\ \text { C } & -0.619075 & 0.754292 & 2.313893 \\ \text { C } & -1.391526 & 1.029802 & 3.456843 \\ \text { C } & 0.548847 & -0.009538 & 2.436965 \\ \text { C } & -1.138918 & 4.119881 & -0.223248 \\ \text { C } & -0.747467 & 3.249920 & 0.811375 \\ \text { C } & -0.031810 & 3.774189 & 1.898692\end{array}$




\begin{tabular}{|c|c|c|c|}
\hline $\mathrm{H}$ & -1.415384 & -4.867209 & 1.996262 \\
\hline $\mathrm{H}$ & -0.917193 & -3.811958 & -0.197893 \\
\hline $\mathrm{H}$ & -3.131126 & -1.944874 & 5.204446 \\
\hline $\mathrm{H}$ & -4.701332 & -2.721613 & 4.752221 \\
\hline $\mathrm{H}$ & -3.294179 & 3.409503 & ๑. 487477 \\
\hline $\mathrm{H}$ & -5.755889 & 3.275294 & ๑. 405914 \\
\hline $\mathrm{H}$ & -7.675658 & -1.113271 & 0.063723 \\
\hline H & -7.545015 & $-\odot .792206$ & 1.837623 \\
\hline $\mathrm{H}$ & -2.029693 & ๑. 630936 & -3.563621 \\
\hline $\mathrm{H}$ & -4.241678 & -2.397747 & -1.437038 \\
\hline $\mathrm{H}$ & -2.082913 & -2.990784 & -3.722953 \\
\hline H & -2.302657 & 1.614691 & 3.373226 \\
\hline $\mathrm{H}$ & 1.153398 & $-\odot .23654 \odot$ & 1.565562 \\
\hline $\mathrm{H}$ & -1.677740 & 3.730998 & -1.082671 \\
\hline $\mathrm{H}$ & ๑. 289452 & 3.125264 & 2.705561 \\
\hline $\mathrm{Rh}$ & ๑. 013791 & ๑. 530931 & -1.104117 \\
\hline $\mathrm{H}$ & 1.233527 & -2.156333 & -1.075814 \\
\hline 0 & 3.121348 & -1.235319 & $\odot .133471$ \\
\hline C & $\odot .782200$ & 1.681127 & -2.553350 \\
\hline S & 4.094156 & $-\odot .246802$ & ๑. 634165 \\
\hline 0 & ๑. 887694 & $\odot .729936$ & -3.318340 \\
\hline C & 3.217605 & 1.003163 & -1.583209 \\
\hline $\mathrm{N}$ & 4.335996 & ๑. 844141 & -0.637220 \\
\hline 0 & 3.800342 & $\odot .462110$ & 1.890812 \\
\hline C & 5.696266 & -1.020614 & ๑. 802297 \\
\hline $\mathrm{H}$ & 2.875687 & $\odot .000170$ & -1.839842 \\
\hline $\mathrm{H}$ & 3.650563 & 1. 430522 & -2.493904 \\
\hline C & 5.133444 & 2.040554 & -0.292554 \\
\hline C & 6.380284 & -0.912307 & 2.012514 \\
\hline C & 6.221045 & -1.762873 & $-\odot .262021$ \\
\hline C & 5.867865 & 2.566439 & -1.496812 \\
\hline H & 5.865263 & 1.732931 & 0.463845 \\
\hline $\mathrm{H}$ & 4.513061 & 2.823255 & $\odot .165066$ \\
\hline $\mathrm{H}$ & 5.950956 & -0.337667 & 2.825990 \\
\hline C & 7.612501 & -1.553697 & 2.152311 \\
\hline C & 7.448958 & -2.393833 & -0.102250 \\
\hline $\mathrm{H}$ & 5.676785 & -1.840768 & -1.197619 \\
\hline C & 5.881434 & 3.847763 & -1.867003 \\
\hline $\mathrm{H}$ & 6.441478 & 1.826465 & -2.054067 \\
\hline H & 8.150966 & -1.470204 & 3. 092412 \\
\hline C & 8.164882 & -2.301120 & 1.105169 \\
\hline H & 7.863326 & -2.969766 & -0.925903 \\
\hline $\mathrm{H}$ & 6.467117 & 4.190186 & -2.715234 \\
\hline $\mathrm{H}$ & 5.327185 & 4.608247 & -1.320095 \\
\hline C & 9.492534 & - 3.001951 & 1.260402 \\
\hline $\mathrm{H}$ & 10.181556 & -2.730540 & $\odot .452151$ \\
\hline H & 9.368020 & -4.091379 & 1.224557 \\
\hline $\mathrm{H}$ & 9.970837 & -2.751556 & 2. 211492 \\
\hline C & $\odot .956486$ & 3.159801 & -2.805708 \\
\hline $\mathrm{H}$ & 1.328495 & 3.348224 & -3.818216 \\
\hline $\mathrm{H}$ & $\odot .033594$ & 3.724372 & -2.656293 \\
\hline C & 1.977077 & 3.327465 & -1.650142 \\
\hline H & 2.954621 & 3.657306 & -2.014652 \\
\hline $\mathrm{H}$ & 1.628718 & 4.032793 & -0.893932 \\
\hline C & 2.034692 & 1.874919 & -1.109887 \\
\hline $\mathrm{H}$ & 1.916871 & 1.813474 & -0.025355 \\
\hline C & $-\odot .267545$ & -4.080892 & -4.094248 \\
\hline $\mathrm{H}$ & -0.683821 & -4.618761 & -4.941167 \\
\hline C & 1.052039 & -4.308555 & -3.698865 \\
\hline $\mathrm{H}$ & 1.664875 & -5.024361 & -4.239405 \\
\hline C & 1.583323 & -3.613581 & -2.609732 \\
\hline $\mathrm{H}$ & 2.608925 & -3.783409 & -2.295418 \\
\hline C & -4.039648 & $\odot .270242$ & -4.251165 \\
\hline $\mathrm{H}$ & -3.982589 & 1.014779 & $-5.040 \odot 29$ \\
\hline C & -5.207463 & $-\odot .475534$ & -4.073501 \\
\hline $\mathrm{H}$ & -6.061854 & -0.310064 & -4.723623 \\
\hline C & -5.275009 & -1.436406 & -3.062060 \\
\hline $\mathrm{H}$ & -6.179791 & -2.022348 & -2.926354 \\
\hline C & ๑. 271435 & 5.137615 & 1.954482 \\
\hline $\mathrm{H}$ & 0.826363 & 5.526640 & 2.803343 \\
\hline C & -0.142184 & 5.995153 & $\odot .935007$ \\
\hline H & ๑. . 086268 & 7.055751 & ๑. 986393 \\
\hline C & -0.852129 & 5.482546 & $-\odot .155080$ \\
\hline $\mathrm{H}$ & -1.177703 & 6.142939 & -0.954047 \\
\hline C & ๑. 946686 & -0.487015 & 3.688007 \\
\hline
\end{tabular}

$\begin{array}{rrrr}\mathrm{H} & 1.862550 & -1.063406 & 3.768959 \\ \mathrm{C} & 0.179473 & -0.209102 & 4.819282 \\ \mathrm{H} & 0.491955 & -0.577336 & 5.792591 \\ \mathrm{C} & -0.990186 & 0.550162 & 4.702492 \\ \mathrm{H} & -1.586011 & 0.775106 & 5.583019\end{array}$

TS15

B3LYP SCF energy: $\quad-3819.28891914$ a.u. B3LYP enthalpy: $\quad-3818.344792$ a.u. B3LYP free energy: $\quad-3818.503334 \mathrm{a} . \mathrm{u}$. M06 SCF energy in solution: $\quad-3819.26594508$ a.u.

M06 enthalpy in solution: $\quad-3818.321818 \mathrm{a} \cdot \mathrm{u}$. Mo6 free energy in solution: $\quad-3818.480360$ a.u.

Imaginary frequency: $\quad-234.4851 \mathrm{~cm}-1$

Cartesian coordinates

\begin{tabular}{|c|c|c|c|}
\hline АТОM & $X$ & Y & Z \\
\hline $\mathrm{P}$ & 1.011514 & -1.656338 & -0.251931 \\
\hline$P$ & 1.425381 & 1.568782 & ๑. 299096 \\
\hline 0 & 5.494204 & -1.604014 & 1.518969 \\
\hline 0 & 5.689023 & -0.862673 & 3.705141 \\
\hline 0 & 5.912941 & ๑. 220327 & -0.926425 \\
\hline 0 & 6.144930 & $-\odot .586451$ & -3.084943 \\
\hline C & 2.750573 & ๑. 873713 & 1.385660 \\
\hline C & 3.623307 & -0.136344 & $\odot .871399$ \\
\hline C & 4.565948 & -0.629141 & 1.759674 \\
\hline C & 4.684162 & $-\odot .189175$ & 3.078242 \\
\hline C & 3.861369 & 0.793110 & 3.583118 \\
\hline C & 2.891904 & 1.313386 & 2.707702 \\
\hline C & 6.255155 & -1.745997 & 2.726476 \\
\hline C & 2.592816 & -1.391903 & -1.155639 \\
\hline C & 2.757379 & -1.881718 & -2.457124 \\
\hline C & 3.927933 & -1.664922 & -3.204625 \\
\hline C & 4.923166 & -0.934361 & -2.593248 \\
\hline C & 4.779341 & -0.451387 & -1.291616 \\
\hline C & 3.639801 & -0.647112 & -0.528256 \\
\hline C & 6.773550 & ๑. 213841 & -2.073784 \\
\hline C & 2. 390072 & 2.189333 & -1.147021 \\
\hline C & 2.001721 & 1.883365 & -2.458576 \\
\hline C & 3.550122 & 2. 956898 & -0.938850 \\
\hline C & $\odot .847701$ & 3.084923 & 1.188727 \\
\hline C & 1.078029 & 4.382498 & 0.708105 \\
\hline C & ๑. . 085233 & 2.926588 & 2.363436 \\
\hline C & 1.537930 & -2.476286 & 1.312031 \\
\hline C & 2.478083 & -3.521257 & 1.277690 \\
\hline C & 1.016446 & -2.067285 & 2.547466 \\
\hline C & -0.447681 & -2.610445 & -2.455548 \\
\hline C & ๑. 115658 & -2.954028 & -1.210850 \\
\hline C & -0.057718 & -4.260102 & -0.726157 \\
\hline $\mathrm{H}$ & 3.958842 & 1.153192 & 4.600985 \\
\hline $\mathrm{H}$ & 2. 252238 & 2.100381 & 3.083806 \\
\hline $\mathrm{H}$ & 6.182447 & -2.778104 & 3. 082864 \\
\hline $\mathrm{H}$ & 7.296695 & -1.462937 & 2.539114 \\
\hline $\mathrm{H}$ & 1.968796 & -2.464304 & -2.914994 \\
\hline $\mathrm{H}$ & 4.041905 & -2.057777 & -4.208520 \\
\hline $\mathrm{H}$ & 6.894758 & 1.238255 & -2.443706 \\
\hline $\mathrm{H}$ & 7.737037 & -0.229351 & -1.805984 \\
\hline $\mathrm{H}$ & 1.131353 & 1.259624 & -2.631841 \\
\hline H & 3.882816 & 3.178964 & $\odot .070960$ \\
\hline $\mathrm{H}$ & 1.650452 & 4.537784 & -0.199281 \\
\hline H & 2.911485 & -3.833604 & $\odot .332058$ \\
\hline $\mathrm{H}$ & ๑. 319662 & -1.235625 & 2.585925 \\
\hline $\mathrm{H}$ & $-\odot .371939$ & -1.594575 & -2.828164 \\
\hline $\mathrm{H}$ & ๑. 351729 & -4.549119 & 0.234881 \\
\hline $\mathrm{Rh}$ & -0.402392 & ๑. 186249 & -0.074230 \\
\hline 0 & -5.952595 & 2.560022 & 0.533306 \\
\hline C & -1.398148 & 1.306289 & -1.683773 \\
\hline S & -5.777883 & 1.367943 & -0.303505 \\
\hline 0 & -1.771920 & $\odot .395562$ & -2.400034 \\
\hline C & -3.308686 & 1.538390 & 0.757097 \\
\hline $\mathrm{N}$ & -4.336655 & ๑. 615083 & ๑. 258792 \\
\hline
\end{tabular}




\begin{tabular}{|c|c|c|c|}
\hline 0 & -5.741045 & 1.491262 & -1.769160 \\
\hline C & -7.022089 & ๑.156903 & 0.118140 \\
\hline $\mathrm{H}$ & -3.842072 & 2.433107 & 1.096468 \\
\hline $\mathrm{H}$ & -2.868691 & 1.098613 & 1.655180 \\
\hline C & -3.926344 & -0.507727 & -0.595399 \\
\hline C & -7.690828 & -0.521824 & -0.900641 \\
\hline C & -7.348034 & -0.051441 & 1.462501 \\
\hline C & -2.736550 & -1.304458 & -0.151212 \\
\hline $\mathrm{H}$ & -4.765102 & -1.219413 & -0.629236 \\
\hline $\mathrm{H}$ & -3.752814 & -0.188979 & -1.628543 \\
\hline $\mathrm{H}$ & -7.435602 & -0.327587 & -1.936826 \\
\hline C & -8.692237 & -1.432870 & -0.561308 \\
\hline C & -8.348402 & $-\odot .963708$ & 1.779255 \\
\hline $\mathrm{H}$ & -6.832132 & ๑. 499566 & 2.241909 \\
\hline C & -2.111311 & -1.365265 & 1.050440 \\
\hline H & -2.427861 & -2.014395 & -0.913443 \\
\hline $\mathrm{H}$ & -9.217495 & -1.963678 & -1.350854 \\
\hline C & -9.037543 & -1.667160 & $\odot .775779$ \\
\hline $\mathrm{H}$ & -8.607276 & -1.129986 & 2.821885 \\
\hline H & -1.424159 & -2.178741 & 1.252746 \\
\hline $\mathrm{H}$ & -2.429800 & $-\odot .785936$ & 1.909436 \\
\hline C & -10.142302 & -2.630120 & 1.137336 \\
\hline $\mathrm{H}$ & -9.867883 & -3.249129 & 1.998551 \\
\hline H & -11.058005 & -2.088648 & 1.407662 \\
\hline $\mathrm{H}$ & -10.386444 & -3.294098 & ๑. 302855 \\
\hline C & -1.257416 & 2.756105 & -2.130275 \\
\hline $\mathrm{H}$ & -1.366366 & 2.895739 & -3.208170 \\
\hline $\mathrm{H}$ & -0.346388 & 3.237363 & -1.771499 \\
\hline C & -2.462049 & 3.094234 & -1.230220 \\
\hline $\mathrm{H}$ & -3.406543 & 2.906108 & -1.747949 \\
\hline H & -2.461263 & 4.106524 & $-\odot .813589$ \\
\hline C & -2.181869 & 2.003101 & -0.164656 \\
\hline H & -0.130604 & 1.928405 & 2.736388 \\
\hline $\mathrm{H}$ & -1.409195 & 2.412114 & ๑. 486984 \\
\hline C & -1.137717 & -3.562077 & -3.206337 \\
\hline $\mathrm{H}$ & -1.561423 & -3.281439 & -4.166267 \\
\hline C & -1.295001 & -4.862759 & -2.719792 \\
\hline $\mathrm{H}$ & -1.836787 & -5.601390 & -3.303424 \\
\hline C & -0.760353 & -5.205417 & -1.477901 \\
\hline H & -0.885741 & -6.211414 & -1.087914 \\
\hline C & -0.404535 & 4.035647 & 3. 051334 \\
\hline $\mathrm{H}$ & -0.982056 & 3.894323 & 3.960428 \\
\hline C & -0.161454 & 5.323969 & 2.566000 \\
\hline $\mathrm{H}$ & -0.549146 & 6.188437 & 3. 096791 \\
\hline C & 0.574270 & 5.492179 & 1.393524 \\
\hline H & ๑. 761892 & 6.489276 & 1.005724 \\
\hline C & 4.284944 & 3.432229 & -2.024831 \\
\hline H & 5.171535 & 4.035811 & -1.851326 \\
\hline C & 3.882024 & 3.132632 & -3.329676 \\
\hline H & 4.454881 & 3.503388 & -4.174976 \\
\hline C & 2.744937 & 2.352041 & -3.543922 \\
\hline H & 2.435123 & 2.104497 & -4.555067 \\
\hline C & 2.864842 & -4.158254 & 2.456605 \\
\hline H & 3.583700 & $-4.97190 \odot$ & 2.417090 \\
\hline C & 2.330252 & -3.751625 & 3.682567 \\
\hline H & 2.633465 & -4.248264 & 4.599842 \\
\hline C & 1. 411361 & -2.701947 & 3.726772 \\
\hline $\mathrm{H}$ & 1.004016 & -2.373421 & 4.678610 \\
\hline
\end{tabular}

TS16

B3LYP SCF energy: $\quad-3819.31840032$ a.u. B3LYP enthalpy:

B3LYP free energy:

-3818.373840 a.u. -3818.530047 a.u.

Mo6 SCF energy in solution: -3819.29297296

a.u.

M06 enthalpy in solution: $\quad-3818.348413$ a.u.

Mo6 free energy in solution: $\quad-3818.504620$

a.u.

Imaginary frequency:

$-320.000 \odot \mathrm{cm}-1$

Cartesian coordinates

$\begin{array}{llcc}\text { ATOM } & X & Y & Z \\ P & -1.405975 & 1.884166 & -0.373098\end{array}$ $-\odot .926180$

\section{$-4.269243$}

$-3.297228$

$-5.484994$

$-6.908458$

$-1.635357$

$-2.761390$

$-3.220934$

$-2.638231$

$-1.556018$

$-1.070493$

$-4.408075$

$-3.127302$

$-4.009194$

$-5.317332$

$-5.704239$

$-4.841507$

$-3.541392$

$-6.770680$

$-2.379416$

$-2.706312$

$-3.167045$

๑. 182899

$-0.066967$

1. 366865

$-1.149904$

$-2.125849$

0. 042013

$-1.506563$

$-1.401919$

$-1.231202$

$-1.105517$

$-0.239454$

$-4.386299$

$-5.343098$

$-3.690473$

$-5.988675$

$-6.824424$

$-7.553280$

$-2.146773$

$-2.939504$

$-0.957247$

$-3.064593$

๑. 801928

$-1.603555$

$-1.129407$

๑. 371168

4. 422136

- 0.008006

4.832986

$-0.852629$

3. 796456

3. 774244

4. 788815

6.478842

4.575632

4. 011771

3. 508283

6. 955428

7. 262964

2. 637639

3. 029552

4. 439897

6. 350191

8. 226877

8. 530541

6.895408

1.527412

3. 173159

8. 603470

9.033312

9.145822

1. 232366

1. 398493

10.423362
$-1.666290$

$\odot .503131$

0.478333

$-1.284641$

$-0.369069$

$-1.351522$

$-0.476960$

$-\odot .285840$

$-0.873178$

$-1.717231$

$-1.944708$

๑. 312284

1. 250611

1. 800447

1. 321837

๑. 268540

- $\odot .281714$

$\odot .155805$

$-1.424345$

$-2.352371$

$-1.946248$

$-3.338425$

- 3.135347

$-4.352167$

$-3.014093$

2. 236583

2. 909406

1. 846675

3. 665187

3. 554340

4. 726414

$-2.190402$

$-2.628533$

1. 283418

$-0.221407$

2. 639057

1. 765776

$-2.391973$

$-1.330348$

$-1.161089$

$-3.655105$

$-4.476596$

3. 201417

1. 300415

2. 774845

4.678575

0.403559

๑. 262289

๑. 150283

$-0.615849$

$\odot .772235$

$-0.990279$

- .233069

$-2.076771$

$-0.142785$

- 0.617957

$-2.037196$

1.184533

1. 134970

$-1.046356$

1. 293059

1. 633666

1. 741959

1. 810935

1.506842

$-0.654563$

- 2. 046841

2. 186970

1. 224421

2. 496592

๑. 623541

$-1.356717$

2. 677932

2. 811154

1. 022036
- $\odot .363904$

131836

4.989842

$\odot .812451$

$-0.767990$

1. 314953

1.446938

. 740499

3. 863143

3. 750329

2. 451131

4.546223

$-0.486453$

$-1.425140$

$-1.608239$

$-0.809139$

$\odot .138948$

๑. 338783

. 193354

$-1.278221$

$-2.579263$

$-0.655594$

$-0.171106$

- 0.828086

๑. 584112

1. 417067

2. 172163

2. 045125

$-2.571358$

$-1.171513$

$-0.416308$

4. 615347

2. 342644

5. 048165

4.754308

$-2.030421$

$-2.334412$

$-0.318788$

๑. 951581

$-3.069956$

๑. 357696

$-1.433034$

1. 710816

1. 490690

$-3.179879$

0.660940

$-1.070984$

2. 500089

$-2.991185$

1. 402202

$-3.577455$

$-1.114127$

๑. 136116

1. 512098

0.888678

$-1.802466$

$-0.886338$

$-0.125870$

1. 197534

0.163770

$-1.363722$

$\odot .746005$

$-0.325694$

1. 792130

0.764087

$-0.259611$

$-0.038428$

$-1.342820$

$-2.306295$

1. 008766

๑. 031255

$-0.816275$

$-2.267167$

$-0.460264$

$-0.401088$ 


\begin{tabular}{|c|c|c|c|}
\hline $\mathrm{H}$ & 11.165516 & $\odot .714479$ & ๑. 347136 \\
\hline $\mathrm{H}$ & 10.701129 & $\odot .547503$ & -1.347691 \\
\hline $\mathrm{H}$ & 10.511667 & 2.106183 & $-\odot .521446$ \\
\hline C & $\odot .835504$ & $-\odot .942751$ & -3.674933 \\
\hline $\mathrm{H}$ & 0.724378 & - ๑ . 807167 & -4.755805 \\
\hline $\mathrm{H}$ & $\odot .352403$ & -1.893076 & -3.411152 \\
\hline C & 2. 298942 & -0.967359 & -3.232081 \\
\hline $\mathrm{H}$ & 2.856804 & -0.166898 & -3.736708 \\
\hline $\mathrm{H}$ & 2.753851 & -1.909093 & -3.573087 \\
\hline H & 1.619845 & -2.074192 & 1.067426 \\
\hline C & 2.403686 & -0.853810 & -1.724917 \\
\hline $\mathrm{H}$ & 1.785104 & -1.605010 & -1.245384 \\
\hline C & -1.471584 & 4.916026 & -3.188261 \\
\hline $\mathrm{H}$ & -1.562960 & 4.980376 & -4.268932 \\
\hline C & -1.313683 & 6.075702 & -2.426525 \\
\hline $\mathrm{H}$ & -1.283615 & 7.048097 & -2.909612 \\
\hline C & -1.188179 & 5.974931 & -1.041658 \\
\hline $\mathrm{H}$ & -1.056725 & 6.868494 & $-\odot .438027$ \\
\hline C & ๑. 266707 & 2.127840 & 3. 393983 \\
\hline $\mathrm{H}$ & 1.193732 & 1.810516 & 3.862514 \\
\hline C & $-\odot .7 \odot 2207$ & 2.811798 & 4.129751 \\
\hline $\mathrm{H}$ & -0.529491 & 3.037714 & 5.178044 \\
\hline C & -1.897552 & 3.200103 & 3.517191 \\
\hline H & -2.654382 & 3.731727 & 4.087455 \\
\hline C & 2.247409 & -4.088571 & ๑. 718422 \\
\hline $\mathrm{H}$ & 3.150441 & -3.957595 & 1.305851 \\
\hline C & 1.972435 & -5.298740 & $\odot .077316$ \\
\hline H & 2.656895 & -6.136298 & 0.177280 \\
\hline C & 0.821033 & -5.423058 & -0.700803 \\
\hline H & $\odot .604845$ & -6.357546 & -1.211001 \\
\hline C & -4.239951 & -3.917977 & -1.331225 \\
\hline H & -4.829289 & -4.688648 & -0.841873 \\
\hline C & -4.552259 & -3.512416 & -2.632506 \\
\hline $\mathrm{H}$ & -5.387334 & -3.965400 & -3.159490 \\
\hline C & -3.788124 & -2.522597 & -3.250466 \\
\hline $\mathrm{H}$ & -4.031581 & -2.192219 & -4.256113 \\
\hline
\end{tabular}

TS17

B3LYP SCF energy: $\quad-3819.33984484$ a.u. B3LYP enthalpy:

B3LYP free energy: -3818.394674 a.u. M06 SCF energy in solution: -3819.31122595 a.u.

M06 enthalpy in solution: $\quad-3818.366055 \mathrm{a} \cdot \mathrm{u}$ M06 free energy in solution: $\quad-3818.526073$ a.u.

Imaginary frequency:

$-308.4628 \mathrm{~cm}-1$

$\begin{array}{lcrr}\text { Cartesian coordinates } & \\ \text { ATOM } & \mathrm{X} & \mathrm{Y} & \mathrm{Z} \\ \text { P } & 2.105616 & 1.702402 & -0.108915 \\ \text { P } & 1.132801 & -1.551981 & -.015235 \\ \text { O } & 6.193853 & -0.451887 & -1.207529 \\ 0 & 6.177036 & -1.692696 & -3.163315 \\ 0 & 5.732796 & -1.709999 & 1.623137 \\ 0 & 6.259972 & -0.501998 & 3.526280 \\ \text { C } & 2.705453 & -1.651293 & -0.934632 \\ \text { C } & 3.876553 & -0.933007 & -0.523788 \\ \text { C } & 4.969405 & -1.039869 & -1.370118 \\ \text { C } & 4.965177 & -1.783698 & -2.550954 \\ \text { C } & 3.850643 & -2.486338 & -2.952243 \\ \text { C } & 2.723485 & -2.401570 & -2.119056 \\ \text { C } & 6.979810 & -0.827611 & -2.347792 \\ \text { C } & 3.424764 & 1.111015 & 1.015916 \\ \text { C } & 3.757352 & 1.831060 & 2.169496 \\ \text { C } & 4.715055 & 1.373515 & 3.091197 \\ \text { C } & 5.317971 & 0.167999 & 2.804461 \\ \text { C } & 5.000071 & -0.554954 & 1.653058 \\ \text { C } & 4.059850 & -0.136091 & 0.726131 \\ \text { C } & 6.629305 & -1.644185 & 2.742133 \\ \text { C } & 1.557847 & -1.967084 & 1.752482 \\ \text { C } & 0.836332 & -1.386706 & 2.806581 \\ \text { C } & 2.552148 & -2.916754 & 2.042554 \\ & & & \end{array}$

\subsection{8}

$-0.058089$

$-0.544450$

2.903388

4. 207118

2. 196843

0.842592

1.621769

1. 937303

3. 842428

1. 842214

7. 244544

7. 873091

3. 276576

4. 969129

6. 524679

7.657591

๑. 066547

3. 127432

0.416455

4. 769132

1.197984

0.586543

2.529039

$-0.478063$

0.078044

$-1.286728$

$-2.078316$

$-1.467987$

$-1.514445$

$-3.486491$

$-2.012136$

$-1.831260$

$-1.067275$

$-0.993078$

$-3.012044$

$-4.535531$

$-3.816002$

$-3.610387$

$-3.531925$

$-3.112002$

$-5.400310$

$-4.084034$

$-5.089625$

$-5.110904$

$-3.084090$

$-6.927350$

$-5.667894$

$-5.361489$

$-6.773140$

$-7.405208$

$-7.962653$

$-8.095131$

$-8.609703$

$-7.620424$

$-8.872824$

$-9.382108$

$-8.531175$

$-8.984277$

$-9.526643$

$-9.892410$

$-10.398846$

$-11.445109$

$-10.376119$

$-10.085958$

1. 491675

1.743734

0.729387

๑. 386160

0.406747

$-0.184684$

$-0.833499$

$-0.948564$

$-1.456676$

$-2.058190$
$-2.959738$

$-4.119863$

$-2.873789$

1.956613

2. 474765

1. 668351

3. 462924

3.373680

4.548997

$-3.077719$

$-2.955806$

0.068480

$-1.365844$

2. 781855

1. 942272

$-2.549472$

$-1.523164$

$-0.649490$

$-3.367960$

$-4.204743$

2. 686651

1. 245790

2. 561383

4.503321

$-1.967715$

0.497157

0.653715

$-0.382024$

0.108637

2. 164569

0.190643

$-1.406267$

$-0.347655$

2. 704573

2. 509711

2. 497602

$-0.417346$

$-0.023419$

1.713003

2. 248936

3.578355

0.730997

$-0.815097$

$-1.232364$

1.896933

1.983263

0.483035

1. 877479

2. 816185

$-0.624063$

1. 808047

$-0.090316$

$-1.461712$

0.840816

$-2.172343$

$-1.895817$

0.387366

1.900689

$-2.962255$

$-0.983530$

1.109464

$-1.463426$

$-1.549672$

$-0.768192$

$-2.449203$

5.786889

6.689740

5.866795

6. 831671

4.701744

4.757093

$-5.180001$

$-6.071456$

$-5.095979$

$-5.923395$
$-0.573022$

.201900

$-1.820373$

$-1.739028$

$-1.822671$

$-2.916111$

1.661216

0.490517

$-0.207570$

$-3.860570$

$-2.413187$

$-2.919048$

$-2.016025$

2. 364585

3. 978335

3. 345843

2.381590

2. 594459

1. 239974

1.172427

$-0.917659$

$-2.861110$

2. 213645

$-1.115331$

$-2.412655$

$-0.084081$

$-1.514376$

0.062395

$-2.573677$

$-1.243203$

$-0.126467$

$-0.295514$

1.133441

$-2.083247$

$-0.327108$

$-1.075243$

0.844319

$-1.151463$

0.087606

$-2.009015$

$-0.920337$

1.179002

1.759078

๑. 361070

0.335230

1. 016131

1.824294

$-0.617182$

0.867743

2. 769578

2. 222309

0.477927

0.242253

$-0.340964$

0.910503

$-0.830429$

$-1.408090$

$-0.122711$

$-1.010089$

$-1.671124$

$-2.040719$

$-2.806444$

$-2.485131$

$-3.652084$

$-3.164989$

0.262234

$-0.286437$

1.428589

1.790100

2. 130431

3. 039808

$-0.271826$

0.337866

$-1.517309$

$-1.882489$ 


$\begin{array}{lrrr}\mathrm{C} & -1.312237 & -3.940113 & -2.288508 \\ \mathrm{H} & -1.804635 & -3.860352 & -3.253325 \\ \mathrm{C} & 2.809366 & -3.279635 & 3.364673 \\ \mathrm{H} & 3.574021 & -4.021035 & 3.578742 \\ \mathrm{C} & 2.085498 & -2.697075 & 4.408561 \\ \mathrm{H} & 2.288753 & -2.982094 & 5.436731 \\ \mathrm{C} & 1.100419 & -1.748467 & 4.128562\end{array}$

$\begin{array}{lrrr}\text { H } & 0.535473 & -1.292378 & 4.936229 \\ \mathrm{C} & 2.783265 & 1.903869 & -4.161092 \\ \mathrm{H} & 2.231189 & 1.674321 & -5.067801 \\ \mathrm{C} & 4.074913 & 2.427909 & -4.239172 \\ \mathrm{H} & 4.528531 & 2.612821 & -5.208718 \\ \mathrm{C} & 4.785882 & 2.712371 & -3.069491 \\ \mathrm{H} & 5.790565 & 3.121901 & -3.127428\end{array}$




\section{X-ray data}
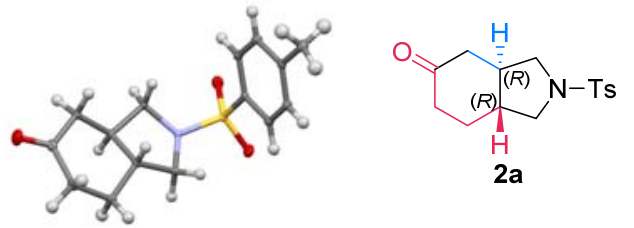

Crystal data and structure refinement for $\mathbf{2 a}$

\begin{tabular}{|c|c|}
\hline Identification code & cu_0767 \\
\hline Empirical formula & $\mathrm{C}_{15} \mathrm{H}_{19} \mathrm{NO}_{3} \mathrm{~S}$ \\
\hline Formula weight & 293.37 \\
\hline Temperature/K & $100(2)$ \\
\hline Crystal system & monoclinic \\
\hline Space group & $\mathrm{P} 2_{1}$ \\
\hline $\mathrm{a} / \AA$ & $8.6148(2)$ \\
\hline $\mathrm{b} / \AA$ & $14.8424(4)$ \\
\hline $\mathrm{c} / \AA$ & $10.8526(3)$ \\
\hline$\alpha /{ }^{\circ}$ & 90 \\
\hline$\beta /{ }^{\circ}$ & $95.3830(10)$ \\
\hline$\gamma /{ }^{\circ}$ & 90 \\
\hline Volume $/ \AA^{3}$ & $1381.54(6)$ \\
\hline Z & 4 \\
\hline$\rho_{\text {calc }} \mathrm{g} / \mathrm{cm}^{3}$ & 1.410 \\
\hline$\mu / \mathrm{mm}^{-1}$ & 2.147 \\
\hline $\mathrm{F}(000)$ & 624.0 \\
\hline Crystal size $/ \mathrm{mm}^{3}$ & $0.17 \times 0.15 \times 0.1$ \\
\hline Radiation & $\mathrm{CuK} \alpha(\lambda=1.54178)$ \\
\hline \multicolumn{2}{|c|}{$2 \Theta$ range for data collection $/{ }^{\circ} 8.182$ to 144.758} \\
\hline Index ranges & $-10 \leq \mathrm{h} \leq 10,-18 \leq \mathrm{k} \leq 18,-13 \leq 1 \leq 13$ \\
\hline Reflections collected & 18690 \\
\hline Independent reflections & $5193\left[\mathrm{R}_{\mathrm{int}}=0.0371, \mathrm{R}_{\mathrm{sigma}}=0.0440\right]$ \\
\hline Data/restraints/parameters & $5193 / 1 / 363$ \\
\hline Goodness-of-fit on $\mathrm{F}^{2}$ & 1.047 \\
\hline Final $\mathrm{R}$ indexes $[\mathrm{I}>=2 \sigma(\mathrm{I})]$ & $\mathrm{R}_{1}=0.0319, \mathrm{wR}_{2}=0.0786$ \\
\hline Final $R$ indexes [all data] & $\mathrm{R}_{1}=0.0339, \mathrm{wR}_{2}=0.0798$ \\
\hline
\end{tabular}


Largest diff. peak/hole / e $\AA^{-3} 0.35 /-0.27$

Flack parameter $\quad 0.066(8)$

\section{References}

(1) Comito, R. J.; Finelli, F. G.; MacMillan, D. W. C. J. Am. Chem. Soc. 2013, 135, 9358.

(2) Crespin, L. N. S.; Greb, A.; Blakemore, D. C.; Ley, S. V. J. Org. Chem. 2017, 82, 13093.

(3) Torres, Ò.; Roglans, A.; Pla-Quintana, A. Adv. Synth. Catal. 2016, 358, 3512.

(4) Kim-Lee, S.-H.; Alonso, I.; Mauleón, P.; Arrayás, R. G.; Carretero, J. C. ACS Cat. 2018, 8, 8993.

(5) Yudasaka, M.; Shimbo, D.; Maruyama, T.; Tada, N.; Itoh, A. Org. Lett. 2019, 21, 1098.

(6) Deng, L.; Jin, L.; Dong, G. Angew. Chem. Int. Ed. 2018, 57, 2702.

(7) Ito, M.; Kawasaki, R.; Kanyiva, K. S.; Shibata, T. Chem. Eur. J. 2018, $24,3721$.

(8) Gaussian 16, Revision B.01, Frisch, M. J.; Trucks, G. W.; Schlegel, H. B.; Scuseria, G. E.; Robb, M. A.; Cheeseman, J. R.; Scalmani, G.; Barone, V.; Petersson, G. A.; Nakatsuji, H.; Li, X.; Caricato, M.; Marenich, A. V.; Bloino, J.; Janesko, B. G.; Gomperts, R.; Mennucci, B.; Hratchian, H. P.; Ortiz, J. V.; Izmaylov, A. F.; Sonnenberg, J. L.; Williams-Young, D.; Ding, F.; Lipparini, F.; Egidi, F.; Goings, J.; Peng, B.; Petrone, A.; Henderson, T.; Ranasinghe, D.; Zakrzewski, V. G.; Gao, J.; Rega, N.; Zheng, G.; Liang, W.; Hada, M.; Ehara, M.; Toyota, K.; Fukuda, R.; Hasegawa, J.; Ishida, M.; Nakajima, T.; Honda, Y.; Kitao, O.; Nakai, H.; Vreven, T.; Throssell, K.; Montgomery, J. A., Jr.; Peralta, J. E.; Ogliaro, F.; Bearpark, M. J.; Heyd, J. J.; Brothers, E. N.; Kudin, K. N.; Staroverov, V. N.; Keith, T. A.; Kobayashi, R.; Normand, J.; Raghavachari, K.; Rendell, A. P.; Burant, J. C.; Iyengar, S. S.; Tomasi, J.; Cossi, M.; Millam, J. M.; Klene, M.; Adamo, C.; Cammi, R.; Ochterski, J. W.; Martin, R. L.; Morokuma, K.; Farkas, O.; Foresman, J. B.; Fox, D. J. Gaussian, Inc., Wallingford CT, 2016.

(9) (a) Becke, A. D. J. Chem. Phys. 1993, 98, 5648. (b) Lee, C.; Yang, W.; Parr, R. G. Phys. Rev. B 1988, $37,785$.

(10) Zhao, Y.; Truhlar, D. G. Theor. Chem. Acc. 2008, 120, 215.

(11) Roy, L. E.; Hay, P. J.; Martin, R. L. J. Chem. Theory Comput. 2008, 4, 1029.

(12) Marenich, A. V.; Cramer, C. J.; Truhlar, D. G. J. Phys. Chem. B 2009, 113, 6378.

(13) Lu, G.; Liu, R. Y.; Yang, Y.; Fang, C.; Lambrecht, D. S.; Buchwald, S. L.; Liu, P. J. Am. Chem. Soc. 2017, 139, 16548.

(14) Grimme, S.; Antony, J.; Ehrlich, S.; Krieg, H. J. Chem. Phys. 2010, 132, 154104.

(15) CYLview, 1.0b; Legault, C. Y., Université de Sherbrooke, 2009 (http://www.cylview.org)

(16) http://www.povray.org

(17) X-ray crystal structures reported in (a) Corkey, B. K.; Toste, F. D. J. Am. Chem. Soc. 2005, 127, 17168-17169. (b) Sevov, C. S.; Zhou, J.; Hartwig, J. F. J. Am. Chem. Soc. 2012, 134, 11960-11963. (c) Sevov, C. S.; Hartwig, J. F. J. Am. Chem. Soc. 2013, 135, 2116-2119. (d) Cirriez, V.; Rasson, C.; Hermant, T.; Petrignet, J.; Díaz Álvarez, J.; Robeyns, K.; Riant, O. Angew. Chem. Int. Ed. 2013, 52, 1785-1788. (e) Xi, Y.; Hartwig, J. F. J. Am. Chem. Soc. 2017, 139, 12758-12772. (f) Kennington, S. C. D.; Taylor, A. J.; Romea, P.; Urpí, F.; Aullón, G.; Font-Bardia, M.; Ferré, L.; Rodrigalvarez, J. Org. Lett. 2019, 21, 305-309;

(18) Ribeiro, R. F.; Marenich, A. V.; Cramer, C. J.; Truhlar, D. G. J. Phys. Chem. B 2011, 115, 14556-14562 


\section{NMR Spectra}

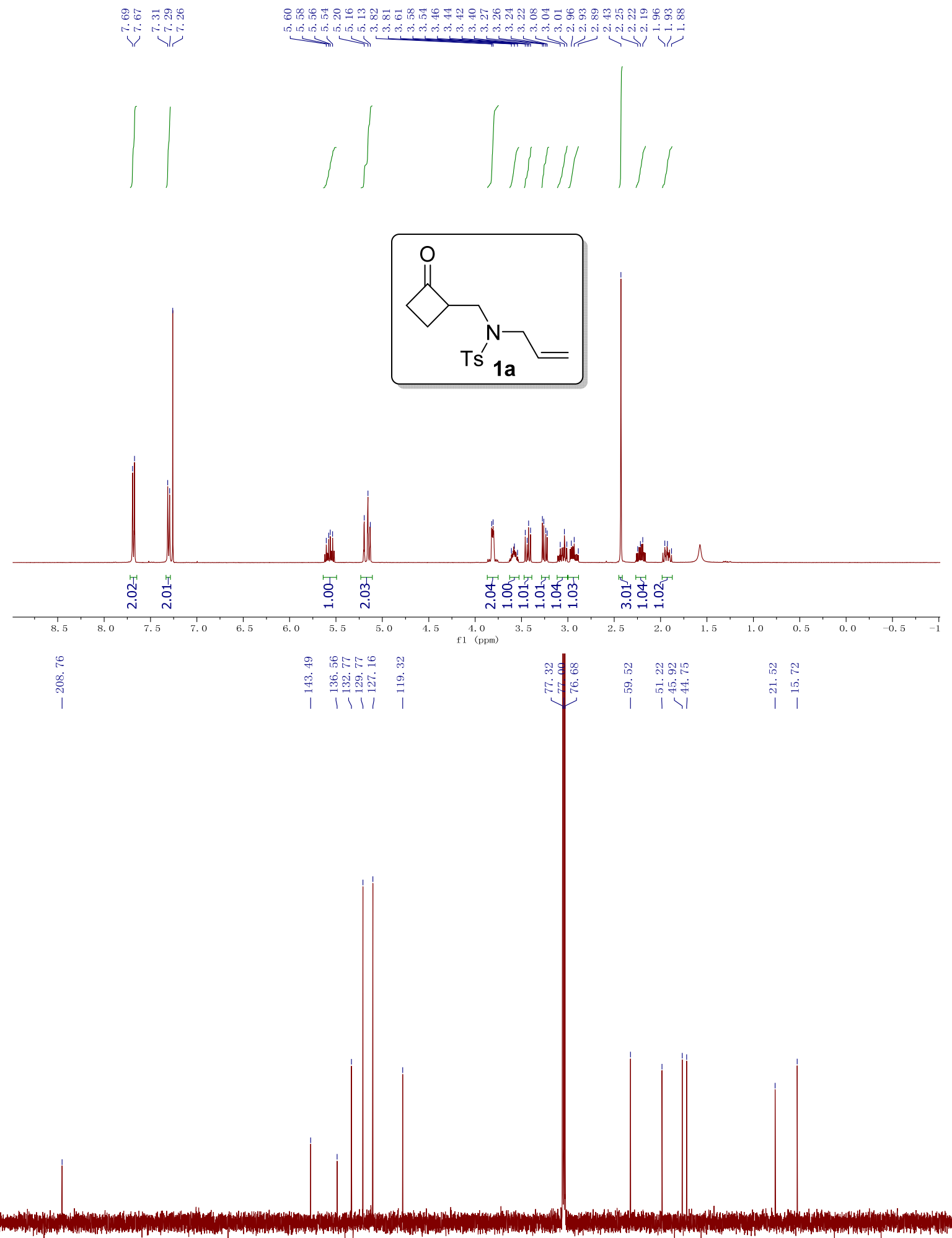

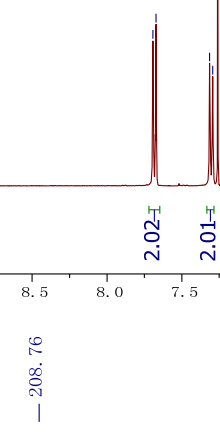

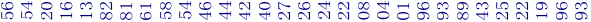



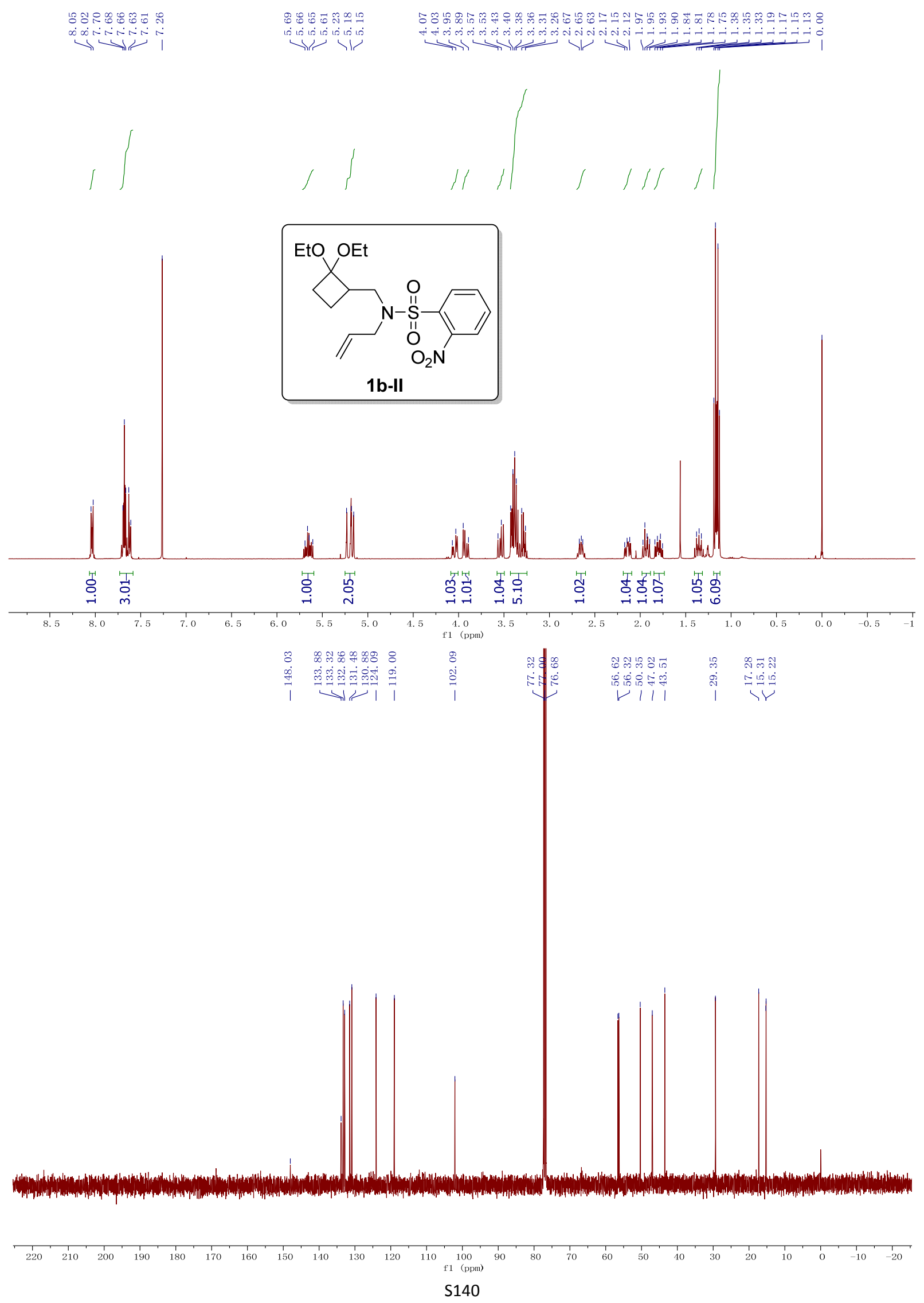

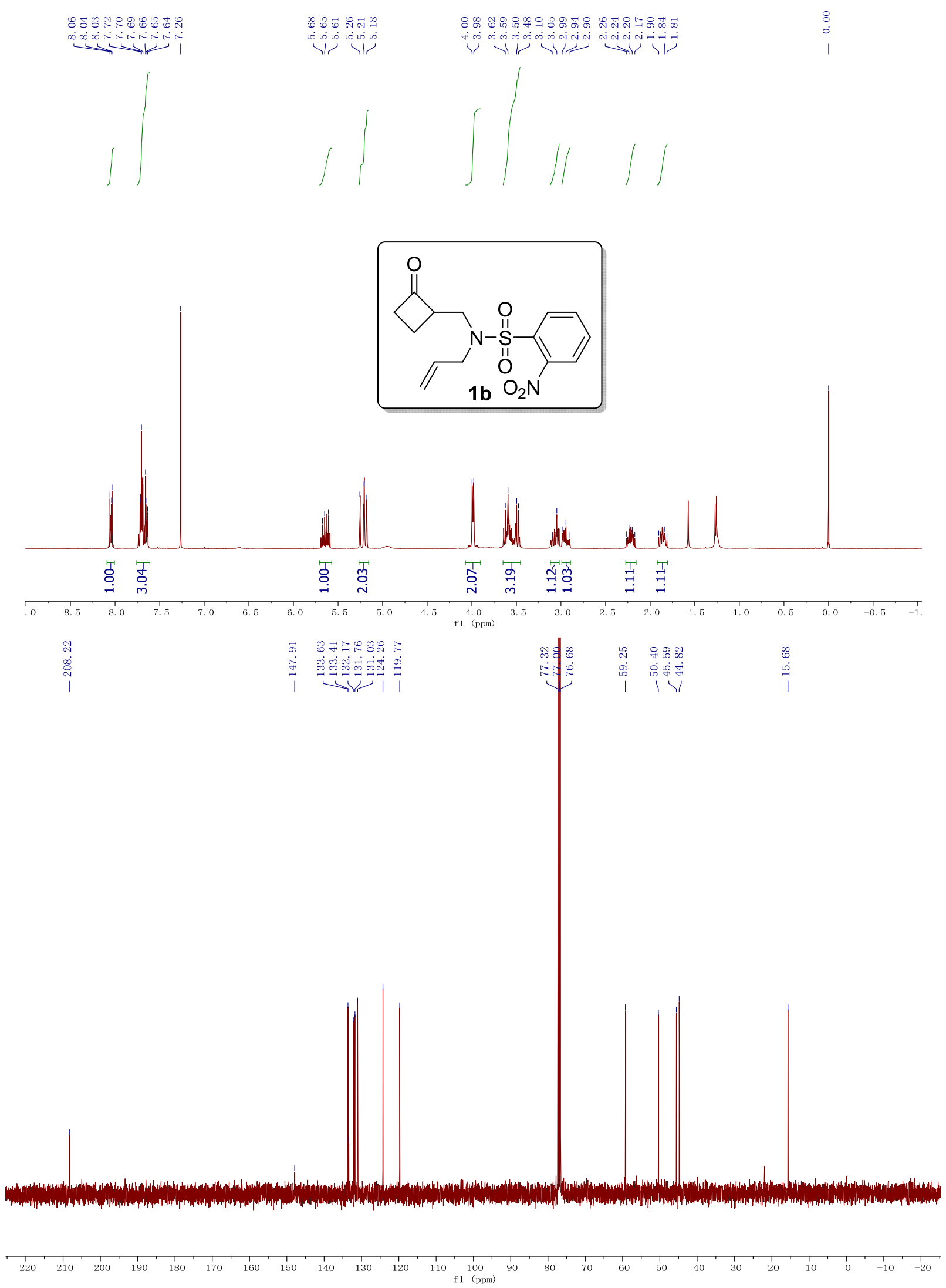


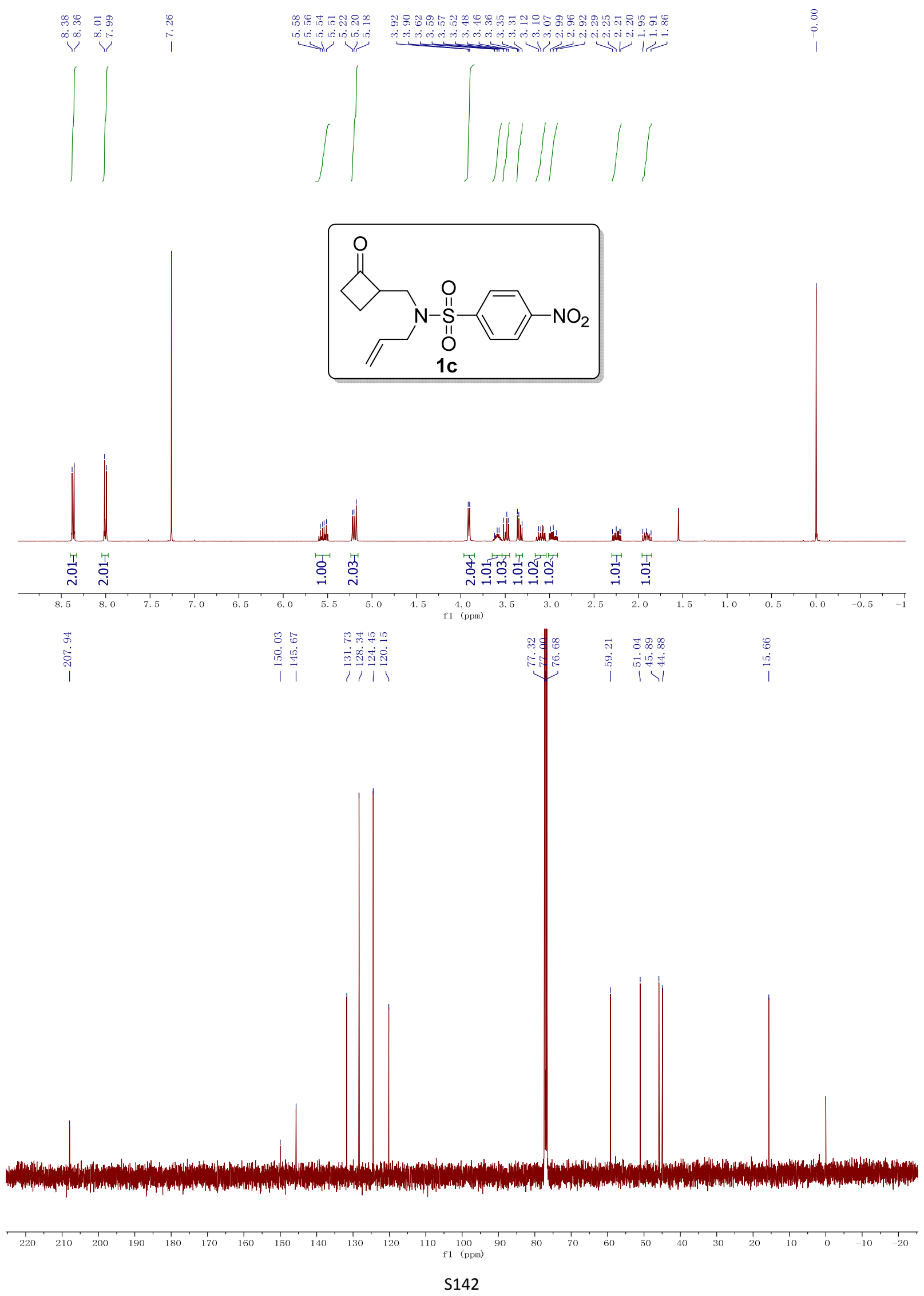




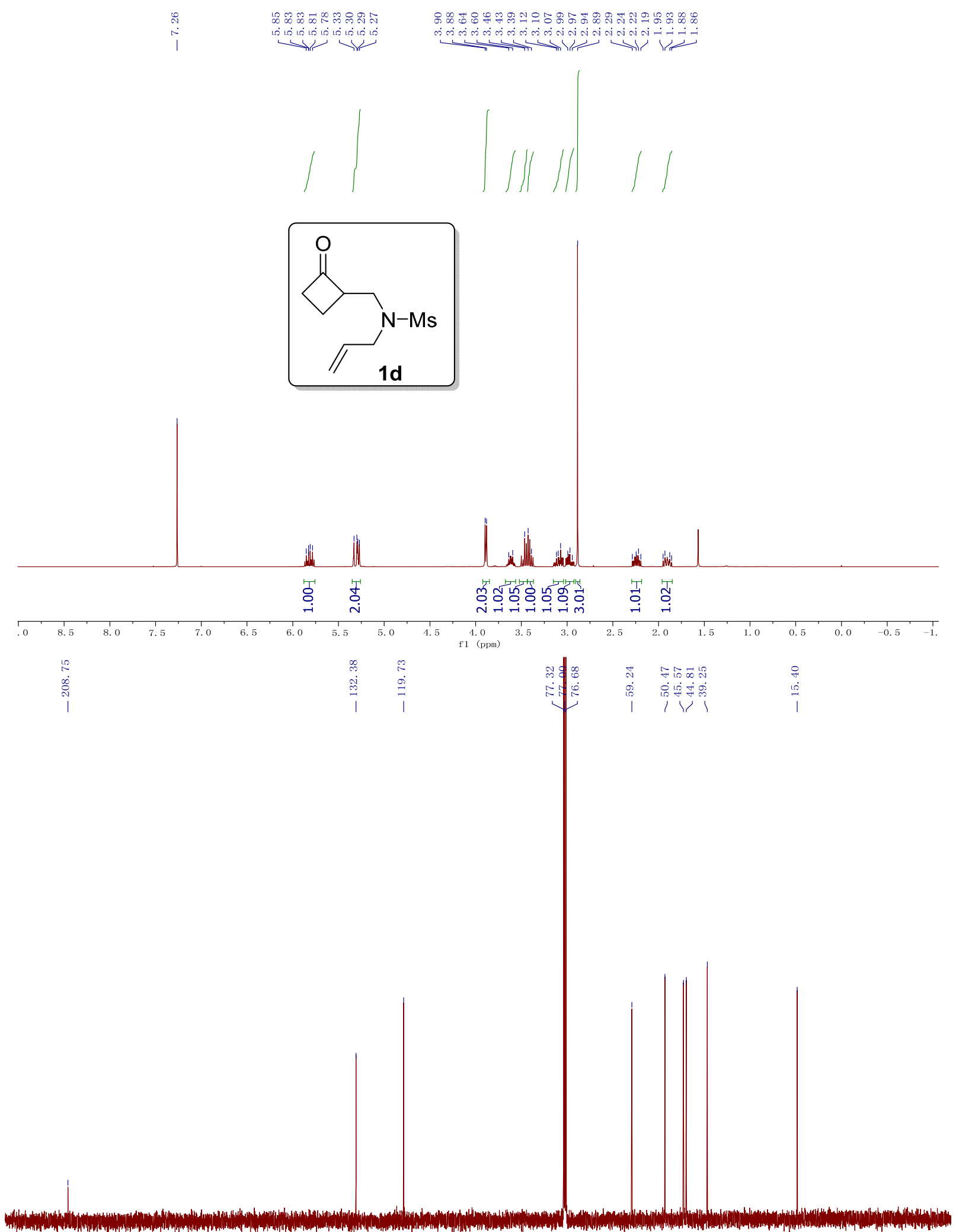

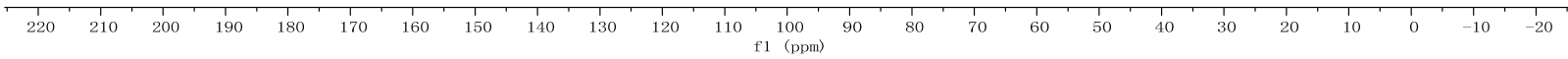
S143 

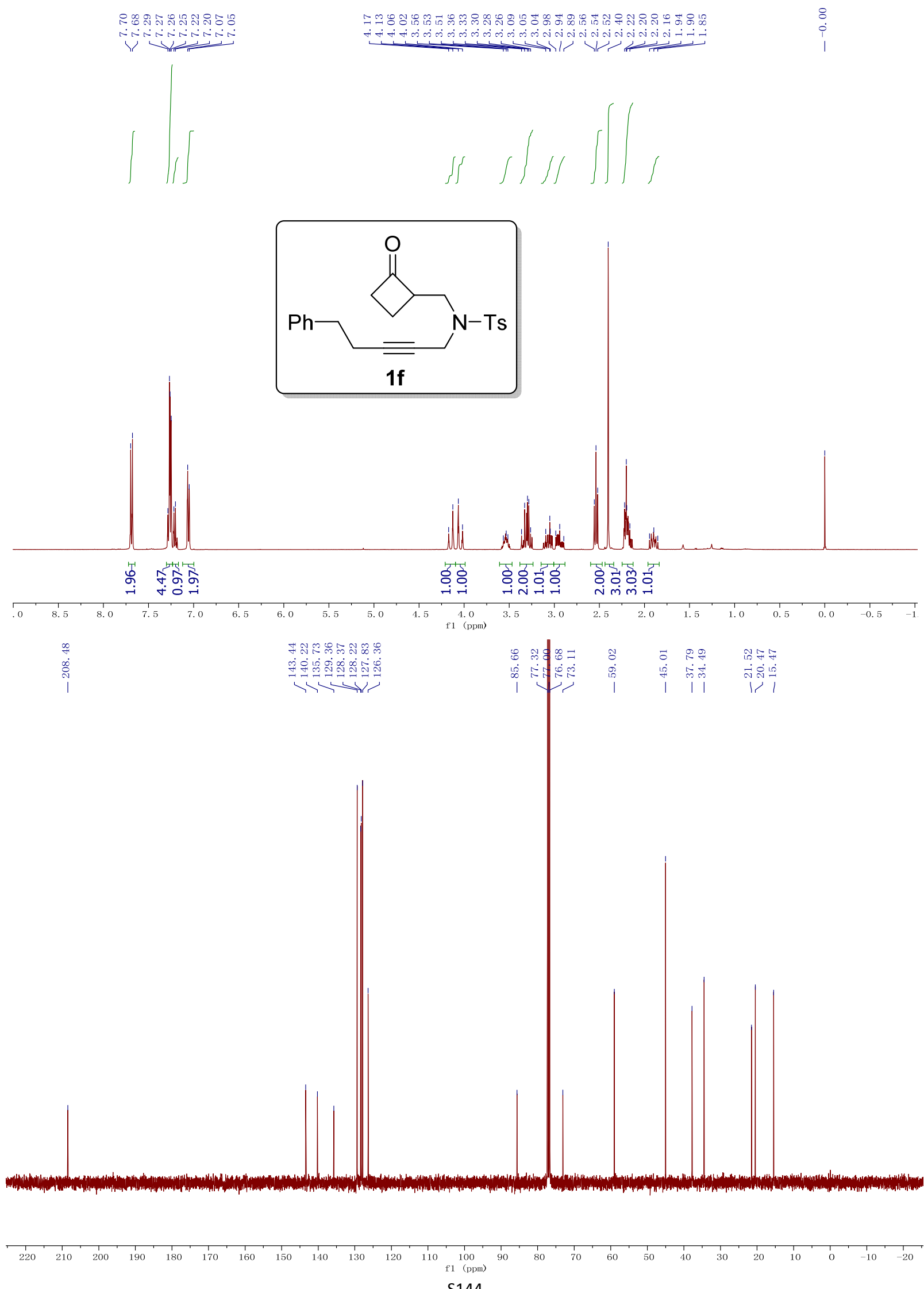

S144 

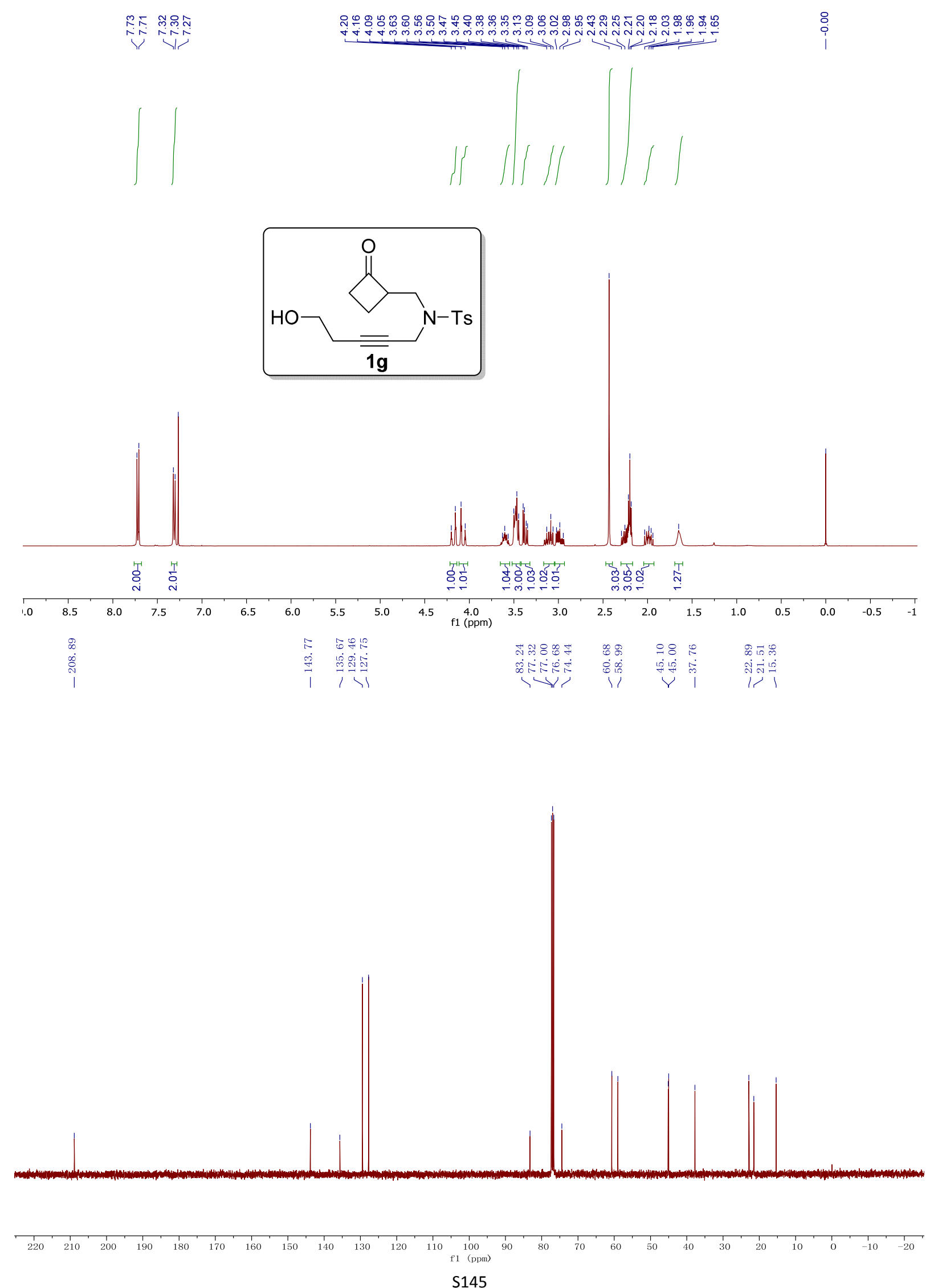

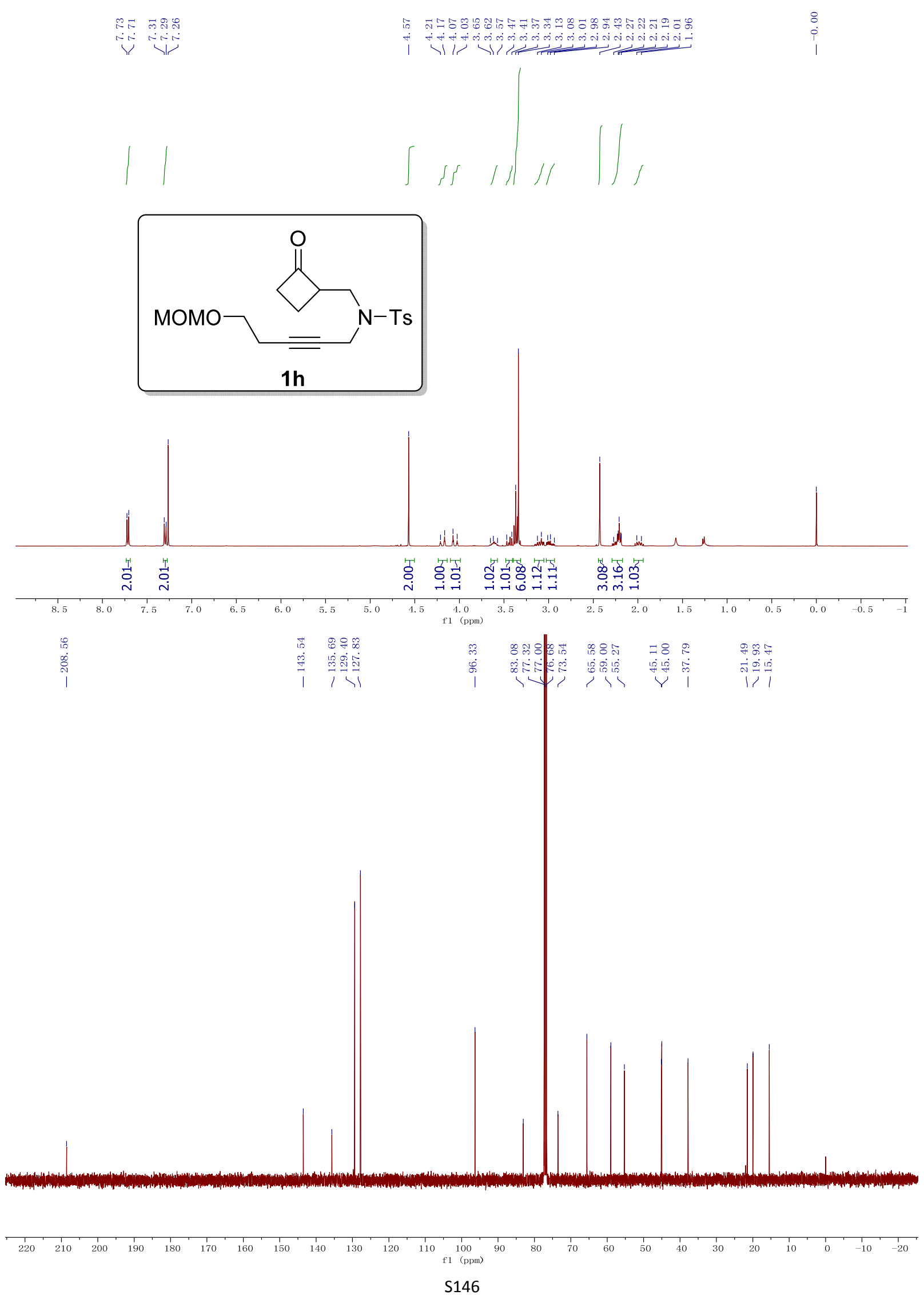

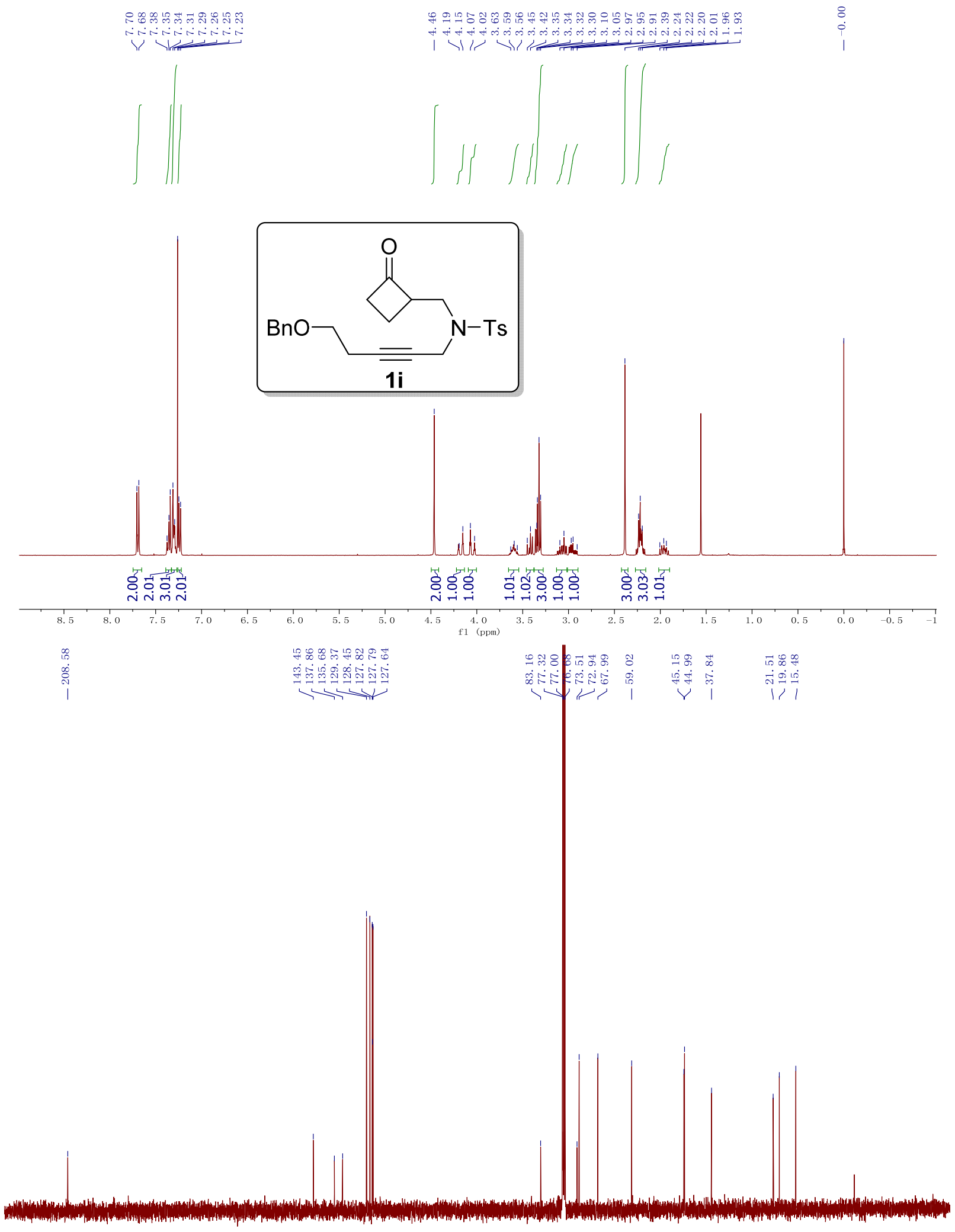

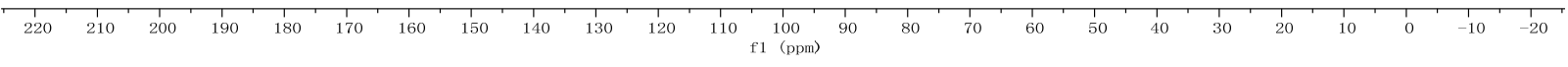




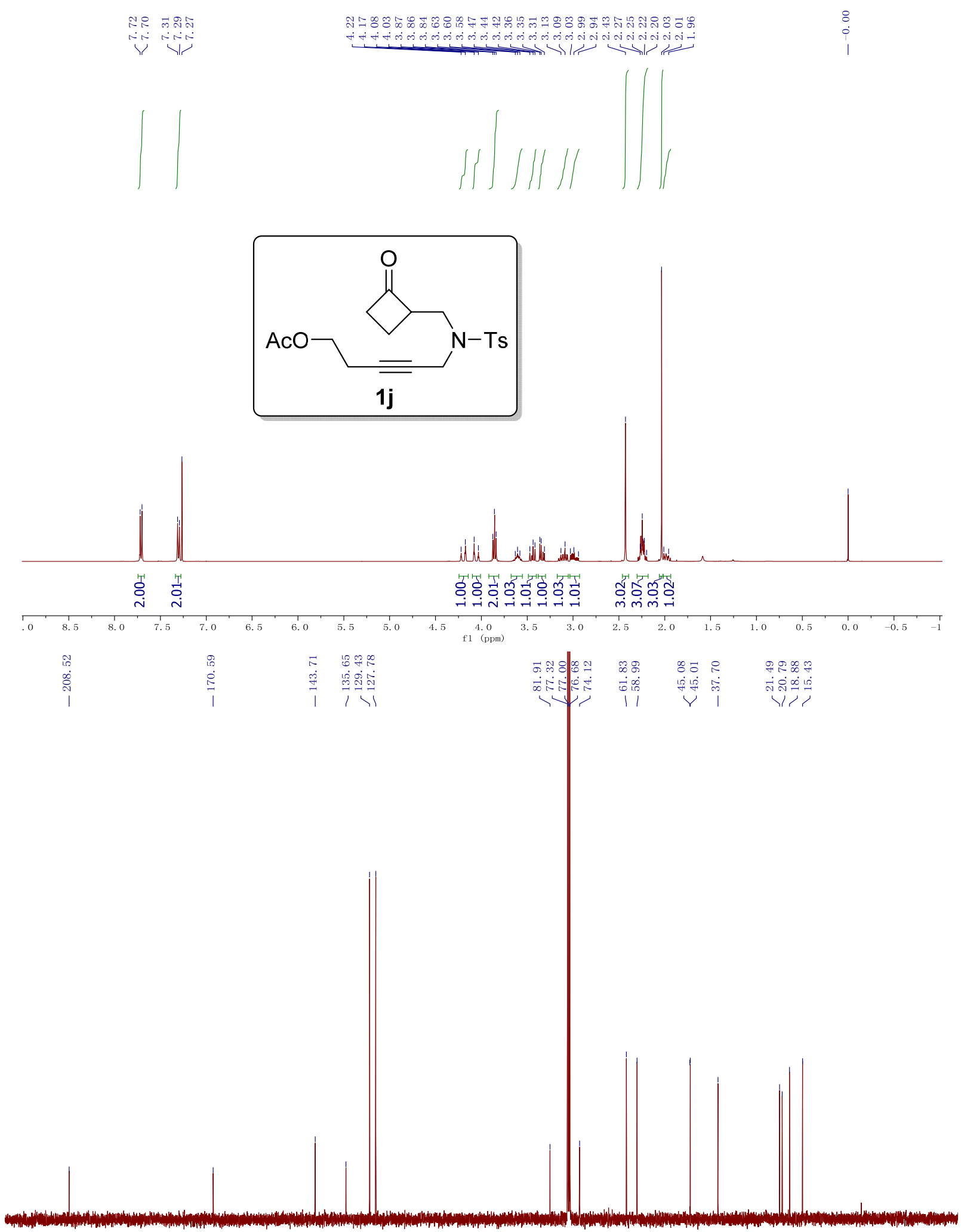

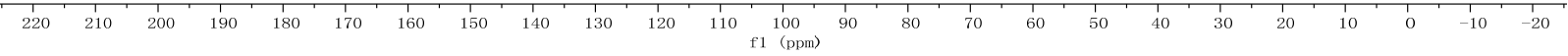



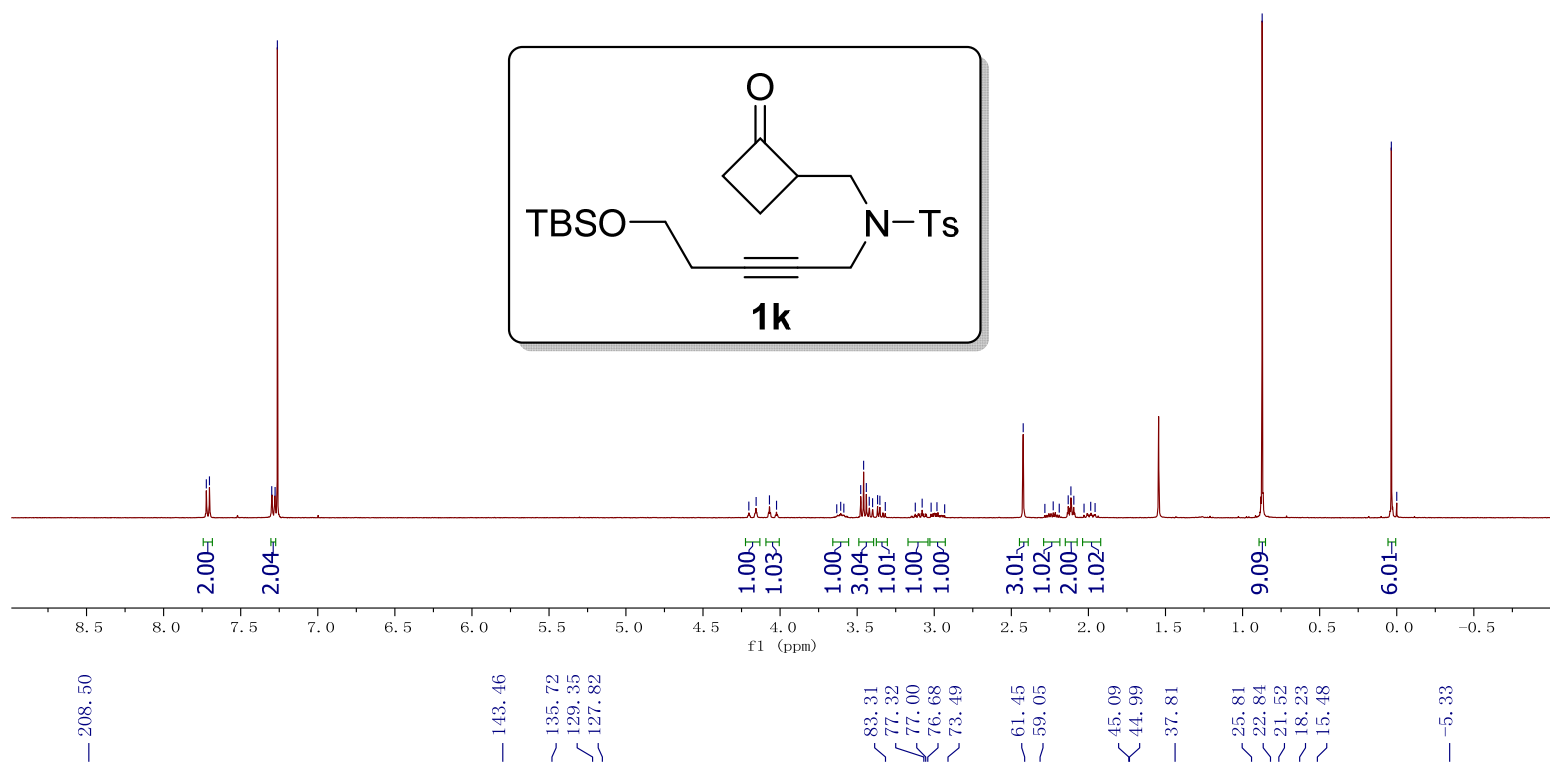

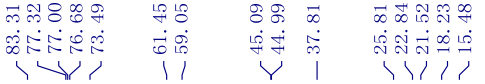

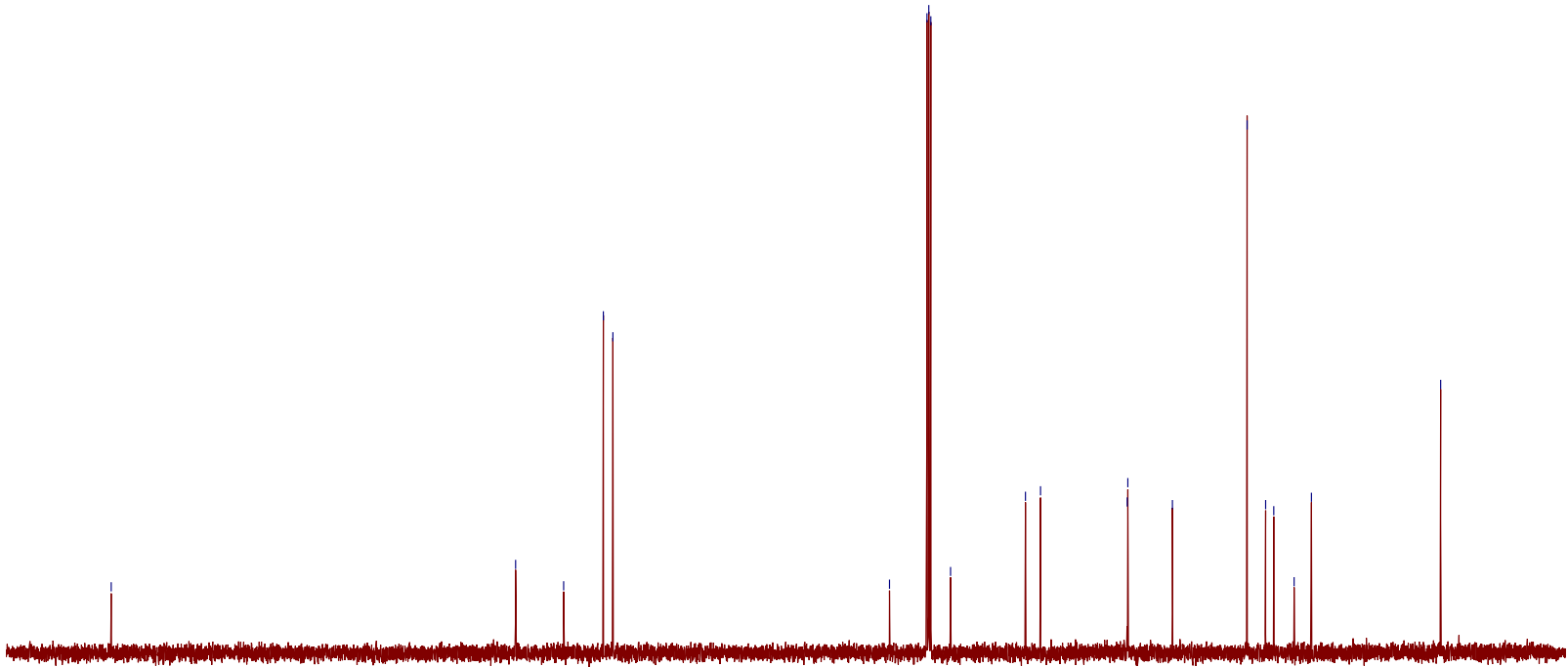

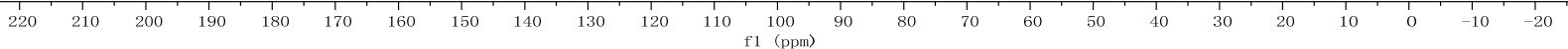


ఇㅠ

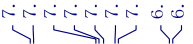

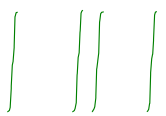

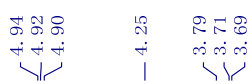

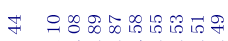

i
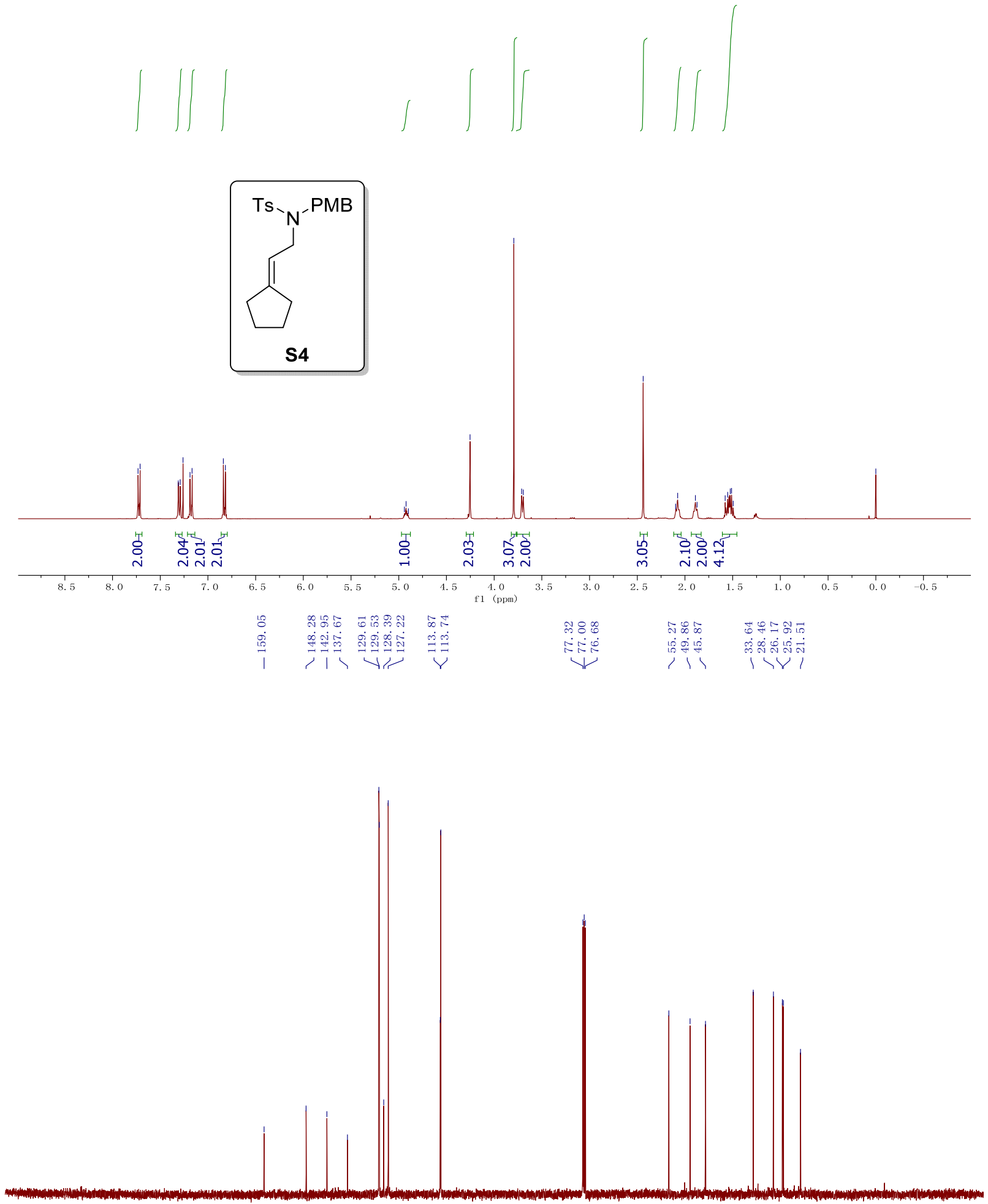

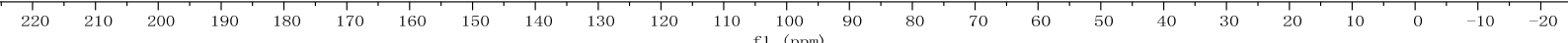
S150 


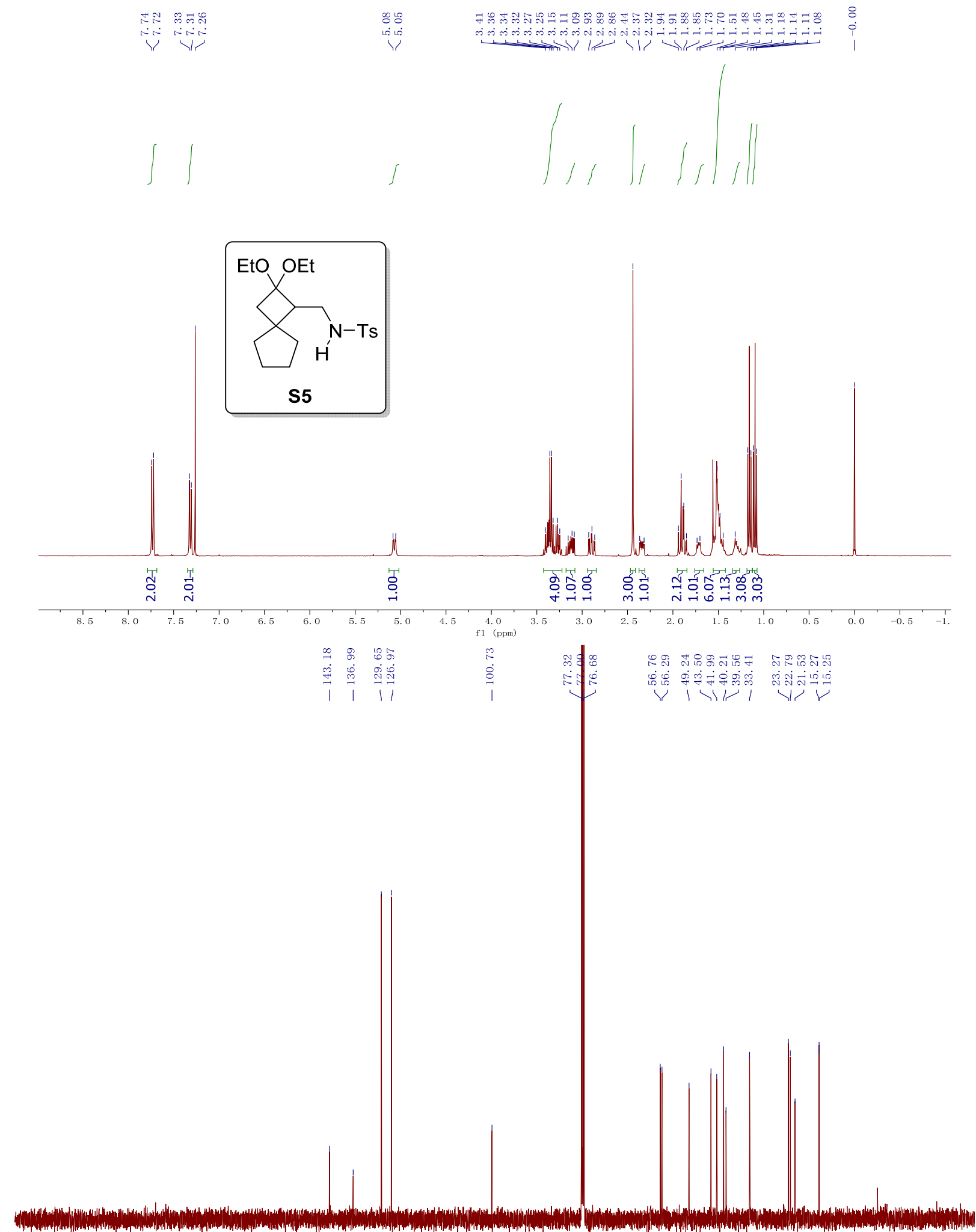

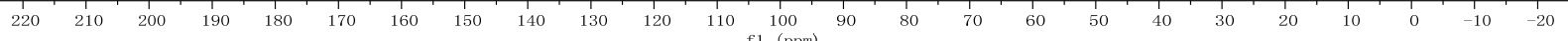

S151 


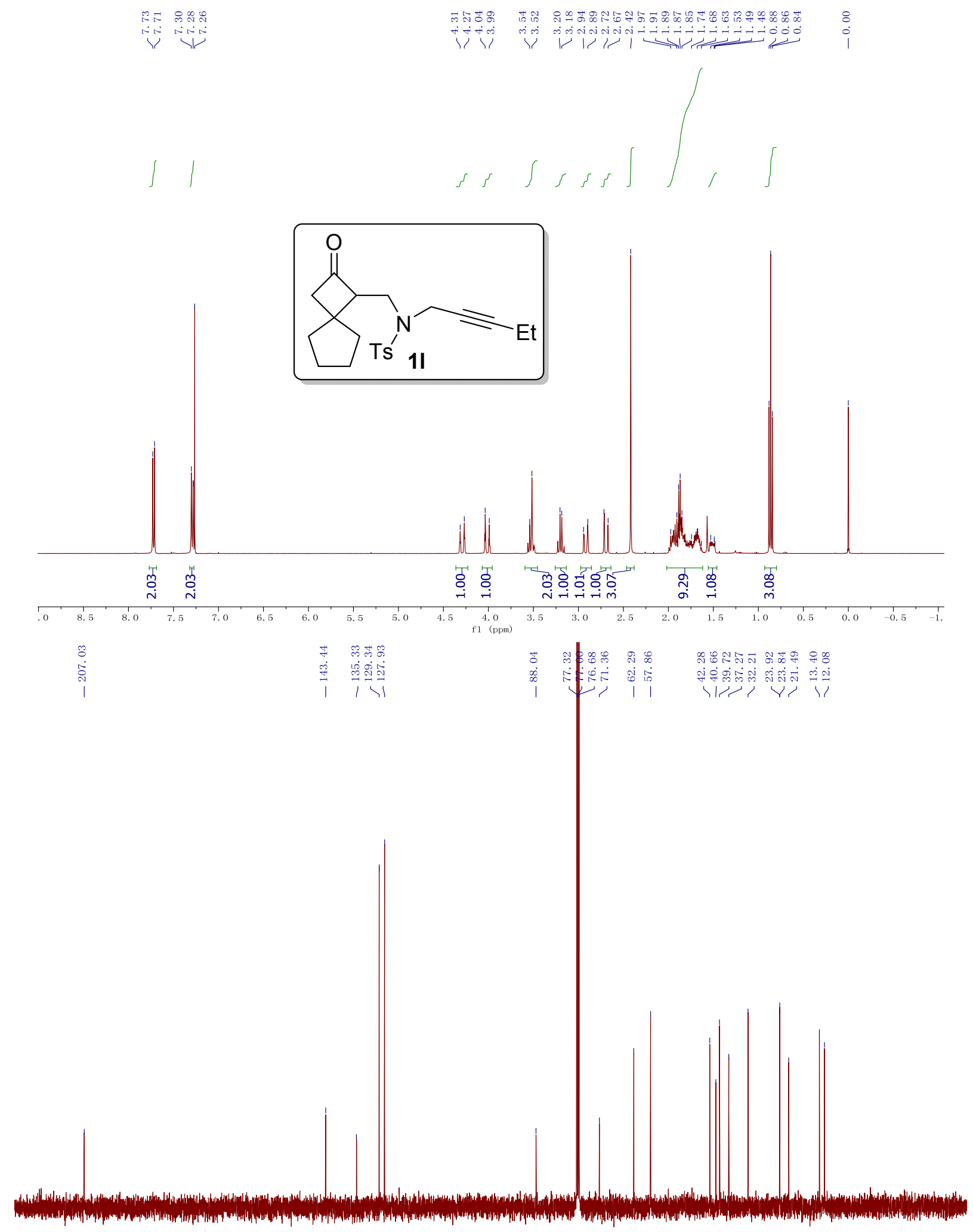

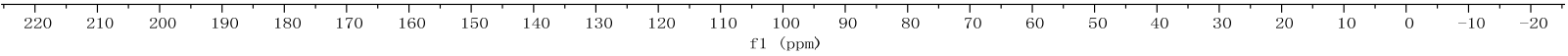



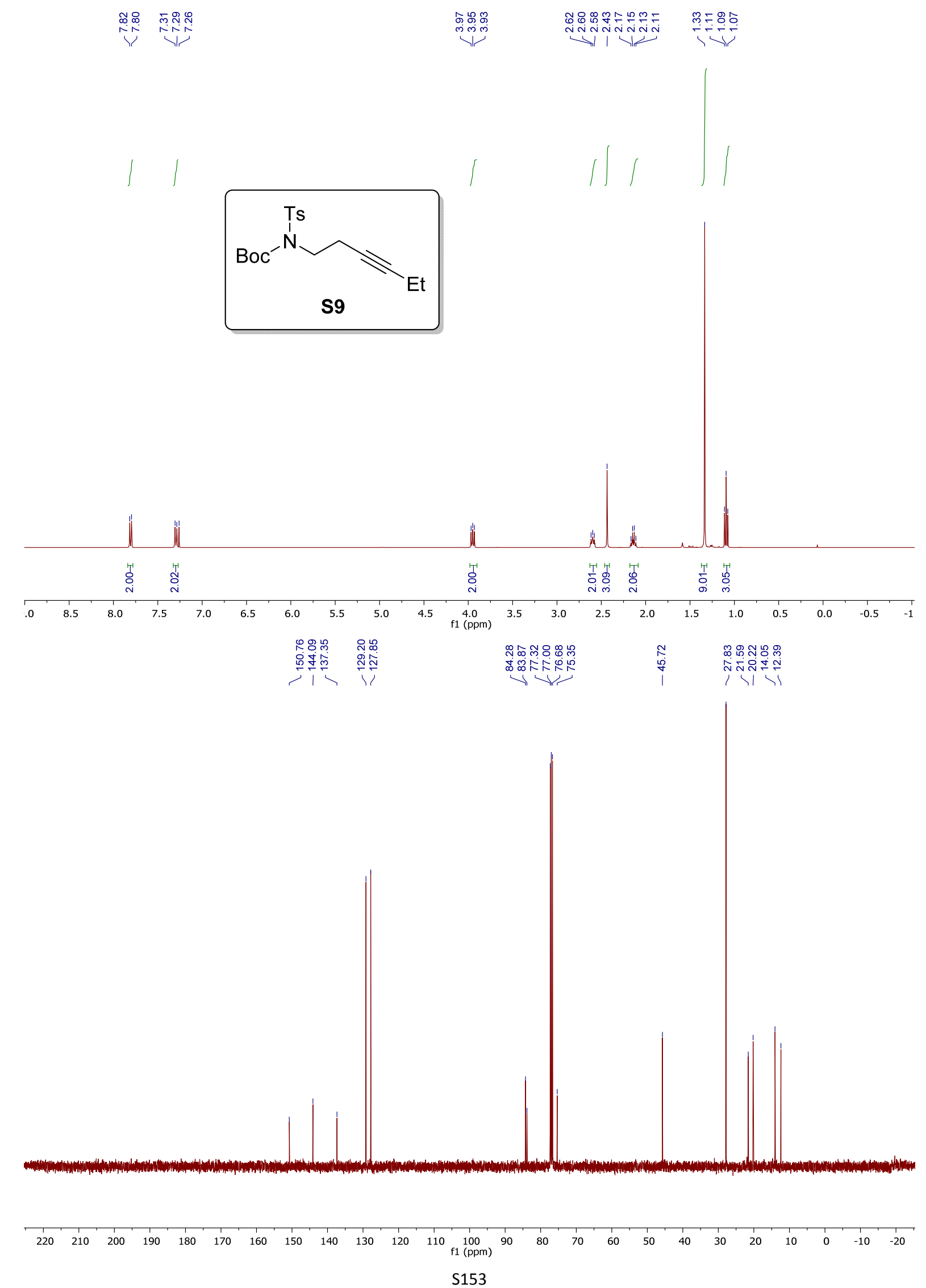

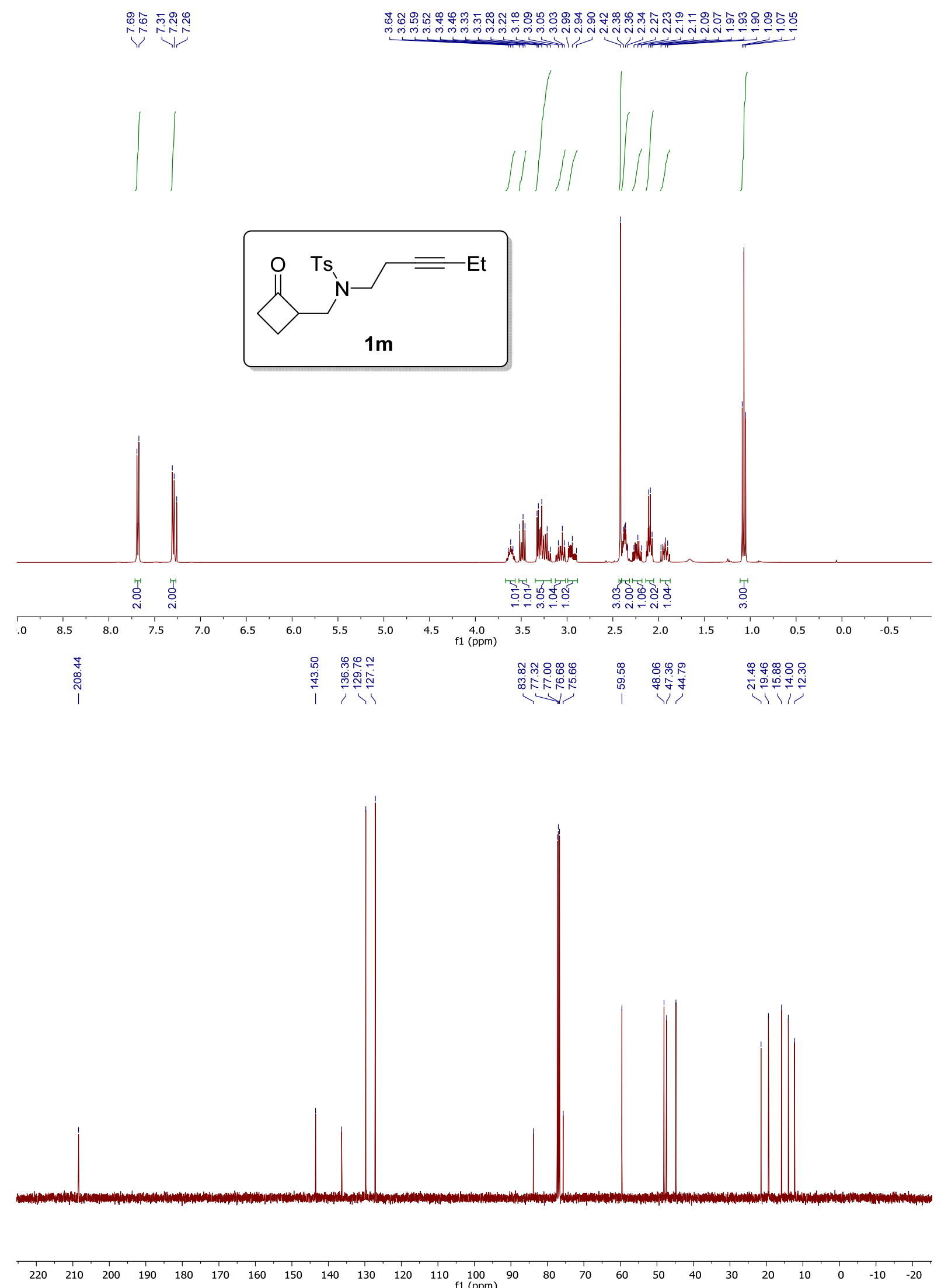

S154 


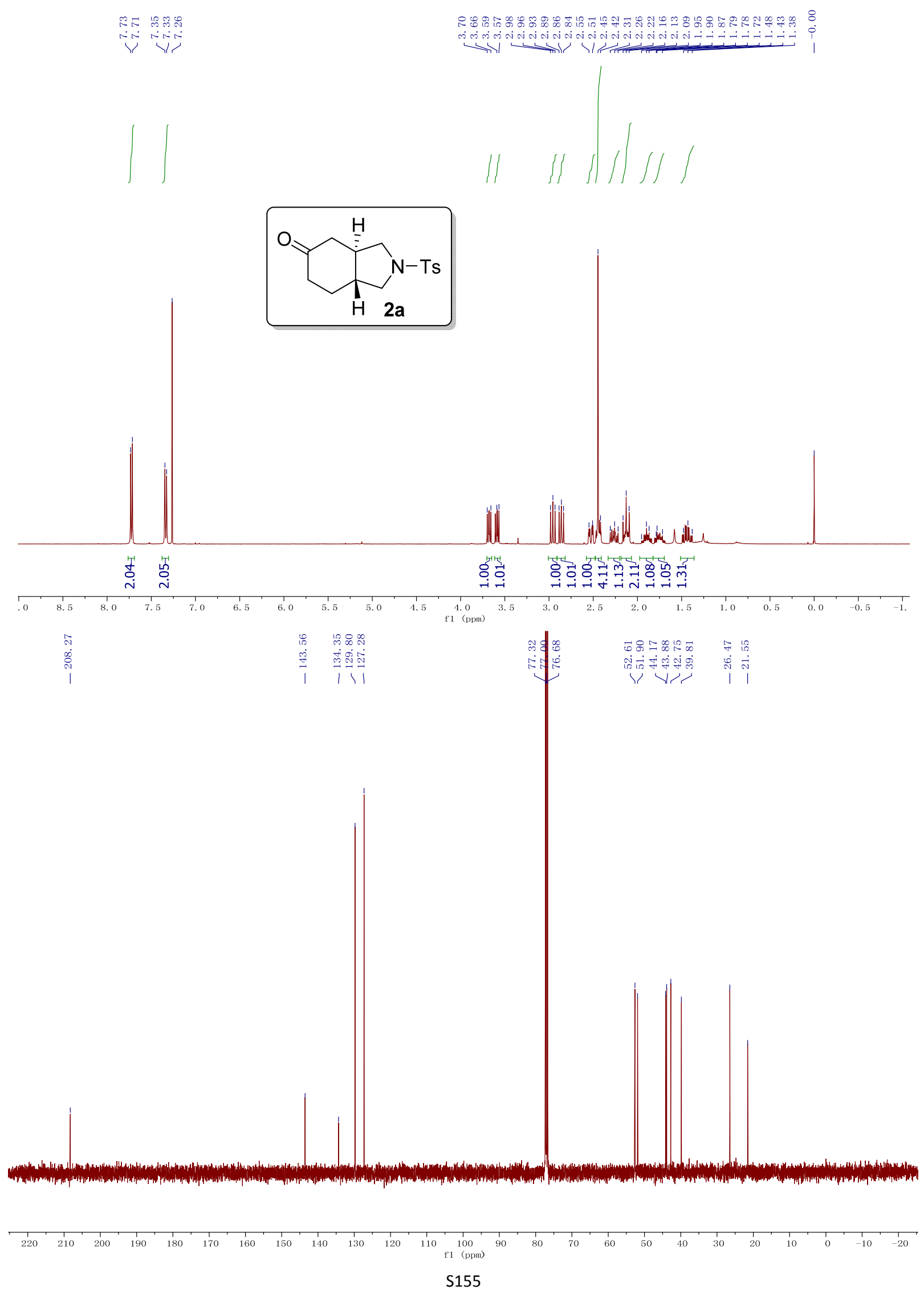



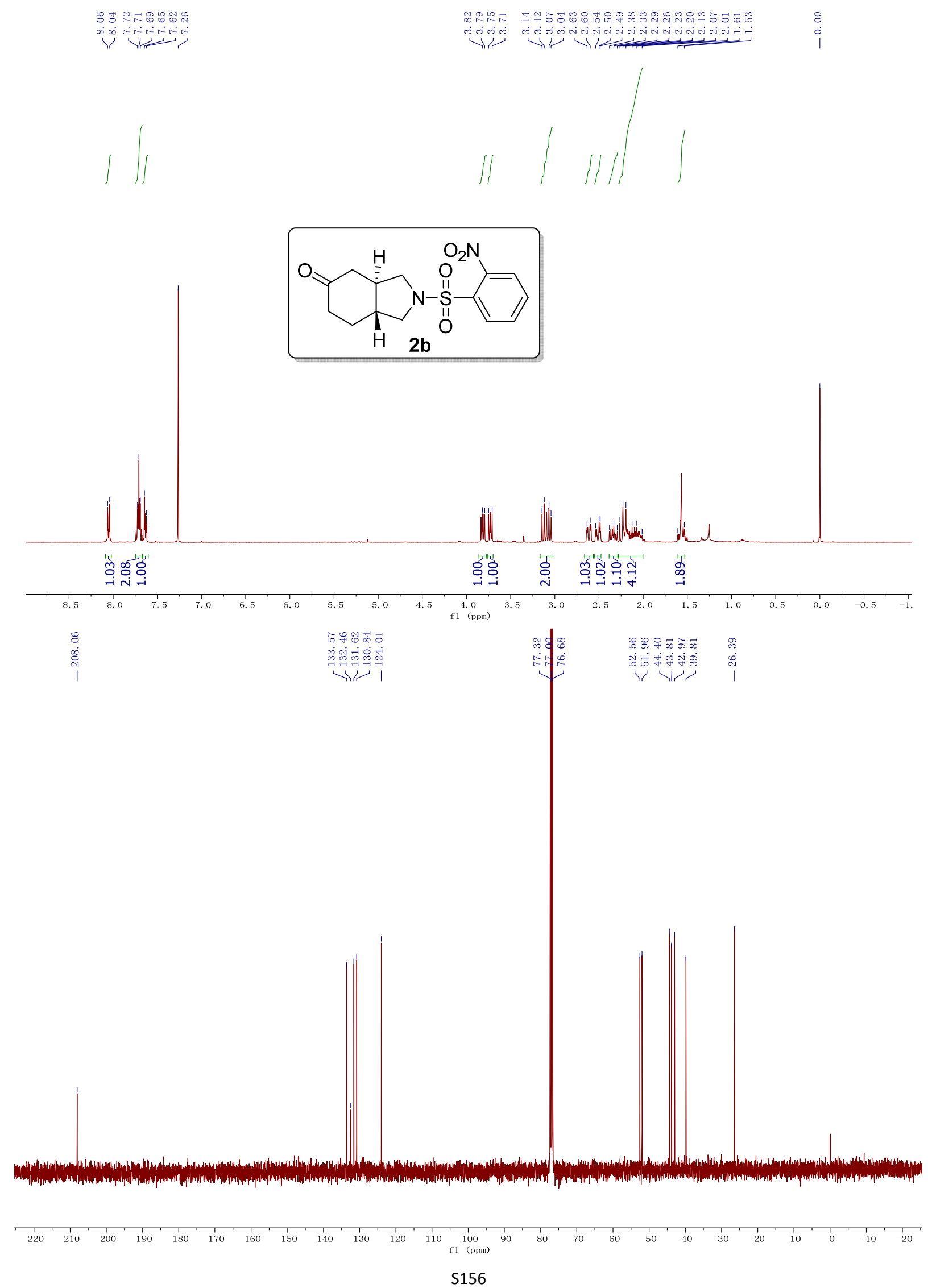

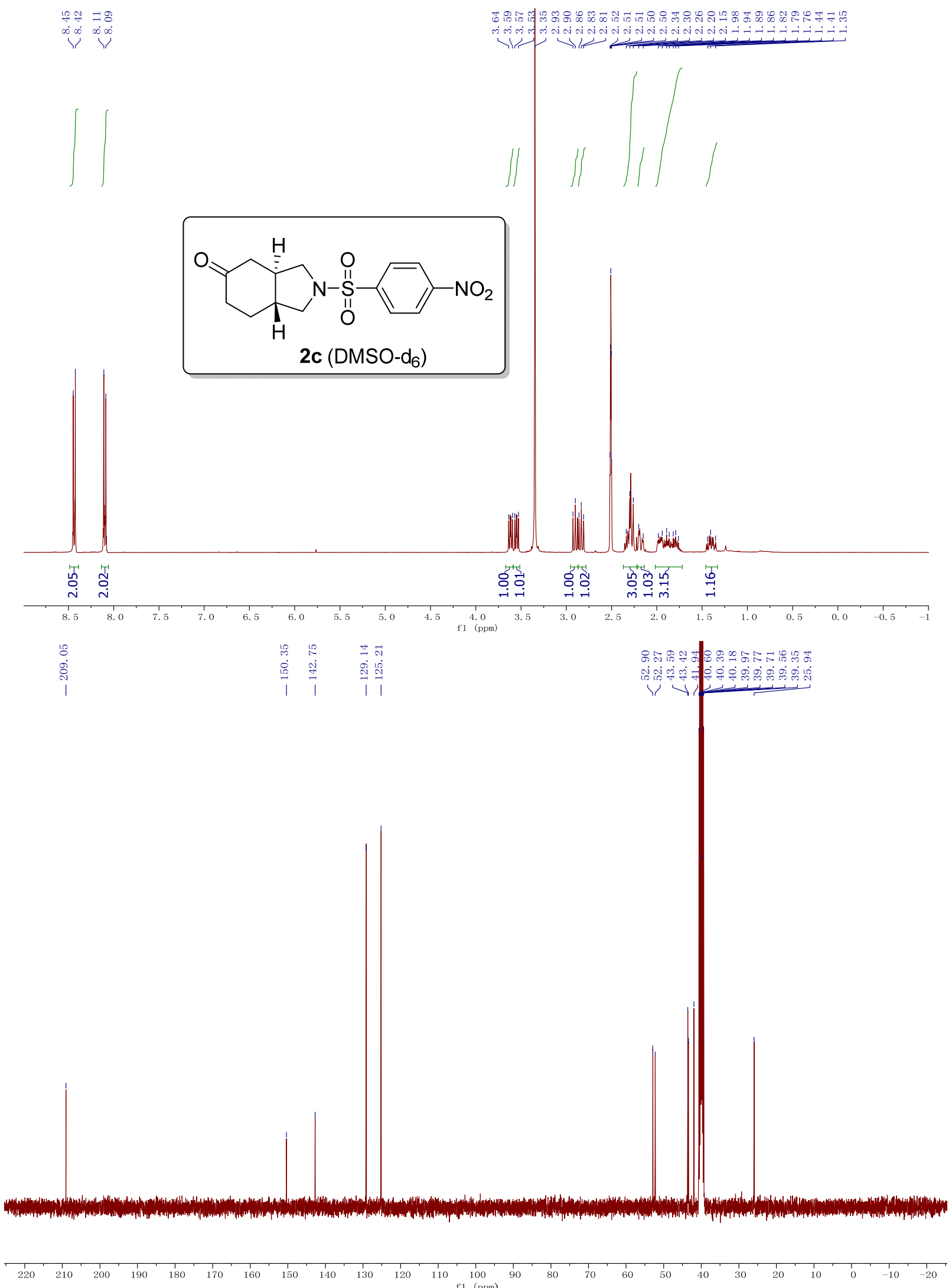

S157 

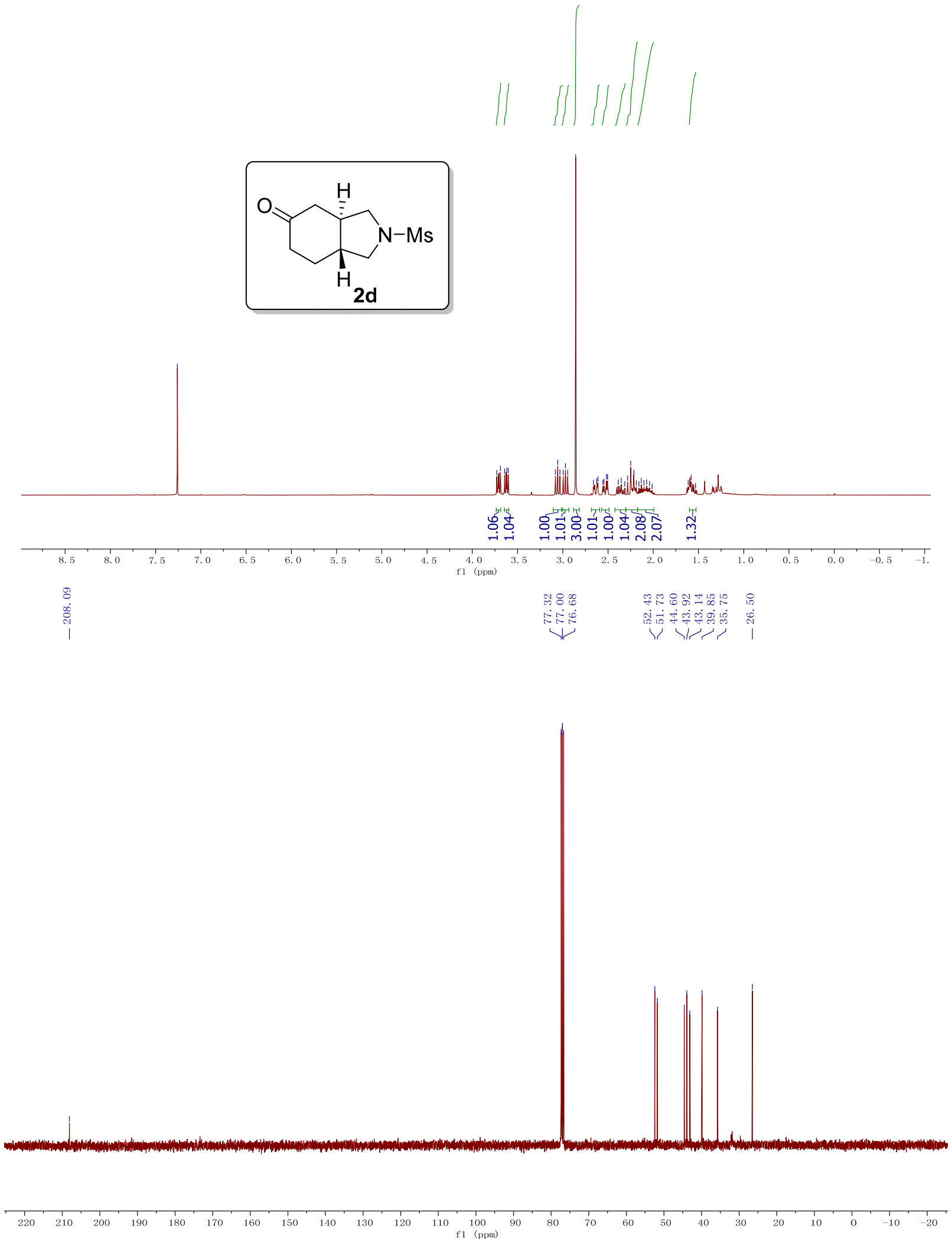


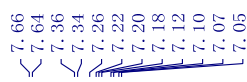

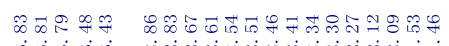

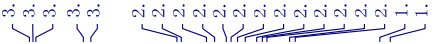

8
$i$
1
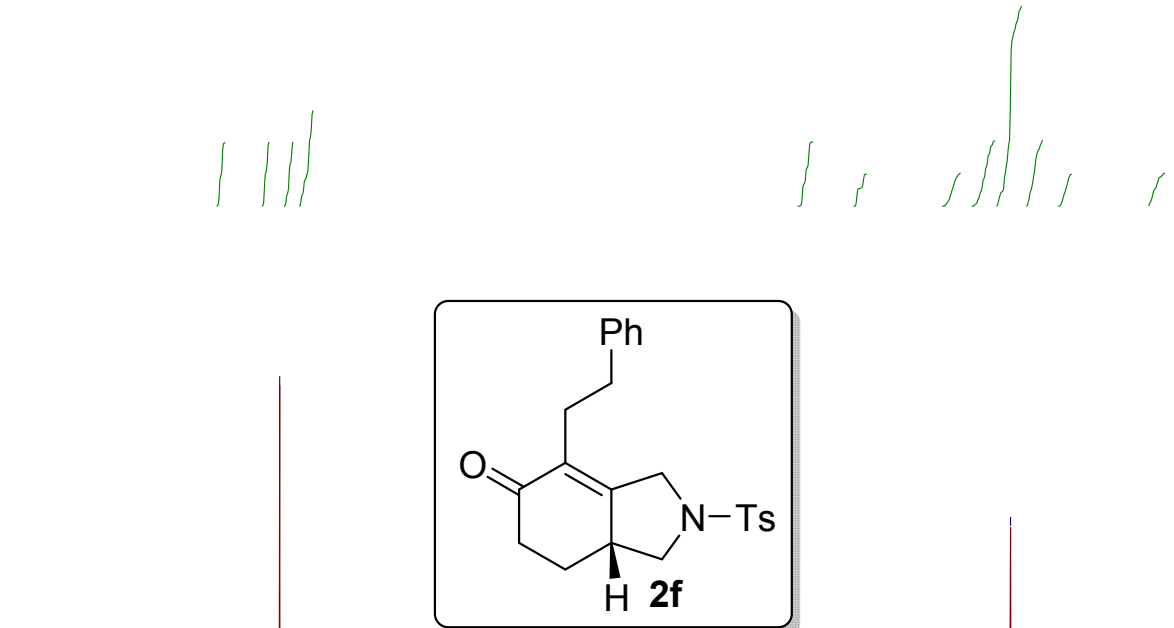

Wh

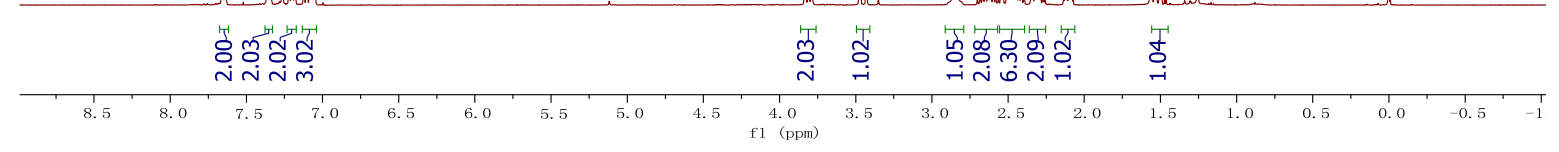

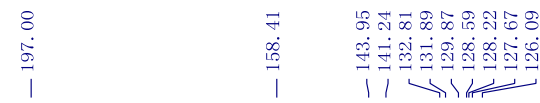

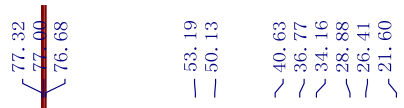

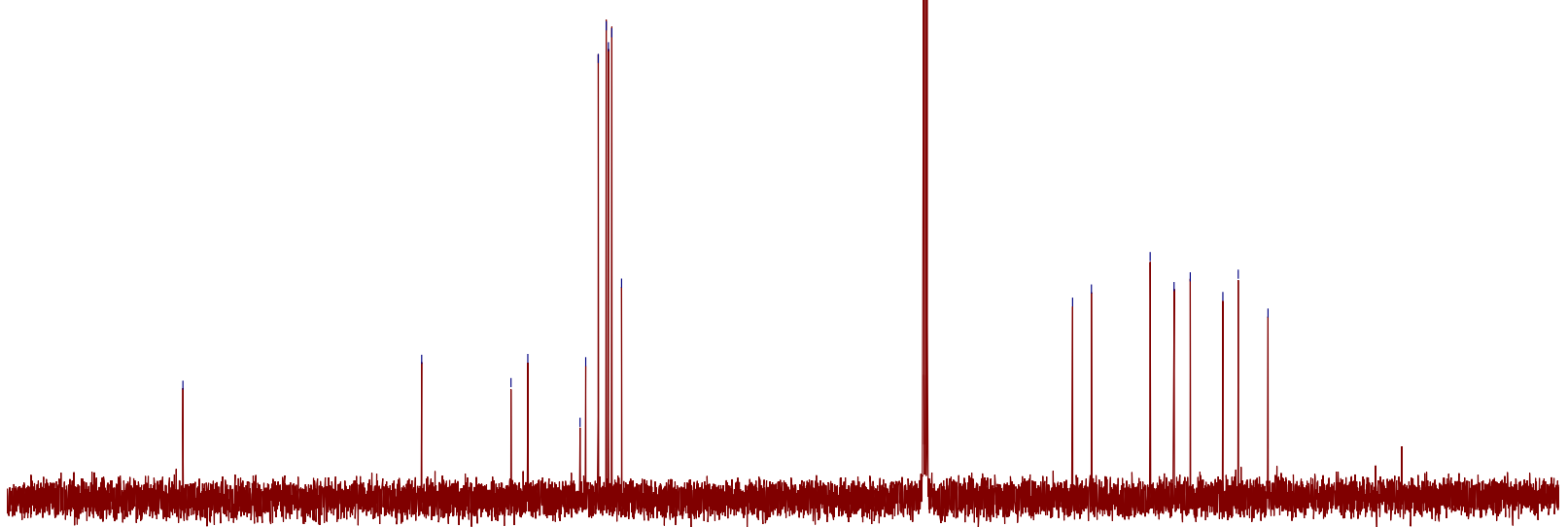

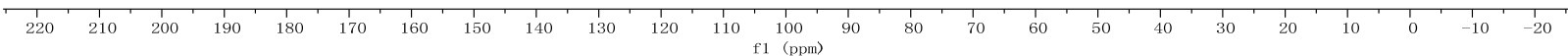



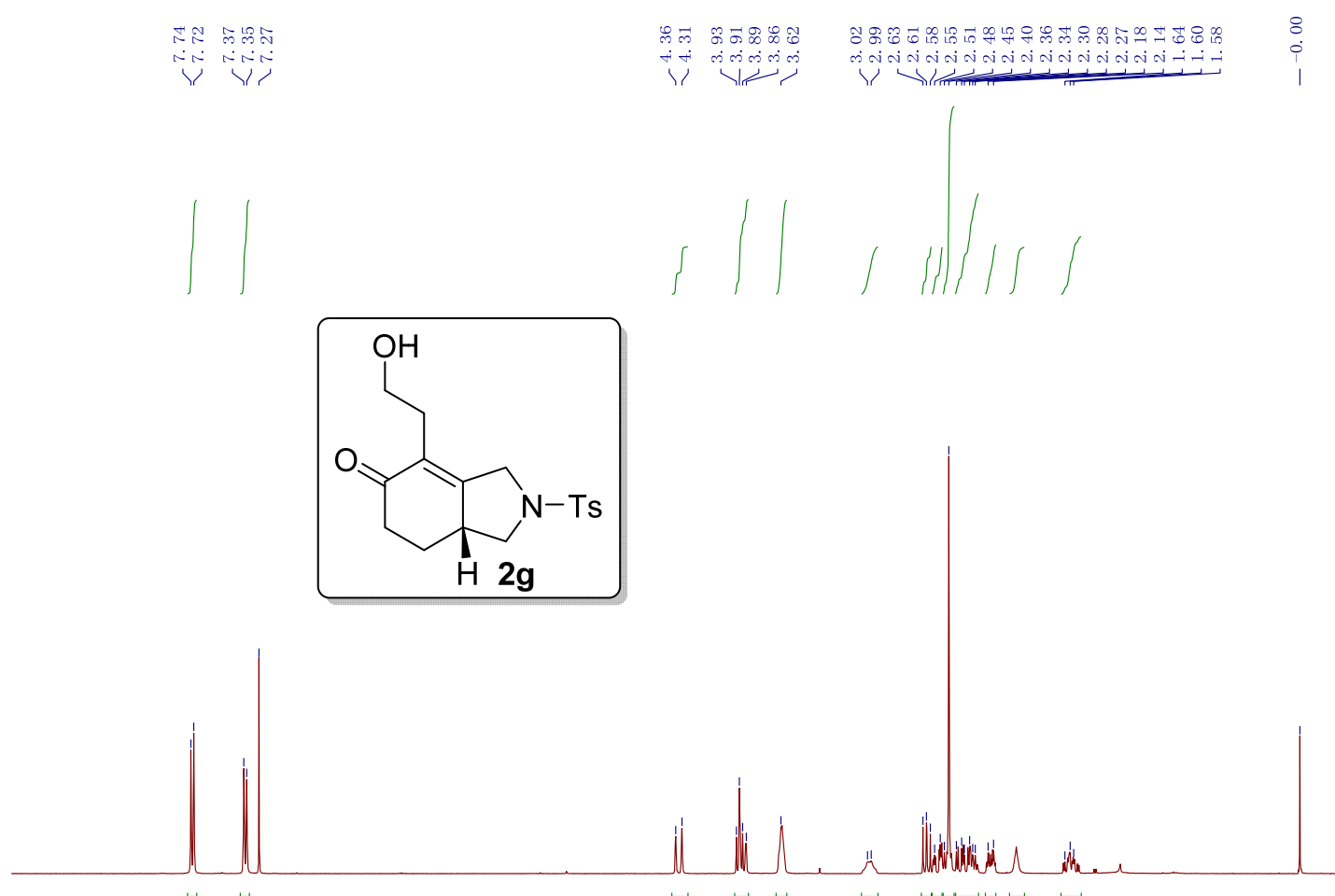

Widncher

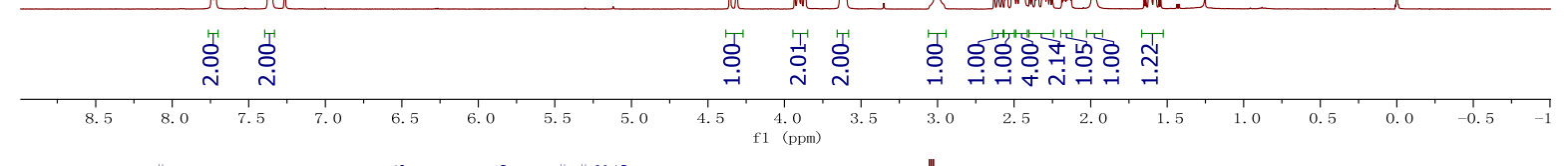

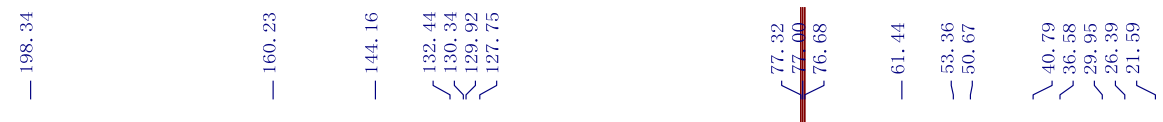

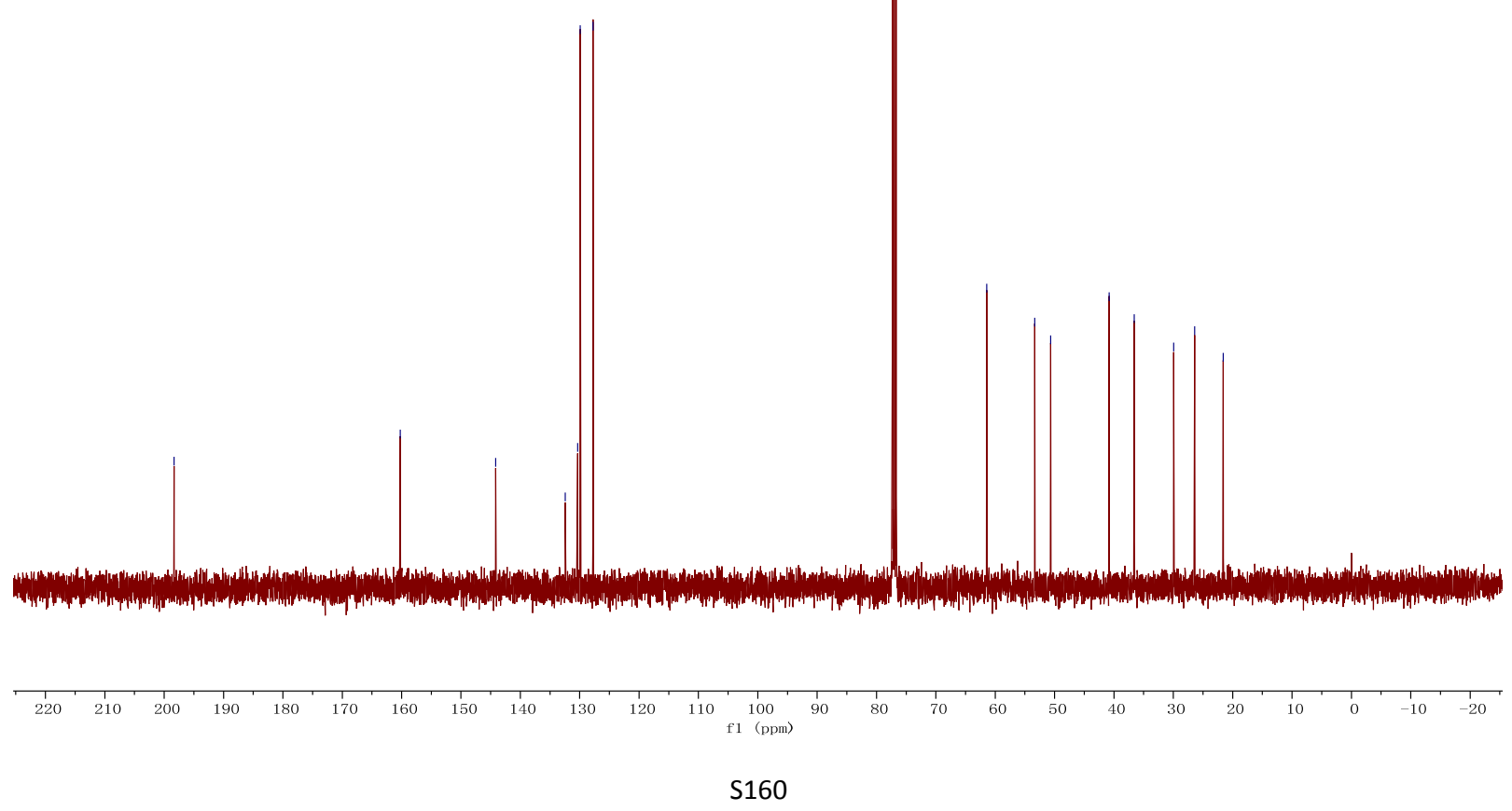



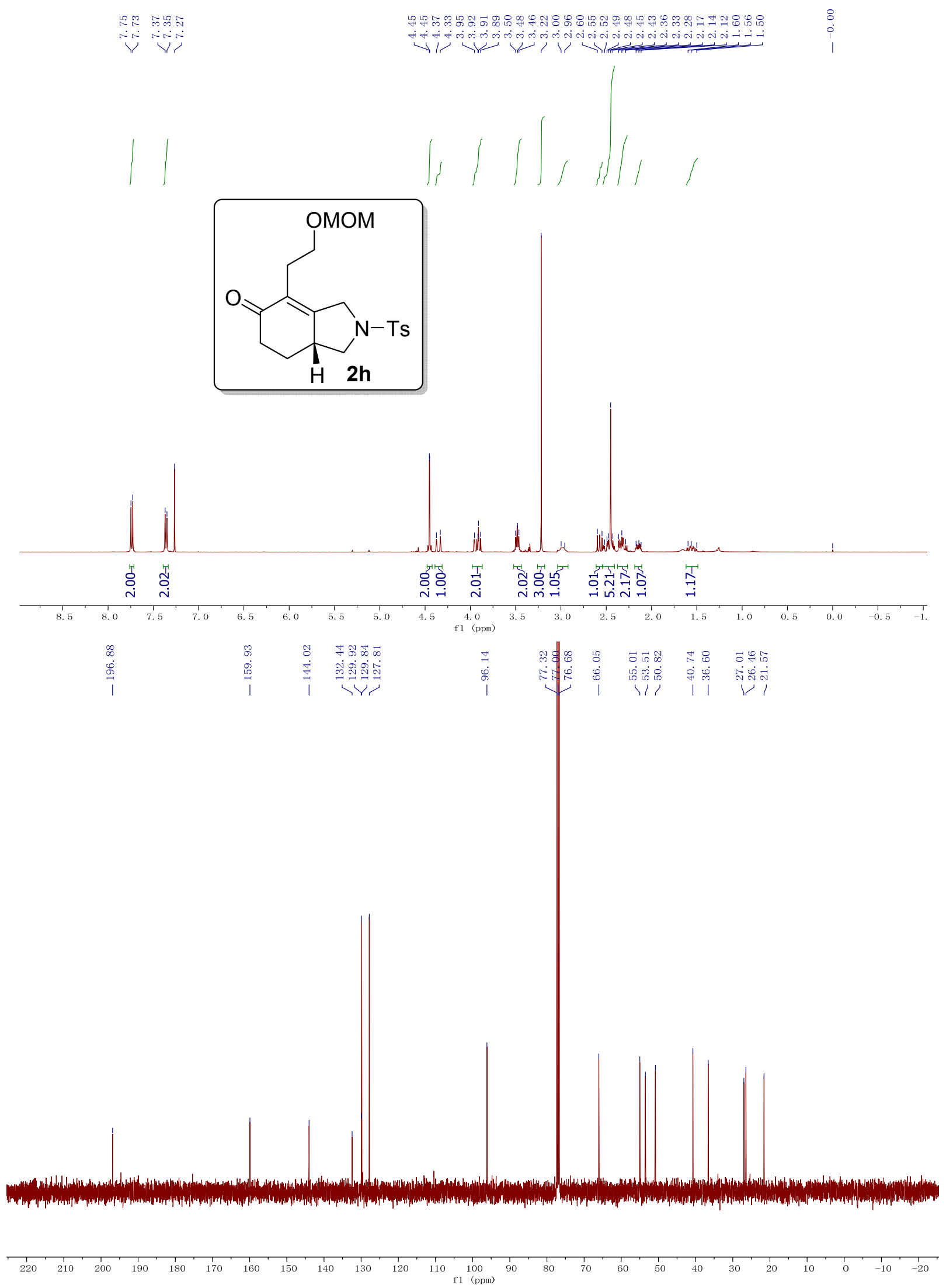

S161 


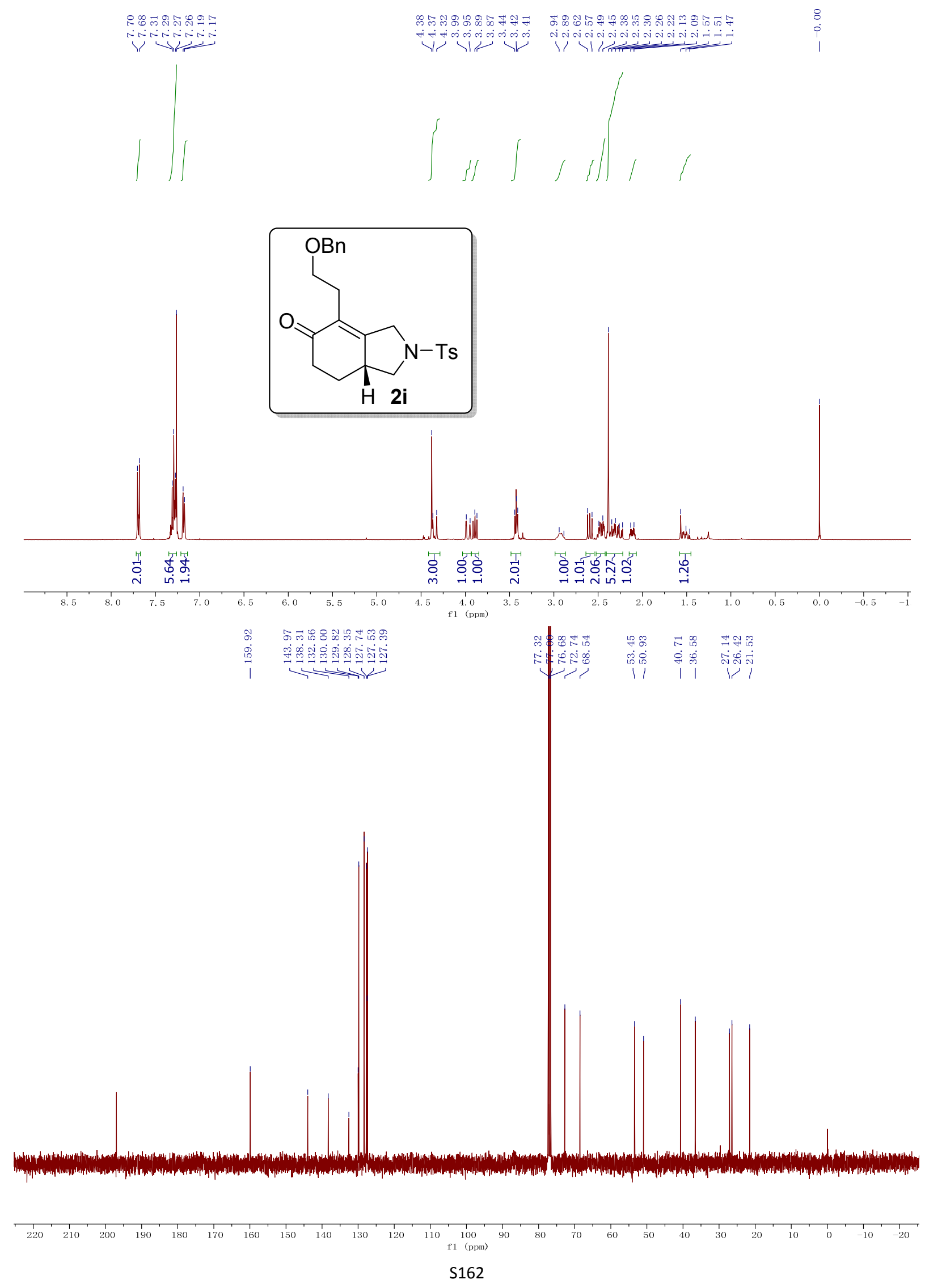




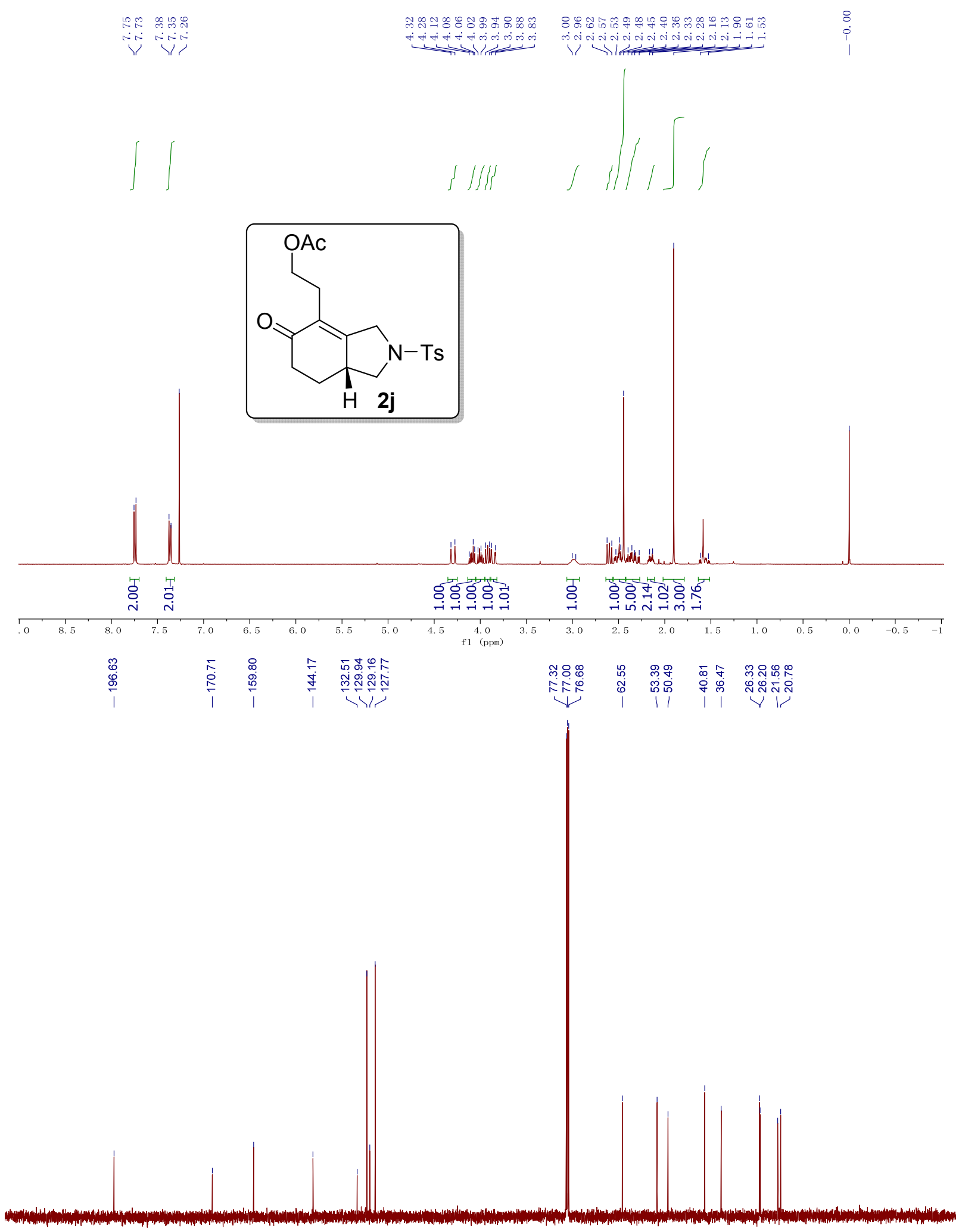

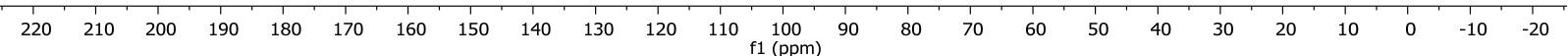

S163 


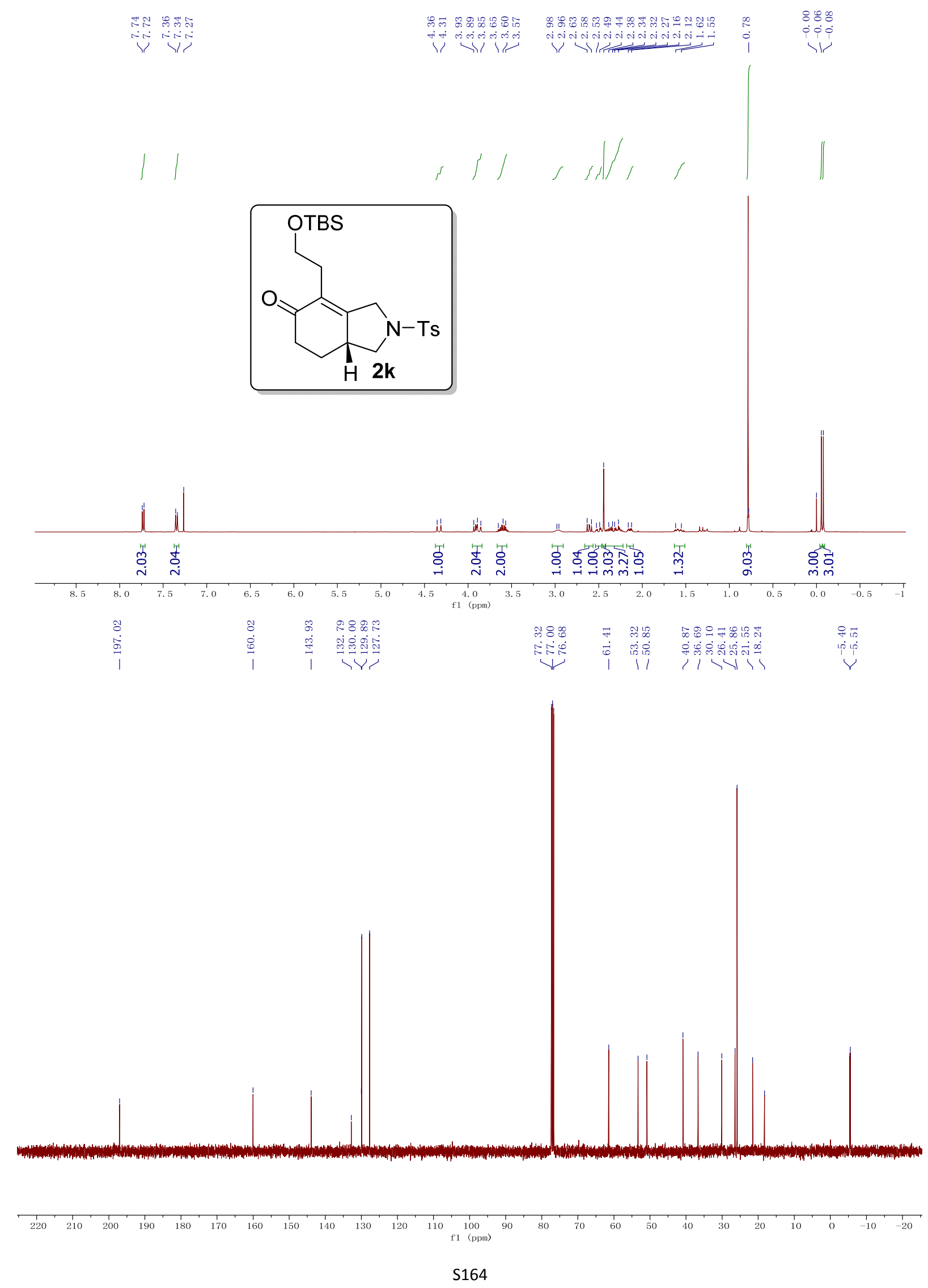



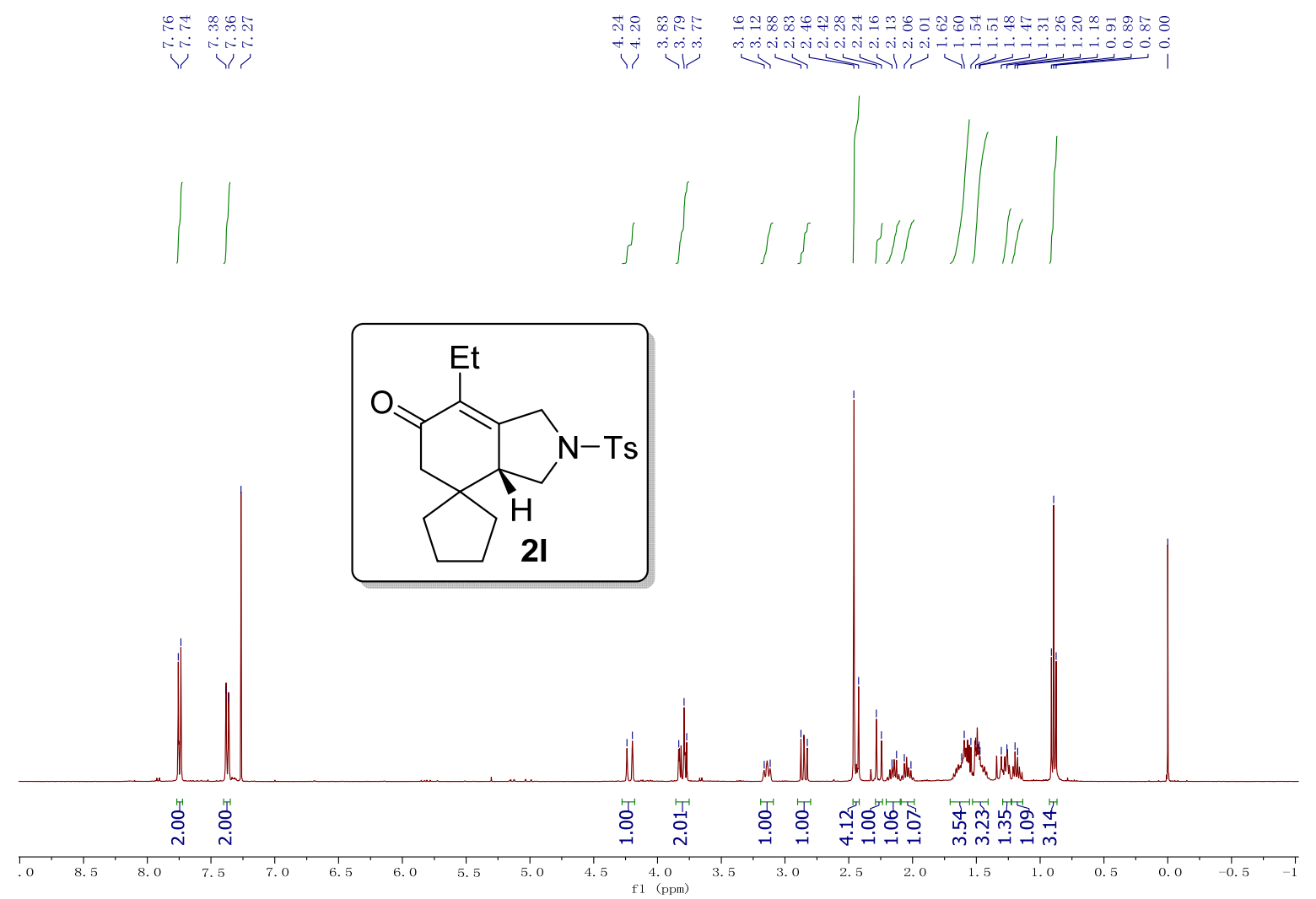

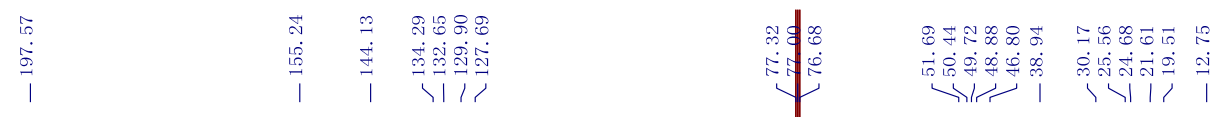
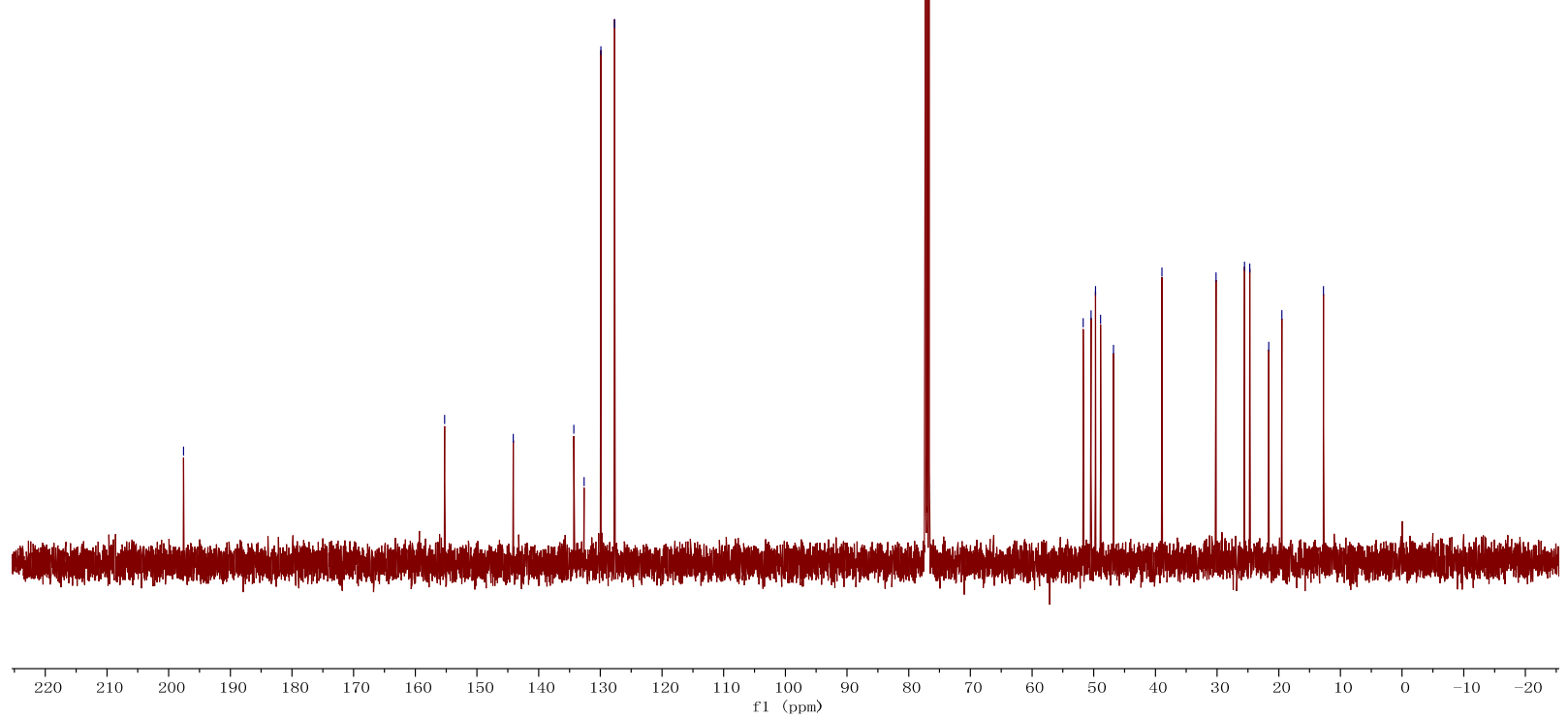

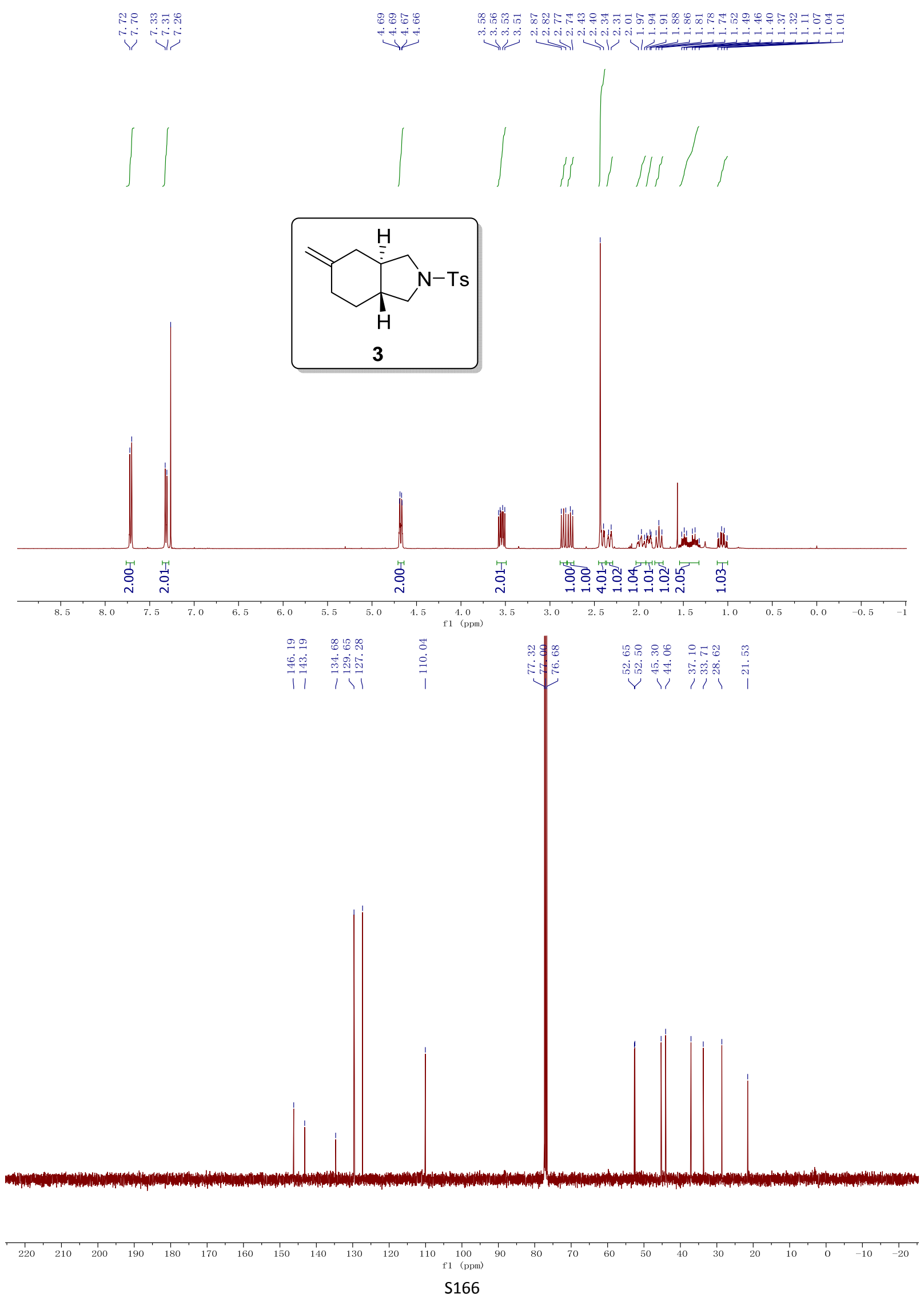

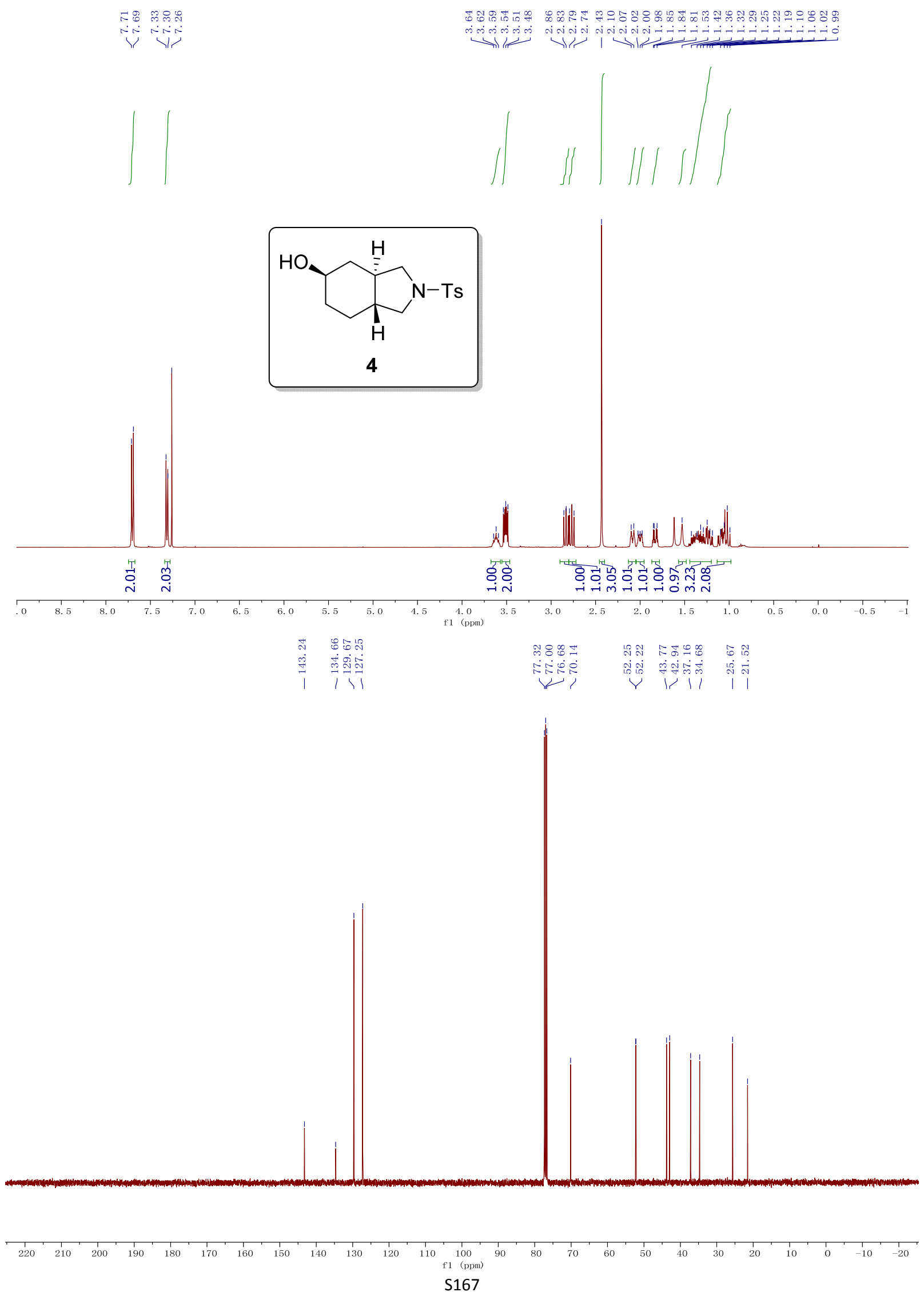


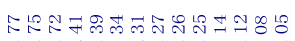

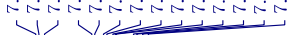

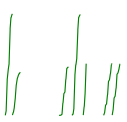

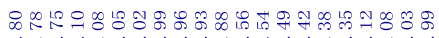

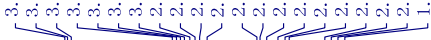

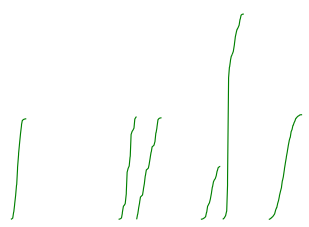

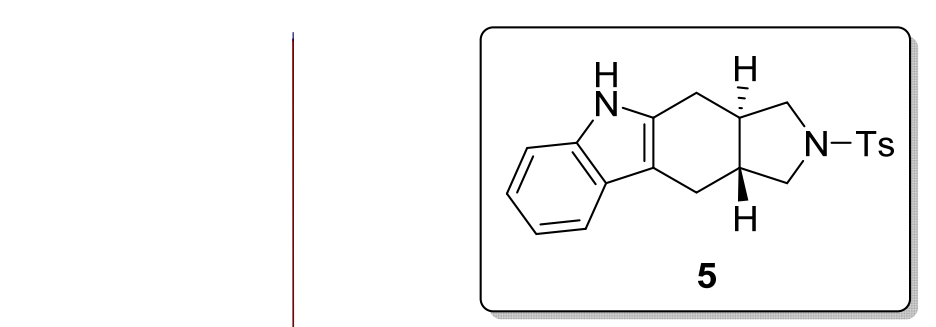

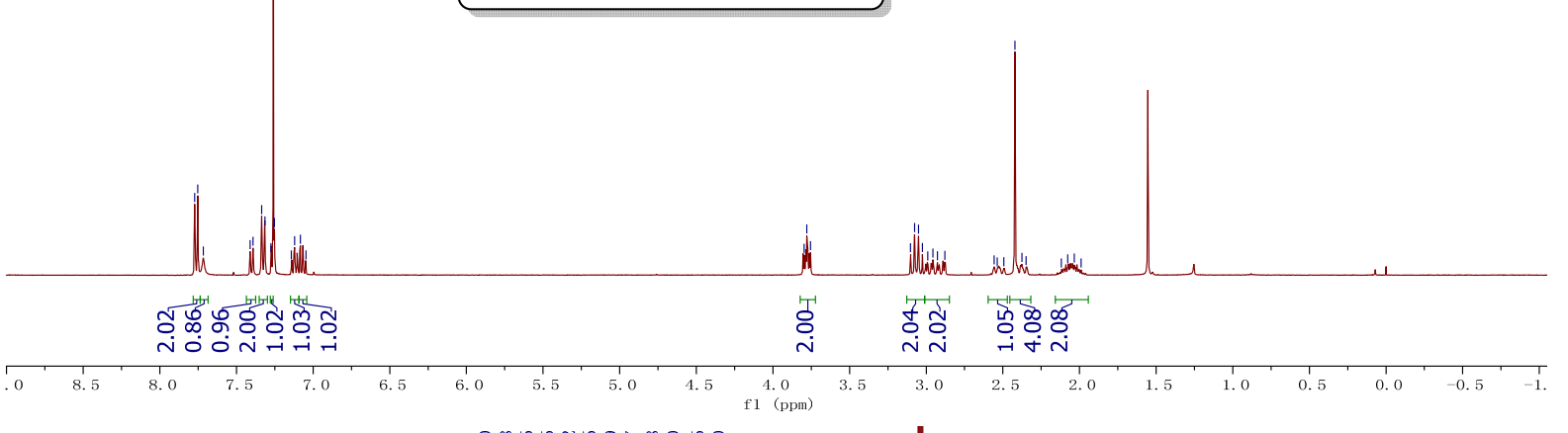

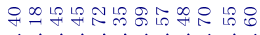

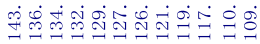

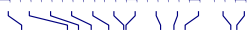

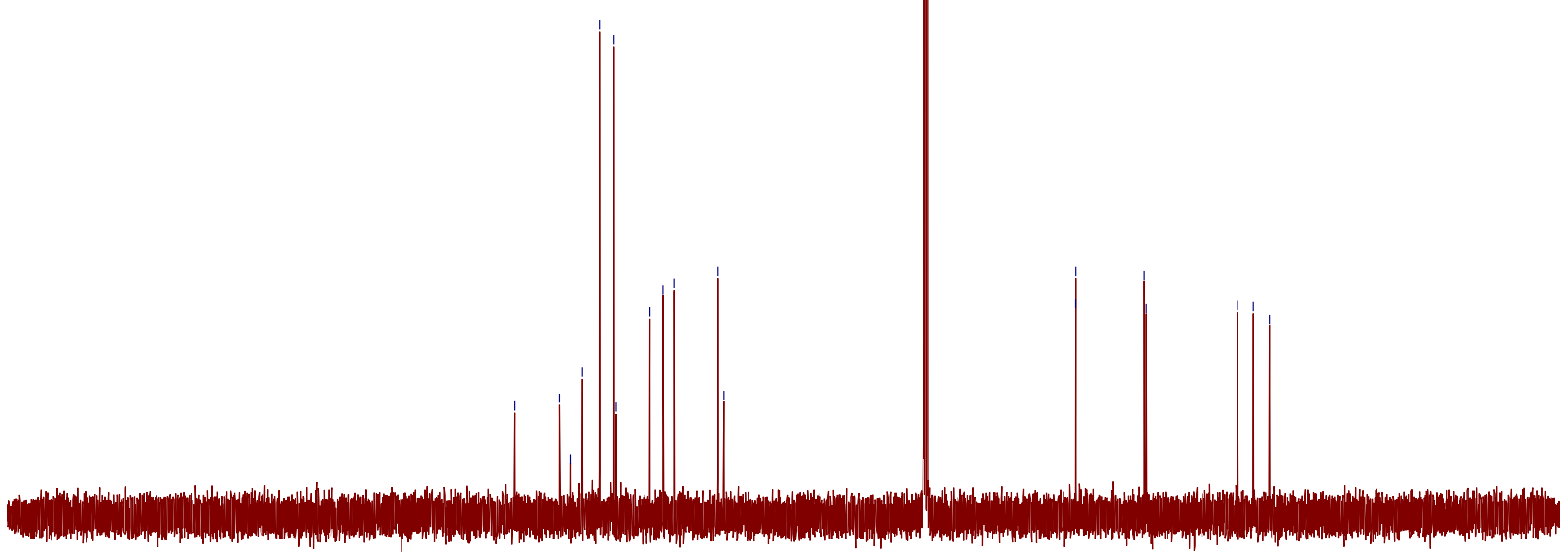

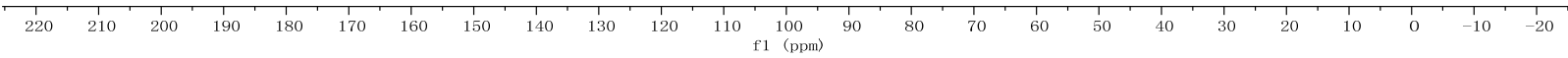
S168 


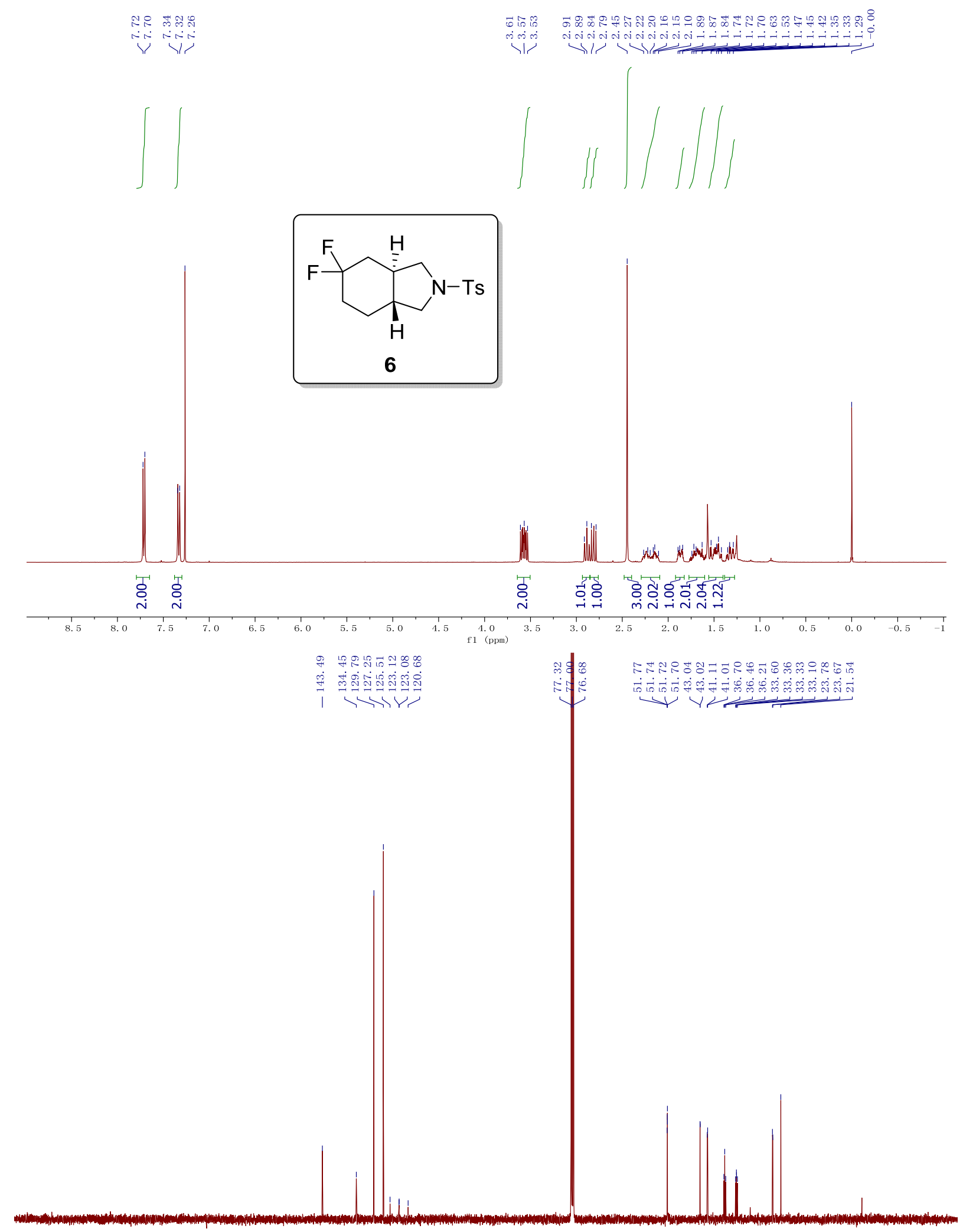

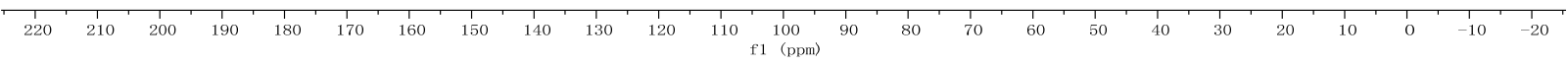
S169 
${ }^{19}$ F NMR spectrum of $\mathbf{6}$
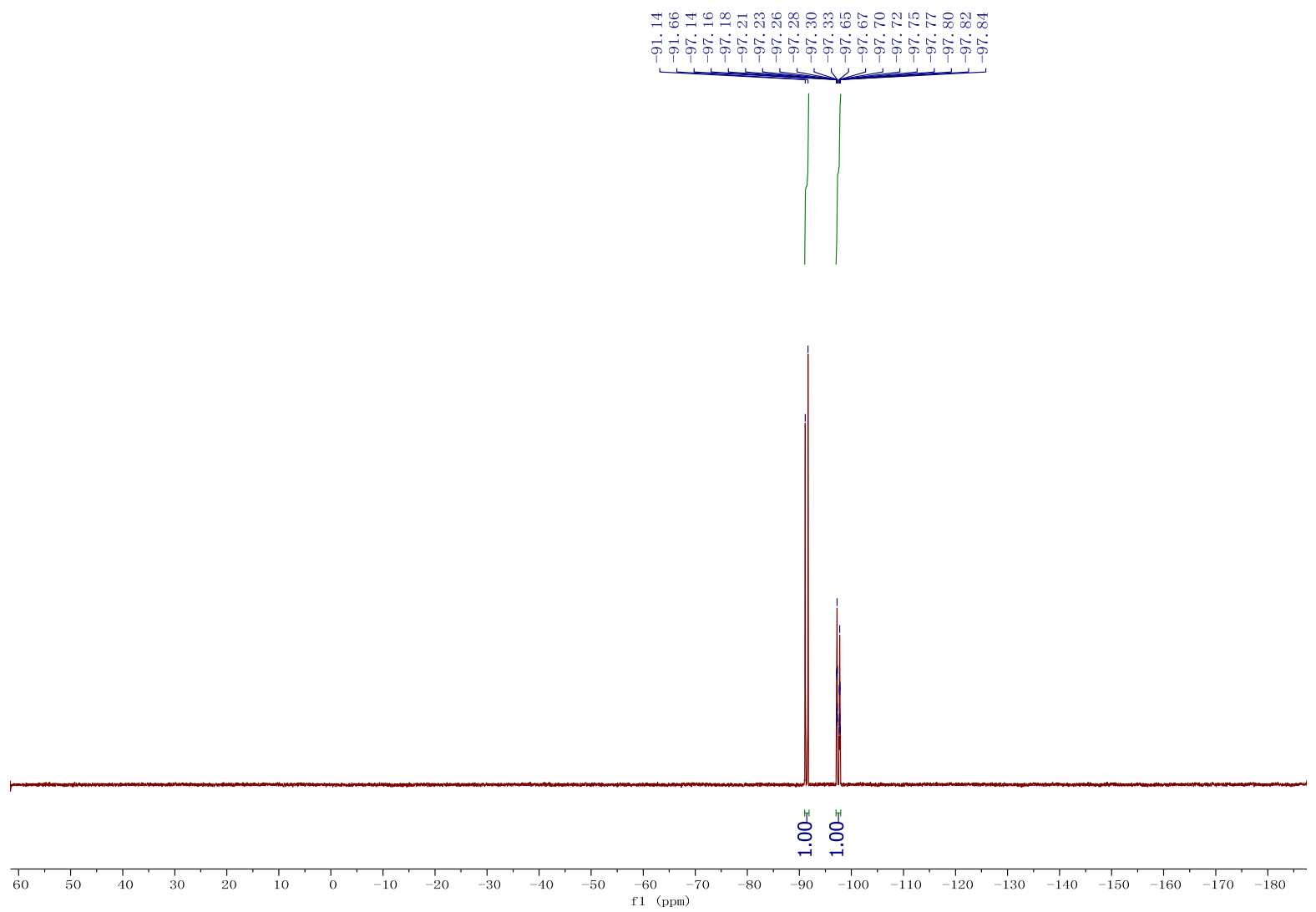

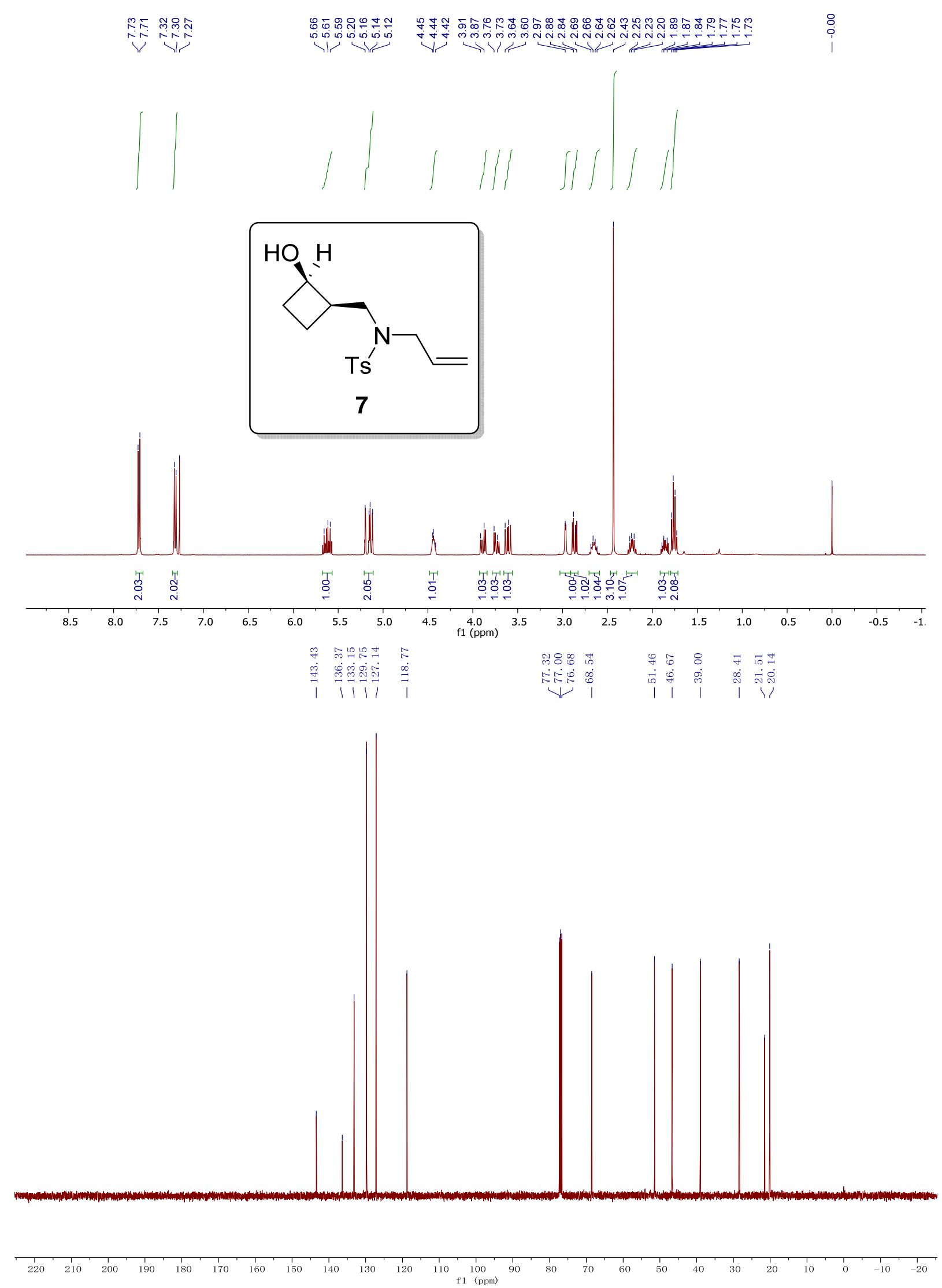
S171 
COSY of 7

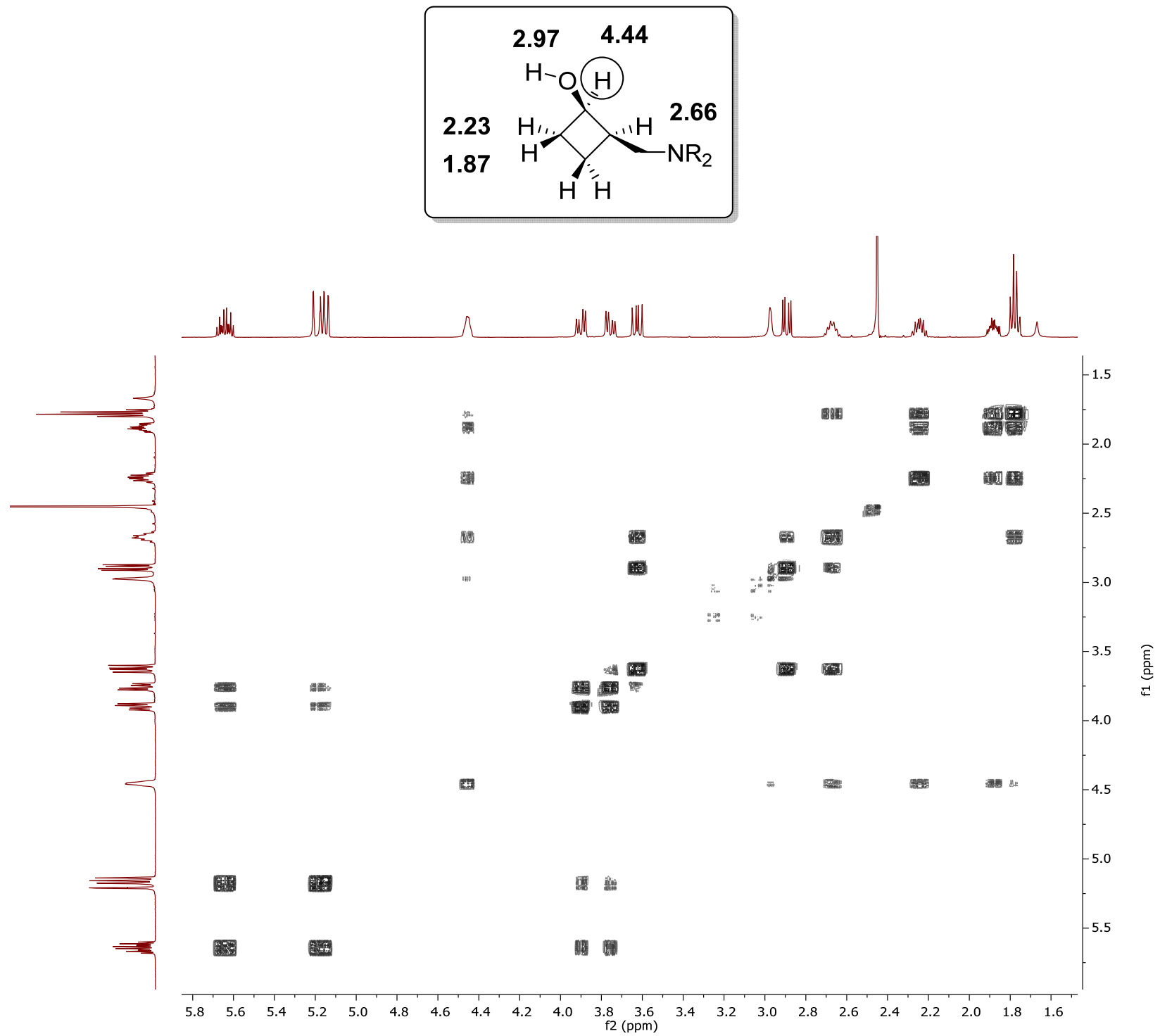


NOE of 7 based on $\delta=4.44$
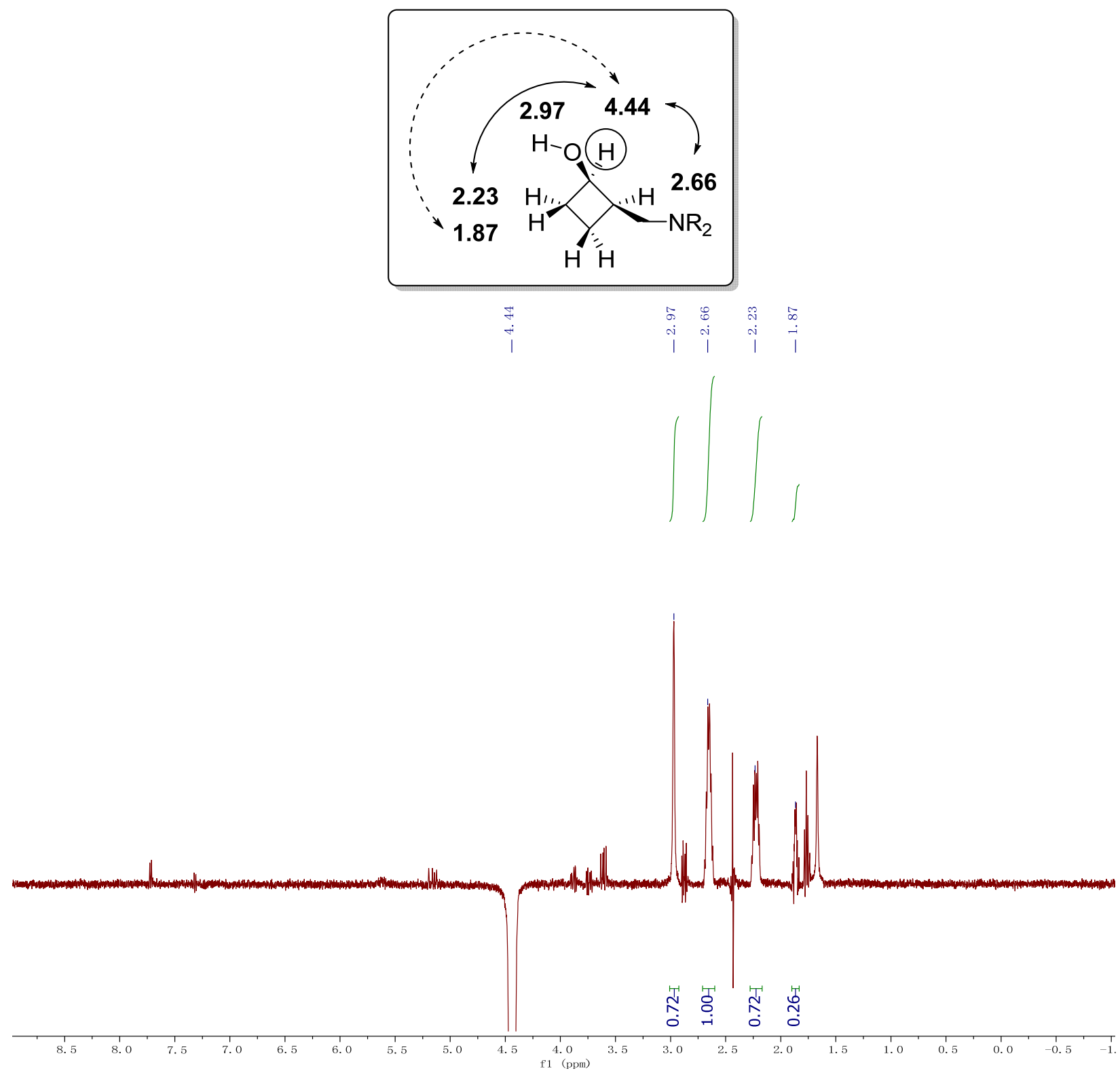

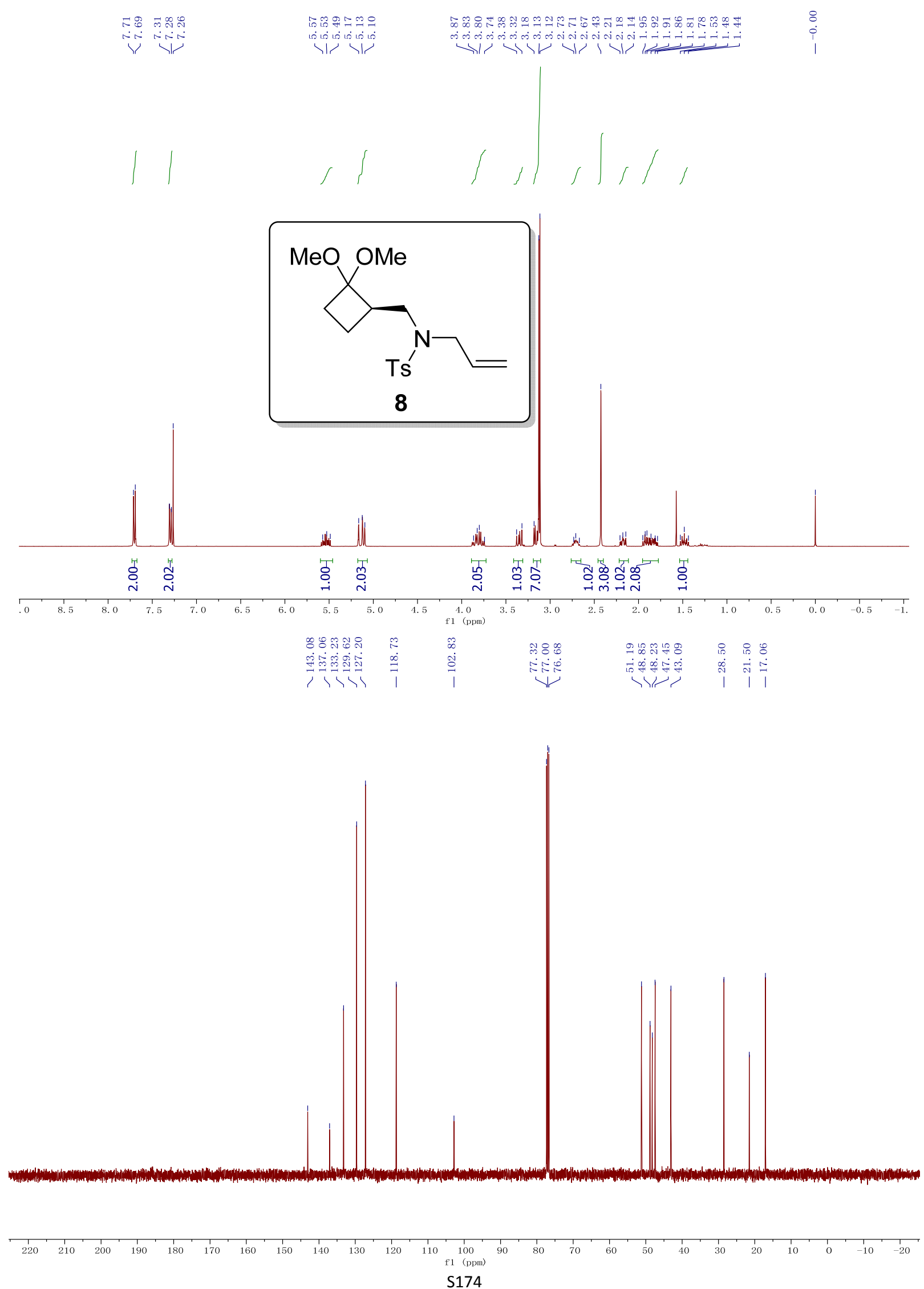


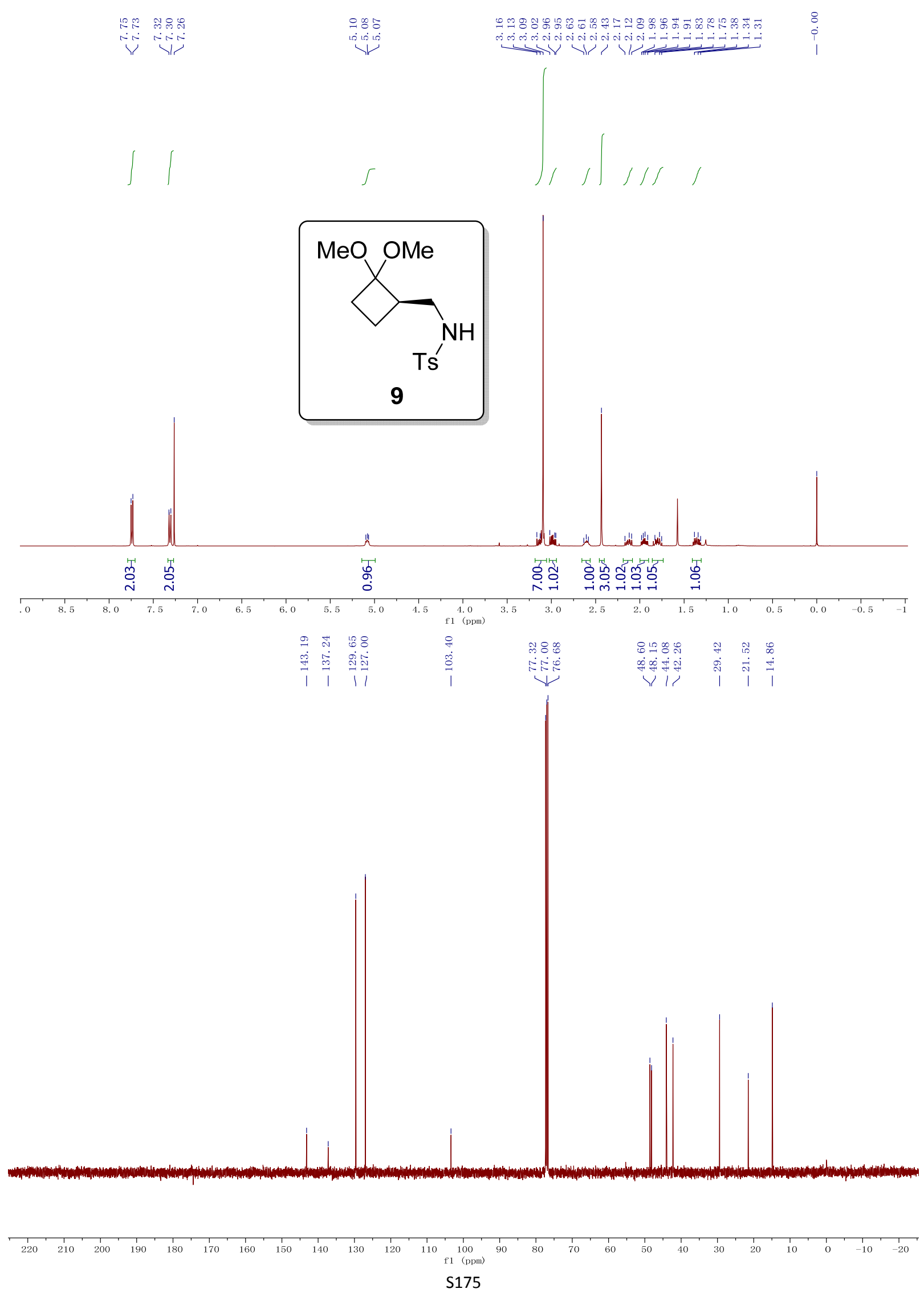

